Prepared for the U.S. Department of Energy

under Contract DE-AC05-76RL01830

\title{
Building Energy Audit Report for Hickam AFB, HI
}

WD Chvála, Jr. DR Dixon

MI De La Rosa

DR Brown

September 2010

Pacific Northwest

NATIONAL LABORATORY

Proudly Operated by Battelle Since 1965 


\title{
DISCLAIMER
}

This report was prepared as an account of work sponsored by an agency of the United States Government. Neither the United States Government nor any agency thereof, nor Battelle Memorial Institute, nor any of their employees, makes any warranty, express or implied, or assumes any legal liability or responsibility for the accuracy, completeness, or usefulness of any information, apparatus, product, or process disclosed, or represents that its use would not infringe privately owned rights. Reference herein to any specific commercial product, process, or service by trade name, trademark, manufacturer, or otherwise does not necessarily constitute or imply its endorsement, recommendation, or favoring by the United States Government or any agency thereof, or Battelle Memorial Institute. The views and opinions of authors expressed herein do not necessarily state or reflect those of the United States Government or any agency thereof.

\author{
PACIFIC NORTHWEST NATIONAL LABORATORY \\ operated by \\ BATTELLE \\ for the \\ UNITED STATES DEPARTMENT OF ENERGY \\ under Contract DE-AC05-76RL01830 \\ Printed in the United States of America \\ Available to DOE and DOE contractors from the \\ Office of Scientific and Technical Information, \\ P.O. Box 62, Oak Ridge, TN 37831-0062; \\ ph: (865) 576-8401 \\ fax: (865) 576-5728 \\ email: reports@adonis.osti.gov \\ Available to the public from the National Technical Information Service, \\ U.S. Department of Commerce, 5285 Port Royal Rd., Springfield, VA 22161 \\ ph: (800) 553-6847 \\ fax: (703) 605-6900 \\ email: orders@ntis.fedworld.gov \\ online ordering: http://www.ntis.gov/ordering.htm
}

This document was printed on recycled paper.

(9/2003) 
PNNL-19633

\section{Building Energy Audit Report for Hickam AFB, HI}

WD Chvála, Jr. $\quad$ DR Dixon

MI De La Rosa

DR Brown

September 2010

Prepared for

U.S. Department of Energy

Federal Energy Management Program

under Contract DE-AC05-76RL01830

Pacific Northwest National Laboratory

Richland, Washington 99352 



\section{Executive Summary}

An assessment of energy efficiency opportunities at Hickam Air Force Base (AFB), HI was performed by a team of engineers from Pacific Northwest National Laboratory (PNNL) under contract to the Department of Energy/Federal Energy Management program (FEMP). The effort used the Facility Energy Decision System (FEDS) model to determine how energy is consumed at Hickam AFB, identify the most cost-effective energy retrofit measures, and calculate the potential energy and cost savings.

A team of engineers from PNNL visited Hickam AFB on 19-29 January 2010 to collect data for the FEDS assessment. During this visit, PNNL engineers collected energy-related information and data from 34 representative buildings, central plants, and other energy systems for input into the FEDS model.

The economic results presented in this report are based on the use of two different sources of capital funds to implement the energy projects; appropriated funds, and alternative financing (e.g., energy savings performance contract [ESPC]). The alternative financing economic input assumptions are for generic ESPC financing to illustrate the differences that the source of capital makes on the technology choices. The FEDS software is capable of performing the comprehensive assessment using other sources of capital (e.g., utility financing) with their distinct economic inputs. Thus, the site is encouraged to re-run the FEDS software using sitespecific alternative financing options and reassess the results. This assessment does not include costs for design; supervision, inspection and overhead (SIOH), or any contingency funds, only the direct capital cost. These additional costs are usually estimated as a \% of direct capital cost. A capital cost multiplier (e.g., typically 1.16 for design and SIOH) can be entered in FEDS and new results produced, or the results can be manually adjusted by increasing capital costs by the appropriate percentage and recalculating net present value (NPV), savings-to-investment ratio (SIR), and payback period.

This report documents the findings of the FEDS assessment and model results for appropriated funds and alternative financing sources of capital for the projects. A complete list of the 135 cost-effective energy- and cost-reducing retrofit measures is included in Appendix C-1 for projects funded using the appropriated funding source of capital. The complete list of 88 costeffective energy and cost-reducing retrofit measures is included in Appendix C-2 for projects funded using the alternative financing source of capital.

Table ES.1 summarizes the results of the energy assessment by retrofit category for appropriated funding sources of capital. Table ES.2 summarizes the results of the energy assessment by retrofit category for alternative financing sources of capital. 
Table ES.1 Summary of Potential Energy and Cost Savings for Hickam AFB Using the Appropriated Funds Source of Capital

\begin{tabular}{|l|r|r|r|r|c|l|}
\hline \multicolumn{1}{|c|}{$\begin{array}{c}\text { Retrofit } \\
\text { Category }\end{array}$} & $\begin{array}{c}\text { Energy } \\
\text { Savings } \\
\text { (MMBtu/yr) }\end{array}$ & $\begin{array}{c}\text { Net Present } \\
\text { Value (\$) }\end{array}$ & $\begin{array}{c}\text { Installed } \\
\text { Cost (\$) }\end{array}$ & $\begin{array}{c}\text { 1st year } \\
\text { savings } \\
(\$)\end{array}$ & $\begin{array}{c}\text { Simple } \\
\text { Payback (yr) }\end{array}$ & SIR \\
\hline Cooling & 14,057 & $2,820,521$ & $5,839,032$ & 829,207 & 7.04 & 2.06 \\
\hline Hot Water & 8,200 & $3,998,220$ & 780,747 & 376,988 & 2.07 & 6.49 \\
\hline Lights & 26,579 & $20,022,961$ & $6,385,181$ & $1,576,090$ & 4.05 & 4.14 \\
\hline Envelope & 5,020 & $3,544,797$ & $1,007,113$ & 273,564 & 3.68 & 4.11 \\
\hline Total & $\mathbf{5 3 , 8 5 6}$ & $\mathbf{3 0 , 3 8 6 , 4 9 9}$ & $\mathbf{1 4 , 0 1 2 , 0 7 3}$ & $\mathbf{3 , 0 5 5 , 8 4 9}$ & $\mathbf{4 . 5 9}$ & $\mathbf{3 . 7 8}$ \\
\hline
\end{tabular}

Table ES.2 Summary of Potential Energy and Cost Savings for Hickam AFB Using the Alternative Financing Source of Capital

\begin{tabular}{|l|r|r|r|r|r|l|}
\hline $\begin{array}{c}\text { Retrofit } \\
\text { Category }\end{array}$ & \multicolumn{1}{c|}{$\begin{array}{c}\text { Energy } \\
\text { Savings } \\
\text { (MMBtu/yr) }\end{array}$} & $\begin{array}{c}\text { Net Present } \\
\text { Value (\$) }\end{array}$ & $\begin{array}{c}\text { Installed } \\
\text { Cost (\$) }\end{array}$ & $\begin{array}{c}\text { 1st year } \\
\text { savings } \\
(\$)\end{array}$ & $\begin{array}{c}\text { Simple } \\
\text { payback } \\
(\mathrm{yr})\end{array}$ & SIR \\
\hline Cooling & 2,195 & 272,865 & 449,371 & 125,096 & 3.59 & 1.60 \\
\hline Hot Water & 7,636 & $1,513,034$ & 580,792 & 352,364 & 1.65 & 3.58 \\
\hline Lights & 21,532 & $4,368,106$ & $3,611,535$ & $1,369,636$ & 2.64 & 2.27 \\
\hline Envelope & 550 & 58,376 & 140,688 & 34,678 & 4.06 & 1.39 \\
\hline Total & $\mathbf{3 1 , 9 1 3}$ & $\mathbf{6 , 2 1 2 , 3 8 1}$ & $\mathbf{4 , 7 8 2 , 3 8 6}$ & $\mathbf{1 , 8 8 1 , 7 7 4}$ & $\mathbf{2 . 5 4}$ & $\mathbf{2 . 3 4}$ \\
\hline
\end{tabular}

For appropriated funds source of capital in Table ES.1, Hickam AFB can save 53,856 MMBtu/year and \$3,055,849/year if all cost-effective retrofits are implemented. The site can reduce its energy consumption by $15.1 \%$ by implementing the 135 cost-effective energy- and cost-reducing projects identified in this report.

For alternative financing source of capital in Table ES.2, Hickam AFB can save 31,913 MMBtu/year and \$1,881,774/year if all cost-effective retrofits are implemented. The site can reduce its energy consumption by $9.1 \%$ by implementing the 88 cost-effective energy- and cost-reducing projects identified in this report.

In addition to this report, the Hickam AFB energy manager will receive a complete record of the FEDS input and output files. The FEDS input files consist of the relevant building and equipment data collected and the assumptions made to perform the complex engineering analysis. The FEDS output files contain considerably more detail in support of future project development. 


\section{Emissions Reduction}

Implementing all the cost-effective building retrofits using appropriated funds will result in a $18 \%$ reduction in greenhouse gas emissions. These reductions are summarized in table ES.3 and included for each building in appendix D.

\section{Table ES.3 Emissions Reduction from Cost-Effective Retrofits}

\begin{tabular}{|l|r|}
\hline \multicolumn{1}{|c|}{ Greenhouse Gas } & \multicolumn{1}{c|}{ Reduction } \\
\hline Sulfur Oxides (lb) & 148,499 \\
\hline Nitrogen Oxides (lb) & 71,453 \\
\hline Carbon Monoxide (lb) & 123,218 \\
\hline Carbon Dioxide (tons) & 15,155 \\
\hline Particulate Matter (lb) & 2,948 \\
\hline Hydrocarbons (lb) & 50,864 \\
\hline
\end{tabular}

\section{Job Creation}

The jobs created from implementation of all the cost-effective retrofits using appropriated funds total 152 job-years. One job-year is equal to $\$ 92,000$ in capital spending for implementation. 


\section{Contents}

Executive Summary ..................................................................................................... iii

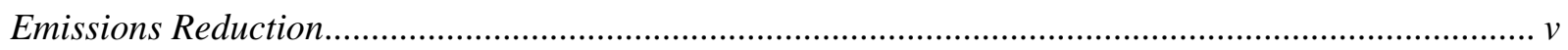

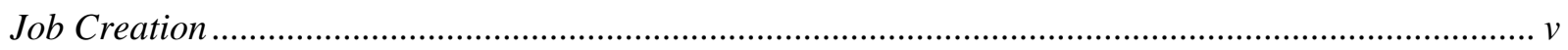

Contents ............................................................................................................................................ vii

Description of ARRA program ........................................................................................ 1

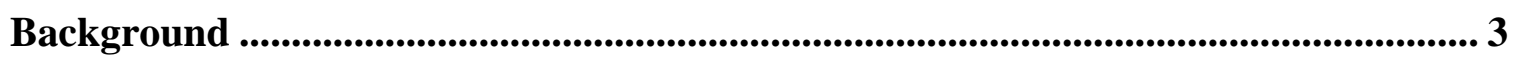

Introduction ....................................................................................................................... 5

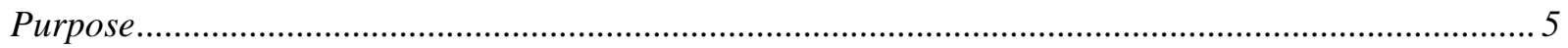

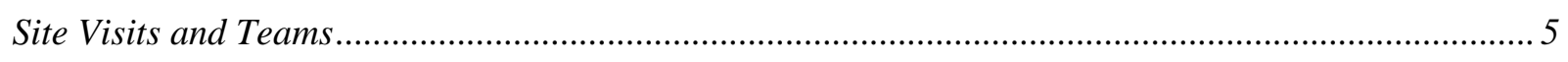

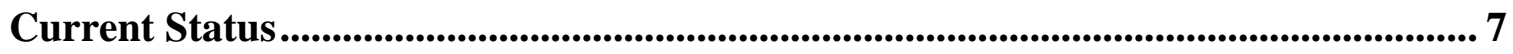

Description of Facilities ................................................................................................................. 9

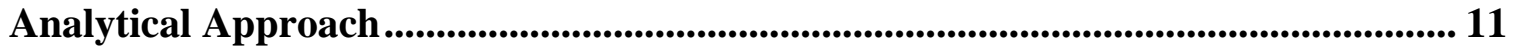

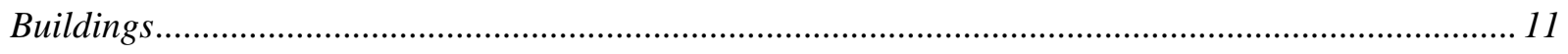

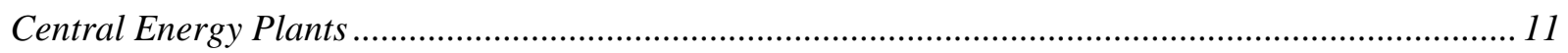

Energy Prices................................................................................................................................... 13

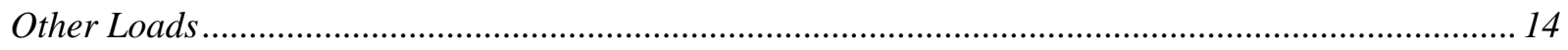

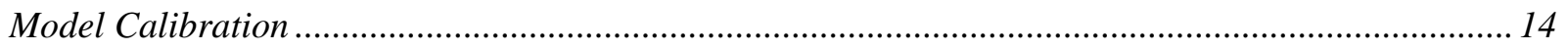

Description of Opportunities Identified .................................................................... 15

Conversion to Water-Cooled Chillers .................................................................. 19

Installation Load Reduction Potential ................................................................................. 21

Recommendations for More In-Depth Assessments ................................................... 23

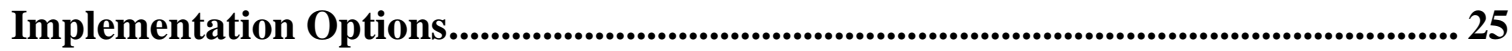

Emissions Reduction ..................................................................................................................... 27

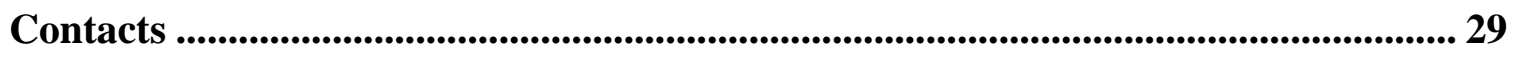

Appendix A FEDS Data Collection Form..................................................................... 33

Appendix B Facility Category Descriptions and Associated Buildings ...................... 37

Appendix C-1 Comprehensive List of Cost-Effective Projects Identified from the FEDS Assessment Using Appropriated Source of Capital...................................................... 43

Appendix C-2 Comprehensive List of Cost-Effective Projects Identified from the FEDS Assessment Using Alternative Financing Sources of Capital ...................................... 55

Appendix D-1 Energy Conservation Measures for Individual Buildings Appropriated

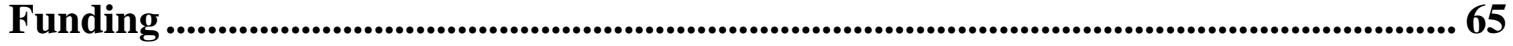

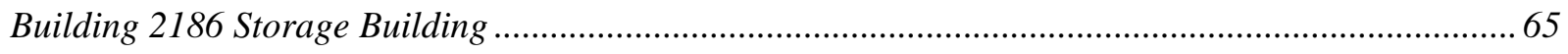




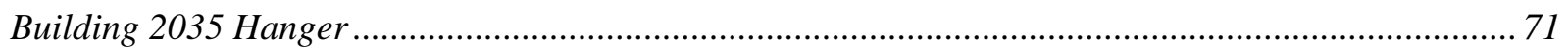

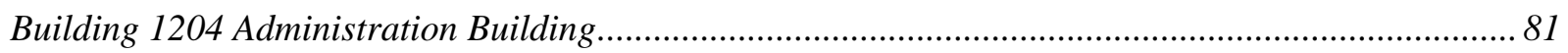

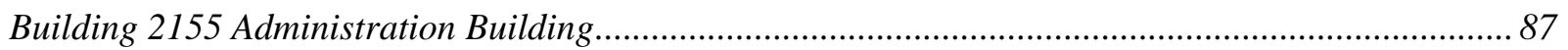

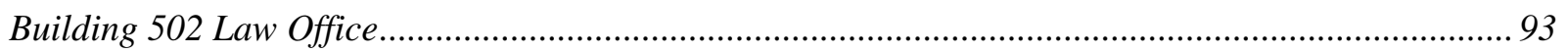

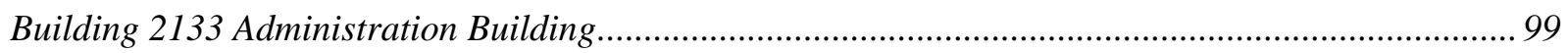

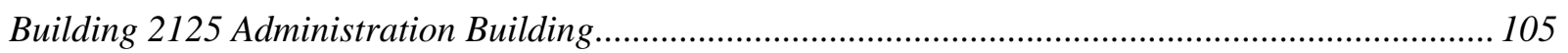

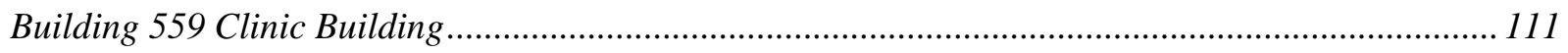

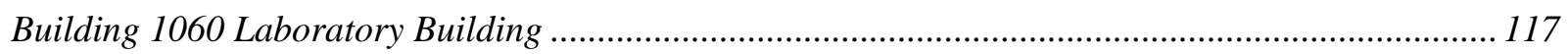

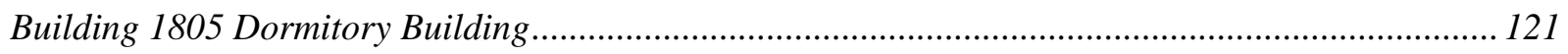

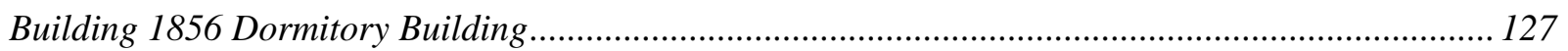

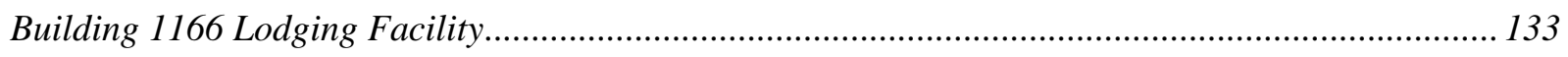

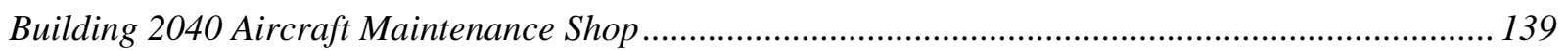

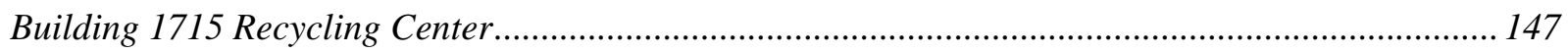

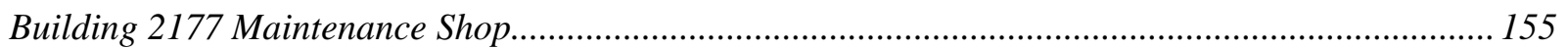

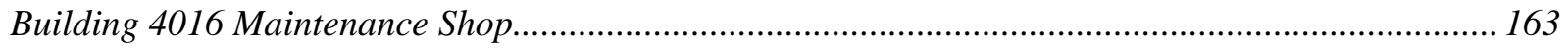

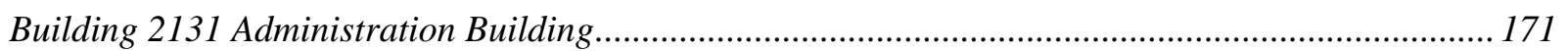

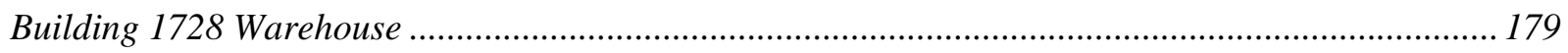

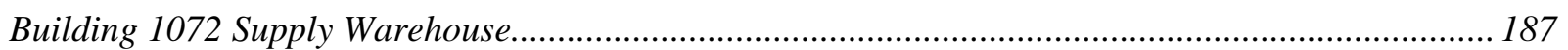

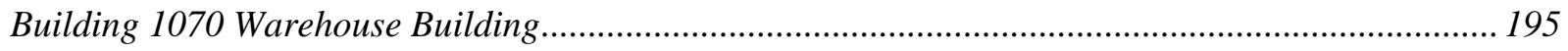

Building 2002 Vehicle Maintenance Building ............................................................................. 203

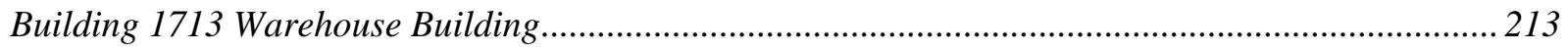

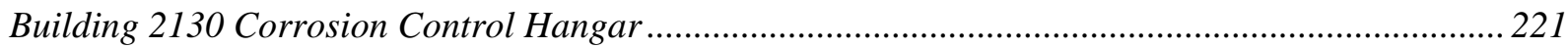

Building 1860 Dining Hall ....................................................................................................... 231

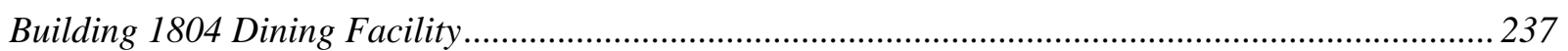

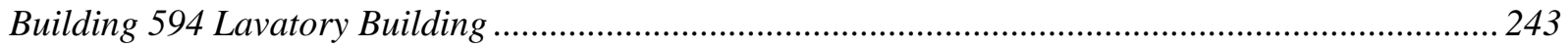

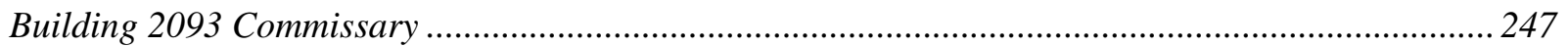

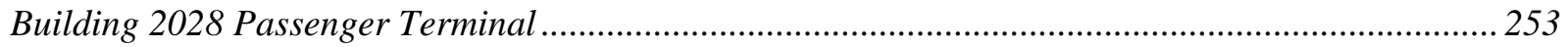

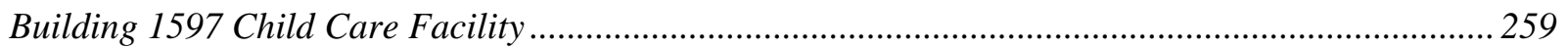

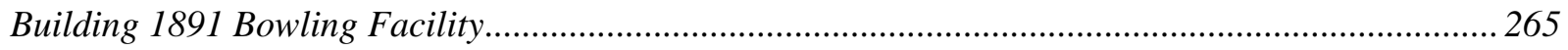

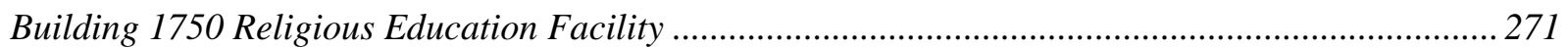

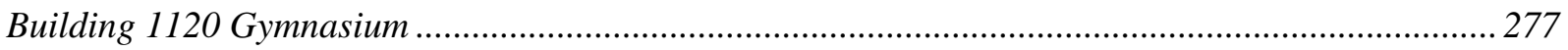

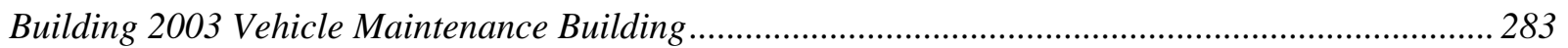

Appendix D-2 Energy Conservation Measures for Individual Buildings Alternative Financing 289

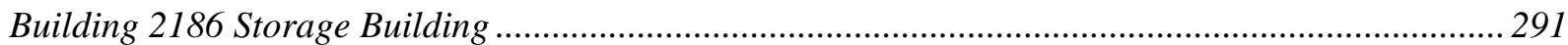

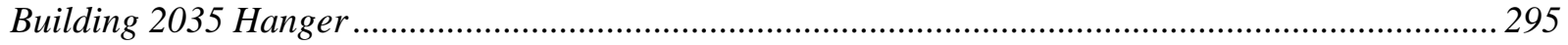

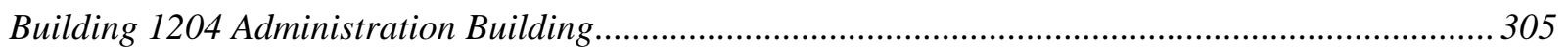

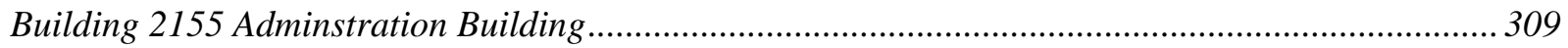

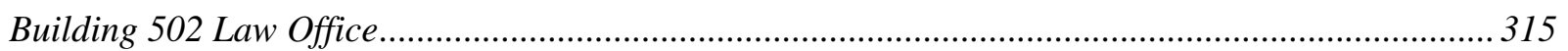

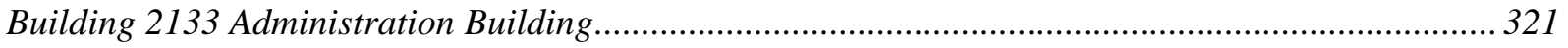

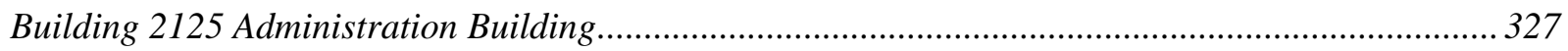




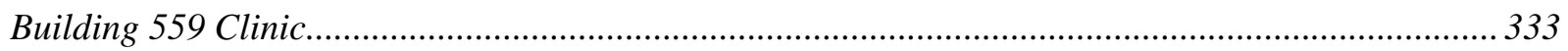

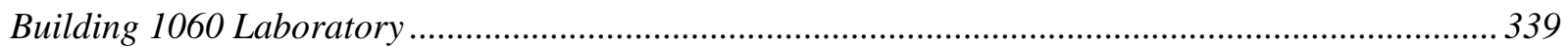

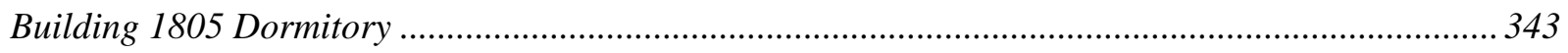

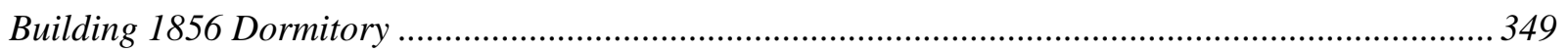

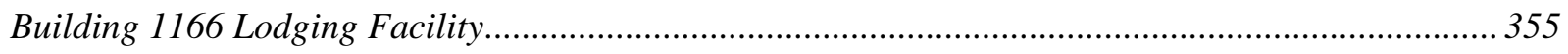

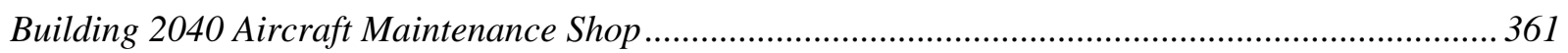

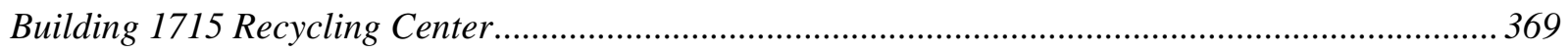

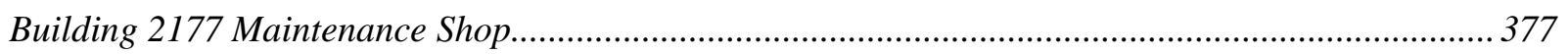

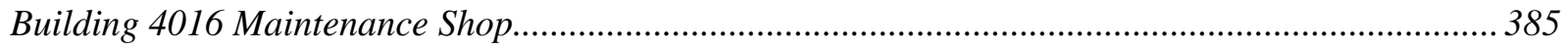

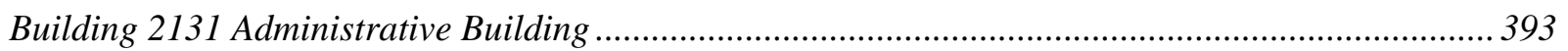

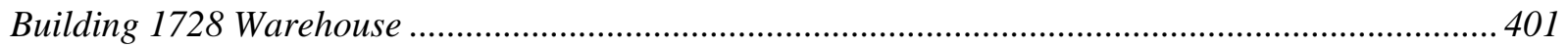

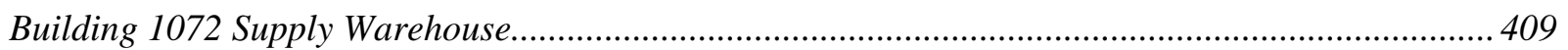

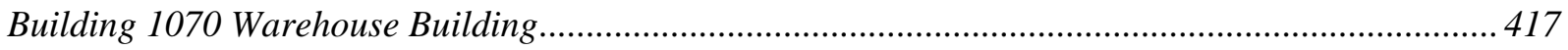

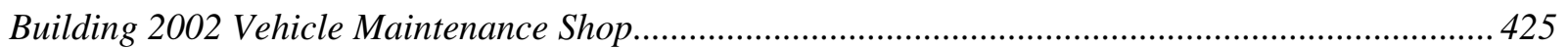

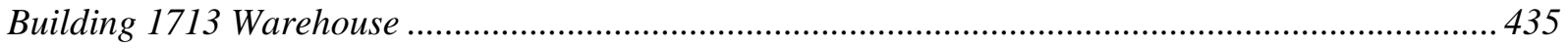

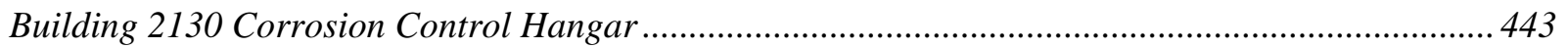

Building 1860 Dining Hall ...................................................................................................... 451

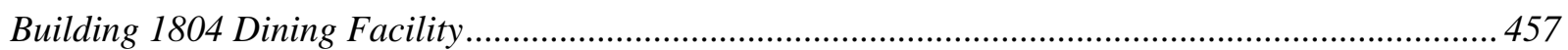

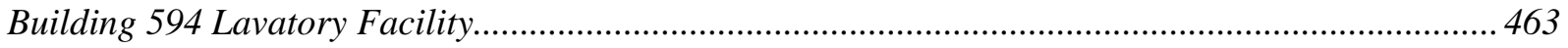

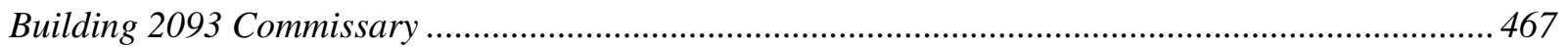

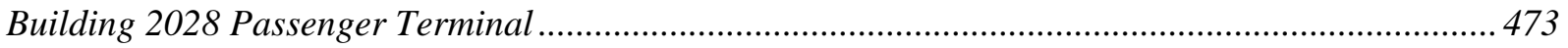

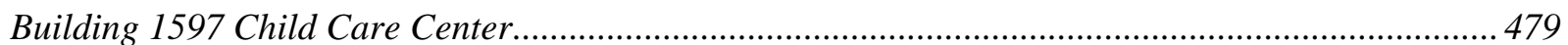

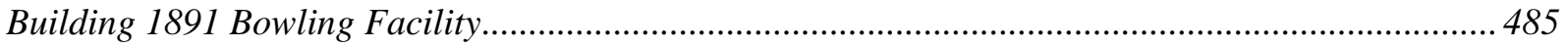

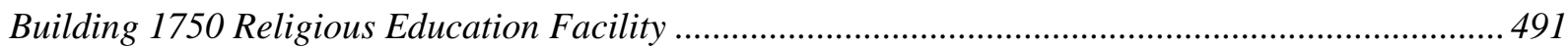

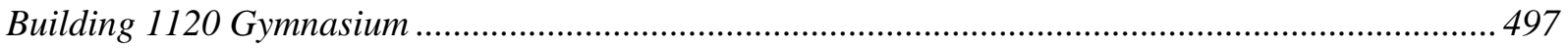

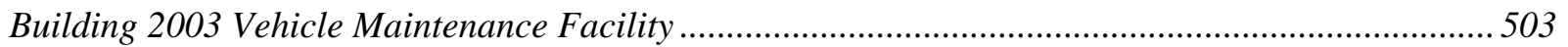

Appendix E Conversion to Water-Cooled Chillers for Building Space Cooling .... 511 


\section{Figures}

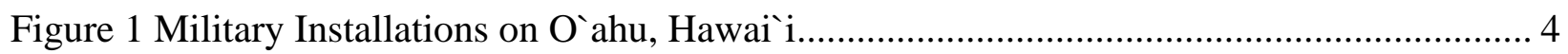

Figure 2 Hickam AFB Energy Reduction Glide Path.................................................................... 7

\section{Tables}

Table ES.1 Summary of Potential Energy and Cost Savings for Hickam AFB Using the Appropriated Funds Source of Capital ................................................................................. iv

Table ES.2 Summary of Potential Energy and Cost Savings for Hickam AFB Using the Alternative Financing Source of Capital................................................................................. iv

Table ES.3 Emissions Reduction from Cost-Effective Retrofits ................................................... v

1 List of Facilities by Facility Category Description ....................................................... 10

2 Central Energy Plants and the Buildings They Serve at Hickam AFB......................... 11

3 Marginal Electricity Rates for Hickam, Pearl, and Smith.............................................. 12

$4 \quad$ FEDS Calibration Results ................................................................................. 12

5a Summary of All Cost-Effective Projects Identified from the FEDS Assessment for Hickam AFB Using Appropriated Source of Capital ..................................................... 16

5b Summary of All Cost-Effective Projects Identified from the FEDS Assessment for Hickam AFB Using Alternative Financing as the Source of Capital ............................ 17

$6 \quad$ Hickam Buildings 2130, 2131, 2133 Existing System Performance and Electricity Cost 20

$7 \quad$ Comparison of Funding Sources ............................................................................. 25

$8 \quad$ Reduction in Greenhouse Gas Emissions ……........................................................... 27

C-1 Comprehensive List of Cost-Effective Projects Using Appropriated Sources of Capital 45

C-2 Comprehensive List of Cost-Effective Projects Using Alternative Financing Sources of

Capital ............................................................................................................................ 57 


\section{Description of ARRA program}

On February 13, 2009, Congress passed the American Recovery and Reinvestment Act (ARRA) of 2009 at the urging of President Obama, who signed it into law 4 days later. A direct response to the economic crisis, the Recovery Act has three immediate goals:

- Create new jobs and save existing ones

- Spur economic activity and invest in long-term growth

- Foster unprecedented levels of accountability and transparency in government spending. ${ }^{1}$

The U.S. Pacific Command (PACOM) is facing significant energy challenges and has identified the need for a comprehensive and integrated approach to addressing these challenges. In a letter dated March 30, 2009, the PACOM Director of Resources and Assessments requested the support of the Department of Energy Federal Energy Management Program (DOE FEMP) in specific assessment, analysis, and training tasks to work toward the accomplishment of PACOM's energy security strategy. An integrated set of ARRA proposals for FEMP assistance requested national laboratory support for the execution of the identified tasks. The resulting 2009-2010 FEMP PACOM scope of work includes renewable energy and efficiency assessments, energy manager training and development, smart grid and islanding feasibility studies, alternative contracting assistance, and technology demonstrations.

In a competitive grant approach across the services and commands, the national laboratories were awarded over \$3,000,000 from DOE FEMP to support PACOM needs. The funds are dedicated to technical assistance projects aimed at bringing the most advanced energy-efficiency, renewable power generation, and microgrid assessments and analyses to Department of Defense (DOD) installations in Hawai i and throughout the Pacific region.

This comprehensive building energy efficiency assessment represents a single task (Task 2.1, FEMP project 237) in the larger PACOM, ARRA-funded energy program.

${ }^{1}$ http://www.recovery.gov/ 


\section{Background}

As the United States' oldest combatant command, PACOM has been a force for peace and a committed partner in the Asia-Pacific region for more than 60 years. With an area of responsibility (AOR) that includes more than 3.4 billion people and encompasses about half the Earth's surface, the Command remains a significant stabilizing influence in the world. PACOM is supported by four component commands: U.S. Pacific Fleet, U.S. Pacific Air Forces, U.S. Army Pacific, and U.S. Marine Corps Forces, Pacific. These commands are headquartered in Hawai'i and have forces stationed and deployed throughout the region.

Home of Headquarters Pacific Air Forces (PACAF) and the 15th Airlift Wing, Hickam Air Force Base is the largest installation in the wing and consists of 2,850 acres of land and facilities valued at more than $\$ 405$ million. Sharing its runways with adjacent Honolulu International Airport (HIA), Hickam and the HIA constitute a single airport complex operated under a jointuse agreement.

The mission of the 15th Airlift Wing is to partner with the Hawaii Air National Guard to provide strategic and tactical airlift capability to PACAF and Air Mobility Command to support local and worldwide missions of combat support and humanitarian or disaster relief. The second mission of the 15th Airlift Wing is to enhance PACAF's power and reach by ensuring world-class en route support, maintaining operational ready forces, and providing superior customer service. The third mission of the wing is to provide airlift support to the commander, Pacific Air Forces and the commander, Pacific Command.

On an average day U.S. military forces in Hawai'i require $3 \mathrm{GW}$ of electricity, representing approximately $10 \%$ of the total electricity needs of the islands. A map of military sites on O'ahu is included in Figure 1. Facilities on other islands include: Pacific Missile Range Facility (PMRF) on Kaua i, Pohakuloa Training Area (PTA) and Kilauea Military Center (KMC) on Hawai i Island, and the Maui High Performance Computing Center (MHPCC) on Maui. In addition to most of these sites, the FEMP PACOM program tasks are performing work in Alaska, Guam, and Japan. 


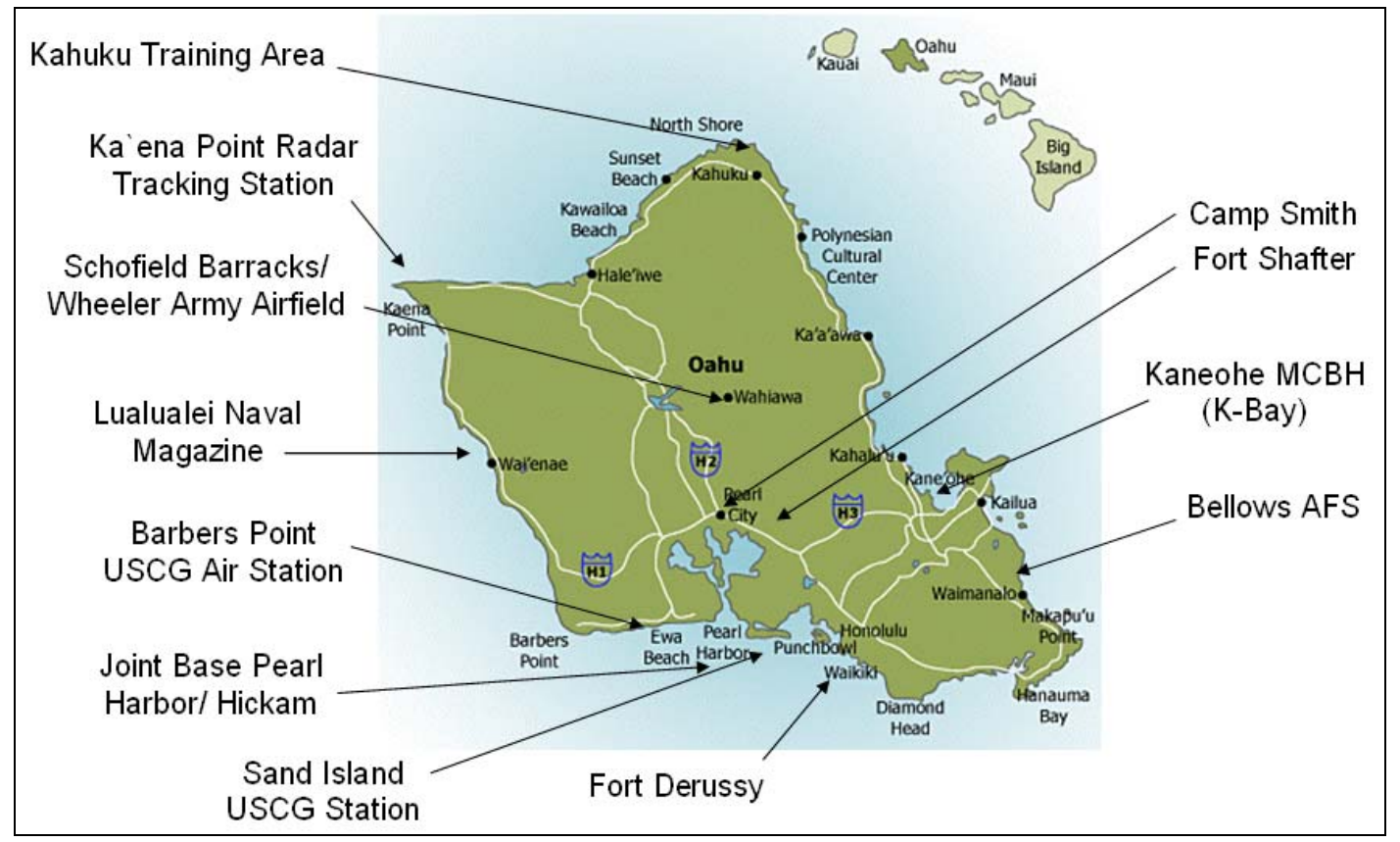

Figure 1 Military Installations on O`ahu, Hawai i 


\section{Introduction}

This report contains the results of a comprehensive building energy efficiency assessment conducted at Hickam AFB, Hawaii, by Pacific Northwest National Laboratory (PNNL). The scope of this activity was based on performing a site-wide energy assessment using the Facility Energy Decision System (FEDS) process to identify cost-effective energy- and cost-reduction projects. In addition, 34 buildings were selected for detailed energy audits of sufficient scope to comply with Energy Independence and Security Act (EISA), section 432 requirements for energy and water evaluations at covered facilities. The results of the FEDS assessment will be used by the installation to develop an implementation plan for the energy conservation measures identified, and outline how Hickam AFB will meet the goals of Executive Order 13423 by FY 2015.

\section{Purpose}

The purpose of this report is to present the findings resulting from the site visit performed January 19-29, 2010, and subsequent modeling and analysis. The objective of the site visit was to collect the necessary data to conduct a detailed site assessment using the FEDS process, which would result in a list of cost-effective, energy- and cost-reduction projects for Hickam AFB.

\section{Site Visits and Teams}

The formal kickoff of the site assessment at Hickam AFB was held on the morning of January 19, 2010. The PNNL team presented an overview of the FEDS assessment process, the data requirements, and schedule for the Hickam AFB work. Participating in this meeting was:

1. Randy Grant - Hickam AFB Energy Manager

2. Jill Sims - Project Manager/Technical lead, SENTECH Hawaii

3. Roger Dunn- Resource Efficiency Manager, Hickam AFB

4. Jared Strebel - Resource Efficiency/Energy Manager, NAVFAC Hawaii

5. Doug Dixon - PNNL

6. Daryl Brown - PNNL

7. Bill Chvála - PNNL

8. Marcus De La Rosa - PNNL 


\section{Current Status}

The Energy Policy Act (EPAct) of 2005 set annual energy reduction goals in British thermal unit (Btu) per gross square foot (sq ft) (Btu/sq ft) of 2\% per year for FY 2006 through FY 2015. The overall goal is 20\% reduction by FY 2015 using FY 2003 as the baseline year. EPAct 2005 goals apply equally to all buildings: standard and industrial. Executive Order (E.O.) 13423 Strengthening Federal Environmental, Energy, and Transportation Management (January 26, 2007), increased the energy reduction goal to 3\% per year or 30\% reduction by FY 2015. In addition, the E.O. established a water reduction goal for federal facilities. Agencies are to reduce water consumption intensity, relative to a FY 2007 baseline, by 2\% annually through FY 2015, or 16\% total by FY 2015.

Hickam AFB is behind the compliance glide path - 6.4\% above the 2003 baseline, compared to the FY 2009 targeted reduction of $9.0 \%$ below the baseline. The historical energy intensity for Hickam AFB Defense Utility Energy Reporting System (DUERS) is shown in Figure 2.

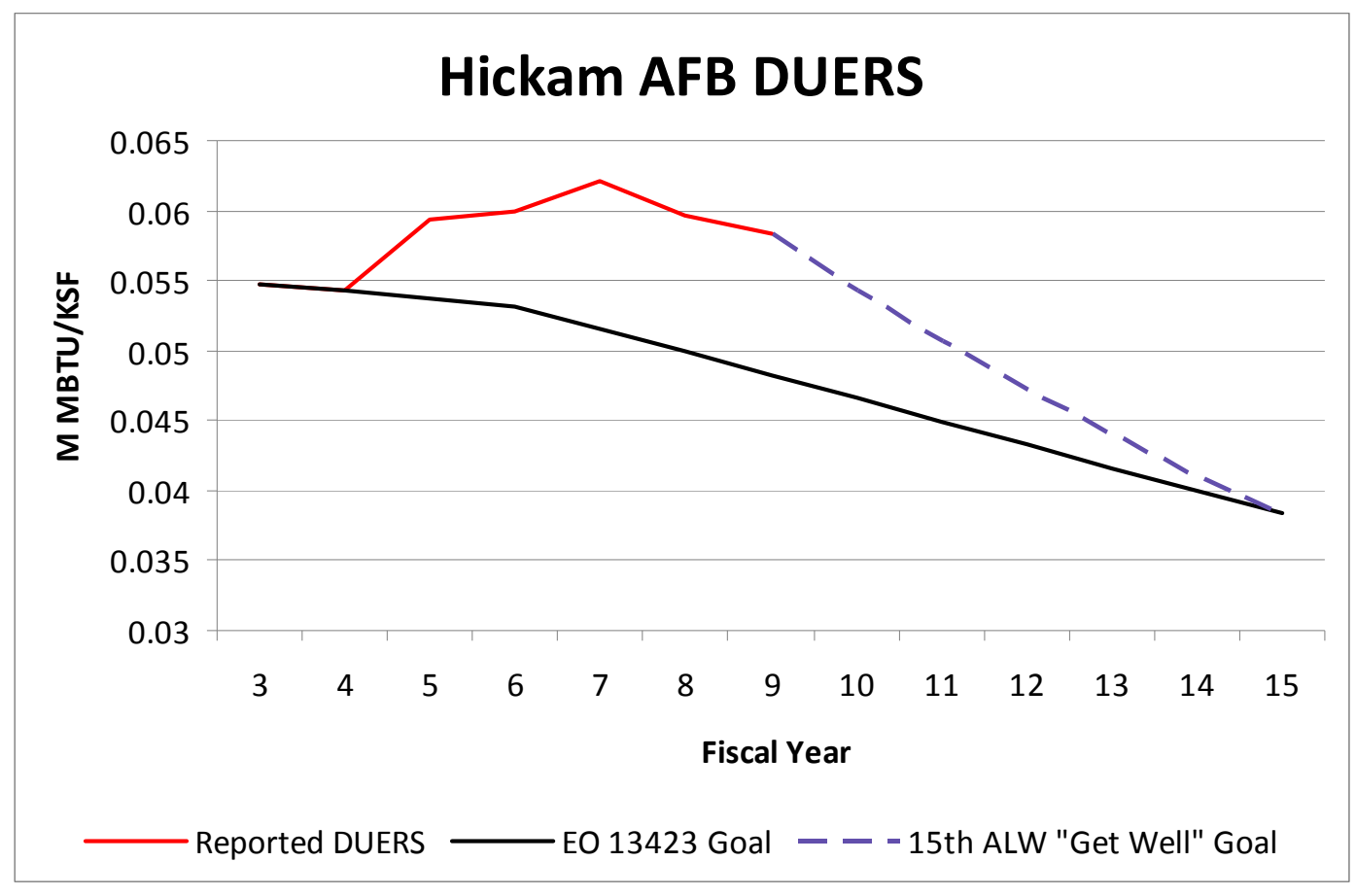

Figure 2 Hickam AFB Energy Reduction Glide Path 


\section{Description of Facilities}

Hickam AFB is a large Air Force installation consisting of 322 buildings totaling approximately 4.87 million square feet, not including the family housing facilities. The scope of the FEDS assessment performed at Hickam AFB included all facilities in the primary cantonment area. Because family housing has been privatized and is metered separately, it was not considered in this analysis.

Table 1 identifies the list of facility categories for the FEDS assessment and the facility proxies for each category. The facilities at Hickam AFB were divided into 30 categories for the purpose of building audits. A complete listing of the facilities (buildings) associated with each FEDS facility category (including subgroups) is provided in Appendix B. 
Table 1. List of Facilities by Facility Category Description

\begin{tabular}{|c|c|c|c|c|}
\hline $\begin{array}{l}\text { FEDS Facility } \\
\text { Category Code }\end{array}$ & Facility Category Description & $\begin{array}{c}\text { Proxy Facility } \\
\text { No. }\end{array}$ & $\begin{array}{l}\text { Facility } \\
\text { Quantity }\end{array}$ & $\begin{array}{c}\text { Category } \\
\text { Area (sq. } \\
\text { ft.) }\end{array}$ \\
\hline 1a & Overhead Protection/Tent Pad & 2186 & 15 & 38,382 \\
\hline $10 \mathrm{a}$ & Large Older Admin/School/HQ & 1102 & 1 & 519,549 \\
\hline $10 \mathrm{~b}$ & Mid-sized Older Admin/School/HQ & 2035 & 6 & 469,042 \\
\hline 10c & Smaller Older Admin/School/HQ & 1204 & 9 & 150,726 \\
\hline $10 \mathrm{~d}$ & Mid-size Newer Admin/School/HQ & 2155 & 3 & 71,322 \\
\hline $10 \mathrm{e}$ & Smaller Newer Admin/School/HQ & 502 & 13 & 60,670 \\
\hline $10 \mathrm{f}$ & Larger Newer Admin/School/HQ & 2133 & 4 & 115,164 \\
\hline $10 \mathrm{~g}$ & Smaller New Admin/School/HQ & 2003,2125 & 21 & 74,987 \\
\hline $21 \mathrm{a}$ & Health Clinic/Vet & 559 & 4 & 93,381 \\
\hline $23 a$ & Hospital/Medical Center & 1060 & 8 & 54,337 \\
\hline $30 \mathrm{a}$ & Dormitory Airman Permanent Party & 1805 & 2 & 121,649 \\
\hline $30 \mathrm{~b}$ & Dormitory Airman Permanent Party & 1856 & 3 & 121,924 \\
\hline $30 \mathrm{c}$ & Dormitory Airman Visiting Quarters & 1166 & 18 & 256,336 \\
\hline $40 \mathrm{a}$ & $\begin{array}{l}\text { Larger Base Engineer Maintenance } \\
\text { Shop }\end{array}$ & $\begin{array}{l}2040,1715 \\
2177\end{array}$ & 8 & 242,165 \\
\hline $40 \mathrm{~b}$ & $\begin{array}{l}\text { Smaller Base Engineer Maintenance } \\
\text { Shop }\end{array}$ & 4016 & 7 & 50,939 \\
\hline $40 \mathrm{c}$ & Shop Aircraft Maintenance & 2131 & 15 & 126,642 \\
\hline $50 a$ & $\begin{array}{l}\text { Warehouse Supply and Equipment } \\
\text { Base }\end{array}$ & 1728,1072 & 10 & $1,012,107$ \\
\hline $50 \mathrm{v}$ & Vehicle Maintenance Shop & 2002 & 11 & 91,158 \\
\hline $50 \mathrm{~b}$ & Exchange Store/Security/MWR & 1713 & 20 & 229,766 \\
\hline 50c & Hanger Aircraft Maintenance & 2130 & 1 & 56,734 \\
\hline $50 \mathrm{~d}$ & $\begin{array}{l}\text { Warehouse Supply and Equipment } \\
\text { Base }\end{array}$ & 1070 & 51 & 162,973 \\
\hline $60 \mathrm{a}$ & Airman Dining Hall & 1860 & 2 & 50,108 \\
\hline $60 \mathrm{~b}$ & Enlisted Open Mess & 1804 & 10 & 100,424 \\
\hline $60 \mathrm{~m}$ & Multipurpose Recreation Building & 594 & 41 & 42,761 \\
\hline 60c & Exchange Sales Store & 2093 & 3 & 305,569 \\
\hline $60 \mathrm{t}$ & Air Passenger Terminal & 2028 & 1 & 46,128 \\
\hline $80 \mathrm{a}$ & Child Care Center & 1597 & 15 & 51,664 \\
\hline $80 \mathrm{~b}$ & Recreation Center & 1891 & 16 & 100,297 \\
\hline $80 \mathrm{c}$ & Religious Education Facility & 1750 & 3 & 11,839 \\
\hline $80 \mathrm{~d}$ & Gymnasium & 1120 & 1 & 46,719 \\
\hline- & Total & 34 bldgs & 322 & $4,875,462$ \\
\hline
\end{tabular}




\section{Analytical Approach}

The general approach was to develop a model of the buildings and other energy-related infrastructure at Hickam AFB, calibrate that model to actual FY 2009 energy use, and then utilize the model to predict energy consumption and identify cost-effective retrofits under typical meterological year (TMY) weather conditions.

\section{Buildings}

Building inventory data for Hickam AFB were obtained from the Air Force Automated Civil Engineering System. A total of 30 building groups were developed to represent the Base and each of the buildings at Hickam AFB was assigned to one of the groups. The mean building size (square footage) and vintage (age) were then calculated for each group based on the building inventory specific to Hickam AFB. Building characteristics were developed from a combination of inferencing relationships within the FEDS model (driven by building type, size, climate, and vintage), walk-through audits of selected buildings at Hickam AFB, and additional building data collected while visiting the Base.

\section{Central Energy Plants}

Any building that provides heating or cooling to more than one building is considered a Central Energy Plant (CEP) in the FEDS analysis. Hickam AFB has has no large central hot water or steam plants.

Hickam AFB has one central air-cooled chiller plant that we could identify (see Table 2). The plant has a combined capacity of 80 tons of cooling, providing cooling to 108,794 square feet of building space. The CEP serves buildings 2130, 2131, and 2133.

Table 2. Central Energy Plants and the Buildings They Serve at Hickam AFB

\begin{tabular}{|c|c|c|c|c|}
\hline $\begin{array}{c}\text { Chilled } \\
\text { Water } \\
\text { Plant }\end{array}$ & $\begin{array}{c}\text { Number of } \\
\text { Chillers }\end{array}$ & $\begin{array}{c}\text { Total } \\
\text { Capacity } \\
\text { (Tons) }\end{array}$ & $\begin{array}{c}\text { Total Floor } \\
\text { Area } \\
\text { (ft }^{2} \text { ) }\end{array}$ & Buildings Served \\
\hline 2134 & 2 & 80 & $108,794 \mathrm{ft}^{2}$ & $2130,2131,2133$ \\
\hline
\end{tabular}




\section{Energy Prices}

Hickam Air Force Base, Pearl Harbor, and Camp Smith (hereinafter, Hickam, Pearl, and Smith) are all served by Hawaiian Electric Company (HECO) under Schedule PP, Large Power Primary Voltage Service. Minor differences in the marginal electricity costs for the three organizations stem from differences in their power factors and the use of Rider M, Off-Peak and Curtailable Services, by Pearl.

The root marginal demand charge for Schedule PP is $\$ 11.85 / \mathrm{kW}$. Energy charges are billed per a declining block structure that is a function of the peak demand. This effectively results in an additional $\$ 2.78 / \mathrm{kW}$ demand charge because an increase in demand shifts more energy into higher-priced blocks. The first $200 \mathrm{kWh} / \mathrm{kW}$ are billed at $\$ 0.121534 / \mathrm{kWh}$, and the second 200 $\mathrm{kWh} / \mathrm{kW}$ are billed at $\$ 0.113702 / \mathrm{kWh}$. All $\mathrm{kWh}$ in excess of $400 \mathrm{kWh} / \mathrm{kW}$ are billed at $\$ 0.110668 / \mathrm{kWh}$. The demand profiles at Hickam, Pearl, and Smith all result in the marginal $\mathrm{kWh}$ being billed at the rate for the third block.

Several adjustments are applied that affect the marginal electricity cost. The total bill is decreased by $0.1 \%$ for each $1 \%$ that power factors are above $85 \%$ (and vice-versa if the power factor is below 85\%). "Interim" increases in the rates established in 2007 and 2009 add 2.82\% to the total bill. Finally, the combination of Public Benefit Funds, Energy Cost, and Integrated Resource Planning surcharges add a little more than $\$ 0.03$ to the cost of each $\mathrm{kWh}$.

The billing demand for each month is the higher of the actual peak demand for that month or the average of peak demand for that month and the peak demand for the previous 11 months. This structure cannot be directly modeled in FEDS, but was found to be equivalent to a 92\% annual demand ratchet, which can be modeled in FEDS.

Pearl utilizes Rider $\mathrm{M}$ to reduce its demand charge by agreeing to reduce its load from 5-9 PM, Monday through Friday. This rider reduces its billing demand by $75 \%$ of the difference between its overall peak demand and its peak demand during the 5-9 PM period. For Pearl, the Rider M billing demand averaged $96 \%$ of its actual peak demand during 2009. This is equivalent to using the actual peak demand as the billing demand and reducing the demand charge by $4 \%$, which was the modeling approach used for FEDS.

The resulting marginal electricity costs are summarized in Table 3.

Table 3. Marginal Electricity Rates for Hickam, Pearl, and Smith

\begin{tabular}{|l|c|c|c|}
\hline & Hickam & Pearl & Smith \\
\hline Demand Charge, \$/kW & 14.92 & 14.24 & 14.86 \\
\hline Energy Charge, \$/kWh & 0.1433 & 0.1426 & 0.1431 \\
\hline Demand Ratchet, \% & $92 \%$ & N/A & $92 \%$ \\
\hline
\end{tabular}

Hickam AFB uses a modest amount of propane and fuel oil, which are delivered regularly. Propane cost is \$32.15 / MMBtu and fuel oil is \$5.10 / Gallon. 


\section{Other Loads}

No comprehensive inventory of exterior lighting was documented. A count of street lights was taken from site plans but runway and taxi lights were not accounted for. Previous experience at other military installations was used to estimate load. This estimate is based on total square footage at Hickam AFB multiplied by typical lighting density found at other military sites. The resulting exterior lighting annual electric consumption was estimated at 2.59 million $\mathrm{kWh}$.

The estimated annual electricity consumption for water pumping (potable water and sewage) was nearly 1.0 million $\mathrm{kWh}$. This estimate is based on assumptions developed at other military installations with similar site characteristics, size, and water consumption. Electricity distribution losses were assumed to be $4 \%$ of purchased electricity.

\section{Model Calibration}

Building energy use was simulated with FEDS and combined with the non-building energy infrastructure characterization to predict the total site energy consumption for FY 2009. Uncertain elements of the modeling assumptions were adjusted until the model's energy consumption prediction matched "reasonably well" with actual energy consumption for FY 2009. Specific model calibration results are shown in Table $4 .^{2}$

Table 4. FEDS Calibration Results

\begin{tabular}{|l|l|c|}
\hline Modeled Element & Fuel Type & Error \\
\hline \multirow{3}{*}{ Total by Fuel Type } & Electricity & $-0.61 \%$ \\
\cline { 2 - 3 } & Propane & $1.50 \%$ \\
\cline { 2 - 3 } & Fuel Oil \#2 & $11.09 \%$ \\
\hline Total Energy & All & $-0.61 \%$ \\
\hline
\end{tabular}

\footnotetext{
${ }^{2}$ For example, an error of $+0.5 \%$ means that the model predicts energy consumption $0.5 \%$ higher than reported consumption.
} 


\section{Description of Opportunities Identified}

The number of conceivable energy conservation measures, fuel-switching opportunities, and renewable-energy projects at federal sites is very large. The FEDS model is used to costeffectively identify energy saving opportunities for the site. FEDS is a software tool that provides a comprehensive method to quickly and objectively identify energy improvements that offer maximum life-cycle cost savings. FEDS determines the optimum set of cost-effective retrofits from a current database of hundreds of proven technologies. These include retrofits for heating, cooling, lighting, motors, building envelope, and hot water systems. Interactive effects are also evaluated as part of the optimization process so that energy savings are not double counted or undercounted. The results are based on life-cycle cost economics consistent with 10 CFR 436.

FEDS identifies the package of retrofits that individually and collectively minimize the life-cycle cost of building energy services, resulting in projects where the net present value (NPV) of the investment is greater than or equal to zero and the savings-to-investment ratio (SIR) is greater than or equal to one. Results are developed for government (appropriated) and alternative (e.g., energy savings performance contract [ESPC] and utility energy services contract [UESC]) financing assumptions.

In general, the discount rate is higher and the economic evaluation life is shorter for alternative financing compared to government financing. The economic life for the latter is set at 25 years with the discount rate adjusted each year in response to market conditions. The currently prescribed government discount rate is 3.0\% in real terms, i.e., in excess of general inflation. Alternative financing assumptions are not prescribed, but set by negotiation between the energy services company (ESCO) and the Federal organization. An economic evaluation life of 10 years and a real discount rate of $10 \%$ are used to represent alternative financing conditions in this assessment, based on a collection of prior site experiences in the Army. This assessment does not include costs for design; supervision, inspection and overhead (SIOH), or any contingency funds, only the direct capital cost. These additional costs are usually estimated as a \% of direct capital cost. A capital cost multiplier (e.g., typically 1.16 for design and SIOH) can be entered in FEDS and new results produced, or the results can be manually adjusted by increasing capital costs by the appropriate percentage and recalculating NPV, SIR, and payback period.

Table 5a summarizes the FEDS results by retrofit category (e.g., cooling) and type (e.g., chillers) using appropriated funding as the source of capital for the projects. Table 5b summarizes the FEDS results by retrofit category using alternative financing as the source of capital for the projects. The complete list of cost-effective energy- and cost-reduction projects resulting from the FEDS modeling and analysis are presented Appendices C-1 (appropriated funds) and C-2 (alternative financing). ${ }^{3}$

\footnotetext{
${ }^{3}$ It should be noted that in addition to this report, the Hickam AFB energy manager will also receive a CD-ROM, which includes all the FEDS input data and output project files. The input data files reflect information collected during the site visits and additional assumptions required to perform the FEDS modeling and assessment. The output project files contain significantly more detailed information to support the list of cost-effective energy projects identified in Appendices C-1 and C-2.
} 
Table 5a. Summary of All Cost-Effective Projects Identified from the FEDS Assessment for Hickam AFB Using Appropriated Sources of Capital (by Retrofit Category and Type)

\begin{tabular}{|c|c|c|c|c|c|c|c|}
\hline $\begin{array}{l}\text { Retrofit } \\
\text { Category }\end{array}$ & Retrofit Technology & $\begin{array}{c}\text { Energy } \\
\text { Savings } \\
\text { (MMBtu/yr) }\end{array}$ & $\begin{array}{l}\text { Net Present } \\
\text { Value (\$) }\end{array}$ & $\begin{array}{l}\text { Installed } \\
\text { Cost }(\$)\end{array}$ & $\begin{array}{c}\text { 1st year } \\
\text { savings (\$) }\end{array}$ & $\begin{array}{c}\text { Simple } \\
\text { Payback } \\
\text { (yr) }\end{array}$ & SIR \\
\hline \multirow{4}{*}{ Cooling } & Packaged ACTotal & 895 & 41,361 & 179,467 & 60,318 & 2.98 & 3.80 \\
\hline & $\begin{array}{l}\text { Water-Cooled Chiller } \\
\text { Total }\end{array}$ & 12,989 & $3,073,646$ & $5,478,947$ & 787,151 & 6.96 & 2.02 \\
\hline & Window AC Total & 173 & 16,816 & 196,484 & 19,857 & 9.89 & 1.20 \\
\hline & Subtotal & 14,057 & $2,820,521$ & $5,839,032$ & 867,326 & 7.04 & 206 \\
\hline \multirow{4}{*}{ Hot Water } & $\begin{array}{l}\text { Distillate Oil Boiler } \\
\text { Total }\end{array}$ & 522 & 340,737 & 56,604 & 14,521 & 3.90 & 7.60 \\
\hline & $\begin{array}{l}\text { Heat Pump Water Heater } \\
\text { Total }\end{array}$ & 7,407 & $3,624,423$ & 708,402 & 351,313 & 2.02 & 6.42 \\
\hline & Misc Measures Total & 271 & 33,060 & 15,741 & 11,154 & 1.41 & 4.69 \\
\hline & Subtotal & 8,200 & $3,998,220$ & 780,747 & 376,988 & 2.07 & 6.49 \\
\hline \multirow{7}{*}{ Lights } & CFL Total & 2,421 & $2,502,424$ & 126,319 & 156,479 & 0.81 & 20.55 \\
\hline & EXIT Lighting Total & 2,908 & $3,242,580$ & 209,262 & 204,437 & 1.02 & 16.45 \\
\hline & Super T8 total & 15,641 & $11,463,234$ & $4,323,525$ & 942,054 & 4.59 & 3.66 \\
\hline & T8 Total & 284 & 160,785 & 237,780 & 23,557 & 10.09 & 1.64 \\
\hline & $\begin{array}{l}\text { High Pressure Sodium } \\
\text { Total }\end{array}$ & 5,122 & $2,471,851$ & $1,411,822$ & 234,220 & 6.03 & 2.76 \\
\hline & Ballast Total & 203 & 182,087 & 76,473 & 15,343 & 4.98 & 3.36 \\
\hline & Subtotal & 26,579 & $20,022,961$ & $6,385,181$ & $1,576,090$ & 4.05 & 4.14 \\
\hline \multirow{3}{*}{ Envelope } & Roof Insulation Total & 4,989 & $3,538,677$ & 983,515 & 271,778 & 3.62 & 4.19 \\
\hline & Windows Total & 31 & 6,102 & 23,598 & 1,786 & 13.2 & 1.30 \\
\hline & Subtotal & 5,020 & $3,544,797$ & $1,007,113$ & 273,564 & 3.68 & 4.11 \\
\hline \multicolumn{2}{|c|}{ Grand Total } & 53,856 & $30,386,499$ & $14,012,073$ & $3,055,849$ & 4.59 & 3.78 \\
\hline
\end{tabular}

From Table 5a, the total cost-effective energy savings is estimated at 53,856 MMBtu/year representing $\$ 3,055,849 /$ year savings with an overall savings to investment ratio (SIR) of 3.78 . This represents 15.1\% in energy savings based on FY 2009 energy data reported to DUERS.

The greatest energy saving potential was found in lighting retrofits. Although T8 lighting is good, advanced T8 lighting can yield additional savings (15,641 MMBtu/year), followed by installation of water cooled chillers (12,989 MMBtu/year). Similiarly, advanced T8 retrofits yields the largest estimated dollar savings (\$942,054/year) and water cooled chillers (\$787,151/year). 
Table 5b. Summary of All Cost-Effective Projects Identified from the FEDS Assessment for Hickam AFB Using Alternative Financing Sources of Capital (by Retrofit Category and Type)

\begin{tabular}{|c|c|c|c|c|c|c|c|}
\hline $\begin{array}{l}\text { Retrofit } \\
\text { Category }\end{array}$ & Retrofit Technology & $\begin{array}{c}\text { Energy } \\
\text { Savings } \\
\text { (MMBtu/yr) }\end{array}$ & $\begin{array}{l}\text { Net Present } \\
\text { Value (\$) }\end{array}$ & $\begin{array}{l}\text { Installed } \\
\text { Cost (\$) }\end{array}$ & $\begin{array}{c}\text { 1st year } \\
\text { savings (\$) }\end{array}$ & $\begin{array}{l}\text { Simple } \\
\text { payback } \\
\text { (yr) }\end{array}$ & SIR \\
\hline \multirow{3}{*}{ Cooling } & Packaged AC Total & 960 & 193,476 & 175,291 & 63,405 & 2.76 & 2.10 \\
\hline & Water-Cooled Chiller Total & 1,235 & 79,389 & 274,080 & 61,691 & 4.44 & 1.28 \\
\hline & Subtotal & 2,195 & 272,865 & 449,371 & 125,096 & 3.59 & 160 \\
\hline \multirow{3}{*}{ Hot Water } & $\begin{array}{l}\text { Heat Pump Water Heater } \\
\text { Total }\end{array}$ & 6,946 & $1,359,000$ & 572,481 & 327,997 & 1.75 & 3.36 \\
\hline & Misc Measures Total & 690 & 154,034 & 8,311 & 24,367 & 0.34 & 13.36 \\
\hline & Subtotal & 7,636 & $1,513,034$ & 580,792 & 352,364 & 165 & 3.58 \\
\hline \multirow{7}{*}{ Lights } & CFL Total & 2,241 & 803,188 & 55,504 & 147,301 & 0.38 & 15.04 \\
\hline & EXIT Lighting Total & 2,927 & $1,009,594$ & 175,183 & 202,065 & 0.87 & 6.86 \\
\hline & Super T8 Lights & 6,969 & $1,118,055$ & $1,504,908$ & 450,038 & 3.34 & 1.77 \\
\hline & T8 Lights & 4,254 & $1,117,475$ & 653,941 & 302,677 & 2.16 & 2.70 \\
\hline & $\begin{array}{l}\text { High Pressure Sodium } \\
\text { Total }\end{array}$ & 5,029 & 301,927 & $1,171,181$ & 255,984 & 4.58 & 1.29 \\
\hline & Ballast Total & 112 & 17,867 & 50,818 & 11,571 & 4.39 & 1.34 \\
\hline & Subtotal & 21,532 & $4,368,106$ & $3,611,535$ & $1,369,636$ & 264 & 227 \\
\hline \multirow{2}{*}{ Envelope } & Roof Insulation Total & 550 & 58,376 & 140,688 & 34,678 & 4.06 & 1.39 \\
\hline & Subtotal & 550 & 58,376 & 140,688 & 34,678 & 4.06 & 139 \\
\hline \multicolumn{2}{|c|}{ Grand Total } & 31,913 & $6,212,381$ & $4,782,386$ & $1,881,774$ & 254 & 234 \\
\hline
\end{tabular}

From Table 5b, the total cost-effective energy savings is estimated at 31,913 MMBtu/year representing \$1,881,774/year savings with an overall savings to investment ratio (SIR) of 2.34. This represents 9.1\% in energy savings based on FY 2009 energy data reported to DUERS.

The greatest energy saving potential was found in advanced T8 lighting measures (6,969 MMBtu/year), followed by heat pump water heater systems (6,946 MMBtu/year). Similiarly, the largest estimated dollar savings was advanced T8 lighting measures (\$450,038/year) followed by heat pump water heater systems $(\$ 327,997 /$ year $)$. 
As would be expected, the total number of cost-effective retrofits is fewer (and installed cost/ capital investment is significantly less) under alternative financing source of capital, and thus, the energy and dollar savings are likewise less. The total number of cost-effective retrofits using appropriated source of capital is 135 and the total number of cost-effective retrofits using alternative financing source of capital is 88. Using appropriated funding will save 21,943 MMBtu/year and $\$ 1,174,075 /$ year more than alternative financing. Utilizing alternative financing reduces the simple payback from 4.59 to 2.54 years because some projects with longer paybacks are eliminated under the alternative financing scenario.

The complete list of cost-effective energy- and cost-reduction projects is given Appendix C-1 for appropriated funds sources of capital and in Appendix C-2 for alternative financing sources of capital. ${ }^{4}$

\footnotetext{
${ }^{4}$ The Hickam AFB energy manager will also receive a CD, that includes all the FEDS input data and output project files. The input data files reflect information collected during the site visits and additional assumptions required to perform the FEDS modeling and assessment.
} 


\section{Conversion to Water-Cooled Chillers}

Water-cooled condensing of cooling equipment refrigerant results in a significant improvement in efficiency compared to air-cooled condensing chillers. This advantage stems from two factors. Condenser water from an evaporative cooling tower is generally cooler than ambient air (except when the relative humidity is very high), and water is a more effective heat transfer fluid than air. The two factors work together to lower the refrigerant condensing temperature, hence improving both theoretical and actual refrigeration cycle efficiency. Combining cooling loads met by multiple smaller cooling units into fewer central units allows additional efficiency gains by using centrifugal compressors, a more efficient technology than alternative compressor types commonly used in smaller cooling equipment. These advantages do come at a price, however. Condensing refrigerant with water requires additional costs associated with a cooling tower, condenser water pumps and piping, and a shell to enclose the water as it passes by the condenser tubing. The condenser pump also represents an additional power consuming device that an aircooled unit does not have. Finally, the distribution of centrally chilled water incurs pumping and piping costs and pumping energy not required by distributed direct expansion coolers (e.g., window air conditioner $[\mathrm{AC}]$ and packaged rooftop AC).

For the reasons noted above, water-cooled chillers offer significant performance advantages over air-cooled equipment that must be weighed against their additional capital costs. During the last few decades, space cooling has become much more common in Hawaiian military facilities because internal heating loads (e.g., personal computers and other office equipment) have increased, building designs have become less suitable for natural ventilation, and occupants expect a more comfortable working environment. The FEDS model generated retrofit recommendations for replacing air-cooled chillers with water-cooled chillers at the building level. The following paragraphs discuss the impact of combining these energy conservation measures (ECMs) into a centralized chilled-water plant. More details of the assessment of water-cooled chillers at Hickam AFB are provided in Appendix E.

Buildings 2130, 2131, and 2133 are currently served by a small central cooling plant comprised of two air-cooled chillers. The proposed retrofit would replace the existing air-cooled chillers with two water-cooled chillers, a cooling tower, and condenser water pumps and piping. The existing chilled water pumps and piping would not change and the electrical service to the central plant should be adequate for the retrofit.

The peak and annual building cooling loads were estimated with the FEDS model and the performance of the existing chillers was estimated from manufacturer's specifications for the two units. From this information, the annual $\mathrm{kWh}$ and peak $\mathrm{kW}$ electrical loads were calculated and then combined with Hickam's electricity rates to calculate the current annual electricity costs. The existing system performance and electricity cost figures are presented in Table 6.

Although the FEDS model estimates a peak of only 61 tons for the three buildings, two 40 -ton water-cooled chillers were assumed for the retrofit to match the existing nameplate capacity of the two air-cooled chillers. In this size range, the water-cooled chillers were assumed to use a rotary screw compressor rated at $0.73 \mathrm{~kW} /$ ton. In addition, the condenser water pump and 
cooling tower fan would be expected to consume $0.12 \mathrm{~kW} /$ ton for a total cooling plant performance of $0.85 \mathrm{~kW} / \mathrm{ton}$. The annual electricity bill for the water-cooled system was calculated to be $\$ 35,360$ based on these assumptions, resulting in an annual savings of about $\$ 15,000$ and a peak electric load reduction of $22 \mathrm{~kW}$.

Table 6. Hickam Buildings 2130, 2131, 2133 Existing System Performance and Electricity Cost

\begin{tabular}{|c|c|c|c|c|c|c|c|}
\hline & $\begin{array}{c}\text { Peak } \\
\text { Load, } \\
\text { Tons }\end{array}$ & $\begin{array}{c}\text { Annual } \\
\text { Load, } \\
\text { Ton- } \\
\text { hours }\end{array}$ & $\begin{array}{c}\text { Annual } \\
\text { Capacity } \\
\text { Factor }\end{array}$ & $\begin{array}{c}\text { Existing } \\
\text { Air } \\
\text { Cooled } \\
\text { kW/ton }\end{array}$ & $\begin{array}{c}\text { Existing } \\
\text { Annual } \\
\text { Electricity } \\
\text { kWh }\end{array}$ & $\begin{array}{c}\text { Existing } \\
\text { Peak } \\
\text { Electricity } \\
\text { kW }\end{array}$ & $\begin{array}{c}\text { Existing } \\
\text { Annual } \\
\text { Electricity } \\
\text { Cost }\end{array}$ \\
\hline 2130 & 18.1 & 73,335 & 0.46 & 1.204 & 88,296 & 21.8 & \\
\hline 2131 & 10.3 & 40,647 & 0.45 & 1.204 & 48,939 & 12.4 & \\
\hline 2133 & 32.7 & 100,092 & 0.35 & 1.204 & 120,511 & 39.3 & \\
\hline Totals & $\mathbf{6 1 . 0}$ & $\mathbf{2 1 4 , 0 7 4}$ & $\mathbf{0 . 4 0}$ & $\mathbf{1 . 2 0 4}$ & $\mathbf{2 5 7 , 7 4 5}$ & $\mathbf{7 3 . 5}$ & $\mathbf{\$ 5 0 , 0 8 7}$ \\
\hline
\end{tabular}

The two new 40-ton water-cooled chillers were estimated to cost $\$ 88,200$ and the cooling tower, condenser pump, and piping an additional $\$ 26,100$. These figures include all direct construction costs, but do not include any allowance for design or SIOH costs. Based on the direct cost, the payback period is 8 years. With an additional 16\% for design and SIOH, the payback period rises to 9 years. 


\section{Installation Load Reduction Potential}

Using the FEDS model, the impact on electric demand can be estimated from implementing all the cost-effective projects at Hickam. The existing peak electric demand from all building loads $^{5}$ at Hickam is $17,545 \mathrm{~kW}$. This peak occurs at 1300 hours during a September weekday. By implementing all the FEDS recommended retrofits, the peak demand can be reduced by 3,294 $\mathrm{kW}$ to $14,251 \mathrm{~kW}$. This represents a $19 \%$ reduction in peak demand.

$\begin{array}{lrr} & \text { Annual Installation Electric Demand } \\ & & \\ \text { Installation Peak Demand: } & \text { Demand }(\mathrm{kW}) & \text { Dollars (2009) } \\ \text { existing } & 17,545 & 2,936,232 \\ \text { post-retrofit } & 14,251 & 2,376,252 \\ \text { difference } & -3,294 & -559,980 \\ \text { \% change } & -19 & -19 \\ \text { Time of Installation } & \text { Existing } & \text { Post-Retrofit } \\ \text { Peak Demand: } & \text { September } & \text { September } \\ \text { Month } & \text { Weekday } & \text { Weekday } \\ \text { Day Type } & 1300 & 1300 \\ \text { Hour } & & \end{array}$

\footnotetext{
${ }^{5}$ The modeled electric demand in FEDS is for all building loads and may not include certain non-building electric loads (e.g., booster pumps, lift stations, transmission losses, etc.).
} 


\section{Recommendations for More In-Depth Assessments}

The FEDS model can provide an unbiased assessment of literally hundreds of energy conservation projects; unfortunately, it is not all-inclusive. While the scope of this project is limited to energy-saving projects included in the FEDS model, the energy-saving opportunities identified below were recognized during the site visit and may be worth additional consideration by the site energy staff. It is recommended that the site consider additional assessment of these potential projects.

Cool Roofs. FEDS does not evaluate the potential savings for cool roof projects.

Building Controls. Recommendations for building controls cannot be easily inferred by the FEDS model engine. A detailed building assessment focused on all heating, ventilation and air conditioning (HVAC) equipment is required to develop project proposals.

Programmable Thermostats. The FEDS model does not consider programmable thermostats in the energy analysis. Programmable thermostats are considered a conservation measure rather than an equipment replacement or building improvement. Programmable thermostats could be a useful conservation measure in smaller commercial buildings or any building that is unoccupied during part of the day. 


\section{Implementation Options}

Hickam AFB would have a number of options for implementing the energy conservation measures (ECMs) identified in this assessment. As shown in Table 7, implementing the building level ECMs using appropriated funds would require an investment of about \$14.0M, and result in 53,856 MMBtu/year representing \$3,055,849/year savings with an overall savings to investment ratio (SIR) of 3.8. Using alternative financing (ESPC or UESC) would result in 31,913 MMBtu/year representing \$1,881,774/year savings with an overall savings to investment ratio (SIR) of 2.3, for an investment cost of $\$ 4.8 \mathrm{M}$. However, the investment cost under alternative financing does not include the financing charges over the life of the project.

The recommended option for implementing the building level ECMs would be to pursue appropriated funds either through the Energy Conservation Investment Program (ECIP) or sustainment, renovation, and modernization (SRM) at the Base level. This would result in the greatest energy and cost savings (see Table 7). The ECIP program within the Air Force may not be an option for these building energy-efficiency ECMs as the focus of the current program is on renewable energy projects. If appropriated funds are not available, then alternative financing would provide the means to get most of the projects implemented without the upfront investment on the part of the Air Force.

Table 7. Comparison of Funding Sources

\begin{tabular}{|l|c|c|r|r|r|c|}
\hline \multicolumn{1}{|c|}{ Funding Source } & $\begin{array}{c}\text { Energy } \\
\text { Savings } \\
(\mathbf{M M B t u} / \mathbf{y r})\end{array}$ & $\begin{array}{c}\text { 1st Year } \\
\text { Savings } \\
(\$ / y r)\end{array}$ & $\begin{array}{c}\text { Installed } \\
\text { Cost (\$) }\end{array}$ & $\begin{array}{l}\frac{\text { Estimated }}{\text { Financing }} \\
\text { Costs (\$) }\end{array}$ & $\begin{array}{c}\text { Total Cost } \\
(\$)\end{array}$ & SIR \\
\hline Appropriated funding & 53,856 & $3,055,849$ & $14,012,073$ & $\mathbf{0}$ & $14,012,073$ & 3.8 \\
\hline Alternative financing & 31,913 & $1,881,774$ & $4,782,386$ & $3,440,614$ & $\mathbf{8 , 2 2 3 , 0 0 0}$ & 2.3 \\
\hline
\end{tabular}

Public benefit funds may be available for some of these ECMs through Hawai'i Energy. Hawai'i Energy operates the new and expanded Hawai'i Energy-Efficiency Programs under contract to the Hawai'i Public Utilities Commission (HPUC) and they are paid for by electric utility ratepayer fees.

\footnotetext{
${ }^{6}$ Assumes alternative financing at an annual interest rate of $6 \%$ for 20 years.
} 


\section{Emissions Reduction}

Implementing all the cost-effective building retrofits using appropriated funds will result in a $18 \%$ reduction in greenhouse gas emissions. These reductions are summarized in table 8 and included for each building in appendix D.

Table 8. Reduction in Greenhouse Gas Emissions

\begin{tabular}{|c|c|}
\hline Greenhouse Gas & Totals \\
\hline \multicolumn{2}{|l|}{ Sulfur Oxides (lb) } \\
\hline existing & 828,151 \\
\hline post-retrofit & 679,652 \\
\hline difference & $-148,499$ \\
\hline$\%$ change & -18 \\
\hline \multicolumn{2}{|l|}{ Nitrogen Oxides (lb) } \\
\hline existing & 397,557 \\
\hline post-retrofit & 326,103 \\
\hline difference & $-71,453$ \\
\hline$\%$ change & -18 \\
\hline \multicolumn{2}{|l|}{ Carbon Monoxide (lb) } \\
\hline existing & 685,129 \\
\hline post-retrofit & 561,911 \\
\hline difference & $-123,218$ \\
\hline$\%$ change & -18 \\
\hline \multicolumn{2}{|l|}{ Carbon Dioxide (tons) } \\
\hline existing & 84,302 \\
\hline post-retrofit & 69,147 \\
\hline difference & $-15,155$ \\
\hline$\%$ change & -18 \\
\hline \multicolumn{2}{|l|}{ Particulate Matter (lb) } \\
\hline existing & 16,428 \\
\hline post-retrofit & 13,481 \\
\hline difference & $-2,948$ \\
\hline$\%$ change & -18 \\
\hline \multicolumn{2}{|l|}{ Hydrocarbons (lb) } \\
\hline existing & 283,022 \\
\hline post-retrofit & 232,157 \\
\hline difference & $-50,864$ \\
\hline \% change & -18 \\
\hline
\end{tabular}




\section{Contacts}

Contact information for assessment team members and site team from PNNL are:

Doug Dixon

Pacific Northwest National Laboratory

902 Battelle Boulevard, MSIN K6-10

Richland, WA 99352

Phone: (509) 372-4253

Email: doug.dixon@pnl.gov

William D. Chvala, Jr.

Pacific Northwest National Laboratory

902 Battelle Boulevard, MSIN K5-08

Richland, WA 99352

Phone: (509) 372-4558

Email: william.chvala@pnl.gov

Daryl Brown

Pacific Northwest National Laboratory

902 Battelle Boulevard, MSIN K6-10

Richland, WA 99352

Phone: (509) 372-4366

Email: daryl.brown@pnl.gov

Marcus De La Rosa

Pacific Northwest National Laboratory

902 Battelle Boulevard, MSIN K5-16

Richland, WA 99352

Phone: (509) 375-2941

Email: marcus.delarosa@pnl.gov 


\section{Appendix A}

\section{FEDS Data Collection Form}




\section{Appendix A \\ FEDS Data Collection Form}

The following form is used to collect FEDS input data during building audits. Note that not all data types indicated on this form are applicable to all buildings. Nor is all the information indicated on this form always available. Where necessary, the FEDS model infers the values for missing data based on other known building characteristics.

FEDS Building Information for

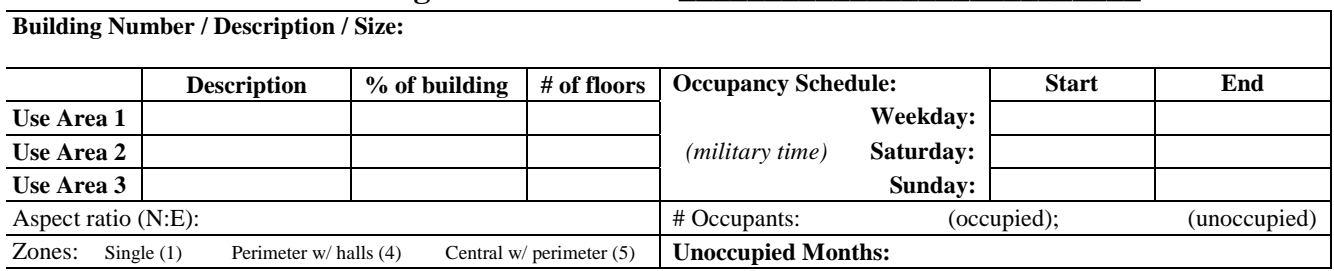

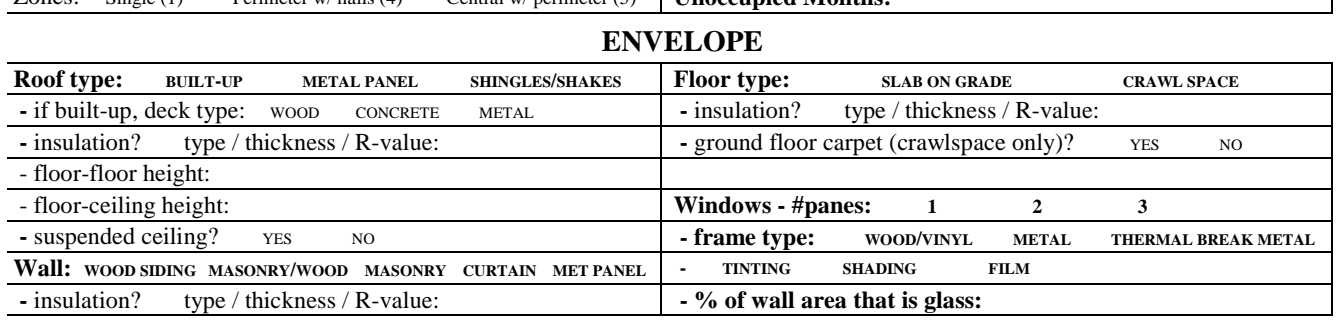

- insulation? type / thickness / R-value:

LIGHTING

\begin{tabular}{|c|c|c|c|c|c|}
\hline $\begin{array}{c}\text { Technology } \\
\text { Type }\end{array}$ & $\begin{array}{l}\text { Fixture Description (size, \#lamps, wattage, } \\
\text { reflectors, ballasts, application, etc.)* }\end{array}$ & $\begin{array}{c}\text { Use Area or \% of } \\
\text { building served }\end{array}$ & $\begin{array}{c}\begin{array}{c}\text { Fixture density } \\
\text { or count }\end{array} \\
\end{array}$ & $\begin{array}{c}\text { Mounting } \\
\text { Method }\end{array}$ & Utilization \\
\hline \multicolumn{6}{|l|}{ Exit Signs } \\
\hline & & & & & \\
\hline & & & & & \\
\hline & & & & & \\
\hline & & & & & \\
\hline & & & & & \\
\hline & & & & & \\
\hline & & Exterior & & -- & \\
\hline & & Exterior & & -- & \\
\hline & & Exterior & & -- & \\
\hline
\end{tabular}

LPS = low pressure sodium EX = exit sign $\quad$ *2-tube T12=2T12; 4-tube T12=4T12; 2-tube T8=2T8; 2-tube T5=2T5; 4-tube Biax=4BIAX

SERVICE HOT WATER

\begin{tabular}{l|l|l|l|}
\hline $\begin{array}{l}\text { Portion of building set served (whole buildings) } \\
\text { (sq. ft, \%, } \text { of buildings, or USE AREA) }\end{array}$ & \multicolumn{1}{|c|}{ System 1: } & System 2: & System 3: \\
\hline Fuel type & & & \\
\hline System type & DISTRIBUTED LOOP & DISTRIBUTED LOOP & DISTRIBUTED \\
\hline Equipment vintage & & & LOOP \\
\hline Tank capacity (gallons, \#tanks) & & & \\
\hline Heating capacity (loop only) & & & \\
\hline Thermostat set point, ${ }^{\circ} \mathrm{F}$ & & & \\
\hline Tank insulation - thickness/R-value & & & \\
\hline Efficiency & & & \\
\hline Loop length (perimeter or stacked service) & & & \\
\hline \#Faucets / aerators installed (\%) & & \\
\hline \#Showers / low-flow showerheads installed (\%) & & & \\
\hline $\begin{array}{l}\text { Note presence of: bottom boards, near tank pipe } \\
\text { insul., tank wrap, heat traps, electronic pilots }\end{array}$ & & & \\
\hline
\end{tabular}

Auditor:

Date:

Sheet of 
HVAC

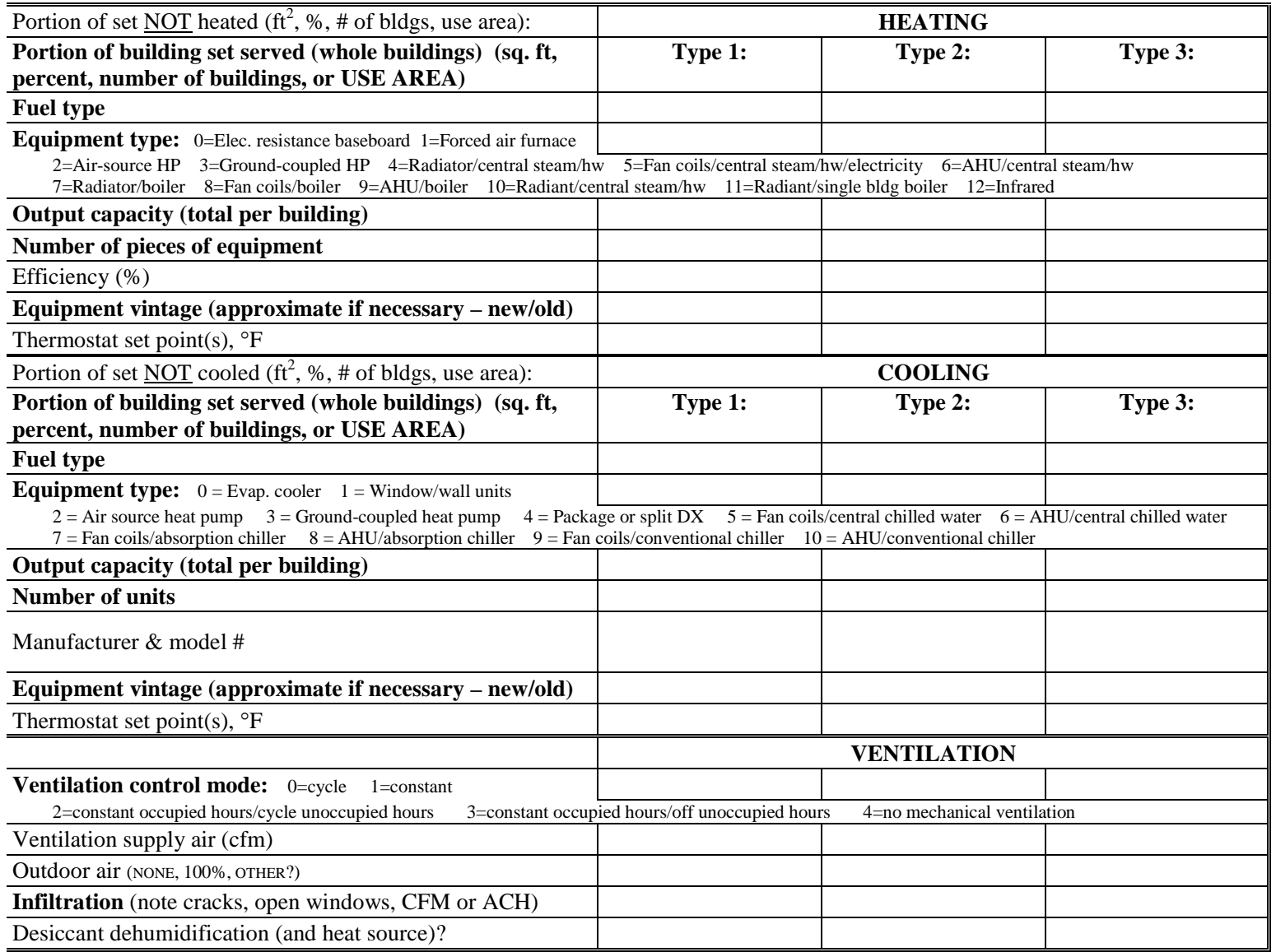

MISC. EQUIPMENT

Refrigeration, food prep, or other - note if irregular. Atypical equipment: description including type, fuel, capacity, utilization.

\begin{tabular}{|c|c|c|c|c|}
\hline \multicolumn{5}{|c|}{ MOTORS } \\
\hline & Type 1: & Type 2: & Type 3: & Type 4: \\
\hline \multicolumn{5}{|l|}{ Horsepower } \\
\hline \multicolumn{5}{|l|}{ \# Motors of this type } \\
\hline \multicolumn{5}{|l|}{ Utilization } \\
\hline Other nameplate data & & & & \\
\hline
\end{tabular}

\section{NOTES/DRAWINGS}




\section{Appendix B}

\section{Facility Category Descriptions and Associated Buildings}




\section{Appendix B \\ Facility Category Descriptions and Associated Buildings}

The following table identifies the buildings in the 30 facility categories defined by the assessment team. The table below includes the FEDS facility category code, the proxy building number(s) audited for the purpose of developing the FEDS model, the proxy building total square footage, the total number of buildings in the category, the total square footage in that category and the percentage of square footage represented by the proxy buildings. Overall, PNNL audited $944,397 \mathrm{ft}^{2}$ of building space out of a total of $4,875,472 \mathrm{ft}^{2}$, or $19 \%$.

\begin{tabular}{|c|c|c|c|c|c|c|}
\hline $\begin{array}{l}\text { Category } \\
\text { Description } \\
\text { [FEDS } \\
\text { Facility } \\
\text { Category } \\
\text { Code] } \\
\end{array}$ & $\begin{array}{c}\text { Proxy } \\
\text { (Audited) } \\
\text { Building } \\
\text { Number }\end{array}$ & $\begin{array}{l}\text { Proxy } \\
\text { Building } \\
\left(\mathrm{ft}^{2}\right)\end{array}$ & $\begin{array}{l}\text { Total } \\
\text { Bldgs. In } \\
\text { Category }\end{array}$ & Non-Audited Buildings in Group & $\begin{array}{l}\text { Total } \\
\text { Area in } \\
\text { Category } \\
\left(\mathrm{ft}^{2}\right)\end{array}$ & $\begin{array}{c}\text { Proxy } \\
\text { Area } \\
\% \text { of } \\
\text { Category }\end{array}$ \\
\hline 1 & 2186 & 2,125 & 15 & $\begin{array}{c}\text { 01754, 2072, 72934, 71949, 71941, } \\
\text { 71942, 72727, 3008, 3393, 4017, 1229, } \\
\text { 1212, 1100, } 2134\end{array}$ & 38,382 & $6 \%$ \\
\hline $10 \mathrm{a}$ & N/A & N/A & 1 & 1102 & 519,549 & $0 \%$ \\
\hline $10 \mathrm{~b}$ & 2035 & 86,391 & 6 & $2060,2045,3440,1200,1050$ & 469,042 & $18 \%$ \\
\hline $10 \mathrm{c}$ & 1204 & 11,374 & 9 & $\begin{array}{c}\text { 1110, 1113, 3225, 2171, 2050, 1001, } \\
\text { 1201, } 1071\end{array}$ & 150,726 & $8 \%$ \\
\hline $10 \mathrm{~d}$ & 2155 & 21,745 & 3 & 1105,3382 & 71,332 & $30 \%$ \\
\hline $10 \mathrm{e}$ & 502 & 9,217 & 13 & $\begin{array}{c}4071,1106,1153,3404,3510,1012 \\
1035,3373,2104,3561,3560,188\end{array}$ & 60,670 & $15 \%$ \\
\hline $10 \mathrm{f}$ & 2133 & 25,764 & 4 & $02140,1850,3386$ & 115,164 & $22 \%$ \\
\hline $10 \mathrm{~g}$ & 2125 & 3,867 & 21 & $\begin{array}{c}\text { 4100, 3417, 3417, 1222, 2003, 2176, } \\
\text { 2070, 1727, 3020, 2167, 3002, 3227, } \\
\text { 192, 4073, 3250, 4070, 3596, 3201, } \\
\text { 3203, 2042 }\end{array}$ & 74,987 & $5 \%$ \\
\hline $21 \mathrm{a}$ & 559 & 78,823 & 4 & $554,1864,3365$ & 93,381 & $84 \%$ \\
\hline $23 a$ & 1060 & 14,920 & 8 & $\begin{array}{c}988,2141,3385,1010,2076,1011, \\
3195\end{array}$ & 54,337 & $27 \%$ \\
\hline $30 a$ & 1805 & 55,187 & 2 & 1843 & 121,649 & $45 \%$ \\
\hline $30 \mathrm{~b}$ & 1856 & 43,187 & 3 & 1852,1854 & 121,924 & $35 \%$ \\
\hline $30 \mathrm{c}$ & 1166 & 25,113 & 18 & $\begin{array}{c}\text { 941, 1156, 1158, 920, 1153, 1166, } \\
\text { 1168, 1172, 725, 727, 728, 920, 922, } \\
\text { 925, 926, 934, 940 }\end{array}$ & 256,336 & $10 \%$ \\
\hline $40 \mathrm{a}$ & 2177 & 3,200 & 8 & $\begin{array}{c}2030,2040,1715,1203,1207,1202, \\
1220\end{array}$ & 242,165 & $1 \%$ \\
\hline
\end{tabular}




\begin{tabular}{|c|c|c|c|c|c|c|}
\hline $\begin{array}{l}\text { Category } \\
\text { Description } \\
\text { [FEDS } \\
\text { Facility } \\
\text { Category } \\
\text { Code] }\end{array}$ & $\begin{array}{c}\text { Proxy } \\
\text { (Audited) } \\
\text { Building } \\
\text { Number }\end{array}$ & $\begin{array}{l}\text { Proxy } \\
\text { Building } \\
\left(\mathrm{ft}^{2}\right)\end{array}$ & $\begin{array}{l}\text { Total } \\
\text { Bldgs. In } \\
\text { Category }\end{array}$ & Non-Audited Buildings in Group & $\begin{array}{l}\text { Total } \\
\text { Area in } \\
\text { Category } \\
\left(\mathrm{ft}^{2}\right)\end{array}$ & $\begin{array}{c}\text { Proxy } \\
\text { Area } \\
\% \text { of } \\
\text { Category }\end{array}$ \\
\hline $40 \mathrm{~b}$ & 4016 & 7,701 & 7 & 3416, 2010, 3402, 3245, 3431, 3422 & 50,939 & $15 \%$ \\
\hline $40 c$ & 2131 & 26,296 & 15 & $\begin{array}{c}\text { 2025, 3004, 3392, 3407, 3407, 3435, } \\
\text { 3426, 3431, 3435, 3247, 2019, 3437, } \\
\text { 3430, } 3434\end{array}$ & 126,642 & $21 \%$ \\
\hline $50 a$ & 1072 & 83,379 & 10 & $\begin{array}{c}1055,1728,4069,1073,1045,3400 \\
3415,2115,3564\end{array}$ & $1,012,107$ & $8 \%$ \\
\hline $50 \mathrm{v}$ & 2002 & 23,981 & 11 & $\begin{array}{c}\text { 4002, 2073, 1720, 2006, 3380, 2001, } \\
\text { 2022, 3425, 4003, 3424 }\end{array}$ & 91,158 & $26 \%$ \\
\hline $50 \mathrm{~b}$ & 1713 & 30,400 & 20 & $\begin{array}{c}\text { 3220, 1722, 1723, 1711, 1714, 1042, } \\
\text { 1710, 2110, 3520, 2116, 1205, 3192, } \\
\text { 2158, 3226, 4032, 3567, 3505, 987, } \\
3381\end{array}$ & 229,766 & $13 \%$ \\
\hline $50 c$ & 2130 & 56,734 & 1 & -- & 56,734 & $100 \%$ \\
\hline $50 \mathrm{~d}$ & 1070 & 62,779 & 51 & $\begin{array}{l}\text { 1219, 3379, 3044, 3594, 4115, 2175, } \\
\text { 1760, 3572, 3584, 3576, 3455, 1043, } \\
\text { 1816, 1844, 4030, 1806, 2187, 2185, } \\
\text { 2037, 14170, 3515, 3525, 4068, 3039, } \\
\text { 2023, 2179, 1223, 2161, 2188, 3436, } \\
\text { 1097, 2069, 4119, 1809, 3571, 2192, } \\
\text { 3577, 3578, 3587, 3589, 3585, 1091, } \\
\text { 1093, 3485, 1810, 1845, 1846, 1847, } \\
1849,2024\end{array}$ & 162,973 & $39 \%$ \\
\hline $60 \mathrm{a}$ & 1860 & 12,941 & 2 & 3417 & 50,108 & $26 \%$ \\
\hline $60 \mathrm{~b}$ & 1804 & 27,579 & 10 & $\begin{array}{c}901,1756,3465,1250,2096,900, \\
2105,905,908\end{array}$ & 100,424 & $27 \%$ \\
\hline $60 \mathrm{~m}$ & 594 & 293 & 41 & $\begin{array}{c}\text { 1028, 1249, 1109, 601, 1046, 2150, } \\
\text { 3406, 4008, 2156, 1217, 3190, 3395, } \\
\text { 1058, 1108, 2154, 1124, 1333, 427, } \\
\text { 3458, 2098, 2157, 1629, 1861, 906, } \\
\text { 2039, 4072, 7475, 924, 3205, 2153, } \\
\text { 180, 3001, 2169, 1281, 2051, 210, } \\
\text { 3389, 918, 3410, 3246 }\end{array}$ & 42,761 & $1 \%$ \\
\hline $60 c$ & 2093 & 115,408 & 3 & 1235,1232 & 305,569 & $38 \%$ \\
\hline $60 \mathrm{t}$ & 2028 & 46,128 & 1 & -- & 46,128 & $100 \%$ \\
\hline $80 \mathrm{a}$ & 1597 & 12,760 & 15 & $\begin{array}{l}\text { 1335, 1654, } 1399,623,1598,1588 \\
\text { 1586, 1656, } 1657,1587,1589,626 \\
627,1655\end{array}$ & 51,664 & $25 \%$ \\
\hline
\end{tabular}




\begin{tabular}{|c|c|c|c|c|c|c|}
\hline $\begin{array}{l}\text { Category } \\
\text { Description } \\
\text { [FEDS } \\
\text { Facility } \\
\text { Category } \\
\text { Code] } \\
\end{array}$ & $\begin{array}{c}\text { Proxy } \\
\text { (Audited) } \\
\text { Building } \\
\text { Number }\end{array}$ & $\begin{array}{l}\text { Proxy } \\
\text { Building } \\
\left(\mathrm{ft}^{2}\right)\end{array}$ & $\begin{array}{c}\text { Total } \\
\text { Bldgs. In } \\
\text { Category } \\
\end{array}$ & Non-Audited Buildings in Group & $\begin{array}{c}\text { Total } \\
\text { Area in } \\
\text { Category } \\
\left(\mathrm{ft}^{2}\right)\end{array}$ & $\begin{array}{c}\text { Proxy } \\
\text { Area } \\
\% \text { of } \\
\text { Category }\end{array}$ \\
\hline $80 \mathrm{~b}$ & 1891 & 3,090 & 16 & $\begin{array}{c}1859,1889,1122,595,1029,3460 \\
501,1095,2094,425,1092,1225, \\
3470,3360,1848\end{array}$ & 100,297 & $3 \%$ \\
\hline $80 c$ & 1750 & 7,296 & 3 & 500,1856 & 11,839 & $62 \%$ \\
\hline $\begin{array}{l}\text { 80d } \\
\text { Totals }\end{array}$ & 1120 & $\begin{array}{l}\mathbf{4 6 , 7 1 9} \\
\mathbf{9 4 4 , 3 9 7}\end{array}$ & $\begin{array}{c}1 \\
322\end{array}$ & -- & $\begin{array}{c}46,719 \\
\mathbf{4 , 8 7 5 , 4 7 2}\end{array}$ & $\begin{array}{l}100 \% \\
\mathbf{1 9 \%}\end{array}$ \\
\hline
\end{tabular}


Appendix C

Comprehensive List of Cost-Effective Projects Identified from the FEDS Assessment Using Appropriated/Alternative Financed Sources of Capital 


\section{Appendix C-1}

\section{Comprehensive List of Cost-Effective Projects Identified from the FEDS Assessment Using Appropriated Source of Capital}

Table C-1 identifies the 135 cost-effective energy- and cost-reducing retrofit projects identified from the FEDS modeling and analysis based on the assumption that the projects will be funded using appropriated source of capital funds. Key energy and economic results are presented for each cost-effective retrofit measure. The projects are grouped by building category. More detail, supporting each line-item project recommendation, is contained in the FEDS input and output files, which are delivered to the site energy manager on a CD in conjunction with this report. 
Table C-1 Comprehensive List of Cost-Effective Projects Using Appropriated Sources of Capital

\begin{tabular}{|c|c|c|c|c|c|c|c|}
\hline Bldg. Set ID & Technology Change & $\begin{array}{l}\text { Energy } \\
\text { Savings } \\
\text { (MMBtu/yr) }\end{array}$ & $\begin{array}{l}\text { Net Present } \\
\text { Value (\$) }\end{array}$ & $\begin{array}{l}\text { Installed } \\
\text { Cost (\$) }\end{array}$ & $\begin{array}{c}\text { 1st year } \\
\text { savings (\$) }\end{array}$ & $\begin{array}{l}\text { Simple } \\
\text { Payback } \\
\text { (yr) }\end{array}$ & SIR \\
\hline 1 & Replace 175W Metal Halide with 4 Super T8 30W Lights & 365 & 409,722 & 62,537 & 27,899 & 2.24 & 7.60 \\
\hline \multirow{10}{*}{$10 b$} & $\begin{array}{l}\text { Wrap Tank with Insulation, Insulate Pipe Near Tank, Aerators, Lower } \\
\text { Tank Temperature }\end{array}$ & - & 65 & 37 & 16 & 2.31 & 5.80 \\
\hline & $\begin{array}{l}\text { Wrap Tank with Insulation, Insulate Pipe Near Tank, Aerators, Lower } \\
\text { Tank Temperature }\end{array}$ & - & 96 & 41 & 22 & 1.86 & 7.40 \\
\hline & Replace LED EXIT Lights with Electroluminescent Panel & - & 417 & 224 & 37 & 6.05 & 2.90 \\
\hline & Replace 4 T12 40W Lights with 3 Super T8 32W Lights & 49 & 39,932 & 13,522 & 3,188 & 4.24 & 4.00 \\
\hline & Replace 175W Metal Halide with 4 Super T8 30W Lights & 36 & 37,250 & 11,463 & 2,885 & 3.97 & 4.20 \\
\hline & $\begin{array}{l}\text { Replace Electric Air-Cooled Chiller with Water-Cooled Chiller and } \\
\text { Cooling Tower (very high efficiency) }\end{array}$ & 1,768 & 531,811 & 593,472 & 108,437 & 5.47 & 2.60 \\
\hline & Wrap Tank with Insulation, Insulate Pipe Near Tank, Aerators & 45 & 11,224 & 2,442 & 2,394 & 1.02 & 13.60 \\
\hline & Replace $75 \mathrm{~W}$ Incandescent Lights with $18 \mathrm{~W}$ CFL Lights & 1,713 & $1,881,018$ & 18,316 & 113,005 & 0.16 & 103.70 \\
\hline & Replace LED EXIT Lights with Electroluminescent Panel & 10 & 22,807 & 11,182 & 1,973 & 5.67 & 3.00 \\
\hline & Replace 3 T8 32W Lights with 3 Super T8 28W Lights & 1,313 & 452,649 & 673,226 & 67,673 & 9.95 & 1.70 \\
\hline \multirow{2}{*}{$10 c$} & $\begin{array}{l}\text { Replace Electric Air-Cooled Chiller with Water-Cooled Chiller and } \\
\text { Cooling Tower (very high efficiency) }\end{array}$ & 1,288 & 276,428 & 576,738 & 76,285 & 7.56 & 1.80 \\
\hline & Replace 2 T8 32W Lights with 2 Super T8 25W Lights & 1,284 & 818,851 & 572,171 & 83,008 & 6.89 & 2.40 \\
\hline
\end{tabular}




\begin{tabular}{|c|c|c|c|c|c|c|c|}
\hline Bldg. Set ID & Technology Change & $\begin{array}{c}\text { Energy } \\
\text { Savings } \\
\text { (MMBtu/yr) }\end{array}$ & $\begin{array}{l}\text { Net Present } \\
\text { Value (\$) }\end{array}$ & $\begin{array}{l}\text { Installed } \\
\text { Cost (\$) }\end{array}$ & $\begin{array}{c}\text { 1st year } \\
\text { savings (\$) }\end{array}$ & $\begin{array}{l}\text { Simple } \\
\text { Payback } \\
\text { (yr) }\end{array}$ & SIR \\
\hline \multirow{3}{*}{$10 d$} & $\begin{array}{l}\text { Replace } 40 \mathrm{~W} \text { Incandescent EXIT Lights with Electroluminescent } \\
\text { Panel }\end{array}$ & 38 & 39,699 & 1,864 & 2,465 & 0.76 & 22.30 \\
\hline & Replace 2 T8 32W Lights with 2 Super T8 25W Lights & 536 & 314,391 & 180,520 & 29,555 & 6.11 & 2.70 \\
\hline & Replace 2 T12 40W Lights with 2 Super T8 32W Lights & 10 & 9,466 & 1,693 & 664 & 2.55 & 6.60 \\
\hline \multirow{4}{*}{$10 \mathrm{e}$} & $\begin{array}{l}\text { Replace Electric Package Unit with Window AC Unit (ultra high } \\
\text { efficiency) }\end{array}$ & 96 & 12,594 & 152,253 & 15,158 & 10.04 & 1.20 \\
\hline & $\begin{array}{l}\text { Replace } 40 \mathrm{~W} \text { Incandescent EXIT Lights with Electroluminescent } \\
\text { Panel }\end{array}$ & 171 & 180,588 & 8,076 & 11,196 & 0.72 & 23.40 \\
\hline & Replace 2 T8 32W Lights with 2 Super T8 25W Lights & 64 & 22,682 & 38,324 & 3,658 & 10.48 & 1.60 \\
\hline & Suspended Ceiling: Increase Insulation by R-19 & 319 & 183,955 & 104,477 & 17,334 & 6.03 & 2.80 \\
\hline \multirow{5}{*}{$10 f$} & $\begin{array}{l}\text { Replace Electric Air-Cooled Chiller with Water-Cooled Chiller and } \\
\text { Cooling Tower (very high efficiency) }\end{array}$ & 821 & 230,892 & 251,246 & 44,753 & 5.61 & 2.50 \\
\hline & Wrap Tank with insulation & 1,020 & 778,332 & 36,477 & 49,939 & 0.73 & 15.60 \\
\hline & $\begin{array}{l}\text { Replace } 40 \mathrm{~W} \text { Incandescent EXIT Lights with Electroluminescent } \\
\text { Panel }\end{array}$ & 52 & 55,454 & 2,485 & 3,439 & 0.72 & 23.30 \\
\hline & Replace 3 T8 32W Lights with 2 Super T8 32W Lights & 1,107 & 807,796 & 279,792 & 65,067 & 4.30 & 3.90 \\
\hline & Replace 2 T8 32W Lights with 2 Super T8 25W Lights & 49 & 20,792 & 26,300 & 2,822 & 9.32 & 1.80 \\
\hline \multirow{2}{*}{$10 \mathrm{~g}$} & Replace LED EXIT Lights with Electroluminescent Panel & 6 & 13,497 & 6,523 & 1,163 & 5.61 & 3.10 \\
\hline & Replace 3 T8 32W Lights with 3 Super T8 25W Lights & 507 & 453,305 & 106,802 & 33,284 & 3.21 & 5.20 \\
\hline
\end{tabular}




\begin{tabular}{|c|c|c|c|c|c|c|c|}
\hline Bldg. Set ID & Technology Change & $\begin{array}{c}\text { Energy } \\
\text { Savings } \\
\text { (MMBtu/yr) }\end{array}$ & $\begin{array}{l}\text { Net Present } \\
\text { Value (\$) }\end{array}$ & $\begin{array}{l}\text { Installed } \\
\text { Cost }(\$)\end{array}$ & $\begin{array}{c}\text { 1st year } \\
\text { savings (\$) }\end{array}$ & $\begin{array}{l}\text { Simple } \\
\text { Payback } \\
\text { (yr) }\end{array}$ & SIR \\
\hline \multirow{5}{*}{$21 a$} & Wrap Tank with insulation, Aerators, LFSHs & 1,622 & $1,018,881$ & 104,979 & 81,745 & 1.28 & 8.80 \\
\hline & $\begin{array}{l}\text { Replace 40W Incandescent EXIT Lights with Electroluminescent } \\
\text { Panel }\end{array}$ & 216 & 234,671 & 11,182 & 14,579 & 0.77 & 22.00 \\
\hline & Replace 4 T12 40W Lights with 3 Super T8 32W Lights & 749 & 641,000 & 147,645 & 47,091 & 3.14 & 5.30 \\
\hline & Replace 2 T8 32W Lights with 2 Super T8 25W Lights & 150 & 128,641 & 51,726 & 10,719 & 4.83 & 3.50 \\
\hline & Attic Ceiling: Increase Insulation by R-13 (blow-in cellulose) & 23 & 4,093 & 17,876 & 1,321 & 13.53 & 1.20 \\
\hline \multirow{4}{*}{$23 a$} & $\begin{array}{l}\text { Replace Electric Air-Cooled Chiller with Water-Cooled Chiller and } \\
\text { Cooling Tower (very high efficiency) }\end{array}$ & 662 & 93,096 & 357,541 & 37,471 & 9.54 & 1.50 \\
\hline & $\begin{array}{l}\text { Replace } 40 \mathrm{~W} \text { Incandescent EXIT Lights with Electroluminescent } \\
\text { Panel }\end{array}$ & 53 & 55,601 & 2,485 & 3,447 & 0.72 & 23.40 \\
\hline & Replace 2 T12 40W Lights with 2 Super T8 30W Lights & 56 & 40,310 & 19,895 & 3,590 & 5.54 & 3.00 \\
\hline & Replace 2 T8 32W Lights with 2 Super T8 25W Lights & 32 & 20,631 & 12,805 & 1,997 & 6.41 & 2.60 \\
\hline \multirow{4}{*}{$30 a$} & $\begin{array}{l}\text { Replace Electric Air-Cooled Chiller with Water-Cooled Chiller and } \\
\text { Cooling Tower (very high efficiency) }\end{array}$ & 619 & 151,454 & 180,106 & 31,976 & 5.63 & 2.50 \\
\hline & Wrap Tank with insulation & 743 & 74,683 & 74,929 & 34,745 & 2.16 & 5.20 \\
\hline & $\begin{array}{l}\text { Replace } 40 \mathrm{~W} \text { Incandescent EXIT Lights with Electroluminescent } \\
\text { Panel }\end{array}$ & 118 & 125,587 & 5,591 & 7,786 & 0.72 & 23.50 \\
\hline & Replace 1 T8 32W Lights with 1 Super T8 25W Lights & 47 & 29,680 & 29,561 & 3,497 & 8.45 & 2.00 \\
\hline $30 b$ & $\begin{array}{l}\text { Replace Electric Air-Cooled Chiller with Water-Cooled Chiller and } \\
\text { Cooling Tower (very high efficiency) }\end{array}$ & 815 & 137,186 & 218,606 & 42,889 & 5.10 & 2.70 \\
\hline
\end{tabular}




\begin{tabular}{|c|c|c|c|c|c|c|c|}
\hline Bldg. Set ID & Technology Change & $\begin{array}{c}\text { Energy } \\
\text { Savings } \\
\text { (MMBtu/yr) }\end{array}$ & $\begin{array}{l}\text { Net Present } \\
\text { Value (\$) }\end{array}$ & $\begin{array}{l}\text { Installed } \\
\text { Cost (\$) }\end{array}$ & $\begin{array}{c}\text { 1st year } \\
\text { savings (\$) }\end{array}$ & $\begin{array}{l}\text { Simple } \\
\text { Payback } \\
\text { (yr) }\end{array}$ & SIR \\
\hline & $\begin{array}{l}\text { Replace Distillate Oil Central Boiler with Central Heat Pump Hot } \\
\text { Water System }\end{array}$ & 454 & 273,922 & 67,621 & 16,516 & 4.09 & 3.80 \\
\hline & $\begin{array}{l}\text { Replace } 40 \mathrm{~W} \text { Incandescent EXIT Lights with Electroluminescent } \\
\text { Panel }\end{array}$ & 184 & 193,347 & 8,386 & 11,977 & 0.70 & 24.10 \\
\hline \multirow{4}{*}{$30 c$} & $\begin{array}{l}\text { Replace Electric Air-Cooled Chiller with Water-Cooled Chiller and } \\
\text { Cooling Tower (very high efficiency) }\end{array}$ & 2832 & 343,656 & $1,529,812$ & 153,440 & 9.97 & 1.4 \\
\hline & Wrap Tank with insulation & 1,713 & 949,321 & 192,544 & 84,474 & 2.28 & 5.00 \\
\hline & Wrap Tank with insulation & 758 & 454,295 & 41,607 & 23,323 & 1.78 & 9.80 \\
\hline & $\begin{array}{l}\text { Replace } 40 \mathrm{~W} \text { Incandescent EXIT Lights with Electroluminescent } \\
\text { Panel }\end{array}$ & 959 & $1,048,312$ & 50,318 & 65,142 & 0.77 & 21.80 \\
\hline \multirow{4}{*}{$40 a$} & $\begin{array}{l}\text { Replace Electric Package Unit with Window AC Unit (ultra high } \\
\text { efficiency) }\end{array}$ & 102 & 5,999 & 57,342 & 6,184 & 9.27 & 1.20 \\
\hline & $\begin{array}{l}\text { Replace Electric Water Heater with Heat Pump Water Heater, } \\
\text { Aerators }\end{array}$ & 5 & 504 & 1,748 & 288 & 6.07 & 1.50 \\
\hline & $\begin{array}{l}\text { Wrap Tank with Insulation, Insulate Pipe Near Tank, Aerators, Lower } \\
\text { Tank Temperature }\end{array}$ & - & 85 & 73 & 22 & 3.32 & 4.20 \\
\hline & $\begin{array}{l}\text { Replace 400W Metal Halide Lights with 310W High Pressure Sodium } \\
\text { Lights }\end{array}$ & 82 & 26,921 & 50,532 & 4,650 & 10.87 & 1.50 \\
\hline \multirow{4}{*}{$40 b$} & $\begin{array}{l}\text { Wrap Tank with Insulation, Insulate Pipe Near Tank, LFSHs, Lower } \\
\text { Tank Temperature }\end{array}$ & 2 & 145 & 1,062 & 105 & 10.11 & 1.40 \\
\hline & $\begin{array}{l}\text { Wrap Tank with Insulation, Insulate Pipe Near Tank, LFSHs, Lower } \\
\text { Tank Temperature }\end{array}$ & 2 & 262 & 1,062 & 128 & 8.30 & 1.70 \\
\hline & $\begin{array}{l}\text { Replace } 40 \mathrm{~W} \text { Incandescent EXIT Lights with Electroluminescent } \\
\text { Panel }\end{array}$ & 33 & 38,642 & 2,174 & 2,415 & 0.90 & 18.80 \\
\hline & $\begin{array}{l}\text { Replace } 40 \mathrm{~W} \text { Incandescent EXIT Lights with Electroluminescent } \\
\text { Panel }\end{array}$ & 48 & 51.025 & 2174 & 3160 & 069 & 24.50 \\
\hline
\end{tabular}




\begin{tabular}{|c|c|c|c|c|c|c|c|}
\hline Bldg. Set ID & Technology Change & $\begin{array}{c}\text { Energy } \\
\text { Savings } \\
\text { (MMBtu/yr) }\end{array}$ & $\begin{array}{l}\text { Net Present } \\
\text { Value (\$) }\end{array}$ & $\begin{array}{l}\text { Installed } \\
\text { Cost (\$) }\end{array}$ & $\begin{array}{c}\text { 1st year } \\
\text { savings (\$) }\end{array}$ & $\begin{array}{l}\text { Simple } \\
\text { Payback } \\
\text { (yr) }\end{array}$ & SIR \\
\hline & Add Insulation to Interior Surface of Metal Roof: 4 inches Fiberglass & 1,297 & $1,005,482$ & 130,584 & 68,276 & 1.9 & 8.70 \\
\hline \multirow{5}{*}{$40 c$} & $\begin{array}{l}\text { Replace Electric Air-Cooled Chiller with Water-Cooled Chiller and } \\
\text { Cooling Tower (very high efficiency) }\end{array}$ & 1,503 & 126,015 & 625,995 & 80,900 & 7.74 & 1.80 \\
\hline & Replace LED EXIT Lights with Electroluminescent Panel & 4 & 9,529 & 4,659 & 824 & 5.65 & 3.00 \\
\hline & $\begin{array}{l}\text { Replace } 400 \text { W Metal Halide Lights with 310W High Pressure Sodium } \\
\text { Lights }\end{array}$ & 2,259 & $1,205,265$ & 634,806 & 110,803 & 5.73 & 2.90 \\
\hline & $\begin{array}{l}\text { Replace 250W Metal Halide Lights with 200W High Pressure Sodium } \\
\text { Lights }\end{array}$ & 245 & 148,979 & 190,109 & 20,025 & 9.49 & 1.80 \\
\hline & Replace LED EXIT Lights with Electroluminescent Panel & 3 & 8,573 & 4,659 & 766 & 6.08 & 2.80 \\
\hline \multirow{7}{*}{$50 a$} & $\begin{array}{l}\text { Wrap Tank with Insulation, Insulate Pipe Near Tank, Aerators, Lower } \\
\text { Tank Temperature }\end{array}$ & 2 & 430 & 335 & 109 & 3.07 & 4.50 \\
\hline & Replace LED EXIT Lights with Electroluminescent Panel & 4 & 12,574 & 6,833 & 1,124 & 6.08 & 2.80 \\
\hline & Replace LED EXIT Lights with Electroluminescent Panel & 5 & 13,149 & 6,833 & 1,158 & 5.90 & 2.90 \\
\hline & Replace 3 T8 32W Lights with 2 Super T8 32W Lights & 1,513 & $1,115,730$ & 146,985 & 75,676 & 1.94 & 8.60 \\
\hline & Replace 3 T8 32W Lights with 3 Super T8 25W Lights & 84 & 79,643 & 36,435 & 6,878 & 5.30 & 3.20 \\
\hline & $\begin{array}{l}\text { Replace 400W Metal Halide Lights with 310W High Pressure Sodium } \\
\text { Lights }\end{array}$ & 2,439 & 990,934 & 500,265 & 90,733 & 5.51 & 3.00 \\
\hline & $\begin{array}{l}\text { Wrap Tank with Insulation, Insulate Pipe Near Tank, Aerators, Lower } \\
\text { Tank Temperature }\end{array}$ & 5 & 1,158 & 335 & 253 & 1.32 & 10.50 \\
\hline $50 b$ & Replace $100 \mathrm{~W}$ Incandescent Lights with $26 \mathrm{~W}$ CFL Lights & 38 & 42,543 & 965 & 2,596 & 0.37 & 45.10 \\
\hline
\end{tabular}




\begin{tabular}{|c|c|c|c|c|c|c|c|}
\hline Bldg. Set ID & Technology Change & $\begin{array}{c}\text { Energy } \\
\text { Savings } \\
\text { (MMBtu/yr) }\end{array}$ & $\begin{array}{l}\text { Net Present } \\
\text { Value (\$) }\end{array}$ & $\begin{array}{l}\text { Installed } \\
\text { Cost (\$) }\end{array}$ & $\begin{array}{c}\text { 1st year } \\
\text { savings (\$) }\end{array}$ & $\begin{array}{l}\text { Simple } \\
\text { Payback } \\
\text { (yr) }\end{array}$ & SIR \\
\hline & Replace LED EXIT Lights with Electroluminescent Panel & 6 & 12,957 & 6,212 & 1,114 & 5.58 & 3.10 \\
\hline & Replace 4 T12 40W Lights with 3 Super T8 32W Lights & 4 & 2,386 & 2,021 & 263 & 7.68 & 2.20 \\
\hline & Add Insulation to Interior Surface of Metal Roof: 4 inches Fiberglass & 670 & 574,453 & 62,828 & 38,300 & 1.64 & 10.1 \\
\hline & Replace LED EXIT Lights with Electroluminescent Panel & 4 & 11,431 & 6,212 & 1,022 & 6.08 & 2.80 \\
\hline & Replace 4 T12 40W Lights with 3 Super T8 32W Lights & 668 & 539,907 & 232,446 & 46,058 & 5.05 & 3.30 \\
\hline \multirow{6}{*}{$50 c$} & $\begin{array}{l}\text { Replace Electric Air-Cooled Chiller with Water-Cooled Chiller and } \\
\text { Cooling Tower (ultra high efficiency) }\end{array}$ & 690 & 296,787 & 158,025 & 33,291 & 4.75 & 3.00 \\
\hline & Wrap Tank with Insulation and Insulate Pipe Near Tank & 1 & 65 & 168 & 27 & 6.22 & 1.40 \\
\hline & Replace LED EXIT Lights with Electroluminescent Panel & - & 627 & 311 & 54 & 5.76 & 3.00 \\
\hline & Replace 3 T8 32W Lights with 2 Super T8 32W Lights & 277 & 201,116 & 36,526 & 14,237 & 2.57 & 6.50 \\
\hline & Replace 2 T8 32W Lights with 2 Super T8 25W Lights & 174 & 90,829 & 49,621 & 8,435 & 5.88 & 2.80 \\
\hline & $\begin{array}{l}\text { Replace 1500W Metal Halide Lights with 1000W High Pressure } \\
\text { Sodium Lights }\end{array}$ & 97 & 99,752 & 36,110 & 8,009 & 4.51 & 3.80 \\
\hline \multirow{3}{*}{$50 d$} & Replace $100 \mathrm{~W}$ Incandescent Lights with $26 \mathrm{~W}$ CFL Lights & 94 & 88,642 & 25,797 & 6,795 & 3.80 & 4.40 \\
\hline & $\begin{array}{l}\text { Replace } 40 \mathrm{~W} \text { Incandescent EXIT Lights with Electroluminescent } \\
\text { Panel }\end{array}$ & 237 & 276,021 & 15,530 & 17,249 & 0.90 & 18.80 \\
\hline & Replace 1 T12 40W Lights with 1 Super T8 32W Lights & 136 & 40,029 & 188,354 & 13,457 & 14.00 & 1.20 \\
\hline
\end{tabular}




\begin{tabular}{|c|c|c|c|c|c|c|c|}
\hline Bldg. Set ID & Technology Change & $\begin{array}{c}\text { Energy } \\
\text { Savings } \\
\text { (MMBtu/yr) }\end{array}$ & $\begin{array}{l}\text { Net Present } \\
\text { Value (\$) }\end{array}$ & $\begin{array}{l}\text { Installed } \\
\text { Cost (\$) }\end{array}$ & $\begin{array}{c}\text { 1st year } \\
\text { savings (\$) }\end{array}$ & $\begin{array}{l}\text { Simple } \\
\text { Payback } \\
\text { (yr) }\end{array}$ & SIR \\
\hline & Add Insulation to Interior Surface of Metal Roof: 4 inches Fiberglass & 902 & 884,636 & 44,561 & 55,844 & 0.80 & 20.90 \\
\hline \multirow{6}{*}{$50 v$} & $\begin{array}{l}\text { Replace } 40 \mathrm{~W} \text { Incandescent EXIT Lights with Electroluminescent } \\
\text { Panel }\end{array}$ & 10 & 10,909 & 512 & 678 & 0.76 & 22.30 \\
\hline & $\begin{array}{l}\text { Replace } 40 \mathrm{~W} \text { Incandescent EXIT Lights with Electroluminescent } \\
\text { Panel }\end{array}$ & 59 & 63,430 & 2,904 & 3,936 & 0.74 & 22.80 \\
\hline & Replace T12 Magnetic Ballasts with T12 Electronic Ballast & 183 & 147,282 & 55,831 & 12,121 & 4.61 & 3.60 \\
\hline & Replace 4 T12 40W Lights with 3 Super T8 32W Lights & 636 & 532,150 & 150,383 & 40,735 & 3.69 & 4.50 \\
\hline & Replace 2 T12 40W Lights with 2 Super T8 32W Lights & 43 & 38,390 & 13,653 & 3,086 & 4.42 & 3.80 \\
\hline & Add Insulation to Interior Surface of Metal Roof: 4 inches Fiberglass & 1,235 & 610,435 & 311,584 & 55,412 & 5.62 & 3.00 \\
\hline \multirow{3}{*}{$60 a$} & $\begin{array}{l}\text { Replace Propane Central Boiler with Conventional Distillate Oil } \\
\text { Boiler, wrap tank with insulation }\end{array}$ & 522 & 340,737 & 56,604 & 14,521 & 3.90 & 7.60 \\
\hline & Replace $25 \mathrm{~W}$ Incandescent Lights with 5W CFL Lights & 206 & 101,167 & 70,815 & 10,326 & 6.86 & 2.40 \\
\hline & $\begin{array}{l}\text { Replace } 40 \mathrm{~W} \text { Incandescent EXIT Lights with Electroluminescent } \\
\text { Panel }\end{array}$ & 41 & 42,011 & 1,864 & 2,604 & 0.72 & 23.50 \\
\hline \multirow{4}{*}{$60 b$} & $\begin{array}{l}\text { Replace Electric Air-Cooled Chiller with Water-Cooled Chiller and } \\
\text { Cooling Tower (very high efficiency) }\end{array}$ & 989 & 353,402 & 760,855 & 96,296 & 7.90 & 1.80 \\
\hline & Wrap Tank with Insulation & 136 & 13,926 & 6,414 & 4,373 & 1.47 & 3.20 \\
\hline & Replace $75 \mathrm{~W}$ Incandescent Lights with 18W CFL Lights & 287 & 293,399 & 8,688 & 17,961 & 0.48 & 34.80 \\
\hline & $\begin{array}{l}\text { Replace } 40 \mathrm{~W} \text { Incandescent EXIT Lights with Electroluminescent } \\
\text { Panel }\end{array}$ & 136 & 143673 & 6212 & 8899 & 070 & 2410 \\
\hline
\end{tabular}




\begin{tabular}{|c|c|c|c|c|c|c|c|}
\hline Bldg. Set ID & Technology Change & $\begin{array}{c}\text { Energy } \\
\text { Savings } \\
\text { (MMBtu/yr) }\end{array}$ & $\begin{array}{l}\text { Net Present } \\
\text { Value (\$) }\end{array}$ & $\begin{array}{l}\text { Installed } \\
\text { Cost (\$) }\end{array}$ & $\begin{array}{c}\text { 1st year } \\
\text { savings (\$) }\end{array}$ & $\begin{array}{l}\text { Simple } \\
\text { Payback } \\
\text { (yr) }\end{array}$ & SIR \\
\hline & Replace 3 T8 32W Lights with 3 Super T8 25W Lights & 77 & 48,227 & 47,123 & 5,640 & 8.36 & 2.00 \\
\hline & Suspended Ceiling: Increase Insulation by R-19 & 191 & 44,282 & 172,935 & 13,054 & 13.25 & 1.30 \\
\hline \multirow{5}{*}{$60 c$} & Replace Electric Water Heater with Heat Pump Water Heater & 455 & 43,139 & 133,662 & 22,976 & 5.82 & 1.60 \\
\hline & $\begin{array}{l}\text { Replace } 40 \mathrm{~W} \text { Incandescent EXIT Lights with Electroluminescent } \\
\text { Panel }\end{array}$ & 53 & 61,092 & 3,355 & 3,814 & 0.88 & 19.20 \\
\hline & $\begin{array}{l}\text { Replace 40W Incandescent EXIT Lights with Electroluminescent } \\
\text { Panel }\end{array}$ & 6 & 6,789 & 373 & 424 & 0.88 & 19.20 \\
\hline & Replace 4 T12 40W Lights with 3 Super T8 32W Lights & 3,470 & $2,737,402$ & 686,561 & 204,230 & 3.36 & 5.00 \\
\hline & Replace 4 T12 40W Lights with 3 Super T8 32W Lights & 366 & 289,499 & 88,090 & 22,512 & 3.91 & 4.30 \\
\hline $60 m$ & $\begin{array}{l}\text { Replace 40W Incandescent EXIT Lights with Electroluminescent } \\
\text { Panel }\end{array}$ & 194 & 226,336 & 12,735 & 14,144 & 0.90 & 18.80 \\
\hline \multirow{5}{*}{$60 t$} & $\begin{array}{l}\text { Replace Electric Air-Cooled Chiller with Water-Cooled Chiller and } \\
\text { Cooling Tower (very high efficiency) }\end{array}$ & 217 & 64,113 & 85,505 & 14,146 & 6.04 & 2.30 \\
\hline & Replace Electric Water Heater with Heat Pump Water Heater & 21 & 3,090 & 3,427 & 982 & 3.49 & 2.70 \\
\hline & $\begin{array}{l}\text { Replace 40W Incandescent EXIT Lights with Electroluminescent } \\
\text { Panel }\end{array}$ & 13 & 14,052 & 621 & 871 & 0.71 & 23.60 \\
\hline & Replace 4 T12 40W Lights with 3 Super T8 32W Lights & 811 & 664,582 & 87,739 & 44,944 & 1.95 & 8.60 \\
\hline & Suspended Ceiling: Increase Insulation by R-19 & 194 & 115,027 & 79,435 & 11,687 & 6.80 & 2.40 \\
\hline $80 a$ & Replace Electric Water Heater with Heat Pump Water Heater & 616 & 28,256 & 51,408 & 36,325 & 1.42 & 6.60 \\
\hline
\end{tabular}




\begin{tabular}{|c|c|c|c|c|c|c|c|}
\hline Bldg. Set ID & Technology Change & $\begin{array}{c}\text { Energy } \\
\text { Savings } \\
\text { (MMBtu/yr) }\end{array}$ & $\begin{array}{l}\text { Net Present } \\
\text { Value }(\$)\end{array}$ & $\begin{array}{l}\text { Installed } \\
\text { Cost }(\$)\end{array}$ & $\begin{array}{l}\text { 1st year } \\
\text { savings (\$) }\end{array}$ & $\begin{array}{l}\text { Simple } \\
\text { Payback } \\
\text { (yr) }\end{array}$ & SIR \\
\hline & Replace LED EXIT Lights with Electroluminescent Panel & 4 & 9,192 & 4,659 & 803 & 5.80 & 3.00 \\
\hline & Replace 4 T12 40W Lights with 4 T8 32W Lights & 58 & 44,649 & 24,602 & 4,113 & 5.98 & 2.80 \\
\hline & Replace 3 T8 32W Lights with 3 Super T8 25W Lights & 399 & 244,588 & 316,737 & 33,176 & 9.55 & 1.80 \\
\hline & $\begin{array}{l}\text { Replace Metal Halide Magnetic Ballast with Metal Halide Electronic } \\
\text { Ballasts }\end{array}$ & 20 & 34,805 & 20,642 & 3,222 & 6.41 & 2.70 \\
\hline \multirow{6}{*}{$80 \mathrm{~b}$} & $\begin{array}{l}\text { Replace existing Package Unit with Single Zone Package Unit (very } \\
\text { high efficiency) }\end{array}$ & 895 & 41,361 & 179,467 & 60,318 & 3 & 3.80 \\
\hline & Wrap Tank with Insulation, Insulate Pipe Near Tank, Aerators & 54 & 2,370 & 3,024 & 2,801 & 1.08 & 13.00 \\
\hline & $\begin{array}{l}\text { Replace 40W Incandescent EXIT Lights with Electroluminescent } \\
\text { Panel }\end{array}$ & 117 & 121,348 & 4,970 & 7,503 & 0.66 & 25.40 \\
\hline & Replace 3 T8 32W Lights with 2 Super T8 32W Lights & 376 & 336,340 & 75,078 & 24,493 & 3.07 & 5.50 \\
\hline & Replace 2 T12 40W Lights with 2 Super T8 30W Lights & 36 & 24,408 & 13,129 & 2,238 & 5.87 & 2.90 \\
\hline & Replace 2 T8 32W Lights with 2 Super T8 25W Lights & 231 & 175,954 & 95,237 & 16,106 & 5.91 & 2.80 \\
\hline \multirow{4}{*}{$80 c$} & Wrap Tank with Insulation & 2 & 193 & 160 & 89 & 1.80 & 2.20 \\
\hline & Replace $75 \mathrm{~W}$ Incandescent Lights with 18W CFL Lights & 73 & 84,112 & 1,564 & 5,098 & 0.31 & 54.80 \\
\hline & Replace $100 \mathrm{~W}$ Incandescent Lights with 26W CFL Lights & 10 & 11,543 & 174 & 698 & 0.25 & 67.40 \\
\hline & $\begin{array}{l}\text { Replace 40W Incandescent EXIT Lights with Electroluminescent } \\
\text { Panel }\end{array}$ & 40 & 42,260 & 1,864 & 2,619 & 0.71 & 23.70 \\
\hline
\end{tabular}




\begin{tabular}{|c|c|c|c|c|c|c|c|}
\hline Bldg. Set ID & Technology Change & $\begin{array}{c}\text { Energy } \\
\text { Savings } \\
\text { (MMBtu/yr) }\end{array}$ & $\begin{array}{l}\text { Net Present } \\
\text { Value (\$) }\end{array}$ & $\begin{array}{l}\text { Installed } \\
\text { Cost (\$) }\end{array}$ & $\begin{array}{c}\text { 1st year } \\
\text { savings (\$) }\end{array}$ & $\begin{array}{l}\text { Simple } \\
\text { Payback } \\
\text { (yr) }\end{array}$ & SIR \\
\hline & Replace 2 T8 32W Lights with 2 Super T8 25W Lights & 7 & 4,563 & 4,027 & 509 & 7.91 & 2.10 \\
\hline & Replace 3 T8 32W Lights with 3 Super T8 25W Lights & 5 & 4,142 & 3,006 & 423 & 7.11 & 2.40 \\
\hline & Replace 2 T12 40W Lights with 2 Super T8 32W Lights & 37 & 28,251 & 9,478 & 2,237 & 4.24 & 4.00 \\
\hline & Insulate Built-up Roof Surface (R-15) and Re-Roof & 158 & 116,314 & 59,235 & 10,550 & 5.61 & 3.00 \\
\hline \multirow{6}{*}{$80 d$} & $\begin{array}{l}\text { Replace Electric Air-Cooled Chiller with Water-Cooled Chiller and } \\
\text { Cooling Tower (very high efficiency) }\end{array}$ & 525 & 155,727 & 112,069 & 27,663 & 4.05 & 3.40 \\
\hline & Wrap Tank with Insulation, Insulate Pipe Near Tank, Aerators & 22 & 3,041 & 588 & 815 & 0.72 & 17.80 \\
\hline & $\begin{array}{l}\text { Replace } 40 \mathrm{~W} \text { Incandescent EXIT Lights with Electroluminescent } \\
\text { Panel }\end{array}$ & 13 & 14,174 & 621 & 878 & 0.71 & 23.80 \\
\hline & Replace 4 T12 40W Lights with 3 Super T8 32W Lights & 120 & 106,019 & 11,663 & 7,027 & 1.66 & 10.10 \\
\hline & Replace 2 T12 40W Lights with 2 Super T8 25W Lights & 43 & 28,117 & 14,429 & 2,541 & 5.68 & 2.90 \\
\hline & $\begin{array}{l}\text { Install Thermal Break Aluminum Frame Double Pane Super Low-e } \\
\text { Window }\end{array}$ & 31 & 6,120 & 23,598 & 1,786 & 13.21 & 1.30 \\
\hline
\end{tabular}




\section{Appendix C-2 \\ Comprehensive List of Cost-Effective Projects Identified from the FEDS Assessment Using Alternative Financing Sources of Capital}

Table C-2 identifies the 88 cost-effective energy- and cost-reducing retrofit projects identified from the FEDS modeling and analysis based on the assumption that they will be funded using alternative financing source of capital funds. Alternative financing includes UESC and ESPC, as well as any other third party financing. Key energy and economic results are presented for each cost-effective retrofit measure. The projects are grouped by building category. 
Table C-2 Comprehensive List of Cost-Effective Projects Using Alternative Financing Sources of Capital

\begin{tabular}{|c|c|c|c|c|c|c|}
\hline FEDS Category & Technology Change & $\begin{array}{c}\text { Energy } \\
\text { Savings } \\
\text { (MMBtu/yr) }\end{array}$ & $\begin{array}{r}1 \text { st yeal } \\
\text { savings }(\end{array}$ & $\begin{array}{l}\text { Installed } \\
\text { Cost }(\$)\end{array}$ & $\begin{array}{l}\text { Net Present } \\
\text { Value }(\$)\end{array}$ & SIR \\
\hline 1 & Replace 175W Metal Halide Lights with 4 Super T8 30W Lights & 365 & 29,120 & 62,537 & 109,318 & 2.70 \\
\hline \multirow{7}{*}{ 10b } & Replace $75 \mathrm{~W}$ Incandescent Lights with 18W CFL Lights & 1,708 & 112,610 & 18,316 & 638,124 & 35.80 \\
\hline & Replace LED EXIT Lights with Electroluminescent EXIT Lights & 10 & 2,156 & 11,182 & 1,872 & 1.20 \\
\hline & Faucet Aerators & 39 & 2,126 & 429 & 11,775 & 28.50 \\
\hline & Faucet Aerators, Lower Tank Temperature & - & 11 & 4 & 60 & 17.20 \\
\hline & Faucet Aerators, Lower Tank Temperature & - & 17 & 8 & 93 & 13.10 \\
\hline & Replace 4 T12 40W Lights with 4 T8 32W Lights & 33 & 2,441 & 6,190 & 8,088 & 2.30 \\
\hline & Replace 175W Metal Halide Lights with 4 Super T8 30W Lights & 35 & 3,182 & 11,463 & 7,323 & 1.60 \\
\hline $10 c$ & Replace 2 T8 32W Lights with 2 Super T8 25W Lights & 1,402 & 116,224 & 572,171 & 110,933 & 1.20 \\
\hline \multirow[t]{2}{*}{$10 \mathrm{~d}$} & Replace 40W Incandescent EXIT Lights with Electroluminescent EXIT Lights & 38 & 2,489 & 1,864 & 12,719 & 7.80 \\
\hline & Replace 2 T12 40W Lights with 2 T8 32W Lights & 10 & 679 & 1,693 & 2,274 & 2.30 \\
\hline \multirow{2}{*}{$10 \mathrm{e}$} & Replace 40W Incandescent EXIT Lights with Electroluminescent EXIT Lights & 173 & 11,434 & 8,076 & 58,838 & 8.30 \\
\hline & Suspended Ceiling: Increase Insulation by R-11 & 301 & 18,388 & 79,922 & 25,635 & 1.30 \\
\hline $10 f$ & $\begin{array}{l}\text { Replace Electric Central Boiler with a Central Heat Pump Hot Water System, } \\
\text { Wrap Tank with Insulation }\end{array}$ & 1,020 & 49,939 & 36,477 & 250,499 & 7.90 \\
\hline
\end{tabular}




\begin{tabular}{|c|c|c|c|c|c|c|}
\hline FEDS Category & Technology Change & $\begin{array}{l}\text { Energy } \\
\text { Savings } \\
\text { (MMBtu/yr) }\end{array}$ & $\begin{array}{c}\text { 1st year } \\
\text { savings (\$) }\end{array}$ & $\begin{array}{l}\text { Installed } \\
\text { Cost (\$) }\end{array}$ & $\begin{array}{l}\text { Net Present } \\
\text { Value (\$) }\end{array}$ & SIR \\
\hline & Replace 40W Incandescent EXIT Lights with Electroluminescent EXIT Lights & 52 & 3,470 & 2,485 & 17,826 & 8.20 \\
\hline & Replace 3 T8 32W Lights with 2 Super T8 32W Lights & 1,107 & 61,136 & 279,792 & 72,136 & 1.30 \\
\hline \multirow[t]{2}{*}{$10 \mathrm{~g}$} & Replace LED EXIT Lights with Electroluminescent EXIT Lights & 6 & 1,270 & 6,523 & 1,162 & 1.20 \\
\hline & Replace 3 T8 32W Lights with 3 Super T8 25W Lights & 507 & 37,937 & 106,802 & 116,142 & 2.10 \\
\hline \multirow{4}{*}{$21 a$} & $\begin{array}{l}\text { Replace Electric Central Boiler with a Central Heat Pump Hot Water System, } \\
\text { Wrap Tank with Insulation, Aerators, LFSH }\end{array}$ & 1,622 & 81,745 & 104,979 & 364,597 & 4.50 \\
\hline & Replace 40W Incandescent EXIT Lights with Electroluminescent EXIT Lights & 217 & 14,717 & 11,182 & 75,065 & 7.70 \\
\hline & Replace 4 T12 40W Lights with 4 T8 32W Lights & 526 & 37,484 & 67,589 & 151,218 & 3.20 \\
\hline & Replace 2 T8 32W Lights with 2 Super T8 25W Lights & 151 & 12,812 & 51,726 & 23,706 & 1.50 \\
\hline \multirow{3}{*}{$23 a$} & Replace 40W Incandescent EXIT Lights with Electroluminescent EXIT Lights & 56 & 3,661 & 2,485 & 18,920 & 8.60 \\
\hline & Replace 2 T8 32W Lights with 2 Super T8 25W Lights & 34 & 2,727 & 12,805 & 3,200 & 1.20 \\
\hline & Replace 2 T12 40W Lights with 2 T8 32W Lights & 46 & 3,444 & 11,833 & 8,330 & 1.70 \\
\hline \multirow{2}{*}{$30 a$} & $\begin{array}{l}\text { Replace Electric Central Boiler with a Central Heat Pump Hot Water System, } \\
\text { Wrap Tank with Insulation }\end{array}$ & 743 & 34,745 & 74,929 & 124,587 & 2.70 \\
\hline & Replace 40W Incandescent EXIT Lights with Electroluminescent EXIT Lights & 117 & 7,810 & 5,591 & 40,123 & 8.20 \\
\hline \multirow{2}{*}{$30 b$} & $\begin{array}{l}\text { Replace Distillate Oil Central Boiler with a Central Heat Pump Hot Water } \\
\text { System }\end{array}$ & 454 & 16,516 & 67,621 & 34,985 & 1.50 \\
\hline & Replace 40W Incandescent EXIT Lights with Electroluminescent EXIT Lights & 184 & 12,055 & 8,386 & 62,138 & 8.40 \\
\hline $30 c$ & Replace Electric Central Boiler with a Central Heat Pump Hot Water System & & & & & \\
\hline
\end{tabular}




\begin{tabular}{|c|c|c|c|c|c|c|}
\hline FEDS Category & Technology Change & $\begin{array}{c}\text { Energy } \\
\text { Savings } \\
\text { (MMBtu/yr) }\end{array}$ & $\begin{array}{c}\text { 1st year } \\
\text { savings (\$) }\end{array}$ & $\begin{array}{l}\text { Installed } \\
\text { Cost (\$) }\end{array}$ & $\begin{array}{l}\text { Net Present } \\
\text { Value (\$) }\end{array}$ & SIR \\
\hline & & 1,712 & 84,422 & 192,033 & 293,507 & 2.50 \\
\hline & $\begin{array}{l}\text { Replace Propane Central Boiler with a Central Heat Pump Hot Water System, } \\
\text { Wrap Tank with Insulation }\end{array}$ & 758 & 23,323 & 41,607 & 131,676 & 4.20 \\
\hline & Replace 40W Incandescent EXIT Lights with Electroluminescent EXIT Lights & 1,061 & 70,862 & 50,318 & 364,412 & 8.20 \\
\hline \multirow{2}{*}{$40 a$} & Faucet Aerators, Lower Tank Temperature & 2 & 98 & 40 & 522 & 14.00 \\
\hline & Faucet Aerators, Lower Tank Temperature & - & 15 & 17 & 67 & 4.90 \\
\hline \multirow{2}{*}{$40 \mathrm{~b}$} & Replace 40W Incandescent EXIT Lights with Electroluminescent EXIT Lights & 33 & 2,443 & 2,174 & 12,192 & 6.60 \\
\hline & Replace 40W Incandescent EXIT Lights with Electroluminescent EXIT Lights & 48 & 3,175 & 2,174 & 16,395 & 8.50 \\
\hline \multirow[t]{2}{*}{$40 c$} & Replace LED EXIT Lights with Electroluminescent EXIT Lights & 5 & 924 & 4,659 & 923 & 1.20 \\
\hline & Replace 400W Metal Halide Light with 310W High Pressure Sodium Light & 2,493 & 128,874 & 634,806 & 105,504 & 1.20 \\
\hline \multirow{4}{*}{$50 a$} & Faucet Aerators, Lower Tank Temperature & 4 & 220 & 89 & 1,175 & 14.30 \\
\hline & Faucet Aerators, Lower Tank Temperature & 1 & 77 & 89 & 349 & 4.90 \\
\hline & Replace 3 T8 32W Lights with 2 Super T8 32W Lights & 1,513 & 79,041 & 146,985 & 309,936 & 3.10 \\
\hline & Replace 400W Metal Halide Light with 310W High Pressure Sodium Light & 2,439 & 119,609 & 500,265 & 188,322 & 1.40 \\
\hline \multirow{2}{*}{$50 \mathrm{~b}$} & Replace 4 T12 40W Lights with 4 T8 32W Lights & 469 & 37,100 & 106,410 & 110,668 & 2.00 \\
\hline & Replace $100 \mathrm{~W}$ Incandescent Lights with 26W CFL Lights & 38 & 2,611 & 965 & 14,194 & 15.70 \\
\hline
\end{tabular}




\begin{tabular}{|c|c|c|c|c|c|c|}
\hline FEDS Category & Technology Change & $\begin{array}{l}\text { Energy } \\
\text { Savings } \\
\text { (MMBtu/yr) }\end{array}$ & $\begin{array}{c}\text { 1st year } \\
\text { savings (\$) }\end{array}$ & $\begin{array}{l}\text { Installed } \\
\text { Cost (\$) }\end{array}$ & $\begin{array}{l}\text { Net Present } \\
\text { Value (\$) }\end{array}$ & SIR \\
\hline & Replace LED EXIT Lights with Electroluminescent EXIT Lights & 6 & 1,216 & 6,212 & 1,142 & 1.20 \\
\hline \multirow{5}{*}{$50 c$} & $\begin{array}{l}\text { Replace Air-Cooled Chiller with Water-Cooled Chiller and Cooling Tower (ultra } \\
\text { high efficiency) }\end{array}$ & 690 & 33,310 & 158,025 & 32,843 & 1.20 \\
\hline & Replace LED EXIT Lights with Electroluminescent EXIT Lights & - & 59 & 311 & 50 & 1.20 \\
\hline & Replace 3 T8 32W Lights with 2 Super T8 32W Lights & 277 & 16,480 & 36,526 & 59,374 & 2.60 \\
\hline & Replace 2 T8 32W Lights with 2 Super T8 25W Lights & 174 & 11,570 & 49,621 & 18,109 & 1.40 \\
\hline & Replace 1500W Metal Halide Light with 1000W High Pressure Sodium Light & 97 & 7,501 & 36,110 & 8,101 & 1.20 \\
\hline \multirow{2}{*}{$50 d$} & Replace $100 \mathrm{~W}$ Incandescent Lights with 26W CFL Lights & 94 & 6,764 & 25,797 & 13,735 & 1.50 \\
\hline & Replace 40W Incandescent EXIT Lights with Electroluminescent EXIT Lights & 237 & 17,450 & 15,530 & 87,088 & 6.60 \\
\hline \multirow{5}{*}{$50 v$} & Replace 40W Incandescent EXIT Lights with Electroluminescent EXIT Lights & 8 & 601 & 512 & 3,019 & 6.90 \\
\hline & Replace 40W Incandescent EXIT Lights with Electroluminescent EXIT Lights & 48 & 3,407 & 2,904 & 17,109 & 6.90 \\
\hline & Replace 4 T12 40W Lights with 4 T8 32W Lights & 366 & 27,067 & 68,843 & 89,460 & 2.30 \\
\hline & Replace 2 T12 40W Lights with 2 T8 32W Lights & 43 & 3,562 & 13,653 & 7,350 & 1.50 \\
\hline & Replace 2 T12 96W Magnetic Ballast with 2 T12 96W Electronic Ballast & 92 & 6,930 & 30,176 & 10,333 & 1.30 \\
\hline \multirow{2}{*}{$60 a$} & Wrap Tank with Insulation & 437 & 14,055 & 536 & 100,131 & 187.90 \\
\hline & Replace 40W Incandescent EXIT Lights with Electroluminescent EXIT Lights & 40 & 2,596 & 1,864 & 13,332 & 8.20 \\
\hline $60 \mathrm{~b}$ & Wrap Tank with Insulation & 136 & 4,373 & 6,414 & 20,924 & 3.00 \\
\hline
\end{tabular}




\begin{tabular}{|c|c|c|c|c|c|c|}
\hline FEDS Category & Technology Change & $\begin{array}{c}\text { Energy } \\
\text { Savings } \\
\text { (MMBtu/yr) }\end{array}$ & $\begin{array}{c}\text { 1st year } \\
\text { savings (\$) }\end{array}$ & $\begin{array}{l}\text { Installed } \\
\text { Cost (\$) }\end{array}$ & $\begin{array}{l}\text { Net Present } \\
\text { Value (\$) }\end{array}$ & SIR \\
\hline & Replace $75 \mathrm{~W}$ Incandescent Lights with $18 \mathrm{~W}$ CFL Lights & 317 & 19,410 & 8,688 & 104,442 & 13.00 \\
\hline & Replace 40W Incandescent EXIT Lights with Electroluminescent EXIT Lights & 151 & 9,649 & 6,212 & 50,154 & 9.10 \\
\hline \multirow{4}{*}{$60 c$} & Replace 40W Incandescent EXIT Lights with Electroluminescent EXIT Lights & 54 & 3,887 & 3,355 & 19,488 & 6.80 \\
\hline & Replace 40W Incandescent EXIT Lights with Electroluminescent EXIT Lights & 6 & 432 & 373 & 2,165 & 6.80 \\
\hline & Replace 4 T12 40W Lights with 4 T8 32W Lights & 2,411 & 165,766 & 314,297 & 656,171 & 3.10 \\
\hline & Replace 4 T12 40W Lights with 4 T8 32W Lights & 258 & 18,490 & 40,326 & 67,979 & 2.70 \\
\hline $60 \mathrm{~m}$ & Replace 40W Incandescent EXIT Lights with Electroluminescent EXIT Lights & 194 & 14,308 & 12,735 & 71,412 & 6.60 \\
\hline \multirow{4}{*}{$60 \mathrm{t}$} & Replace Electric Water Heater with Heat Pump Water Heater & 21 & 982 & 3,427 & 2,196 & 1.60 \\
\hline & Replace 40W Incandescent EXIT Lights with Electroluminescent EXIT Lights & 14 & 943 & 621 & 4,888 & 8.90 \\
\hline & Replace 4 T12 40W Lights with 3 Super T8 32W Lights & 911 & 51,498 & 87,739 & 210,894 & 3.40 \\
\hline & Suspended Ceiling: Increase Insulation by R-11 & 249 & 16,290 & 60,766 & 32,741 & 1.50 \\
\hline \multirow{2}{*}{$80 a$} & Replace Electric Water Heater with Heat Pump Water Heater & 616 & 36,325 & 51,408 & 156,953 & 4.10 \\
\hline & $\begin{array}{l}\text { Replace 150W Metal Halide Magnetic Ballast with 150W Metal Halide } \\
\text { Electronic Ballast }\end{array}$ & 20 & 4,641 & 20,642 & 7,534 & 1.40 \\
\hline \multirow[t]{2}{*}{$80 \mathrm{~b}$} & Replace Electric Package Unit with Single Zone Package Unit (high efficiency) & 960 & 63,405 & 175,291 & 193,476 & 2.10 \\
\hline & Faucet Aerators & 48 & 2,503 & 229 & 14,140 & 62.80 \\
\hline
\end{tabular}




\begin{tabular}{|c|c|c|c|c|c|c|}
\hline FEDS Category & Technology Change & $\begin{array}{l}\text { Energy } \\
\text { Savings } \\
\text { (MMBtu/yr) }\end{array}$ & $\begin{array}{c}\text { 1st year } \\
\text { savings (\$) }\end{array}$ & $\begin{array}{l}\text { Installed } \\
\text { Cost (\$) }\end{array}$ & $\begin{array}{l}\text { Net Present } \\
\text { Value (\$) }\end{array}$ & SIR \\
\hline & Replace 40W Incandescent EXIT Lights with Electroluminescent EXIT Lights & 116 & 7,522 & 4,970 & 38,996 & 8.80 \\
\hline & Replace 4 T8 32W Lights with 3 Super T8 32W Lights & 373 & 21,152 & 75,078 & 47,084 & 1.60 \\
\hline & Replace 2 T12 40W Lights with 2 T8 32W Lights & 27 & 1,958 & 7,809 & 3,652 & 1.50 \\
\hline \multirow{5}{*}{$80 c$} & Wrap Tank with Insulation & 2 & 89 & 160 & 288 & 2.10 \\
\hline & Replace $75 \mathrm{~W}$ Incandescent Lights with $18 \mathrm{~W}$ CFL Lights & 73 & 5,174 & 1,564 & 28,614 & 19.30 \\
\hline & Replace $100 \mathrm{~W}$ Incandescent Lights with 26W CFL Lights & 11 & 732 & 174 & 4,079 & 24.50 \\
\hline & Replace 40W Incandescent EXIT Lights with Electroluminescent EXIT Lights & 40 & 2,645 & 1,864 & 13,615 & 8.30 \\
\hline & Replace 2 T12 40W Lights with 2 T8 32W Lights & 37 & 2,627 & 9,478 & 6,027 & 1.60 \\
\hline \multirow{5}{*}{$80 d$} & $\begin{array}{l}\text { Replace Air-Cooled Chiller with Water-Cooled Chiller and Cooling Tower (very } \\
\text { high efficiency) }\end{array}$ & 545 & 28,381 & 116,055 & 46,546 & 1.40 \\
\hline & Wrap Tank with Insulation, Aerators & 21 & 783 & 296 & 4,510 & 16.20 \\
\hline & Replace 40W Incandescent EXIT Lights with Electroluminescent EXIT Lights & 13 & 884 & 621 & 4,551 & 8.30 \\
\hline & Replace 4 T12 40W Lights with 3 Super T8 32W Lights & 120 & 7,159 & 11,663 & 29,900 & 3.60 \\
\hline & Replace 2 T12 40W Lights with 2 T8 32W Lights & 28 & 2,059 & 5,820 & 6,258 & 2.10 \\
\hline
\end{tabular}




\section{Appendix D}

\section{Building Details}

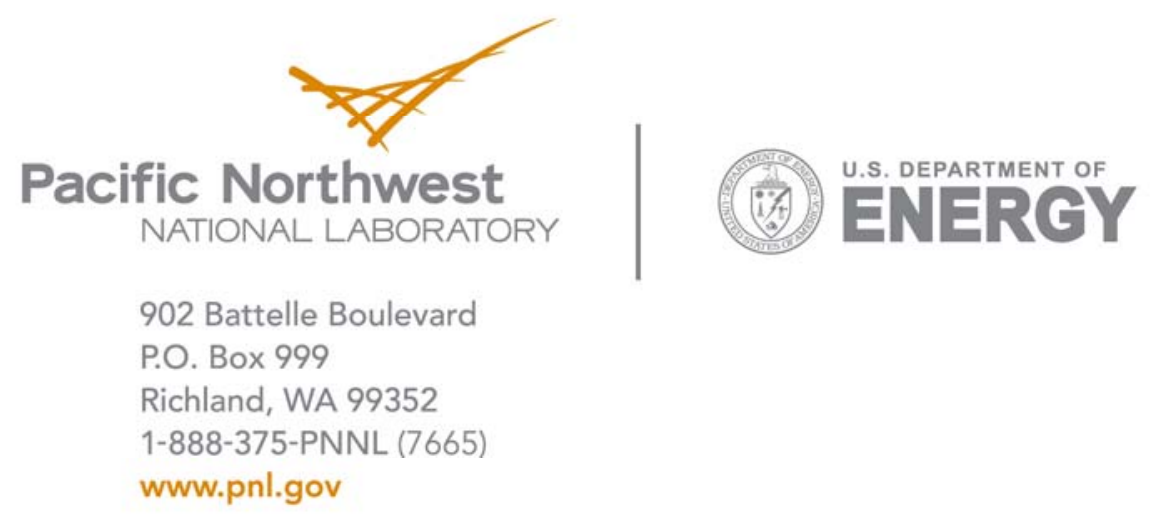




\section{Appendix D-1 \\ Energy Conservation Measures for Individual Buildings Appropriated Funding}

The following information identifies the cost-effective energy- and cost-reducing retrofit projects using appropriated funding for the buildings visited during the FEDS modeling and analysis. Key energy and economic results are presented for each cost-effective retrofit measure.

\section{Building 2186 Storage Building}

The following information identifies the cost-effective energy- and cost-reducing retrofit projects for building 2186 identified from the FEDS modeling and analysis. Key energy and economic results are presented for each cost-effective retrofit measure.

\section{Facility Description 2186}

Building 2186 is overhead storage for landscaping equipment built in 1986. 2186 has some lighting but no cooling or building envelope. Building 2186 is 2,125 sf. 


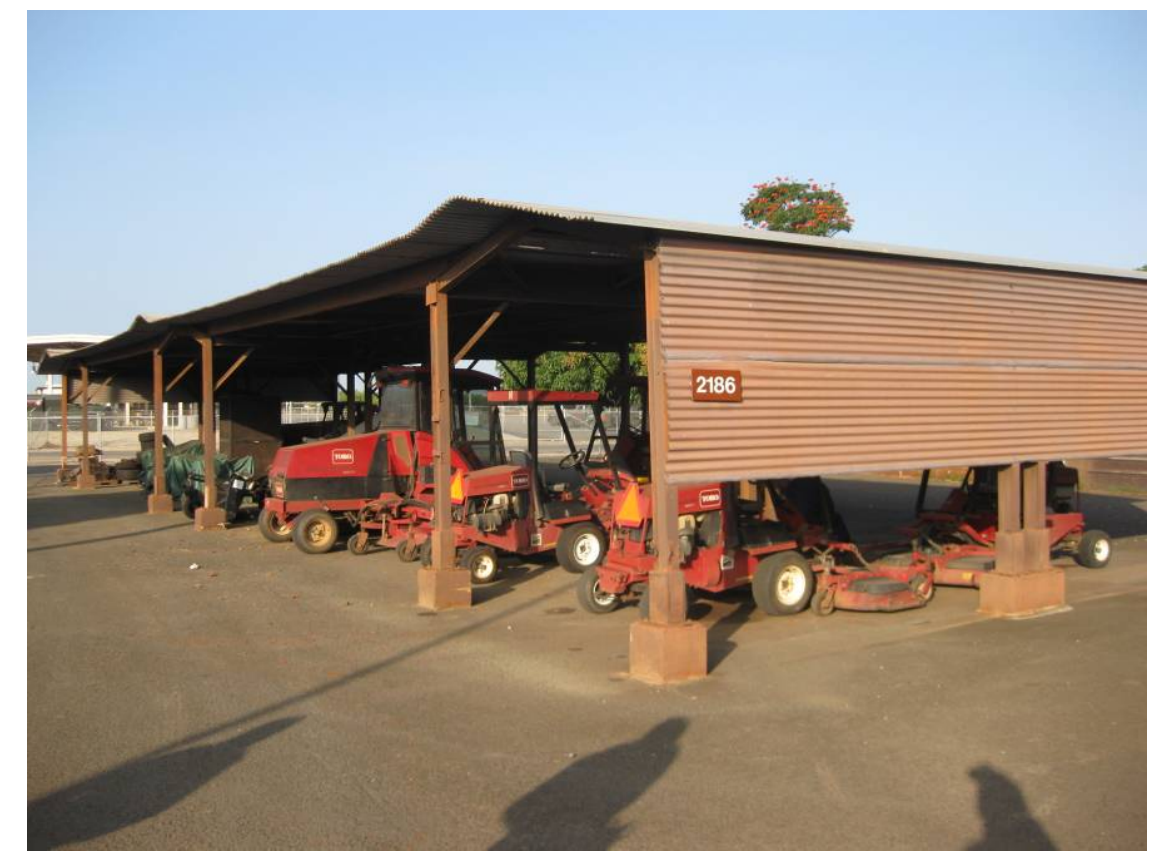

\section{Appropriated Funding Results}

FEDS did not find any life cycle cost effective retrofits using appropriated funding. 


\section{Appropriated Funding Energy Consumption by Fuel Type}

The modeled energy consumption for a typical year was 2,451 kwh before retrofits and 2,451 kwh after proposed retrofits are implemented. The energy use intensity goes from 3.9 MBtu/Ksf to 3.9 MBtu/Ksf after retrofits.

\begin{tabular}{|c|c|c|c|c|}
\hline \multirow[b]{2}{*}{ Fuel } & \multicolumn{3}{|c|}{ Covered lighting 2186} & \multirow[b]{2}{*}{$\begin{array}{l}\text { Dollars } \\
(2009)^{*}\end{array}$} \\
\hline & Energy & $\begin{array}{c}\text { Energy } \\
\text { Intensity } \\
\text { (user units/1000ft2) }\end{array}$ & $\begin{array}{c}\text { Energy } \\
\text { Intensity } \\
(\text { MBtu/1000ft2) }\end{array}$ & \\
\hline \multicolumn{5}{|c|}{ Electricity (kWh) } \\
\hline existing & 2,451 & $1,153.2$ & 3.9 & 434 \\
\hline post-retrofit & 2,451 & $1,153.2$ & 3.9 & 431 \\
\hline difference & 0 & ०.० & $\odot . \odot$ & -3 \\
\hline$\%$ change & $\odot$ & $\odot$ & $\odot$ & -1 \\
\hline \multicolumn{5}{|l|}{ Total (MBtu) } \\
\hline existing & 8 & 3.9 & 3.9 & 434 \\
\hline post-retrofit & 8 & 3.9 & 3.9 & 431 \\
\hline difference & $\odot$ & $\odot . \odot$ & $\odot . \odot$ & -3 \\
\hline$\%$ change & $\odot$ & $\odot$ & 0 & -1 \\
\hline
\end{tabular}




\section{Appropriated Funding Energy Consumption by End Use}

Motors and miscellaneous equipment is the largest load in the building with 2,451 kwh/year.

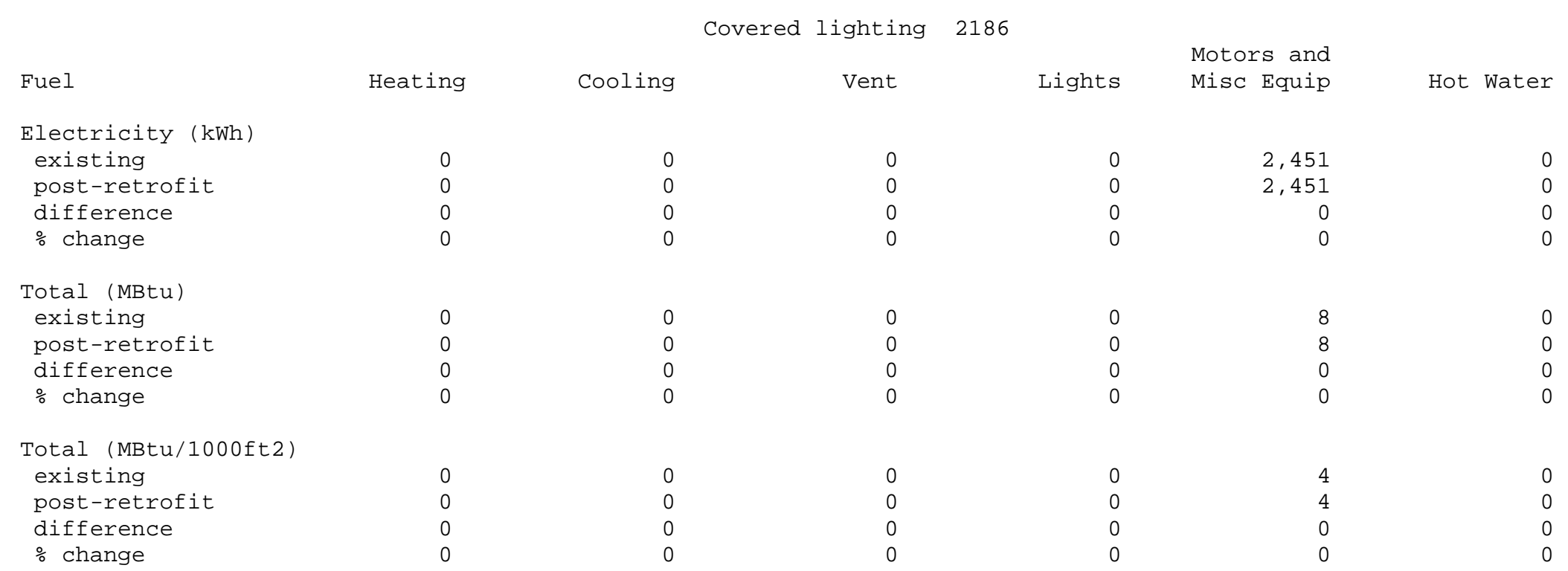




\section{Appropriated Funding Emission Reduction}

The emission reductions from implemented the proposed retrofits are as follows:

Covered lighting 2186

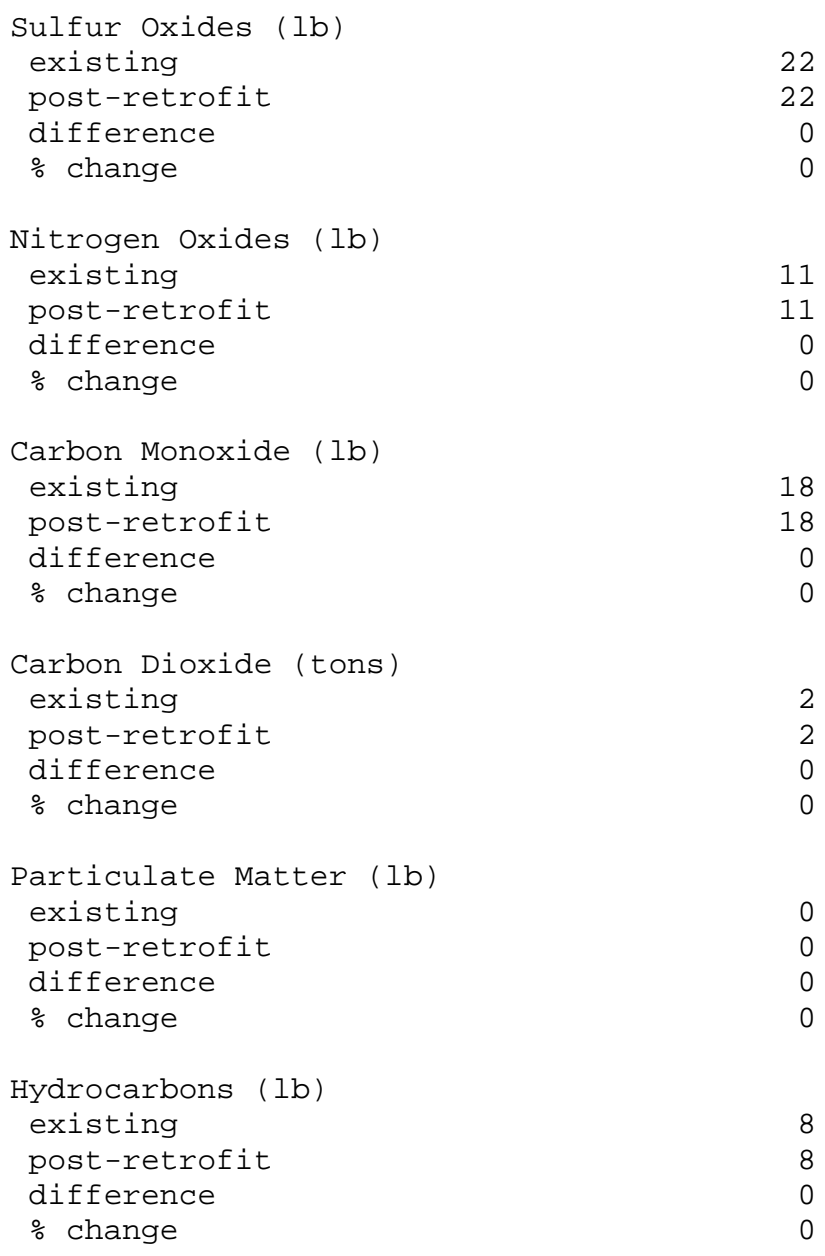




\section{Building 2035 Hanger}

The following information identifies the cost-effective energy- and cost-reducing retrofit projects for building 2035 identified from the FEDS modeling and analysis. Key energy and economic results are presented for each cost-effective retrofit measure.

\section{Facility Description 2035}

Building 2035 is a hangar with two high-bay spaces originally built in 1937. One of the high-bays has been converted to office space where an administration building has been built inside the hanger. This building inside a building is cooled by air cooled chillers and receives little to no solar radiation. The other high-bay is used to store and transport aircraft parts and has a small office space served by an electric DX, or package unit. Building 2035 is 86,391 sf.

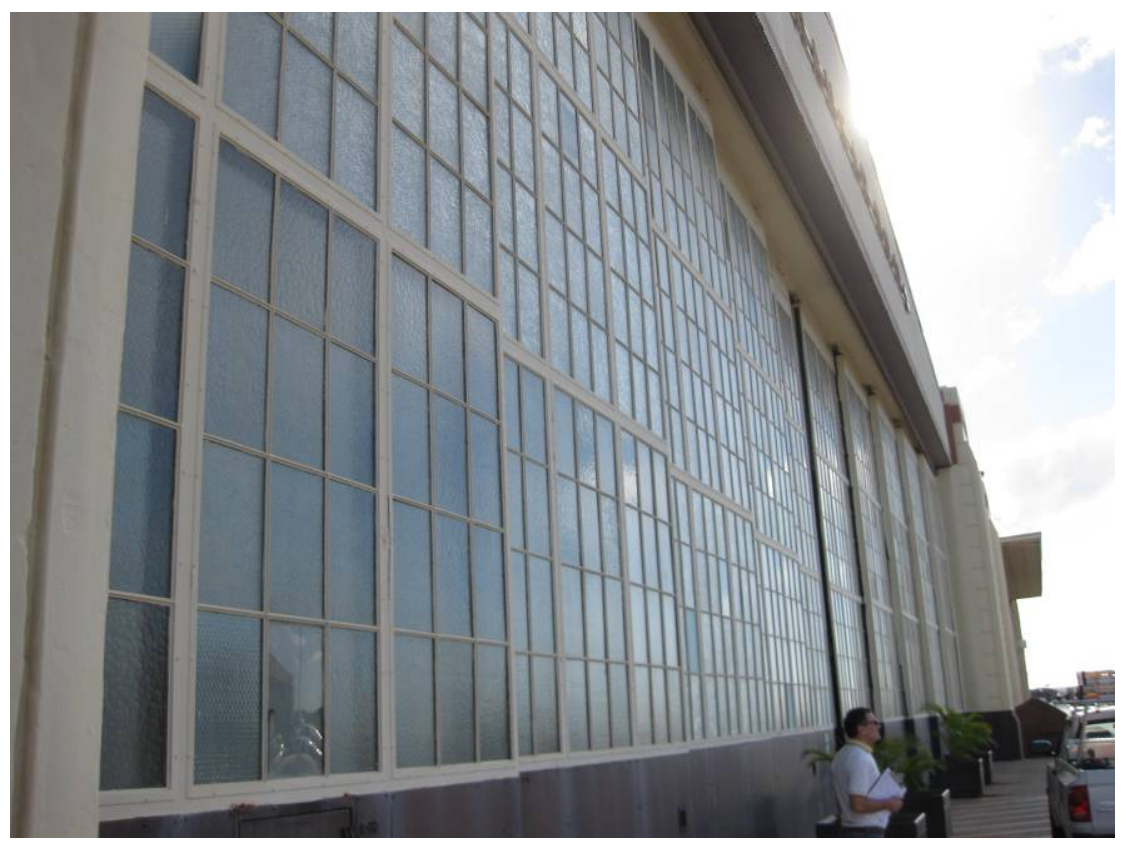




\section{Appropriated Funding Results}

A FEDS analysis using appropriated funding suggests replacing the air cooled chiller with a very high efficiency water cooled chiller for hangar 13. 32W T8 fluorescent lamps are suggested to be replaced with 28W SuperT8 lamps as well as other lighting retrofits. Suspended ceiling insulation is recommended to be increased as well as upgrades to the hot water system for hangar 13 . The FEDS analysis suggested replacing the lighting for hangar 11 as well as various upgrades to the hot water system.

Appropriated funding FEDS results for hangar 13 building 2035:

\begin{tabular}{|c|c|c|c|c|c|c|c|c|}
\hline Bldg. Set Description & $\begin{array}{l}\text { End } \\
\text { Use }\end{array}$ & Existing Technology & Retrofit Technology & $\begin{array}{l}\text { Energy } \\
\text { Savings } \\
\text { (MMBtu/yr) }\end{array}$ & $\begin{array}{l}\text { 1st year } \\
\text { savings } \\
(\$ / y r)\end{array}$ & $\begin{array}{l}\text { Installed } \\
\text { Cost (\$) }\end{array}$ & $\begin{array}{l}\text { Net } \\
\text { Present } \\
\text { Value (\$) }\end{array}$ & SIR \\
\hline $\begin{array}{l}\text { Large } 1930 \text { 's admin space } \\
2035 \text { hangar } 13\end{array}$ & Cooling & $\begin{array}{l}\text { Electric Air-Cooled Chiller } \\
\{C 1\}\end{array}$ & $\begin{array}{l}\text { Water-Cooled Reciprocating } \\
\text { Electric Chiller (very high } \\
\text { efficiency) and Cooling Tower }\end{array}$ & 255 & 16,564 & 94,307 & 79,487 & 2.5 \\
\hline $\begin{array}{l}\text { Large } 1930 \text { 's admin space } \\
2035 \text { hangar } 13\end{array}$ & Lights & $\begin{array}{l}\text { FL237: FL 2X4 3F32T8 ELC3 } \\
\text { REF }\end{array}$ & $\begin{array}{l}\text { FL296: FL 2X4 3F28ST8 ELC3 } \\
\text { REF }\end{array}$ & 20 & 1,038 & 10,283 & 7,011 & 1.7 \\
\hline $\begin{array}{l}\text { Large } 1930 \text { 's admin space } \\
2035 \text { hangar } 13\end{array}$ & Lights & EX6: EXIT - LED & $\begin{array}{l}\text { EX12: EXIT - } \\
\text { ELECTROLUMINESCENT PANEL } \\
\text { RETRO KIT }\end{array}$ & 2 & 330 & 1,864 & 3,809 & 3.0 \\
\hline $\begin{array}{l}\text { Large } 1930 \text { 's admin space } \\
2035 \text { hangar } 13\end{array}$ & Lights & IN8: INC 75 CEIL & $\begin{array}{l}\text { CF5: CFL } 18 \text { INTEGRAL UNIT } \\
\text { ELC }\end{array}$ & 317 & 20,921 & 3,373 & 348,237 & \#\#\#\# \\
\hline $\begin{array}{l}\text { Large } 1930 \text { 's admin space } \\
2035 \text { hangar } 13\end{array}$ & $\begin{array}{l}\text { Hot } \\
\text { Water }\end{array}$ & Electric Water Heater & $\begin{array}{l}\text { Wrap Tank with Insulation, } \\
\text { Insulate Pipe Near Tank, } \\
\text { Aerators }\end{array}$ & 8 & 451 & 526 & 2,091 & 11.9 \\
\hline
\end{tabular}


Appropriated funding FEDS results for hangar 11 building 2035:

\begin{tabular}{|c|c|c|c|c|c|c|c|c|}
\hline Bldg. Set Description & $\begin{array}{l}\text { End } \\
\text { Use }\end{array}$ & Existing Technology & Retrofit Technology & $\begin{array}{l}\text { Energy } \\
\text { Savings } \\
\text { (MMBtu/yr) }\end{array}$ & $\begin{array}{l}\text { 1st year } \\
\text { savings } \\
(\$ / y r)\end{array}$ & $\begin{array}{l}\text { Installed } \\
\text { Cost (\$) }\end{array}$ & $\begin{array}{l}\text { Net } \\
\text { Present } \\
\text { Value (\$) }\end{array}$ & SIR \\
\hline $\begin{array}{l}1930 \text { 's wharehouse space } \\
\text { hangar } 11\end{array}$ & Lights & FL1: FL 2X4 4F40T12 STD2 & $\begin{array}{l}\text { FL280: FL 2X4 3F32ST8 ELC3 } \\
\text { REF (FIX REPL) }\end{array}$ & 9 & 565 & 2,491 & 6,986 & 3.8 \\
\hline $\begin{array}{l}1930 \text { 's wharehouse space } \\
\text { hangar } 11\end{array}$ & Lights & MH4: MH 175 PEND & $\begin{array}{l}\text { FL289: FL 2X4 4F30ST8 ELC2 } \\
\text { REF }\end{array}$ & 6 & 513 & 2,111 & 6,558 & 4.1 \\
\hline $\begin{array}{l}1930 \text { 's wharehouse space } \\
\text { hangar } 11\end{array}$ & $\begin{array}{l}\text { Hot } \\
\text { Water }\end{array}$ & Electric Water Heater & $\begin{array}{l}\text { Wrap Tank with Insulation, } \\
\text { Insulate Pipe Near Tank, } \\
\text { Aerators, Lower Tank } \\
\text { Temperature }\end{array}$ & - & 2 & 6 & 11 & 5.9 \\
\hline $\begin{array}{l}\text { 1930's wharehouse space } \\
\text { hangar } 11\end{array}$ & $\begin{array}{l}\text { Hot } \\
\text { Water }\end{array}$ & Electric Water Heater & $\begin{array}{l}\text { Wrap Tank with Insulation, } \\
\text { Insulate Pipe Near Tank, } \\
\text { Aerators, Lower Tank } \\
\text { Temperature }\end{array}$ & - & 4 & 8 & 17 & 7.1 \\
\hline
\end{tabular}




\section{Appropriated Funding Energy Consumption by Fuel Type}

The modeled energy consumption for the administration space for a typical year was 795,887 kwh before retrofits and 593,955 kwh after proposed retrofits are implemented. The energy use intensity goes from $62.9 \mathrm{MBtu} / \mathrm{Ksf}$ to $46.9 \mathrm{MBtu} / \mathrm{Ksf}$ after retrofits.

Large 1930 's admin space 2035 hangar 13

Fuel

Electricity (kWh)

existing

post-retrofit

difference

$\%$ change

Total (MBtu)

existing

post-retrofit

difference

$\%$ change
Energy

795,887

593,955

$-201,932$

$-25$

2, 716

2,027

-689
-25
Energy

Intensity

(user units/1000ft2)

$18,425.9$

$13,750.9$

$-4,675.0$

$-25$

62.9

46.9

$-16.0$

$-25$
Energy

Intensity (MBtu/1000ft2)

Dollars

$(20 \odot 9)$ *

141,087

$\begin{array}{rr}46.9 & 104,498 \\ -16.0 & -36,588\end{array}$

$\begin{array}{rr}46.9 & 104,498 \\ -16.0 & -36,588\end{array}$

$-25$

62.9

46.9

$-16.0$

$-25$
141,087

104,498

$-36,588$

* Dollar values for electricity include both energy and demand components. 
The modeled energy consumption for the highbay space in the building for a typical year was 62,619 kwh before retrofits and 58,228 kwh after proposed retrofits are implemented. The energy use intensity goes from $4.9 \mathrm{MBtu} / \mathrm{Ksf}$ to $4.6 \mathrm{MBtu} / \mathrm{Ksf}$ after retrofits.

Large 1930's warehouse space 2035 hangar 11

Fuel

Electricity (kWh)

existing

post-retrofit

difference

$\%$ change

Total (MBtu)

existing

post-retrofit

difference

$\%$ change
Energy

62,619

58,228

$-4,391$

$-7$

214
199
-15
-7

Energy

Intensity

(user units/1000ft2)

$1,449.6$

$1,348.0$

$-101.6$

$-7$

4.9

4.6

-0.3
-7
Energy

Intensity

(MBtu/1000ft2)

Dollars

$(2009)$ *

11,100

10,244

$-856$

$-7$

11,100

10,244

$\begin{array}{rr}4.6 & 10,244 \\ -0.3 & -856 \\ -7 & -8\end{array}$

* Dollar values for electricity include both energy and demand components. 


\section{Appropriated Funding Energy Consumption by End Use}

Space cooling is the largest load in the administration space of the building with 253,896 kWh/year, followed by motors and miscellaneous equipment with 137,454 kWh/year.

Large 1930 's admin space 2035 hangar 13

Fuel

Electricity ( kWh)

existing

post-retrofit

difference

$\%$ change

Total (MBtu)

existing

post-retrofit

difference

$\%$ change

Heating

Cooling

Vent

Lights

Motors and

Misc Equip

Hot Water

253,896
137,454
$-116,442$
-46

867
469
-397
-46

69,266
57,522
$-11,744$

$-17$

226,334
155,064

$-71,270$

$-31$

240,705
240,705

$\odot$

772

236

196
-40
-17

-40
-17

529
-243

$-31$

Total (MBtu/1000ft2)

existing

post-retrofit

difference

$\%$ change 
Lighting is the largest load in the highbay space of the building with 53,825 $\mathrm{kWh}$ /year, followed by motors and miscellaneous equipment with $8,705 \mathrm{kWh} /$ year.

1930's warehouse space 2035 hangar 11

Fuel Heating
Electricity (kWh)
existing
post-retrofit
difference
$\%$ change
Total (MBtu)
existing
post-retrofit
difference
\% change

Cooling Vent Lights

Motors and Misc Equip

Hot water *

Total (MBtu/1000ft2)

existing

post-retrofit

difference

$\%$ change

$\begin{array}{ll}\odot & 0 \\ 0 & 0 \\ 0 & 0 \\ 0 & 0\end{array}$

$\odot$
$\odot$
$\odot$
$\odot$

$\odot$

53,825

49,471

$\begin{array}{rr}0 & -4,354 \\ 0 & -8\end{array}$

8,705

705
0

$\begin{array}{ll}0 & 0 \\ 0 & 0 \\ 0 & 0 \\ 0 & 0\end{array}$

184

169

$-15$

$\begin{array}{lr}0 & 169 \\ 0 & -15 \\ 0 & -8\end{array}$

30
30
$\odot$
$\odot$

88
52
-36
-41

$\begin{array}{ll}\odot & \odot \\ \odot & \odot \\ \odot & \odot \\ \odot & 0\end{array}$

$\begin{array}{rr}0 & 4 \\ 0 & 4 \\ 0 & 0 \\ 0 & -8\end{array}$

1
1
0
$\odot$

0

$-41$

* Energy consumption values for both distributed and central SHW are reported for Hot Water annual energy use. 


\section{Appropriated Funding Emission Reduction}

The emission reductions from implemented the proposed retrofits are as follows:

Large 1930 's admin space 2035 hangar 13

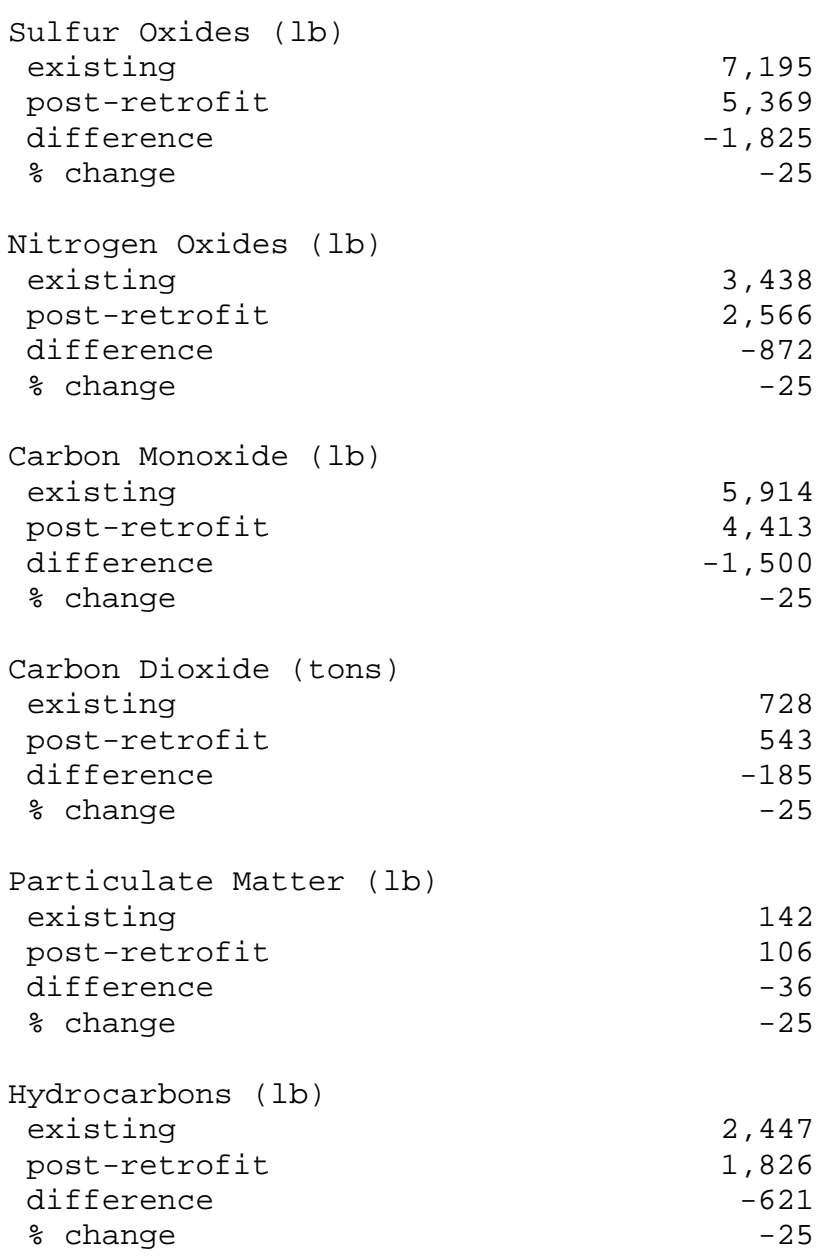


Large 1930's warehouse space hangar 11

Sulfur oxides ( $1 b$ )

existing

post-retrofit

566

difference

526

$-40$

$\%$ change

$-7$

Nitrogen oxides (lb)

existing

post-retrofit

271

difference

252

$-19$

$\%$ change

$-7$

Carbon Monoxide (lb)

existing

post-retrofit

465

post-retrofit
difference

433

$\%$ change

-33
-7

Carbon Dioxide (tons)

existing

post-retrofit

difference

$\%$ change

57

-4
-7

Particulate Matter (lb)

existing

post-retrofit

difference

$\%$ change

11

10

-1
-7

Hydrocarbons (lb)

existing

post-retrofit

difference

193
179

$-14$ 


\section{Building 1204 Administration Building}

The following information identifies the cost-effective energy- and cost-reducing retrofit projects for building 1204 identified from the FEDS modeling and analysis. Key energy and economic results are presented for each cost-effective retrofit measure.

\section{Facility Description 1204}

Building 1204 is a small admin building built in 1939. This building is served by an air cooled chiller and has little to no insulation in its building envelope. Building 1204 is 11,374 sf.

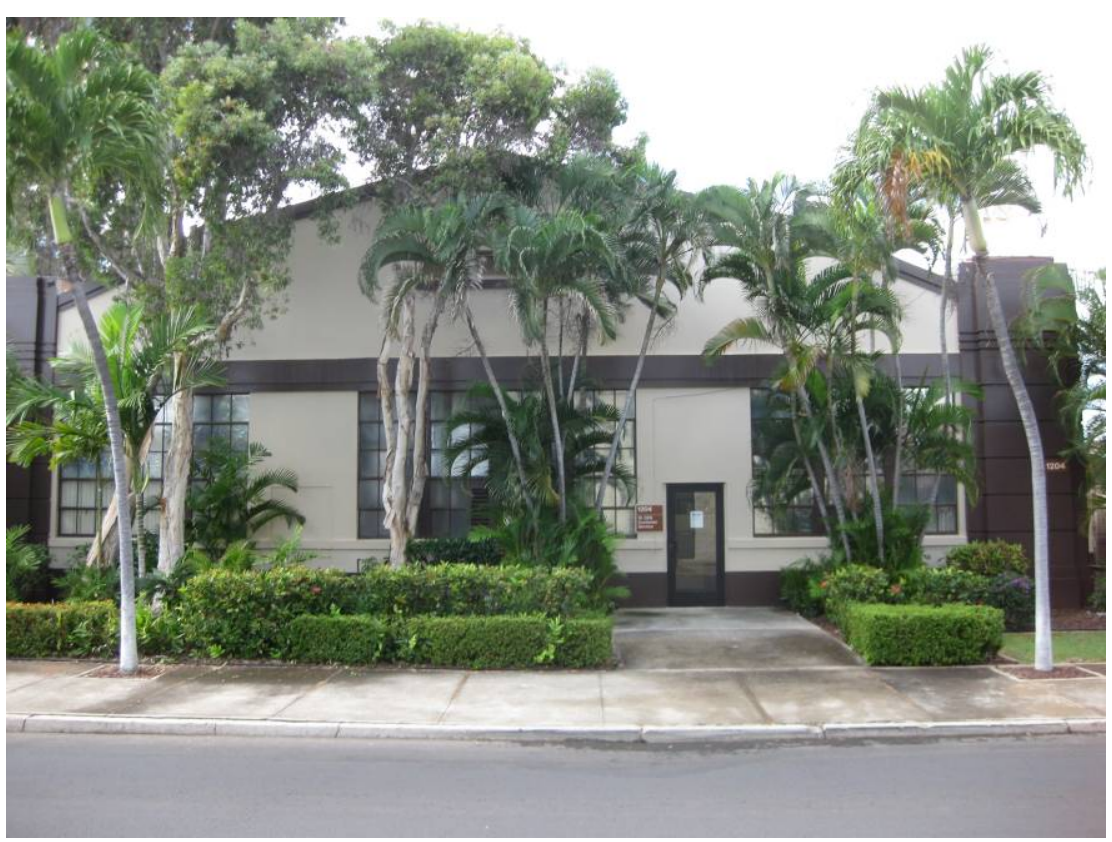




\section{Appropriated Funding Results}

A FEDS analysis using appropriated funding suggests replacing the air cooled chiller with a high efficiency water cooled chiller. This analysis also suggests replacing 32W T8 fluorescent lamps with 25W SuperT8 lamps.

Appropriated funding FEDS results for building 1204:

\begin{tabular}{|c|c|c|c|c|c|c|c|c|}
\hline Bldg. Set Description & $\begin{array}{l}\text { End } \\
\text { Use }\end{array}$ & Existing Technology & Retrofit Technology & $\begin{array}{l}\text { Energy } \\
\text { Savings } \\
\text { (MMBtu/yr) }\end{array}$ & $\begin{array}{l}\text { 1st year } \\
\text { savings } \\
\text { (\$/yr) }\end{array}$ & $\begin{array}{l}\text { Installed } \\
\text { Cost (\$) } \\
\end{array}$ & $\begin{array}{l}\text { Net } \\
\text { Present } \\
\text { Value (\$) } \\
\end{array}$ & SIR \\
\hline Small 1040's admin 1204 & Cooling & $\begin{array}{l}\text { Electric Air-Cooled Chiller } \\
\{\mathrm{C} 1\}\end{array}$ & $\begin{array}{l}\text { Water-Cooled Reciprocating } \\
\text { Electric Chiller (very high } \\
\text { efficiency) and Cooling Tower }\end{array}$ & 97 & 6,275 & 52,914 & 19,891 & 1.7 \\
\hline Small 1040's admin 1204 & Lights & FL39: FL 2X4 2F32T8 EEF2 & $\begin{array}{l}\text { FL303: FL 2X4 2F25ST8 ELC2 } \\
\text { REF }\end{array}$ & 57 & 3,684 & 25,464 & 36,259 & 2.4 \\
\hline
\end{tabular}




\section{Appropriated Funding Energy Consumption by Fuel Type}

The modeled energy consumption for a typical year was 250,798 kwh before retrofits and 204,306 kwh after proposed retrofits are implemented. The energy use intensity goes from 75.3 MBtu/Ksf to 61.3 MBtu/Ksf after retrofits.

Small 1040's admin 1204

Fuel

Energy

250,798

204,306

$-46,492$

post-retrofit

difference

$\%$ change

Total (MBtu)

existing

post-retrofit

difference

$\%$ change

* Dollar values for electricity include

Energy

(user units/1000ft2)
Energy
Intensity
(MBtu/1000ft2)

Dollars

$(2009)$ *

75.3

61.3

$-14.0$

$-19$

44,459

35,945

$-8,514$

$17,962.5$
$-4,087.6$

$-19$

75.3

61.3

$-14.0$

$-19$

75.3

61.3

$-14.0$

$-19$ 


\section{Appropriated Funding Energy Consumption by End Use}

Space cooling is the largest load in the building with $83,381 \mathrm{kWh} /$ year, followed by ventilation with $72,592 \mathrm{kWh} / \mathrm{year}$.

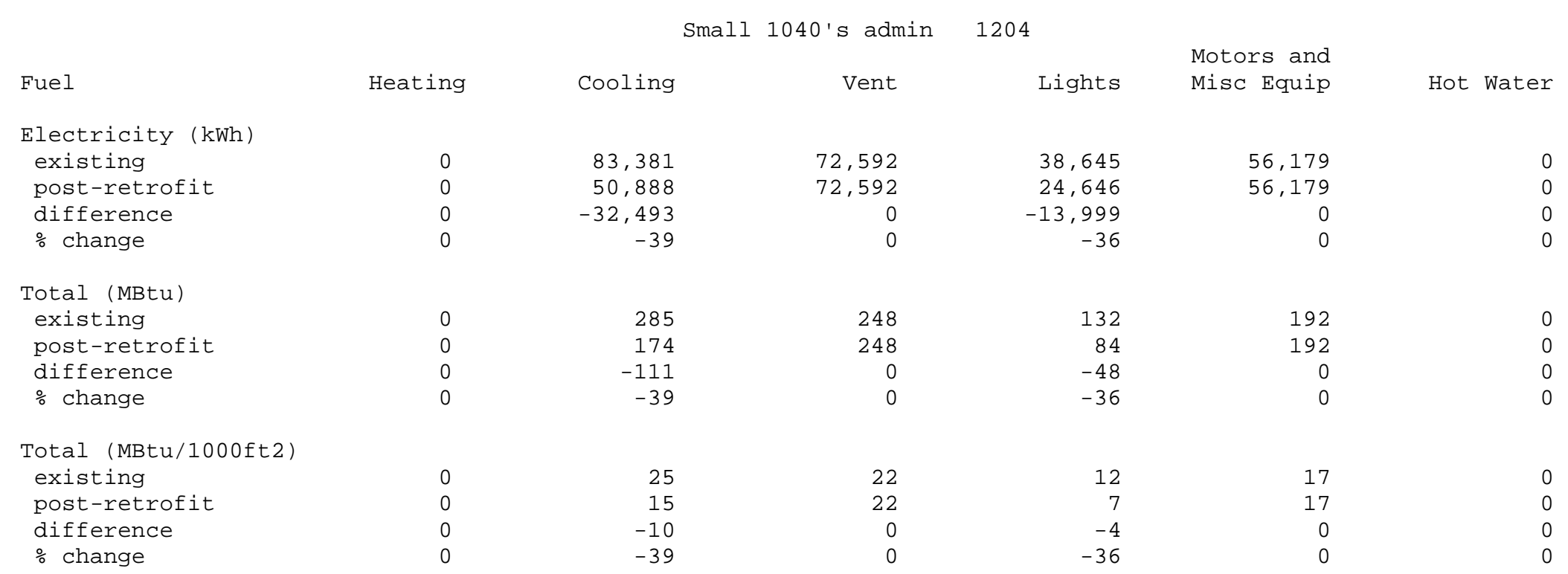




\section{Appropriated Funding Emission Reduction}

The emission reductions from implemented the proposed retrofits are as follows:

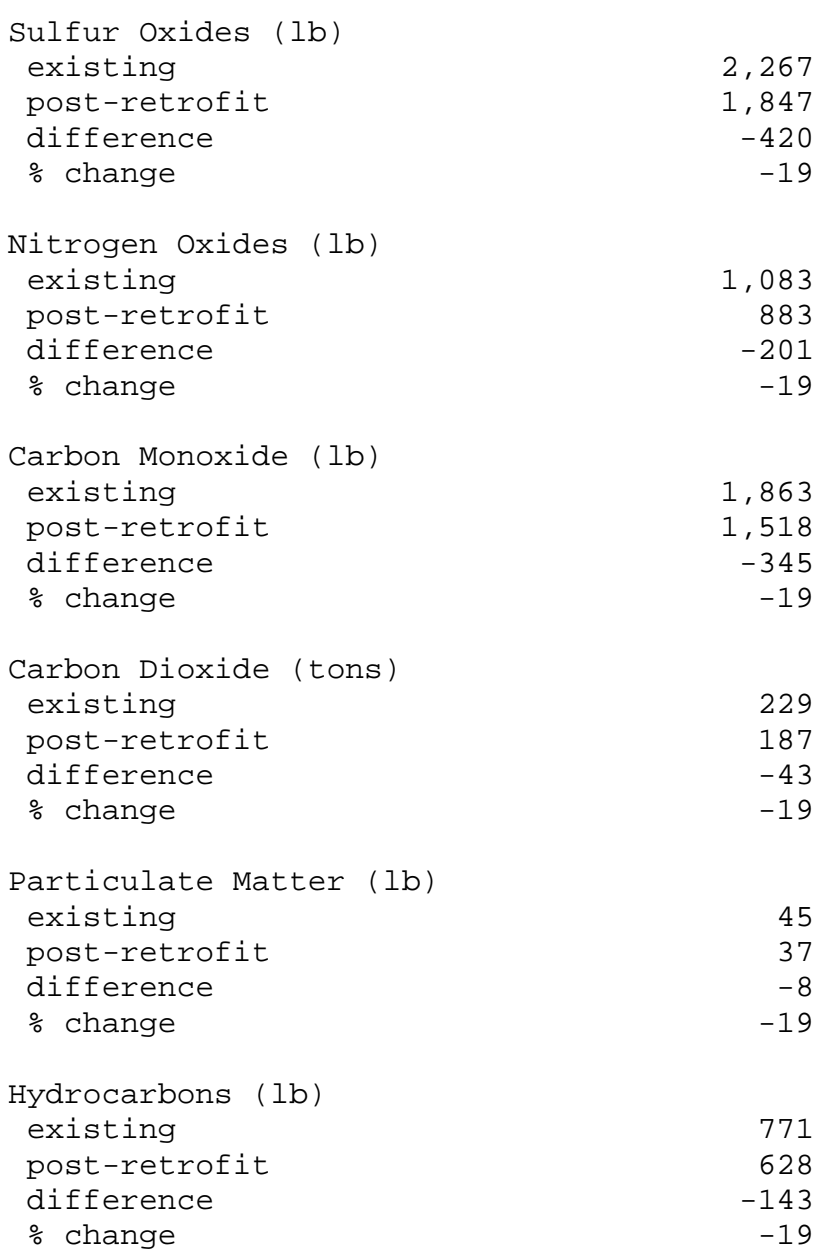




\section{Building 2155 Administration Building}

The following information identifies the cost-effective energy- and cost-reducing retrofit projects for building 2155 identified from the FEDS modeling and analysis. Key energy and economic results are presented for each cost-effective retrofit measure.

\section{Facility Description 2155}

Building 2155 is a weapons systems management facility built in 1968. This building is cooled by a DX, or package unit and has little to no insulation in the building envelope. Building 2155 is 21,745 sf.

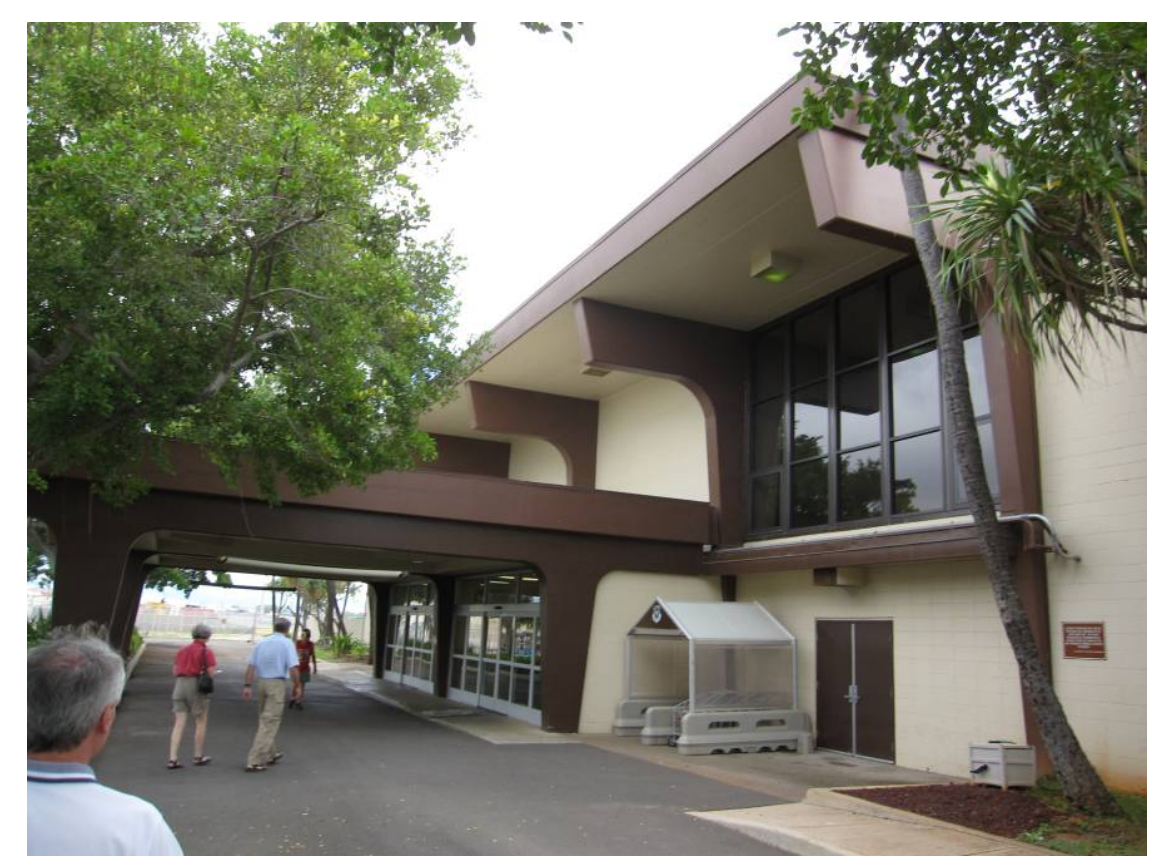




\section{Appropriated Funding Results}

A FEDS analysis using appropriated funding suggests replacing some of the lighting in the building as well as increasing the insulation in the suspended ceiling.

Appropriated funding FEDS results for building 2155:

\begin{tabular}{|c|c|c|c|c|c|c|c|c|}
\hline Bldg. Set Description & $\begin{array}{l}\text { End } \\
\text { Use }\end{array}$ & Existing Technology & Retrofit Technology & $\begin{array}{l}\text { Energy } \\
\text { Savings } \\
\text { (MMBtu/yr) }\end{array}$ & $\begin{array}{l}\text { 1st year } \\
\text { savings } \\
(\$ / y r)\end{array}$ & $\begin{array}{l}\text { Installed } \\
\text { Cost (\$) }\end{array}$ & $\begin{array}{l}\text { Net } \\
\text { Present } \\
\text { Value (\$) }\end{array}$ & SIR \\
\hline $\begin{array}{l}\text { medium 1960's admin } \\
2155\end{array}$ & Lights & FL51: FL 2X4 2F32T8 ELC2 & $\begin{array}{l}\text { FL303: FL 2X4 2F25ST8 ELC2 } \\
\text { REF }\end{array}$ & 23 & 1,289 & 7,449 & 14,120 & 2.9 \\
\hline $\begin{array}{l}\text { medium 1960's admin } \\
2155\end{array}$ & Lights & EX1: EXIT - INC $(2 \times 20)$ & $\begin{array}{l}\text { EX12: EXIT - } \\
\text { ELECTROLUMINESCENT PANEL } \\
\text { RETRO KIT }\end{array}$ & 13 & 858 & 621 & 13,848 & 23.3 \\
\hline $\begin{array}{l}\text { medium 1960's admin } \\
2155\end{array}$ & Lights & FL4: FL 1X4 2F40T12 STD2 & FL52: FL 1X4 2F32T8 ELC2 & 3 & 184 & 451 & 2,656 & 6.9 \\
\hline $\begin{array}{l}\text { medium 1960's admin } \\
2155\end{array}$ & Roof & Roof Insulation R-Value 8.90 & $\begin{array}{l}\text { Suspended Ceiling: Increase } \\
\text { Insulation by R-19 }\end{array}$ & 35 & 2,334 & 37,446 & 1,386 & 1.0 \\
\hline
\end{tabular}




\section{Appropriated Funding Energy Consumption by Fuel Type}

The modeled energy consumption for a typical year was 314,110 kwh before retrofits and 292,777 kwh after proposed retrofits are implemented. The energy use intensity goes from 49.3 MBtu/Ksf to 46.0 MBtu/Ksf after retrofits.

Medium 1960 's admin 2155

Fuel

Electricity (kWh)

existing

post-retrofit

difference

$\%$ change

Total (MBtu)

existing

post-retrofit

difference

$\%$ change

* Dollar values for electricity include both

\section{Energy}

$$
\begin{array}{r}
314,110 \\
292,777 \\
-21,333 \\
-7
\end{array}
$$

1,072

999

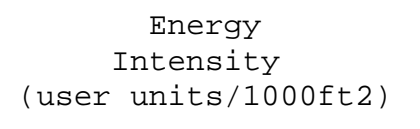
$14,445.2$
$13,464.1$

-981.1
-7

49.3

46.0

$-3.3$

$-7$
Energy

Dollars

(MBtu/1000ft2) (2009)*

49.3

46.0

-3.3
-7

5,682

51,510

$-4,172$
-7

49.3

46.0

$-3.3$

$-7$
55,682

51,510

$-4,172$

列 


\section{Appropriated Funding Energy Consumption by End Use}

Motors and miscellaneous equipment is the largest load in the building with $145,710 \mathrm{kWh} /$ year, followed by space cooling with 92,995 kWh/year.

\begin{tabular}{|c|c|c|c|c|c|c|}
\hline & & & $960^{\prime} \mathrm{s}$ ac & & & \\
\hline Fuel & Heating & Cooling & Vent & Lights & $\begin{array}{l}\text { Motors and } \\
\text { Misc Equip }\end{array}$ & Hot Water \\
\hline Electricity (kv & & & & & & \\
\hline existing & $\odot$ & 92,995 & 52,915 & 22,491 & 145,710 & $\odot$ \\
\hline post-retrofit & $\odot$ & 80,242 & 52,944 & 13,881 & 145,710 & $\odot$ \\
\hline difference & $\odot$ & $-12,753$ & 29 & $-8,610$ & 0 & $\odot$ \\
\hline$\%$ change & $\odot$ & -14 & $\odot$ & -38 & $\odot$ & $\odot$ \\
\hline Total (MBtu) & & & & & & \\
\hline existing & $\odot$ & 317 & 181 & 77 & 497 & $\odot$ \\
\hline post-retrofit & $\odot$ & 274 & 181 & 47 & 497 & $\odot$ \\
\hline difference & $\odot$ & -44 & $\odot$ & -29 & $\odot$ & $\odot$ \\
\hline$\%$ change & $\odot$ & -14 & $\odot$ & -38 & $\odot$ & $\odot$ \\
\hline Total (MBtu/100 & & & & & & \\
\hline existing & $\odot$ & 15 & 8 & 4 & 23 & $\odot$ \\
\hline post-retrofit & $\odot$ & 13 & 8 & 2 & 23 & $\odot$ \\
\hline difference & $\odot$ & -2 & $\odot$ & -1 & $\odot$ & $\odot$ \\
\hline$\%$ change & $\odot$ & -14 & $\odot$ & -38 & $\odot$ & $\odot$ \\
\hline
\end{tabular}




\section{Appropriated Funding Emission Reduction}

The emission reductions from implemented the proposed retrofits are as follows:

Medium 1960 's admin 2155

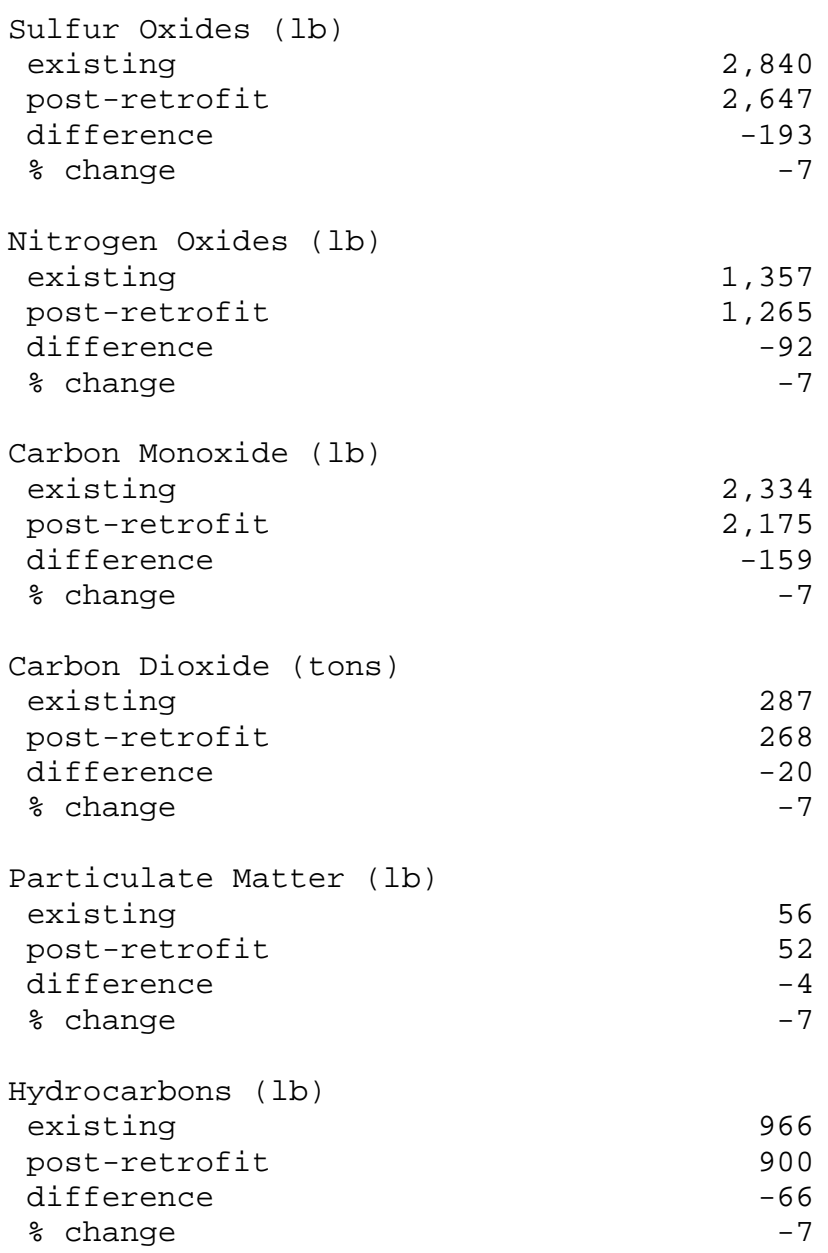




\section{Building 502 Law Office}

The following information identifies the cost-effective energy- and cost-reducing retrofit projects for building 502 identified from the FEDS modeling and analysis. Key energy and economic results are presented for each cost-effective retrofit measure.

\section{Facility Description 502}

Building 502 is a small law office building built in 1971 that is served by two separate electric DX units with a courtroom in the center of the office space. Building 502 is 9,217 sf.

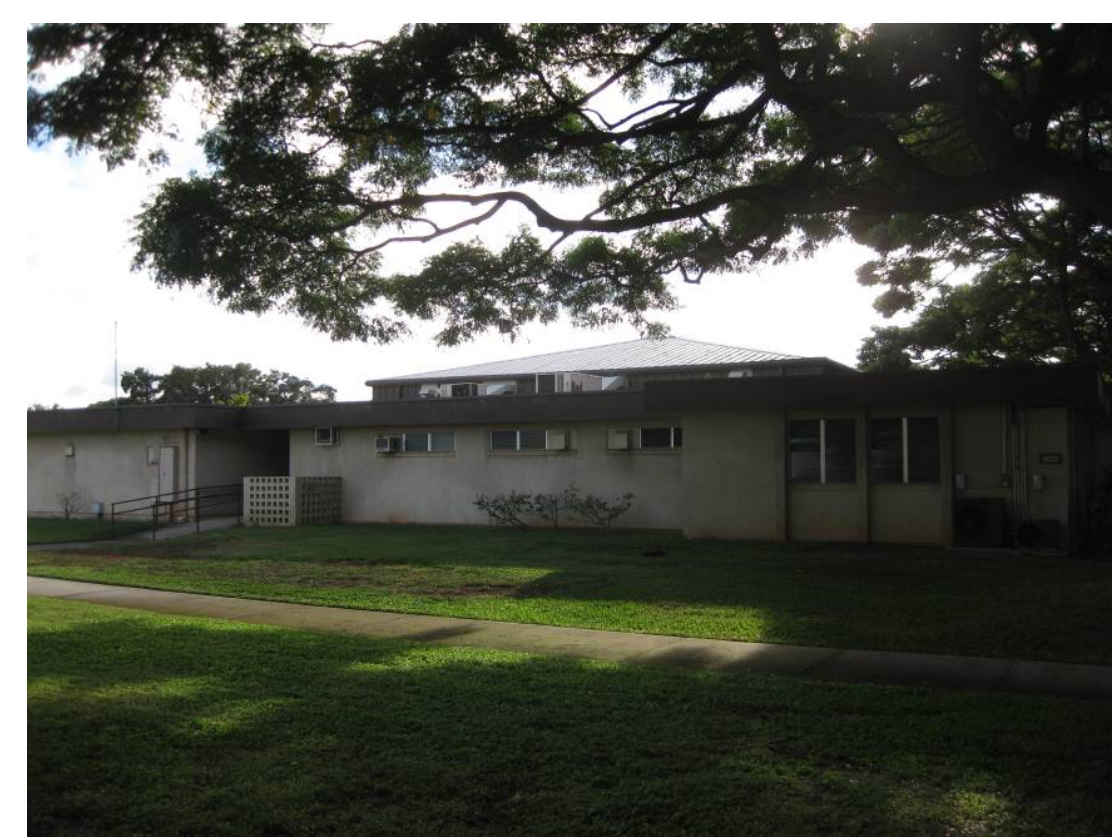




\section{Appropriated Funding Results}

A FEDS analysis using appropriated funding suggests replacing the package unit with high efficiency window units. The FEDS analysis also suggests upgrading the lighting from 32W T8 lamps to 25W Super T8 lamps and replacing the exit lights with electroluminescent panels. The electric water heater is suggested to be replaced by a heat pump water heater and insulation in the suspended ceiling is recommended to be increased.

Appropriated funding FEDS results for building 502:

\begin{tabular}{|c|c|c|c|c|c|c|c|c|}
\hline Bldg. Set Description & $\begin{array}{l}\text { End } \\
\text { Use }\end{array}$ & Existing Technology & Retrofit Technology & $\begin{array}{l}\text { Energy } \\
\text { Savings } \\
\text { (MMBtu/yr) }\end{array}$ & $\begin{array}{c}\text { 1st year } \\
\text { savings } \\
(\$ / y r)\end{array}$ & $\begin{array}{l}\text { Installed } \\
\text { Cost (\$) }\end{array}$ & $\begin{array}{l}\text { Net } \\
\text { Present } \\
\text { Value (\$) } \\
\end{array}$ & SIR \\
\hline small 1960s admin 502 & Cooling & Electric Package Unit $\{\mathrm{C} 1\}$ & $\begin{array}{l}\text { Window Unit AC (ultra high } \\
\text { efficiency) }\end{array}$ & 13 & 1,546 & 15,573 & 1,191 & 1.1 \\
\hline small 1960s admin 502 & Lights & EX1: EXIT - INC $(2 \times 20)$ & $\begin{array}{l}\text { EX12: EXIT - } \\
\text { ELECTROLUMINESCENT PANEL } \\
\text { RETRO KIT }\end{array}$ & 13 & 862 & 621 & 13,893 & 23.4 \\
\hline small 1960s admin 502 & Lights & FL51: FL 2X4 2F32T8 ELC2 & $\begin{array}{l}\text { FL303: FL 2X4 2F25ST8 ELC2 } \\
\text { REF }\end{array}$ & 10 & 556 & 5,822 & 3,445 & 1.6 \\
\hline small 1960s admin 502 & $\begin{array}{l}\text { Hot } \\
\text { Water }\end{array}$ & Electric Water Heater & $\begin{array}{l}\text { Heat Pump Water Heater } \\
\text { (Com) }\end{array}$ & 3 & 139 & 1,285 & 2 & 1.0 \\
\hline small 1960s admin 502 & Roof & Roof Insulation R-Value 0.00 & $\begin{array}{l}\text { Suspended Ceiling: Increase } \\
\text { Insulation by R-19 }\end{array}$ & 48 & 2,817 & 15,872 & 31,000 & 3.0 \\
\hline
\end{tabular}




\section{Appropriated Funding Energy Consumption by Fuel Type}

The modeled energy consumption for a typical year was 143,177 kwh before retrofits and 116,778 kwh after proposed retrofits are implemented. The energy use intensity goes from 53.0 MBtu/Ksf to 43.2 MBtu/Ksf after retrofits.

Fuel

Electricity (kWh)

existing

post-retrofit

difference

$\%$ change

Total (MBtu)

existing

post-retrofit

difference

$\%$ change
Energy

143,177
116,778
$-26,399$
-18

489
399
-90
-18

Small 1960s admin 502

Energy
Intensity
(user units/10

$15,534.1$
$12,669.9$
$-2,864.2$
-18

53.0
43.2
-9.8
-18

Dollars

$(2009)$ *

53.0
43.2
-9.8
-18


53.0
43.2
-9.8
-18

25,381

20,545

$-4,836$

$-19$

25,381

20,545

$-4,836$ 


\section{Appropriated Funding Energy Consumption by End Use}

Space cooling is the largest load in the building with 58,890 $\mathrm{kWh} /$ year, followed by motors and miscellaneous equipment with 45,525 $\mathrm{kWh} /$ year.

Fuel

Electricity (kWh)

existing

post-retrofit

difference

$\%$ change

Total (MBtu)

existing

post-retrofit

difference

$\%$ change

Total (MBtu/1000ft2)

existing

post-retrofit

difference

$\%$ change

Heating

Small 1960s admin 502

Cooling

Vent

Lights

Motors and

Misc Equip

Hot Water
39,561

$-19,329$

$-33$

201

135
-66

$-33$

22

15

$-7$
13,327

8,333

$-4,995$

24, 575

$-1,332$

$-5$

$-37$

45
28
-17

-17
-37

5
3
-2

-2
-37
45,525

45,525

$$
\begin{aligned}
& 0 \\
& 0
\end{aligned}
$$

155

155

$\odot$

17

17
$\odot$
0
860

116

$-744$

$-87$ 


\section{Appropriated Funding Emission Reduction}

The emission reductions from implemented the proposed retrofits are as follows:

Small 1960s admin 502

\begin{tabular}{|c|c|}
\hline \multicolumn{2}{|l|}{ Sulfur Oxides (lb) } \\
\hline existing & 1,294 \\
\hline post-retrofit & 1,056 \\
\hline difference & -239 \\
\hline$\%$ change & -18 \\
\hline \multicolumn{2}{|l|}{ Nitrogen Oxides (lb) } \\
\hline existing & 619 \\
\hline post-retrofit & $5 \odot 4$ \\
\hline difference & -114 \\
\hline$\%$ change & -18 \\
\hline \multicolumn{2}{|l|}{ Carbon Monoxide (lb) } \\
\hline existing & 1,064 \\
\hline post-retrofit & 868 \\
\hline difference & -196 \\
\hline$\%$ change & -18 \\
\hline \multicolumn{2}{|l|}{ Carbon Dioxide (tons) } \\
\hline existing & 131 \\
\hline post-retrofit & 107 \\
\hline difference & -24 \\
\hline$\%$ change & -18 \\
\hline \multicolumn{2}{|l|}{ Particulate Matter (lb) } \\
\hline existing & 26 \\
\hline post-retrofit & 21 \\
\hline difference & -5 \\
\hline$\%$ change & -18 \\
\hline \multicolumn{2}{|l|}{ Hydrocarbons (lb) } \\
\hline existing & 440 \\
\hline post-retrofit & 359 \\
\hline difference & -81 \\
\hline$\%$ change & -18 \\
\hline
\end{tabular}




\section{Building 2133 Administration Building}

The following information identifies the cost-effective energy- and cost-reducing retrofit projects for building 2133 identified from the FEDS modeling and analysis. Key energy and economic results are presented for each cost-effective retrofit measure.

\section{Facility Description 2133}

Building 2133 is a weapon systems management facility built in 2005. 2133 is cooled by an air cooled chiller and has some insulation in its building envelope. Building 2133 is 25,764 sf.

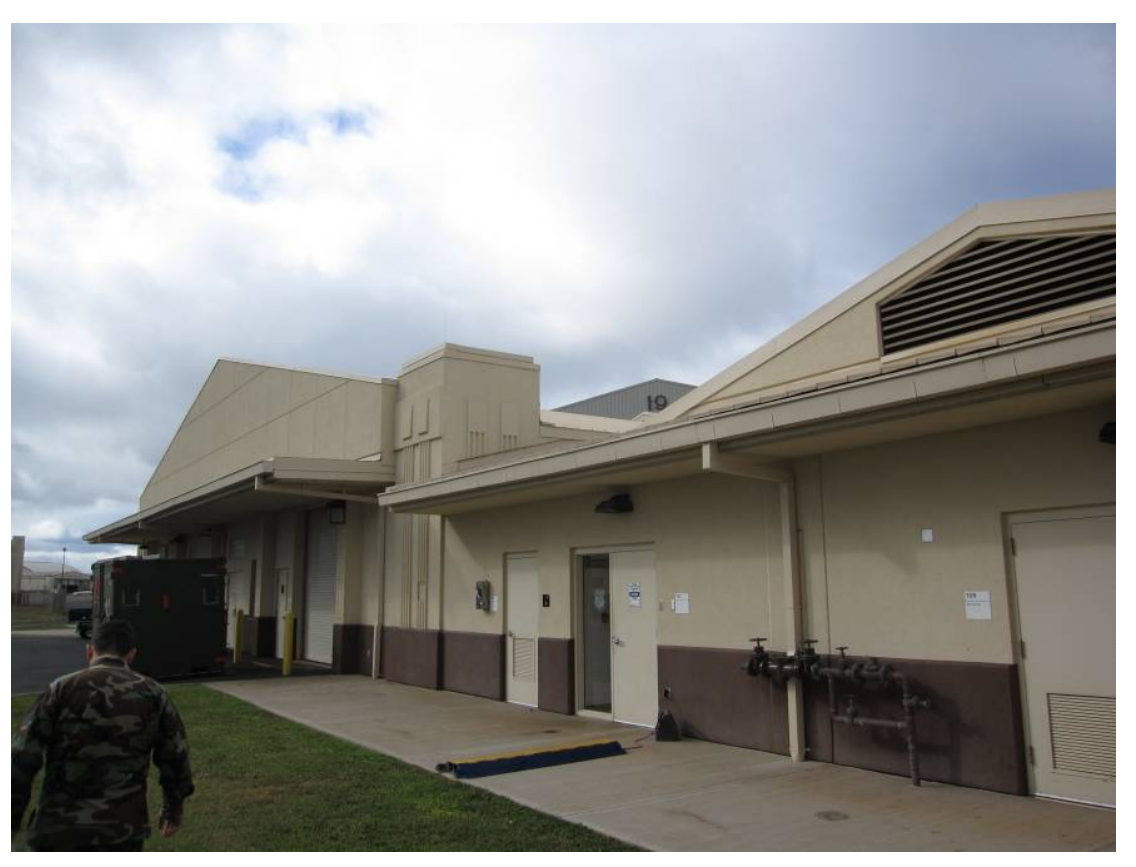




\section{Appropriated Funding Results}

A FEDS analysis using appropriated funding suggests replacing the air cooled chiller with a very high efficiency water cooled chiller. FEDS also suggests replacing some of the lights and replacing the electric central boiler with a central heat pump water heater.

Appropriated funding FEDS results for building 2133:

\begin{tabular}{|c|c|c|c|c|c|c|c|c|}
\hline Bldg. Set Description & $\begin{array}{l}\text { End } \\
\text { Use }\end{array}$ & Existing Technology & Retrofit Technology & $\begin{array}{l}\text { Energy } \\
\text { Savings } \\
\text { (MMBtu/yr) }\end{array}$ & $\begin{array}{l}\text { 1st year } \\
\text { savings } \\
(\$ / y r)\end{array}$ & $\begin{array}{l}\text { Installed } \\
\text { Cost (\$) }\end{array}$ & $\begin{array}{l}\text { Net } \\
\text { Present } \\
\text { Value (\$) } \\
\end{array}$ & SIR \\
\hline $\begin{array}{l}\text { medium 2000's admin } \\
2133\end{array}$ & Cooling & $\begin{array}{l}\text { Electric Air-Cooled Chiller } \\
\{\mathrm{C} 1\}\end{array}$ & $\begin{array}{l}\text { Water-Cooled Reciprocating } \\
\text { Electric Chiller (very high } \\
\text { efficiency) and Cooling Tower }\end{array}$ & 180 & 9,823 & 58,197 & 48,779 & 2.3 \\
\hline $\begin{array}{l}\text { medium 2000's admin } \\
2133\end{array}$ & Lights & FL236: FL 2X4 3F32T8 ELC3 & $\begin{array}{l}\text { FL279: FL 2X4 2F32ST8 ELC2 } \\
\text { REF }\end{array}$ & 246 & 14,500 & 62,594 & 179,762 & 3.9 \\
\hline $\begin{array}{l}\text { medium 2000's admin } \\
2133\end{array}$ & Lights & EX1: EXIT - INC $(2 \times 20)$ & $\begin{array}{l}\text { EX12: EXIT - } \\
\text { ELECTROLUMINESCENT PANEL } \\
\text { RETRO KIT }\end{array}$ & 13 & 857 & 621 & 13,807 & 23.2 \\
\hline $\begin{array}{l}\text { medium 2000's admin } \\
2133\end{array}$ & Lights & CF20: CFL 2-13 + BLST UNIT & FL53: FL 1X4 1F32T8 ELC1 & - & 72 & 1,208 & 46 & 1.0 \\
\hline $\begin{array}{l}\text { medium 2000's admin } \\
2133\end{array}$ & Lights & FL51: FL 2X4 2F32T8 ELC2 & $\begin{array}{l}\text { FL303: FL 2X4 2F25ST8 ELC2 } \\
\text { REF }\end{array}$ & 11 & 629 & 5,884 & 4,605 & 1.8 \\
\hline
\end{tabular}




\section{Appropriated Funding Energy Consumption by Fuel Type}

The modeled energy consumption for a typical year was 586,408 kwh before retrofits and 373,475 kwh after proposed retrofits are implemented. The energy use intensity goes from 77.7 MBtu/Ksf to 49.5 MBtu/Ksf after retrofits.

Medium 2000's admin 2133

Fuel

Electricity (kWh)

existing

post-retrofit

difference

$\%$ change

Total (MBtu)

existing

post-retrofit

difference

$\%$ change
Energy

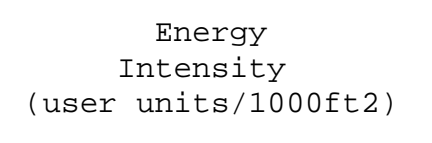

586,408

373,475

$-212,933$

$-36$
2, 001
1,275
$-727$

$22,760.8$

8

$8,264.8$

77.7

49.5

$-28.2$

$-36$
Energy

Intensity

(MBtu/1000ft2)

lars

(2009)*

77.7

49.5

$-28.2$

$-36$

77.7

49.5

$-28.2$

$-36$
103,952

65,708

$-38,245$

245
-37

103,952

65,708

$-38,245$

* Dollar values for electricity include both energy and demand components. 


\section{Appropriated Funding Energy Consumption by End Use}

Space cooling is the largest load in the building with $170,993 \mathrm{kWh} /$ year, followed by motors and miscellaneous equipment with $132,355 \mathrm{kWh} /$ year.

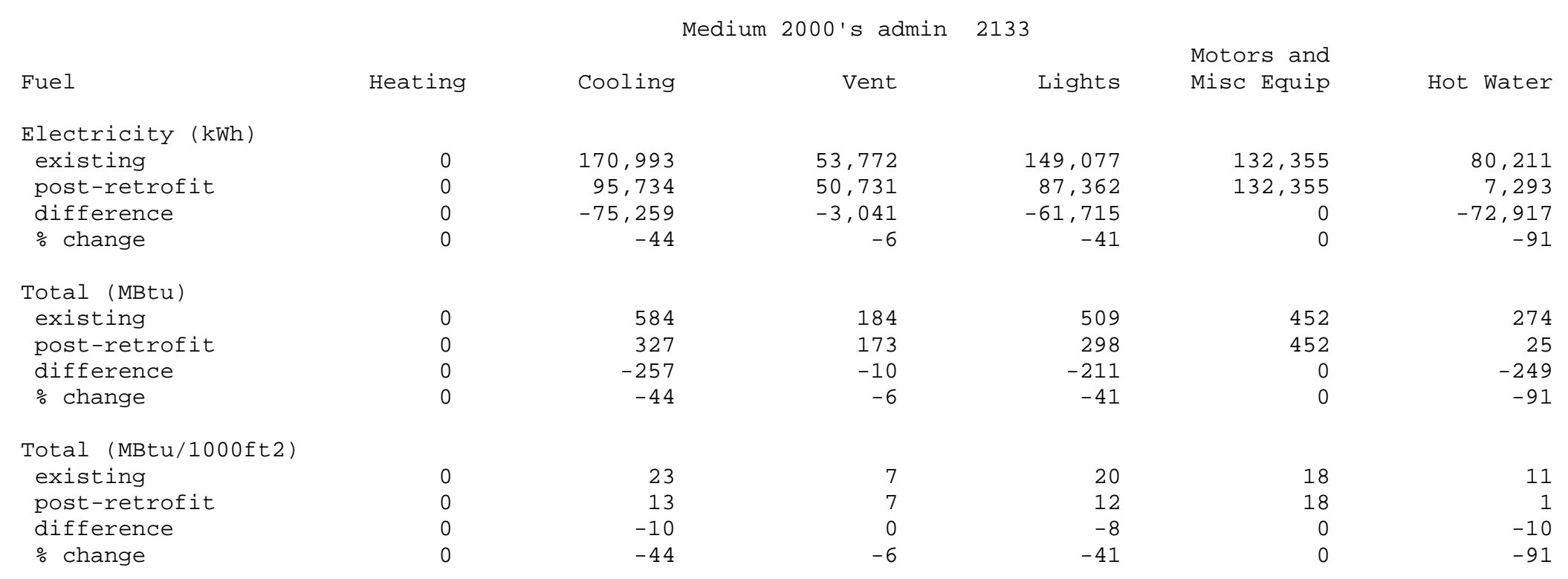




\section{Appropriated Funding Emission Reduction}

The emission reductions from implemented the proposed retrofits are as follows:

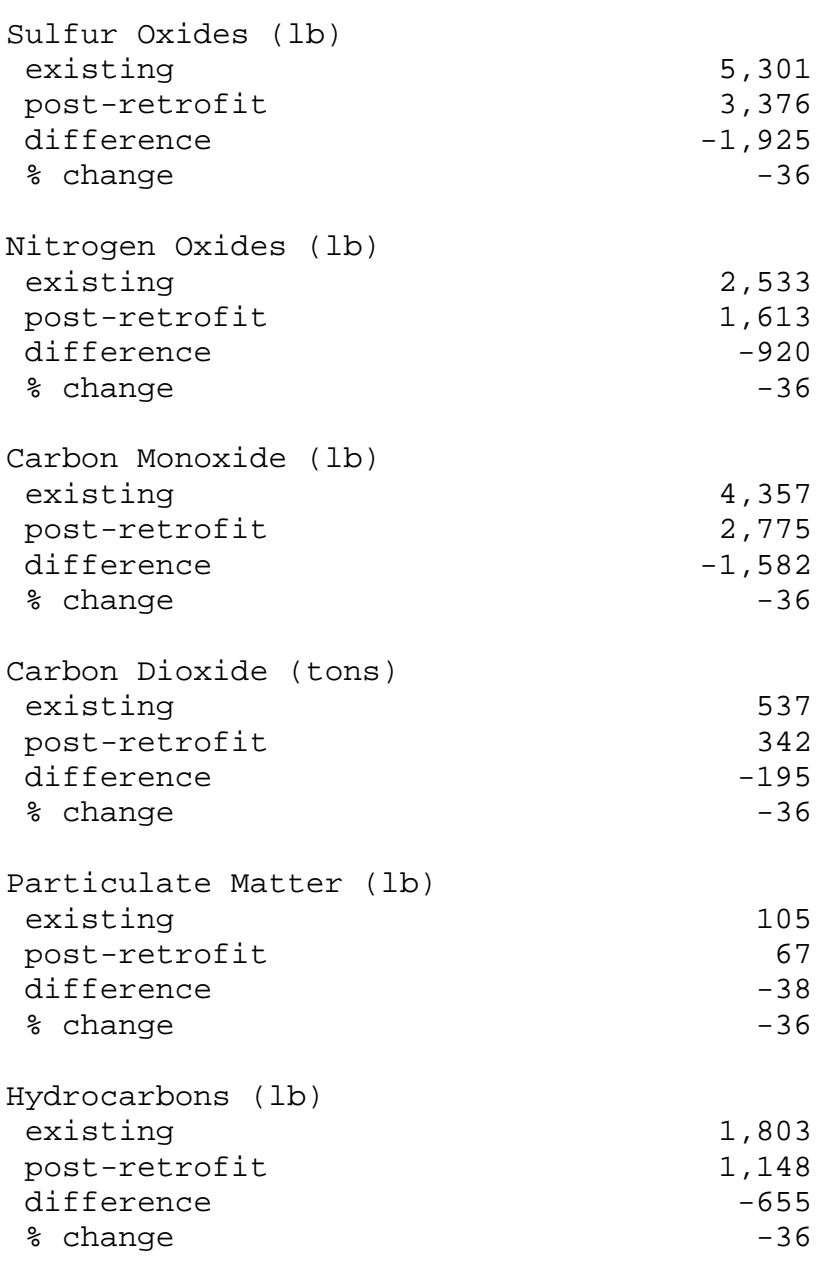




\section{Building 2125 Administration Building}

The following information identifies the cost-effective energy- and cost-reducing retrofit projects for building 2125 identified from the FEDS modeling and analysis. Key energy and economic results are presented for each cost-effective retrofit measure.

\section{Facility Description 2125}

Building 2125 is an administration building built in 1994. This petroleum operations building is cooled by an electric package unit and has little to no insulation in its building envelope. Building 2125 is 3,867 sf.

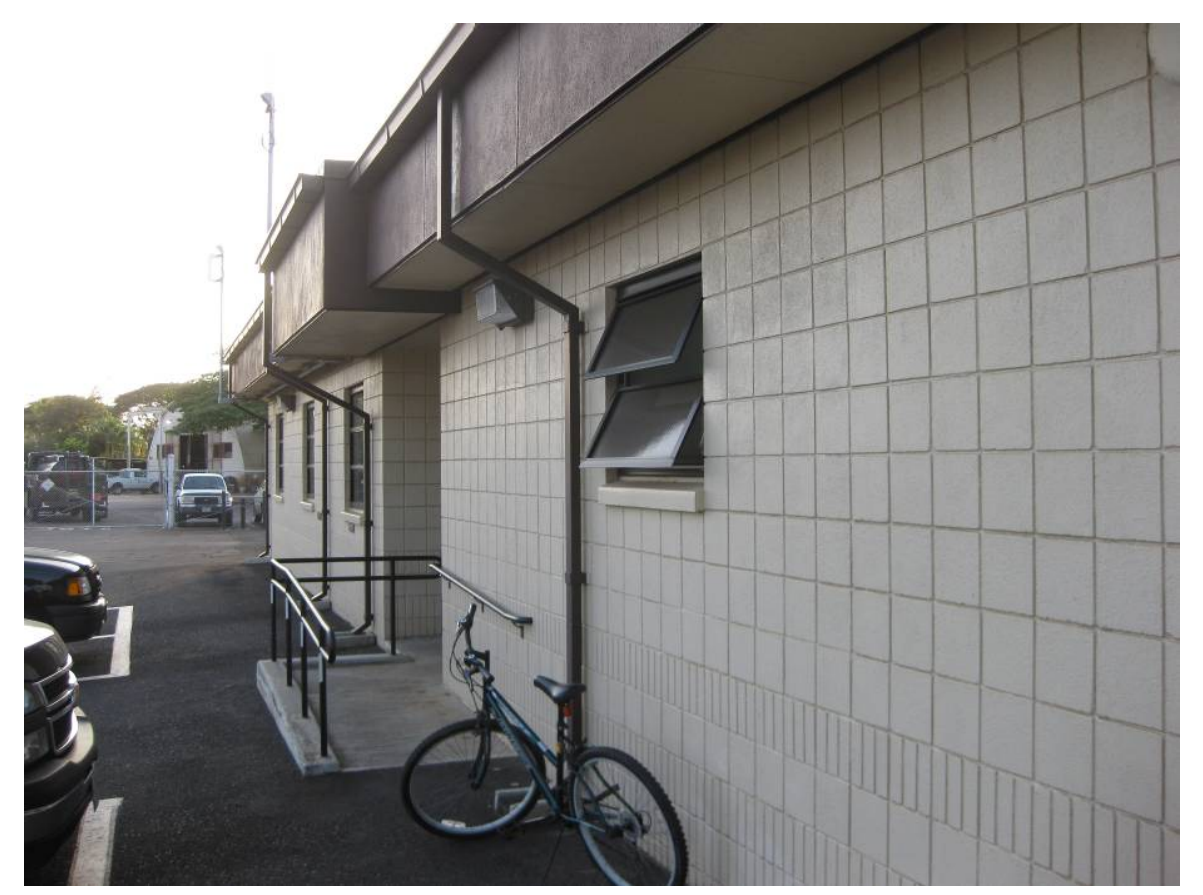




\section{Appropriated Funding Results}

A FEDS analysis using appropriated funding suggests replacing the 32W T8 lighting with 25W Super T8 lighting as well as replacing the exit lighting and increasing the insulation in the roof to 4 inches of fiberglass.

Appropriated funding FEDS results for building 2125:

\begin{tabular}{|c|c|c|c|c|c|c|c|c|}
\hline Bldg. Set Description & $\begin{array}{l}\text { End } \\
\text { Use }\end{array}$ & Existing Technology & Retrofit Technology & $\begin{array}{l}\text { Energy } \\
\text { Savings } \\
\text { (MMBtu/yr) }\end{array}$ & $\begin{array}{l}\text { 1st year } \\
\text { savings } \\
(\$ / y r)\end{array}$ & $\begin{array}{l}\text { Installed } \\
\text { Cost (\$) }\end{array}$ & $\begin{array}{l}\text { Net } \\
\text { Present } \\
\text { Value (\$) } \\
\end{array}$ & SIR \\
\hline Small 1990's admin 2125 & Lights & FL38: FL 2X4 3F32T8 EEF1,2 & $\begin{array}{l}\text { FL304: FL 2X4 3F25ST8 ELC3 } \\
\text { REF }\end{array}$ & 26 & 1,720 & 5,508 & 23,438 & 5.3 \\
\hline Small 1990's admin 2125 & Lights & EX6: EXIT - LED & $\begin{array}{l}\text { EX12: EXIT - } \\
\text { ELECTROLUMINESCENT PANEL } \\
\text { RETRO KIT }\end{array}$ & - & 56 & 311 & 643 & 3.1 \\
\hline
\end{tabular}




\section{Appropriated Funding Energy Consumption by Fuel Type}

The modeled energy consumption for a typical year was 56,331 kwh before retrofits and 48,664 kwh after proposed retrofits are implemented. The energy use intensity goes from 48.7 MBtu/Ksf to 43.0 MBtu/Ksf after retrofits.

Small 1990 's admin 2125

Fuel

Energy

Energy
Intensity
(user units/1000ft2)

$$
\begin{array}{ll}
\text { Energy } & \\
\text { Intensity } & \text { Dollars } \\
\text { (MBtu/1000ft2) } & (2009)^{*}
\end{array}
$$

Electricity (kWh)

existing

post-retrofit

56,331

48,664

$14,567.1$
$12,584.4$
$-1,982.7$

difference

$-7,667$

$-1,982.7$
-14

$$
\begin{array}{r}
49.7 \\
43.0 \\
-6.8 \\
-14
\end{array}
$$

9,963

8,555

$-1,408$

Total (MBtu)

existing

post-retrofit

difference

$$
\begin{array}{r}
49.7 \\
43.0 \\
-6.8 \\
-14
\end{array}
$$
49.7
43.0
$-6.8$
$-14$

$-26$

9,963

8,555

$-1,408$

* Dollar values for electricity include both energy and demand components. 


\section{Appropriated Funding Energy Consumption by End Use}

Space cooling is the largest load in the building with 18,138 kWh/year, followed by lights with 16,214 kWh/year.

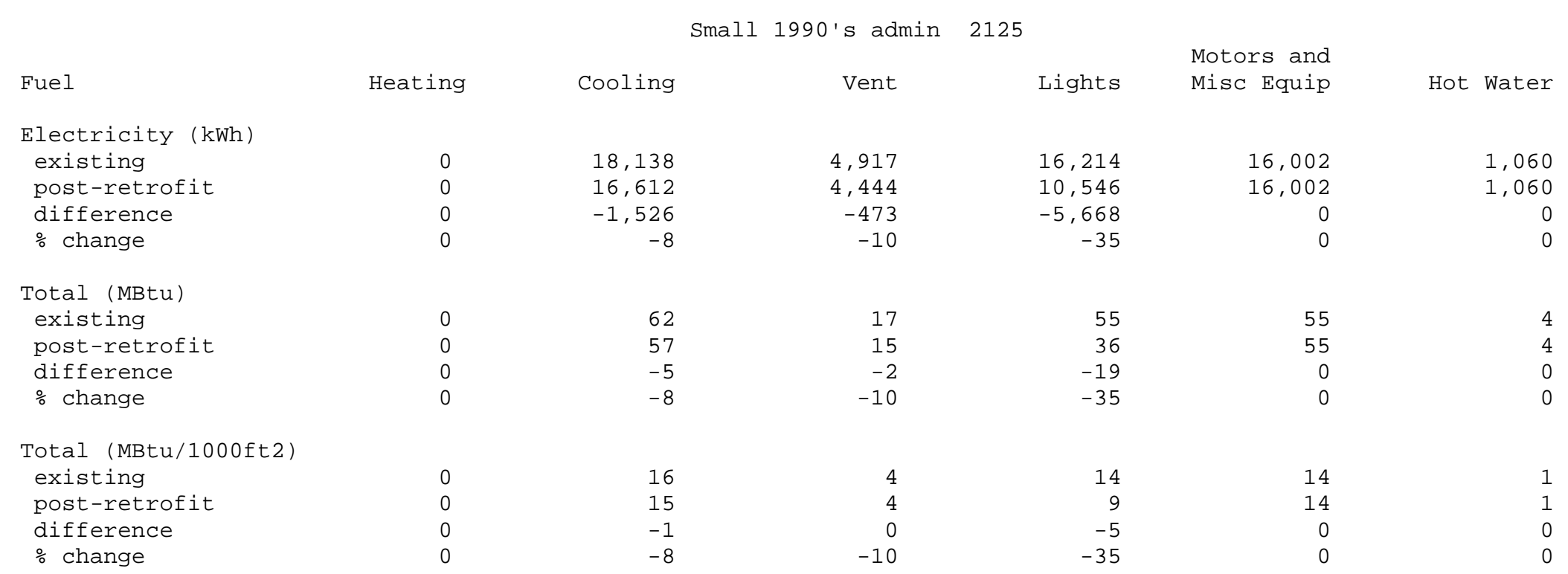




\section{Appropriated Funding Emission Reduction}

The emission reductions from implemented the proposed retrofits are as follows:

Small 1990's admin 2125

$\begin{array}{lr}\text { Sulfur oxides (lb) } & \\ \text { existing } & 509 \\ \text { post-retrofit } & 440 \\ \text { difference } & -69 \\ \text { \% change } & -14 \\ & \\ \text { Nitrogen 0xides (lb) } & 243 \\ \text { existing } & 210 \\ \text { post-retrofit } & -33 \\ \text { difference } & -14 \\ \text { \% change } & \\ & \\ \text { Carbon Monoxide (lb) } & 419 \\ \text { existing } & 362 \\ \text { post-retrofit } & -57 \\ \text { difference } & -14 \\ \text { \% change } & \\ \text { Carbon Dioxide (tons) } & 52 \\ \text { existing } & 45 \\ \text { post-retrofit } & -7 \\ \text { difference } & -14 \\ \text { \% change } & \\ \text { Particulate Matter (lb) } & -10 \\ \text { existing } & -14 \\ \text { post-retrofit } & \\ \text { difference } & 173 \\ \text { \% change } & -24 \\ \text { Hydrocarbons (lb) } & -14 \\ \text { existing } & \\ \text { post-retrofit } & \\ \text { difference } & \\ \text { \% change } & \\ & \end{array}$




\section{Building 559 Clinic Building}

The following information identifies the cost-effective energy- and cost-reducing retrofit projects for building 559 identified from the FEDS modeling and analysis. Key energy and economic results are presented for each cost-effective retrofit measure.

\section{Facility Description 559}

Building 559 is the air force clinic built in 1942. This building is cooled by water cooled chillers and has an electric central hot water system. Building 559 is 78,823 sf.

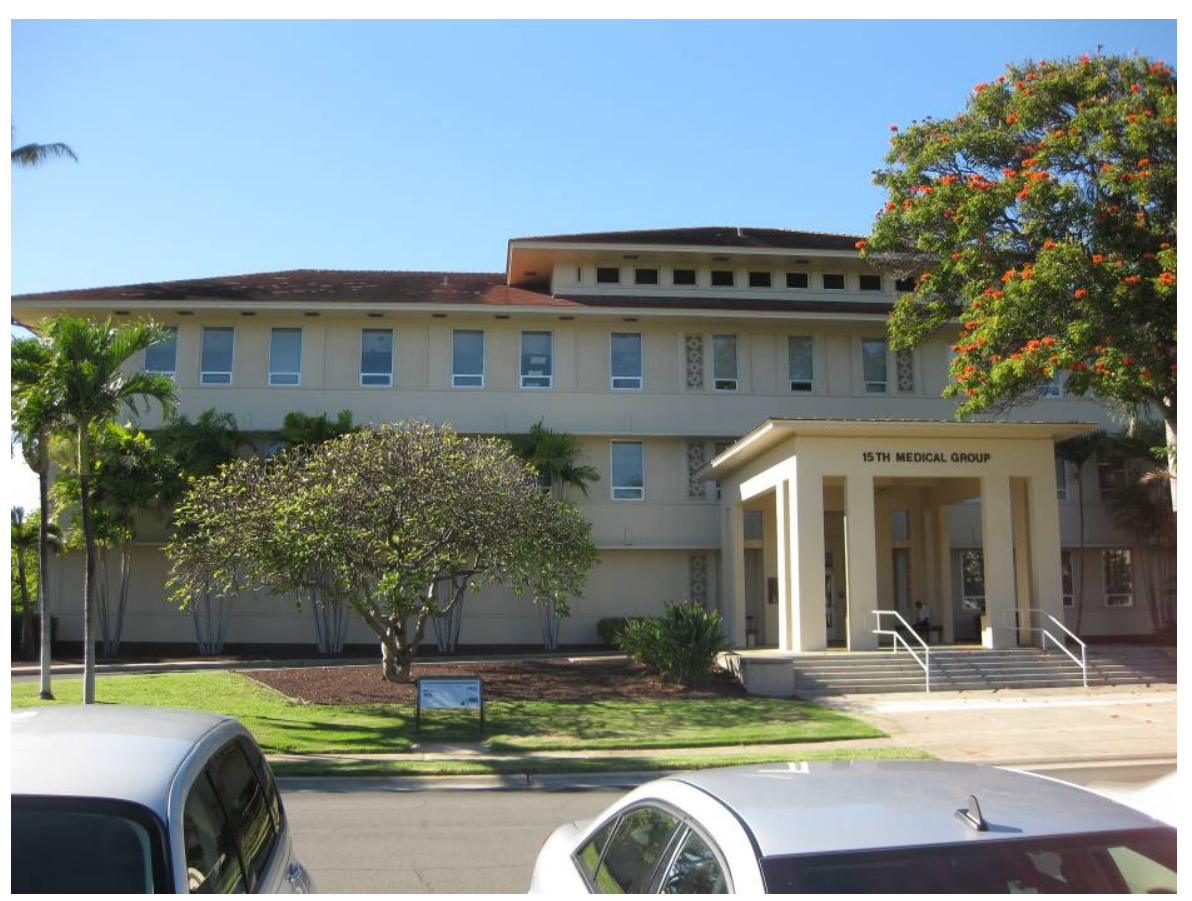




\section{Appropriated Funding Results}

A FEDS analysis using appropriated funding suggests several lighting upgrades as well as replacing the electric central boiler with a central heat pump system. Increasing the insulation of the attic by using blow-in cellulose is also suggested.

Appropriated funding FEDS results for building 559:

\begin{tabular}{|c|c|c|c|c|c|c|c|c|}
\hline Bldg. Set Description & $\begin{array}{l}\text { End } \\
\text { Use }\end{array}$ & Existing Technology & Retrofit Technology & $\begin{array}{l}\text { Energy } \\
\text { Savings } \\
\text { (MMBtu/yr) }\end{array}$ & $\begin{array}{c}\text { 1st year } \\
\text { savings } \\
(\$ / y r)\end{array}$ & $\begin{array}{l}\text { Installed } \\
\text { Cost (\$) }\end{array}$ & $\begin{array}{l}\text { Net } \\
\text { Present } \\
\text { Value (\$) } \\
\end{array}$ & SIR \\
\hline Medical facilities 559 & Lights & FL1: FL 2X4 4F40T12 STD2 & $\begin{array}{l}\text { FL280: FL 2X4 3F32ST8 ELC3 } \\
\text { REF (FIX REPL) }\end{array}$ & 633 & 39,807 & 124,627 & 542,013 & 5.3 \\
\hline Medical facilities 559 & Lights & EX1: EXIT - INC $(2 \times 20)$ & $\begin{array}{l}\text { EX12: EXIT - } \\
\text { ELECTROLUMINESCENT PANEL } \\
\text { RETRO KIT }\end{array}$ & 54 & 3,653 & 2,795 & 58,803 & 22.0 \\
\hline Medical facilities 559 & Lights & FL39: FL 2X4 2F32T8 EEF2 & $\begin{array}{l}\text { FL303: FL 2X4 2F25ST8 ELC2 } \\
\text { REF }\end{array}$ & 127 & 9,090 & 43,662 & 109,270 & 3.5 \\
\hline Medical facilities 559 & $\begin{array}{l}\text { Hot } \\
\text { Water }\end{array}$ & Electric Central Boiler & $\begin{array}{l}\text { Central Heat Pump Hot Water } \\
\text { System, Wrap Tank, Aerators, } \\
\text { LFSHs }\end{array}$ & 939 & 47,853 & 74,876 & 579,478 & 7.2 \\
\hline Medical facilities 559 & Roof & $\begin{array}{l}\text { Roof Insulation R-Value } \\
11.00\end{array}$ & $\begin{array}{l}\text { Attic Ceiling: Increase } \\
\text { Insulation by R-13 (blow-in } \\
\text { cellulose) }\end{array}$ & 19 & 1,137 & 15,089 & 3,830 & 1.3 \\
\hline
\end{tabular}




\section{Appropriated Funding Energy Consumption by Fuel Type}

The modeled energy consumption for a typical year was 1,458,222 kwh before retrofits and 938,860 kwh after proposed retrofits are implemented. The energy use intensity goes from 63.6 MBtu/Ksf to 41.1 MBtu/Ksf after retrofits.

\begin{tabular}{|c|c|c|c|c|}
\hline \multirow[b]{2}{*}{ Fuel } & \multicolumn{3}{|c|}{ Medical facilities } & \multirow[b]{2}{*}{$\begin{array}{l}\text { Dollars } \\
(2009) \text { * }\end{array}$} \\
\hline & Energy & $\begin{array}{c}\text { Energy } \\
\text { Intensity } \\
\text { (user units/1000ft2) }\end{array}$ & $\begin{array}{c}\text { Energy } \\
\text { Intensity } \\
\text { (MBtu/1000ft2) }\end{array}$ & \\
\hline \multicolumn{5}{|c|}{ Electricity (kWh) } \\
\hline existing & $1,458,222$ & $18,500 . \odot$ & 63.1 & 258,498 \\
\hline post-retrofit & 938,860 & $11,911.0$ & 40.7 & 165,179 \\
\hline difference & $-519,361$ & $-6,589.0$ & -22.5 & $-93,319$ \\
\hline$\%$ change & -36 & -36 & -36 & -36 \\
\hline \multicolumn{5}{|c|}{ other Fuels (MBtu) } \\
\hline existing & 34 & $\odot .4$ & 0.4 & 1,100 \\
\hline post-retrofit & 34 & 0.4 & 0.4 & 1,100 \\
\hline difference & 0 & 0.0 & 0.0 & 0 \\
\hline$\%$ change & $\odot$ & 0 & 0 & $\odot$ \\
\hline \multicolumn{5}{|l|}{ Total (MBtu) } \\
\hline existing & 5,011 & 63.6 & 63.6 & 259,599 \\
\hline post-retrofit & 3,239 & 41.1 & 41.1 & 166,280 \\
\hline difference & $-1,773$ & -22.5 & -22.5 & $-93,319$ \\
\hline$\%$ change & -35 & -35 & -35 & -36 \\
\hline
\end{tabular}

* Dollar values for electricity include both energy and demand components. 


\section{Appropriated Funding Energy Consumption by End Use}

Motors and miscellaneous equipment is the largest load in the building with 396,160 kWh/year, followed by lighting with 363,057 $\mathrm{kWh} /$ year.

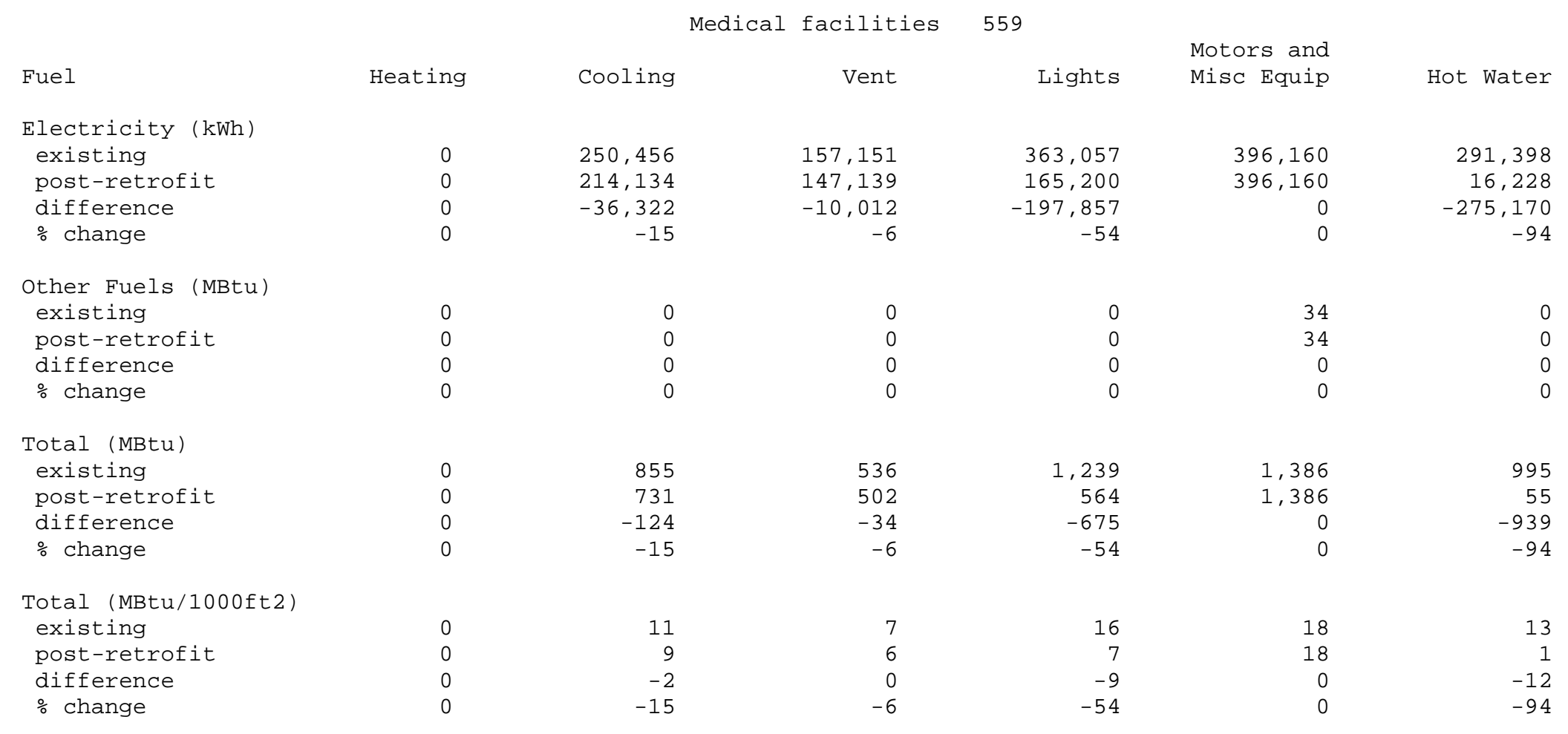




\section{Appropriated Funding Emission Reduction}

The emission reductions from implemented the proposed retrofits are as follows:

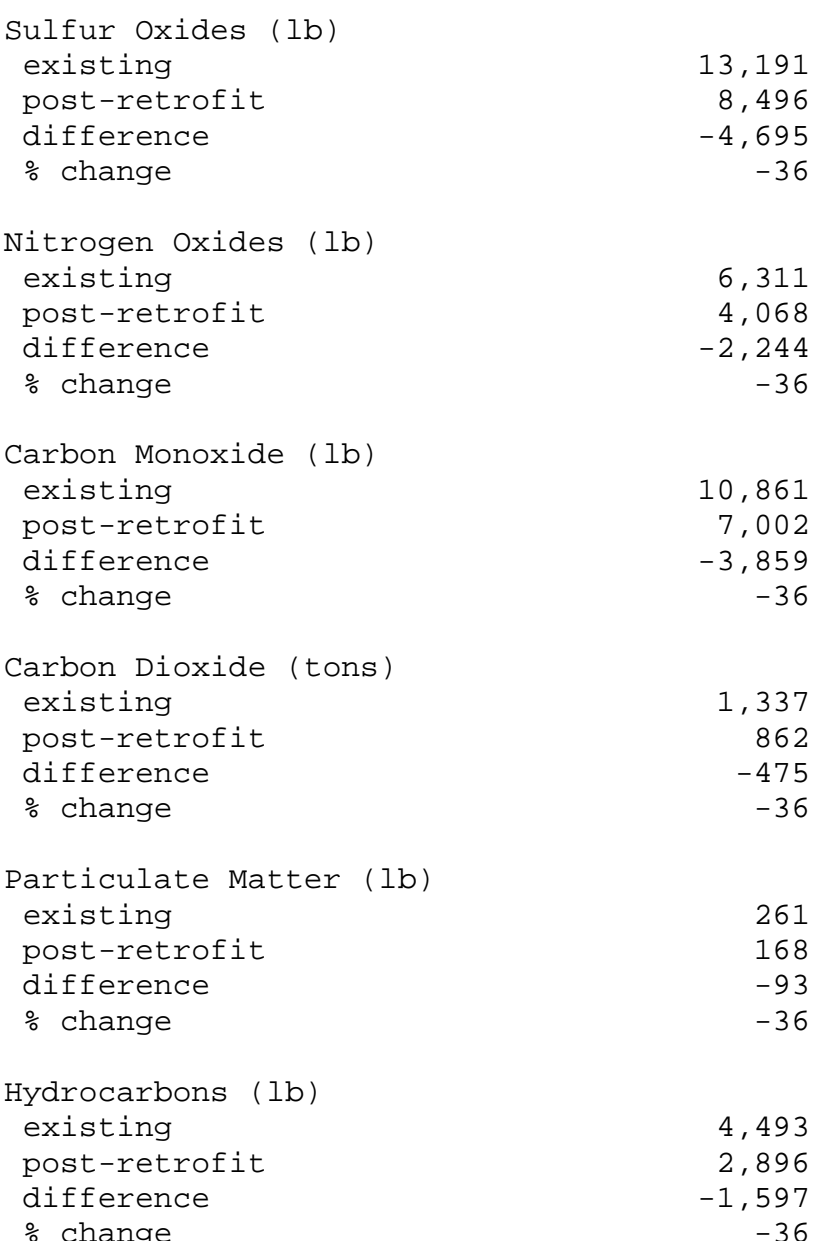




\section{Building 1060 Laboratory Building}

The following information identifies the cost-effective energy- and cost-reducing retrofit projects for building 1060 identified from the FEDS modeling and analysis. Key energy and economic results are presented for each cost-effective retrofit measure.

\section{Facility Description 1060}

Building 1060 is a lab built in 1943. This lab is cooled by an air cooled chiller and has an electric water heater. 1060 is 14,920 sf.

$<$ no picture is available $>$

\section{Appropriated Funding Results}

A FEDS analysis using appropriated funding suggests replacing the air cooled chiller with a water cooled chiller. The analysis also suggests replacing several of the lighting technologies in the building including the exit lights, T12 and T8 lights.

Appropriated funding FEDS results for building 1060:

\begin{tabular}{|c|c|c|c|c|c|c|c|c|}
\hline Bldg. Set Description & $\begin{array}{l}\text { End } \\
\text { Use }\end{array}$ & Existing Technology & Retrofit Technology & $\begin{array}{l}\text { Energy } \\
\text { Savings } \\
\text { (MMBtu/yr) }\end{array}$ & $\begin{array}{l}\text { 1st year } \\
\text { savings } \\
(\$ / y r)\end{array}$ & $\begin{array}{l}\text { Installed } \\
\text { Cost }(\$)\end{array}$ & $\begin{array}{l}\text { Net } \\
\text { Present } \\
\text { Value (\$) }\end{array}$ & SIR \\
\hline Labs 1060 & Cooling & $\begin{array}{l}\text { Electric Air-Cooled Chiller } \\
\{\mathrm{C} 1\}\end{array}$ & $\begin{array}{l}\text { Water-Cooled Reciprocating } \\
\text { Electric Chiller (very high } \\
\text { efficiency) and Cooling Tower }\end{array}$ & 139 & 7,949 & 58,769 & 29,552 & 1.9 \\
\hline Labs 1060 & Lights & EX1: EXIT - INC (2x20) & $\begin{array}{l}\text { EX12: EXIT - } \\
\text { ELECTROLUMINESCENT PANEL } \\
\text { RETRO KIT }\end{array}$ & 13 & 868 & 621 & 14,008 & 23.5 \\
\hline Labs 1060 & Lights & FL39: FL 2X4 2F32T8 EEF2 & $\begin{array}{l}\text { FL303: FL 2X4 2F25ST8 ELC2 } \\
\text { REF }\end{array}$ & 9 & 550 & 3,516 & 5,698 & 2.6 \\
\hline Labs 1060 & Lights & FL3: FL 2X4 2F40T12 STD2 & $\begin{array}{l}\text { FL283: FL 2X4 2F30ST8 ELC2 } \\
\text { (FIX REPL) }\end{array}$ & 15 & 990 & 5,463 & 11,141 & 3.0 \\
\hline
\end{tabular}




\section{Appropriated Funding Energy Consumption by Fuel Type}

The modeled energy consumption for a typical year was 292,009 kwh before retrofits and 239,726 kwh after proposed retrofits are implemented. The energy use intensity goes from 66.8 MBtu/Ksf to 54.8 MBtu/Ksf after retrofits.

\begin{tabular}{|c|c|c|c|c|}
\hline \multirow[b]{2}{*}{ Fuel } & \multirow[b]{2}{*}{ Energy } & Labs & \multirow[b]{2}{*}{$\begin{array}{l}\text { Energy } \\
\text { Intensity } \\
\text { (MBtu/1000ft2) }\end{array}$} & \multirow[b]{2}{*}{$\begin{array}{l}\text { Dollars } \\
(2009) \text { * }\end{array}$} \\
\hline & & $\begin{array}{c}\text { Energy } \\
\text { Intensity } \\
\text { (user units/1000ft2) }\end{array}$ & & \\
\hline \multicolumn{5}{|c|}{ Electricity (kWh) } \\
\hline existing & 292,009 & $19,571.7$ & 66.8 & 51,764 \\
\hline post-retrofit & 239,726 & $16,067.4$ & 54.8 & 42,176 \\
\hline difference & $-52,284$ & $-3,504.3$ & -12.0 & $-9,588$ \\
\hline$\%$ change & -18 & -18 & -18 & -19 \\
\hline \multicolumn{5}{|l|}{ Total (MBtu) } \\
\hline existing & 997 & 66.8 & 66.8 & 51,764 \\
\hline post-retrofit & 818 & 54.8 & 54.8 & 42,176 \\
\hline difference & -178 & -12.0 & -12.0 & $-9,588$ \\
\hline$\%$ change & -18 & -18 & -18 & -19 \\
\hline
\end{tabular}

* Dollar values for electricity include both energy and demand components. 


\section{Appropriated Funding Energy Consumption by End Use}

Space cooling is the largest load in the building with $116,645 \mathrm{kWh} /$ year, followed by motors and miscellaneous equipment with 77,382 kWh/year.

Fuel
Electricity (kWh)
existing
post-retrofit
difference
\% change
Total (MBtu)
existing
post-retrofit
difference
\% change
Total (MBtu/1000ft2)
existing
post-retrofit
difference
$\%$ change

$\begin{array}{rr}\text { Heating } & \text { Cooling } \\ & \\ \odot & 116,645 \\ \odot & 73,090 \\ \odot & -43,555 \\ \odot & -37 \\ & \\ \odot & 398 \\ \odot & 249 \\ \odot & -149 \\ \odot & -37 \\ & \\ \odot & 27 \\ \odot & 17 \\ \odot & -10 \\ \odot & -37\end{array}$

\begin{abstract}
Labs 1060
\end{abstract}

$$
\text { Vent }
$$

Lights

Misc Equip

Hot Water

52,192
51,672
-520
-1

178
176
-2
-1

12
12
0
-1

44,584
36,375
$-8,208$
-18

152
124
-28
-18

77,382
77,382
0
0

1,207

1,207

$\odot$
$\odot$

264

264

$\odot$
$\odot$

4
4
$\odot$
$\odot$

18

18
0

$-1$ 


\section{Appropriated Funding Emission Reduction}

The emission reductions from implemented the proposed retrofits are as follows:

Labs 1060

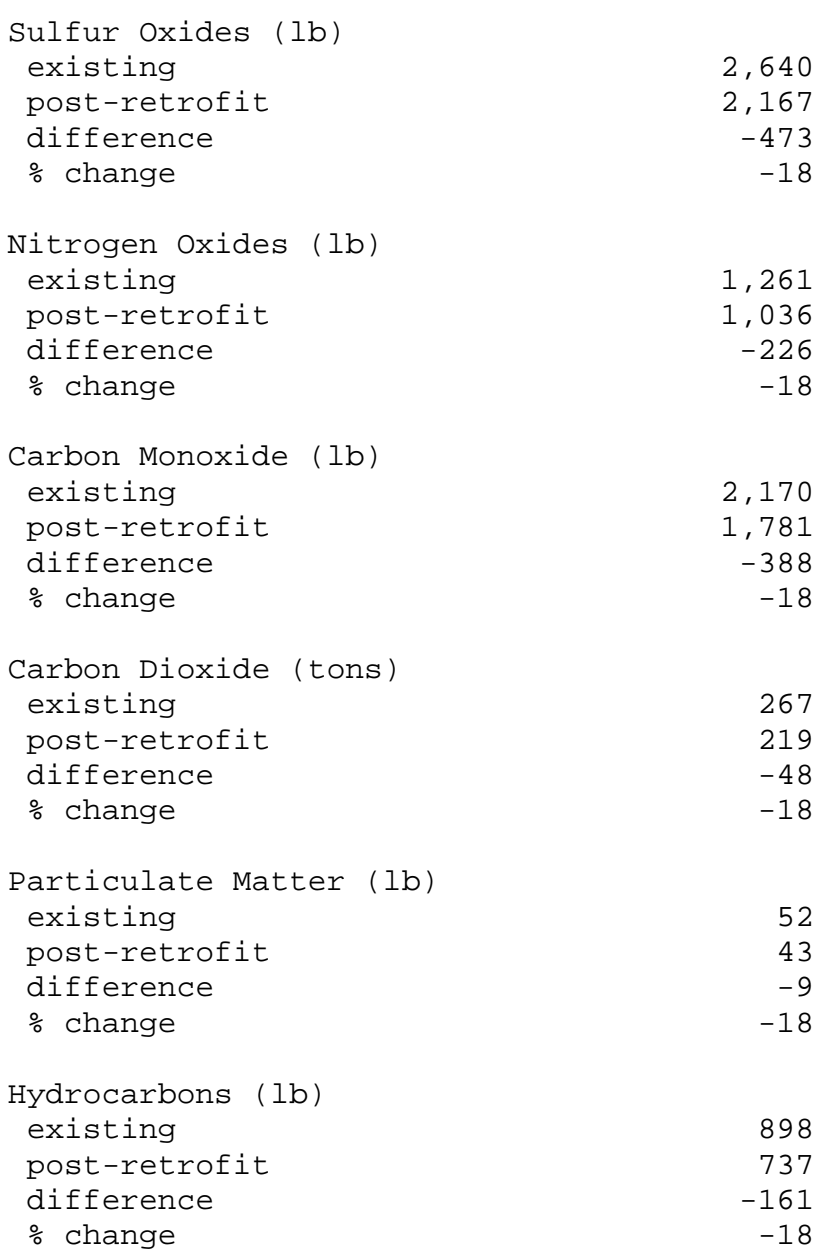




\section{Building 1805 Dormitory Building}

The following information identifies the cost-effective energy- and cost-reducing retrofit projects for building 1805 identified from the FEDS modeling and analysis. Key energy and economic results are presented for each cost-effective retrofit measure.

\section{Facility Description 1805}

Building 1805 is a dormitory built in 1970. The dormitory is cooled by an air cooled chiller and has little to no insulation in its building envelope. This building has a desuperheater system, providing some of the hot water to the building. 1805 is 55,187 sf.

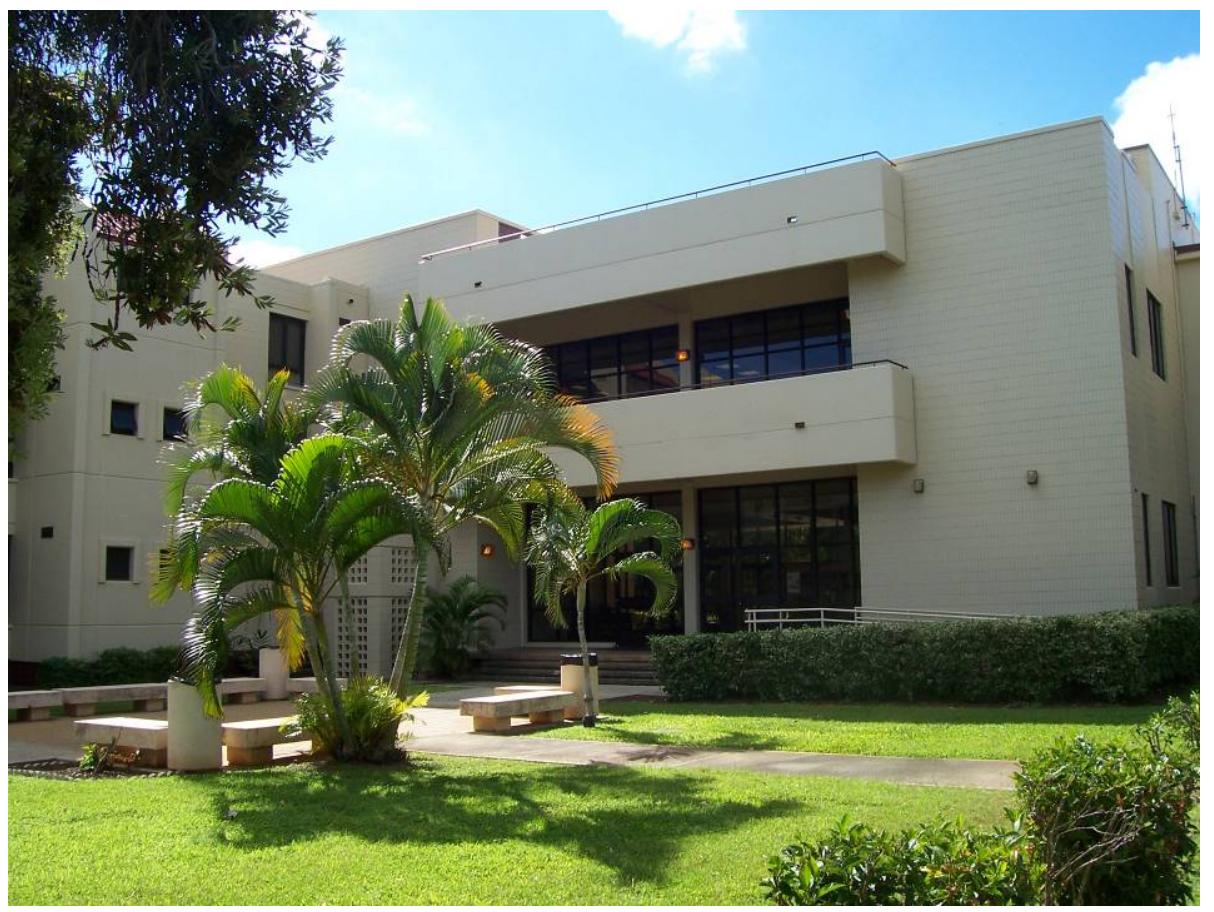




\section{Appropriated Funding Results}

A FEDS analysis using appropriated funding suggests replacing the air cooled chiller with a water cooled chiller as well as replacing some of the lighting technologies and replacing the electric central boiler with a central heat pump. Increasing the perimeter insulation as well as replacing the windows was also suggested.

Appropriated funding FEDS analysis results for building 1805:

\begin{tabular}{|c|c|c|c|c|c|c|c|c|}
\hline Bldg. Set Description & $\begin{array}{l}\text { End } \\
\text { Use }\end{array}$ & Existing Technology & Retrofit Technology & $\begin{array}{l}\text { Energy } \\
\text { Savings } \\
\text { (MMBtu/yr) } \\
\end{array}$ & $\begin{array}{l}\text { 1st year } \\
\text { savings } \\
(\$ / y r)\end{array}$ & $\begin{array}{l}\text { Installed } \\
\text { Cost (\$) }\end{array}$ & $\begin{array}{l}\text { Net } \\
\text { Present } \\
\text { Value (\$) }\end{array}$ & SIR \\
\hline Dorms 1970's 1805 & Cooling & $\begin{array}{l}\text { Electric Air-Cooled Chiller } \\
\{C 1\}\end{array}$ & $\begin{array}{l}\text { Water-Cooled Reciprocating } \\
\text { Electric Chiller (very high } \\
\text { efficiency) and Cooling Tower }\end{array}$ & 172 & 9,749 & 87,783 & 27,443 & 1.5 \\
\hline Dorms 1970's 1805 & Lights & EX1: EXIT - INC $(2 \times 20)$ & $\begin{array}{l}\text { EX12: EXIT - } \\
\text { ELECTROLUMINESCENT PANEL } \\
\text { RETRO KIT }\end{array}$ & 58 & 3,862 & 2,795 & 62,275 & 23.3 \\
\hline Dorms 1970's 1805 & Lights & FL41: FL 1X4 1F32T8 EEF1 & $\begin{array}{l}\text { FL302: FL 1X4 1F25ST8 ELC1 } \\
\text { REF }\end{array}$ & 23 & 1,693 & 14,346 & 14,333 & 2.0 \\
\hline
\end{tabular}




\section{Appropriated Funding Energy Consumption by Fuel Type}

The modeled energy consumption for a typical year was 515,140 kwh before retrofits and 334,549 kwh after proposed retrofits are implemented. The energy use intensity goes from 31.9 MBtu/Ksf to 20.7 MBtu/Ksf after retrofits.

Dorms 1970 's 1805

Fuel

Electricity (kWh)

existing

post-retrofit

difference

$\%$ change

Total (MBtu)

existing

post-retrofit

difference

$\%$ change
Energy

515,140

441,113

$-74,027$

$-14$

1,758

1,506

$-253$

Energy
Intensity
(user units/100०ft2)

$9,334.4$

$7,993.1$

$-1,341.4$

$-14$

31.9

27.3

$-4.6$

$-14$
Energy

Intensity

(MBtu/1000ft2)

Dollars

(2009) *

31.9
27.3
-4.6
-14


31.9
27.3
-4.6
-14

91,072

77,967

$-13,105$

$-14$

91,072

77,967

$-13,105$

$-14$

* Dollar values for electricity include both energy and demand components. 


\section{Appropriated Funding Energy Consumption by End Use}

Space cooling is the largest load in the building with 188,139 kWh/year, followed by hot water with 121,790 kWh/year.

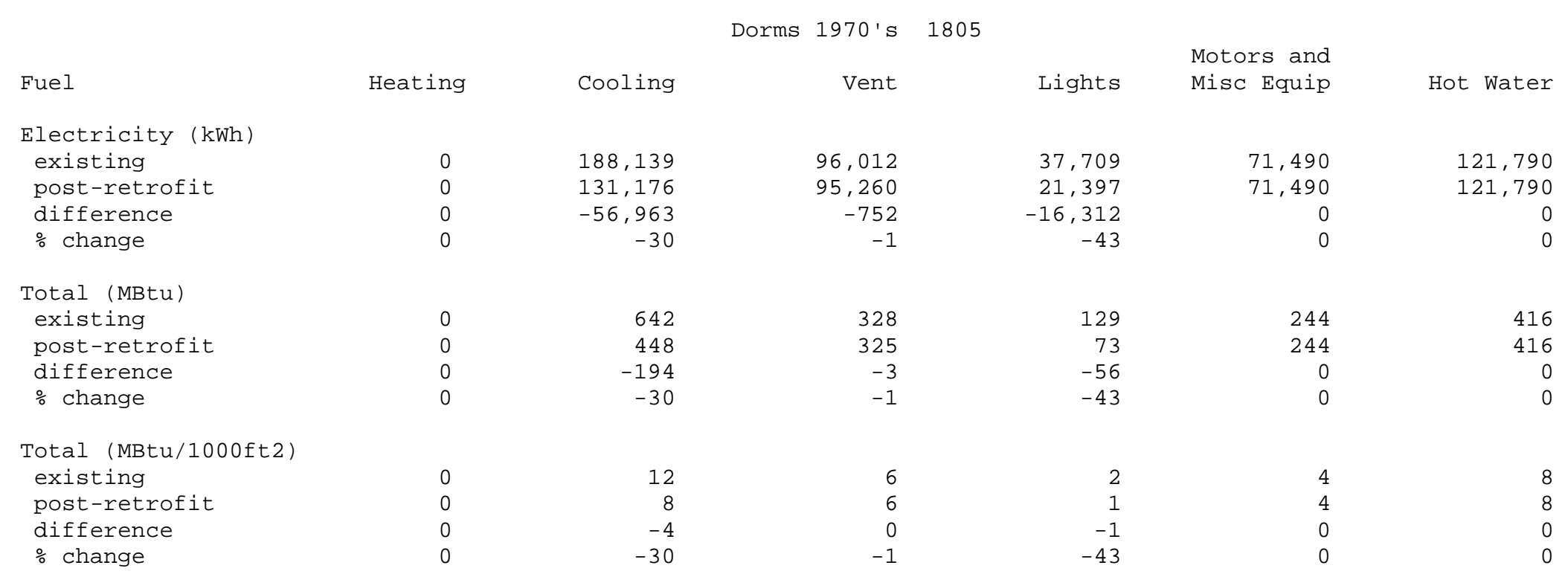




\section{Appropriated Funding Emission Reduction}

The emission reductions from implemented the proposed retrofits are as follows:

Sulfur Oxides (lb)

existing

post-retrofit

Dorms 1970's 1805

difference

$\%$ change

3,988

-669
-14

Nitrogen Oxides (lb)

existing

post-retrofit

2,225

difference

1,906

$\%$ change

-320
-14

Carbon Monoxide (lb)

existing

post-retrofit

828

difference

3,278

$-550$

$\%$ change

$-14$

Carbon Dioxide (tons)

existing

post-retrofit

471

post-retro

404

$\%$ change

$-14$

Particulate Matter (lb)

existing

post-retrofit

difference

92

79

$-13$

$\%$ change

1,584

(lb)

existing

1,356
-228

post-retrofit

$\%$ change

$-14$ 


\section{Building 1856 Dormitory Building}

The following information identifies the cost-effective energy- and cost-reducing retrofit projects for building 1856 identified from the FEDS modeling and analysis. Key energy and economic results are presented for each cost-effective retrofit measure.

\section{Facility Description 1856}

Building 1856 is a dormitory built in 1995. The dormitory is cooled by an electric air cooled chiller and has substantial roofing and wall insulation in its building envelope. The central hot water system runs on diesel fuel and works in conjunction with a desuperheater. Building 1856 is 43,187 sf.

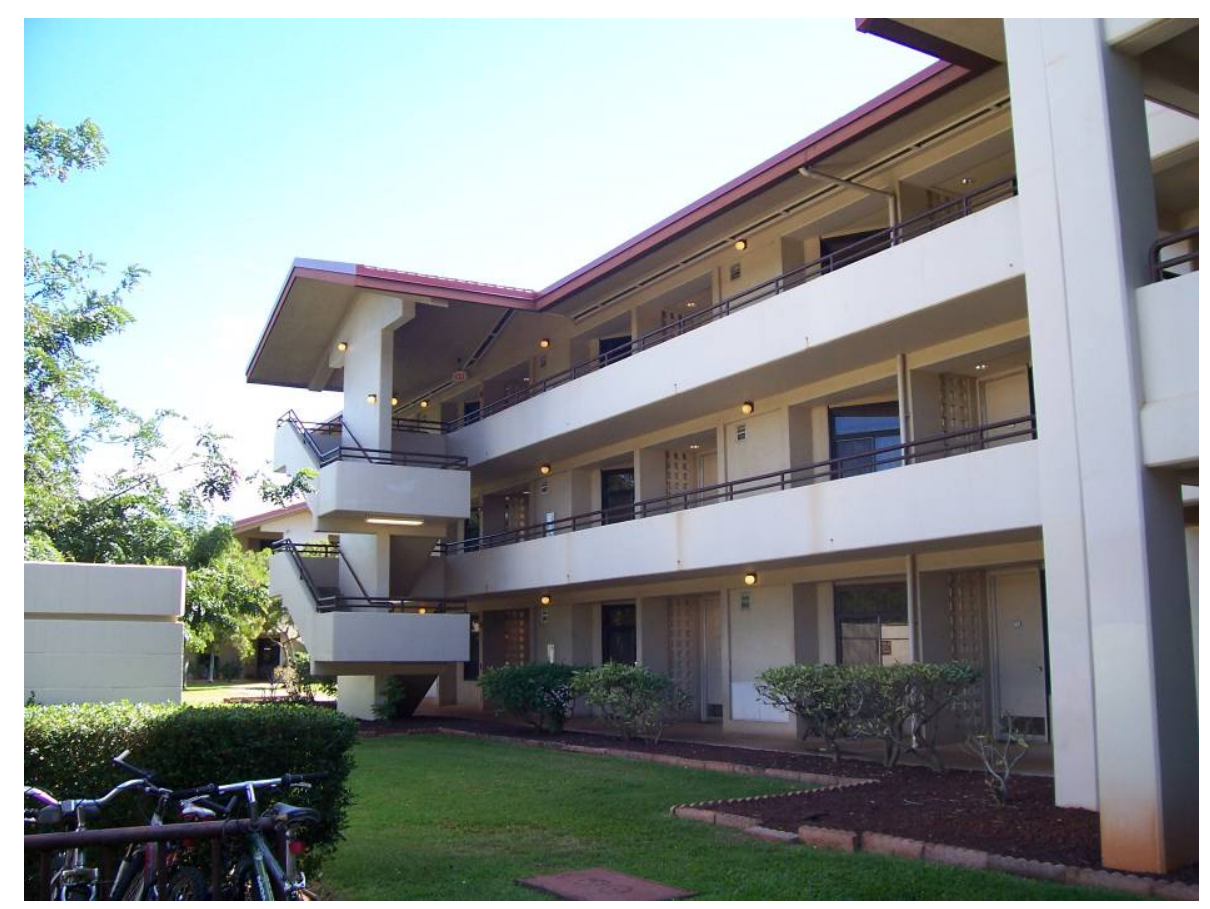




\section{Appropriated Funding Results}

A FEDS analysis using appropriated funding suggests replacing the air cooled chiller with a very high efficiency water cooled chiller. The distillate oil, or diesel, central hot water boiler is suggested to be replaced with a central heat pump hot water system. An increase in the perimeter insulation is suggested as well as replacing the exit lighting.

Appropriated funding FEDS analysis results for building 1856:

\begin{tabular}{|c|c|c|c|c|c|c|c|c|}
\hline Bldg. Set Description & $\begin{array}{l}\text { End } \\
\text { Use }\end{array}$ & Existing Technology & Retrofit Technology & $\begin{array}{l}\text { Energy } \\
\text { Savings } \\
\text { (MMBtu/yr) }\end{array}$ & $\begin{array}{c}\text { 1st year } \\
\text { savings } \\
(\$ / y r)\end{array}$ & $\begin{array}{l}\text { Installed } \\
\text { Cost (\$) }\end{array}$ & $\begin{array}{l}\text { Net } \\
\text { Present } \\
\text { Value (\$) } \\
\end{array}$ & SIR \\
\hline $\begin{array}{l}\text { Dorms } 1990 \text { 's } 1856 \text { - heat } \\
\text { recovery }\end{array}$ & Cooling & $\begin{array}{l}\text { Electric Air-Cooled Chiller } \\
\{\mathrm{C} 1\}\end{array}$ & $\begin{array}{l}\text { Water-Cooled Reciprocating } \\
\text { Electric Chiller (very high } \\
\text { efficiency) and Cooling Tower }\end{array}$ & 286 & 15,031 & 75,277 & 48,558 & 2.8 \\
\hline $\begin{array}{l}\text { Dorms 1990's } 1856 \text { - heat } \\
\text { recovery }\end{array}$ & Lights & EX1: EXIT - INC $(2 \times 20)$ & $\begin{array}{l}\text { EX12: EXIT - } \\
\text { ELECTROLUMINESCENT PANEL } \\
\text { RETRO KIT }\end{array}$ & 62 & 3,994 & 2,795 & 64,466 & 24.1 \\
\hline $\begin{array}{l}\text { Dorms } 1990 \text { 's } 1856 \text { - heat } \\
\text { recovery }\end{array}$ & $\begin{array}{l}\text { Hot } \\
\text { Water }\end{array}$ & Distillate Oil Central Boiler & $\begin{array}{l}\text { Central Heat Pump Hot Water } \\
\text { System }\end{array}$ & 151 & 5,505 & 22,540 & 91,307 & 3.8 \\
\hline
\end{tabular}




\section{Appropriated Funding Energy Consumption by Fuel Type}

The modeled energy consumption for a typical year was 418,237 kwh before retrofits and 320,744 kwh after proposed retrofits are implemented. The modeled distillate oil consumption for a typical year was 1,252 gallons before retrofits and 0 gallons after proposed retrofits are implemented. The energy use intensity goes from 37.1 MBtu/Ksf to 25.3 MBtu/Ksf after retrofits.

\begin{tabular}{|c|c|c|c|c|}
\hline \multirow[b]{2}{*}{ Fuel } & \multirow[b]{2}{*}{ Energy } & Dorms 1990 's & \multirow[b]{2}{*}{$\begin{array}{c}\text { Energy } \\
\text { Intensity } \\
\text { (MBtu/1000ft2) }\end{array}$} & \multirow[b]{2}{*}{$\begin{array}{l}\text { Dollars } \\
(2009) \text { * }\end{array}$} \\
\hline & & $\begin{array}{c}\text { Energy } \\
\text { Intensity } \\
\text { (user units/1000ft2) }\end{array}$ & & \\
\hline \multicolumn{5}{|c|}{ Electricity (kWh) } \\
\hline existing & 418,237 & $9,684.3$ & 33.1 & 73,940 \\
\hline post-retrofit & 320,744 & $7,426.9$ & 25.3 & 56,404 \\
\hline difference & $-97,494$ & $-2,257.5$ & -7.7 & $-17,536$ \\
\hline$\%$ change & -23 & -23 & -23 & -24 \\
\hline \multicolumn{5}{|c|}{ Distillate Oil (gal) } \\
\hline existing & 1,252 & 29.0 & 4.0 & 6,385 \\
\hline post-retrofit & 0 & 0.0 & $\odot . \odot$ & 0 \\
\hline difference & $-1,252$ & -29.0 & -4.0 & $-6,385$ \\
\hline$\%$ change & -100 & -100 & -100 & -100 \\
\hline \multicolumn{5}{|l|}{ Total (MBtu) } \\
\hline existing & 1,601 & 37.1 & 37.1 & 80,325 \\
\hline post-retrofit & 1,095 & 25.3 & 25.3 & 56,404 \\
\hline difference & -506 & -11.7 & -11.7 & $-23,921$ \\
\hline$\%$ change & -32 & -32 & -32 & -30 \\
\hline
\end{tabular}

* Dollar values for electricity include both energy and demand components. 


\section{Appropriated Funding Energy Consumption by End Use}

Space cooling is the largest load in the building with 233,630 kWh/year, followed by ventilation with 66,184 kWh/year.

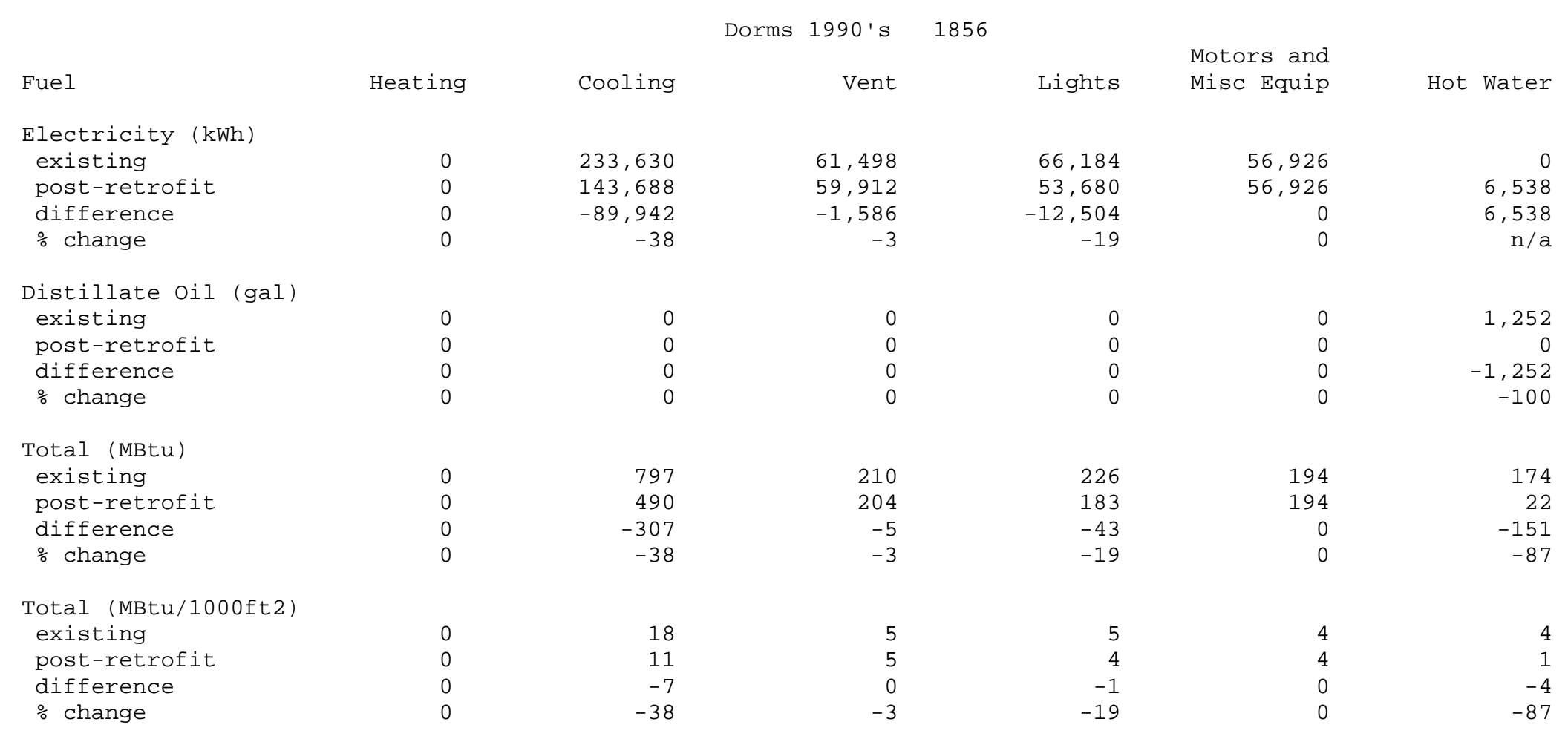




\section{Appropriated Funding Emission Reduction}

The emission reductions from implemented the proposed retrofits are as follows:

Dorms 1990 's 1856

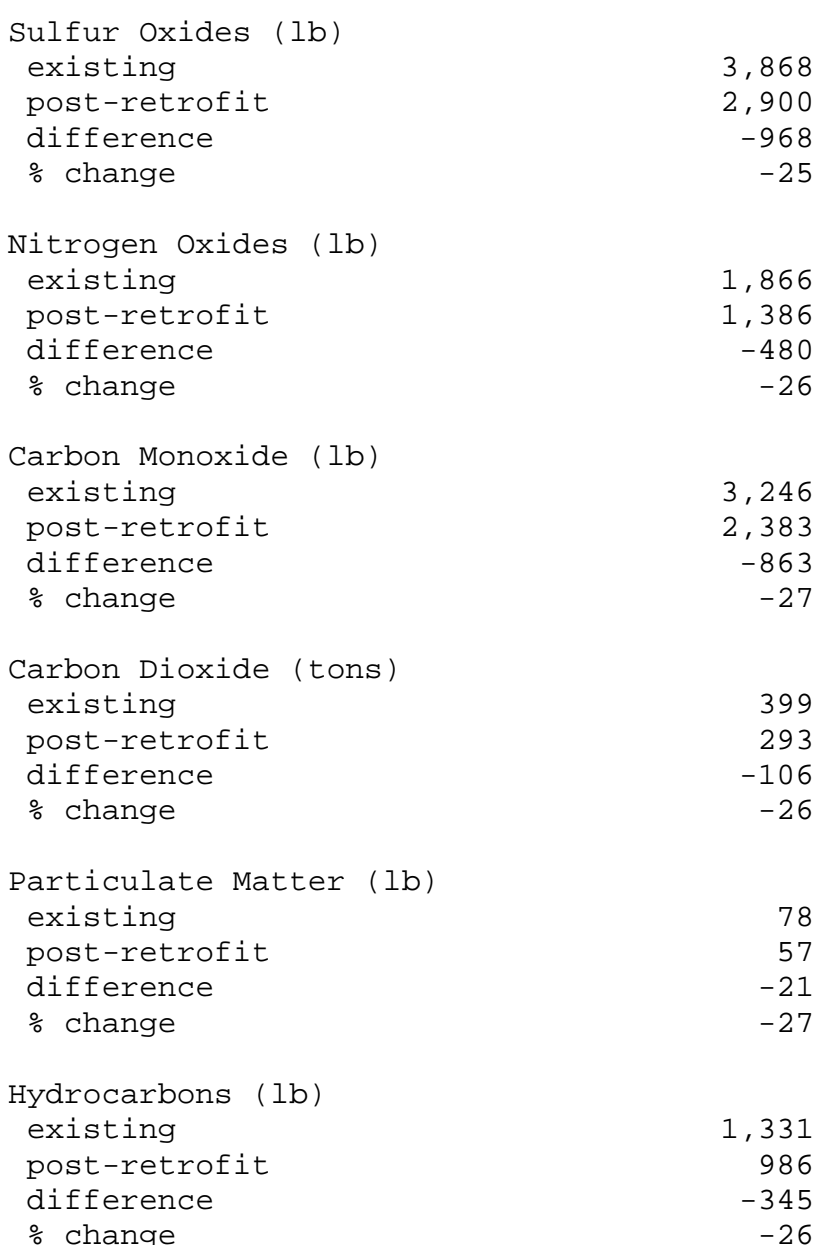




\section{Building 1166 Lodging Facility}

The following information identifies the cost-effective energy- and cost-reducing retrofit projects for building 1166 identified from the FEDS modeling and analysis. Key energy and economic results are presented for each cost-effective retrofit measure.

\section{Facility Description 1166}

Building 1166 is a hotel style building used as a temporary lodging facility and was built in 1968 . The building is cooled by an air cooled chiller and has little to no insulation in the building envelope. Building 1166 is 25,113 sf.

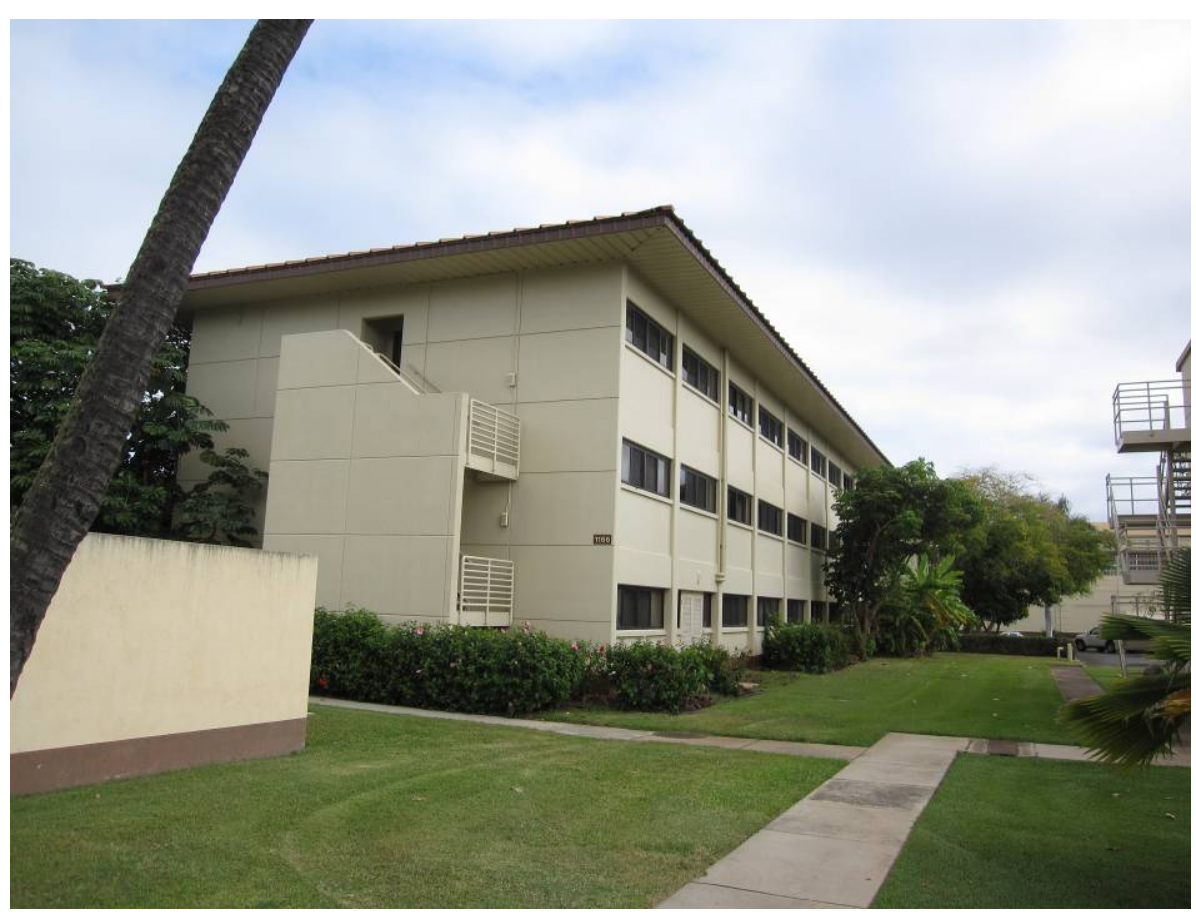




\section{Appropriated Funding Results}

A FEDS analysis using appropriated funding suggests replacing the air cooled chiller with a water cooled chiller. The analysis also suggests lighting retrofits as well as replacing the current electric and propane hot water boilers with a central heat pump hot water system. Increasing the roof insulation on the interior surface of the roof was also suggested.

Appropriated funding FEDS analysis results for building 1166:

\begin{tabular}{|c|c|c|c|c|c|c|c|c|}
\hline Bldg. Set Description & $\begin{array}{l}\text { End } \\
\text { Use }\end{array}$ & Existing Technology & Retrofit Technology & $\begin{array}{l}\text { Energy } \\
\text { Savings } \\
\text { (MMBtu/yr) } \\
\end{array}$ & $\begin{array}{l}\text { 1st year } \\
\text { savings } \\
(\$ / y r)\end{array}$ & $\begin{array}{l}\text { Installed } \\
\text { Cost (\$) }\end{array}$ & $\begin{array}{l}\text { Net } \\
\text { Present } \\
\text { Value (\$) } \\
\end{array}$ & SIR \\
\hline Lodging facilities 1166 & Cooling & $\begin{array}{l}\text { Electric Air-Cooled Chiller } \\
\{C 1\}\end{array}$ & $\begin{array}{l}\text { Water-Cooled Reciprocating } \\
\text { Electric Chiller (very high } \\
\text { efficiency) and Cooling Tower }\end{array}$ & 197 & 10,822 & 95,816 & 31,149 & 1.6 \\
\hline Lodging facilities 1166 & Lights & EX1: EXIT - INC (2x20) & $\begin{array}{l}\text { EX12: EXIT - } \\
\text { ELECTROLUMINESCENT PANEL } \\
\text { RETRO KIT }\end{array}$ & 52 & 3,577 & 2,795 & 57,532 & 21.6 \\
\hline
\end{tabular}




\section{Appropriated Funding Energy Consumption by Fuel Type}

The modeled energy consumption for a typical year was 425,234 kwh before retrofits and 315,695 kwh after proposed retrofits are implemented. The modeled other fuels (propane) consumption for a typicalyear was $48 \mathrm{MBtu}$ before retrofits and $0 \mathrm{MBtu}$ after proposed retrofits are implemented. The energy use intensity goes from 59.7 MBtu/Ksf to $42.9 \mathrm{MBtu} / \mathrm{Ksf}$ after retrofits.

Fuel

Electricity (kWh)

existing

post-retrofit

difference

$\%$ change

Other Fuels (MBtu)

existing

post-retrofit

difference

$\%$ change

Total (MBtu)

existing

post-retrofit

difference

$\%$ change
Lodging facilities 1166

Energy
Intensity
(user units/1000ft2)

Energy

Intensity Dollars (MBtu/1000ft2) (2009)*

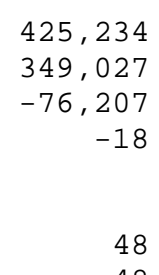

\section{1,499}

1,239

-260
-17
1.9

\section{8}

47.4

$-10.4$

$-18$

1.9

1.9

0.0

0.0
0
59.7
49.3

$-10.4$

$-17$
59.7

49.3

$-10.4$

$-17$
75,177

61,691

$-13,487$

$-18$

1,531

1,531

$\odot$

76,708

63,221

$-13,487$

* Dollar values for electricity include both energy and demand components. 


\section{Appropriated Funding Energy Consumption by End Use}

Space cooling is the largest load in the building with 116,650 kWh/year, followed by ventilation with 117,053 kWh/year.

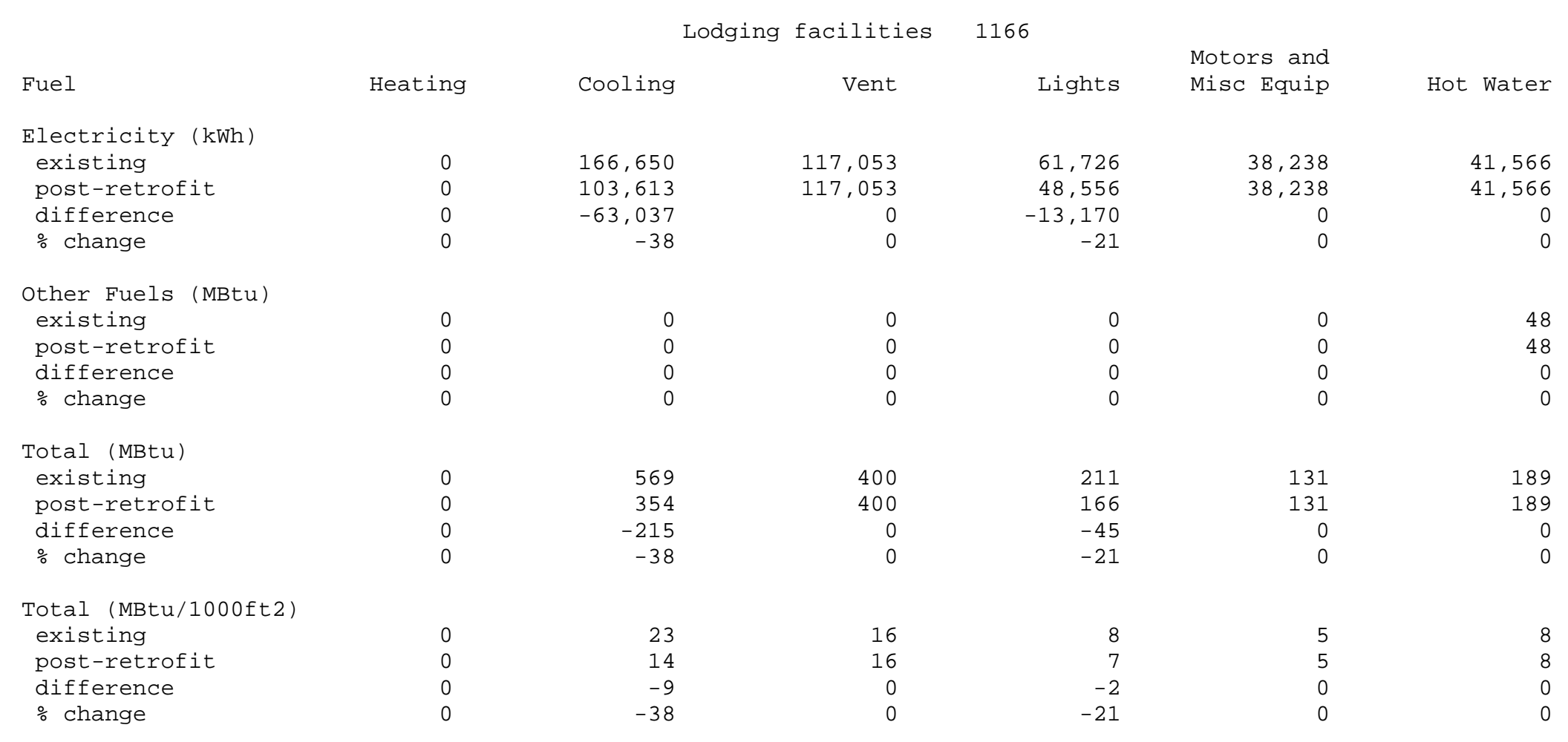




\section{Appropriated Funding Emission Reduction}

The emission reductions from implemented the proposed retrofits are as follows:

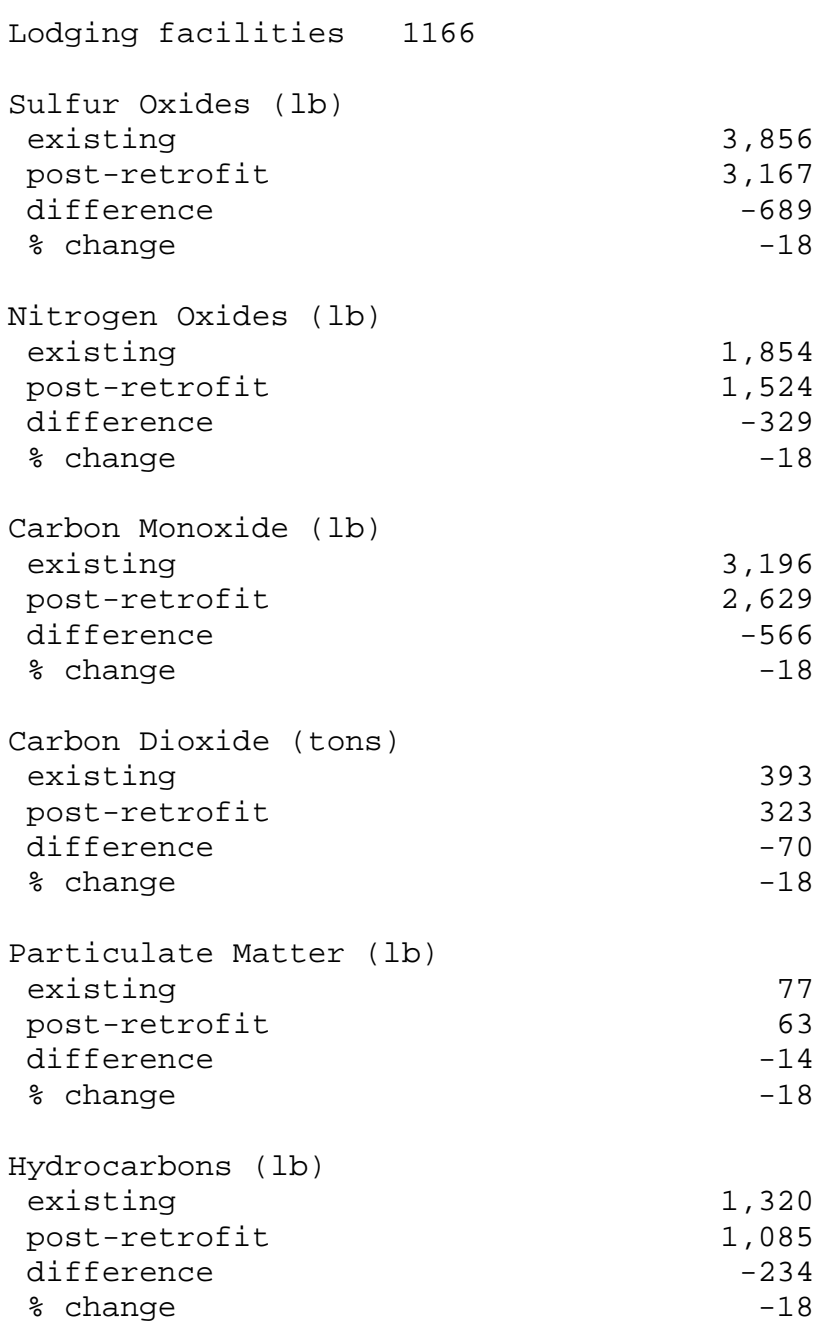




\section{Building 2040 Aircraft Maintenance Shop}

The following information identifies the cost-effective energy- and cost-reducing retrofit projects for building 2040 identified from the FEDS modeling and analysis. Key energy and economic results are presented for each cost-effective retrofit measure.

\section{Facility Description 2040}

Building 2040 is an aircraft maintenance shop built in 1937. 2040 is cooled by an air cooled chiller and has little to no insulation in its building envelope. Building 2040 is 77,439 sf.

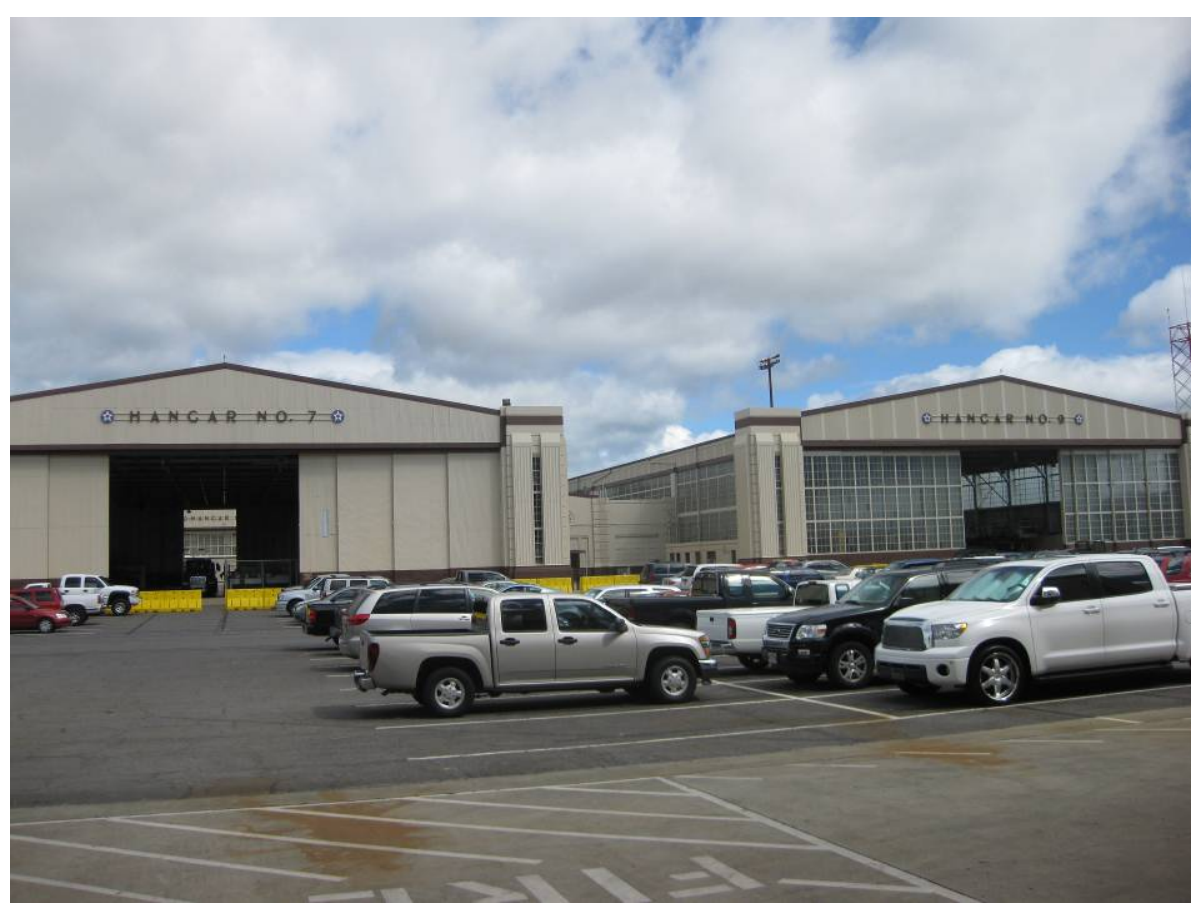




\section{Appropriated Funding Results}

A FEDS analysis using appropriated funding suggests replacing T12 lighting with Super T8 lighting. It was also suggested to make changes to the hot water system including reducing the temperature, installing aerators and increasing insulation in the conditioned space. FEDS had no life cycle cost effective retrofits for the unconditioned space.

Appropriated funding FEDS analysis results for building 2040 conditioned space:

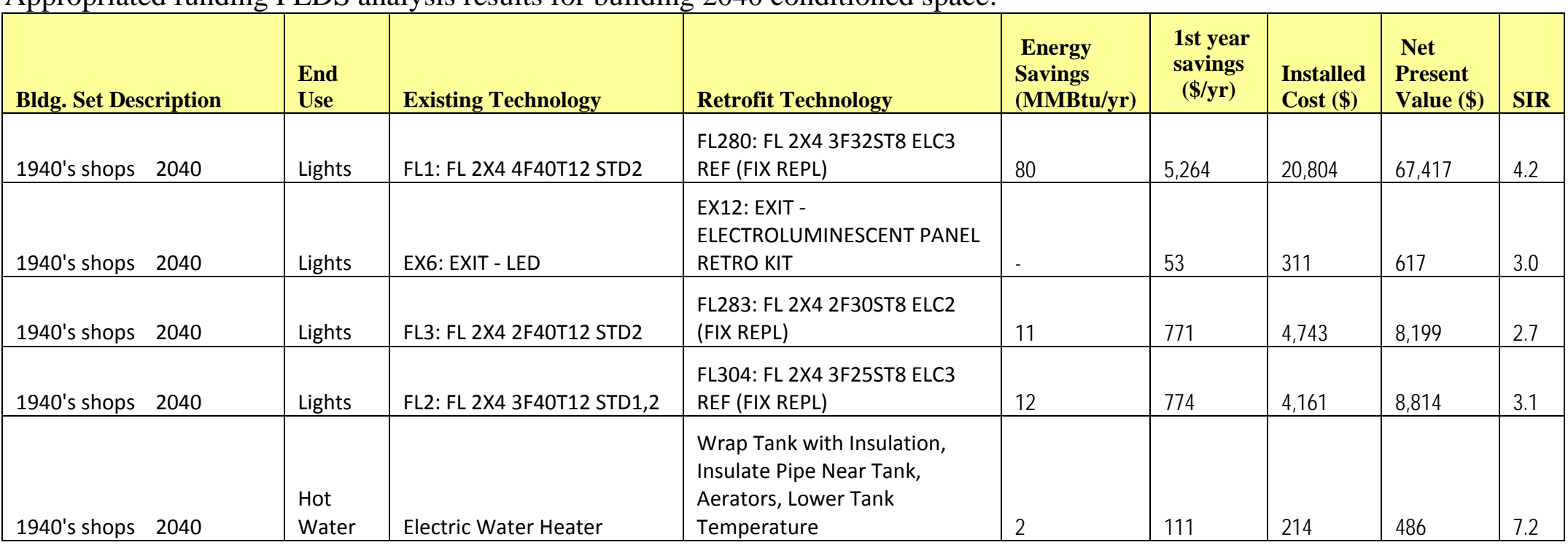




\section{Appropriated Funding Energy Consumption by Fuel Type}

The modeled energy consumption for a typical year was 352,637 kwh. No proposed retrofits were suggested for the unconditioned space. The energy use intensity is $18.3 \mathrm{MBtu} / \mathrm{Ksf}$.

\begin{tabular}{|c|c|c|c|c|}
\hline \multirow[b]{2}{*}{ Fuel } & 1940 's shops & \multicolumn{2}{|c|}{2040 unconditioned space } & \multirow[b]{2}{*}{$\begin{array}{l}\text { Dollars } \\
(20 \odot 9)^{*}\end{array}$} \\
\hline & Energy & $\begin{array}{c}\text { Energy } \\
\text { Intensity } \\
\text { (user units/1000ft2) }\end{array}$ & $\begin{array}{c}\text { Energy } \\
\text { Intensity } \\
\text { (MBtu/1000ft2) }\end{array}$ & \\
\hline \multicolumn{5}{|c|}{ Electricity (kWh) } \\
\hline existing & 352,637 & $5,362.8$ & 18.3 & 62,343 \\
\hline post-retrofit & 352,637 & $5,362.8$ & 18.3 & 62,013 \\
\hline difference & $\odot$ & $\odot . \odot$ & $\odot . \odot$ & -330 \\
\hline$\%$ change & $\odot$ & $\odot$ & 0 & -1 \\
\hline \multicolumn{5}{|l|}{ Total (MBtu) } \\
\hline existing & 1,204 & 18.3 & 18.3 & 62,343 \\
\hline post-retrofit & 1,204 & 18.3 & 18.3 & 62,013 \\
\hline difference & $-1=0$ & 0.0 & 0.0 & -330 \\
\hline$\%$ change & $\odot$ & 0 & $\odot$ & -1 \\
\hline
\end{tabular}

* Dollar values for electricity include both energy and demand components. 
The modeled energy consumption for a typical year was 156,928 kwh before retrofits and 125,990 kwh after proposed retrofits are implemented. The energy use intensity goes from 46.2 MBtu/Ksf to 37.1 MBtu/Ksf after retrofits.

\section{0 's shops \\ 2040}

Fuel

Energy

Electricity $(\mathrm{kWh})$

existing

post-retrofit

difference

$\%$ change

Total (MBtu)

existing

post-retrofit

difference

$\%$ change

* Dollar values for electricity include both energy and demand components.

\section{Energy \\ Intensity Dollars

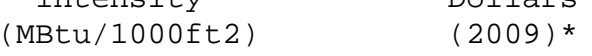

Intensity
(user units/1000ft2)

$13,523.7$

$10,857.4$

$-2,666.2$

$-20$
46.2
37.1
$-9.1$
$-20$

46. 2

37.1

$-9.1$

$-20$

46.2

37.1

$-9.1$

$-20$
27,743

22,156

$-5,587$

27,743

22,156

$-5,587$ $-20$ 


\section{Appropriated Funding Energy Consumption by End Use}

Motors and miscellaneous equipment is the largest load in the unconditioned space of the building with 308,331 kWh/year, followed by lighting with $44,307 \mathrm{kWh} /$ year.

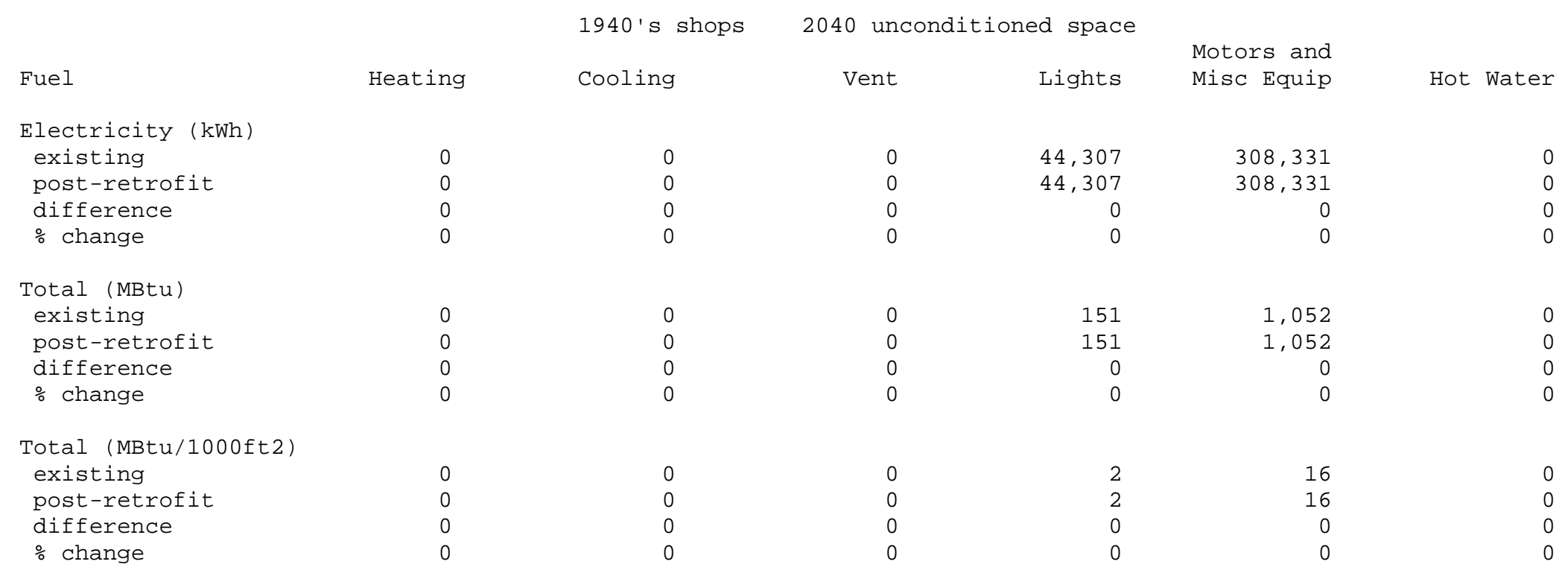


Motors and miscellaneous equipment is the largest load in the conditioned space of the building with 56,167 kWh/year, followed by space cooling with 52,533 kWh/year.

\begin{tabular}{|c|c|c|c|c|c|c|}
\hline Fuel & Heating & 1940 's & $\begin{array}{r}2040 \text { co } \\
\text { Vent }\end{array}$ & ed space & $\begin{array}{l}\text { Motors and } \\
\text { Misc Equip }\end{array}$ & Hot Water \\
\hline \multicolumn{7}{|c|}{ Electricity (kWh) } \\
\hline existing & 0 & 52,533 & 2,983 & 43,930 & 56,167 & 1,315 \\
\hline post-retrofit & 0 & 47,075 & 2,665 & 19,342 & 56,167 & 741 \\
\hline difference & $\odot$ & $-5,459$ & -318 & $-24,588$ & 0 & -574 \\
\hline$\%$ change & $\odot$ & -10 & -11 & -56 & $\odot$ & -44 \\
\hline \multicolumn{7}{|l|}{ Total (MBtu) } \\
\hline existing & 0 & 179 & 10 & 150 & 192 & 4 \\
\hline post-retrofit & 0 & 161 & 9 & 66 & 192 & 3 \\
\hline difference & 0 & -19 & -1 & -84 & 0 & -2 \\
\hline$\%$ change & 0 & -10 & -11 & -56 & 0 & -44 \\
\hline \multicolumn{7}{|c|}{ Total (MBtu/100๑ft2) } \\
\hline existing & $\odot$ & 15 & 1 & 13 & 17 & 0 \\
\hline post-retrofit & $\odot$ & 14 & 1 & 6 & 17 & $\odot$ \\
\hline difference & 0 & -2 & 0 & -7 & 0 & 0 \\
\hline$\%$ change & 0 & -10 & -11 & -56 & 0 & -44 \\
\hline
\end{tabular}




\section{Appropriated Funding Emission Reduction}

The emission reductions from implemented the proposed retrofits are as follows:

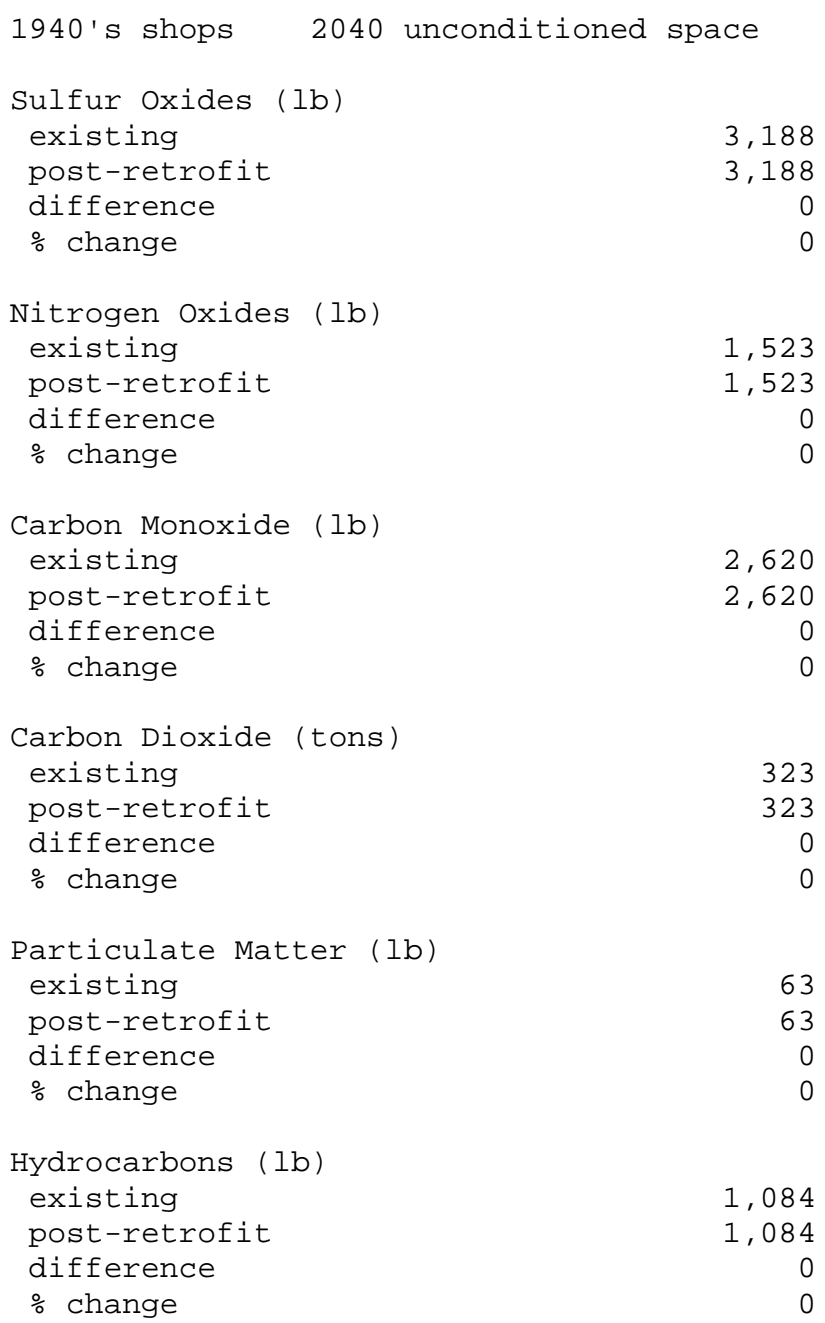


Sulfur Oxides (lb)

existing

post-retrofit

1,419

difference

1,139

$-280$

$\%$ change

$-20$

Nitrogen 0xides (lb)

existing

post-retrofit

678

544

difference

544
-134

$\%$ change

$-20$

Carbon Monoxide (lb)

existing

post-retrofit

166

difference

936

$\%$ change

230

Carbon Dioxide (tons)

existing

post-retrofit 115

difference $\quad-28$

$\%$ change

Particulate Matter (lb)

existing

post-retrofit

$\%$ change

-6
-20

Hydrocarbons ( $1 b)$

existing

post-retrofi

483

difference

387

$\%$ change

$-20$ 


\section{Building 1715 Recycling Center}

The following information identifies the cost-effective energy- and cost-reducing retrofit projects for building 1715 identified from the FEDS modeling and analysis. Key energy and economic results are presented for each cost-effective retrofit measure.

\section{Facility Description 1715}

1715 is a recycling center built in 1944. The majority of the space is unconditioned, with a small office that is served by an electric package unit. The building was modeled as two linked buildings, one conditioned, one unconditioned. Building 1715 is 30,400 sf.

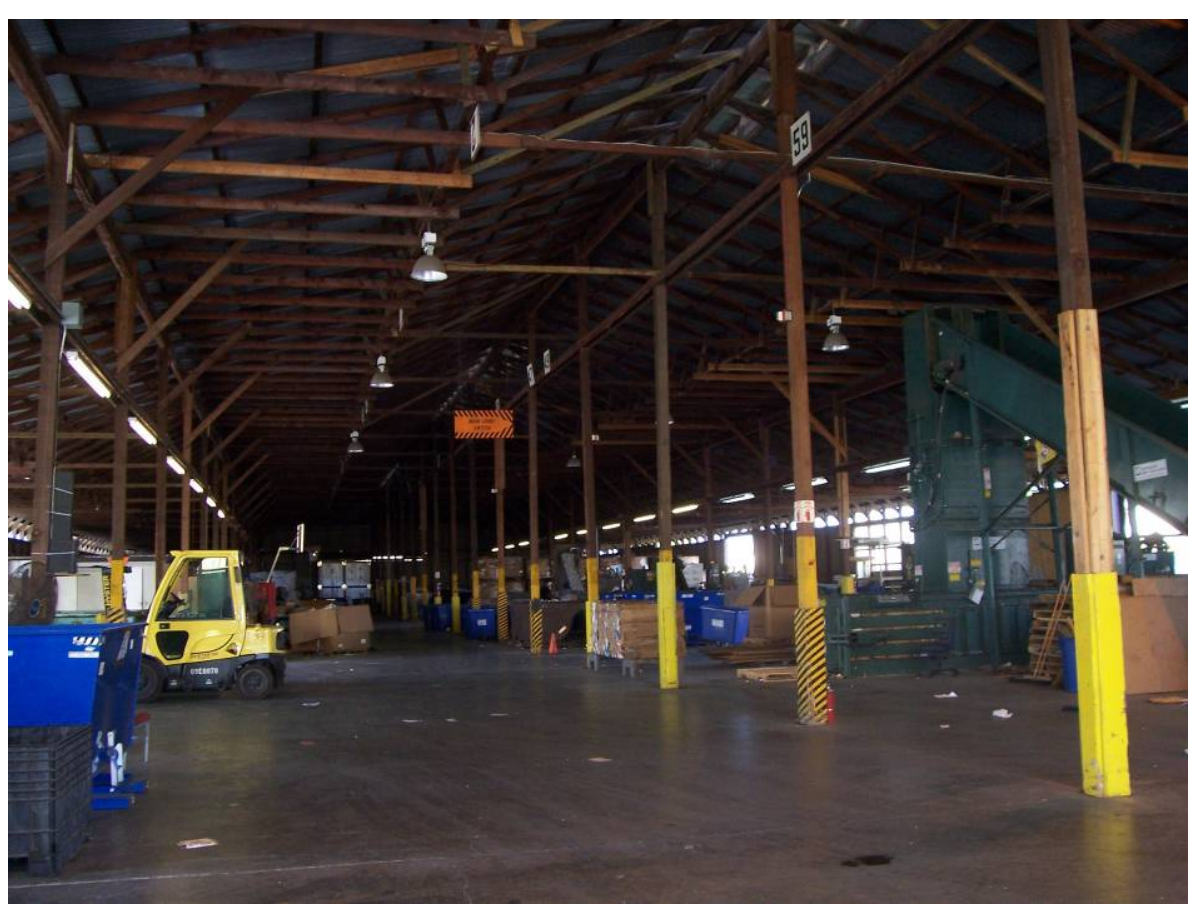




\section{Appropriated Funding Results}

A FEDS analysis using appropriated funding suggests replacing lights in the unconditioned space as well as replacing lights in the conditioned space.

Appropriated funding FEDS results for building 1715 unconditioned space:

\begin{tabular}{|c|c|c|c|c|c|c|c|c|}
\hline Bldg. Set Description & $\begin{array}{l}\text { End } \\
\text { Use }\end{array}$ & Existing Technology & Retrofit Technology & $\begin{array}{l}\text { Energy } \\
\text { Savings } \\
\text { (MMBtu/yr) }\end{array}$ & $\begin{array}{c}\text { 1st year } \\
\text { savings } \\
(\$ / y r)\end{array}$ & $\begin{array}{l}\text { Installed } \\
\text { Cost (\$) }\end{array}$ & $\begin{array}{l}\text { Net } \\
\text { Present } \\
\text { Value (\$) } \\
\end{array}$ & SIR \\
\hline 1940's shops 1715 & Lights & FL62: FL 1X8 2F96T12 STD2 & $\begin{array}{l}\text { FL131: FL 1X8 2F96T12ES ELC2 } \\
\text { REF (FIX REPL) }\end{array}$ & 22 & 1,613 & 10,748 & 16,329 & 2.5 \\
\hline 1940's shops & Lights & EX6: EXIT - LED & $\begin{array}{l}\text { EX12: EXIT - } \\
\text { ELECTROLUMINESCENT PANEL } \\
\text { RETRO KIT }\end{array}$ & - & 102 & 621 & 1,143 & 2.8 \\
\hline
\end{tabular}

Appropriated funding FEDS results for building 1715 conditioned space:

\begin{tabular}{|c|c|c|c|c|c|c|c|c|}
\hline Bldg. Set Description & $\begin{array}{l}\text { End } \\
\text { Use }\end{array}$ & Existing Technology & Retrofit Technology & $\begin{array}{l}\text { Energy } \\
\text { Savings } \\
\text { (MMBtu/yr) }\end{array}$ & $\begin{array}{l}\text { 1st year } \\
\text { savings } \\
(\$ / y r)\end{array}$ & $\begin{array}{l}\text { Installed } \\
\text { Cost }(\$)\end{array}$ & $\begin{array}{l}\text { Net } \\
\text { Present } \\
\text { Value (\$) }\end{array}$ & SIR \\
\hline 1940's shops 1715 & Lights & FL37: FL 2X4 4F32T8 EEF2 & $\begin{array}{l}\text { FL280: FL 2X4 3F32ST8 ELC3 } \\
\text { REF }\end{array}$ & 115 & 7,656 & 25,173 & 103,216 & 5.1 \\
\hline 1940's shops 1715 & Lights & EX6: EXIT - LED & $\begin{array}{l}\text { EX12: EXIT - } \\
\text { ELECTROLUMINESCENT PANEL } \\
\text { RETRO KIT }\end{array}$ & - & 54 & 311 & 626 & 3.0 \\
\hline
\end{tabular}




\section{Appropriated Funding Energy Consumption by Fuel Type}

The modeled energy consumption for the unconditioned space in the building a typical year was 147,909 kwh before retrofits and 141,258 kwh after proposed retrofits are implemented. The energy use intensity goes from 18.0 MBtu/Ksf to 17.2 MBtu/Ksf after retrofits.

\begin{tabular}{|c|c|c|c|c|}
\hline & & 1715 unconditi & d space & \\
\hline Fuel & Energy & $\begin{array}{c}\text { Energy } \\
\text { Intensity } \\
\text { (user units/1000ft2) }\end{array}$ & $\begin{array}{c}\text { Energy } \\
\text { Intensity } \\
\text { (MBtu/1000ft2) }\end{array}$ & $\begin{array}{l}\text { Dollars } \\
(2009) \text { * }\end{array}$ \\
\hline Electricity (kv & & & & \\
\hline existing & 147,909 & $5,288.5$ & 18.0 & 26,220 \\
\hline post-retrofit & 141,258 & $5,050.7$ & 17.2 & 24,852 \\
\hline difference & $-6,651$ & -237.8 & $-\odot .8$ & $-1,367$ \\
\hline$\%$ change & -4 & -4 & -4 & -5 \\
\hline Total (MBtu) & & & & \\
\hline existing & 505 & 18.0 & 18.0 & 26,220 \\
\hline post-retrofit & 482 & 17.2 & 17.2 & 24,852 \\
\hline difference & -23 & -0.8 & -0.8 & $-1,367$ \\
\hline$\%$ change & -4 & -4 & -4 & -5 \\
\hline
\end{tabular}

* Dollar values for electricity include both energy and demand components. 
The modeled energy consumption for the conditioned space in the building a typical year was 332,402 kwh before retrofits and 299,027 kwh after proposed retrofits are implemented. The energy use intensity goes from 466.5 MBtu/Ksf to 424.6 MBtu/Ksf after retrofits.

1940's shops 1715 conditioned space

Fuel

Electricity (kWh)

existing

post-retrofit

difference

$\%$ change

Total (MBtu)

existing

post-retrofit

difference

$\%$ change
Energy

$$
\begin{array}{r}
332,402 \\
299,027 \\
-33,375 \\
-10 \\
\\
1,134 \\
1,021 \\
-114 \\
-10
\end{array}
$$

\section{Energy \\ Intensity \\ (user units/1000ft2)}

$136,678.6$

$122,955.2$

$-13,723.4$

$-10$

466.5

419.6

$-46.8$

$-10$

\section{Energy}

Intensity

(MBtu/1000ft2)

Dollars

(2009) *

466.5

419.6

$-46.8$

$-10$

466.5

419.6

$-46.8$

$-10$
58,765

52,585

$-6,180$

$$
-11
$$

58,765

52,585

$-6,180$

$-11$

* Dollar values for electricity include both energy and demand components. 


\section{Appropriated Funding Energy Consumption by End Use}

Motors and miscellaneous equipment is the largest load in the unconditioned space of the building with 131,142 kWh/year, followed by lighting with $16,767 \mathrm{kWh} /$ year.

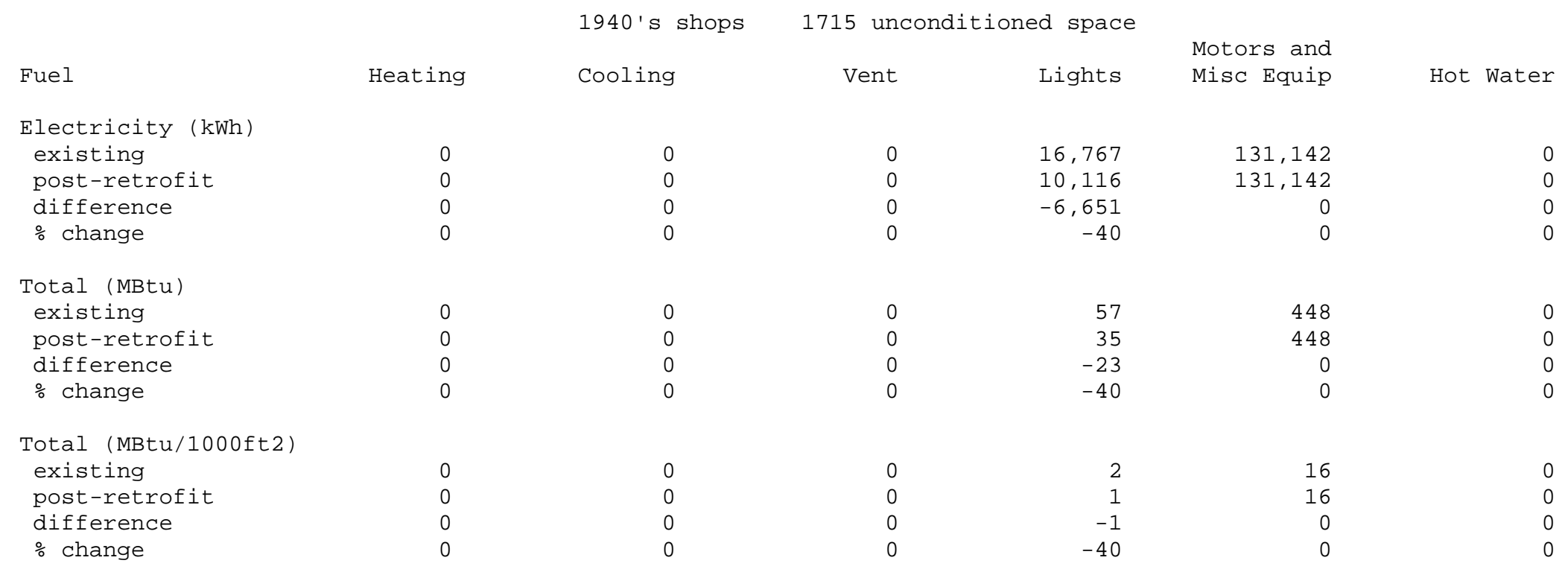


Lighting is the largest load in the conditioned space of the building with 234,179 $\mathrm{kWh} / \mathrm{year}$, followed by space cooling with 82,158 kWh/year.

Fuel
Electricity (kWh)
existing
post-retrofit
difference
$\%$ change
Total (MBtu)
existing
post-retrofit
difference
\% change
Total (MBtu/100०ft2)
existing
post-retrofit
difference
$\%$ change

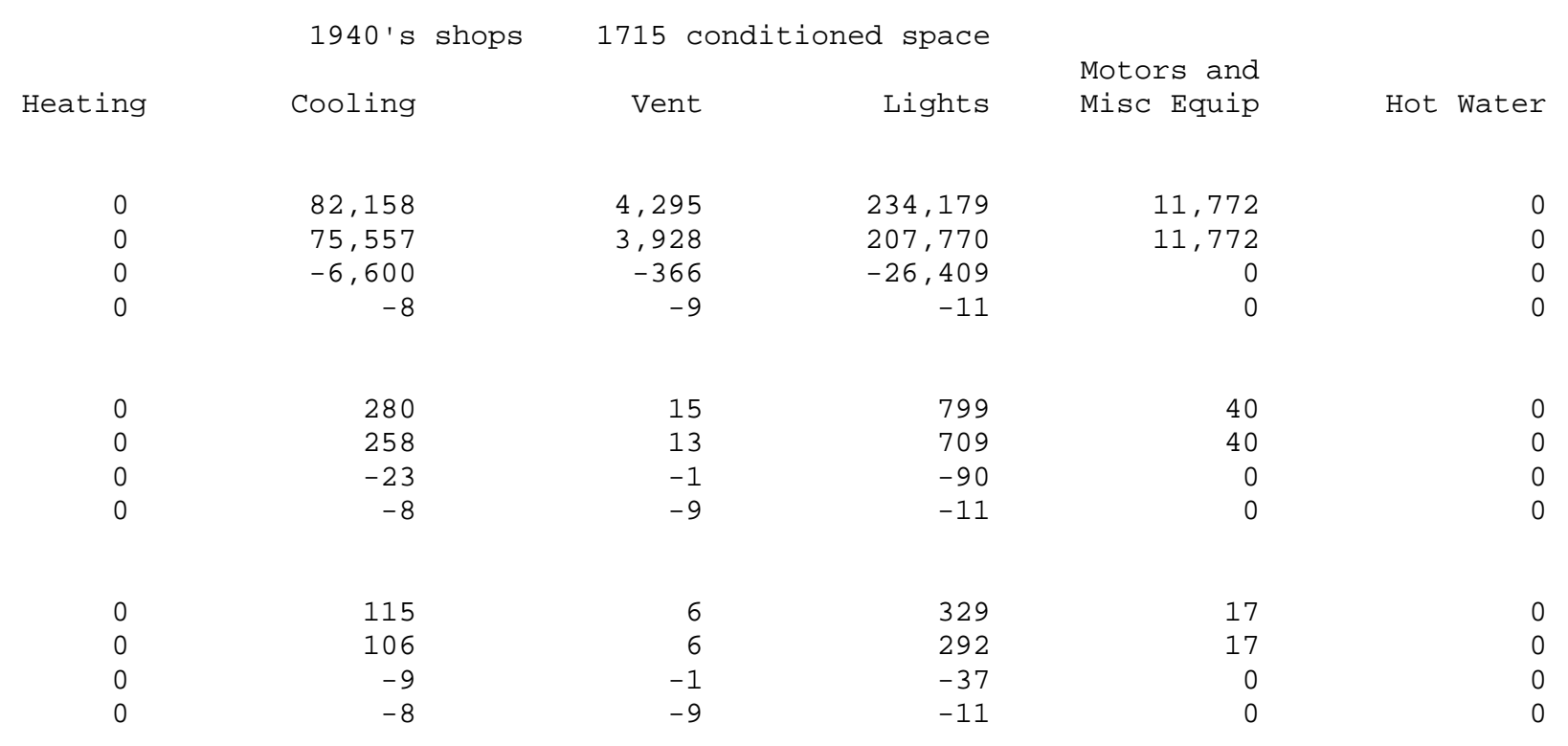




\section{Appropriated Funding Emission Reduction}

The emission reductions from implemented the proposed retrofits are as follows:

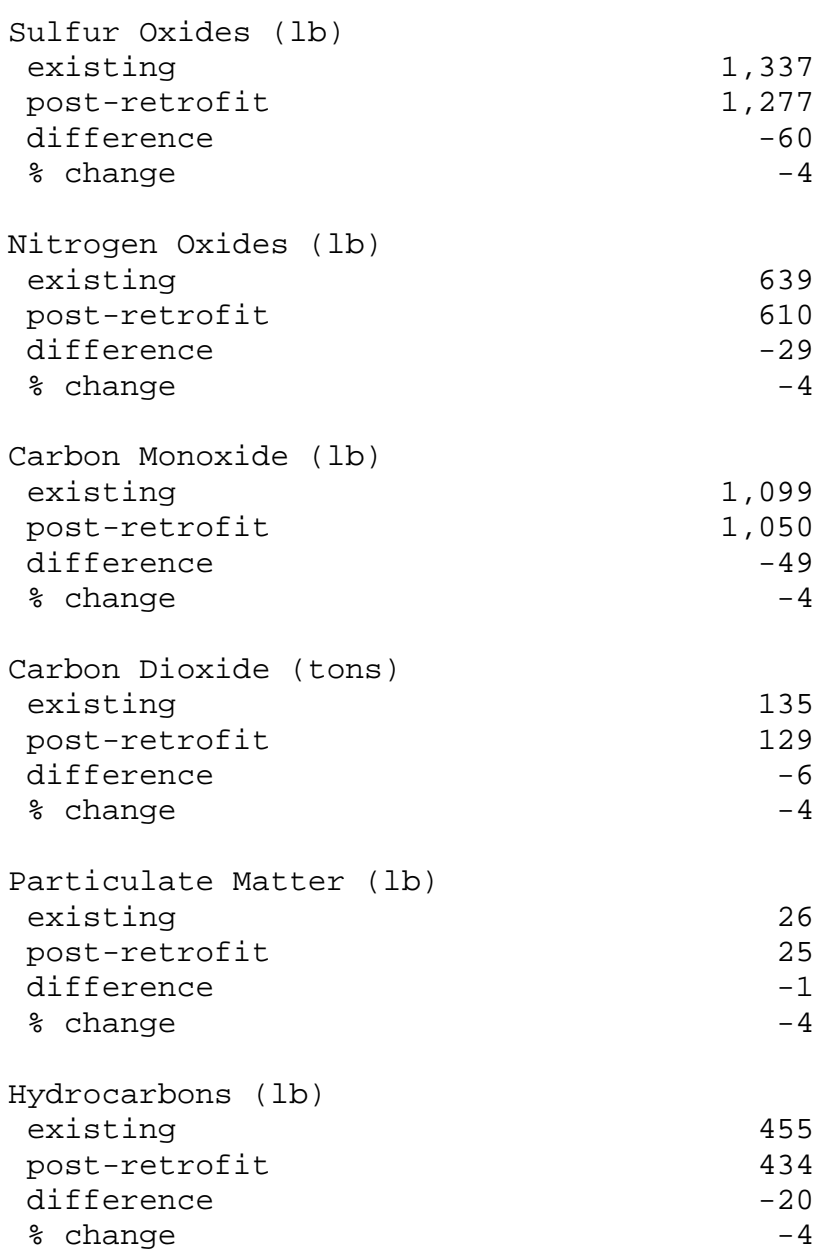


Sulfur Oxides (lb)

existing

post-retrofit

3,005

difference

2,703

$\%$ change

$-10$

Nitrogen 0xides (lb)

existing

post-retrofit

436

1,292

$-144$

difference

$-10$

Carbon Monoxide (lb)

existing

post-retrofit

difference

2,470

2,222

$-248$

\% change

Carbon Dioxide (tons)

existing

post-retrofit

304

difference

274

$\%$ change

-31
-10

Particulate Matter (lb)

existing

post-retrofit

59

difference

53
-6

\% change

Hydrocarbons (1b)

existing

1,022

post-retrofit

difference

920

$\%$ change

-103
-10 


\section{Building 2177 Maintenance Shop}

The following information identifies the cost-effective energy- and cost-reducing retrofit projects for building 2177 identified from the FEDS modeling and analysis. Key energy and economic results are presented for each cost-effective retrofit measure.

\section{Facility Description 2177}

2177 is a base engineer maintenance shop built in 1944. This building is partially cooled. Building 2177 is 3,200 sf.

$<$ no photo is available $>$

\section{Appropriated Funding Results}

A FEDS analysis using appropriated funding suggests replacing T12 lighting with T8 lighting in the unconditioned spaces. Upgrades to the hot water system include insulating the tank and pipes as well as installing aerators and lowering the tank temperature for the unconditioned spaces. For the conditioned spaces FEDS suggests replacing the lighting, and upgrading the hot water system.

Appropriated funding FEDS results for building 2177 unconditioned spaces:

\begin{tabular}{|c|c|c|c|c|c|c|c|c|}
\hline Bldg. Set Description & $\begin{array}{l}\text { End } \\
\text { Use }\end{array}$ & Existing Technology & Retrofit Technology & $\begin{array}{l}\text { Energy } \\
\text { Savings } \\
\text { (MMBtu/yr) }\end{array}$ & $\begin{array}{l}\text { 1st year } \\
\text { savings } \\
(\$ / y r)\end{array}$ & $\begin{array}{l}\text { Installed } \\
\text { Cost (\$) }\end{array}$ & $\begin{array}{l}\text { Net } \\
\text { Present } \\
\text { Value (\$) }\end{array}$ & SIR \\
\hline 1940's shops & Lights & FL4: FL 1X4 2F40T12 STD2 & FL52: FL 1X4 2F32T8 ELC2 & 7 & 526 & 2,437 & 6,431 & 3.6 \\
\hline 1940's shops & $\begin{array}{l}\text { Hot } \\
\text { Water }\end{array}$ & Electric Water Heater & $\begin{array}{l}\text { Wrap Tank with Insulation, } \\
\text { Insulate Pipe Near Tank, } \\
\text { Aerators, Lower Tank } \\
\text { Temperature }\end{array}$ & - & 1 & 6 & 7 & 4.2 \\
\hline
\end{tabular}


Appropriated funding FEDS results for building 2177 conditioned spaces:

\begin{tabular}{|c|c|c|c|c|c|c|c|c|}
\hline Bldg. Set Description & $\begin{array}{l}\text { End } \\
\text { Use }\end{array}$ & Existing Technology & Retrofit Technology & $\begin{array}{l}\text { Energy } \\
\text { Savings } \\
\text { (MMBtu/yr) } \\
\end{array}$ & $\begin{array}{l}\text { 1st year } \\
\text { savings } \\
(\$ / y r)\end{array}$ & $\begin{array}{l}\text { Installed } \\
\text { Cost (\$) }\end{array}$ & $\begin{array}{l}\text { Net } \\
\text { Present } \\
\text { Value (\$) } \\
\end{array}$ & SIR \\
\hline 1940's shops 2177 & Lights & EX6: EXIT - LED & $\begin{array}{l}\text { EX12: EXIT - } \\
\text { ELECTROLUMINESCENT PANEL } \\
\text { RETRO KIT }\end{array}$ & - & 7 & 43 & 89 & 3.1 \\
\hline 1940's shops 2177 & Lights & MH5: MH 250 PEND & FL309: FL 2X3 6F40BX ELC2 REF & 4 & 242 & 1,703 & 2,332 & 2.4 \\
\hline 1940 's shops 2177 & $\begin{array}{l}\text { Hot } \\
\text { Water }\end{array}$ & Electric Water Heater & $\begin{array}{l}\text { Wrap Tank with Insulation, } \\
\text { Insulate Pipe Near Tank, } \\
\text { Aerators, Lower Tank } \\
\text { Temperature }\end{array}$ & - & 3 & 9 & 11 & 4.3 \\
\hline
\end{tabular}




\section{Appropriated Funding Energy Consumption by Fuel Type}

The modeled energy consumption for a typical year was 13,067 kwh before retrofits and 11,096 kwh after proposed retrofits are implemented. The energy use intensity goes from 27.9 MBtu/Ksf to 23.7 MBtu/Ksf after retrofits.

\begin{tabular}{|c|c|c|c|c|}
\hline Fuel & Energy & $\begin{array}{c}\text { Energy } \\
\text { Intensity } \\
\text { (user units/1000ft2) }\end{array}$ & $\begin{array}{c}\text { Energy } \\
\text { Intensity } \\
\text { (MBtu/1000ft2) }\end{array}$ & $\begin{array}{l}\text { Dollars } \\
(2009) \text { * }\end{array}$ \\
\hline \multicolumn{5}{|c|}{ Electricity (kWh) } \\
\hline existing & 13,067 & $8,166.8$ & 27.9 & 2,311 \\
\hline post-retrofit & 11,096 & $6,935.2$ & 23.7 & 1,951 \\
\hline difference & $-1,971$ & $-1,231.6$ & -4.2 & -360 \\
\hline$\%$ change & -15 & -15 & -15 & -16 \\
\hline \multicolumn{5}{|l|}{ Total (MBtu) } \\
\hline existing & 45 & 27.9 & 27.9 & 2,311 \\
\hline post-retrofit & 38 & 23.7 & 23.7 & 1,951 \\
\hline difference & -7 & -4.2 & -4.2 & -360 \\
\hline \% change & -15 & -15 & -15 & -16 \\
\hline
\end{tabular}

* Dollar values for electricity include both energy and demand components. 
The modeled energy consumption for a typical year was 29,056 kwh before retrofits and 27,936 kwh after proposed retrofits are implemented. The energy use intensity goes from 62.0 MBtu/Ksf to 59.6 MBtu/Ksf after retrofits.

\begin{tabular}{|c|c|c|c|c|}
\hline Fuel & Energy & $\begin{array}{c}\text { Energy } \\
\text { Intensity } \\
\text { (user units/1000ft2) }\end{array}$ & $\begin{array}{c}\text { Energy } \\
\text { Intensity } \\
\text { (MBtu/1000ft2) }\end{array}$ & $\begin{array}{l}\text { Dollars } \\
\text { (2009) * }\end{array}$ \\
\hline \multicolumn{5}{|c|}{ Electricity (kWh) } \\
\hline existing & 29,056 & $18,159.8$ & 62.0 & 5,139 \\
\hline post-retrofit & 27,936 & $17,460.1$ & 59.6 & 4,911 \\
\hline difference & $-1,119$ & -699.6 & -2.4 & -228 \\
\hline$\%$ change & -4 & -4 & -4 & -4 \\
\hline \multicolumn{5}{|l|}{ Total (MBtu) } \\
\hline existing & 99 & 62.0 & 62.0 & 5,139 \\
\hline post-retrofit & 95 & 59.6 & 59.6 & 4,911 \\
\hline difference & -4 & -2.4 & -2.4 & -228 \\
\hline$\%$ change & -4 & -4 & -4 & -4 \\
\hline
\end{tabular}

* Dollar values for electricity include both energy and demand components. 


\section{Appropriated Funding Energy Consumption by End Use}

Motors and miscellaneous equipment is the largest load in the unconditioned space of the building with 7,502 kWh/year, followed by lighting with 5,536 kWh/year.

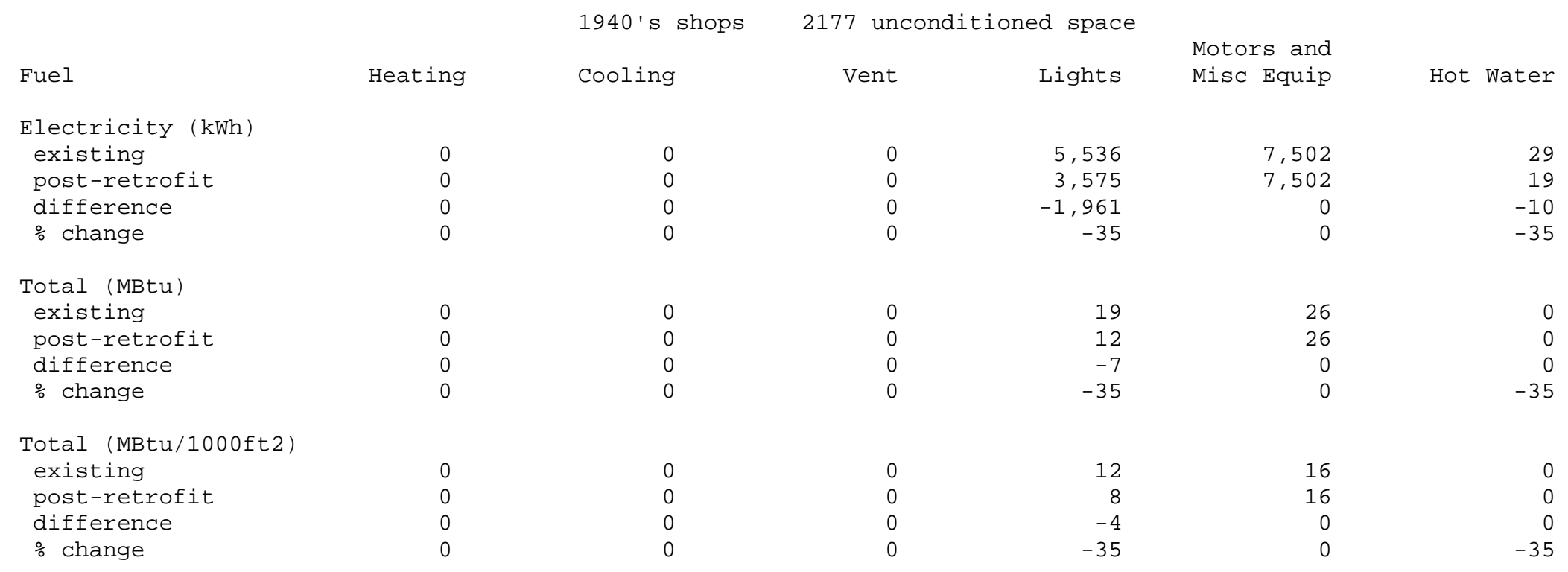


Space cooling is the largest load in the conditioned space of the building with 10,796 kWh/year, followed by lighting with 8,210 kWh/year.

Fuel
Electricity (kWh)
existing
post-retrofit
difference
$\%$ change
Total (MBtu)
existing
post-retrofit
difference
\% change
Total (MBtu/1000ft2)
existing
post-retrofit
difference
\% change

$\begin{array}{cr} & \\ \text { Heating } & \text { Cooling } \\ & \\ 0 & 10,796 \\ 0 & 10,550 \\ 0 & -246 \\ 0 & -2 \\ & \\ 0 & 37 \\ 0 & 36 \\ 0 & -1 \\ 0 & -2 \\ & \\ 0 & \\ 0 & 23 \\ 0 & 23 \\ 0 & -1 \\ & -2\end{array}$

2177 conditioned space

Vent

Lights

8,210

2,268

2,211

$-3$

7, 408

-801
-10
28
25
-3
-10

Motors and

Misc Equip

Hot Water

$$
\begin{array}{r}
7,745 \\
7,745 \\
0 \\
0
\end{array}
$$

23

-16
-41

37

36
-1

$-2$

\begin{tabular}{l}
8 \\
8 \\
\hline
\end{tabular}

$-3$

18
16

16
-2

$-10$

$\begin{array}{rr}26 & 0 \\ 26 & 0 \\ 0 & 0 \\ 0 & -41\end{array}$

$\begin{array}{rr}17 & 0 \\ 17 & 0 \\ 0 & 0 \\ 0 & -41\end{array}$




\section{Appropriated Funding Emission Reduction}

The emission reductions from implemented the proposed retrofits are as follows:

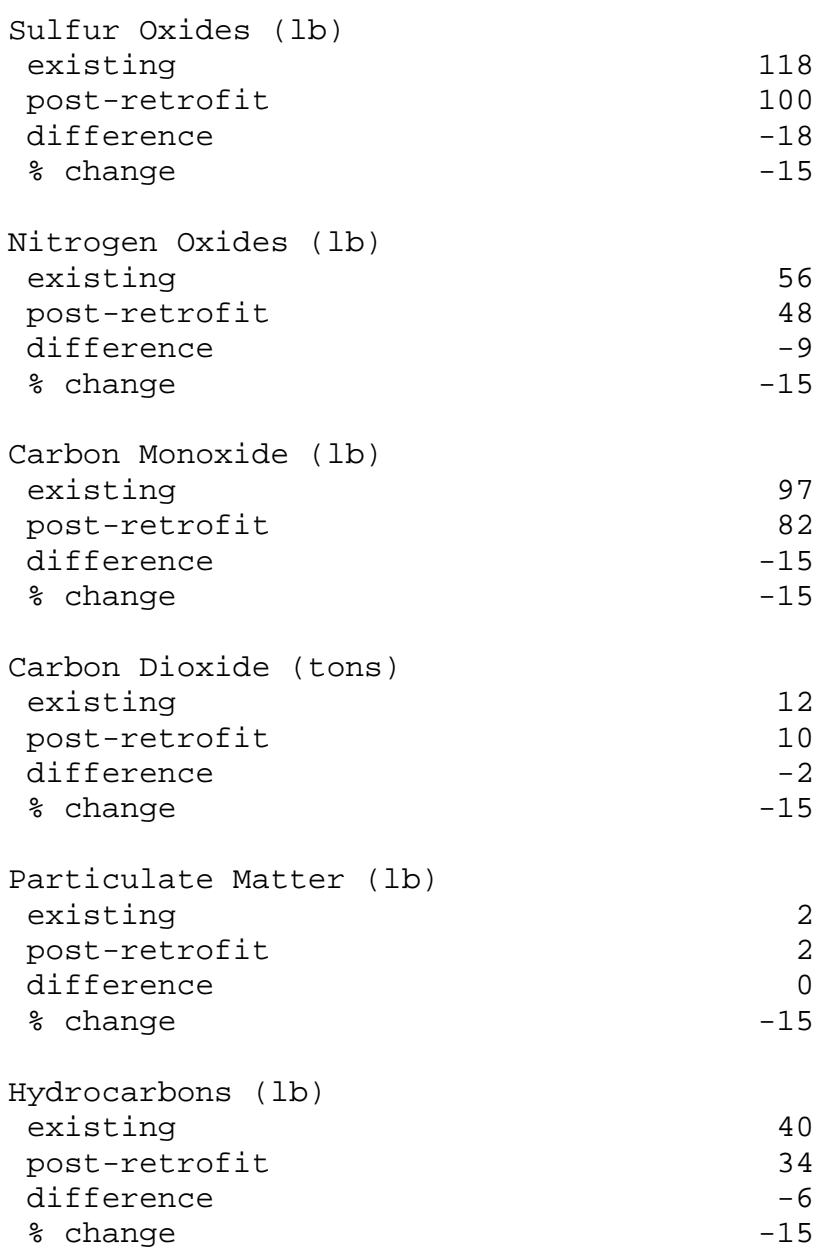


Sulfur oxides (lb)

existing

post-retrofit

difference

$\%$ change

263

253

$-10$

$-4$

Nitrogen 0xides (lb)

existing

post-retrofit

126

121

difference

$\%$ change

$-5$

Carbon Monoxide (lb)

existing

post-retrofit

difference

$\%$ change

Carbon Dioxide (tons)

existing

post-retrofit

difference

$\%$ change

-1
-4

Particulate Matter (lb)

existing

post-retrofit

difference

$\%$ change

Hydrocarbons (lb)

existing

post-retrofit

difference

89 


\section{Building 4016 Maintenance Shop}

The following information identifies the cost-effective energy- and cost-reducing retrofit projects for building 4016 identified from the FEDS modeling and analysis. Key energy and economic results are presented for each cost-effective retrofit measure.

\section{Facility Description 4016}

Building 4016 is a base engineer maintenance shop built in 1973. 4016 is cooled by multiple package units and has little to no insulation in its building enveloped. Building 4016 is 7,701 sf.

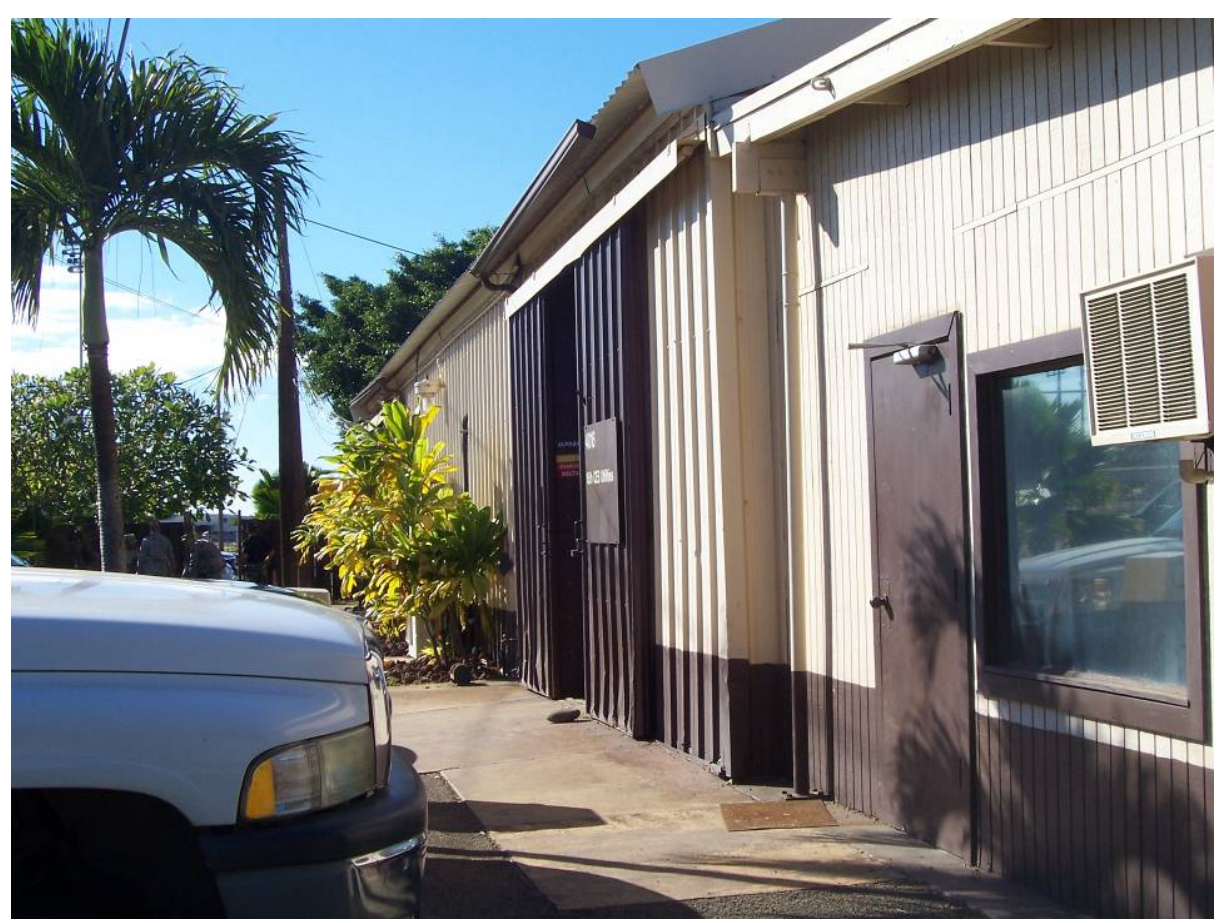




\section{Appropriated Funding Results}

A FEDS analysis using appropriated funding suggests replacing the electric package unit with an ultra high efficiency window AC unit in the conditioned space. The EXIT lights are suggested to be replaced and upgrades to the hot water heater are also suggested. To the unconditioned space FEDS suggests replacing T8 lights with Super T8 lighting, replacing the EXIT lighting and making various improvements to the hot water system.

Appropriated funding FEDS results for building 4016 conditioned spaces:

\begin{tabular}{|c|c|c|c|c|c|c|c|c|}
\hline Bldg. Set Description & $\begin{array}{l}\text { End } \\
\text { Use }\end{array}$ & Existing Technology & Retrofit Technology & $\begin{array}{l}\text { Energy } \\
\text { Savings } \\
\text { (MMBtu/yr) }\end{array}$ & $\begin{array}{c}\text { 1st year } \\
\text { savings } \\
(\$ / y r)\end{array}$ & $\begin{array}{l}\text { Installed } \\
\text { Cost (\$) }\end{array}$ & $\begin{array}{l}\text { Net } \\
\text { Present } \\
\text { Value (\$) } \\
\end{array}$ & SIR \\
\hline $\begin{array}{l}\text { 1970's shops } 4016 \\
\text { 1970's shops } 4016\end{array}$ & $\begin{array}{l}\text { Lights } \\
\text { Hot } \\
\text { Water }\end{array}$ & $\begin{array}{l}\text { EX1: EXIT - INC }(2 \times 20) \\
\text { Electric Water Heater }\end{array}$ & $\begin{array}{l}\text { EX12: EXIT - } \\
\text { ELECTROLUMINESCENT PANEL } \\
\text { RETRO KIT } \\
\text { Wrap Tank with Insulation, } \\
\text { Insulate Pipe Near Tank, LFSHs, } \\
\text { Lower Tank Temperature }\end{array}$ & 14 & 881 & 621 & 14,215 & 23.9 \\
\hline
\end{tabular}

Appropriated funding FEDS results for building 4016 unconditioned spaces:

\begin{tabular}{|c|c|c|c|c|c|c|c|c|}
\hline Bldg. Set Description & $\begin{array}{l}\text { End } \\
\text { Use }\end{array}$ & Existing Technology & Retrofit Technology & $\begin{array}{l}\text { Energy } \\
\text { Savings } \\
\text { (MMBtu/yr) }\end{array}$ & $\begin{array}{l}\text { 1st year } \\
\text { savings } \\
(\$ / y r)\end{array}$ & $\begin{array}{l}\text { Installed } \\
\text { Cost (\$) }\end{array}$ & $\begin{array}{l}\text { Net } \\
\text { Present } \\
\text { Value (\$) }\end{array}$ & SIR \\
\hline 1970's shops 4016 & Lights & EX1: EXIT - INC (2×20) & $\begin{array}{l}\text { EX12: EXIT - } \\
\text { ELECTROLUMINESCENT PANEL } \\
\text { RETRO KIT } \\
\text { FL302: FL 1X4 1F25ST8 }\end{array}$ & 5 & 344 & 311 & 5,520 & 18.8 \\
\hline $\begin{array}{l}\text { 1970's shops } 4016 \\
\text { 1970's shops } 4016\end{array}$ & $\begin{array}{l}\text { Lights } \\
\text { Hot } \\
\text { Water }\end{array}$ & $\begin{array}{l}\text { FL41: FL 1X4 1F32T8 EEF1 } \\
\text { Electric Water Heater }\end{array}$ & $\begin{array}{l}\text { ELC1REF } \\
\text { Wrap Tank with Insulation, } \\
\text { Insulate Pipe Near Tank, LFSHs, } \\
\text { Lower Tank Temperature }\end{array}$ & 1 & 124 & 869 & 1,230 & 2.4 \\
\hline
\end{tabular}




\section{Appropriated Funding Energy Consumption by Fuel Type}

The modeled energy consumption for the conditioned spaces of the building for a typical year was 67,485 kwh before retrofits and $63,631 \mathrm{kwh}$ after proposed retrofits are implemented. The energy use intensity goes from 39.9 MBtu/Ksf to $37.6 \mathrm{MBtu} / \mathrm{Ksf}$ after retrofits.

\begin{tabular}{|c|c|c|c|c|}
\hline \multirow[b]{2}{*}{ Fuel } & 1970 's shops & \multicolumn{2}{|c|}{4016 conditioned space } & \multirow[b]{2}{*}{$\begin{array}{l}\text { Dollars } \\
(2009)\end{array}$} \\
\hline & Energy & $\begin{array}{c}\text { Energy } \\
\text { Intensity } \\
\text { (user units/10๑०ft2) }\end{array}$ & $\begin{array}{c}\text { Energy } \\
\text { Intensity } \\
\text { (MBtu/1000ft2) }\end{array}$ & \\
\hline \multicolumn{5}{|c|}{ Electricity (kWh) } \\
\hline existing & 67,485 & $11,685.8$ & 39.9 & 11,936 \\
\hline post-retrofit & 63,631 & $11,018.3$ & 37.6 & 11,186 \\
\hline difference & $-3,855$ & -667.5 & -2.3 & -750 \\
\hline$\%$ change & -6 & -6 & -6 & -6 \\
\hline \multicolumn{5}{|l|}{ Total (MBtu) } \\
\hline existing & 230 & 39.9 & 39.9 & 11,936 \\
\hline post-retrofit & 217 & 37.6 & 37.6 & 11,186 \\
\hline difference & -13 & -2.3 & -2.3 & $\begin{array}{r}+1+100 \\
-750\end{array}$ \\
\hline$\%$ change & -6 & -6 & -6 & -6 \\
\hline
\end{tabular}

* Dollar values for electricity include both energy and demand components. 
The modeled energy consumption for the unconditioned spaces of the building for a typical year was 12,772 kwh before retrofits and $10,903 \mathrm{kwh}$ after proposed retrofits are implemented. The energy use intensity goes from $22.6 \mathrm{MBtu} / \mathrm{Ksf}$ to $19.3 \mathrm{MBtu} / \mathrm{Ksf}$ after retrofits.

1970 's shops 4016 unconditioned space

Fuel

Electricity (kWh)

existing

post-retrofit

difference

$\%$ change

Total (MBtu)

existing

post-retrofit

difference

$\%$ change
Energy

12,772

10,903

$-1,869$
-15

44
37
-6
-15

Energy
Intensity
(user units/1000ft2)

\section{$6,634.9$ \\ $5,663.7$ \\ $-971.2$}

$-15$
22.6
19.3
$-3.3$
$-15$

\section{Energy}

Intensity

(MBtu/1000ft2)

Dollars

(2009) *

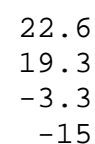

2,259

1,917

$-342$

$-15$

2,259

1,917

$-342$

$-15$

* Dollar values for electricity include both energy and demand components. 


\section{Appropriated Funding Energy Consumption by End Use}

Motors and miscellaneous equipment is the largest load in the conditioned space of the building with 27,381 kWh/year, followed by space cooling with 23,540 kWh/year.

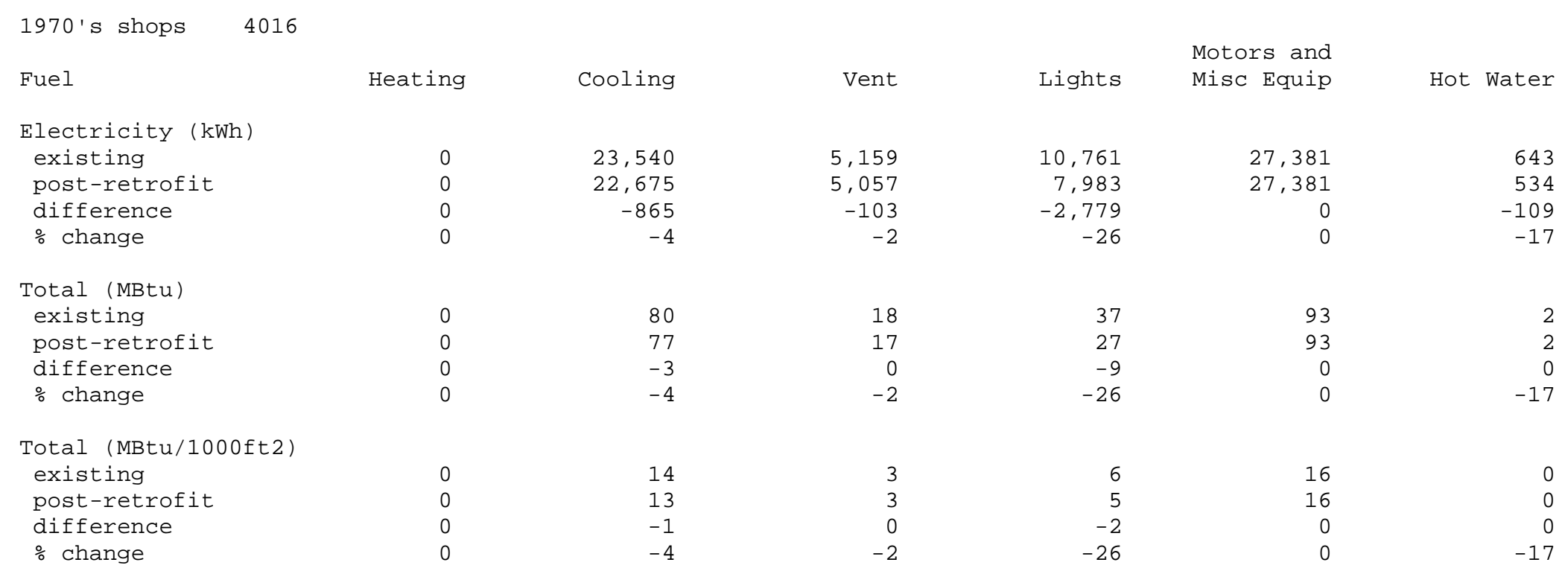


Motors and miscellaneous equipment is the largest load in the unconditioned space of the building with 8,620 kWh/year, followed by lighting with 3,738 kWh/year.

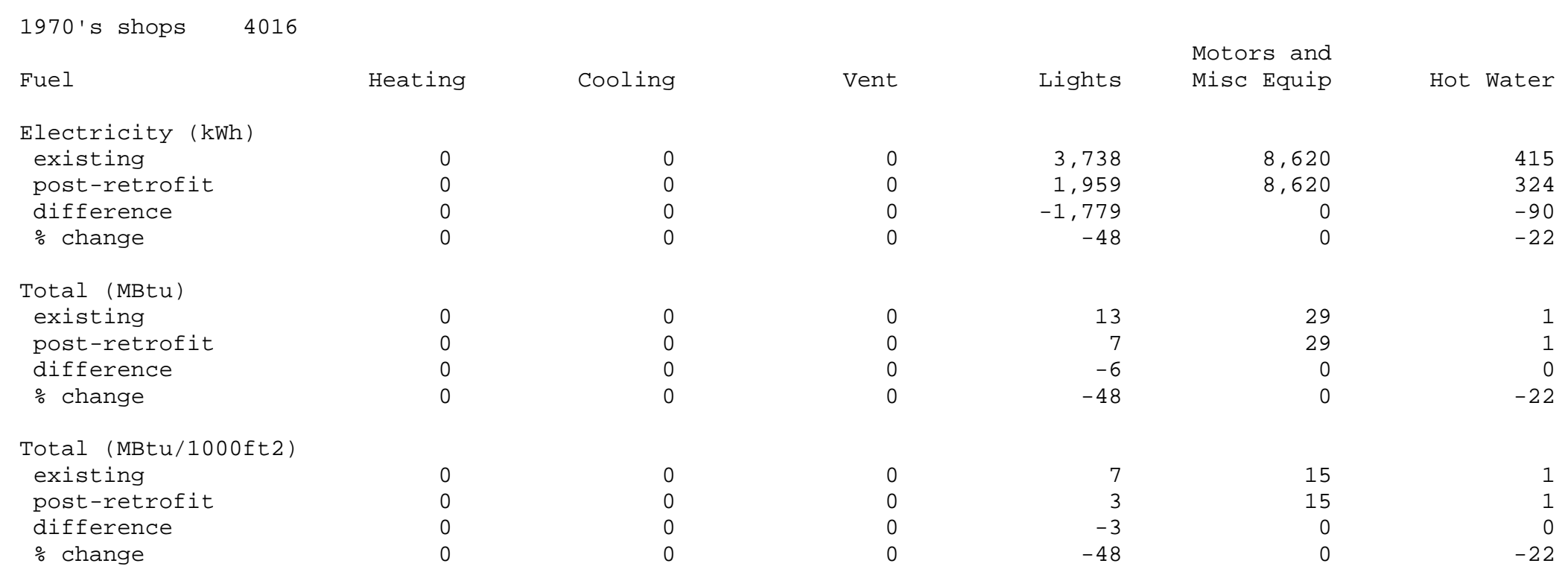




\section{Appropriated Funding Emission Reduction}

The emission reductions from implemented the proposed retrofits are as follows:

1970 's shops

4016 conditioned space

$\begin{array}{lr}\begin{array}{l}\text { Sulfur oxides (lb) } \\ \text { existing }\end{array} & 610 \\ \text { post-retrofit } & 575 \\ \text { difference } & -35 \\ \text { \% change } & -6 \\ & \\ \text { Nitrogen oxides (lb) } & 292 \\ \text { existing } & 275 \\ \text { post-retrofit } & -17 \\ \text { difference } & -6 \\ \text { \% change } & \\ & \\ \text { Carbon Monoxide (lb) } & 501 \\ \text { existing } & 473 \\ \text { post-retrofit } & -29 \\ \text { difference } & -6 \\ \text { \% change } & \\ \text { Carbon Dioxide (tons) } & \\ \text { existing } & 62 \\ \text { post-retrofit } & 58 \\ \text { difference } & -4 \\ \text { \% change } & -6 \\ \text { Particulate Matter (lb) } & \\ \text { existing } & \\ \text { post-retrofit } & 12 \\ \text { difference } & 11 \\ \text { \% change } & -1 \\ \text { Hydrocarbons (lb) } & -6 \\ \text { existing } & \\ \text { post-retrofit } & \\ \text { difference } & \\ \text { \% change } & -12\end{array}$


Sulfur Oxides (lb)

existing

post-retrofit

115

difference

99

$\%$ change

-17
-15

Nitrogen 0xides (lb)

existing

post-retrofit

5

difference

47

$-8$

Carbon Monoxide (lb)

existing

post-retrofit

difference

$\%$ change

Carbon Dioxide (tons)

existing

post-retrofit

difference

10
-2

$\%$ change

Particulate Matter (lb)

existing

post-retrofit

difference

$\%$ change

2

2

$-15$

Hydrocarbons (lb)

existing

post-retrofit

difference

34

$\%$ change

$-15$ 


\section{Building 2131 Administration Building}

The following information identifies the cost-effective energy- and cost-reducing retrofit projects for building 2131 identified from the FEDS modeling and analysis. Key energy and economic results are presented for each cost-effective retrofit measure.

\section{Facility Description 2131}

Building 2131 is a building with some administration space as well as some lab-space and unconditioned high-bay space. Building 2131 was built in 2008 and is 26,296 sf.

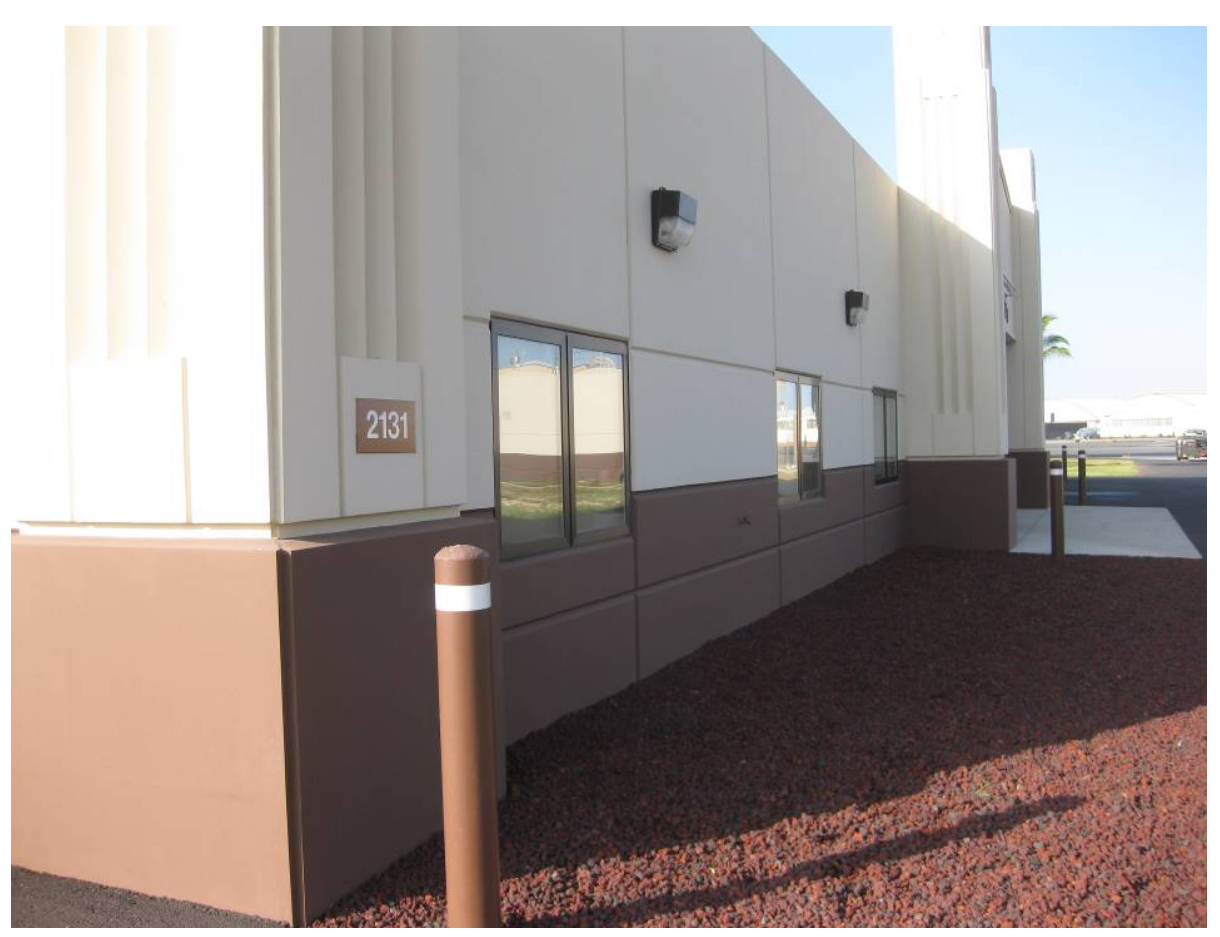




\section{Appropriated Funding Results}

A FEDS analysis using appropriated funding suggests replacing the air cooled chiller with a water cooled chiller as well as replacing the EXIT lights with electroluminescent panels for the administration and laboratory space. FEDS also suggests replacing the air cooled chiller with a very high efficiency water cooled chiller and replacing the EXIT and metal halide lighting

Appropriated funding FEDS results for building 2131 administration and laboratory space:

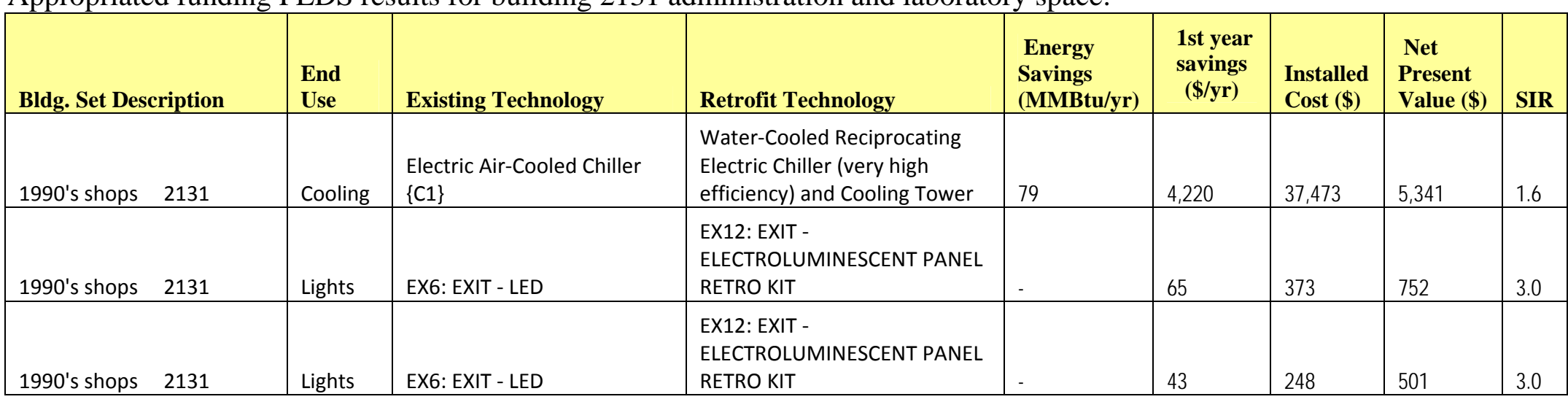

Appropriated funding FEDS results for building 2131 high bay space:

\begin{tabular}{|c|c|c|c|c|c|c|c|c|}
\hline Bldg. Set Description & $\begin{array}{l}\text { End } \\
\text { Use }\end{array}$ & Existing Technology & Retrofit Technology & $\begin{array}{l}\text { Energy } \\
\text { Savings } \\
\text { (MMBtu/yr) }\end{array}$ & $\begin{array}{l}\text { 1st year } \\
\text { savings } \\
(\$ / y r)\end{array}$ & $\begin{array}{l}\text { Installed } \\
\text { Cost (\$) }\end{array}$ & $\begin{array}{l}\text { Net } \\
\text { Present } \\
\text { Value (\$) } \\
\end{array}$ & SIR \\
\hline $\begin{array}{l}1990 \text { 's shop highbay space } \\
2131\end{array}$ & Lights & EX6: EXIT - LED & $\begin{array}{l}\text { EX12: EXIT - } \\
\text { ELECTROLUMINESCENT PANEL } \\
\text { RETRO KIT }\end{array}$ & - & 102 & 621 & 1,143 & 2.8 \\
\hline $\begin{array}{l}1990 \text { 's shop highbay space } \\
2131\end{array}$ & Lights & MH13: MH 250 WALL & HS26: HPS 200 WALL & 5 & 396 & 3,457 & 3,247 & 1.9 \\
\hline
\end{tabular}




\section{Appropriated Funding Energy Consumption by Fuel Type}

The modeled energy consumption for administration and laboratory spaces of the building for a typical year was 221,784 kwh before retrofits and 198,333 kwh after proposed retrofits are implemented. The energy use intensity goes from 57.6 MBtu/Ksf to 51.5 $\mathrm{MBtu} / \mathrm{Ksf}$ after retrofits.

1990 's shops administration and laboratory space 2131

Fuel

$$
\text { Energy }
$$

Energy

$\begin{array}{cc}\text { Intensity } & \text { Intensity } \\ \text { (user units/1000ft2) } & \text { (MBtu/1000ft2 }\end{array}$

Dollars $(2009)^{*}$

Electricity (kWh)

existing

post-retrofit

difference

221,784

198,333

$16,868.3$

$15,084.7$

$-1,783.6$

$\%$ change

Total (MBtu)

existing

post-retrofit

difference

757
677
-80
-11

57.6

51.5

-6.1
-11

57.6

51.5

$-6.1$

$-11$

39,316

34,894

$-4,422$

\% change

* Dollar values for electricity include both energy and demand components. 
The modeled energy consumption for high bay spaces of the building for a typical year was 228,359 kwh before retrofits and 195,226 kwh after proposed retrofits are implemented. The energy use intensity goes from 59.3 MBtu/Ksf to $50.7 \mathrm{MBtu} / \mathrm{Ksf}$ after retrofits.

1990 's shop highbay space 2131

Fuel

Electricity (kWh)

existing

post-retrofit

difference

$\%$ change

Total (MBtu)

existing

post-retrofit

difference

$\%$ change
Energy

152,074

150,489

$-1,585$

$-1$

519
514
-5
-1

Energy

Intensity

(user units/1000ft2)

$11,570.7$

$11,450.1$

$-120.6$

$-1$

39.5
39.1
-0.4
-1

Energy

Intensity (MBtu/1000ft2)

39.5

39.1

$-0.4$

39.5

39.1

$-0.4$

$-1$
Dollars

$(2009)$ *

26,965

26,689

26,965

26,689

$-276$

* Dollar values for electricity include both energy and demand components. 


\section{Appropriated Funding Energy Consumption by End Use}

Motors and miscellaneous equipment is the largest load in the building with 84,806 kWh/year, followed by space cooling with 62,357 kWh/year.

1990 's shops administration and laboratory space 2131

Fuel Heating
Electricity (kWh)
existing
post-retrofit
difference
\% change
Total (MBtu)
existing
post-retrofit
difference
\% change
Total (MBtu/1000ft2)
existing
post-retrofit
difference
\% change

\begin{tabular}{rr} 
& \\
$\odot$ & \\
$\odot$ & 62,357 \\
$\odot$ & 39,030 \\
$\odot$ & $-23,327$ \\
& -37 \\
$\odot$ & \\
$\odot$ & \\
$\odot$ & 213 \\
$\odot$ & 133 \\
& -80 \\
$\odot$ & -37 \\
$\odot$ & \\
$\odot$ & \\
$\odot$ & 16 \\
& 10 \\
& -6 \\
\hline
\end{tabular}

Vent

Lights

Motors and

Misc Equip

Hot Water *

14,183
14,175
-8
0

48
48
0
0

4
4
0
0

57,107
56,992
-116
0

195
195
0
0

15
15
0
$\odot$
84,806
84,806

0
0

3,330

3,330

0
0

$\begin{array}{rr}27 & 14,175 \\ -37 & -8 \\ & 0\end{array}$

116

289

289
289

0
$\odot$
11
11
0

$\begin{array}{ll}6 & 4 \\ 10 & 4 \\ 6 & 0 \\ 37 & 0\end{array}$

15
15
$\odot$
0

22
22
$\odot$
$\odot$

1
1
0
0 
Motors and miscellaneous equipment is the largest load in the building with 92,181 kWh/year, followed by space cooling with 57,524 kWh/year.

Annual Energy Use by Building Set, Fuel Type, and End Use 1990 's shop highbay space 2131

Fuel

Electricity (kWh)

existing

post-retrofit

difference

$\%$ change

Total (MBtu)

existing

post-retrofit

difference

\% change

Total (MBtu/1000ft2)

existing

post-retrofit

difference

$\%$ change

\section{Heating}

Cooling

Vent

$\odot$

$\odot$

$\odot$

0

$\odot$

8

$\odot$
Lights
57,524
55,940
$-1,585$

$-3$

196

191

-5
-3

15

15
0
Motors and

Misc Equip
92,181
92,181

$\odot$
$\odot$

315

315

$$
\odot
$$

24
24
0
Hot Water

2,368

2,368

$\odot$
0

8

8

0

1

1

0 


\section{Appropriated Funding Emission Reduction}

The emission reductions from implemented the proposed retrofits are as follows:

1990 's shops administration and laboratory space 2131

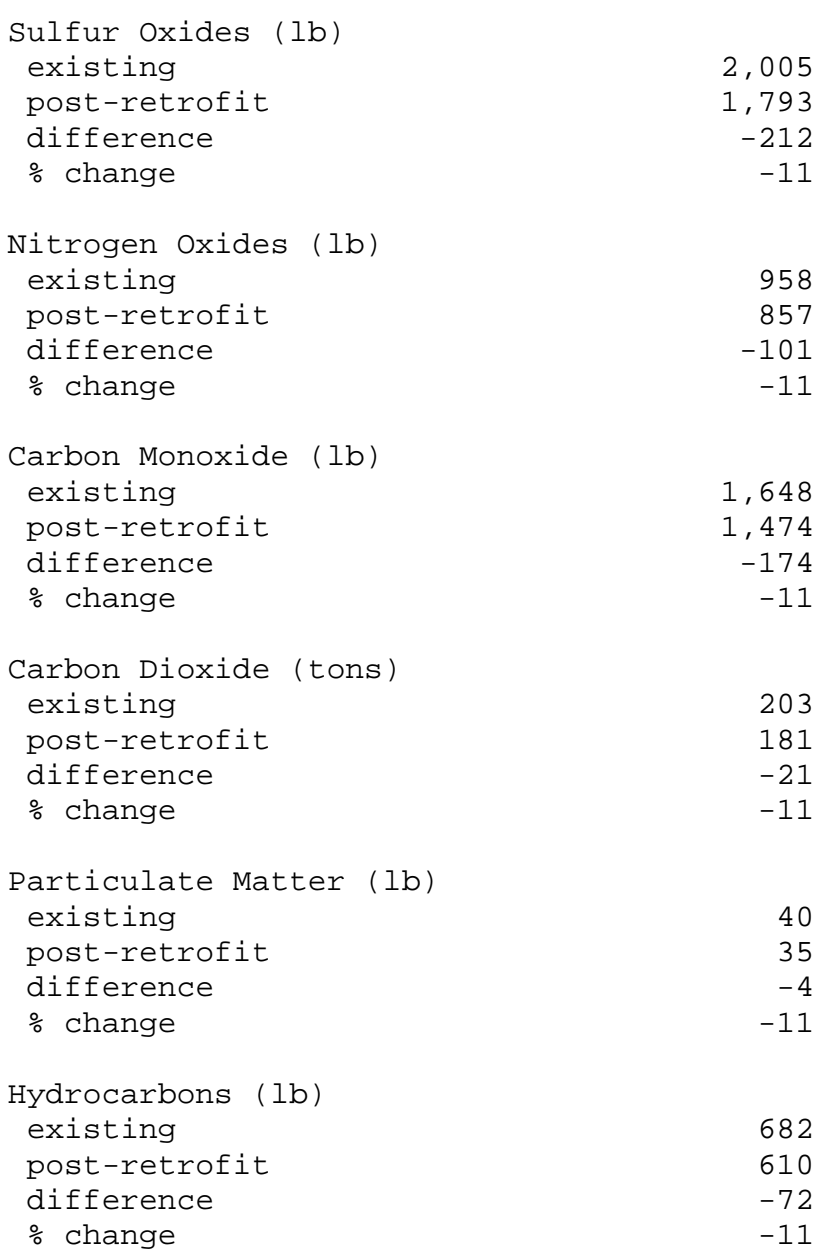


Sulfur oxides (lb)

existing

post-retrofit

difference

$\%$ change

1,375

$-14$

Nitrogen 0xides (lb)

existing

post-retrofit

difference

$\%$ change

Carbon Monoxide (lb)

existing

post-retrofit

difference

$\%$ change

Carbon Dioxide (tons)

existing

post-retrofit

difference

$\%$ change

657

650
-7

1,130

1,118

$-12$

139

138

Particulate Matter (lb)

existing

post-retrofit

difference

$\%$ change

Hydrocarbons (lb)

existing

post-retrofit

difference

$\%$ change
$-1$

$-1$

$-1$

$-1$

's shop highbay space 2131

27
27
0
-1


468
463
-5
-1 


\section{Building 1728 Warehouse}

The following information identifies the cost-effective energy- and cost-reducing retrofit projects for building 1728 identified from the FEDS modeling and analysis. Key energy and economic results are presented for each cost-effective retrofit measure.

\section{Facility Description 1728}

1728 is a warehouse building built in 1993. This building partially unconditioned with the office space being served by an electric air cooled chiller. Building 1728 is 140,383 sf.

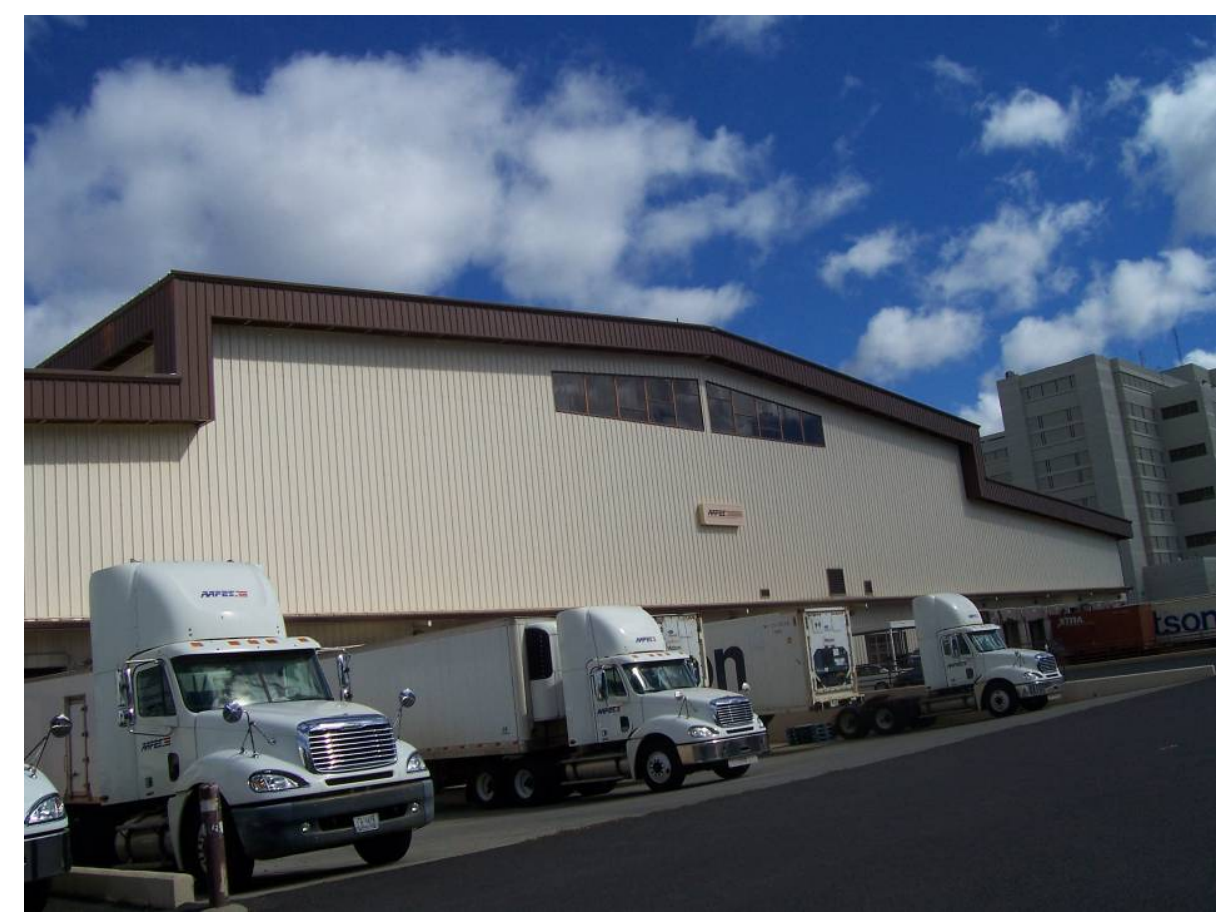

\section{Appropriated Funding Results}


A FEDS analysis using appropriated funding suggests replacing lights in the conditioned and unconditioned spaces. For the conditioned space, it is recommended to increase roof insulation as well as increasing the insulation on the hot water tank, the hot water system pipes and installing aerators.

Appropriated funding FEDS results for building 1728 unconditioned space:

\begin{tabular}{|c|c|c|c|c|c|c|c|c|}
\hline Bldg. Set Description & $\begin{array}{l}\text { End } \\
\text { Use }\end{array}$ & Existing Technology & Retrofit Technology & $\begin{array}{l}\text { Energy } \\
\text { Savings } \\
\text { (MMBtu/yr) }\end{array}$ & $\begin{array}{c}\text { 1st year } \\
\text { savings } \\
(\$ / y r)\end{array}$ & $\begin{array}{l}\text { Installed } \\
\text { Cost (\$) }\end{array}$ & $\begin{array}{l}\text { Net } \\
\text { Present } \\
\text { Value (\$) } \\
\end{array}$ & SIR \\
\hline $\begin{array}{l}1050 \text { 's Wharehouse/storage } \\
1728\end{array}$ & Lights & MH6: MH 400 PEND & HS18: HPS 310 PEND & 175 & 6,807 & 38,531 & 73,510 & 2.9 \\
\hline $\begin{array}{l}1050 \text { 's Wharehouse/storage } \\
1728\end{array}$ & Lights & EX6: EXIT - LED & $\begin{array}{l}\text { EX12: EXIT - } \\
\text { ELECTROLUMINESCENT PANEL } \\
\text { RETRO KIT }\end{array}$ & - & 102 & 621 & 1,143 & 2.8 \\
\hline $\begin{array}{l}1050 \text { 's Wharehouse/storage } \\
1728\end{array}$ & Lights & HS13: HPS 70 PEND & $\begin{array}{l}\text { FL279: FL 2X4 2F32ST8 ELC2 } \\
\text { REF }\end{array}$ & 10 & 1,043 & 9,512 & 8,235 & 1.9 \\
\hline
\end{tabular}

Appropriated funding FEDS results for building 1728 conditioned space:

\begin{tabular}{|c|c|c|c|c|c|c|c|c|}
\hline Bldg. Set Description & $\begin{array}{l}\text { End } \\
\text { Use }\end{array}$ & Existing Technology & Retrofit Technology & $\begin{array}{l}\text { Energy } \\
\text { Savings } \\
\text { (MMBtu/yr) }\end{array}$ & $\begin{array}{l}\text { 1st year } \\
\text { savings } \\
(\$ / y r)\end{array}$ & $\begin{array}{l}\text { Installed } \\
\text { Cost }(\$)\end{array}$ & $\begin{array}{l}\text { Net } \\
\text { Present } \\
\text { Value (\$) }\end{array}$ & SIR \\
\hline $\begin{array}{l}\text { 1050's } \\
\text { Wharehouse/storage } 1728\end{array}$ & Lights & EX6: EXIT - LED & $\begin{array}{l}\text { EX12: EXIT - } \\
\text { ELECTROLUMINESCENT PANEL } \\
\text { RETRO KIT }\end{array}$ & - & 51 & 311 & 571 & 2.8 \\
\hline $\begin{array}{l}\text { 1050's } \\
\text { Wharehouse/storage } 1728\end{array}$ & Lights & FL37: FL 2X4 4F32T8 EEF2 & $\begin{array}{l}\text { FL280: FL 2X4 3F32ST8 ELC3 } \\
\text { REF }\end{array}$ & 55 & 3,213 & 3,312 & 50,587 & 16.3 \\
\hline
\end{tabular}




\section{Appropriated Funding Energy Consumption by Fuel Type}

The modeled energy consumption for the unconditioned space for a typical year was 493,902 kwh before retrofits and 439,598 kwh after proposed retrofits are implemented. The energy use intensity goes from $12.2 \mathrm{MBtu} / \mathrm{Ksf}$ to $10.9 \mathrm{MBtu} / \mathrm{Ksf}$ after retrofits.

1050's Wharehouse/storage 1728 unconditioned space

Fuel

Energy

493,902
439,598
$-54,304$
-11

1,686
1,500
-185
-11

$\begin{gathered}\text { Energy } \\ \text { Intensity }\end{gathered}$
(user units/10

$3,584.7$
$3,190.5$
-394.1
-11

12.2
10.9
-1.3
-11

\section{Energy \\ Intensity}

(MBtu/1000ft2)

Dollars

$(2009)$ *

Electricity (kWh)

existing

post-retrofit

difference

$\%$ change

Total (MBtu)

existing

difference

$-11$
12.2

10.9

$-1.3$

$-11$

12.2

10.9

$-1.3$

$-11$
87,317

77,305

$-10,011$

$-11$

87,317

77,305

$-10,011$

$-11$

* Dollar values for electricity include both energy and demand components. 
The modeled energy consumption for the unconditioned space for a typical year was 96,903 kwh before retrofits and 80,766 kwh after proposed retrofits are implemented. The energy use intensity goes from 127.2 MBtu/Ksf to $106.0 \mathrm{MBtu} / \mathrm{Ksf}$ after retrofits.

\section{0 's Wharehouse/storage 1728 unconditioned space}

Fuel

Electricity (kWh)

existing

post-retrofit

difference

$\%$ change

Total (MBtu)

existing

post-retrofit

difference

$\%$ change
Energy

$$
\begin{array}{r}
96,903 \\
80,766 \\
-16,137 \\
-17
\end{array}
$$

331
276
-55
-17

Energy

Intensity

(user units/1000ft2)

$37,256.0$

$31,051.9$

$-6,204.1$

$-17$

127.2
106.0
-21.2

-21.2
-17
Energy

Intensity

(MBtu/1000ft2)

127.2

106.0

$-21.2$

17

127.2

106.0

$-21.2$

$-17$
Dollars

(2009) *

17,131

14,203

$-2,928$

$-17$

17,131

14,203

$-2,928$

* Dollar values for electricity include both energy and demand components. 


\section{Appropriated Funding Energy Consumption by End Use}

Lighting is the largest load in the unconditioned space of the building with 253,372 kWh/year, followed by motors and miscellaneous equipment with 240,530 kWh/year.

1050 's Wharehouse/storage 1728 unconditioned space

Fuel Heating
Electricity (kWh)
existing
post-retrofit
difference
$\%$ change
Total (MBtu)
existing
post-retrofit
difference
$\%$ change
Total (MBtu/1000ft2)
existing
post-retrofit
difference
$\%$ change

Cooling Ment Motors and

Misc Equip

240,530

240,530

199,068

$-54,304$

$-21$

865
679
-185
-21

821
821
0
0

$\odot$

6
6
$\odot$
$\odot$
Hot Water

$\%$ change

$\begin{array}{ll}\odot & \odot \\ \odot & \odot \\ \odot & \odot \\ \odot & \odot\end{array}$


Motors and miscellaneous equipment is the largest load in the conditioned space of the building with 31,893 kWh/year, followed by space cooling with 28,814 kWh/year.

1050 's Wharehouse/storage

Fuel

Electricity (kWh)

existing

post-retrofit

difference

$\%$ change

Total (MBtu)

existing

post-retrofit

difference

$\%$ change

Heating

Cooling
$\begin{array}{r}28,814 \\ 24,548 \\ -4,266 \\ -15 \\ \\ 98 \\ 84 \\ -15 \\ -15 \\ \\ 38 \\ 32 \\ -6 \\ -15\end{array}$

Vent

9,289
8,639
-649

-649
-7

32

29

-2
-7

Total (MBtu/100๑ft2)

existing

post-retrofit

difference

$\%$ change

15
-15
1728 conditioned space

Motors and

Lights Misc Equip

Hot Water

26,560
15,339
$-11,222$

$-11,222$
-42

31,893

31,893

$$
\odot
$$

347

347

0

91
52
-38
-42

109

109

$\odot$
$\odot$

42
42
0
0

\author{
.
}




\section{Appropriated Funding Emission Reduction}

The emission reductions from implemented the proposed retrofits are as follows:

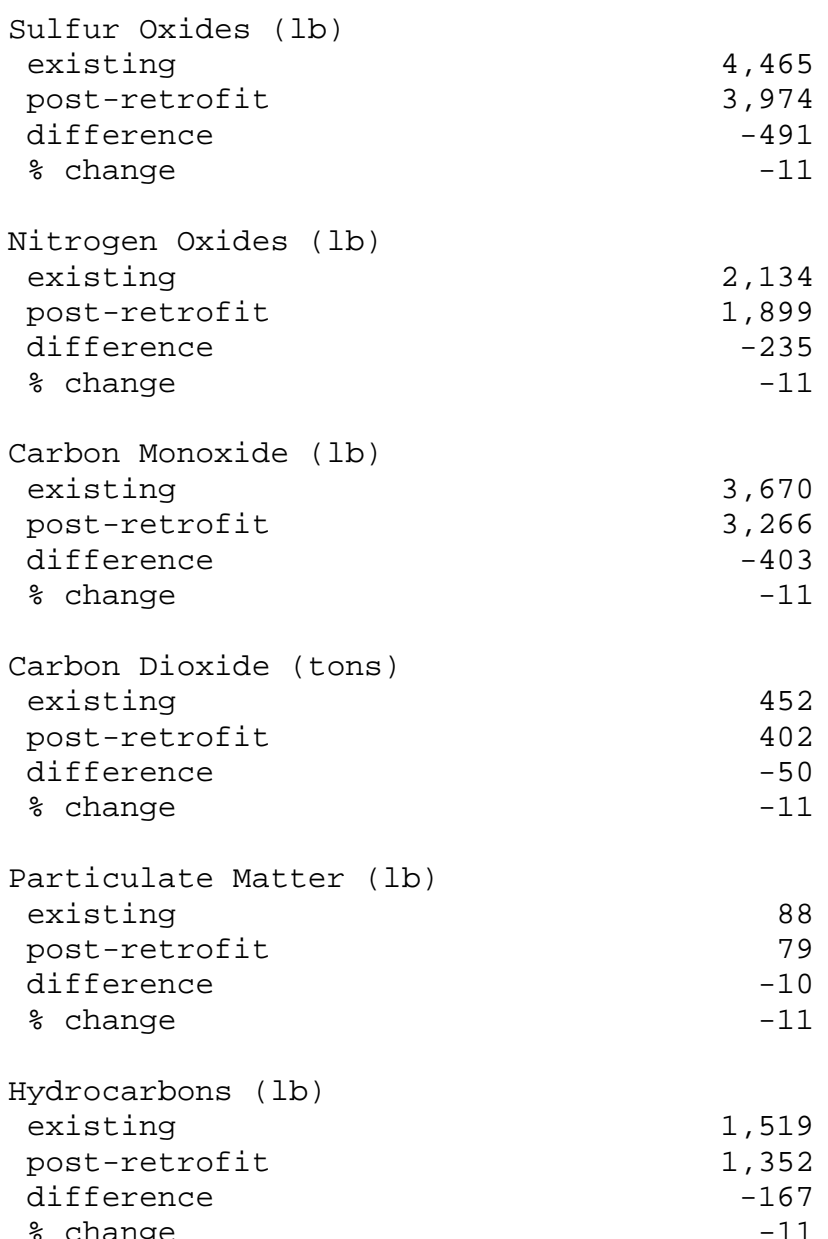


Sulfur Oxides (lb)

existing

post-retrofit

876

difference

$\%$ change

730

$-146$

$-17$

Nitrogen Oxides (lb)

existing

post-retrofit

419

post-retrof

349

difference

$-70$

$\%$ change

$-17$

Carbon Monoxide (lb)

existing

post-retrofit

720

difference

600

$\%$ change

$-120$

Carbon Dioxide (tons)

existing

post-retrofit

difference

$\%$ change

74

$-15$

Particulate Matter (lb)

existing

post-retrofit

difference

$\%$ change

17

14

$-3$

Hydrocarbons (1b)

existing

post-retrofi

298

difference

248

$\%$ change

$-17$ 


\section{Building 1072 Supply Warehouse}

The following information identifies the cost-effective energy- and cost-reducing retrofit projects for building 1072 identified from the FEDS modeling and analysis. Key energy and economic results are presented for each cost-effective retrofit measure.

\section{Facility Description 1072}

1072 is a warehouse building built in 1941. The warehouse is largely unconditioned but has a few small offices that are conditioned by DX units. Building 1072 is 83,379 sf.

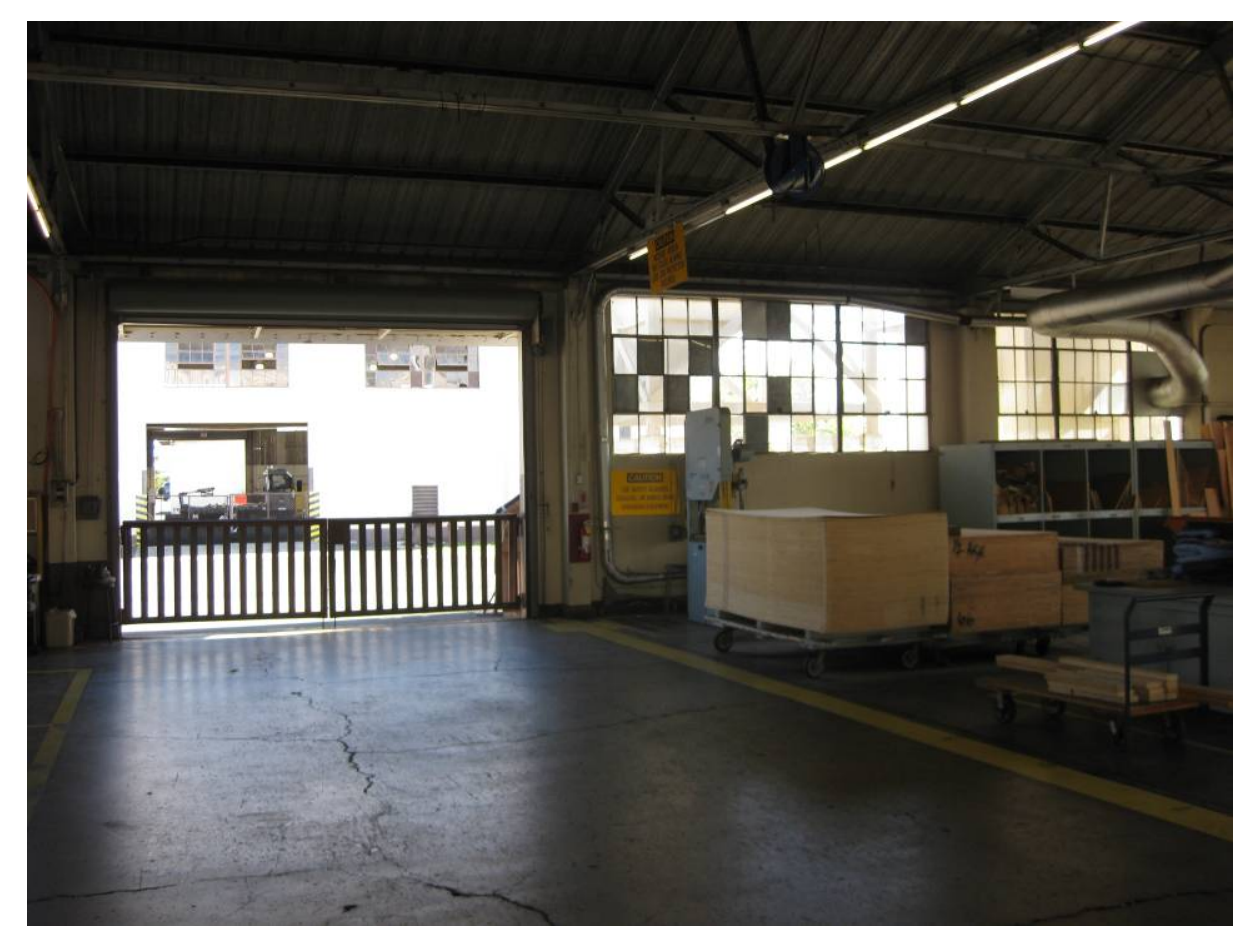

\section{Appropriated Funding Results}


A FEDS analysis using appropriated funding suggests replacing lights in the conditioned and unconditioned spaces of the building. FEDS also suggests adding insulation to the interior of the roof and replacing the single pane windows with double pane, super low-e windows in the conditioned space.

Appropriated funding FEDS results for building 1072 unconditioned space:

\begin{tabular}{|c|c|c|c|c|c|c|c|c|}
\hline Bldg. Set Description & $\begin{array}{l}\text { End } \\
\text { Use }\end{array}$ & Existing Technology & Retrofit Technology & $\begin{array}{l}\text { Energy } \\
\text { Savings } \\
\text { (MMBtu/yr) } \\
\end{array}$ & $\begin{array}{c}\text { 1st year } \\
\text { savings } \\
(\$ / y r)\end{array}$ & $\begin{array}{l}\text { Installed } \\
\text { Cost (\$) }\end{array}$ & $\begin{array}{l}\text { Net } \\
\text { Present } \\
\text { Value (\$) } \\
\end{array}$ & SIR \\
\hline $\begin{array}{l}\text { 1050's } \\
\text { Wharehouse/storage } 1072\end{array}$ & Lights & MH6: MH 400 PEND & HS18: HPS 310 PEND & 69 & 2,679 & 15,160 & 28,922 & 2.9 \\
\hline $\begin{array}{l}\text { 1050's } \\
\text { Wharehouse/storage } 1072\end{array}$ & Lights & EX6: EXIT - LED & $\begin{array}{l}\text { EX12: EXIT - } \\
\text { ELECTROLUMINESCENT PANEL } \\
\text { RETRO KIT }\end{array}$ & - & 102 & 621 & 1,143 & 2.8 \\
\hline $\begin{array}{l}\text { 1050's } \\
\text { Wharehouse/storage } 1072\end{array}$ & Lights & FL236: FL 2X4 3F32T8 ELC3 & $\begin{array}{l}\text { FL279: FL 2X4 2F32ST8 ELC2 } \\
\text { REF }\end{array}$ & 128 & 6,814 & 13,362 & 100,543 & 8.5 \\
\hline
\end{tabular}

Appropriated funding FEDS results for building 1072 conditioned space:

\begin{tabular}{|c|c|c|c|c|c|c|c|c|}
\hline Bldg. Set Description & $\begin{array}{l}\text { End } \\
\text { Use }\end{array}$ & Existing Technology & Retrofit Technology & $\begin{array}{l}\text { Energy } \\
\text { Savings } \\
\text { (MMBtu/yr) }\end{array}$ & $\begin{array}{l}\text { 1st year } \\
\text { savings } \\
(\$ / y r)\end{array}$ & $\begin{array}{l}\text { Installed } \\
\text { Cost (\$) }\end{array}$ & $\begin{array}{l}\text { Net } \\
\text { Present } \\
\text { Value (\$) }\end{array}$ & SIR \\
\hline $\begin{array}{l}\text { 1050's } \\
\text { Wharehouse/storage } 1072\end{array}$ & Lights & EX6: EXIT - LED & $\begin{array}{l}\text { EX12: EXIT - } \\
\text { ELECTROLUMINESCENT PANEL } \\
\text { RETRO KIT }\end{array}$ & - & 56 & 311 & 661 & 3.1 \\
\hline $\begin{array}{l}\text { 1050's } \\
\text { Wharehouse/storage } 1072\end{array}$ & Lights & FL236: FL 2X4 3F32T8 ELC3 & $\begin{array}{l}\text { FL279: FL 2X4 2F32ST8 ELC2 } \\
\text { REF }\end{array}$ & 40 & 2,099 & 3,158 & 31,829 & 11.1 \\
\hline $\begin{array}{l}\text { 1050's } \\
\text { Wharehouse/storage } 1072\end{array}$ & Roof & Roof Insulation R-Value 0.00 & $\begin{array}{l}\text { Add Insulation to Interior } \\
\text { Surface of Metal Roof: } 4 \text { inches } \\
\text { of Fiberglass }\end{array}$ & 302 & 15,861 & 22,799 & 241,103 & 11.6 \\
\hline
\end{tabular}




\section{Appropriated Funding Energy Consumption by Fuel Type}

The modeled energy consumption for the unconditioned space fo the building for a typical year was 404,132 kwh before retrofits and 346,253 kwh after proposed retrofits are implemented. The energy use intensity goes from $17.5 \mathrm{MBtu} / \mathrm{Ksf}$ to $15.0 \mathrm{MBtu} / \mathrm{Ksf}$ after retrofits.

1050's Wharehouse/storage 1072

Fuel

\section{Energy}

$$
\begin{aligned}
& 404,132 \\
& 346,253 \\
& -57,879
\end{aligned}
$$

post-retrofit

difference

$\%$ change

Total (MBtu)

existing

post-retrofit

difference

$\%$ change

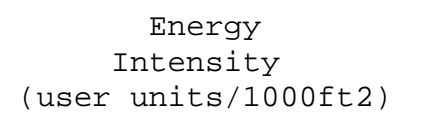

$5,134.6$

$4,399.2$

$-735.4$

$-14$

1,379
1,182
-198
-14
17.5

15.0

$-2.5$
Energy

Intensity

(MBtu/1000ft2)

17.5

15.0

$-2.5$

$-14$

17.5

15.0

-2.5
-14
Dollars (2009) *

71,446

60,890

$-10,556$

$-15$

71,446

60,890

$-10,556$

* Dollar values for electricity include both energy and demand components. 
The modeled energy consumption for the conditioned space fo the building for a typical year was $163,410 \mathrm{kwh}$ before retrofits and 64,784 kwh after proposed retrofits are implemented. The energy use intensity goes from 83.6 MBtu/Ksf to 33.1 MBtu/Ksf after retrofits.

1050's Wharehouse/storage 1072

Fuel

Energy

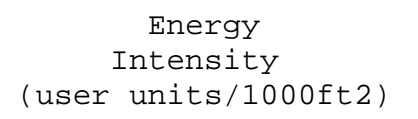

Energy

Dollars

Electricity (kWh)

existing

post-retrofit

difference

$\%$ change

163,410
64,784

64,784
$-98,626$

$$
\begin{array}{r}
24,499.2 \\
9,712.7 \\
-14,786.4 \\
-60
\end{array}
$$

83.6

33.1

$-50.5$

$-60$

$(2009)$ *

Total (MBtu)

existing

post-retrofit

difference

83.6

33.1

$-50.5$

83.6

33.1

$-50.5$

$-60$

28,889

11,393

$-17,497$

497
-61

$\%$ change

28,889

11,393

$-61$

* Dollar values for electricity include both energy and demand components. 


\section{Appropriated Funding Energy Consumption by End Use}

Lighting is the largest load in the unconditioned space of the building with $266,729 \mathrm{kWh} / \mathrm{year}$, followed by motors and miscellaneous equipment with $137,403 \mathrm{kWh} /$ year.

1050's Wharehouse/storage 1072 unconditioned space

\begin{tabular}{|c|c|c|c|c|c|c|}
\hline Fuel & Heating & Cooling & Vent & Lights & $\begin{array}{l}\text { Motors and } \\
\text { Misc Equip }\end{array}$ & Hot Water \\
\hline \multicolumn{7}{|c|}{ Electricity (kWh) } \\
\hline existing & $\odot$ & $\odot$ & $\odot$ & 266,729 & 137,403 & $\odot$ \\
\hline post-retrofit & $\odot$ & $\odot$ & $\odot$ & 208,850 & 137,403 & $\odot$ \\
\hline difference & $\odot$ & $\odot$ & $\odot$ & $-57,879$ & 0 & $\odot$ \\
\hline$\%$ change & $\odot$ & $\odot$ & $\odot$ & -22 & $\odot$ & $\odot$ \\
\hline \multicolumn{7}{|l|}{ Total (MBtu) } \\
\hline existing & $\odot$ & $\odot$ & $\odot$ & 910 & 469 & $\odot$ \\
\hline post-retrofit & $\odot$ & $\odot$ & $\odot$ & 713 & 469 & $\odot$ \\
\hline difference & $\odot$ & $\odot$ & $\odot$ & -198 & 0 & $\odot$ \\
\hline$\%$ change & $\odot$ & $\odot$ & $\odot$ & -22 & $\odot$ & 0 \\
\hline \multicolumn{7}{|c|}{ Total (MBtu/100๑ft2) } \\
\hline existing & $\odot$ & $\odot$ & $\odot$ & 12 & 6 & $\odot$ \\
\hline post-retrofit & 0 & 0 & 0 & 9 & 6 & 0 \\
\hline difference & $\odot$ & $\odot$ & $\odot$ & -3 & $\odot$ & $\odot$ \\
\hline$\%$ change & $\odot$ & 0 & 0 & -22 & $\odot$ & 0 \\
\hline
\end{tabular}


Space cooling is the largest load in the unconditioned space of the building with 79,200 kWh/year, followed by ventilation with 53,432 kWh/year.

1050 's Wharehouse/storage 1072 conditioned space

\begin{tabular}{|c|c|c|c|c|c|}
\hline Heating & Cooling & Vent & Lights & $\begin{array}{l}\text { Motors and } \\
\text { Misc Equip }\end{array}$ & Hot water \\
\hline$\odot$ & $79,20 \odot$ & 53,432 & 19,134 & 11,644 & $\odot$ \\
\hline$\odot$ & 25,266 & 16,793 & 11,081 & 11,644 & $\odot$ \\
\hline$\odot$ & $-53,934$ & $-36,639$ & $-8,052$ & 0 & $\odot$ \\
\hline$\odot$ & -68 & -69 & -42 & $\odot$ & $\odot$ \\
\hline 0 & 270 & 182 & 65 & 40 & $\odot$ \\
\hline 0 & 86 & 57 & 38 & 40 & $\odot$ \\
\hline 0 & -184 & -125 & -27 & 0 & $\odot$ \\
\hline$\odot$ & -68 & -69 & -42 & $\odot$ & $\odot$ \\
\hline$\odot$ & 41 & 27 & 10 & 6 & $\odot$ \\
\hline$\odot$ & 13 & 9 & 6 & 6 & $\odot$ \\
\hline$\odot$ & -28 & -19 & -4 & $\odot$ & $\odot$ \\
\hline 0 & -68 & -69 & -42 & $\odot$ & $\odot$ \\
\hline
\end{tabular}




\section{Appropriated Funding Emission Reduction}

The emission reductions from implemented the proposed retrofits are as follows:

1050 's Wharehouse/storage

1072 unconditioned space

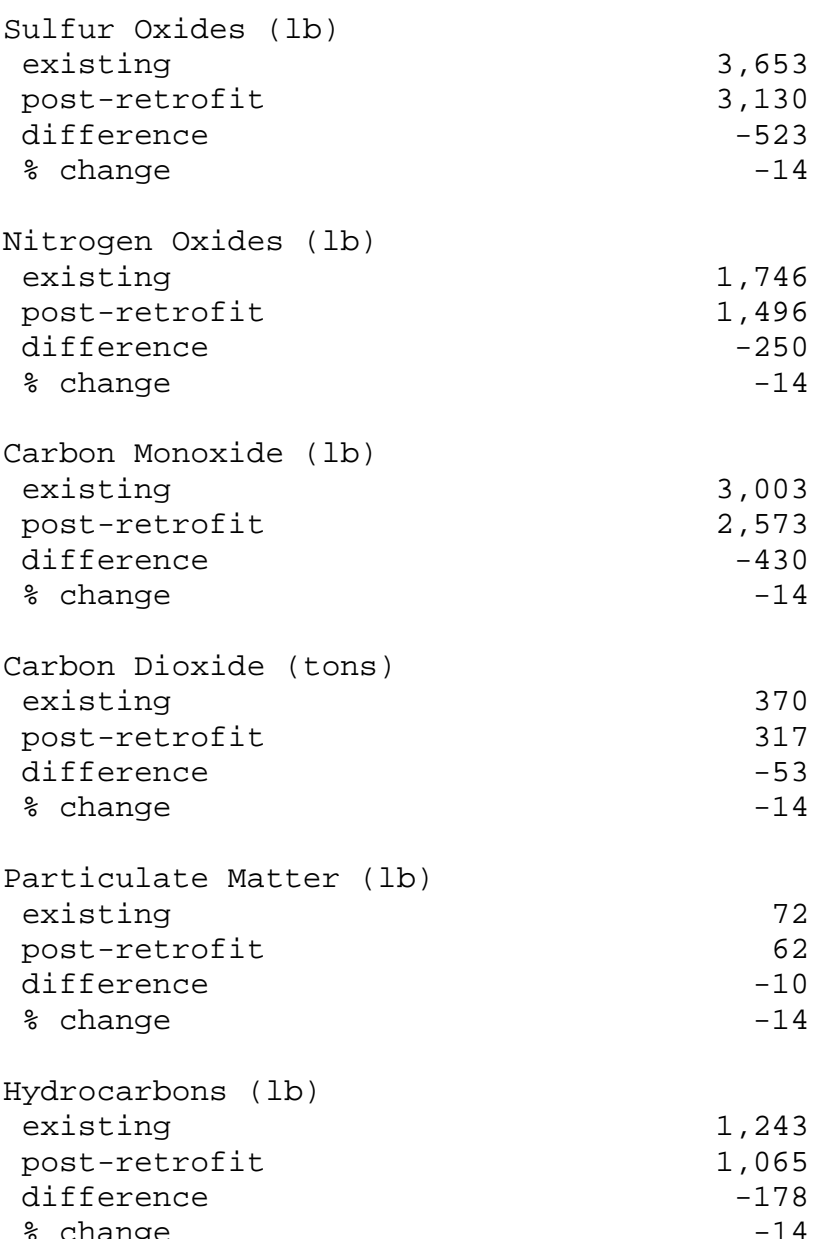


Sulfur oxides (lb)

existing

post-retrofit

difference

$\%$ change

Nitrogen oxides (lb)

existing

post-retrofit

difference

\% change

Carbon Monoxide ( $1 b$ )

existing

post-retrofit

difference

$\%$ change

Carbon Dioxide (tons)

existing

post-retrofit

difference

$\%$ change

1,477

586

$-892$

$-60$

706

280

$-426$

$-60$

1,214

481

-733
-60

$-60$

150

59

$-90$

$-60$

Particulate Matter (lb)

existing

post-retrofit

difference

$\%$ change

$-18$

$-60$

Hydrocarbons (lb)

existing

post-retrofit

503

199

$-303$

$-60$ 


\section{Building 1070 Warehouse Building}

The following information identifies the cost-effective energy- and cost-reducing retrofit projects for building 1070 identified from the FEDS modeling and analysis. Key energy and economic results are presented for each cost-effective retrofit measure.

\section{Facility Description 1070}

1070 is a warehouse building with some conditioned hazmat storage built in 1941 . Building 1070 is mostly unconditioned storage with a small office that is conditioned by a small DX unit. Building 1070 is 62,779 sf.

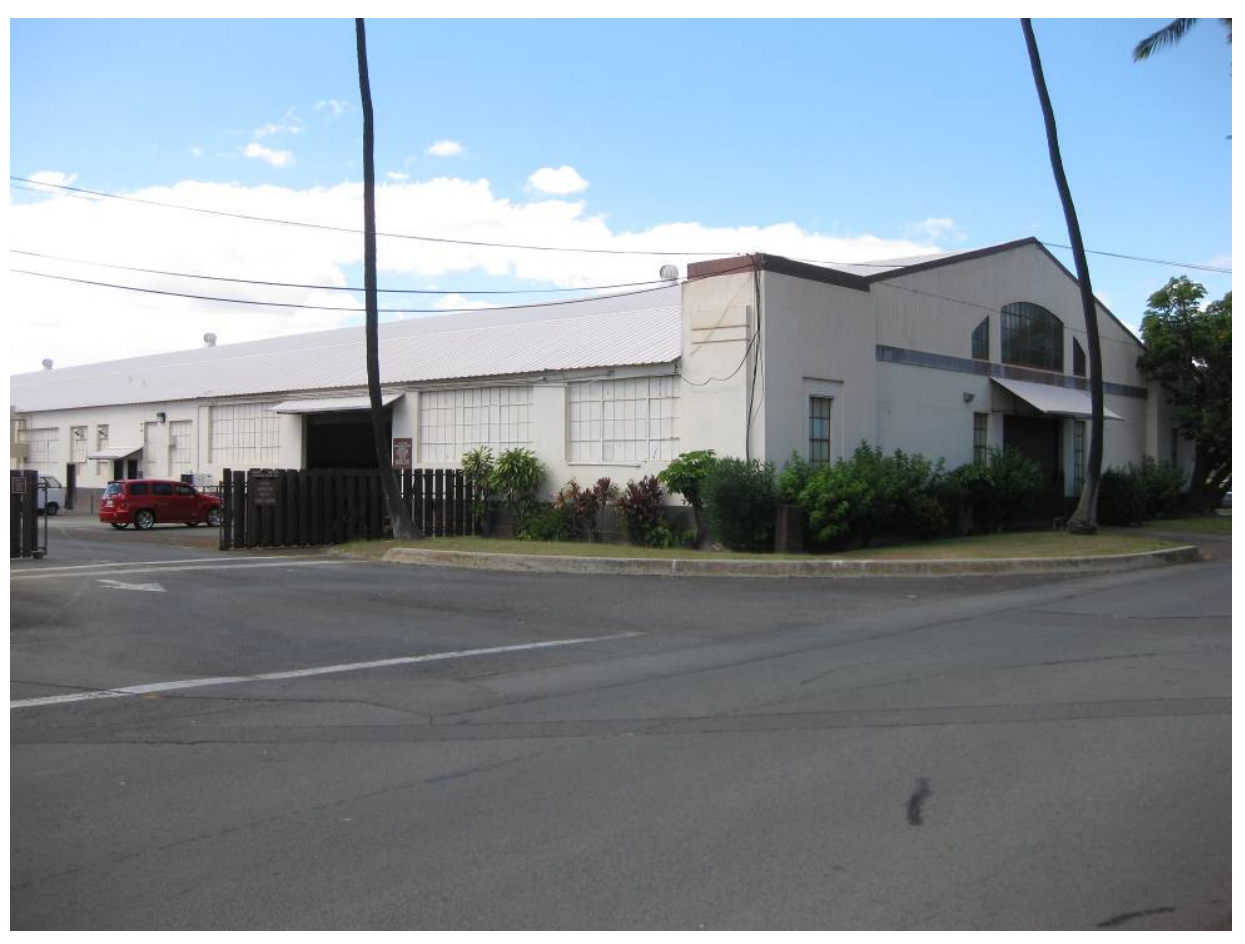




\section{Appropriated Funding Results}

A FEDS analysis using appropriated funding suggests replacing lighting in the unconditioned space as well as replacing the EXIT lighting fixtures in the conditioned and unconditioned spaces. FEDS also suggests increasing the insulation in the roof of the conditioned office space.

Appropriated funding FEDS results for building 1070 unconditioned space:

\begin{tabular}{|c|c|c|c|c|c|c|c|c|}
\hline Bldg. Set Description & $\begin{array}{l}\text { End } \\
\text { Use }\end{array}$ & Existing Technology & Retrofit Technology & $\begin{array}{l}\text { Energy } \\
\text { Savings } \\
\text { (MMBtu/yr) }\end{array}$ & $\begin{array}{c}\text { 1st year } \\
\text { savings } \\
(\$ / y r)\end{array}$ & $\begin{array}{l}\text { Installed } \\
\text { Cost (\$) }\end{array}$ & $\begin{array}{l}\text { Net } \\
\text { Present } \\
\text { Value (\$) } \\
\end{array}$ & SIR \\
\hline small storage & Lights & EX1: EXIT - INC $(2 \times 20)$ & $\begin{array}{l}\text { EX12: EXIT - } \\
\text { ELECTROLUMINESCENT PANEL } \\
\text { RETRO KIT }\end{array}$ & 5 & 344 & 311 & 5,520 & 18.8 \\
\hline small storage & Lights & IN27: INC 100 WALL & $\begin{array}{l}\text { CF9: CFL } 26 \text { INTEGRAL UNIT } \\
\text { ELC }\end{array}$ & 9 & 666 & 2,530 & 8,694 & 4.4 \\
\hline small storage 1070 & Lights & FL5: FL 1X4 1F40T12 STD1 & FL53: FL 1X4 1F32T8 ELC1 & 2 & 177 & 2,467 & 524 & 1.2 \\
\hline
\end{tabular}

Appropriated funding FEDS results for building 1070 conditioned space:

\begin{tabular}{|c|c|c|c|c|c|c|c|c|}
\hline Bldg. Set Description & $\begin{array}{l}\text { End } \\
\text { Use }\end{array}$ & Existing Technology & Retrofit Technology & $\begin{array}{l}\text { Energy } \\
\text { Savings } \\
\text { (MMBtu/yr) }\end{array}$ & $\begin{array}{c}\text { 1st year } \\
\text { savings } \\
(\$ / y r)\end{array}$ & $\begin{array}{l}\text { Installed } \\
\text { Cost (\$) }\end{array}$ & $\begin{array}{l}\text { Net } \\
\text { Present } \\
\text { Value (\$) }\end{array}$ & SIR \\
\hline $\begin{array}{l}\text { small storage } 1070 \\
\text { small storage } 1070\end{array}$ & $\begin{array}{l}\text { Lights } \\
\text { Roof }\end{array}$ & $\begin{array}{l}\text { EX1: EXIT - INC }(2 \times 20) \\
\text { Roof Insulation R-Value } 0.00\end{array}$ & $\begin{array}{l}\text { EX12: EXIT - } \\
\text { ELECTROLUMINESCENT PANEL } \\
\text { RETRO KIT } \\
\text { Add Insulation to Interior } \\
\text { Surface of Metal Roof: } 4 \text { inches } \\
\text { Fiberglass }\end{array}$ & 6 & 420 & 311 & $\begin{array}{l}6,786 \\
12,902\end{array}$ & 22.8 \\
\hline
\end{tabular}




\section{Appropriated Funding Energy Consumption by Fuel Type}

The modeled energy consumption for unconditioned spaces in the building for a typical year was 15,732 kwh before retrofits and 11,110 kwh after proposed retrofits are implemented. The modeled other fuels (propane) consumption for the unconditioned spaces in the building for a typical year was $382 \mathrm{MBtu}$ before retrofits and $382 \mathrm{MBtu}$ after proposed retrofits are implemented. The energy use intensity goes from 7.1 MBtu/Ksf to 6.8 MBtu/Ksf after retrofits.

\begin{tabular}{|c|c|c|c|c|}
\hline \multirow[b]{2}{*}{ Fuel } & \multirow[b]{2}{*}{ Energy } & \multicolumn{2}{|c|}{1070 unconditioned space } & \multirow[b]{2}{*}{$\begin{array}{l}\text { Dollars } \\
(20 \odot 9) \text { * }\end{array}$} \\
\hline & & $\begin{array}{c}\text { Energy } \\
\text { Intensity } \\
\text { (user units/1000ft2) }\end{array}$ & $\begin{array}{l}\text { Energy } \\
\text { Intensity } \\
(\text { MBtu/1000ft2) }\end{array}$ & \\
\hline \multicolumn{5}{|c|}{ Electricity (kWh) } \\
\hline existing & 15,732 & 255.7 & 0.9 & 2,781 \\
\hline post-retrofit & 11,110 & 180.6 & 0.6 & 1,954 \\
\hline difference & $-4,623$ & -75.1 & $-\odot .3$ & -828 \\
\hline$\%$ change & -29 & -29 & -29 & -30 \\
\hline \multicolumn{5}{|c|}{ Other Fuels (MBtu) } \\
\hline existing & 382 & 6.2 & 6.2 & 12,278 \\
\hline post-retrofit & 382 & 6.2 & 6.2 & 12,278 \\
\hline difference & $\odot$ & $\odot . \odot$ & $\odot .0$ & 0 \\
\hline$\%$ change & $\odot$ & 0 & 0 & $\odot$ \\
\hline \multicolumn{5}{|l|}{ Total (MBtu) } \\
\hline existing & 436 & 7.1 & 7.1 & $15,06 \odot$ \\
\hline post-retrofit & 420 & 6.8 & 6.8 & 14,232 \\
\hline difference & -16 & -0.3 & $-\odot .3$ & -828 \\
\hline$\%$ change & -4 & -4 & -4 & -5 \\
\hline
\end{tabular}

* Dollar values for electricity include both energy and demand components. 
The modeled energy consumption for conditioned spaces in the building for a typical year was 25,042 kwh before retrofits and 17,627 kwh after proposed retrofits are implemented. The modeled other fules (propane) consumption for the unconditioned spaces in the building for a typical year was $8 \mathrm{MBtu}$ before retrofits and $8 \mathrm{MBtu}$ after proposed retrofits are implemented. The energy use intensity goes from 74.3 MBtu/Ksf to 54.1 MBtu/Ksf after retrofits.

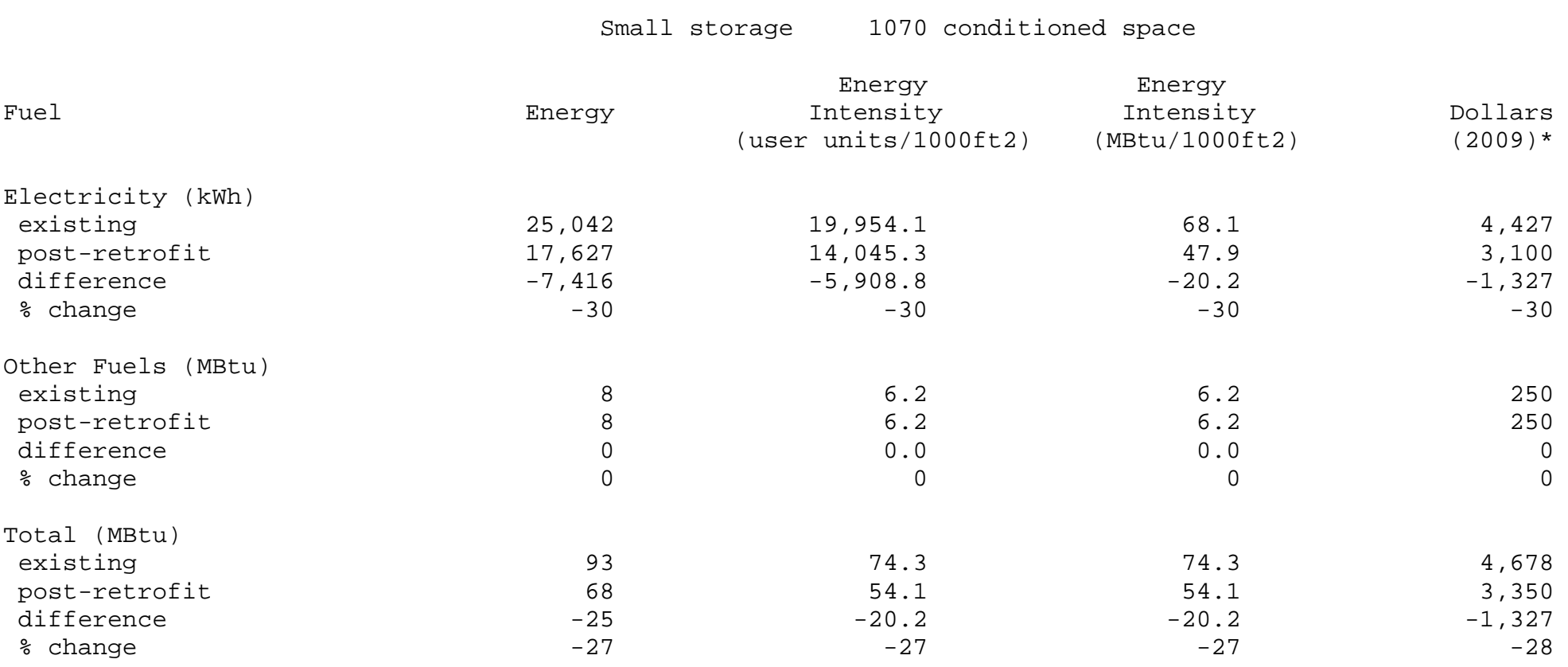




\section{Appropriated Funding Energy Consumption by End Use}

Lighting is the largest load in the unconditioned space of the building with $14,736 \mathrm{kWh} /$ year, followed by motors and miscellaneous equipment with $997 \mathrm{kWh} /$ year.

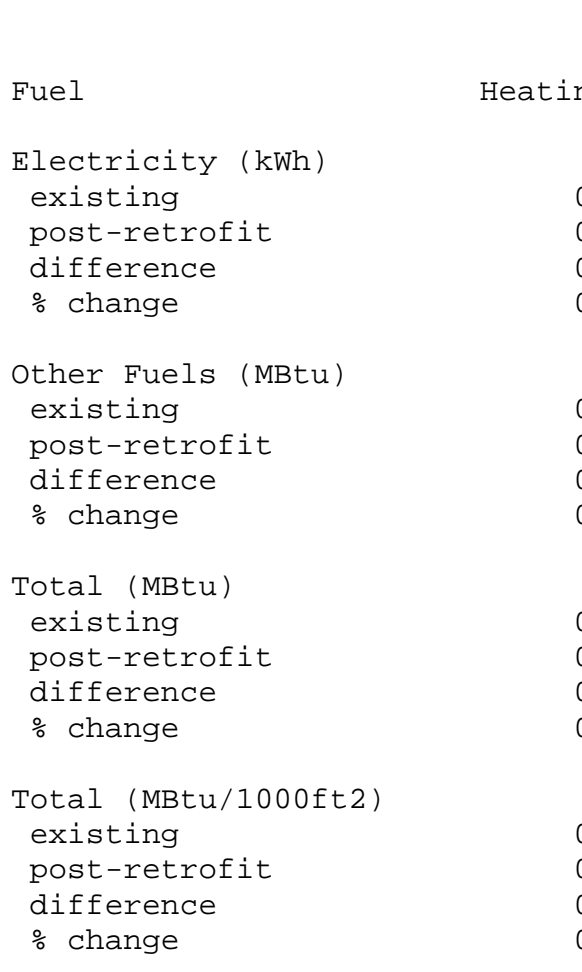

\section{small storage \\ 1070 unconditioned space}

Cooling

$\odot$

$\odot$

$\odot$

$\odot$

$\odot$
$\odot$
$\odot$
$\odot$

$\begin{array}{ll}\odot & \odot \\ \odot & \odot\end{array}$

14,736
10,113
$-4,623$
-31

$\odot$

$\odot$

Vent Lights Misc Equip

Hot Water

997

997

0
$\odot$

$\odot$

382

382

○

385

385

$\begin{array}{rr}50 & 385 \\ 35 & 0 \\ -16 & 0\end{array}$

$\odot$

0

$\begin{array}{rr}1 & 6 \\ 1 & 6 \\ 0 & 0 \\ -31 & 0\end{array}$

$\odot$
$\odot$
$\odot$
$\odot$ 
Space cooling is the largest load in the conditioned space of the building with $12,471 \mathrm{kWh} /$ year, followed by motors and miscellaneous equipment with 6,323 kWh/year.

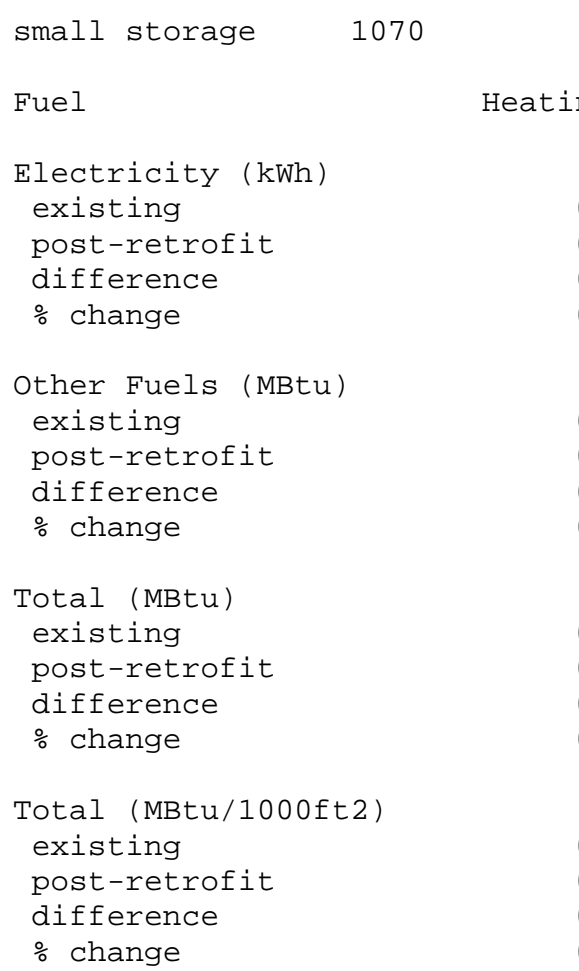

$\begin{array}{rr}\text { Cooling } & \text { Ven } \\ 12,471 & 3,43 \\ 7,504 & 2,37 \\ -4,967 & -1,06 \\ -40 & -31\end{array}$

Motors and
Lights $\quad$ Misc Equip

Hot Water

$\begin{array}{rrrrrr}\odot & 12,471 & 3,435 & 2,814 & 6,323 & 0 \\ \odot & 7,504 & 2,375 & 1,424 & 6,323 & 0 \\ \odot & -4,967 & -1,060 & -1,389 & 0 & 0 \\ \odot & -40 & -31 & -49 & 0 & 0\end{array}$

$\begin{array}{ll}\odot & \odot \\ \odot & \odot \\ \odot & \odot \\ \odot & \odot\end{array}$

$\begin{array}{rr}\odot & 43 \\ \odot & 26 \\ \odot & -17\end{array}$




\section{Appropriated Funding Emission Reduction}

The emission reductions from implemented the proposed retrofits are as follows:
Small storage
1070 unconditioned space

\begin{tabular}{|c|c|}
\hline Sulfur oxides (lb) & \\
\hline existing & 238 \\
\hline post-retrofit & 196 \\
\hline difference & -42 \\
\hline$\%$ change & -18 \\
\hline Nitrogen Oxides (lb) & \\
\hline existing & 202 \\
\hline post-retrofit & 182 \\
\hline difference & -20 \\
\hline$\%$ change & -10 \\
\hline Carbon Monoxide (lb) & \\
\hline existing & 407 \\
\hline post-retrofit & 373 \\
\hline difference & -34 \\
\hline$\%$ change & -8 \\
\hline Carbon Dioxide (tons) & \\
\hline existing & 47 \\
\hline post-retrofit & 43 \\
\hline difference & -4 \\
\hline$\%$ change & -9 \\
\hline Particulate Matter (lb) & \\
\hline existing & 7 \\
\hline post-retrofit & 6 \\
\hline difference & -1 \\
\hline$\%$ change & -12 \\
\hline Hydrocarbons (lb) & \\
\hline existing & 144 \\
\hline post-retrofit & 130 \\
\hline difference & -14 \\
\hline$\%$ change & -10 \\
\hline
\end{tabular}




\section{Small storage}

228

xides (lb)

existing

post-retrofit

difference

$\%$ change

161

$-67$

$-29$

Nitrogen oxides (lb)

existing

post-retrofit

difference

\% change

111

79

$-32$

$-29$

Carbon Monoxide (lb)

existing

post-retrofit

192

137

$-55$

$\%$ change

$-29$

Carbon Dioxide (tons)

existing

post-retrofit

difference

$\%$ change

24

17

$-7$

$-29$

Particulate Matter (lb)

existing

post-retrofit

difference

$\%$ change

$$
\begin{array}{r}
5 \\
3 \\
-1 \\
-29
\end{array}
$$

Hydrocarbons (lb)

existing

post-retrofit

difference

$\%$ change

79

$-23$

$-29$
1070 conditioned space 


\section{Building 2002 Vehicle Maintenance Building}

The following information identifies the cost-effective energy- and cost-reducing retrofit projects for building 2002 identified from the FEDS modeling and analysis. Key energy and economic results are presented for each cost-effective retrofit measure.

\section{Facility Description 2002}

2002 is a vehicle maintenance building with conditioned admin and unconditioned workshop space built in 1940. Building 2002 generally has fluorescent lighting, an electric hot water system and its administration spaces are cooled by an electric package, or DX, unit. Building 2002 is 23,981 sf. 


\section{Appropriated Funding Results}

A FEDS analysis using appropriated funding suggests replacing the EXIT lighting as well as replacing the T12 Fluorescent lighting in the unconditioned spaces. In the conditioned spaces FEDS suggests replacing EXIT lighting, T12 Flourescent lighting, and increasing the insulation on the interior of the metal roof.

Appropriated funding FEDS results for building 2002 unconditioned spaces:

\begin{tabular}{|c|c|c|c|c|c|c|c|c|}
\hline Bldg. Set Description & $\begin{array}{l}\text { End } \\
\text { Use }\end{array}$ & Existing Technology & Retrofit Technology & $\begin{array}{l}\text { Energy } \\
\text { Savings } \\
\text { (MMBtu/yr) }\end{array}$ & $\begin{array}{l}\text { 1st year } \\
\text { savings } \\
(\$ / y r)\end{array}$ & $\begin{array}{l}\text { Installed } \\
\text { Cost (\$) }\end{array}$ & $\begin{array}{l}\text { Net } \\
\text { Present } \\
\text { Value (\$) } \\
\end{array}$ & SIR \\
\hline $\begin{array}{l}\text { Vehicle maintenance } \\
2002\end{array}$ & Lights & FL62: FL 1X8 2F96T12 STD2 & $\begin{array}{l}\text { FL131: FL 1X8 2F96T12ES ELC2 } \\
\text { REF (FIX REPL) }\end{array}$ & 13 & 888 & 5,076 & 9,820 & 2.9 \\
\hline $\begin{array}{l}\text { Vehicle maintenance } \\
2002\end{array}$ & Lights & EX1: EXIT - INC $(2 \times 20)$ & $\begin{array}{l}\text { EX12: EXIT - } \\
\text { ELECTROLUMINESCENT PANEL } \\
\text { RETRO KIT }\end{array}$ & 1 & 106 & 93 & 1,695 & 19.2 \\
\hline $\begin{array}{l}\text { Vehicle maintenance } \\
2002\end{array}$ & Lights & FL1: FL 2X4 4F40T12 STD2 & $\begin{array}{l}\text { FL280: FL 2X4 3F32ST8 ELC3 } \\
\text { REF (FIX REPL) }\end{array}$ & 30 & 1,998 & 8,916 & 24,600 & 3.8 \\
\hline $\begin{array}{l}\text { Vehicle maintenance } \\
2002\end{array}$ & Lights & EX1: EXIT - INC $(2 \times 20)$ & $\begin{array}{l}\text { EX12: EXIT - } \\
\text { ELECTROLUMINESCENT PANEL } \\
\text { RETRO KIT }\end{array}$ & 8 & 599 & 528 & 9,603 & 19.2 \\
\hline $\begin{array}{l}\text { Vehicle maintenance } \\
2002\end{array}$ & Lights & FL4: FL 1X4 2F40T12 STD2 & FL52: FL 1X4 2F32T8 ELC2 & 4 & 280 & 1,241 & 3,490 & 3.8 \\
\hline
\end{tabular}


Appropriated funding FEDS results for building 2002 conditioned spaces:

\begin{tabular}{|c|c|c|c|c|c|c|c|c|}
\hline Bldg. Set Description & $\begin{array}{l}\text { End } \\
\text { Use }\end{array}$ & Existing Technology & Retrofit Technology & $\begin{array}{l}\text { Energy } \\
\text { Savings } \\
\text { (MMBtu/yr) }\end{array}$ & $\begin{array}{l}\text { 1st year } \\
\text { savings } \\
(\$ / y r)\end{array}$ & $\begin{array}{l}\text { Installed } \\
\text { Cost (\$) }\end{array}$ & $\begin{array}{l}\text { Net } \\
\text { Present } \\
\text { Value (\$) } \\
\end{array}$ & SIR \\
\hline $\begin{array}{l}\text { Vehicle maintenance } \\
2002\end{array}$ & Lights & EX1: EXIT - INC $(2 \times 20)$ & $\begin{array}{l}\text { EX12: EXIT - } \\
\text { ELECTROLUMINESCENT PANEL } \\
\text { RETRO KIT }\end{array}$ & 1 & 64 & 47 & 1028 & 23.1 \\
\hline $\begin{array}{l}\text { Vehicle maintenance } \\
2002\end{array}$ & Lights & EX1: EXIT - INC $(2 \times 20)$ & $\begin{array}{l}\text { EX12: EXIT - } \\
\text { ELECTROLUMINESCENT PANEL } \\
\text { RETRO KIT }\end{array}$ & 5 & 364 & 264 & 5873 & 23.2 \\
\hline $\begin{array}{l}\text { Vehicle maintenance } \\
2002\end{array}$ & Lights & FL62: FL 1X8 2F96T12 STD2 & $\begin{array}{l}\text { FL131:FL 1X8 2F96T12ES } \\
\text { ELC2REF (FIX REPL) }\end{array}$ & 3 & 220 & 1015 & 2674 & 3.8 \\
\hline $\begin{array}{l}\text { Vehicle maintenance } \\
2002\end{array}$ & Lights & FL1:FL2X4 4F40T12 STD2 & $\begin{array}{l}\text { FL280: FL2X4 3F32ST8 ELC3 } \\
\text { REF (FIX REPL) }\end{array}$ & 8 & 497 & 1783 & 6558 & 4.7 \\
\hline $\begin{array}{l}\text { Vehicle maintenance } \\
2002\end{array}$ & Lights & FL4: FL 1X4 2F40T12 STD2 & FL52: FL 1X4 2F32T8 ELC2 & 1 & 56 & 248 & 698 & 3.8 \\
\hline $\begin{array}{l}\text { Vehicle maintenance } \\
2002\end{array}$ & Roof & Roof Insulation R-Value 0.00 & $\begin{array}{l}\text { Add Insulation to Interior } \\
\text { surface of Metal Roof: } 4 \text { inches } \\
\text { Fiberglass }\end{array}$ & 129 & 6,404 & 16,393 & 90,163 & 6.5 \\
\hline
\end{tabular}




\section{Appropriated Funding Energy Consumption by Fuel Type}

The modeled energy consumption for a typical year for the unconditioned space was 125,846 kwh before retrofits and 112,380 kwh after proposed retrofits are implemented. The energy use intensity goes from $22.4 \mathrm{MBtu} / \mathrm{Ksf}$ to $20.0 \mathrm{MBtu} / \mathrm{Ksf}$ after retrofits.

Vehicle maintenance $20 \odot 2$ unconditioned space

Fuel

Electricity (kWh)

existing

post-retrofit

difference

$\%$ change

Total (MBtu)

existing

post-retrofit

difference

$\%$ change

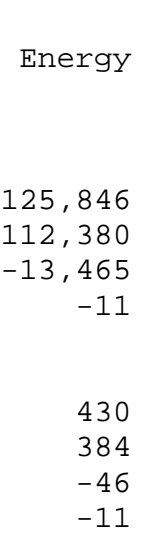

Energy
Intensity
(user units/10

$6,559.9$
$5,858.0$
-701.9
-11

22.4
20.0
-2.4
-11

Energy
Intensity
MBtu/1000ft2)

Dollars

$(2009)^{\star}$

22,248

19,763

$-2,486$

20.0

$-2.4$

$-11$

$-11$

22.4
20.0
-2.4
-11

22,248

19,763

$-2,486$

$-11$

* Dollar values for electricity include both energy and demand components. 
The modeled energy consumption for a typical year for the conditioned space was 98,451 kwh before retrofits and 56,191 kwh after proposed retrofits are implemented. The energy use intensity goes from $70.1 \mathrm{MBtu} / \mathrm{Ksf}$ to $40.0 \mathrm{MBtu} / \mathrm{Ksf}$ after retrofits.

Vehicle maintenance 2002 conditioned spaces

Fuel

Electricity $(\mathrm{kWh})$

existing

post-retrofit

difference

$\%$ change

Total (MBtu)

existing

post-retrofit

difference

$\%$ change
Energy

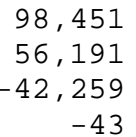

336
192
-144

$-43$

\section{Energy}

Intensity

(user units/1000ft2)

Intensity

(MBtu/1000ft2)

$$
\begin{array}{r}
20,527.7 \\
11,716.3 \\
-8,811.4 \\
-43
\end{array}
$$

\section{1}

40.

$-30.1$

$-43$
70.1
40.0
-30.1
-43

70.1
40.0
-30.1

$-43$
Dollars

$(2009)$ *

17,405

9,882

$-7,524$

17,405

9,882

$-7,524$

* Dollar values for electricity include both energy and demand components. 


\section{Appropriated Funding Energy Consumption by End Use}

Motors and miscellaneous equipment is the largest load in the building with 94,120 kWh/year, followed by lighting with 31 ,616 $\mathrm{kWh} /$ year.

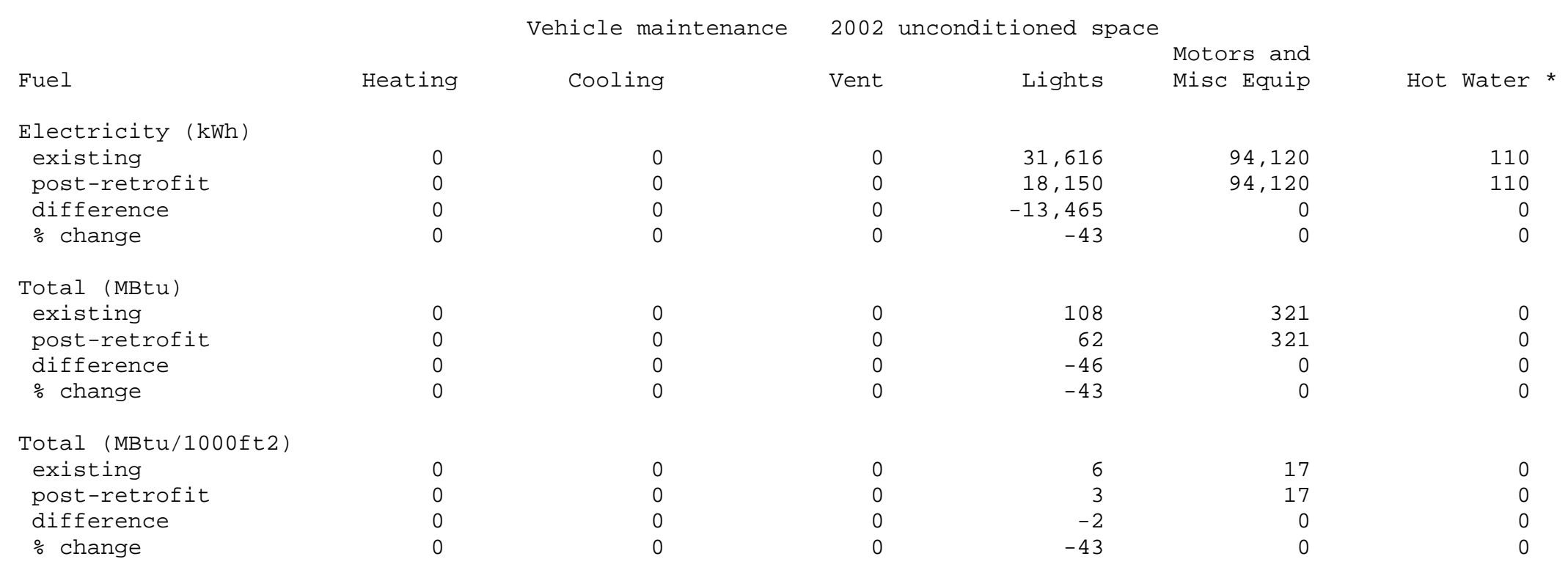

* Energy consumption values for both distributed and central SHW are reported for Hot water annual energy use. 
Space cooling is the largest load in the building with 59,237 kWh/year, followed by motors and miscellaneous equipment with 23,530 kWh/year.

Vehicle maintenance 2002 conditioned space

Fuel Heating
Electricity (kWh)
existing
post-retrofit
difference
$\%$ change
Total (MBtu)
existing
post-retrofit
difference
\% change

$\begin{array}{rr}\text { Cooling } & \text { Vent } \\ 59,237 & 6,969 \\ 25,090 & 2,917 \\ -34,147 & -4,052 \\ -58 & -58 \\ & \\ 202 & 24 \\ 86 & 1 \\ -117 & -14 \\ -58 & -58\end{array}$

$20 \odot 2$ conditioned space Motors and

Lights

Misc Equip
8,605
4,544
$-4,061$

$-47$

29
16
-14
29
16
-14
$-47$

$-58$

6
3
-3
-47

$-47$

23,530
23,530
0
0

80
80
0
0

17
17
0
0

Hot Water *

110
110
0
0


0
0
0
0


0
0
0
0

change

* Energy consumption values for both distributed and central SHW are reported for Hot Water annual energy use. 


\section{Appropriated Funding Emission Reduction}

The emission reductions from implemented the proposed retrofits are as follows:

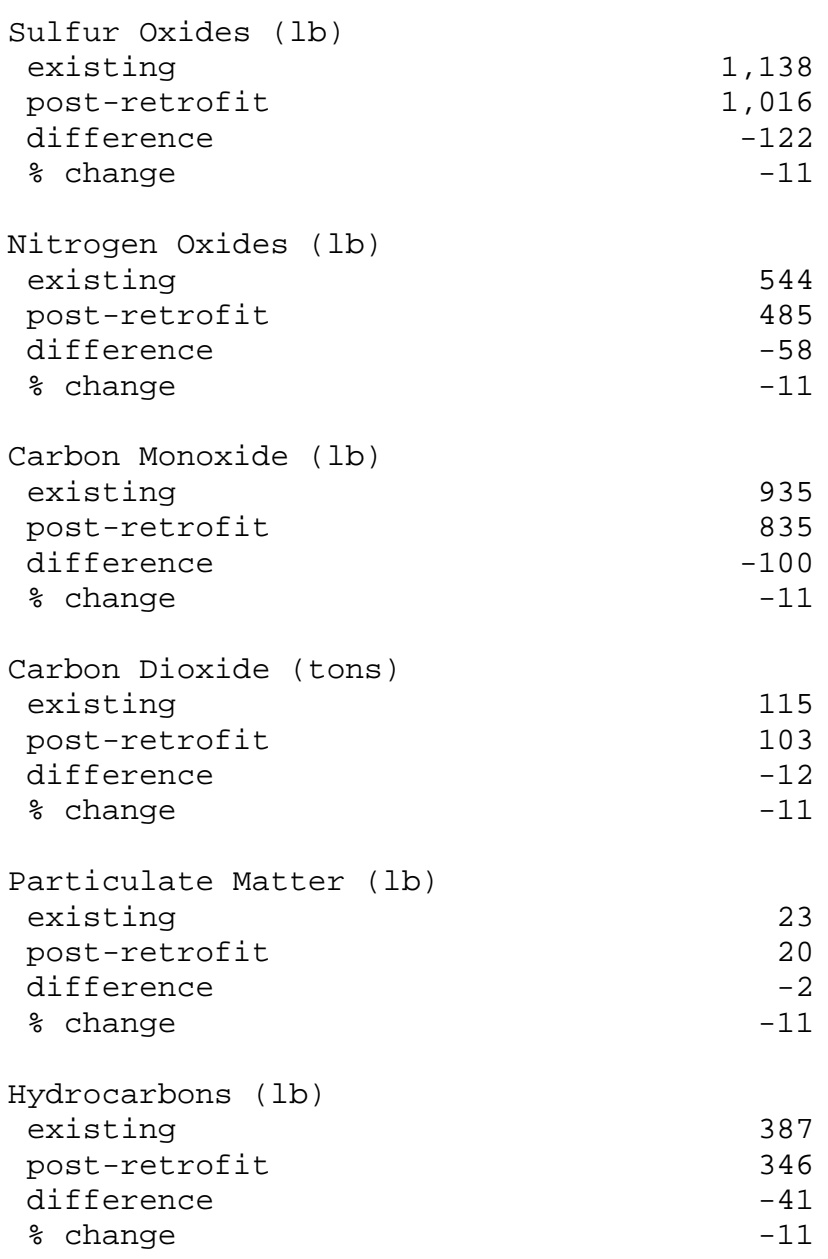


Sulfur oxides (lb)

existing

890

post-retrofit

508

difference

$-382$

$\%$ change

$-43$

Nitrogen 0xides (lb)

existing

post-retrofit

243

difference

$-183$

$\%$ change

$-43$

Carbon Monoxide (lb)

existing

post-retrofit

418

difference

$\%$ change

Carbon Dioxide (tons)

existing

post-retrofit

difference

51

$\%$ change

Particulate Matter (lb)

existing

post-retrofit

difference

$\%$ change

$-43$

Hydrocarbons (lb)

existing

difference -130

$\%$ change

$-43$ 


\section{Building 1713 Warehouse Building}

The following information identifies the cost-effective energy- and cost-reducing retrofit projects for building 1713 identified from the FEDS modeling and analysis. Key energy and economic results are presented for each cost-effective retrofit measure.

\section{Facility Description 1713}

1713 is a warehouse building built in 1944. 1713 is the main recycling center on base and has a small conditioned office space served by an electric package, or DX, unit. Building 1713 is 30,400 sf.

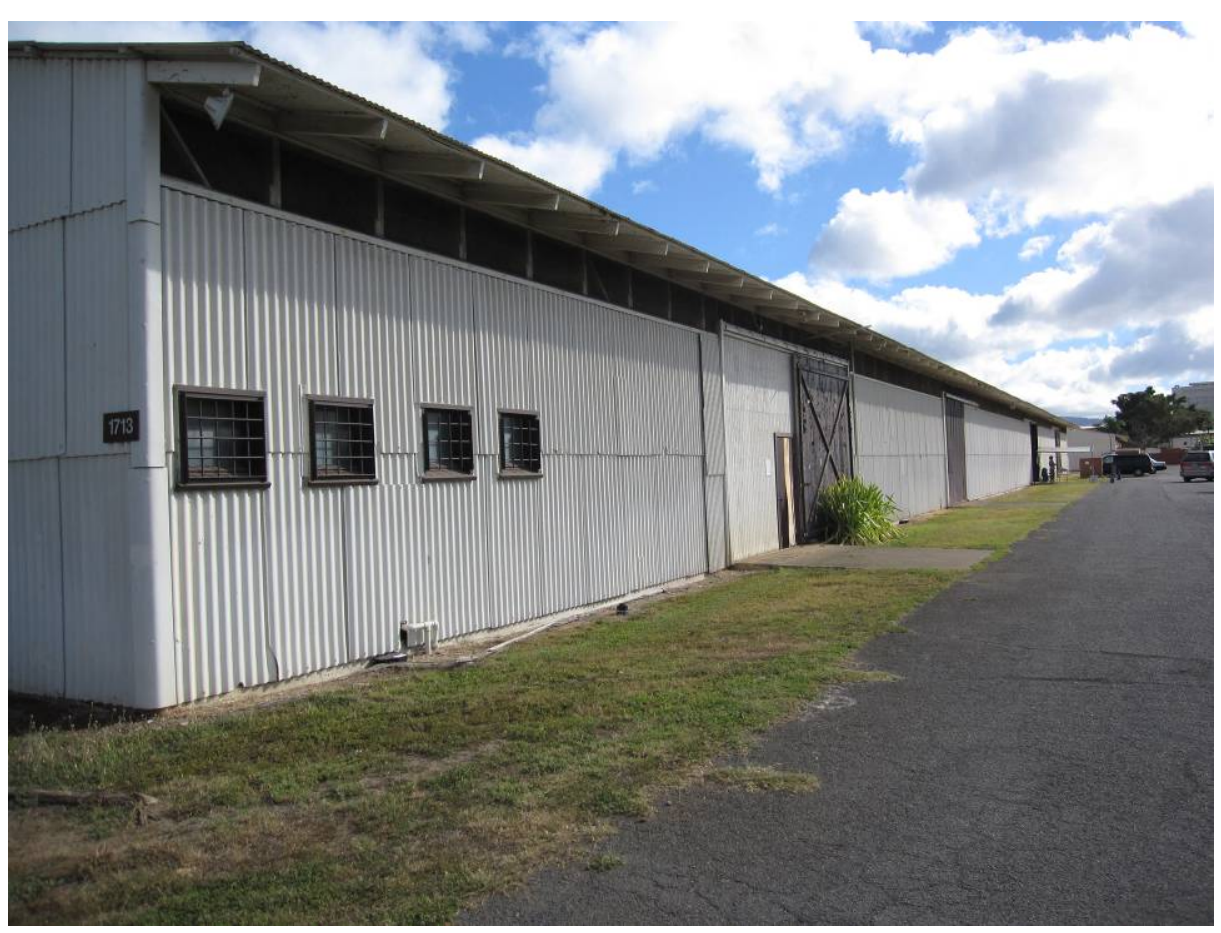

\section{Appropriated Funding Results}


A FEDS analysis using appropriated funding suggests replacing the lighting in the building in the conditioned and unconditioned spaces as well as increasing the interior insulation of the roof for the conditioned space only.

Appropriated funding FEDS results for building 1713 unconditioned space:

\begin{tabular}{|c|c|c|c|c|c|c|c|c|}
\hline Bldg. Set Description & $\begin{array}{l}\text { End } \\
\text { Use }\end{array}$ & Existing Technology & Retrofit Technology & $\begin{array}{l}\text { Energy } \\
\text { Savings } \\
\text { (MMBtu/yr) }\end{array}$ & $\begin{array}{l}\text { 1st year } \\
\text { savings } \\
\text { (\$/yr) }\end{array}$ & $\begin{array}{l}\text { Installed } \\
\text { Cost (\$) }\end{array}$ & $\begin{array}{l}\text { Net } \\
\text { Present } \\
\text { Value (\$) }\end{array}$ & SIR \\
\hline 1940 's storage & Lights & FL4: FL 1X4 2F40T12 STD2 & FL52: FL 1X4 2F32T8 ELC2 & 26 & 2,334 & 24,875 & 14,590 & 1.6 \\
\hline 1940's storage 1713 & Lights & EX6: EXIT - LED & $\begin{array}{l}\text { EX12: EXIT - } \\
\text { ELECTROLUMINESCENT PANEL } \\
\text { RETRO KIT }\end{array}$ & - & 51 & 311 & 572 & 2.8 \\
\hline
\end{tabular}

Appropriated funding FEDS results for building 1713 conditioned space:

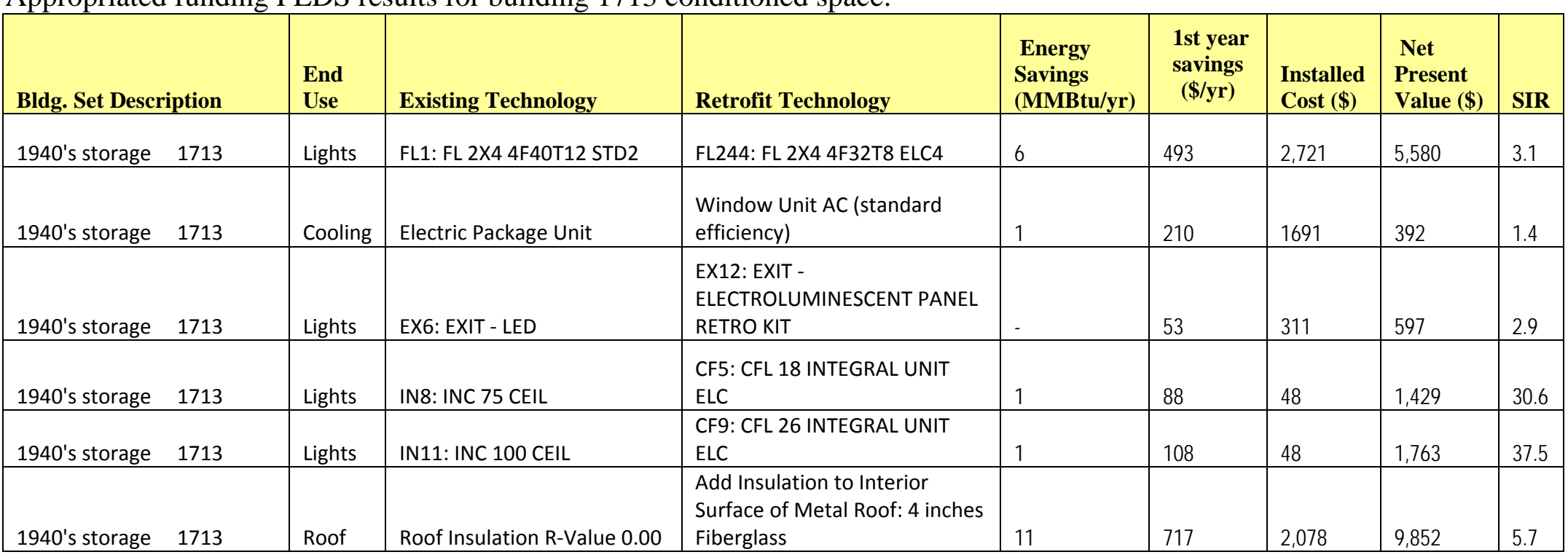




\section{Appropriated Funding Energy Consumption by Fuel Type}

The modeled energy consumption for the unconditioned space in the building for a typical year was 22,232 kwh before retrofits and 14,495 kwh after proposed retrofits are implemented. The energy use intensity goes from $2.5 \mathrm{MBtu} / \mathrm{Ksf}$ to $1.7 \mathrm{MBtu} / \mathrm{Ksf}$ after retrofits.

\begin{tabular}{|c|c|c|c|c|}
\hline & $1940^{\prime} s$ & 1713 uncondi & ned space & \\
\hline Fuel & Energy & $\begin{array}{c}\text { Energy } \\
\text { Intensity } \\
\text { (user units/1000ft2) }\end{array}$ & $\begin{array}{c}\text { Energy } \\
\text { Intensity } \\
\text { (MBtu/1000ft2) }\end{array}$ & $\begin{array}{l}\text { Dollars } \\
(2009) \text { * }\end{array}$ \\
\hline Electricity (kw & & & & \\
\hline existing & 22,232 & 746.3 & 2.5 & 3,941 \\
\hline post-retrofit & 14,495 & 486.5 & 1.7 & 2,550 \\
\hline difference & $-7,738$ & -259.7 & -0.9 & $-1,391$ \\
\hline$\%$ change & -35 & -35 & -35 & -35 \\
\hline Total (MBtu) & & & & \\
\hline existing & 76 & 2.5 & 2.5 & 3,941 \\
\hline post-retrofit & 49 & 1.7 & 1.7 & 2,550 \\
\hline difference & -26 & -0.9 & $-\odot .9$ & $-1,391$ \\
\hline$\%$ change & -35 & -35 & -35 & -35 \\
\hline
\end{tabular}

* Dollar values for electricity include both energy and demand components. 
The modeled energy consumption for the conditioned space in the building for a typical year was 10,421 kwh before retrofits and 3,445 kwh after proposed retrofits are implemented. The energy use intensity goes from $58.5 \mathrm{MBtu} / \mathrm{Ksf}$ to $19.3 \mathrm{MBtu} / \mathrm{Ksf}$ after retrofits.

\section{0's storage 1713 conditioned space}

Fuel

Electricity (kWh)

existing

post-retrofit

difference

$\%$ change

Total (MBtu)

existing

post-retrofit

difference

$\%$ change
Energy

10,421

3,445

$-6,976$

$-67$

36
12
-24
-67

Energy

Intensity

(user units/1000ft2)

$17,140.2$

$5,666.8$

$-11,473.4$

$-67$

58.5

19.3

$-39.2$

$-67$

$\begin{array}{ll}\text { Energy } & \\ \text { Intensity } & \text { Dollars } \\ \text { (MBtu/1000ft2) } & (2009)^{*}\end{array}$

58.5

19.3

$-39.2$

$-67$

58.5

19.3

19.3
-39.2

$-67$
1,842

606

$-67$

1,842

606

$-1,236$
-67

* Dollar values for electricity include both energy and demand components. 


\section{Appropriated Funding Energy Consumption by End Use}

Lighting is the largest load in the unconditioned space of the building with 21,755 kWh/year, followed by motors and miscellaneous equipment with $477 \mathrm{kWh} /$ year.

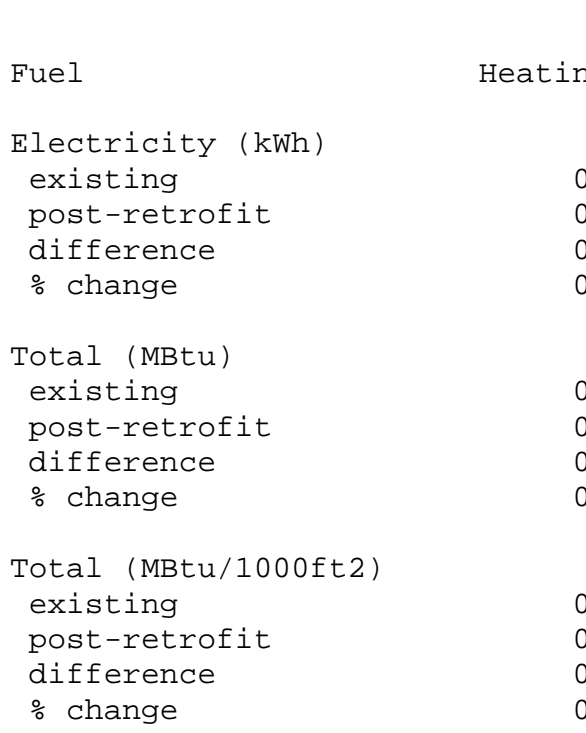

\section{0 's storage \\ 1713 unconditioned space}

Cooling

$\odot$

$\odot$

$\odot$

$\odot$

$\odot$

$\odot$

21,755
14,017
$-7,738$
-36

74
48
-26
-36

2
2
-1
-36

$0 \quad 14,017$

$\odot$

36

$\odot$

$-36$

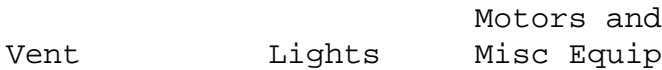

Hot Water

$\%$ change

477

477

$\odot$

$\odot$

\section{2}

2
0

$\odot$

0

$\odot$

$\odot$

0
0
0
0 
Space cooling is the largest load in the building with 5,318 kWh/year, followed by lighting with 4,901 kWh/year.

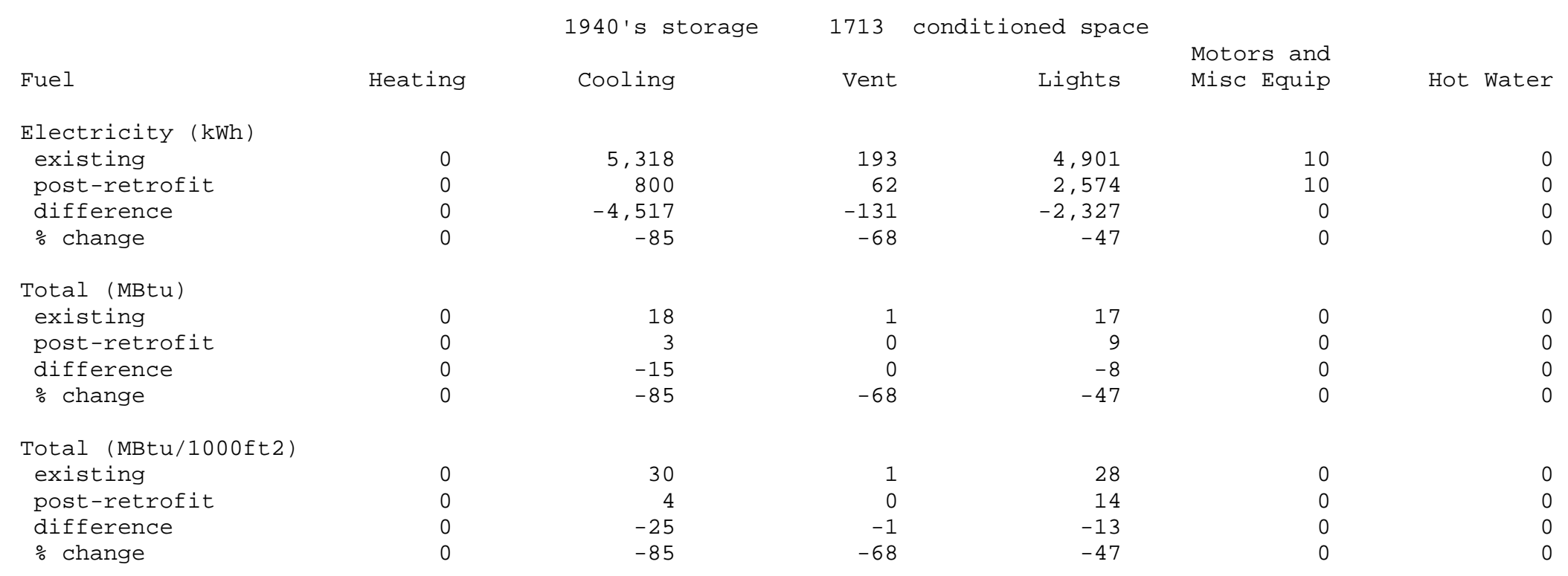




\section{Appropriated Funding Emission Reduction}

The emission reductions from implemented the proposed retrofits are as follows:

Sulfur oxides (lb)

existing

post-retrofit

201

difference

131

$\%$ change

$-35$

Nitrogen 0xides (lb)

existing

post-retrofit

difference

$\%$ change

Carbon Monoxide (lb)

existing

post-retrofit

difference

$\%$ change

165

108

-57
-35

Carbon Dioxide (tons)

existing

post-retrofit

difference

$2 \odot$

difference

13
-7

change

Particulate Matter (lb)

existing

post-retrofit

difference

$\%$ change

4
3
-1
-35

Hydrocarbons (lb)

existing

post-retrofit $\quad 45$

68

difference -24

$\%$ change

$-35$ 
Sulfur oxides (lb)

existing

post-retrofit

94

difference

31

$\%$ change

Nitrogen 0xides (lb)

existing

post-retrofit

45

difference

15

$\%$ change

$-67$

Carbon Monoxide (lb)

existing

post-retrofit

difference

$\%$ change

Carbon Dioxide (tons)

existing

post-retrofit

difference

10

$\%$ change

$-6$

$-67$

Particulate Matter (lb)

existing

post-retrofit

difference

2

$\%$ change

-1
-67

Hydrocarbons (lb)

existing

post-retrofit

difference

$\%$ change

11
-21

$-67$ 


\section{Building 2130 Corrosion Control Hangar}

The following information identifies the cost-effective energy- and cost-reducing retrofit projects for building 2130 identified from the FEDS modeling and analysis. Key energy and economic results are presented for each cost-effective retrofit measure.

\section{Facility Description 2130}

2130 is a corrosion control facility for aircraft built in 2008. Building 2130 cleans aircraft of corrosion causing agents and has a large ventilation system to aid its mission. Building 2130 is 56,734 sf.

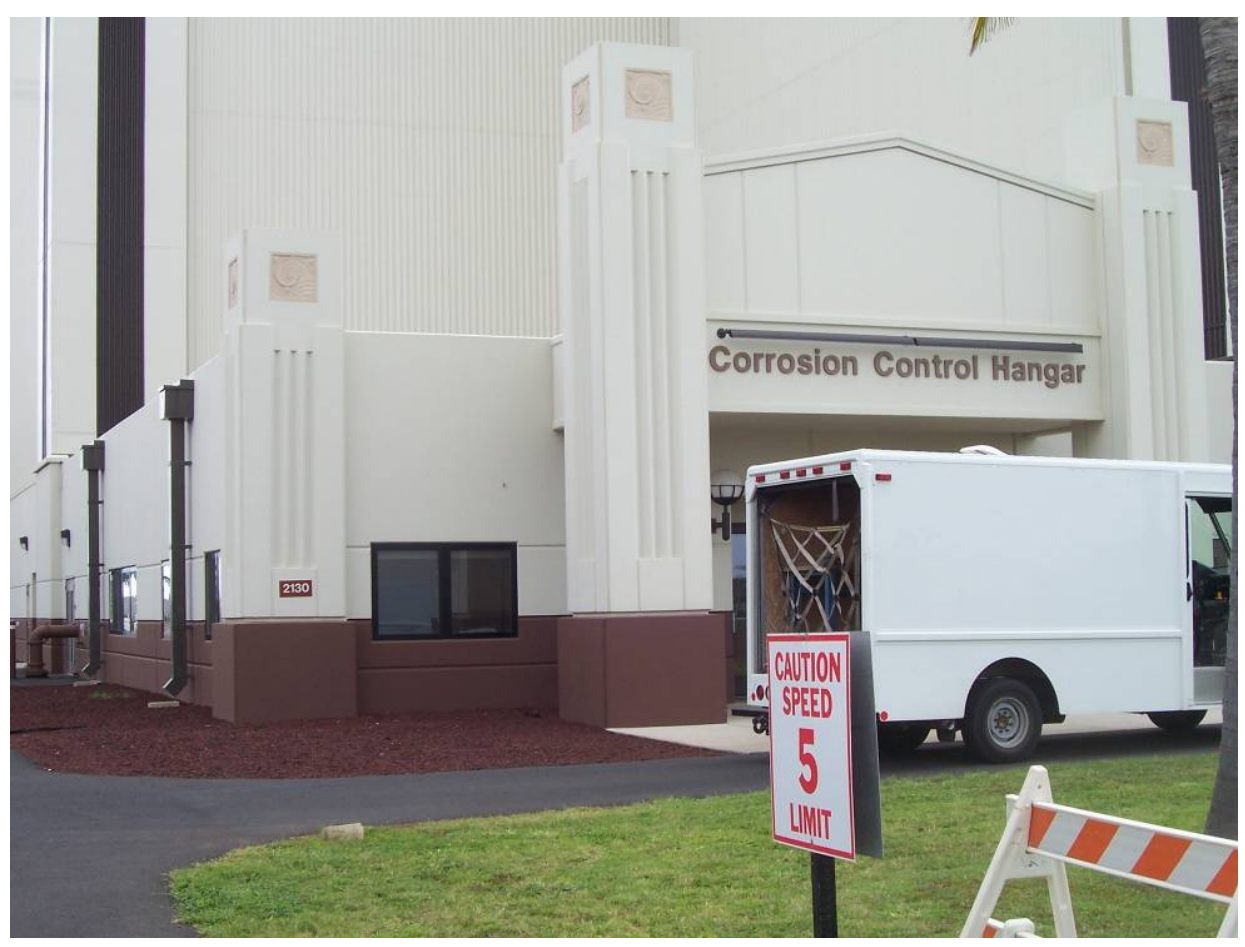




\section{Appropriated Funding Results}

A FEDS analysis using appropriated funding suggests replacing the lighting in the building . FEDS also suggests various upgrades to the hot water system for the unconditioned space. For the conditioned space FEDS suggests replacing the air cooled chiller with a very high efficiency water cooled chiller, insulating the hot water system and repacing some fo the lighting.

Appropriated funding FEDS results for building 2130 unconditioned space:

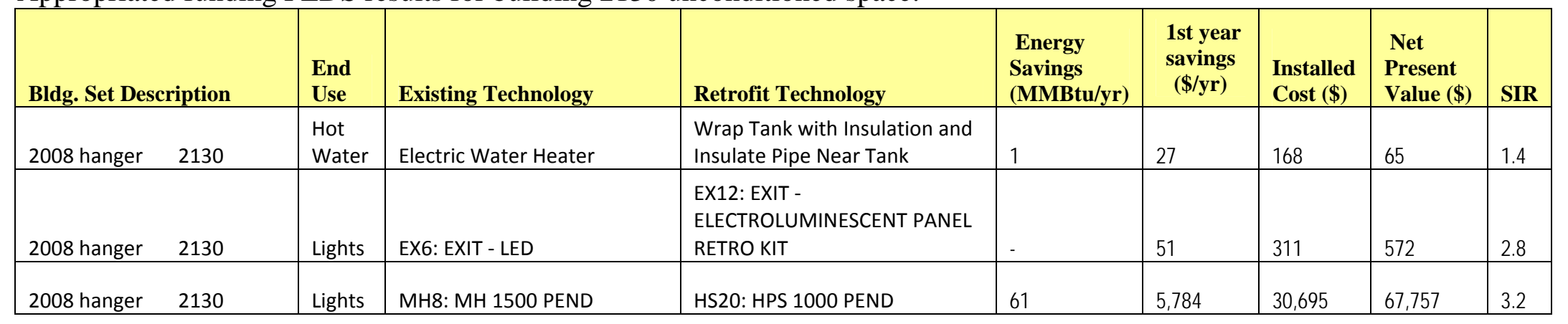


Appropriated funding FEDS results for building 2130 conditioned space:

\begin{tabular}{|c|c|c|c|c|c|c|c|c|}
\hline Bldg. Set Description & $\begin{array}{l}\text { End } \\
\text { Use }\end{array}$ & Existing Technology & Retrofit Technology & $\begin{array}{l}\text { Energy } \\
\text { Savings } \\
\text { (MMBtu/yr) }\end{array}$ & $\begin{array}{l}\text { 1st year } \\
\text { savings } \\
(\$ / y r)\end{array}$ & $\begin{array}{l}\text { Installed } \\
\text { Cost (\$) }\end{array}$ & $\begin{array}{l}\text { Net } \\
\text { Present } \\
\text { Value (\$) } \\
\end{array}$ & SIR \\
\hline $\begin{array}{l}2008 \text { hanger conditioned } \\
\text { space } 2130\end{array}$ & Cooling & $\begin{array}{l}\text { Electric Air-Cooled Chiller } \\
\{C 1\}\end{array}$ & $\begin{array}{l}\text { Water-Cooled Reciprocating } \\
\text { Electric Chiller (very high } \\
\text { efficiency) and Cooling Tower }\end{array}$ & 120 & 7,819 & 44,112 & 64,450 & 2.5 \\
\hline $\begin{array}{l}2008 \text { hanger conditioned } \\
\text { space } 2130\end{array}$ & $\begin{array}{l}\text { Hot } \\
\text { Water }\end{array}$ & Electric Water Heater & $\begin{array}{l}\text { Wrap Tank with Insulation and } \\
\text { Insulate Pipe Near Tank }\end{array}$ & 1 & 27 & 168 & 65 & 1.4 \\
\hline $\begin{array}{l}2008 \text { hanger conditioned } \\
\text { space } 2130\end{array}$ & Lights & EX6: EXIT - LED & $\begin{array}{l}\text { EX12: EXIT - } \\
\text { ELECTROLUMINESCENT PANEL } \\
\text { RETRO KIT }\end{array}$ & 1 & 107 & 627 & 1,229 & 3.0 \\
\hline $\begin{array}{l}2008 \text { hanger conditioned } \\
\text { space } 2130\end{array}$ & Lights & FL236: FL 2X4 3F32T8 ELC3 & $\begin{array}{l}\text { FL279: FL 2X4 2F32ST8 ELC2 } \\
\text { REF }\end{array}$ & 109 & 5,596 & 14,473 & 78,914 & 6.5 \\
\hline $\begin{array}{l}2008 \text { hanger conditioned } \\
\text { space } 2130\end{array}$ & Lights & FL51: FL 2X4 2F32T8 ELC2 & $\begin{array}{l}\text { FL303: FL 2X4 2F25ST8 ELC2 } \\
\text { REF }\end{array}$ & 67 & 3,263 & 19,435 & 34,889 & 2.8 \\
\hline
\end{tabular}




\section{Appropriated Funding Energy Consumption by Fuel Type}

The modeled energy consumption for the unconditioned space for a typical year was 194,224 kwh before retrofits and 176,113 kwh after proposed retrofits are implemented. The energy use intensity goes from $13.7 \mathrm{MBtu} / \mathrm{Ksf}$ to $12.5 \mathrm{MBtu} / \mathrm{Ksf}$ after retrofits.

2008 hanger unconditione space

2130

Fuel

Energy

194,224

176,113

$-18,111$
-9

post-retrofit

difference

$\%$ change

Total (MBtu)

existing

post-retrofit

difference

$\%$ change

$$
\begin{gathered}
\text { Energy } \\
\text { Intensity } \\
\text { (user units/1000ft2) }
\end{gathered}
$$

$$
\begin{array}{r}
4,027.4 \\
3,651.9 \\
-375.5 \\
-9
\end{array}
$$

663
601
-62
-9

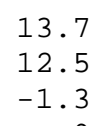

12.5
-1.3

$-9$
Energy

Intensity

(MBtu/1000ft2)

Dollars

$(2009)$ *

$\begin{array}{rr}13.7 & 34,438 \\ 12.5 & 31,233 \\ -1.3 & -3,205 \\ -9 & -9 \\ & \\ 13.7 & 34,438 \\ 12.5 & 31,233 \\ -1.3 & -3,205 \\ -9 & -9\end{array}$

$-9$

* Dollar values for electricity include both energy and demand components. 
The modeled energy consumption for the conditioned space for a typical year was 410,499 kwh before retrofits and 218,352 kwh after proposed retrofits are implemented. The energy use intensity goes from 164.6 MBtu/Ksf to $127.7 \mathrm{MBtu} / \mathrm{Ksf}$ after retrofits.

2008 hanger

conditioned space 2130

Fuel

Energy

Energy
Intensity
(user units/1000ft2)

$$
\begin{aligned}
& \text { Energy } \\
& \text { Intensity }
\end{aligned}
$$

(MBtu/1000ft2)

\section{Dollars}

$(2009)^{*}$

\section{Electricity (kWh)}

existing

post-retrofit

410,499

318,352

$48,237.3$

$37,409.2$

$-10,828.1$

164.6
127.7
-37.0

72,786

$-92,147$

$-22$

$-22$

-37.0
-22

459

$-16,328$

1,401
1,087
-314
-22

164.6

127.7

$-37.0$

164.6

127.7

$-37.0$

72,786

56,459

$-16,328$

22

* Dollar values for electricity include both energy and demand components. 


\section{Appropriated Funding Energy Consumption by End Use}

Motors and miscellaneous equipment is the largest load in the unconditioned space with 117,194 kWh/year, followed by lighting with 73,546 kWh/year.

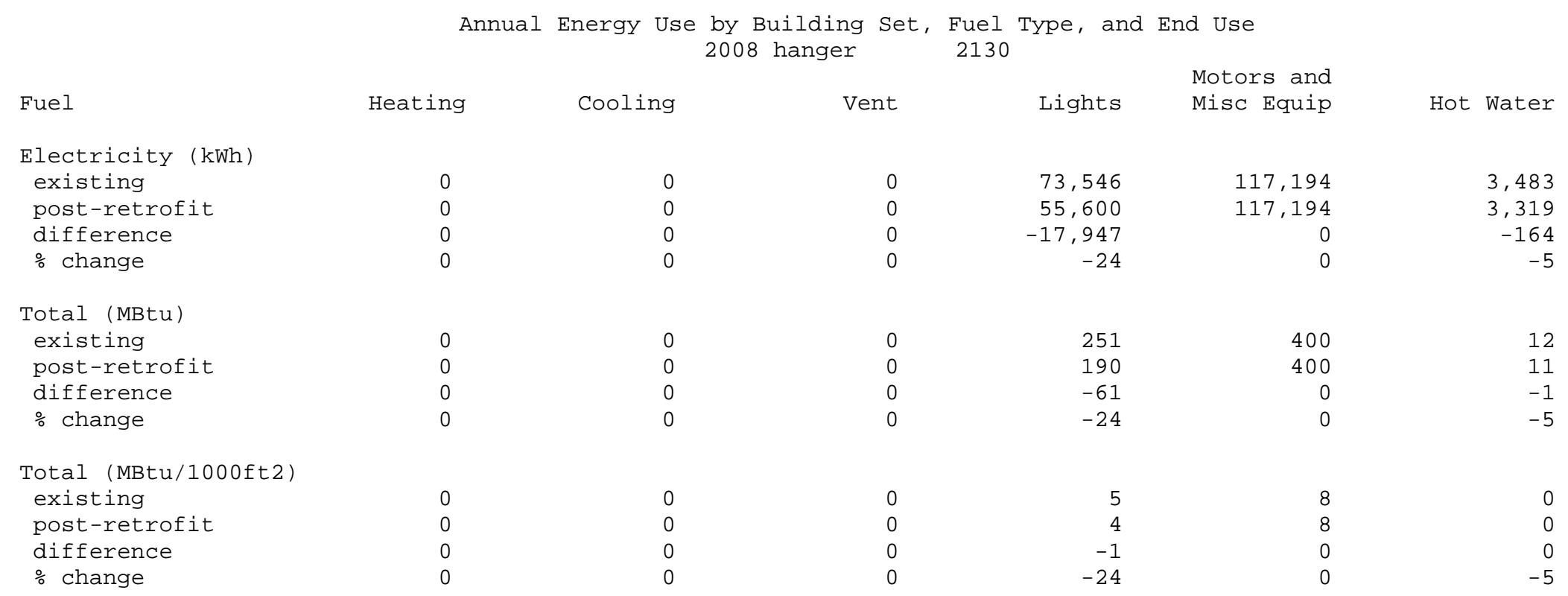


Lighting is the largest load in the conditioned space with 172,239 kWh/year, followed by space cooling with 120,545 kWh/year.

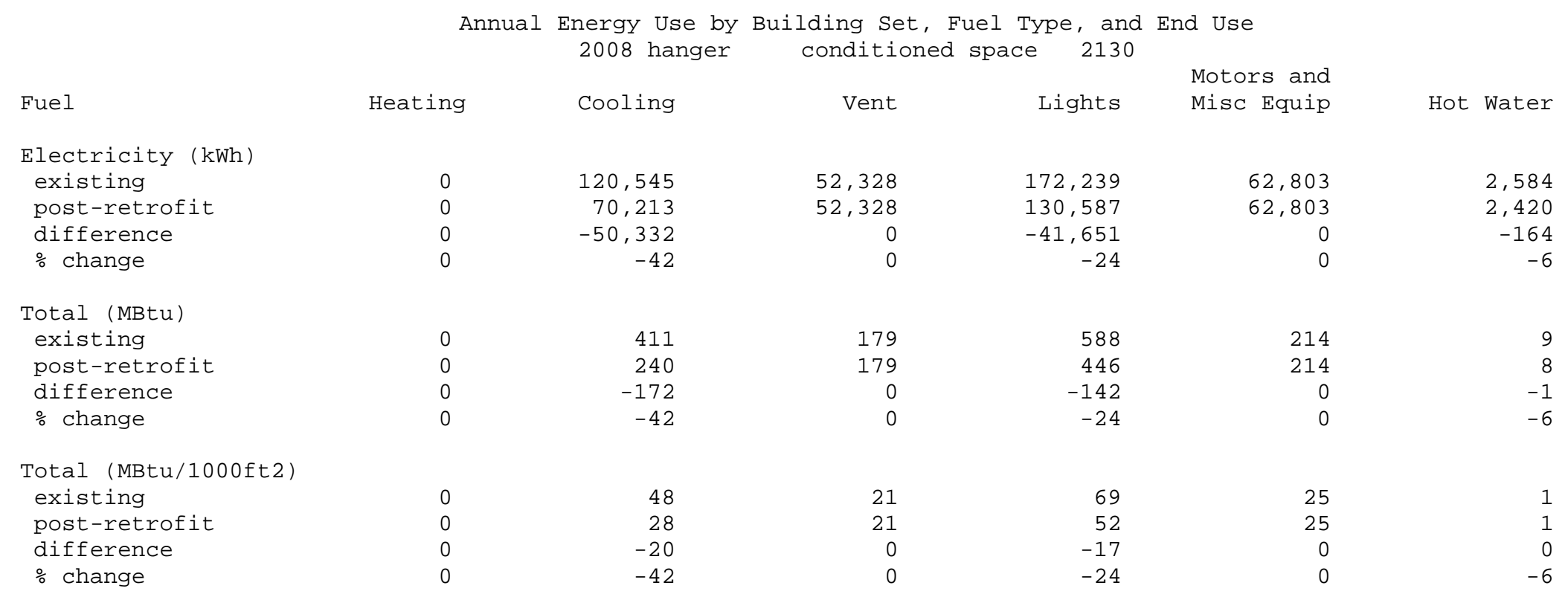




\section{Appropriated Funding Emission Reduction}

The emission reductions from implemented the proposed retrofits are as follows:

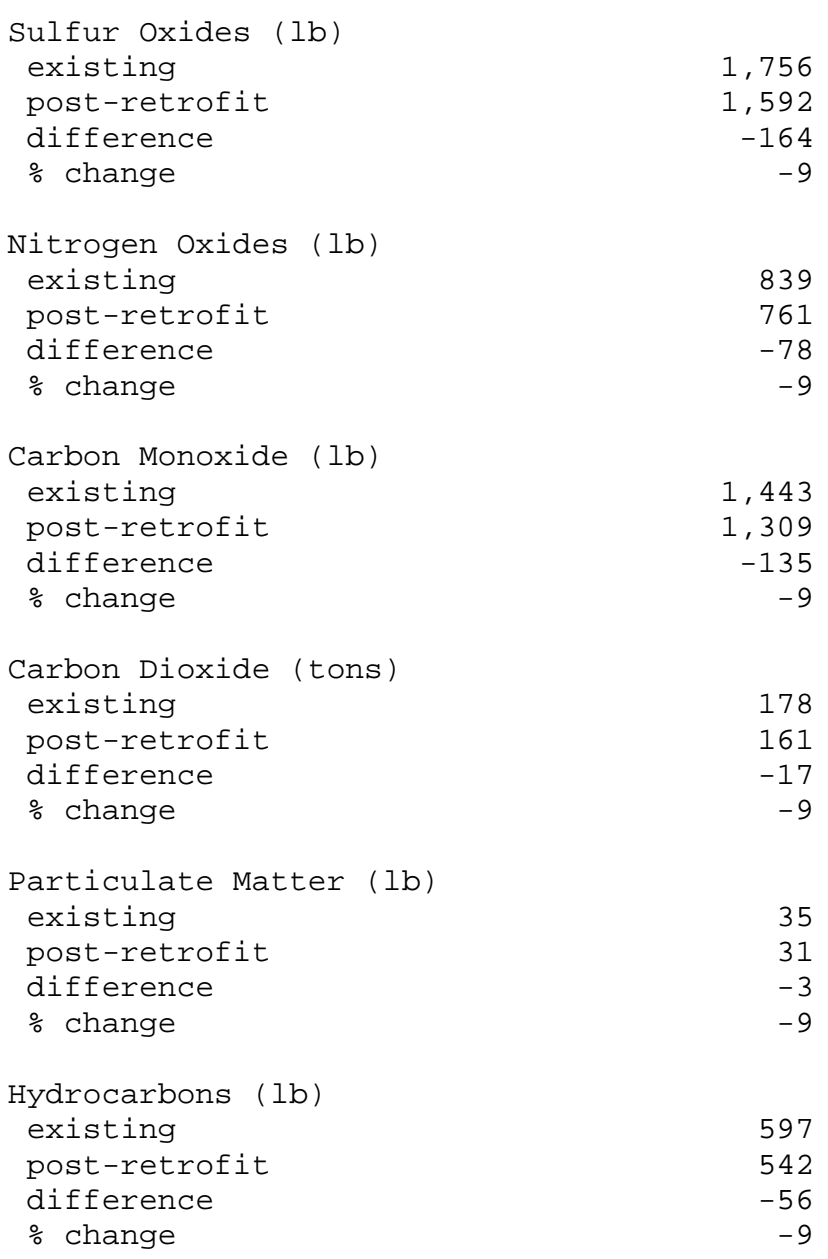


Sulfur oxides (lb)

existing

post-retrofit

3,711

difference

2,878
-833

$\%$ change

$-22$

Nitrogen 0xides (lb)

existing

post-retrofit

, 773

1,375

$-398$

difference

$-22$

Carbon Monoxide (lb)

existing

post-retrofit

difference

3,050

2,365

$-685$

\% change

$-22$

Carbon Dioxide (tons)

existing

post-retrofit

376

291

difference

$-84$

$\%$ change

$-22$

Particulate Matter (lb)

existing

post-retrofit

difference

$\%$ change

$-16$

Hydrocarbons (lb)

existing

post-retrofit

difference

1,262

979

$\%$ change

$-22$ 


\section{Building 1860 Dining Hall}

The following information identifies the cost-effective energy- and cost-reducing retrofit projects for building 1860 identified from the FEDS modeling and analysis. Key energy and economic results are presented for each cost-effective retrofit measure.

\section{Facility Description 1860}

1860 is a dining hall built in 1969. It is lighted mostly by T8's and is cooled by an electric air cooled chiller Building 1860 is 12,941 sf.

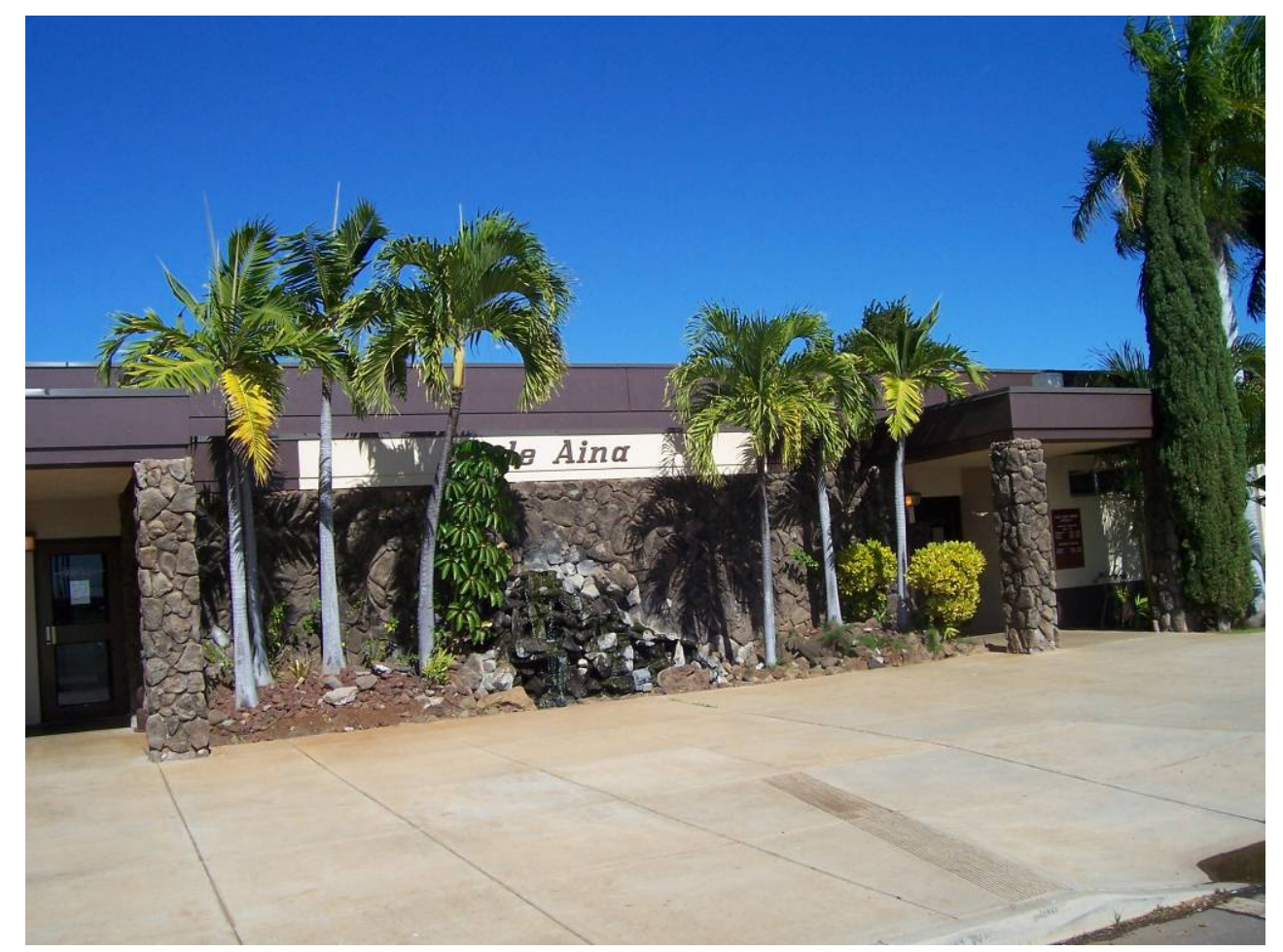




\section{Appropriated Funding Results}

A FEDS analysis using appropriated funding suggests replacing incandescent lights with CFL lights, replacing the air cooled chiller with a water cooled reciprocating chiller and replacing the propane water heater with a conventional distillate oil boiler.

Appropriated funding FEDS results for building 1860:

\begin{tabular}{|c|c|c|c|c|c|c|c|c|}
\hline Bldg. Set Description & $\begin{array}{l}\text { End } \\
\text { Use }\end{array}$ & Existing Technology & Retrofit Technology & $\begin{array}{l}\text { Energy } \\
\text { Savings } \\
\text { (MMBtu/yr) } \\
\end{array}$ & $\begin{array}{l}\text { 1st year } \\
\text { savings } \\
(\$ / y r)\end{array}$ & $\begin{array}{l}\text { Installed } \\
\text { Cost (\$) } \\
\end{array}$ & $\begin{array}{l}\text { Net } \\
\text { Present } \\
\text { Value (\$) } \\
\end{array}$ & SIR \\
\hline Dining Hall 1860 & Cooling & $\begin{array}{l}\text { Electric Air-Cooled Chiller } \\
\{C 1\}\end{array}$ & $\begin{array}{l}\text { Water-Cooled Reciprocating } \\
\text { Electric Chiller (very high } \\
\text { efficiency) and Cooling Tower }\end{array}$ & 174 & 10,591 & 57,337 & 11,791 & 2.6 \\
\hline Dining Hall 1860 & Lights & IN18: INC 25 WALL & CF14: CFL 5 + BLST UNIT & 12 & 642 & 4,340 & 6,348 & 2.5 \\
\hline Dining Hall 1860 & Lights & EX1: EXIT - INC $(2 \times 20)$ & $\begin{array}{l}\text { EX12: EXIT - } \\
\text { ELECTROLUMINESCENT PANEL } \\
\text { RETRO KIT }\end{array}$ & 13 & 869 & 621 & 14,011 & 23.6 \\
\hline Dining Hall 1860 & $\begin{array}{l}\text { Hot } \\
\text { Water }\end{array}$ & Other Fuels Central Boiler & $\begin{array}{l}\text { Conventional Distillate Oil } \\
\text { Boiler - 86.5\% Combustion } \\
\text { Efficiency, Wrap Tank }\end{array}$ & 240 & 7,636 & 22,413 & 163,808 & 9.0 \\
\hline Dining Hall 1860 & Lights & EX1: EXIT - INC (2x20) & $\begin{array}{l}\text { EX12: EXIT - } \\
\text { ELECTROLUMINESCENT PANEL } \\
\text { RETRO KIT }\end{array}$ & 14 & 927 & 621 & 14,985 & 25.1 \\
\hline Dining Hall 1860 & $\begin{array}{l}\text { Hot } \\
\text { Water }\end{array}$ & Other Fuels Central Boiler & $\begin{array}{l}\text { Conventional Distillate Oil } \\
\text { Boiler - } 83 \% \text { Combustion } \\
\text { Efficiency, Wrap Tank }\end{array}$ & 230 & 7,536 & 15,786 & 164,000 & 12.4 \\
\hline Dining Hall 1860 & Window & $\begin{array}{l}\text { Metal Frame Single Pane } \\
\text { Window }\end{array}$ & $\begin{array}{l}\text { Install Aluminum Frame } \\
\text { Double Pane Super Low-e } \\
\text { Window }\end{array}$ & 26 & 1,607 & 23,991 & 2,756 & 1.1 \\
\hline
\end{tabular}




\section{Appropriated Funding Energy Consumption by Fuel Type}

The modeled energy consumption for a typical year was 485,332 kwh before retrofits and 413,895 kwh after proposed retrofits are implemented. The energy use intensity goes from 203.1 MBtu/Ksf to 148.0 MBtu/Ksf after retrofits.

\begin{tabular}{|c|c|c|c|c|}
\hline & & Dining Hall 1860 & & \\
\hline Fuel & Energy & $\begin{array}{c}\text { Energy } \\
\text { Intensity } \\
\text { (user units/1000ft2) }\end{array}$ & $\begin{array}{c}\text { Energy } \\
\text { Intensity } \\
\text { (MBtu/1000ft2) }\end{array}$ & $\begin{array}{l}\text { Dollars } \\
(2009) \text { * }\end{array}$ \\
\hline Electricity (kh & & & & \\
\hline existing & 485,332 & $37,503.4$ & 128.0 & 86,015 \\
\hline post-retrofit & 413,895 & $31,983.2$ & 109.1 & 72,819 \\
\hline difference & $-71,437$ & $-5,520.2$ & -18.8 & $-13,196$ \\
\hline$\%$ change & -14 & -14 & -14 & -15.3 \\
\hline Distillate 0il & & & & \\
\hline existing & $\odot$ & 0.0 & $\odot .0$ & $\odot$ \\
\hline post-retrofit & 2,222 & 171.7 & 171.7 & 11,333 \\
\hline difference & 2,222 & 171.7 & 171.7 & 11,333 \\
\hline$\%$ change & $\mathrm{n} / \mathrm{a}$ & $\mathrm{n} / \mathrm{a}$ & $\mathrm{n} / \mathrm{a}$ & $\mathrm{n} / \mathrm{a}$ \\
\hline other Fuels (MB & & & & \\
\hline existing & 971 & 75.0 & 75.0 & 31,223 \\
\hline post-retrofit & 116 & $15 . \odot$ & 15.0 & 6,225 \\
\hline difference & -408 & -52.5 & -52.5 & $-13,111$ \\
\hline$\%$ change & -78 & -78 & -78 & -78 \\
\hline Total (MBtu) & & & & \\
\hline existing & 2,628 & 203.1 & 203.1 & 117,208 \\
\hline post-retrofit & 1,915 & 148.0 & 148.0 & 90,377 \\
\hline difference & -443 & -55.1 & -55.1 & $-26,831$ \\
\hline$\%$ change & -27 & -27 & -27 & -23 \\
\hline
\end{tabular}

* Dollar values for electricity include both energy and demand components. 


\section{Appropriated Funding Energy Consumption by End Use}

Space cooling is the largest load in the building with 221,654 kWh/year, followed by motors and miscellaneous equipment with $160,038 \mathrm{kWh} /$ year.

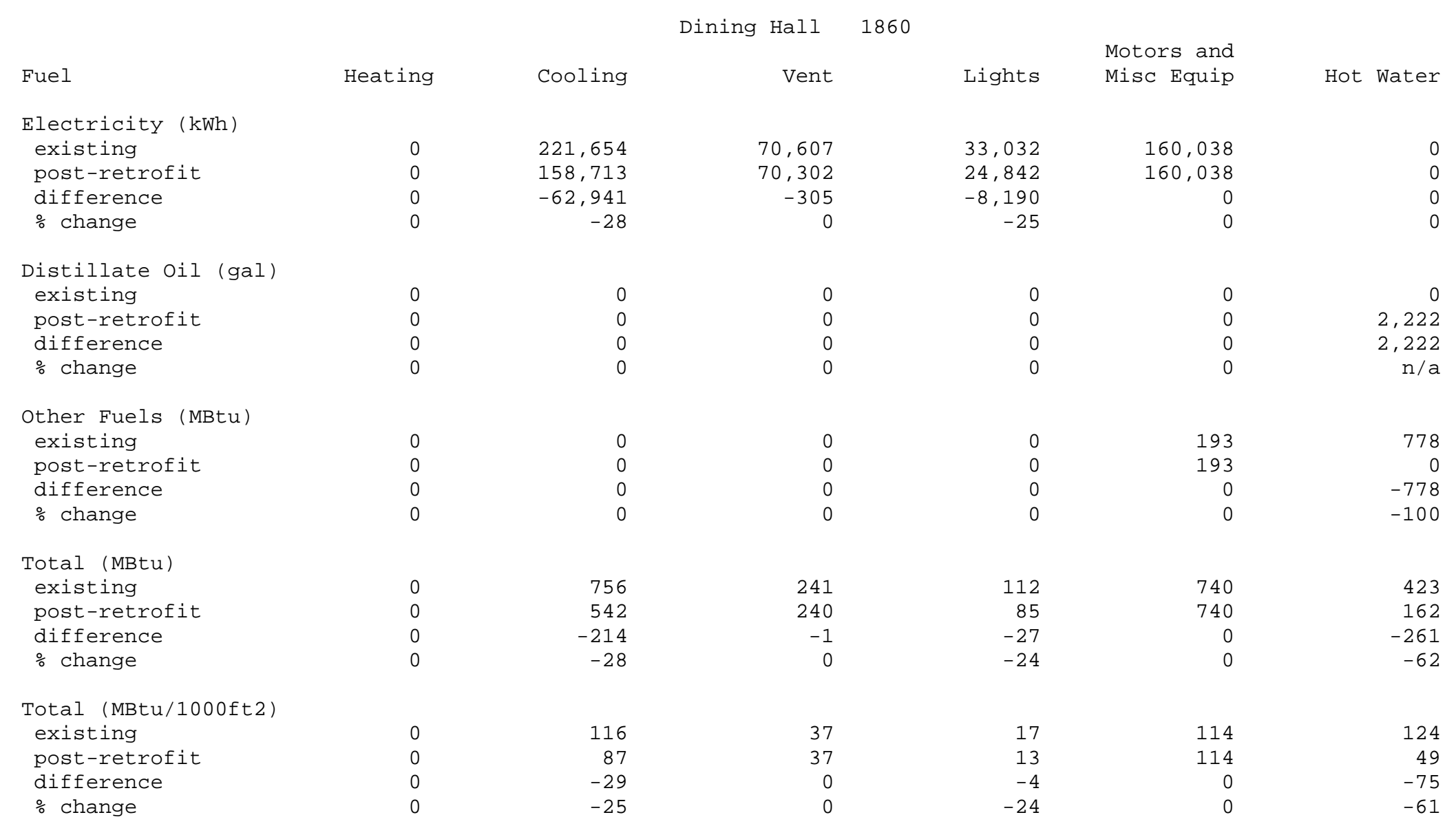




\section{Appropriated Funding Emission Reduction}

The emission reductions from implemented the proposed retrofits are as follows:

Dining Hall 1860

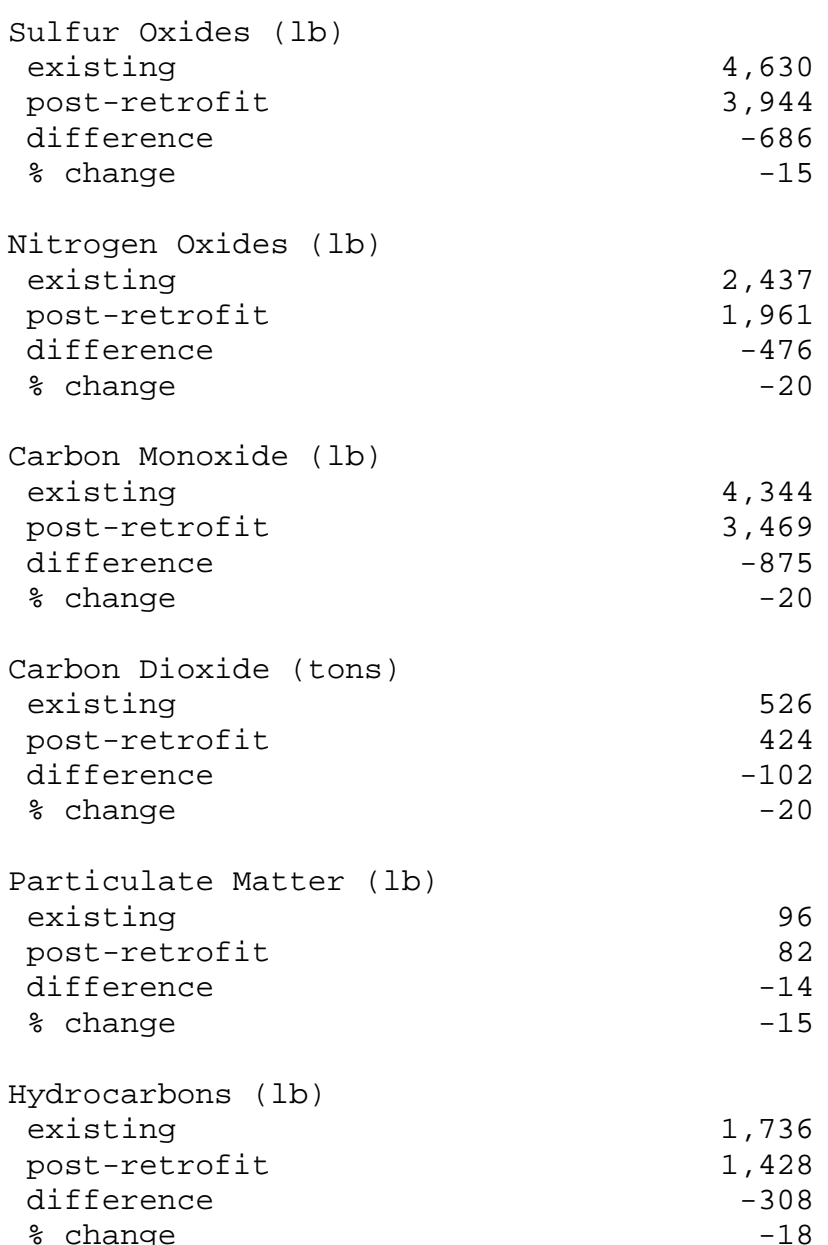




\section{Building 1804 Dining Facility}

The following information identifies the cost-effective energy- and cost-reducing retrofit projects for building 1804 identified from the FEDS modeling and analysis. Key energy and economic results are presented for each cost-effective retrofit measure.

\section{Facility Description 1804}

1804 is an open mess facility built in 2003. Building 1804 has incandescent and 32W T8 lilghts, an electric air cooled chiller and little to no insulation in its building envelope. Building 1804 is 27,579 sf.

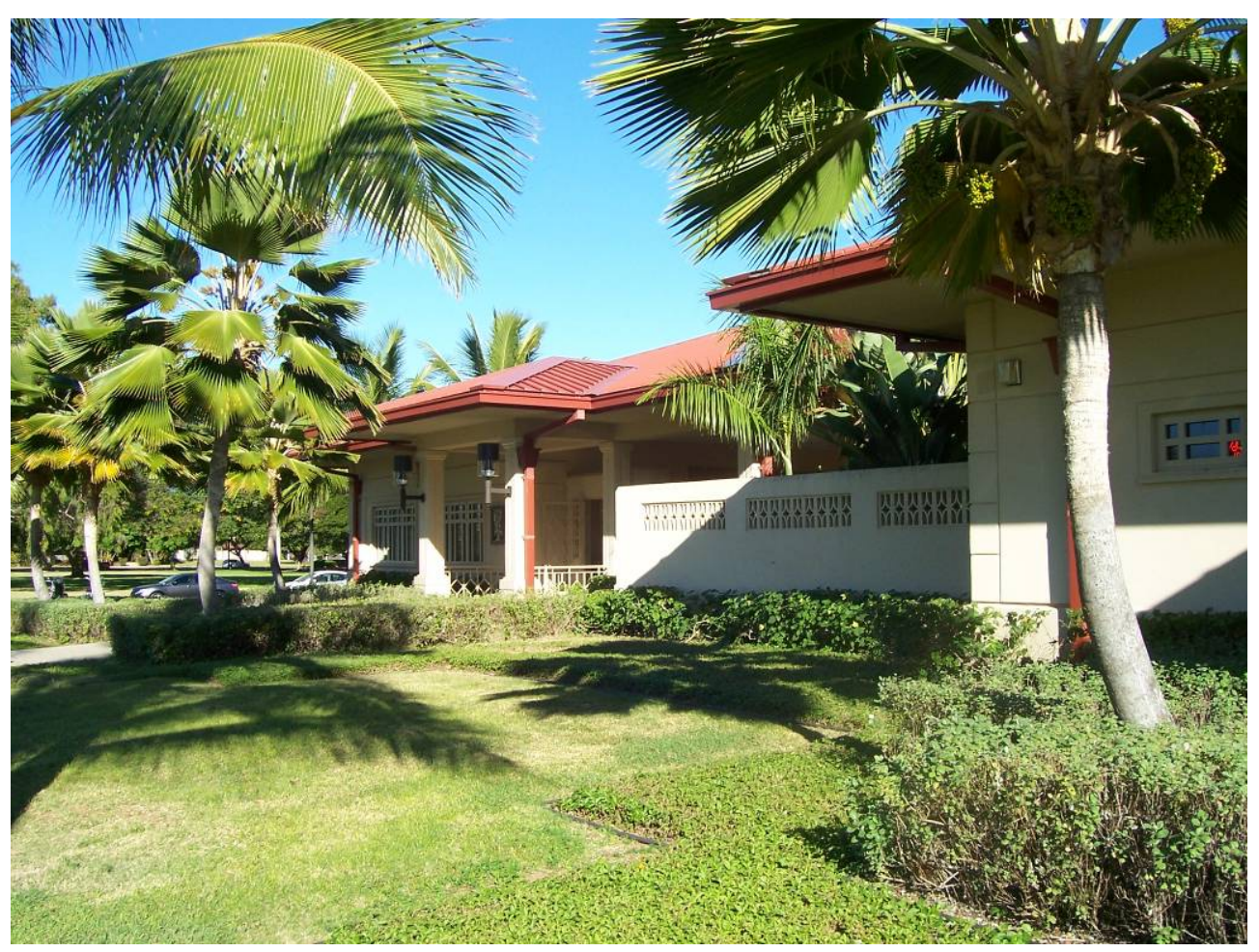




\section{Appropriated Funding Results}

A FEDS analysis using appropriated funding suggests replacing the air cooled chiller with a very high efficiency air cooled chiller. Incandescent lights are suggested to be replaced by CFL lights, FEDS suggests increasing the insulation on the hot water tank and increasing insulation in the suspended ceiling.

Appropriated funding FEDS results for building 1804:

\begin{tabular}{|c|c|c|c|c|c|c|c|c|c|}
\hline \multicolumn{2}{|c|}{ Bldg. Set Description } & $\begin{array}{l}\text { End } \\
\text { Use }\end{array}$ & Existing Technology & Retrofit Technology & $\begin{array}{l}\text { Energy } \\
\text { Savings } \\
\text { (MMBtu/yr) }\end{array}$ & $\begin{array}{c}\text { 1st year } \\
\text { savings } \\
(\$ / y r)\end{array}$ & $\begin{array}{l}\text { Installed } \\
\text { Cost (\$) }\end{array}$ & $\begin{array}{l}\text { Net } \\
\text { Present } \\
\text { Value (\$) } \\
\end{array}$ & SIR \\
\hline Dining & 1804 & Cooling & $\begin{array}{l}\text { Electric Air-Cooled Chiller } \\
\{\mathrm{C} 1\}\end{array}$ & $\begin{array}{l}\text { Air-Cooled Electric Chiller (very } \\
\text { high efficiency) }\end{array}$ & 142 & 60,113 & 304,293 & 333,778 & 2.9 \\
\hline Dining & 1804 & Lights & EX1: EXIT - INC $(2 \times 20)$ & $\begin{array}{l}\text { EX12: EXIT - } \\
\text { ELECTROLUMINESCENT PANEL } \\
\text { RETRO KIT }\end{array}$ & 143 & 9,245 & 6,212 & 149,431 & 25.1 \\
\hline Dining & 1804 & Lights & IN25: INC 75 WALL & $\begin{array}{l}\text { CF5: CFL } 18 \text { INTEGRAL UNIT } \\
\text { ELC }\end{array}$ & 312 & 19,296 & 8,978 & 315,418 & 36.1 \\
\hline Dining & 1804 & Lights & FL38: FL 2X4 3F32T8 EEF1,2 & $\begin{array}{l}\text { FL304: FL 2X4 3F25ST8 ELC3 } \\
\text { REF }\end{array}$ & 22 & 1,598 & 12,941 & 14,068 & 2.1 \\
\hline Dining & 1804 & $\begin{array}{l}\text { Hot } \\
\text { Water }\end{array}$ & Other Fuels Water Heater & Wrap Tank with Insulation & 45 & 1,457 & 2,138 & 4,640 & 3.2 \\
\hline Dining & 1804 & Roof & Roof Insulation R-Value 8.90 & $\begin{array}{l}\text { Suspended Ceiling: Increase } \\
\text { Insulation by R-19 }\end{array}$ & 64 & 4,362 & 47,492 & 25,081 & 1.5 \\
\hline
\end{tabular}




\section{Appropriated Funding Energy Consumption by Fuel Type}

The modeled energy consumption for a typical year was 1,001,869 kwh before retrofits and 795,914 kwh after proposed retrofits are implemented. The modeled other fuels (propane) consumption for a typical year was 881 gallons before retrofits and 836 gallons after proposed retrofits are implemented. The energy use intensity goes from $155.9 \mathrm{MBtu} / \mathrm{Ksf}$ to $128.8 \mathrm{MBtu} / \mathrm{Ksf}$ after retrofits.

\begin{tabular}{|c|c|c|c|c|}
\hline \multirow[b]{2}{*}{ Fuel } & \multicolumn{2}{|r|}{ Dining } & \multirow[b]{2}{*}{$\begin{array}{c}\text { Energy } \\
\text { Intensity } \\
\text { (MBtu/1000ft2) }\end{array}$} & \multirow[b]{2}{*}{$\begin{array}{l}\text { Dollars } \\
(2009)\end{array}$} \\
\hline & Energy & $\begin{array}{c}\text { Energy } \\
\text { Intensity } \\
\text { (user units/1000ft2) }\end{array}$ & & \\
\hline \multicolumn{5}{|c|}{ Electricity (kWh) } \\
\hline existing & 1, $\odot \odot 1,869$ & $36,327.2$ & 124.0 & 177,601 \\
\hline post-retrofit & 795,914 & $28,859.4$ & 98.5 & 140,030 \\
\hline difference & $-205,955$ & $-7,467.8$ & -25.5 & $-37,571$ \\
\hline$\%$ change & -21 & -21 & -21 & -21 \\
\hline \multicolumn{5}{|c|}{ other Fuels (MBtu) } \\
\hline existing & 881 & 32.0 & 32.0 & 28,333 \\
\hline post-retrofit & 836 & 30.3 & 30.3 & 26,875 \\
\hline difference & -45 & -1.6 & -1.6 & $-1,457$ \\
\hline$\%$ change & -5 & -5 & -5 & -5 \\
\hline \multicolumn{5}{|l|}{ Total (MBtu) } \\
\hline existing & 4,301 & 155.9 & 155.9 & 205,934 \\
\hline post-retrofit & 3,552 & 128.8 & 128.8 & 166,906 \\
\hline difference & -748 & -27.1 & -27.1 & $-39,028$ \\
\hline$\%$ change & -17 & -17 & -17 & -19 \\
\hline
\end{tabular}

* Dollar values for electricity include both energy and demand components. 


\section{Appropriated Funding Energy Consumption by End Use}

Space cooling is the largest load in the building with $387,047 \mathrm{kWh} /$ year, followed by motors and miscellaneous equipment with $316,839 \mathrm{kWh} /$ year.

\begin{tabular}{|c|c|c|c|c|c|c|}
\hline Fuel & Heating & Cooling & Vent & Lights & $\begin{array}{l}\text { Motors and } \\
\text { Misc Equip }\end{array}$ & Hot Water \\
\hline \multicolumn{7}{|c|}{ Electricity (kWh) } \\
\hline existing & $\odot$ & 387,047 & 100,190 & 197,794 & 316,839 & $\odot$ \\
\hline post-retrofit & $\odot$ & 287,902 & 86,126 & 105,048 & 316,839 & $\odot$ \\
\hline difference & $\odot$ & $-99,145$ & $-14,063$ & $-92,746$ & 0 & $\odot$ \\
\hline$\%$ change & $\odot$ & -26 & -14 & -47 & $\odot$ & $\odot$ \\
\hline \multicolumn{7}{|c|}{ Other Fuels (MBtu) } \\
\hline existing & $\odot$ & $\odot$ & $\odot$ & $\odot$ & 725 & 156 \\
\hline post-retrofit & $\odot$ & $\odot$ & $\odot$ & $\odot$ & 725 & 111 \\
\hline difference & $\odot$ & $\odot$ & $\odot$ & $\odot$ & 0 & -45 \\
\hline$\%$ change & $\odot$ & $\odot$ & $\odot$ & $\odot$ & $\odot$ & -29 \\
\hline \multicolumn{7}{|l|}{ Total (MBtu) } \\
\hline existing & $\odot$ & 1,321 & 342 & 675 & 1,806 & 156 \\
\hline post-retrofit & $\odot$ & 983 & 294 & 359 & 1,806 & 111 \\
\hline difference & 0 & -338 & -48 & -317 & 0 & $\begin{array}{l} + \pm 1 \\
-45\end{array}$ \\
\hline$\%$ change & $\odot$ & -26 & -14 & -47 & $\odot$ & -29 \\
\hline \multicolumn{7}{|c|}{ Total (MBtu/1000ft2) } \\
\hline existing & $\odot$ & 48 & 12 & 24 & 66 & 6 \\
\hline post-retrofit & $\odot$ & 36 & 11 & 13 & 66 & 4 \\
\hline difference & $\odot$ & -12 & -2 & -11 & 0 & -2 \\
\hline$\%$ change & $\odot$ & -26 & -14 & -47 & $\odot$ & -29 \\
\hline
\end{tabular}




\section{Appropriated Funding Emission Reduction}

The emission reductions from implemented the proposed retrofits are as follows:

Dining

1804

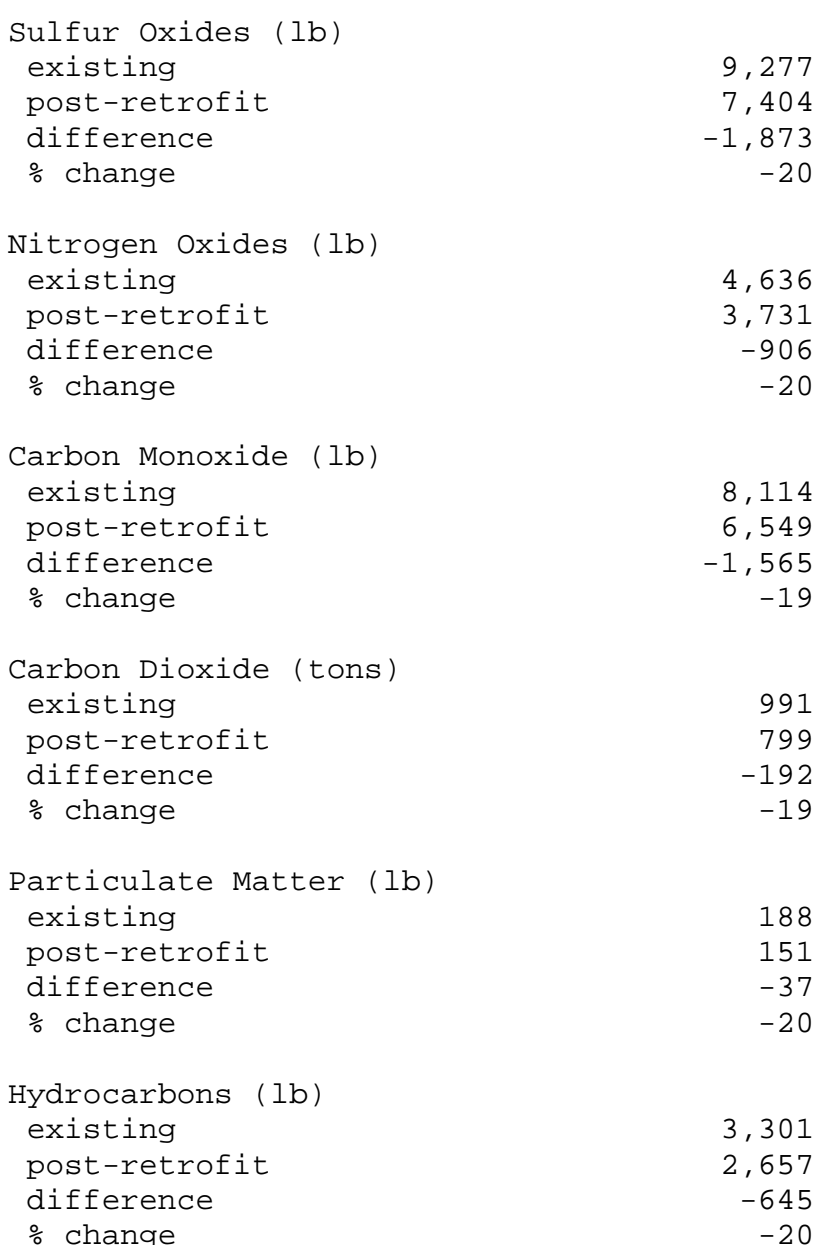




\section{Building 594 Lavatory Building}

The following information identifies the cost-effective energy- and cost-reducing retrofit projects for building 594 identified from the FEDS modeling and analysis. Key energy and economic results are presented for each cost-effective retrofit measure.

\section{Facility Description 594}

594 is a lavatory by the baseball fields built in 1977. Building 594 is not cooled and has very little lighting. Building 594 is 293 sf.

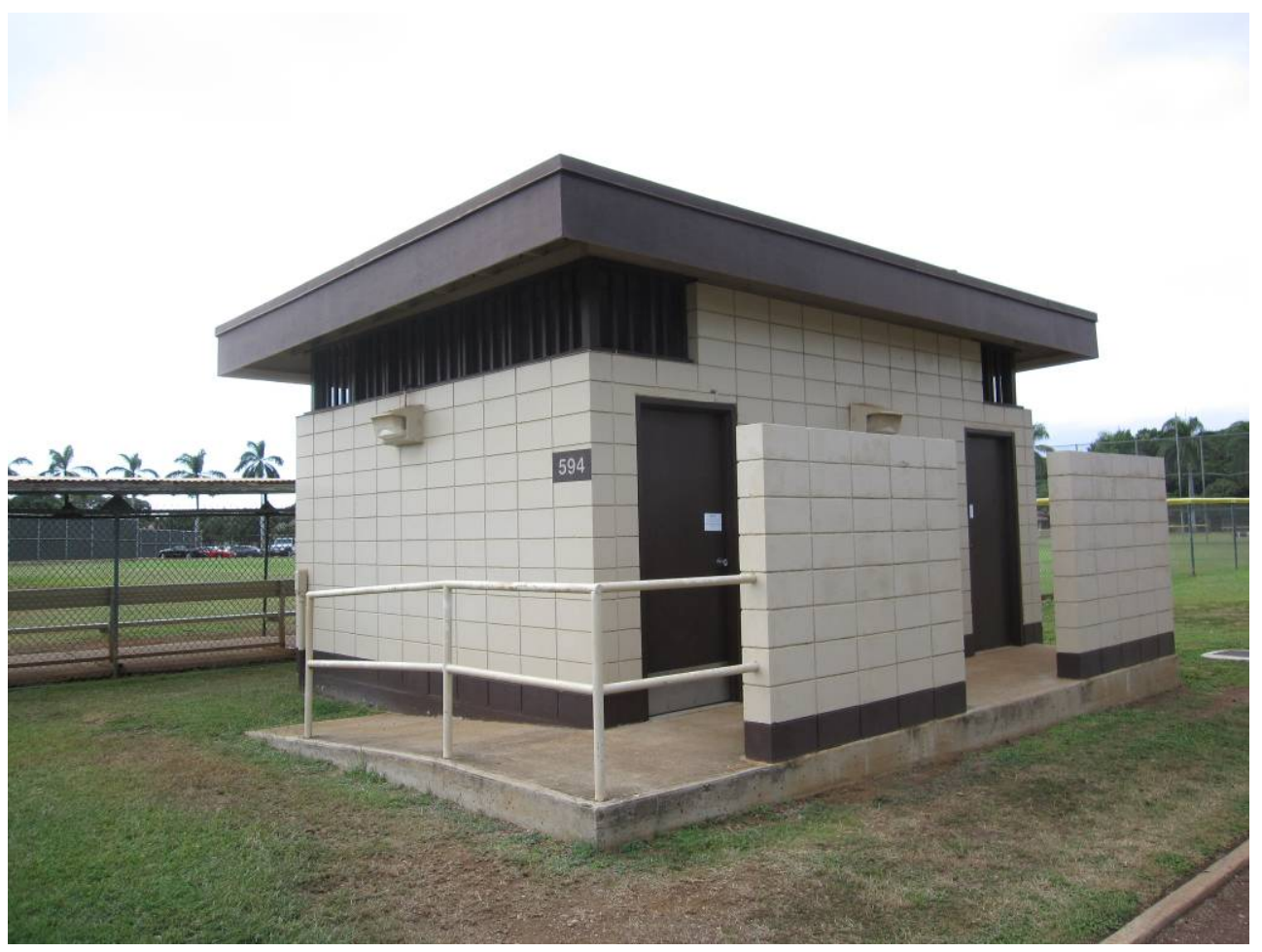




\section{Appropriated Funding Results}

FEDS did not find any life cycle cost effective retrofits using appropriated funding.

\section{Appropriated Funding Energy Consumption by Fuel Type}

The modeled energy consumption for a typical year was 2,429 kwh before retrofits and 1,040 kwh after proposed retrofits are implemented. The energy use intensity goes from $34.9 \mathrm{MBtu} / \mathrm{Ksf}$ to $18.7 \mathrm{MBtu} / \mathrm{Ksf}$ after retrofits.

\begin{tabular}{|c|c|c|c|c|}
\hline \multirow[b]{2}{*}{ Fuel } & \multicolumn{2}{|c|}{ sanitary latrines/small storag } & \multirow{2}{*}{$\begin{array}{c}594 \\
\\
\text { Energy } \\
\text { Intensity } \\
\text { (MBtu/1000ft2) }\end{array}$} & \multirow[b]{2}{*}{$\begin{array}{l}\text { Dollars } \\
\text { (2009)* }\end{array}$} \\
\hline & Energy & $\begin{array}{c}\text { Energy } \\
\text { Intensity } \\
\text { (user units/1000ft2) }\end{array}$ & & \\
\hline \multicolumn{5}{|c|}{ Electricity (kWh) } \\
\hline existing & 2,429 & $8,290.3$ & 28.3 & 431 \\
\hline post-retrofit & 1,040 & $3,548.5$ & 12.1 & 183 \\
\hline difference & $-1,389$ & $-4,741.8$ & -16.2 & -248 \\
\hline$\%$ change & -57 & -57 & -57 & -58 \\
\hline \multicolumn{5}{|c|}{ Other Fuels (MBtu) } \\
\hline existing & 2 & 6.6 & 6.6 & 63 \\
\hline post-retrofit & 2 & 6.6 & 6.6 & 63 \\
\hline difference & $\odot$ & $\odot . \odot$ & $\odot .0$ & $\odot$ \\
\hline$\%$ change & $\odot$ & 0 & 0 & $\odot$ \\
\hline \multicolumn{5}{|l|}{ Total (MBtu) } \\
\hline existing & 10 & 34.9 & 34.9 & 493 \\
\hline post-retrofit & 5 & 18.7 & 18.7 & 245 \\
\hline difference & -5 & -16.2 & -16.2 & -248 \\
\hline$\%$ change & -46 & -46 & -46 & -50 \\
\hline
\end{tabular}

* Dollar values for electricity include both energy and demand components. 


\section{Appropriated Funding Energy Consumption by End Use}

Lighting is the largest load in the building with 2,091 kWh/year, followed by motors and miscellaneous equipment with 338 $\mathrm{kWh} /$ year.

Fuel
Electricity (kWh)
existing
post-retrofit
difference
\% change
Total (MBtu)
existing
post-retrofit
difference
\% change
Total (MBtu/100\%ft2)
existing
post-retrofit
difference
$\%$ change

$\begin{array}{llll}\text { Sanitary latrines/small storage } & 594 & \\ \text { Cooling } & \text { Vent } & \text { Lights } & \text { Motors and } \\ & \text { Misc Equip }\end{array}$

Hot water

\begin{tabular}{|c|c|c|c|}
\hline 0 & $\odot$ & $\odot$ & 2,091 \\
\hline$\odot$ & 0 & $\odot$ & 702 \\
\hline$\odot$ & $\odot$ & $\odot$ & $-1,389$ \\
\hline$\odot$ & $\odot$ & $\odot$ & -66 \\
\hline$\odot$ & $\odot$ & $\odot$ & 7 \\
\hline$\odot$ & $\odot$ & $\odot$ & 2 \\
\hline$\odot$ & $\odot$ & $\odot$ & -5 \\
\hline$\odot$ & $\odot$ & $\odot$ & -66 \\
\hline$\odot$ & $\odot$ & $\odot$ & 24 \\
\hline 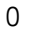 & 0 & $\odot$ & 8 \\
\hline$\theta$ & $\odot$ & $\odot$ & -16 \\
\hline & $\odot$ & $\odot$ & -66 \\
\hline
\end{tabular}

338
338
$\odot$
0

3
3
$\odot$
0

11
11
$\odot$
0




\section{Appropriated Funding Emission Reduction}

The emission reductions from implemented the proposed retrofits are as follows:

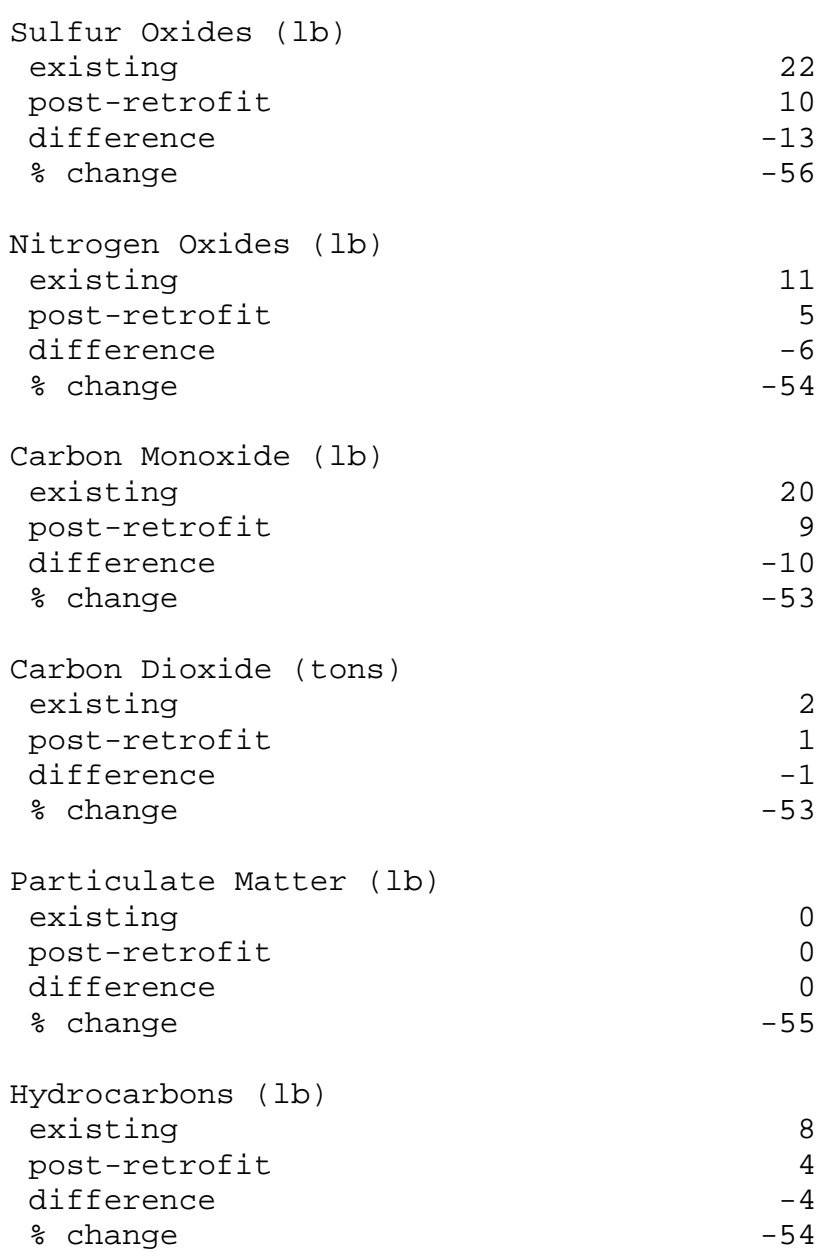




\section{Building 2093 Commissary}

The following information identifies the cost-effective energy- and cost-reducing retrofit projects for building 2093 identified from the FEDS modeling and analysis. Key energy and economic results are presented for each cost-effective retrofit measure.

\section{Facility Description 2093}

2093 is the commissary and was built in 1975. Building 2093 has large conditioned service spaces as well as large unconditioned storage spaces. Building 2093 is 115,408 sf.

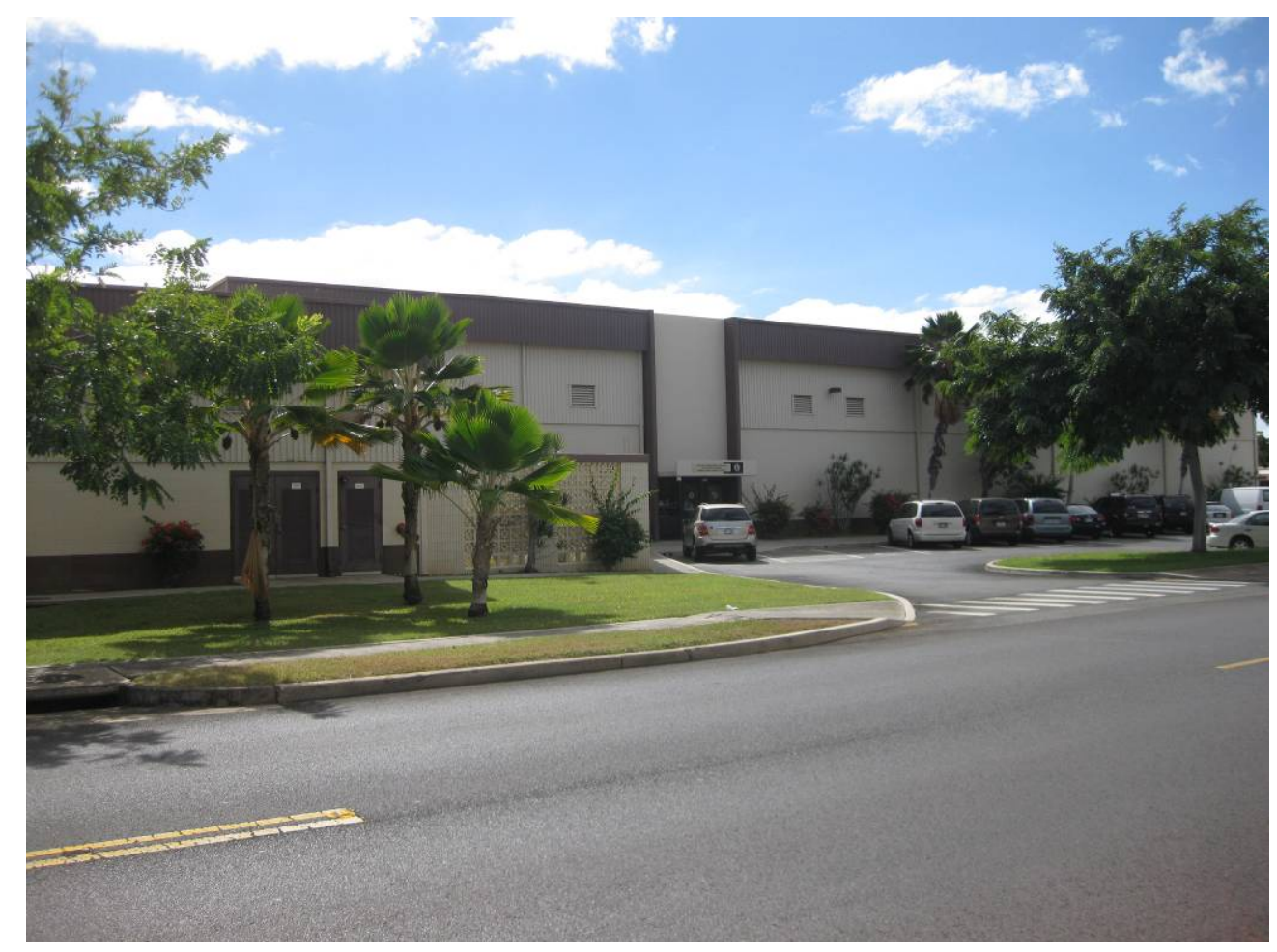




\section{Appropriated Funding Results}

A FEDS analysis using appropriated funding suggests replacing the water cooled chiller with an ultra high efficiency water cooled chiller. FEDS suggests replacing the electric water heater with a heat pump water heater and replacing some of the lighting.

Appropriated funding FEDS results for building 2093:

\begin{tabular}{|c|c|c|c|c|c|c|c|c|}
\hline Bldg. Set Description & $\begin{array}{l}\text { End } \\
\text { Use }\end{array}$ & Existing Technology & Retrofit Technology & $\begin{array}{l}\text { Energy } \\
\text { Savings } \\
\text { (MMBtu/yr) }\end{array}$ & $\begin{array}{l}\text { 1st year } \\
\text { savings } \\
\text { (\$/yr) }\end{array}$ & $\begin{array}{l}\text { Installed } \\
\text { Cost (\$) }\end{array}$ & $\begin{array}{l}\text { Net } \\
\text { Present } \\
\text { Value (\$) } \\
\end{array}$ & SIR \\
\hline $\begin{array}{l}\text { Comissary, large sales } \\
2093\end{array}$ & Cooling & $\begin{array}{l}\text { Electric Water-Cooled } \\
\text { Reciprocating Chiller }\{\mathrm{C} 1\}\end{array}$ & $\begin{array}{l}\text { Water-Cooled Centrifugal } \\
\text { Electric Chiller (ultra high } \\
\text { efficiency) }\end{array}$ & 743 & 45,994 & 284,148 & 205,262 & 2.3 \\
\hline $\begin{array}{l}\text { Comissary, large sales } \\
2093\end{array}$ & Lights & FL1: FL 2X4 4F40T12 STD2 & $\begin{array}{l}\text { FL280: FL 2X4 3F32ST8 ELC3 } \\
\text { REF (FIX REPL) }\end{array}$ & 1,603 & 91,420 & 259,302 & $1,271,569$ & 5.9 \\
\hline $\begin{array}{l}\text { Comissary, large sales } \\
2093\end{array}$ & Lights & EX1: EXIT - INC $(2 \times 20)$ & $\begin{array}{l}\text { EX12: EXIT - } \\
\text { ELECTROLUMINESCENT PANEL } \\
\text { RETRO KIT }\end{array}$ & 22 & 1,453 & 1,118 & 23,392 & 21.9 \\
\hline $\begin{array}{l}\text { Comissary, large sales } \\
2093\end{array}$ & Lights & FL200: FL 1X8 1F96T8 EEF1 & FL250: FL 1X8 1F96T8 ELC1 & 63 & 7,061 & 76,308 & 44,218 & 1.6 \\
\hline $\begin{array}{l}\text { Comissary, large sales } \\
2093\end{array}$ & Lights & FL1: FL 2X4 4F40T12 STD2 & $\begin{array}{l}\text { FL280: FL 2X4 3F32ST8 ELC3 } \\
\text { REF (FIX REPL) }\end{array}$ & 164 & 9,787 & 33,270 & 130,720 & 4.9 \\
\hline $\begin{array}{l}\text { Comissary, large sales } \\
2093\end{array}$ & Lights & EX1: EXIT - INC $(2 \times 20)$ & $\begin{array}{l}\text { EX12: EXIT - } \\
\text { ELECTROLUMINESCENT PANEL } \\
\text { RETRO KIT }\end{array}$ & 2 & 162 & 124 & 2,600 & 21.9 \\
\hline $\begin{array}{l}\text { Comissary, large sales } \\
2093\end{array}$ & $\begin{array}{l}\text { Hot } \\
\text { Water }\end{array}$ & Electric Water Heater & $\begin{array}{l}\text { Heat Pump Water Heater } \\
\text { (Com) }\end{array}$ & 168 & 8,496 & 47,981 & 16,739 & 1.6 \\
\hline
\end{tabular}




\section{Appropriated Funding Energy Consumption by Fuel Type}

The modeled energy consumption for a typical year was 6,735,356 kwh before retrofits and 5,877,351 kwh after proposed retrofits are implemented. The energy use intensity goes from 199.2 MBtu/Ksf to $173.8 \mathrm{MBtu} / \mathrm{Ksf}$ after retrofits.

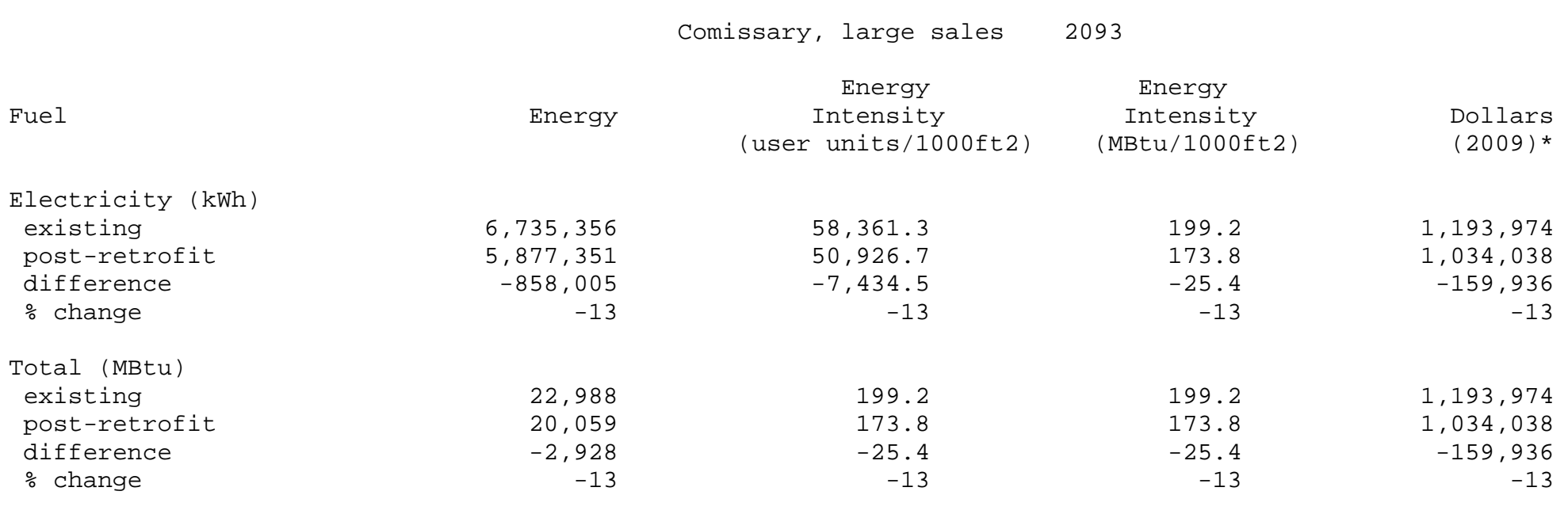

* Dollar values for electricity include both energy and demand components. 


\section{Appropriated Funding Energy Consumption by End Use}

Motors and miscellaneous equipment is the largest load in the building with 4,432,117 kWh/year, followed by lighting with 1,096,681 $\mathrm{kWh} /$ year.

\begin{tabular}{|c|c|c|c|c|c|c|}
\hline Fuel & Heating & Cooling & large s & Lights & $\begin{array}{l}\text { Motors and } \\
\text { Misc Equip }\end{array}$ & Hot Water * \\
\hline \multicolumn{7}{|c|}{ Electricity (kWh) } \\
\hline existing & $\odot$ & 778,012 & 369,229 & $1,096,681$ & $4,432,117$ & 59,317 \\
\hline post-retrofit & $\odot$ & 418,662 & 352,544 & 663,912 & $4,432,117$ & 10,116 \\
\hline difference & $\odot$ & $-359,350$ & $-16,685$ & $-432,769$ & 0 & $-49,201$ \\
\hline$\%$ change & $\odot$ & -46 & -5 & -39 & $\odot$ & -83 \\
\hline \multicolumn{7}{|l|}{ Total (MBtu) } \\
\hline existing & $\odot$ & 2,655 & 1,260 & 3,743 & 15,127 & 202 \\
\hline post-retrofit & 0 & 1,429 & 1,203 & 2,266 & 15,127 & 35 \\
\hline difference & $\odot$ & $-1,226$ & -57 & $-1,477$ & $\odot$ & -168 \\
\hline$\%$ change & $\odot$ & -46 & -5 & -39 & $\odot$ & -83 \\
\hline \multicolumn{7}{|c|}{ Total (MBtu/1000ft2) } \\
\hline existing & $\odot$ & 23 & 11 & 32 & 131 & 2 \\
\hline post-retrofit & $\odot$ & 12 & 10 & 20 & 131 & $\odot$ \\
\hline difference & $\odot$ & -11 & $\odot$ & -13 & $\odot$ & -1 \\
\hline$\%$ change & $\odot$ & -46 & -5 & -39 & $\odot$ & -83 \\
\hline
\end{tabular}

* Energy consumption values for both distributed and central SHW are reported for Hot water annual energy use. 


\section{Appropriated Funding Emission Reduction}

The emission reductions from implemented the proposed retrofits are as follows:

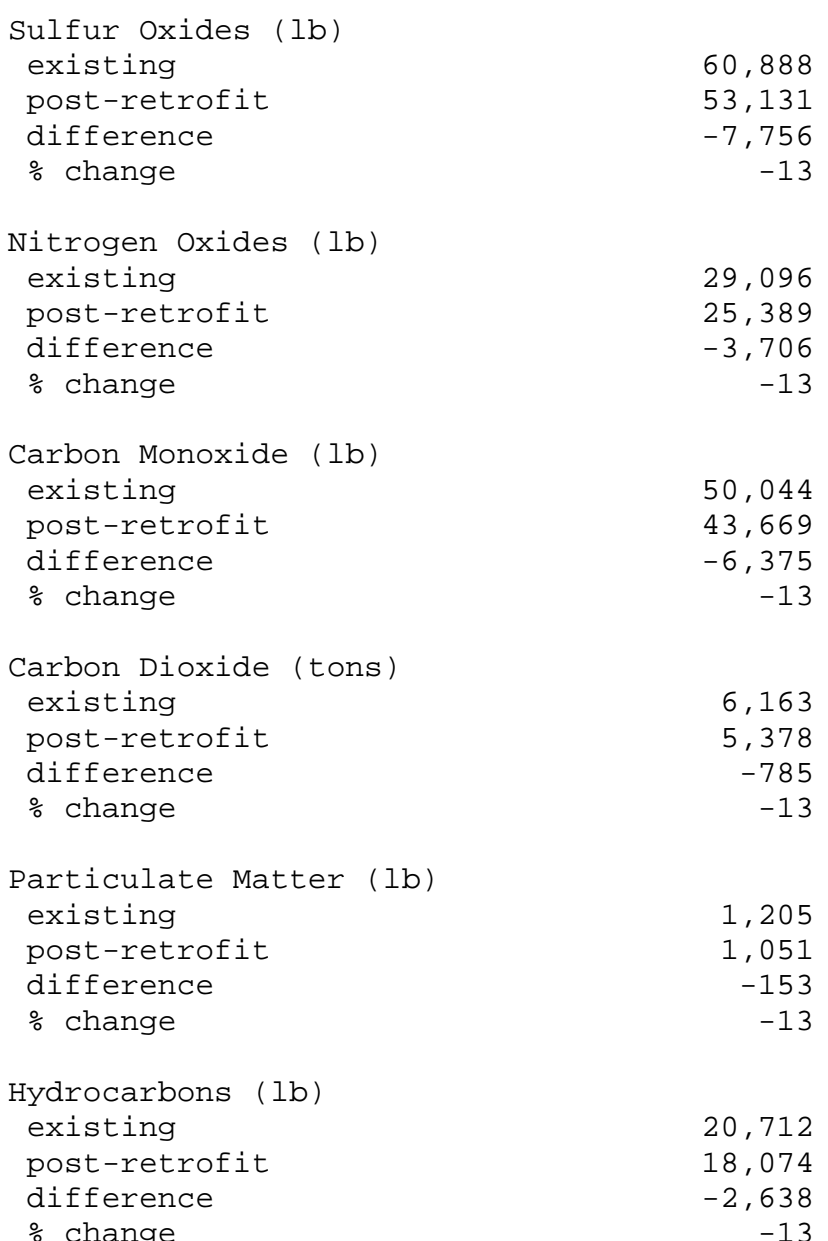




\section{Building 2028 Passenger Terminal}

The following information identifies the cost-effective energy- and cost-reducing retrofit projects for building 2028 identified from the FEDS modeling and analysis. Key energy and economic results are presented for each cost-effective retrofit measure.

\section{Facility Description 2028}

2028 is the air passenger terminal built in 1973. Building 2028 has a water cooled reciprocating chiller, metal halide, fluorescent, incandescent and high pressure sodium lights and little to no insulation in the building envelope. Building 2028 is 46,128 sf.

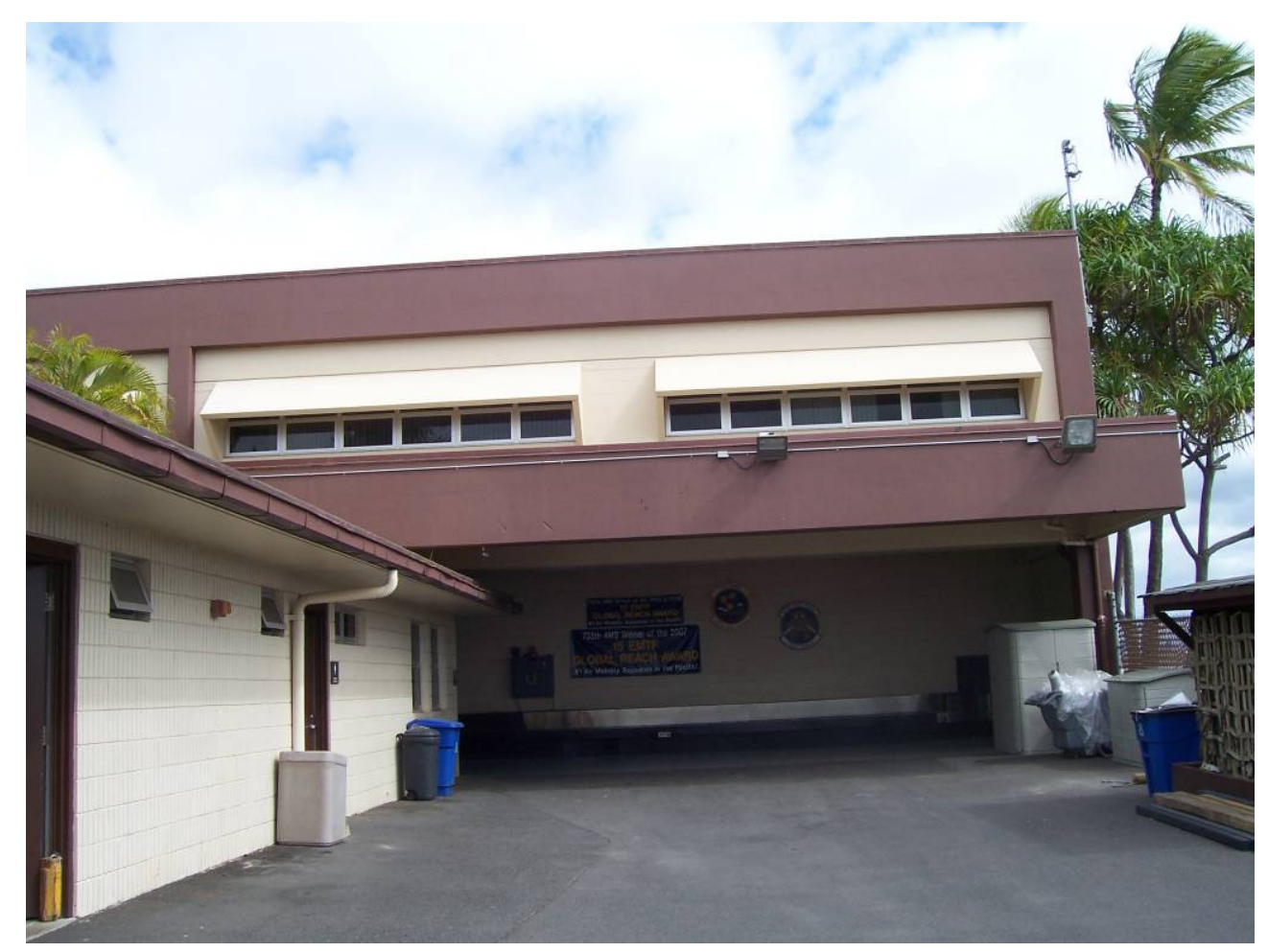




\section{Appropriated Funding Results}

A FEDS analysis using appropriated funding suggests replacing the lighting, replacing the electric water heater with a heat pump water heater and increasing the insulation in the suspended ceiling.

Appropriated funding FEDS results for building 2028:

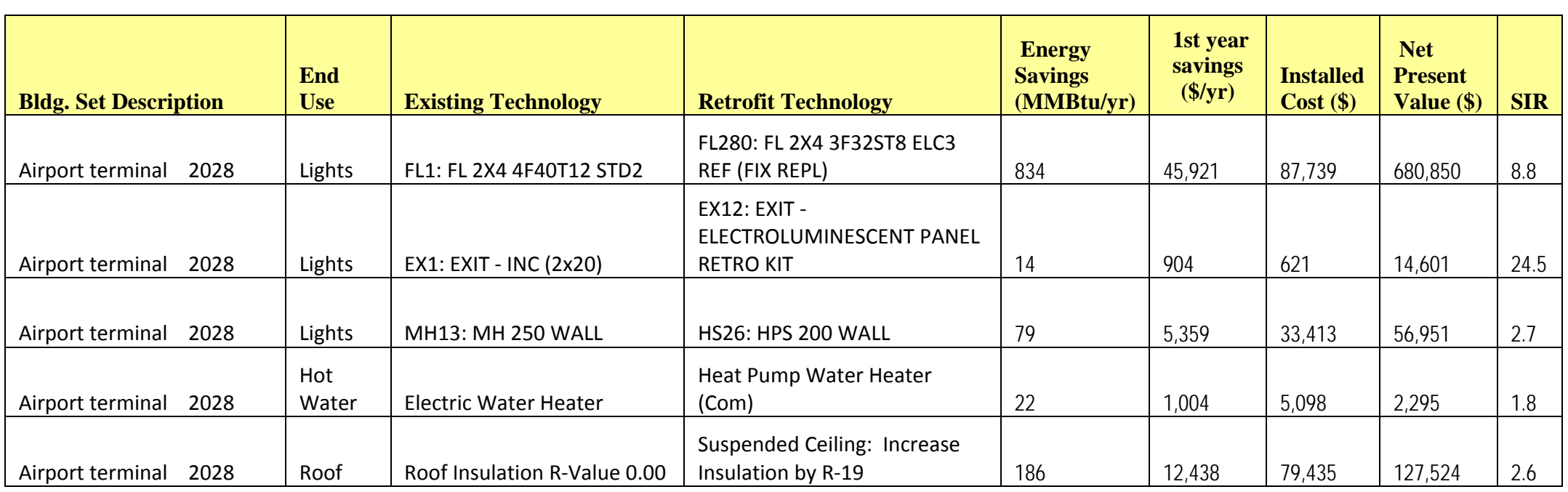




\section{Appropriated Funding Energy Consumption by Fuel Type}

The modeled energy consumption for a typical year was 804,699 kwh before retrofits and 482,604 kwh after proposed retrofits are implemented. The energy use intensity goes from 59.5 MBtu/Ksf to 35.7 MBtu/Ksf after retrofits.

Airport terminal 2028

Fuel

Electricity (kWh)

existing

post-retrofit

difference

$\%$ change

Total (MBtu)

existing

post-retrofit

difference

$\%$ change
Energy

Energy
Intensity
(user units/1000ft2)

804,699

482,604

$-322,095$

$-40$

2,746

1,647

$-1,099$

$-40$

$$
\begin{array}{r}
17,444.9 \\
10,462.3 \\
-6,982.6 \\
-40
\end{array}
$$

59.5

35.7

$-23.8$

$-40$
Energy

Intensity

(MBtu/1000ft2)

Dollars

(2009) *

59.5

35.7

$-23.8$

$-40$

142,649

84,907

$-57,741$

$-40$

59.5

35.7

$-23.8$

$-40$

142,649

84,907

$-57,741$

$-40$

* Dollar values for electricity include both energy and demand components. 


\section{Appropriated Funding Energy Consumption by End Use}

Lighting is the largest load in the building with $410,212 \mathrm{kWh} /$ year, followed by space cooling with 235,351 kWh/year.

\begin{tabular}{|c|c|c|c|c|c|c|}
\hline \multirow[b]{2}{*}{ Fuel } & \multirow[b]{2}{*}{ Heating } & \multicolumn{2}{|c|}{ Airport terminal } & 028 & \multirow[b]{2}{*}{$\begin{array}{l}\text { Motors and } \\
\text { Misc Equip }\end{array}$} & \multirow[b]{2}{*}{ Hot Water } \\
\hline & & Cooling & Vent & Lights & & \\
\hline \multicolumn{7}{|c|}{ Electricity (kWh) } \\
\hline existing & 0 & 235,351 & 67,184 & 410,212 & 84,592 & 7,360 \\
\hline post-retrofit & 0 & 139,143 & 39,140 & 218,736 & 84,592 & 994 \\
\hline difference & 0 & $-96,209$ & $-28,044$ & $-191,476$ & 0 & $-6,366$ \\
\hline$\%$ change & 0 & -41 & -42 & -47 & 0 & -86 \\
\hline \multicolumn{7}{|l|}{ Total (MBtu) } \\
\hline existing & 0 & 803 & 229 & 1,400 & 289 & 25 \\
\hline post-retrofit & 0 & 475 & 134 & 747 & 289 & 3 \\
\hline difference & 0 & -328 & -96 & -654 & 0 & -22 \\
\hline$\%$ change & 0 & -41 & -42 & -47 & 0 & -86 \\
\hline \multicolumn{7}{|c|}{ Total (MBtu/1000ft2) } \\
\hline existing & $\Theta$ & 17 & 5 & 30 & 6 & 1 \\
\hline post-retrofit & 0 & 10 & 3 & 16 & 6 & 0 \\
\hline difference & 0 & -7 & -2 & -14 & 0 & 0 \\
\hline$\%$ change & 0 & -41 & -42 & -47 & $\theta$ & -86 \\
\hline
\end{tabular}




\section{Appropriated Funding Emission Reduction}

The emission reductions from implemented the proposed retrofits are as follows:

Airport terminal

2028

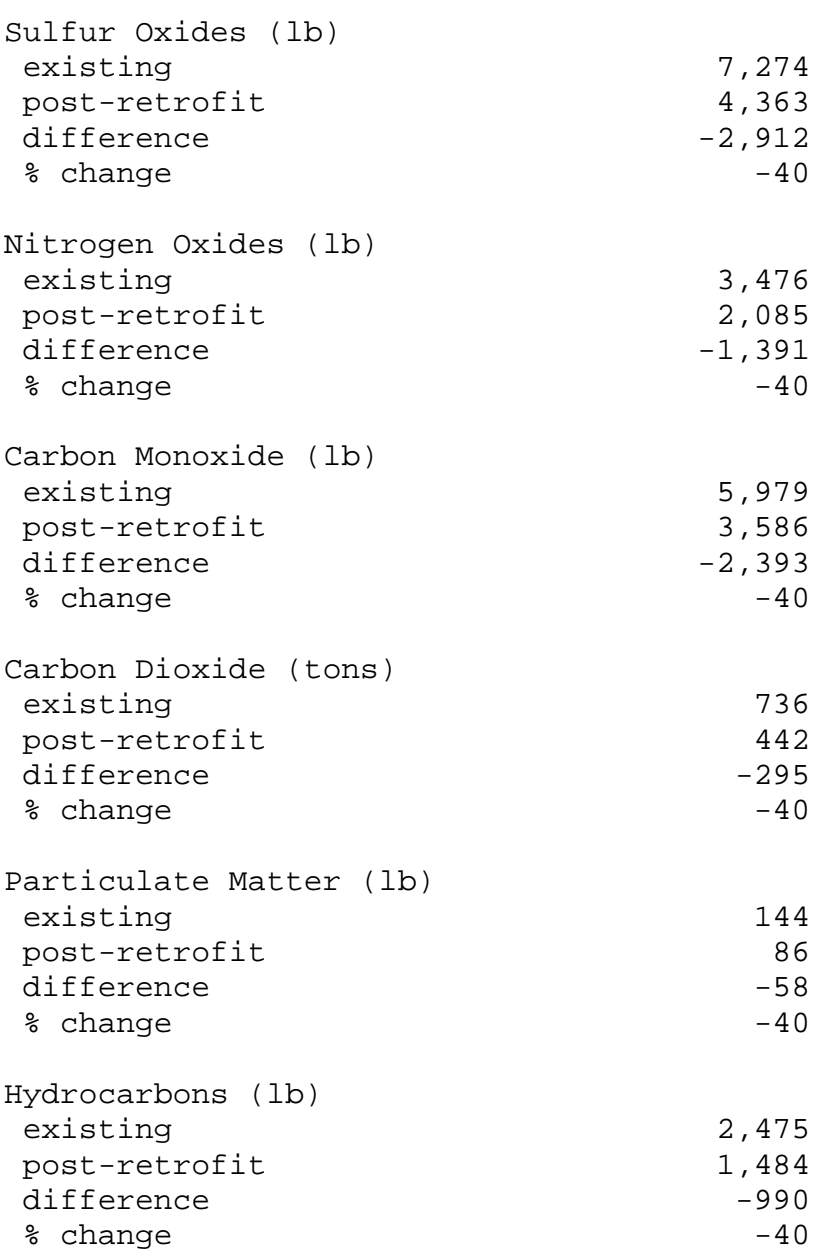




\section{Building 1597 Child Care Facility}

The following information identifies the cost-effective energy- and cost-reducing retrofit projects for building 1597 identified from the FEDS modeling and analysis. Key energy and economic results are presented for each cost-effective retrofit measure.

\section{Facility Description 1597}

1597 is a child care center built 1985. Building 1597 is conditioned by an electric air cooled chiller, has many fluorescent and some metal halide lights and has some insulation in the building envelope. Building 1597 is 12,760 sf.

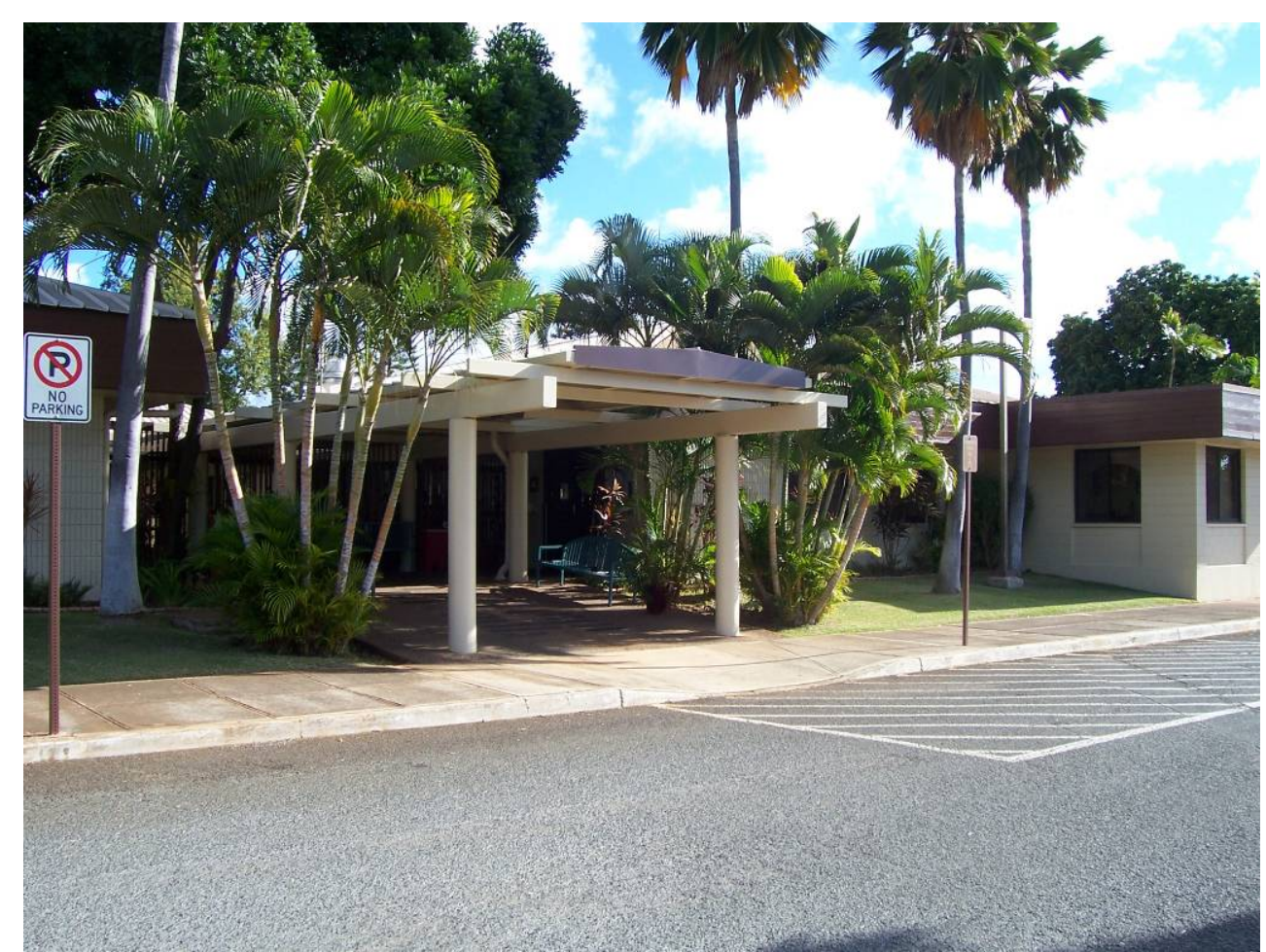




\section{Appropriated Funding Results}

A FEDS analysis using appropriated funding suggests replacing some of the lighting as well as replacing the electric water heater with a heat pump water heater.

Appropriated funding FEDS results for building 1597:

\begin{tabular}{|c|c|c|c|c|c|c|c|c|}
\hline Bldg. Set Description & $\begin{array}{l}\text { End } \\
\text { Use }\end{array}$ & Existing Technology & Retrofit Technology & $\begin{array}{l}\text { Energy } \\
\text { Savings } \\
\text { (MMBtu/yr) }\end{array}$ & $\begin{array}{c}\text { 1st year } \\
\text { savings } \\
(\$ / y r)\end{array}$ & $\begin{array}{l}\text { Installed } \\
\text { Cost (\$) }\end{array}$ & $\begin{array}{l}\text { Net } \\
\text { Present } \\
\text { Value (\$) } \\
\end{array}$ & SIR \\
\hline youth Center 1597 & Lights & FL38: FL 2X4 3F32T8 EEF1,2 & $\begin{array}{l}\text { FL304: FL 2X4 3F25ST8 ELC3 } \\
\text { REF }\end{array}$ & 24 & 1,956 & 18,356 & 14,731 & 1.8 \\
\hline youth Center 1597 & Lights & EX6: EXIT - LED & $\begin{array}{l}\text { EX12: EXIT - } \\
\text { ELECTROLUMINESCENT PANEL } \\
\text { RETRO KIT }\end{array}$ & - & 106 & 621 & 1,216 & 3.0 \\
\hline youth Center & Lights & MH40: MH 150 HE WALL & MH67: MH 150 HE WALL ELC & 1 & 215 & 1,376 & 2,320 & 2.7 \\
\hline youth Center & Lights & FL41: FL 1X4 1F32T8 EEF1 & $\begin{array}{l}\text { FL302: FL 1X4 1F25ST8 ELC1 } \\
\text { REF }\end{array}$ & - & 42 & 652 & 55 & 1.1 \\
\hline youth Center & Lights & FL38: FL 2X4 3F32T8 EEF1,2 & $\begin{array}{l}\text { FL304: FL 2X4 3F25ST8 ELC3 } \\
\text { REF }\end{array}$ & 7 & 562 & 2,760 & 6,697 & 3.4 \\
\hline youth Center 1597 & $\begin{array}{l}\text { Hot } \\
\text { Water }\end{array}$ & Electric Water Heater & $\begin{array}{l}\text { Heat Pump Water Heater } \\
\text { (Com) }\end{array}$ & 42 & 2,455 & 6,854 & 1,579 & 3.4 \\
\hline
\end{tabular}




\section{Appropriated Funding Energy Consumption by Fuel Type}

The modeled energy consumption for a typical year was 200,246 kwh before retrofits and 177,850 kwh after proposed retrofits are implemented. The energy use intensity goes from 53.6 MBtu/Ksf to 47.6 MBtu/Ksf after retrofits.

\begin{tabular}{|c|c|c|c|c|}
\hline \multirow[b]{2}{*}{ Fuel } & \multicolumn{3}{|c|}{ Youth Center $\quad 1597$} & \multirow[b]{2}{*}{$\begin{array}{l}\text { Dollars } \\
(2009) \text { * }\end{array}$} \\
\hline & Energy & $\begin{array}{c}\text { Energy } \\
\text { Intensity } \\
\text { (user units/1000ft2) }\end{array}$ & $\begin{array}{c}\text { Energy } \\
\text { Intensity } \\
\text { (MBtu/1000ft2) }\end{array}$ & \\
\hline \multicolumn{5}{|c|}{ Electricity (kWh) } \\
\hline existing & $20 \odot, 246$ & $15,693.2$ & 53.6 & 35,497 \\
\hline post-retrofit & 177,850 & $13,938.1$ & 47.6 & 31,290 \\
\hline difference & $-22,395$ & $-1,755.1$ & -6.0 & $-4,207$ \\
\hline$\%$ change & -11 & -11 & -11 & -12 \\
\hline \multicolumn{5}{|l|}{ Total (MBtu) } \\
\hline existing & 683 & 53.6 & 53.6 & 35,497 \\
\hline post-retrofit & 607 & 47.6 & 47.6 & 31,290 \\
\hline difference & -76 & -6.0 & -6.0 & $-4,207$ \\
\hline$\%$ change & -11 & -11 & -11 & -12 \\
\hline
\end{tabular}

* Dollar values for electricity include both energy and demand components. 


\section{Appropriated Funding Energy Consumption by End Use}

Space cooling is the largest load in the building with 84,926 kWh/year, followed by motors and miscellaneous equipment with 36,565 $\mathrm{kWh} /$ year.

\begin{tabular}{|c|c|c|c|c|c|c|}
\hline Fuel & Heating & Cooling & Center & Lights & $\begin{array}{l}\text { Motors and } \\
\text { Misc Equip }\end{array}$ & Hot Water \\
\hline \multicolumn{7}{|c|}{ Electricity (kWh) } \\
\hline existing & $\odot$ & 84,926 & 36,501 & 27,943 & 36,565 & 14,310 \\
\hline post-retrofit & $\odot$ & 83,699 & 35,937 & 19,739 & 36,565 & 1,910 \\
\hline difference & $\odot$ & $-1,227$ & -564 & $-8,204$ & 0 & $-12,400$ \\
\hline$\%$ change & $\odot$ & -1 & -2 & -29 & $\odot$ & -87 \\
\hline \multicolumn{7}{|l|}{ Total (MBtu) } \\
\hline existing & $\odot$ & 290 & 125 & 95 & 125 & 49 \\
\hline post-retrofit & $\odot$ & 286 & 123 & 67 & 125 & 7 \\
\hline difference & $\odot$ & -4 & -2 & -28 & 0 & -42 \\
\hline$\%$ change & $\odot$ & -1 & -2 & -29 & $\odot$ & -87 \\
\hline \multicolumn{7}{|c|}{ Total (MBtu/100๑ft2) } \\
\hline existing & $\odot$ & 23 & 10 & 7 & 10 & 4 \\
\hline post-retrofit & $\odot$ & 22 & 10 & 5 & 10 & 1 \\
\hline difference & $\odot$ & $\odot$ & $\odot$ & -2 & $\odot$ & -3 \\
\hline$\%$ change & $\odot$ & -1 & -2 & -29 & $\odot$ & -87 \\
\hline
\end{tabular}




\section{Appropriated Funding Emission Reduction}

The emission reductions from implemented the proposed retrofits are as follows:

Youth Center

1597

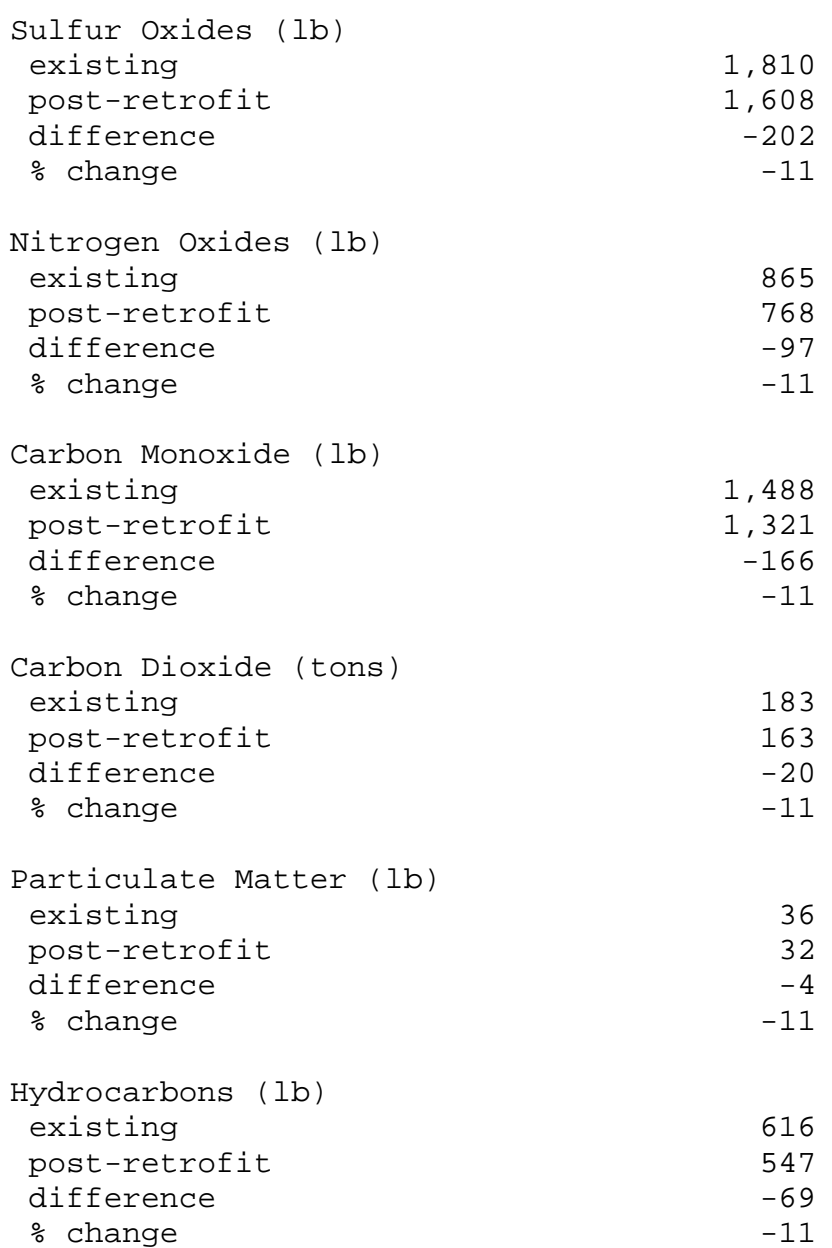




\section{Building 1891 Bowling Facility}

The following information identifies the cost-effective energy- and cost-reducing retrofit projects for building 1971 identified from the FEDS modeling and analysis. Key energy and economic results are presented for each cost-effective retrofit measure.

\section{Facility Description 1891}

1891 is a bowling facility built in 1971. The facility was once a gymnasium, but has since been converted to a bowling center.

Building 1891 is cooled by an electric package unit, has fluorescent, incandescent, and metal halide lights and some insulation in the building envelope. Building 1891 is 3,090 sf.

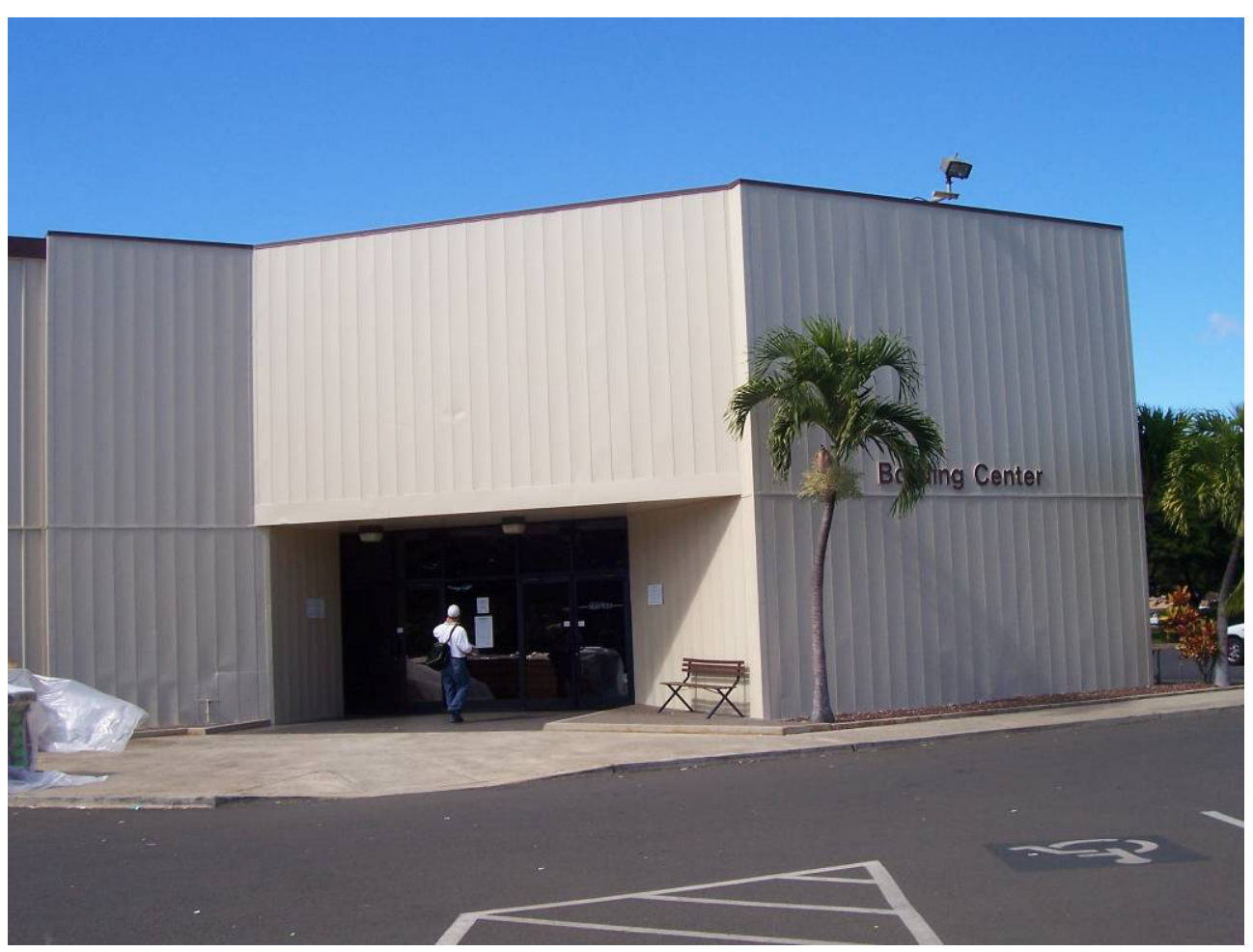




\section{Appropriated Funding Results}

A FEDS analysis using appropriated funding suggests replacing the electric package unit with a very high efficiency single zone package unit. FEDS also suggests delamping 4 tube T8 fixtures to 3 tube T8 fixtures as well as increasing insulation in the attic ceiling, and various improvements to the electric hot water system.

Appropriated funding FEDS results for building 1891:

\begin{tabular}{|c|c|c|c|c|c|c|c|c|}
\hline Bldg. Set Description & $\begin{array}{l}\text { End } \\
\text { Use } \\
\end{array}$ & Existing Technology & Retrofit Technology & $\begin{array}{l}\text { Energy } \\
\text { Savings } \\
\text { (MMBtu/yr) }\end{array}$ & $\begin{array}{c}\text { 1st year } \\
\text { savings } \\
(\$ / y r)\end{array}$ & $\begin{array}{l}\text { Installed } \\
\text { Cost (\$) }\end{array}$ & $\begin{array}{l}\text { Net } \\
\text { Present } \\
\text { Value (\$) } \\
\end{array}$ & SIR \\
\hline $\begin{array}{l}\text { misc recreation bldgs } \\
1891\end{array}$ & Cooling & Electric Package Unit $\{\mathrm{C} 1\}$ & $\begin{array}{l}\text { Single Zone Packaged AC Unit } \\
\text { (very high efficiency / small) }\end{array}$ & 108 & 9,969 & 30,233 & 6,907 & 3.8 \\
\hline $\begin{array}{l}\text { misc recreation bldgs } \\
1891\end{array}$ & Lights & EX1: EXIT - INC $(2 \times 20)$ & $\begin{array}{l}\text { EX12: EXIT - } \\
\text { ELECTROLUMINESCENT PANEL } \\
\text { RETRO KIT }\end{array}$ & 7 & 464 & 311 & 7,525 & 25.2 \\
\hline $\begin{array}{l}\text { misc recreation bldgs } \\
1891\end{array}$ & Lights & FL37: FL 2X4 4F32T8 EEF2 & $\begin{array}{l}\text { FL280: FL 2X4 3F32ST8 ELC3 } \\
\text { REF }\end{array}$ & 22 & 1,440 & 4,416 & 19,784 & 5.5 \\
\hline $\begin{array}{l}\text { misc recreation bldgs } \\
1891\end{array}$ & Lights & FL39: FL 2X4 2F32T8 EEF2 & $\begin{array}{l}\text { FL303: FL 2X4 2F25ST8 ELC2 } \\
\text { REF }\end{array}$ & 14 & 1,007 & 5,952 & 11,004 & 2.8 \\
\hline $\begin{array}{l}\text { misc recreation bldgs } \\
1891\end{array}$ & $\begin{array}{l}\text { Hot } \\
\text { Water }\end{array}$ & Electric Water Heater & $\begin{array}{l}\text { Wrap Tank with Insulation, } \\
\text { Insulate Pipe Near Tank, } \\
\text { Aerators }\end{array}$ & 3 & 175 & 189 & 148 & 13.0 \\
\hline $\begin{array}{l}\text { misc recreation bldgs } \\
1891\end{array}$ & Roof & $\begin{array}{l}\text { Roof Insulation R-Value } \\
11.00\end{array}$ & $\begin{array}{l}\text { Attic Ceiling: Increase } \\
\text { Insulation by R-30 (blow-in } \\
\text { cellulose) }\end{array}$ & 13 & 740 & 3,705 & 8,601 & 3.3 \\
\hline
\end{tabular}




\section{Appropriated Funding Energy Consumption by Fuel Type}

The modeled energy consumption for a typical year was 158,242 kwh before retrofits and 100,281 kwh after proposed retrofits are implemented. The energy use intensity goes from 176.2 MBtu/Ksf to $112.2 \mathrm{MBtu} / \mathrm{Ksf}$ after retrofits.

Fuel

Electricity (kWh)

existing

post-retrofit

difference

$\%$ change

other Fuels (MBtu)

existing

post-retrofit

difference

$\%$ change

Total (MBtu)

existing

post-retrofit

difference

$\%$ change
Energy

158,242

100,281

$-57,961$

$-37$

4
4
0
0

545

347
-198

$-36$
Misc recreation bldgs 1891

Energy
Intensity
(user units/1000ft2)

Energy

Intensity

(MBtu/1000ft2)

Dollars

$(2009)$ *

$51,211.1$
$32,453.5$
$-18,757.6$
-37

174.8

110.8

$-64.0$

$-37$

28,052

17,643

$-10,408$

1.4

1.4

0.0

1.4
1.4

0

176.2
112.2
-64.0
-36

176.2

112.2

$-64.0$

$-36$
$-37$

143

143

$$
\odot
$$

28,195

17,787

$-10,408$

* Dollar values for electricity include both energy and demand components. 


\section{Appropriated Funding Energy Consumption by End Use}

Space cooling is the largest load in the building with $82,546 \mathrm{kWh} /$ year, followed by lighting with $38,734 \mathrm{kWh} /$ year.

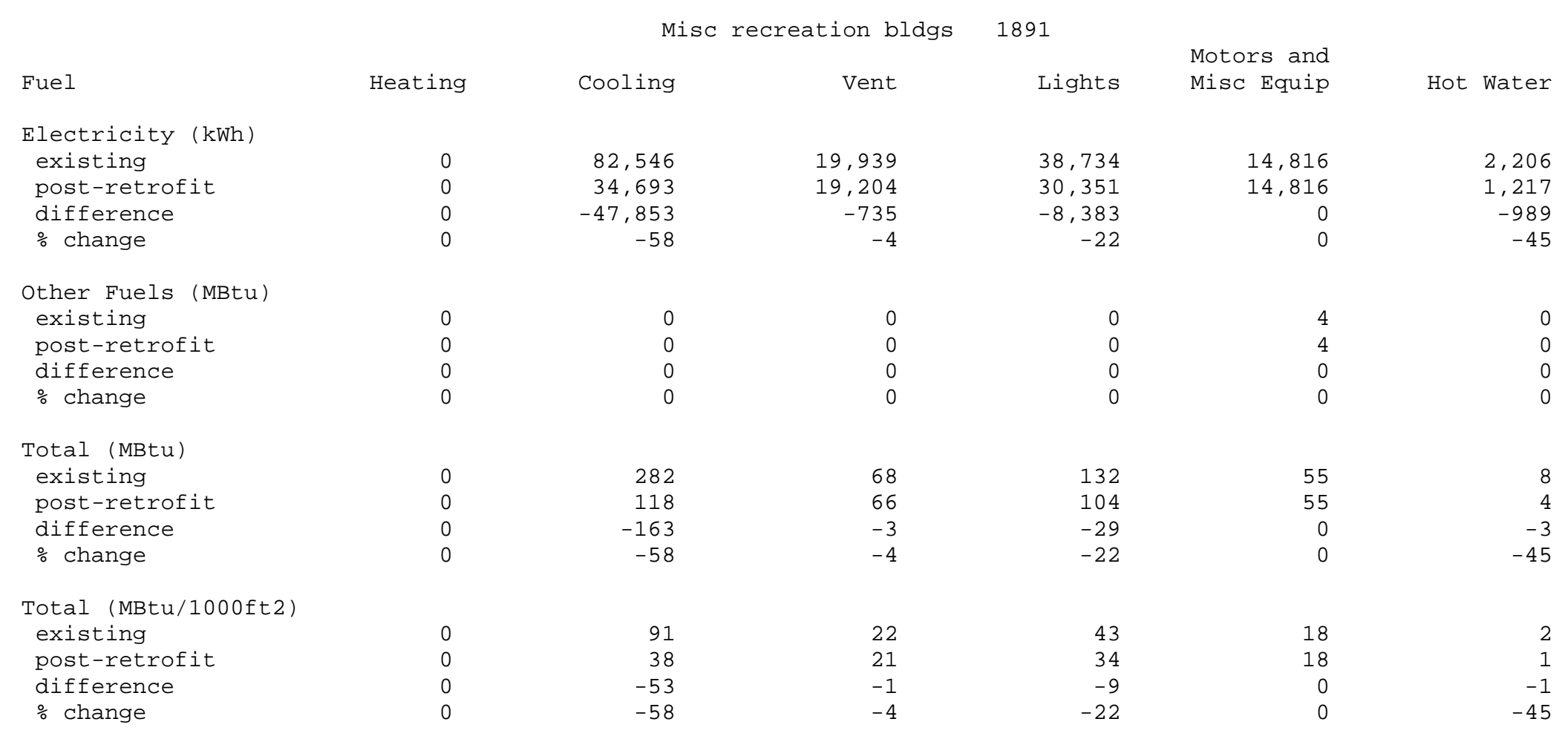




\section{Appropriated Funding Emission Reduction}

The emission reductions from implemented the proposed retrofits are as follows:

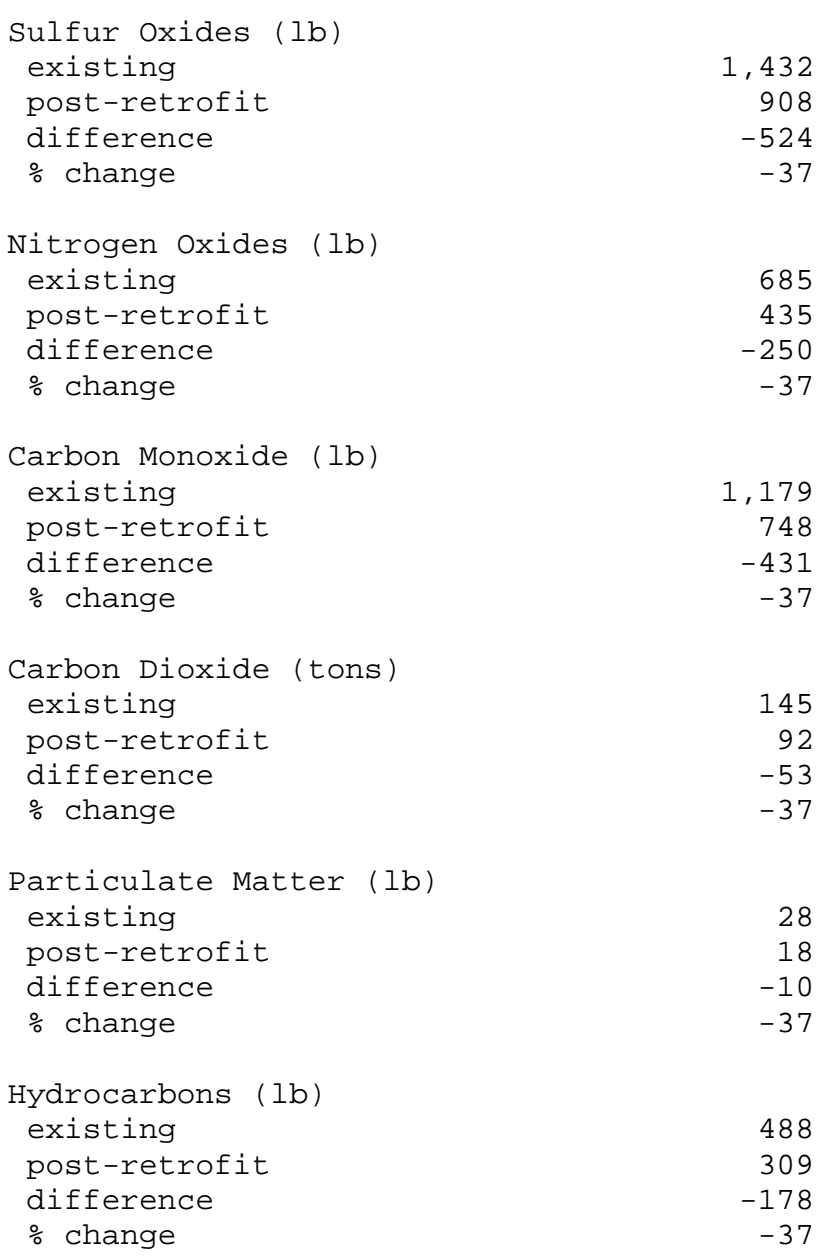




\section{Building 1750 Religious Education Facility}

The following information identifies the cost-effective energy- and cost-reducing retrofit projects for building 1750 identified from the FEDS modeling and analysis. Key energy and economic results are presented for each cost-effective retrofit measure.

\section{Facility Description 1750}

1750 is a religious education facility built in 1977. Building 1750 is conditioned by an electric package unit. The building has incandescent, fluorescent, and metal halide lights and has little to no insulation in the building envelope. Building 1750 is 7,296 sf.

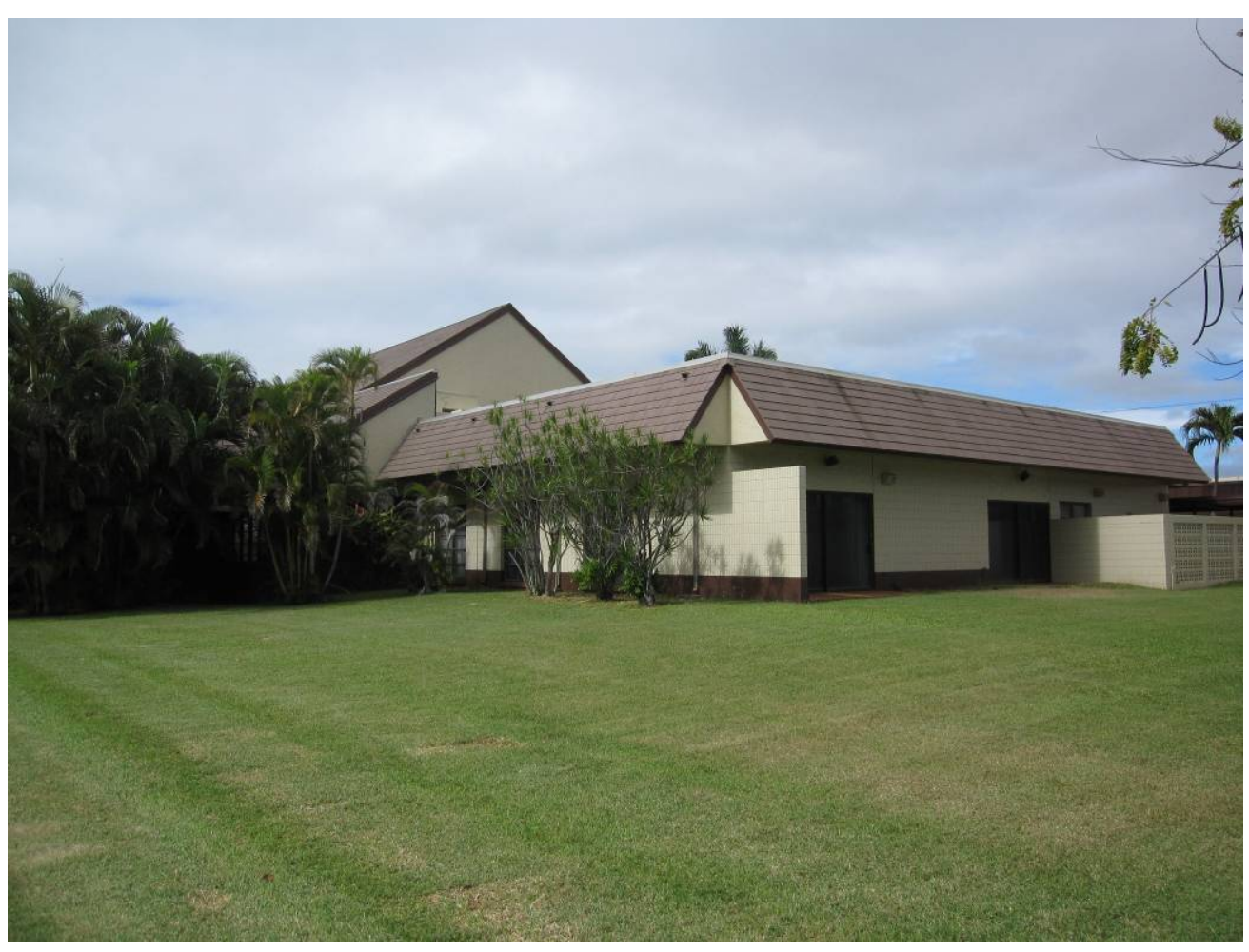




\section{Appropriated Funding Results}

A FEDS analysis using appropriated funding suggests replacing the lighting in the building, increasing the insulation in the roof and increasing the insulation on the hot water tank.

Appropriated funding FEDS results for building 1750:

\begin{tabular}{|c|c|c|c|c|c|c|c|c|}
\hline Bldg. Set Description & $\begin{array}{l}\text { End } \\
\text { Use }\end{array}$ & Existing Technology & Retrofit Technology & $\begin{array}{l}\text { Energy } \\
\text { Savings } \\
\text { (MMBtu/yr) }\end{array}$ & $\begin{array}{c}\text { 1st year } \\
\text { savings } \\
\text { (\$/yr) }\end{array}$ & $\begin{array}{l}\text { Installed } \\
\text { Cost (\$) }\end{array}$ & $\begin{array}{l}\text { Net } \\
\text { Present } \\
\text { Value (\$) } \\
\end{array}$ & SIR \\
\hline Religious facilities 1750 & Lights & FL38: FL 2X4 3F32T8 EEF1,2 & $\begin{array}{l}\text { FL304: FL 2X4 3F25ST8 ELC3 } \\
\text { REF }\end{array}$ & 3 & 256 & 1,853 & 2,473 & 2.3 \\
\hline Religious facilities 1750 & Lights & EX1: EXIT - INC $(2 \times 20)$ & $\begin{array}{l}\text { EX12: EXIT - } \\
\text { ELECTROLUMINESCENT PANEL } \\
\text { RETRO KIT }\end{array}$ & 13 & 851 & 621 & 13,734 & 23.1 \\
\hline Religious facilities 1750 & Lights & FL39: FL 2X4 2F32T8 EEF2 & $\begin{array}{l}\text { FL303: FL 2X4 2F25ST8 ELC2 } \\
\text { REF }\end{array}$ & 4 & 305 & 2,482 & 2,654 & 2.1 \\
\hline Religious facilities 1750 & Lights & IN8: INC 75 CEIL & $\begin{array}{l}\text { CF5: CFL } 18 \text { INTEGRAL UNIT } \\
\text { ELC }\end{array}$ & 23 & 1,611 & 502 & 26,565 & 53.9 \\
\hline Religious facilities 1750 & Lights & IN11: INC 100 CEIL & $\begin{array}{l}\text { CF9: CFL } 26 \text { INTEGRAL UNIT } \\
\text { ELC }\end{array}$ & 3 & 223 & 58 & 3,682 & 64.6 \\
\hline Religious facilities 1750 & Lights & FL4: FL 1X4 2F40T12 STD2 & FL52: FL 1X4 2F32T8 ELC2 & 16 & 932 & 3,949 & 11,771 & 4.0 \\
\hline Religious facilities 1750 & $\begin{array}{l}\text { Hot } \\
\text { Water }\end{array}$ & Distillate Oil Water Heater & Wrap Tank with Insulation & 1 & 30 & 53 & 64 & 2.2 \\
\hline Religious facilities 1750 & Roof & Roof Insulation R-Value 0.00 & $\begin{array}{l}\text { Insulate Built-up Roof Surface } \\
\text { (R-15) and Re-Roof }\end{array}$ & 94 & 6,283 & 36,504 & 68,040 & 2.9 \\
\hline
\end{tabular}




\section{Appropriated Funding Energy Consumption by Fuel Type}

The modeled energy consumption for a typical year was 133,294 kwh before retrofits and 87,822 kwh after proposed retrofits are implemented. The modeled distillate oil consumption for a typical year was 64 gallons before retrofits and 58 gallons after proposed retrofits are implemented. The energy use intensity goes from $63.6 \mathrm{MBtu} / \mathrm{Ksf}$ to $42.2 \mathrm{MBtu} / \mathrm{Ksf}$ after retrofits.

Religious facilities 1750

\begin{tabular}{|c|c|c|c|c|}
\hline Fuel & Energy & $\begin{array}{c}\text { Energy } \\
\text { Intensity } \\
\text { (user units/1000ft2) }\end{array}$ & $\begin{array}{l}\text { Energy } \\
\text { Intensity } \\
\text { (MBtu/100\%ft2) }\end{array}$ & $\begin{array}{l}\text { Dollars } \\
\text { (2009)* }\end{array}$ \\
\hline \multicolumn{5}{|c|}{ Electricity (kWh) } \\
\hline existing & 133,294 & $18,269.4$ & 62.4 & 23,629 \\
\hline post-retrofit & 87,822 & $12,037.0$ & 41.1 & 15,451 \\
\hline difference & $-45,472$ & $-6,232.4$ & -21.3 & $-8,178$ \\
\hline$\%$ change & -34 & -34 & -34 & -35 \\
\hline \multicolumn{5}{|c|}{ Distillate Oil (gal) } \\
\hline existing & 64 & 8.7 & 1.2 & 324 \\
\hline post-retrofit & 58 & 7.9 & 1.1 & 294 \\
\hline difference & -6 & -0.8 & -0.1 & -30 \\
\hline$\%$ change & -9 & -9 & -9 & -9 \\
\hline \multicolumn{5}{|l|}{ Total (MBtu) } \\
\hline existing & 464 & 63.6 & 63.6 & 23,953 \\
\hline post-retrofit & 308 & 42.2 & 42.2 & 15,745 \\
\hline difference & -156 & -21.4 & -21.4 & $-8,208$ \\
\hline$\%$ change & -34 & -34 & -34 & -34 \\
\hline
\end{tabular}

* Dollar values for electricity include both energy and demand components. 


\section{Appropriated Funding Energy Consumption by End Use}

Space cooling is the largest load in the building with $60,705 \mathrm{kWh} /$ year, followed by lighting with $31,784 \mathrm{kWh} /$ year.

\begin{tabular}{|c|c|c|c|c|c|c|}
\hline \multirow[b]{2}{*}{ Fuel } & \multicolumn{4}{|c|}{ Religious facilities } & & \multirow[b]{2}{*}{ Hot Water } \\
\hline & Heating & Cooling & Vent & Lights & $\begin{array}{l}\text { Motors and } \\
\text { Misc Equip }\end{array}$ & \\
\hline \multicolumn{7}{|c|}{ Electricity (kWh) } \\
\hline existing & 0 & 60,705 & 29,785 & 31,784 & 11,019 & $\odot$ \\
\hline post-retrofit & $\odot$ & 30,632 & 29,151 & 17,020 & 11,019 & $\odot$ \\
\hline difference & $\odot$ & $-30,073$ & -635 & $-14,764$ & 0 & $\odot$ \\
\hline$\%$ change & $\odot$ & -50 & -2 & -46 & $\odot$ & $\odot$ \\
\hline \multicolumn{7}{|c|}{ Distillate Oil (gal) } \\
\hline existing & $\odot$ & $\odot$ & $\odot$ & $\odot$ & $\odot$ & 64 \\
\hline post-retrofit & $\odot$ & $\odot$ & $\odot$ & $\odot$ & $\odot$ & 58 \\
\hline difference & $\odot$ & $\odot$ & $\odot$ & $\odot$ & $\odot$ & -6 \\
\hline$\%$ change & $\odot$ & $\odot$ & $\odot$ & $\odot$ & $\odot$ & -9 \\
\hline \multicolumn{7}{|l|}{ Total (MBtu) } \\
\hline existing & $\odot$ & 207 & 102 & 108 & 38 & 9 \\
\hline post-retrofit & $\odot$ & 105 & 99 & 58 & 38 & 8 \\
\hline difference & $\odot$ & -103 & -2 & -50 & $\odot$ & -1 \\
\hline$\%$ change & $\odot$ & -50 & -2 & -46 & $\odot$ & -9 \\
\hline \multicolumn{7}{|c|}{ Total (MBtu/100๑ft2) } \\
\hline existing & $\odot$ & 28 & 14 & 15 & 5 & 1 \\
\hline post-retrofit & $\odot$ & 14 & 14 & 8 & 5 & 1 \\
\hline difference & $\odot$ & -14 & 0 & -7 & $\odot$ & 0 \\
\hline$\%$ change & $\odot$ & -50 & -2 & -46 & $\odot$ & -9 \\
\hline
\end{tabular}




\section{Appropriated Funding Emission Reduction}

The emission reductions from implemented the proposed retrofits are as follows:

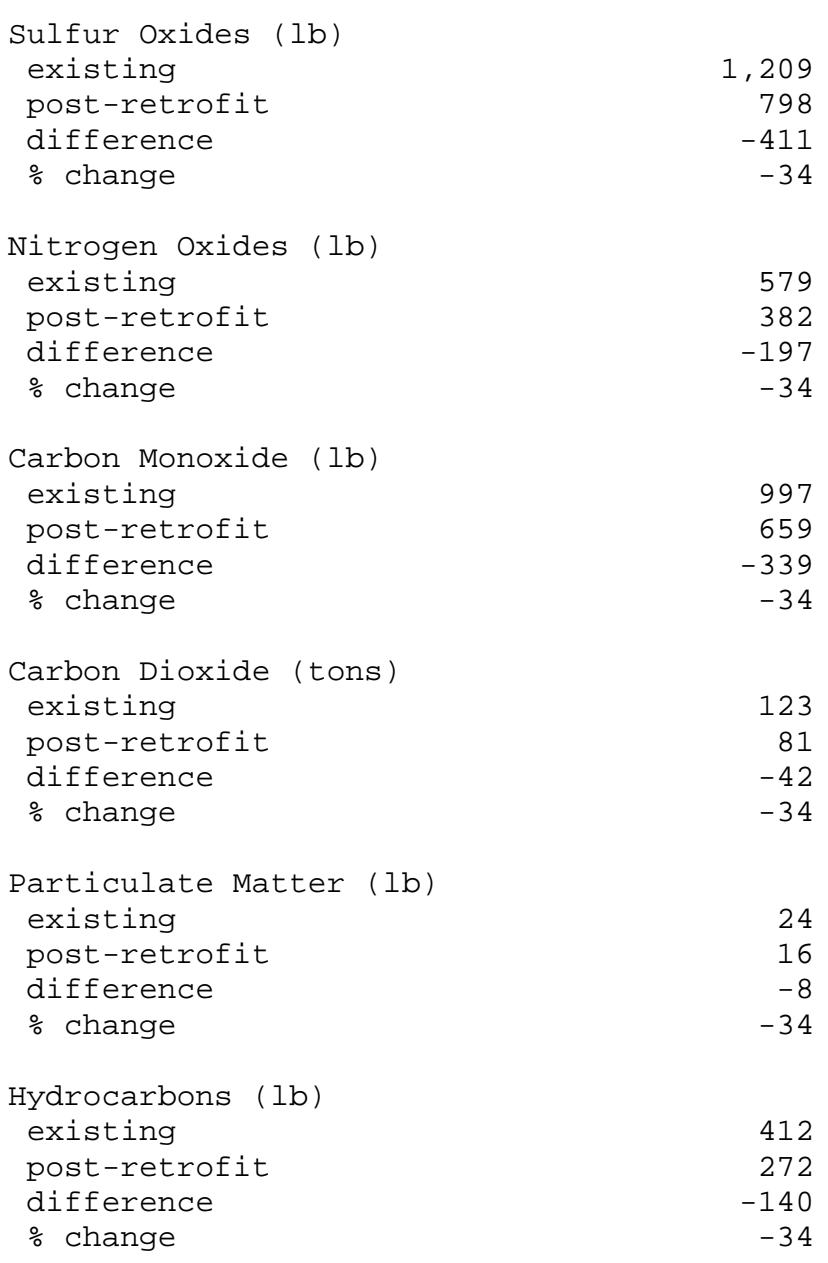




\section{Building 1120 Gymnasium}

The following information identifies the cost-effective energy- and cost-reducing retrofit projects for building 1120 identified from the FEDS modeling and analysis. Key energy and economic results are presented for each cost-effective retrofit measure.

\section{Facility Description 1120}

1120 is the main gymnasium built in 1949. Building 1120 is conditioned by an electric air cooled chiller. It has fluorescent, metal halide and high pressure sodium lights as well as little to no insulation in its building envelope. Building 1120 is 46,719 sf.

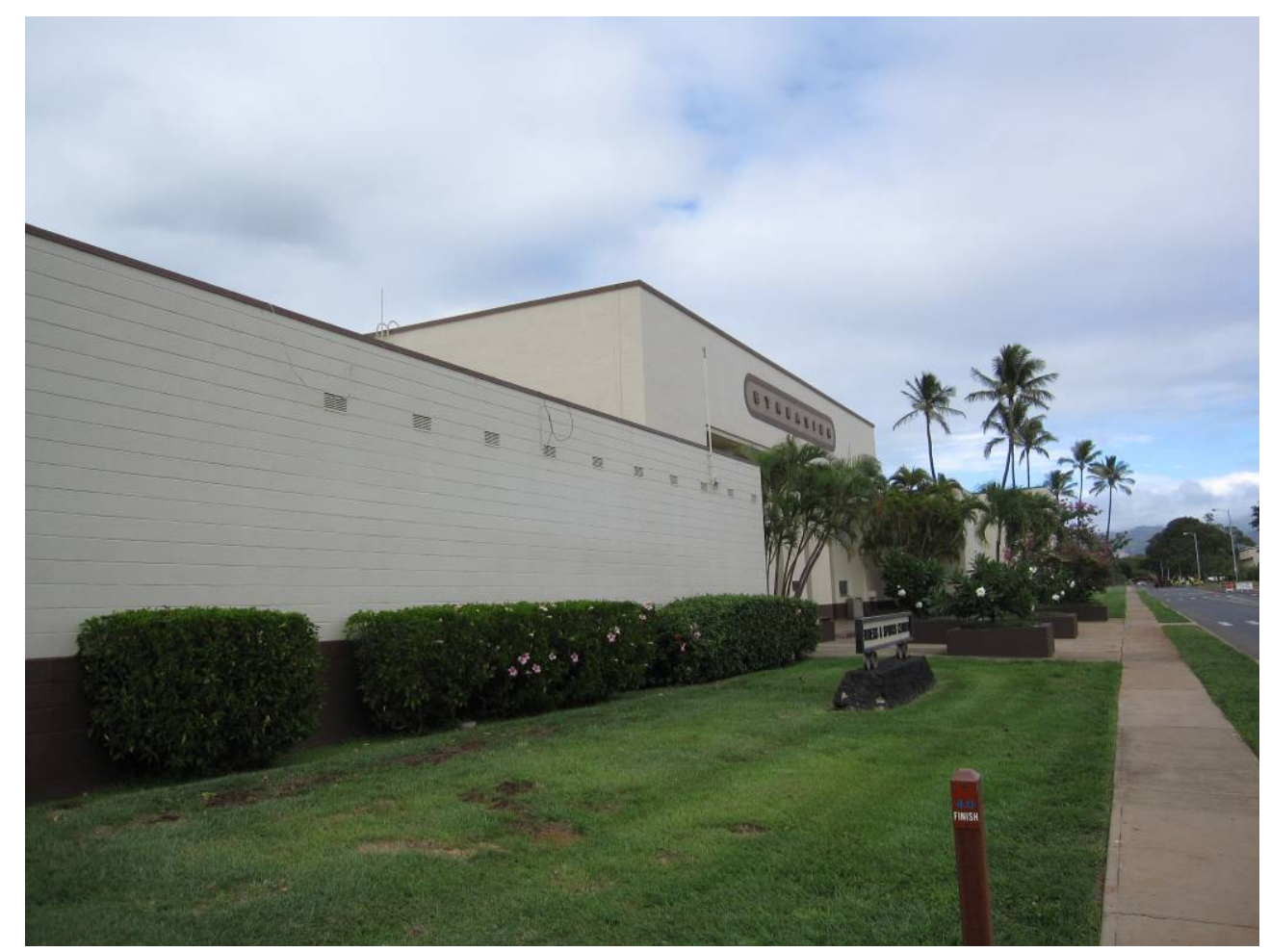




\section{Appropriated Funding Results}

A FEDS analysis using appropriated funding suggests replacing the electric air cooled chiller with a very high efficiency water cooled chiller. FEDS also suggests replacing some of the lighting, installing double pane super low-e windows, and making various improvements to the hot water system.

Appropriated funding FEDS results fobuilding 1750:

\begin{tabular}{|c|c|c|c|c|c|c|c|c|}
\hline Bldg. Set Description & $\begin{array}{l}\text { End } \\
\text { Use }\end{array}$ & Existing Technology & Retrofit Technology & $\begin{array}{l}\text { Energy } \\
\text { Savings } \\
\text { (MMBtu/yr) }\end{array}$ & $\begin{array}{l}\text { 1st year } \\
\text { savings } \\
(\$ / y r)\end{array}$ & $\begin{array}{l}\text { Installed } \\
\text { Cost (\$) }\end{array}$ & $\begin{array}{l}\text { Net } \\
\text { Present } \\
\text { Value (\$) }\end{array}$ & SIR \\
\hline gymnasium 1120 & Cooling & $\begin{array}{l}\text { Electric Air-Cooled Chiller } \\
\{\text { C1 }\}\end{array}$ & $\begin{array}{l}\text { Water-Cooled Reciprocating } \\
\text { Electric Chiller (very high } \\
\text { efficiency) and Cooling Tower }\end{array}$ & 525 & 27,663 & 112,069 & 155,721 & 3.4 \\
\hline gymnasium 1120 & Lights & FL3: FL 2X4 2F40T12 STD2 & $\begin{array}{l}\text { FL303: FL 2X4 2F25ST8 ELC2 } \\
\text { REF (FIX REPL) }\end{array}$ & 43 & 2,541 & 14,429 & 28,120 & 2.9 \\
\hline gymnasium 1120 & Lights & EX1: EXIT - INC $(2 \times 20)$ & $\begin{array}{l}\text { EX12: EXIT - } \\
\text { ELECTROLUMINESCENT PANEL } \\
\text { RETRO KIT }\end{array}$ & 13 & 878 & 621 & 14,174 & 23.8 \\
\hline gymnasium 1120 & Lights & FL1: FL 2X4 4F40T12 STD2 & $\begin{array}{l}\text { FL280: FL 2X4 3F32ST8 ELC3 } \\
\text { REF (FIX REPL) }\end{array}$ & 120 & 7,027 & 11,663 & 106,029 & 10.1 \\
\hline gymnasium 1120 & $\begin{array}{l}\text { Hot } \\
\text { Water }\end{array}$ & Distillate Oil Water Heater & $\begin{array}{l}\text { Wrap Tank with Insulation, } \\
\text { Insulate Pipe Near Tank, } \\
\text { Aerators }\end{array}$ & 22 & 815 & 588 & 3,041 & 17.8 \\
\hline
\end{tabular}




\section{Appropriated Funding Energy Consumption by Fuel Type}

The modeled energy consumption for a typical year was 1,054,786 kwh before retrofits and 827,778 kwh after proposed retrofits are implemented. The modeled distillate oil consumption for a typical year was 356 gallons before retrofits and 196 gallons after proposed retrofits are implemented. The energy use intensity goes from 78.1 MBtu/Ksf to 61.1 MBtu/Ksf after retrofits.

\begin{tabular}{|c|c|c|c|c|}
\hline \multirow[b]{2}{*}{ Fuel } & \multirow[b]{2}{*}{ Energy } & Gymnasium & \multirow[b]{2}{*}{$\begin{array}{c}\text { Energy } \\
\text { Intensity } \\
\text { (MBtu/1000ft2) }\end{array}$} & \multirow[b]{2}{*}{$\begin{array}{l}\text { Dollars } \\
(2009) \text { * }\end{array}$} \\
\hline & & $\begin{array}{c}\text { Energy } \\
\text { Intensity } \\
\text { (user units/1000ft2) }\end{array}$ & & \\
\hline \multicolumn{5}{|c|}{ Electricity (kWh) } \\
\hline existing & $1,054,786$ & $22,577.2$ & 77.1 & 186,981 \\
\hline post-retrofit & 827,778 & $17,718.2$ & 60.5 & 145,636 \\
\hline difference & $-227,008$ & $-4,859.0$ & -16.6 & $-41,345$ \\
\hline$\%$ change & -22 & -22 & -22 & -22 \\
\hline \multicolumn{5}{|c|}{ Distillate Oil (gal) } \\
\hline existing & 356 & 7.6 & 1.1 & 1,814 \\
\hline post-retrofit & 196 & 4.2 & 0.6 & 999 \\
\hline difference & -160 & -3.4 & $-\odot .5$ & -815 \\
\hline$\%$ change & -45 & -45 & -45 & -45 \\
\hline \multicolumn{5}{|l|}{ Total (MBtu) } \\
\hline existing & 3,649 & 78.1 & 78.1 & 188,795 \\
\hline post-retrofit & 2,852 & 61.1 & 61.1 & 146,635 \\
\hline difference & -797 & -17.1 & $-17 \cdot 1$ & $-42,160$ \\
\hline$\%$ change & -22 & -22 & -22 & -22 \\
\hline
\end{tabular}

* Dollar values for electricity include both energy and demand components. 


\section{Appropriated Funding Energy Consumption by End Use}

Space cooling is the largest load in the building with $445,860 \mathrm{kWh} /$ year, followed by motors and miscellaneous equipment with $256,750 \mathrm{kWh} /$ year.

\begin{tabular}{|c|c|c|c|c|c|c|}
\hline \multirow[b]{2}{*}{ Fuel } & \multirow[b]{2}{*}{ Heating } & \multicolumn{2}{|c|}{ Gymnasium } & 1120 & & \multirow[b]{2}{*}{ Hot Water } \\
\hline & & Cooling & Vent & Lights & $\begin{array}{l}\text { Motors and } \\
\text { Misc Equip }\end{array}$ & \\
\hline \multicolumn{7}{|c|}{ Electricity (kWh) } \\
\hline existing & $\odot$ & 445,860 & 141,105 & 211,070 & 256,750 & $\odot$ \\
\hline post-retrofit & $\odot$ & 276,537 & 122,765 & 171,725 & 256,750 & $\odot$ \\
\hline difference & $\odot$ & $-169,323$ & $-18,340$ & $-39,345$ & 0 & 0 \\
\hline$\%$ change & $\odot$ & -38 & -13 & -19 & $\odot$ & $\odot$ \\
\hline \multicolumn{7}{|c|}{ Distillate Oil (gal) } \\
\hline existing & $\odot$ & $\odot$ & $\odot$ & $\odot$ & $\odot$ & 356 \\
\hline post-retrofit & $\odot$ & $\odot$ & $\odot$ & $\odot$ & $\odot$ & 196 \\
\hline difference & $\odot$ & $\odot$ & $\odot$ & $\odot$ & $\odot$ & -160 \\
\hline$\%$ change & $\odot$ & $\odot$ & $\odot$ & $\odot$ & $\odot$ & -45 \\
\hline \multicolumn{7}{|l|}{ Total (MBtu) } \\
\hline existing & $\odot$ & 1,522 & 482 & 720 & 876 & 49 \\
\hline post-retrofit & $\odot$ & 944 & 419 & 586 & 876 & 27 \\
\hline difference & $\odot$ & -578 & -63 & -134 & $\odot$ & -22 \\
\hline$\%$ change & $\odot$ & -38 & -13 & -19 & $\odot$ & -45 \\
\hline \multicolumn{7}{|c|}{ Total (MBtu/1000ft2) } \\
\hline existing & $\odot$ & 33 & 10 & 15 & 19 & 1 \\
\hline post-retrofit & 0 & 20 & 9 & 13 & 19 & 1 \\
\hline difference & $\odot$ & -12 & -1 & -3 & 0 & 0 \\
\hline$\%$ change & $\odot$ & -38 & -13 & -19 & $\odot$ & -45 \\
\hline
\end{tabular}




\section{Appropriated Funding Emission Reduction}

The emission reductions from implemented the proposed retrofits are as follows:

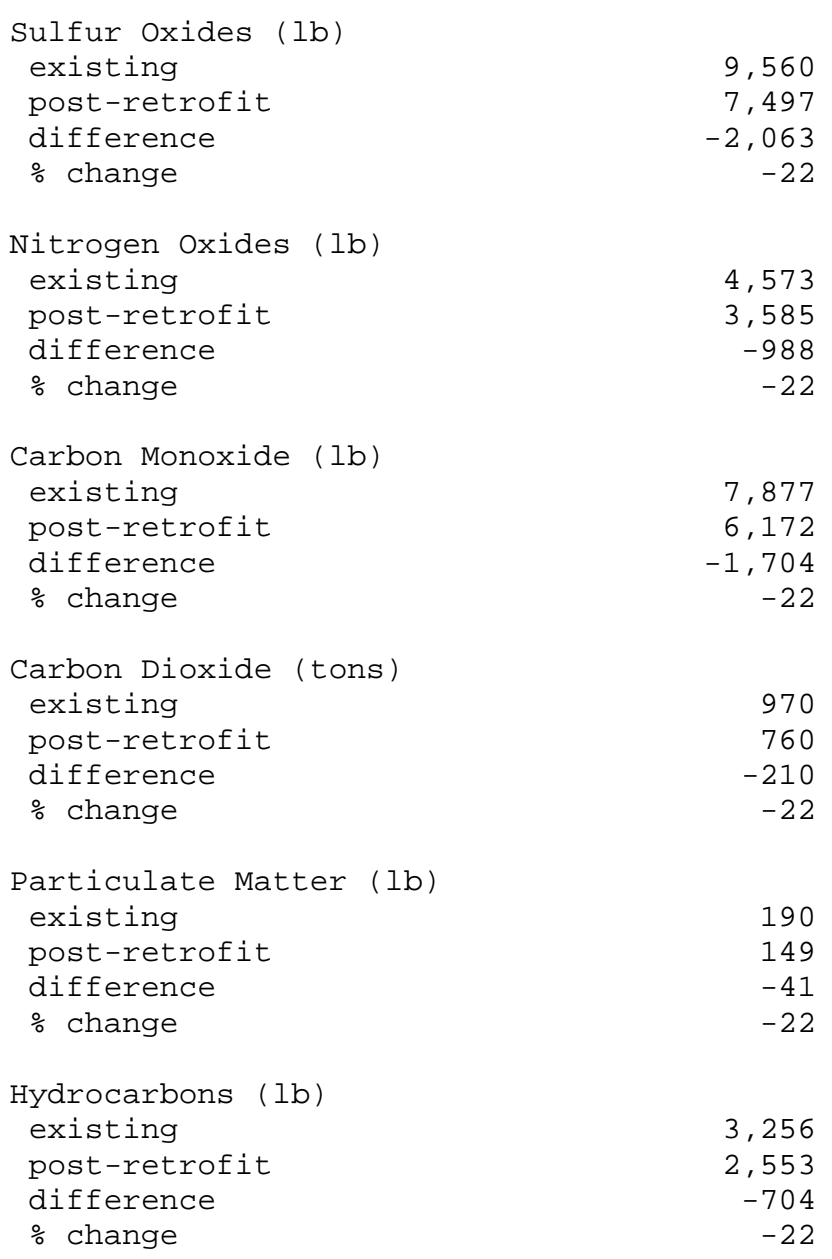




\section{Building 2003 Vehicle Maintenance Building}

The following information identifies the cost-effective energy- and cost-reducing retrofit projects for building 2003 identified from the FEDS modeling and analysis. Key energy and economic results are presented for each cost-effective retrofit measure.

\section{Facility Description 2003}

2003 is a vehicle maintenance administration facility built in 1994. Building 2003 is conditioned by an electric package unit, is lit by 32 watt fluorescent T8's and has little to no insulation in its building envelope. Building 2003 is 6,848 sf. 


\section{Appropriated Funding Results}

A FEDS analysis using appropriated funding suggests replacing the fluorescent lighting, the EXIT lighting, increasing the insulation in the roof and replacing the electric water heater with a heat pump water heater.

Appropriated funding FEDS results for building 2003:

\begin{tabular}{|c|c|c|c|c|c|c|c|c|}
\hline Bldg. Set Description & $\begin{array}{l}\text { End } \\
\text { Use }\end{array}$ & Existing Technology & Retrofit Technology & $\begin{array}{l}\text { Energy } \\
\text { Savings } \\
\text { (MMBtu/yr) }\end{array}$ & $\begin{array}{c}\text { 1st year } \\
\text { savings } \\
(\$ / y r)\end{array}$ & $\begin{array}{l}\text { Installed } \\
\text { Cost (\$) }\end{array}$ & $\begin{array}{l}\text { Net } \\
\text { Present } \\
\text { Value (\$) } \\
\end{array}$ & SIR \\
\hline Small 1990's admin & Lights & FL39: FL 2X4 2F32T8 EEF2 & $\begin{array}{l}\text { FL303: FL 2X4 2F25ST8 ELC2 } \\
\text { REF }\end{array}$ & 98 & 5,891 & 14,843 & 83,966 & 6.7 \\
\hline Small 1990's admin & Lights & EX6: EXIT - LED & $\begin{array}{l}\text { EX12: EXIT - } \\
\text { ELECTROLUMINESCENT PANEL } \\
\text { RETRO KIT }\end{array}$ & 1 & 113 & 621 & 1,318 & 3.1 \\
\hline Small 1990's admin & Lights & FL41: FL 1X4 1F32T8 EEF1 & $\begin{array}{l}\text { FL302: FL 1X4 1F25ST8 ELC1 } \\
\text { REF }\end{array}$ & 4 & 324 & 2,310 & 3,185 & 2.4 \\
\hline Small 1990's admin & $\begin{array}{l}\text { Hot } \\
\text { Water }\end{array}$ & Electric Water Heater & $\begin{array}{l}\text { Heat Pump Water Heater } \\
\text { (Com) }\end{array}$ & 5 & 212 & 1,714 & 137 & 1.1 \\
\hline Small 1990's admin & Roof & Roof Insulation R-Value 0.00 & $\begin{array}{l}\text { Add Insulation to Interior } \\
\text { Surface of Metal Roof: } 4 \\
\text { inches Fiberglass }\end{array}$ & 299 & 16,487 & 23,407 & 250,910 & 11.7 \\
\hline
\end{tabular}




\section{Appropriated Funding Energy Consumption by Fuel Type}

The modeled energy consumption for a typical year was 234,145 kwh before retrofits and 120,033 kwh after proposed retrofits are implemented. The energy use intensity goes from 116.7 MBtu/Ksf to 59.8 MBtu/Ksf after retrofits.

Small 1990's admin 2003

Fuel

Energy

Electricity (kWh)

existing

post-retrofit

difference

$\%$ change

Total (MBtu)

existing

post-retrofit

difference

$\%$ change

234,145
120,033
$-114,112$
-49

799
410
-389
-49

$$
\begin{gathered}
\text { Energy } \\
\text { Intensity }
\end{gathered}
$$$$
\text { (MBtu/1000ft2) }
$$

\section{Dollars}

$(2009)$ *

$34,191.8$

$16,663.6$

$-49$

116.7

59.8

$-56.9$

$-49$

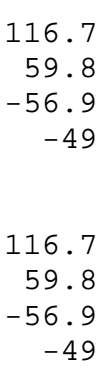

116.7
59.8

56.9

41,507

21,118

$-20,389$

$-49$

41,507

21,118

$-20,389$

* Dollar values for electricity include both energy and demand components. 


\section{Appropriated Funding Energy Consumption by End Use}

Space cooling is the largest load in the building with $91,143 \mathrm{kWh} /$ year, followed by ventilation with $56,887 \mathrm{kWh} / \mathrm{year}$.

\begin{tabular}{|c|c|c|c|c|c|c|}
\hline \multirow[b]{2}{*}{ Fuel } & \multirow[b]{2}{*}{ Heating } & \multicolumn{3}{|c|}{ Small 1990 's admin 2003} & \multirow[b]{2}{*}{$\begin{array}{l}\text { Motors and } \\
\text { Misc Equip }\end{array}$} & \multirow[b]{2}{*}{ Hot Water } \\
\hline & & Cooling & Vent & Lights & & \\
\hline \multicolumn{7}{|c|}{ Electricity (kWh) } \\
\hline existing & 0 & 91,143 & 56,887 & 56,227 & 28,339 & 1,550 \\
\hline post-retrofit & 0 & 34,654 & 21,727 & 35,104 & 28,339 & 209 \\
\hline difference & $\odot$ & $-56,490$ & $-35,159$ & $-21,122$ & 0 & $-1,341$ \\
\hline$\%$ change & 0 & -62 & -62 & -38 & 0 & -87 \\
\hline \multicolumn{7}{|l|}{ Total (MBtu) } \\
\hline existing & 0 & 311 & 194 & 192 & 97 & 5 \\
\hline post-retrofit & 0 & 118 & 74 & 120 & 97 & 1 \\
\hline difference & $\odot$ & -193 & -120 & -72 & 0 & -5 \\
\hline \% change & 0 & -62 & -62 & -38 & 0 & -87 \\
\hline \multicolumn{7}{|c|}{ Total (MBtu/100๑ft2) } \\
\hline existing & 0 & 45 & 28 & 28 & 14 & 1 \\
\hline post-retrofit & $\odot$ & 17 & 11 & 17 & 14 & 0 \\
\hline difference & 0 & -28 & -18 & -11 & 0 & -1 \\
\hline$\%$ change & $\Theta$ & -62 & -62 & -38 & 0 & -87 \\
\hline
\end{tabular}




\section{Appropriated Funding Emission Reduction}

The emission reductions from implemented the proposed retrofits are as follows:

Small 1990's admin 2003

Sulfur Oxides (lb)

existing

post-retrofit

difference

2,117

1,085

$-1,032$

$\%$ change

$-49$

Nitrogen Oxides (lb)

existing

post-retrofit

difference

$\%$ change

1,011

519

$-493$

Carbon Monoxide (lb)

existing

post-retrofit

difference

1,740

892

$-848$

$\%$ change

$-49$

Carbon Dioxide (tons)

existing

post-retrofit

110

$-104$

differenc

$-49$

Particulate Matter (lb)

existing

post-retrofit

42

difference

21

$\%$ change

$-49$

Hydrocarbons (1b)

existing

post-retrofit

720

difference

369

$\%$ change

(




\section{Appendix D-2}

\section{Energy Conservation Measures for Individual Buildings Alternative Financing}

The following information identifies the cost-effective energy- and cost-reducing retrofit projects using alternative financing for the buildings visited during the FEDS modeling and analysis. Key energy and economic results are presented for each cost-effective retrofit measure 


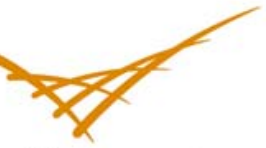




\section{Building 2186 Storage Building}

The following information identifies the cost-effective energy- and cost-reducing retrofit projects for building 2186 identified from the FEDS modeling and analysis. Key energy and economic results are presented for each cost-effective retrofit measure.

\section{Facility Description 2186}

Building 2186 is overhead storage for landscaping equipment built in 1986. 2186 has some lighting but no cooling or building envelope. Building 2186 is 2,125 sf.

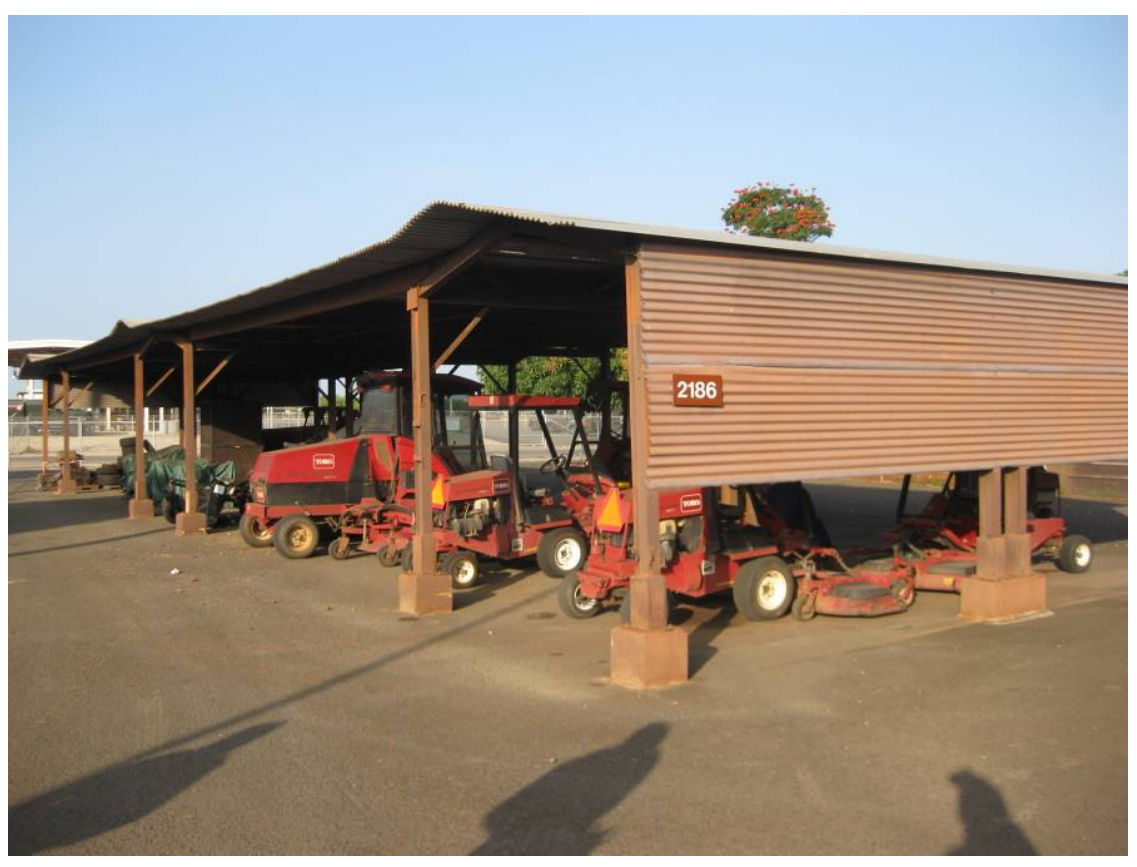




\section{Alternative Financing Results}

FEDS did not find any life cycle cost effective retrofits using alternative financing.

\section{Alternative Financing Energy Consumption by Fuel Type}

The modeled energy consumption for a typical year was 2,451 kwh before retrofits and 2,451 kwh after proposed retrofits are implemented. The energy use intensity goes from 3.9 MBtu/Ksf to 3.9 MBtu/Ksf after retrofits.

Covered lighting 2186

Fuel

Electricity (kWh)

existing

post-retrofit

difference

$\%$ change

Total (MBtu)

existing

post-retrofit

difference

\% change
Energy

2,451

2,451$$
\odot
$$

8

8

$\odot$

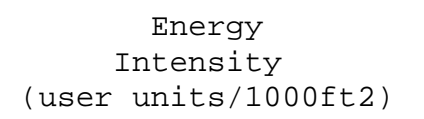

(user units/1000ft2)

$$
\begin{array}{r}
1,153.2 \\
1,153.2 \\
0.0
\end{array}
$$

3.9

3.9
$\odot .0$

$\odot$

$\begin{array}{ll}\text { Energy } & \text { Dollars } \\ \text { Intensity } & (2009)^{*}\end{array}$

3.9

3. 9

0.0

3.9

3.9

$\odot . \odot$
$(2009)^{*}$

434

433

-1
0

434

433

$-1$

* Dollar values for electricity include both energy and demand components. 


\section{Alternative Financing Energy Consumption by End Use}

Annual Energy Use by Building Set, Fuel Type, and End Use $\begin{array}{cc}\text { Building Set } \ldots & 1 \\ \text { Covered lighting } 2186\end{array}$

Fuel

Heating

Cooling

Electricity (kWh)

existing

post-retrofit

difference

$\%$ change

Total (MBtu)

existing

post-retrofit

difference

$\%$ change

Total (MBtu/1000ft2)

existing

post-retrofit

difference

$\%$ change
Vent

Lights

Misc Equip

2,451

2,451

0
$\odot$

8

8

$\odot$

4
4
$\odot$
$\odot$
Hot Water 


\section{Alternative Financing Emission Reduction}

The emission reductions from implemented the proposed retrofits are as follows:

Covered lighting 2186

Sulfur Oxides (lb)

existing

post-retrofit

difference

$\%$ change

Nitrogen 0xides (lb)

existing

post-retrofit

difference

$\%$ change

Carbon Monoxide (lb)

existing

post-retrofit

difference

$\%$ change

18

18

$\odot$

Carbon Dioxide (tons)

existing

post-retrofit

difference

$\%$ change

Particulate Matter (lb)

existing

post-retrofit

difference

$\%$ change

Hydrocarbons ( $1 b$ )

existing

post-retrofit

difference

$\%$ change 


\section{Building 2035 Hanger}

The following information identifies the cost-effective energy- and cost-reducing retrofit projects for building 2035 identified from the FEDS modeling and analysis. Key energy and economic results are presented for each cost-effective retrofit measure.

\section{Facility Description 2035}

Building 2035 is a hangar with two high-bay spaces originally built in 1937. One of the high-bays has been converted to office space where an administration building has been built inside the hanger. This building inside a building is cooled by air cooled chillers and receives little to no solar radiation. The other high-bay is used to store and transport aircraft parts and has a small office space served by an electric DX, or package unit. Building 2035 is 86,391 sf.

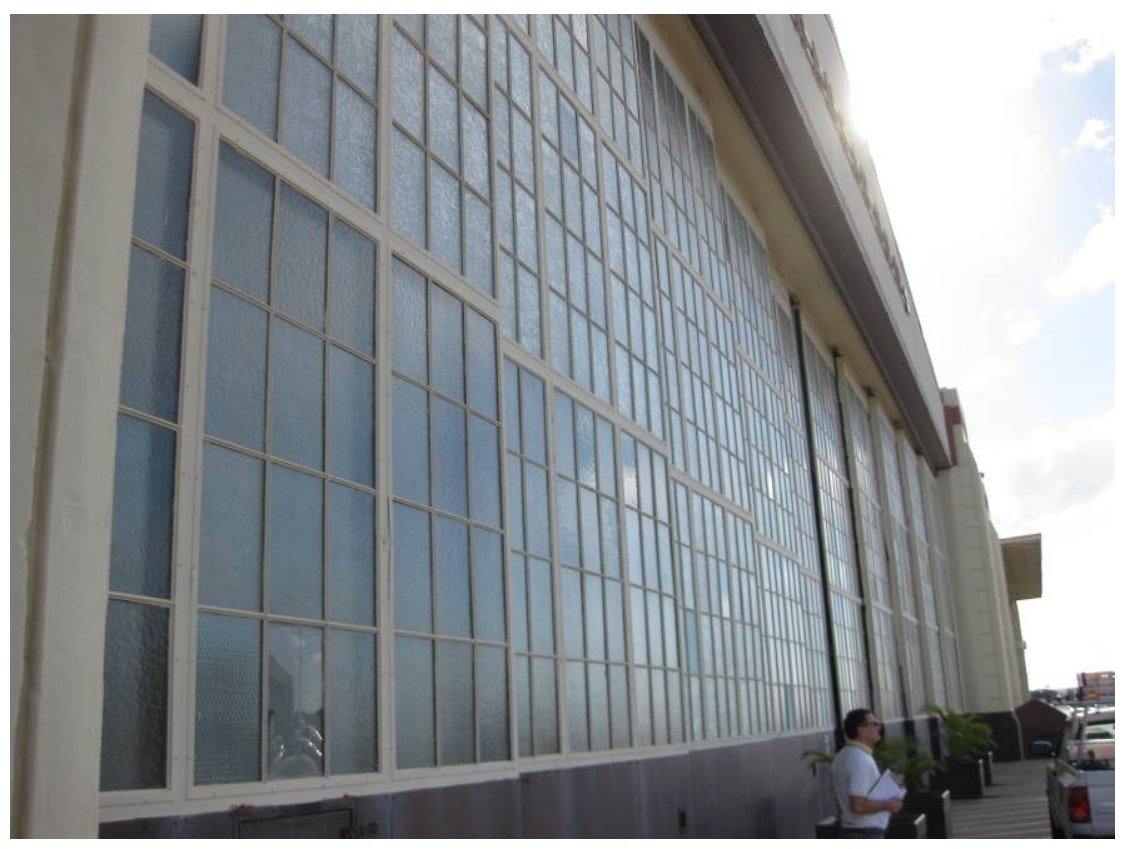




\section{Alternative Financing Results}

A FEDS analysis using alternative financing for hangar 13 suggests replacing the air cooled chiller with a standard efficiency water cooled reciprocating chiller, replacing the incandescent lights with CFLs, installing aerators, and replacing LED EXIT signs with electroluminescent signs. The FEDS analysis suggests for hangar 11 installing aerators, lowering the hot water tank temperature, replacing LED EXIT lights with electroluminescent signs, T12 lights and metal halide lights.

Alternative financing FEDS results for building 2035 hangar 13:

\begin{tabular}{|c|c|c|c|c|c|c|c|c|}
\hline Bldg. Set Description & $\begin{array}{l}\text { End } \\
\text { Use } \\
\end{array}$ & Existing Technology & Retrofit Technology & $\begin{array}{l}\text { Energy } \\
\text { Savings } \\
\text { (MMBtu/yr) }\end{array}$ & $\begin{array}{l}\text { 1st Year } \\
\text { Savings } \\
(\$ / y r)\end{array}$ & $\begin{array}{l}\text { Installed } \\
\text { Cost (\$) }\end{array}$ & $\begin{array}{l}\text { Net } \\
\text { Present } \\
\text { Value (\$) } \\
\end{array}$ & SIR \\
\hline $\begin{array}{l}\text { Large } 1930 \text { 's admin space } \\
2035 \text { hangar } 13\end{array}$ & Cooling & $\begin{array}{l}\text { Electric Air-Cooled Chiller } \\
\{\mathrm{C} 1\}\end{array}$ & $\begin{array}{l}\text { Water-Cooled Reciprocating } \\
\text { Electric Chiller (standard } \\
\text { efficiency) and Cooling Tower }\end{array}$ & 227 & 14,916 & 85,130 & 1,520 & 1.0 \\
\hline $\begin{array}{l}\text { Large } 1930 \text { 's admin space } \\
2035 \text { hangar } 13\end{array}$ & $\begin{array}{l}\text { Hot } \\
\text { Water }\end{array}$ & Electric Water Heater & Faucet Aerators & 7 & 392 & 79 & 2,169 & 28.6 \\
\hline $\begin{array}{l}\text { Large } 1930 \text { 's admin space } \\
2035 \text { hangar } 13\end{array}$ & Lights & IN8: INC 75 CEIL & $\begin{array}{l}\text { CF5: CFL } 18 \text { INTEGRAL UNIT } \\
\text { ELC }\end{array}$ & 322 & 21,166 & 3,373 & 119,968 & 36.6 \\
\hline $\begin{array}{l}\text { Large } 1930 \text { 's admin space } \\
2035 \text { hangar } 13\end{array}$ & Lights & EX6: EXIT - LED & $\begin{array}{l}\text { EX12: EXIT - } \\
\text { ELECTROLUMINESCENT PANEL } \\
\text { RETRO KIT }\end{array}$ & 2 & 361 & 1,864 & 323 & 1.2 \\
\hline
\end{tabular}


Alternative financing FEDS results for building 2035 hangar 11:

\begin{tabular}{|c|c|c|c|c|c|c|c|c|}
\hline Bldg. Set Description & $\begin{array}{l}\text { End } \\
\text { Use }\end{array}$ & Existing Technology & Retrofit Technology & $\begin{array}{l}\text { Energy } \\
\text { Savings } \\
\text { (MMBtu/yr) }\end{array}$ & $\begin{array}{c}\text { 1st Year } \\
\text { Savings } \\
\text { (\$/yr) }\end{array}$ & $\begin{array}{l}\text { Installed } \\
\text { Cost (\$) }\end{array}$ & $\begin{array}{l}\text { Net } \\
\text { Present } \\
\text { Value (\$) }\end{array}$ & SIR \\
\hline $\begin{array}{l}1930 \text { 's wharehouse space } \\
\text { hangar } 11\end{array}$ & $\begin{array}{l}\text { Hot } \\
\text { Water }\end{array}$ & Electric Water Heater & $\begin{array}{l}\text { Faucet Aerators, Lower Tank } \\
\text { Temperature }\end{array}$ & - & 3 & 2 & 16 & 9.0 \\
\hline $\begin{array}{l}1930 \text { 's wharehouse space } \\
\text { hangar } 11\end{array}$ & Lights & FL1: FL 2X4 4F40T12 STD2 & FL244: FL 2X4 4F32T8 ELC4 & 6 & 446 & 1,140 & 1,466 & 2.3 \\
\hline $\begin{array}{l}\text { 1930's wharehouse space } \\
\text { hangar } 11\end{array}$ & Lights & MH4: MH 175 PEND & $\begin{array}{l}\text { FL289: FL 2X4 4F30ST8 ELC2 } \\
\text { REF }\end{array}$ & 6 & 581 & 2,111 & 1,321 & 1.6 \\
\hline
\end{tabular}




\section{Alternative Financing Energy Consumption by Fuel Type}

The modeled energy consumption for hangar 13 for a typical year was 795,887 kwh before retrofits and 624,153 kwh after proposed retrofits are implemented. The energy use intensity goes from $62.9 \mathrm{MBtu} / \mathrm{Ksf}$ to $49.3 \mathrm{MBtu} / \mathrm{Ksf}$ after retrofits.

Large $1930^{\prime}$ 's admin space 2035 hangar 13

Fuel

Electricity ( kWh)

existing

post-retrofit

difference

$\%$ change

Total (MBtu)

existing

post-retrofit

difference

$\%$ change
Energy

795,887

624,153

$-171,734$

$-22$

\section{2,716}

2,130

-586
-22
Energy

Intensity

(user units/1000ft2)

$18,425.9$

$14,450.0$

$-3,975.9$

$-22$

62.9

49.3

$-13.6$

$-22$
Energy

Intensity

(MBtu/1000ft2)

Dollars

$(2009)$ *

141,004

$\begin{array}{rr}49.3 & 110,284 \\ -13.6 & -30,720 \\ -22 & -22\end{array}$

$\begin{array}{rr}49.3 & 110,284 \\ -13.6 & -30,720 \\ -22 & -22\end{array}$

$-22$

141,004

110,284

$-30,720$

49.3

$-13.6$

$-22$

* Dollar values for electricity include both energy and demand components. 
The modeled energy consumption for hangar 11 for a typical year was 62,619 kwh before retrofits and 58,981 kwh after proposed retrofits are implemented. The energy use intensity goes from 4.9 MBtu/Ksf to 4.7 MBtu/Ksf after retrofits.

1930 's warehouse space hangar 11

Fuel

Electricity $(\mathrm{kWh})$

existing

post-retrofit

difference

$\%$ change

Total (MBtu)

existing

post-retrofit

difference

$\%$ change
Energy

62,619

58,981

$-3,638$

$-6$

214
201
-12
-6
Energy

Intensity

(user units/1000ft2)

$1,449.6$

$1,365.4$

$-84.2$

4. 9

$-0.3$

$-6$
Energy

Intensity (MBtu/1000ft2)

$\begin{array}{rr}4.9 & 11,094 \\ 4.7 & 10,422 \\ -\odot .3 & -672 \\ -6 & -6\end{array}$

$\begin{array}{rr}4.9 & 11,094 \\ 4.7 & 10,422 \\ -0.3 & -672 \\ -6 & -6\end{array}$

* Dollar values for electricity include both energy and demand components. 


\section{Alternative Financing Energy Consumption by End Use}

Annual Energy Use by Building Set, Fuel Type, and End Use Large $1930^{\prime}$ 's admin space 2035 hangar 13

Fuel Heating
Electricity (kWh)
existing
post-retrofit
difference
$\%$ change
Total (MBtu)
existing
post-retrofit
difference
$\%$ change
Total (MBtu/1000ft2)
existing
post-retrofit
difference
$\%$ change

$\begin{array}{lr} & \\ & \\ \odot & \\ \odot & 253,896 \\ \odot & 159,419 \\ \odot & -94,477 \\ \odot & -37 \\ & \\ \odot & 867 \\ \odot & 544 \\ \odot & -322 \\ \odot & -37 \\ & \\ \odot & 20 \\ \odot & 13 \\ \odot & -7 \\ \odot & -37\end{array}$

Vent
69,266
60,916
8,350
-12

236
208
-28
-12


5
5
-1
-12

Lights

Motors and

Misc Equip

Hot Water

226,334
159,538
$-66,796$
-30

772
545
-228
-30

18
13
-5
-30

240,705

240,705

$\odot$

5,687

3,575

$-2,112$
-37

$\begin{array}{rr}822 & 19 \\ 822 & 12 \\ 0 & -7 \\ 0 & -37\end{array}$

19

19
19
0

0
0
0
-37 
Annual Energy Use by Building Set, Fuel Type, and End Use Large 1930 's warehouse space 2035 hangar 11

Fuel

Electricity (kWh)

existing

post-retrofit

difference

$\%$ change

Total (MBtu)

existing

post-retrofit

difference

$\%$ change

Total (MBtu/1000ft2)

existing

post-retrofit

difference

$\%$ change
Heating

Cooling

Vent

Lights

Motors and

Misc Equip

Hot Water *

53,825
50,215
$-3,611$

$-3,611$
-7

171

$\odot$

$\odot$

$\odot$
0

0

$\odot$

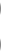

0

$\odot$
-12
-7

$\begin{array}{rr}8,705 & 88 \\ 8,705 & 61 \\ 0 & -27 \\ 0 & -31\end{array}$

30

30
0

0

$\odot$
0

$-31$

4
4
$\odot$
-7

1

1
0

$\odot$ 


\section{Alternative Financing Emission Reduction}

The emission reductions from implemented the proposed retrofits are as follows:

Large 1930 's admin space 2035 hangar 13

Sulfur Oxides (lb)

existing

post-retrofit

7,195

difference

5,642

$\%$ change

$-1,552$
-22

Nitrogen oxides (lb)

existing

post-retrofit

difference

3,438

2,696

$-742$

$\%$ change

$-22$

Carbon Monoxide (lb)

existing

post-retrofit

5,914

difference

4,638

$\%$ change

$-1,276$
-22

Carbon Dioxide (tons)

existing

post-retrofit

728

difference

571

$\%$ change

$-157$

$-22$

Particulate Matter (lb)

existing

post-retrofit

142

difference

112

$\%$ change

-31
-22

Hydrocarbons (lb)

existing

post-retrofit

2,447

1,919

$\%$ change

-528
-22 
Sulfur 0xides (lb)

existing

566

post-retrofit

533

difference

$-33$

$\%$ change

271

255

$-16$

ting

post-retrofit

$\%$ change

465

438
-27

existing

post-retrofit

difference

$-6$

Carbon Dioxide (tons)

existing

post-retrofit

difference

$\%$ change

54

-3
-6

Particulate Matter (lb)

existing

post-retrofit

difference

$\%$ change

11

11

$-1$

Hydrocarbons (1b)

existing

193

post-retrofit

difference

$\%$ change

-11
-6 


\section{Building 1204 Administration Building}

The following information identifies the cost-effective energy- and cost-reducing retrofit projects for building 1204 identified from the FEDS modeling and analysis. Key energy and economic results are presented for each cost-effective retrofit measure.

\section{Facility Description 1204}

Building 1204 is a small admin building built in 1939. This building is served by an air cooled chiller and has little to no insulation in its building envelope. Building 1204 is 11,374 sf.

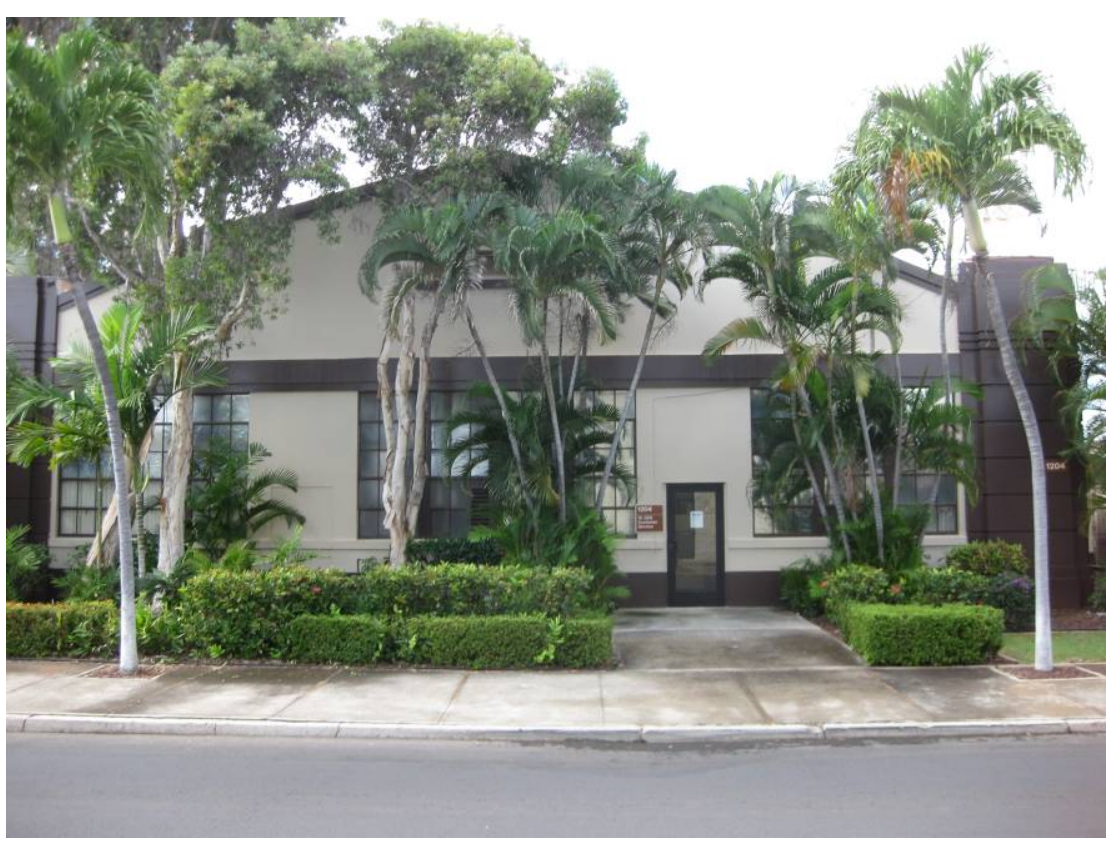




\section{Alternative Financing Results}

A FEDS analysis using alternative financing suggests replacing 32W T8 lighting with 25W Super T8 lighting.

Alternative financing FEDS results for building 1204:

\begin{tabular}{|c|c|c|c|c|c|c|c|c|}
\hline Bldg. Set Description & $\begin{array}{l}\text { End } \\
\text { Use }\end{array}$ & Existing Technology & Retrofit Technology & $\begin{array}{l}\text { Energy } \\
\text { Savings } \\
\text { (MMBtu/yr) }\end{array}$ & $\begin{array}{c}\text { 1st Year } \\
\text { Savings } \\
(\$ / y r)\end{array}$ & $\begin{array}{l}\text { Installed } \\
\text { Cost (\$) }\end{array}$ & $\begin{array}{l}\text { Net } \\
\text { Present } \\
\text { Value (\$) } \\
\end{array}$ & SIR \\
\hline Small 1040's admin 1204 & Lights & FL39: FL 2X4 2F32T8 EEF2 & $\begin{array}{l}\text { FL303: FL 2X4 2F25ST8 ELC2 } \\
\text { REF }\end{array}$ & 62 & 5,156 & 25,464 & 4,841 & 1.2 \\
\hline
\end{tabular}

\section{Alternative Financing Energy Consumption by Fuel Type}

The modeled energy consumption for a typical year was 250,798 kwh before retrofits and 232,667 kwh after proposed retrofits are implemented. The energy use intensity goes from $75.3 \mathrm{MBtu} / \mathrm{Ksf}$ to $69.8 \mathrm{MBtu} / \mathrm{Ksf}$ after retrofits.

Small 1040 's admin 1204

\begin{tabular}{|c|c|c|c|c|}
\hline Fuel & Energy & $\begin{array}{c}\text { Energy } \\
\text { Intensity } \\
\text { (user units/1000ft2) }\end{array}$ & $\begin{array}{l}\text { Energy } \\
\text { Intensity } \\
\text { (MBtu/1000ft2) }\end{array}$ & $\begin{array}{l}\text { Dollars } \\
(2009) \text { * }\end{array}$ \\
\hline \multicolumn{5}{|c|}{ Electricity (kWh) } \\
\hline existing & 250,798 & $22,050.1$ & 75.3 & 44,433 \\
\hline post-retrofit & 232,667 & $20,456.1$ & 69.8 & 41,111 \\
\hline difference & $-18,131$ & $-1,594.0$ & -5.4 & $-3,322$ \\
\hline$\%$ change & -7 & -7 & -7 & -7 \\
\hline \multicolumn{5}{|l|}{ Total (MBtu) } \\
\hline existing & 856 & 75.3 & 75.3 & 44,433 \\
\hline post-retrofit & 794 & 69.8 & 69.8 & 41,111 \\
\hline difference & -62 & -5.4 & -5.4 & $-3,322$ \\
\hline$\%$ change & -7 & -7 & -7 & -7 \\
\hline
\end{tabular}

* Dollar values for electricity include both energy and demand components. 


\section{Alternative Financing Energy Consumption by End Use}

Space cooling is the largest load in the building with $83,381 \mathrm{kWh} /$ year, followed by ventilation with $72,592 \mathrm{kWh} / \mathrm{year}$.

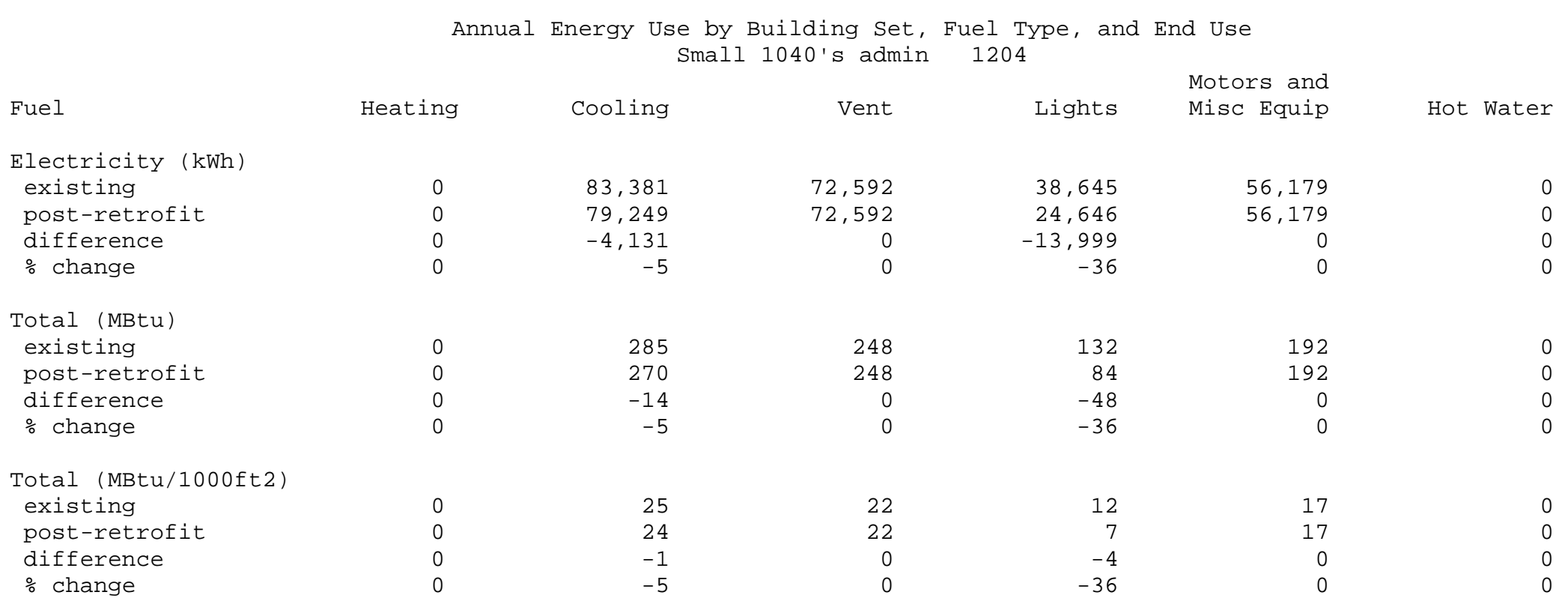




\section{Alternative Financing Emission Reduction}

The emission reductions from implemented the proposed retrofits are as follows:

Small $1040^{\prime} \mathrm{s}$ admin

Sulfur Oxides (lb)

existing

post-retrofit

difference

$\%$ change

$-164$

Nitrogen Oxides (lb)

existing

post-retrofit

difference

, 083

1,005

$\%$ change

-78
-7

Carbon Monoxide (lb)

existing

post-retrofit

1,863

difference

1,729

$\%$ change

135
-7

Carbon Dioxide (tons)

existing

post-retrofit

229

difference

213

$\%$ change

$-17$

Particulate Matter (lb)

existing

post-retrofit

difference

$\%$ change

Hydrocarbons (lb)

existing

post-retrofit

difference

$\%$ change 


\section{Building 2155 Adminstration Building}

The following information identifies the cost-effective energy- and cost-reducing retrofit projects for building 2155 identified from the FEDS modeling and analysis. Key energy and economic results are presented for each cost-effective retrofit measure.

\section{Facility Description 2155}

Building 2155 is a weapons systems management facility built in 1968. This building is cooled by a DX, or package unit and has little to no insulation in the building envelope. Building 2155 is 21,745 sf.

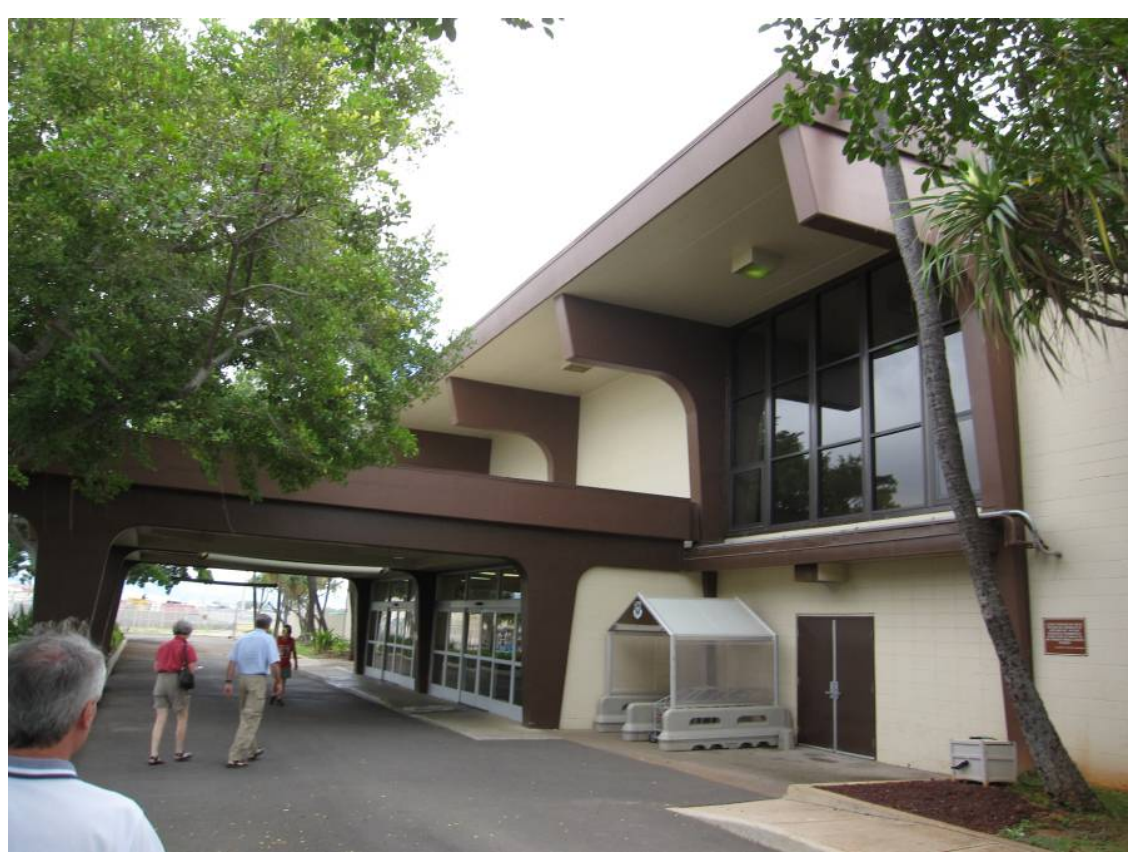




\section{Alternative Financing Results}

A FEDS analysis using alternative financing suggests replacing several lighting technologies.

Alternative financing FEDS results for building 2155:

\begin{tabular}{|c|c|c|c|c|c|c|c|c|}
\hline Bldg. Set Description & $\begin{array}{l}\text { End } \\
\text { Use }\end{array}$ & Existing Technology & Retrofit Technology & $\begin{array}{l}\text { Energy } \\
\text { Savings } \\
\text { (MMBtu/yr) }\end{array}$ & $\begin{array}{l}\text { 1st Year } \\
\text { Savings } \\
\text { (\$/yr) }\end{array}$ & $\begin{array}{l}\text { Installed } \\
\text { Cost (\$) }\end{array}$ & $\begin{array}{l}\text { Net } \\
\text { Present } \\
\text { Value (\$) } \\
\end{array}$ & SIR \\
\hline medium 1960's admin 2155 & Lights & EX1: EXIT - INC (2x20) & $\begin{array}{l}\text { EX12: EXIT - } \\
\text { ELECTROLUMINESCENT PANEL } \\
\text { RETRO KIT }\end{array}$ & 13 & 855 & 621 & 4,382 & 8.1 \\
\hline medium 1960's admin 2155 & Lights & FL51: FL 2X4 2F32T8 ELC2 & $\begin{array}{l}\text { FL303: FL 2X4 2F25ST8 ELC2 } \\
\text { REF }\end{array}$ & 23 & 1,494 & 7,449 & 1,288 & 1.2 \\
\hline medium 1960's admin 2155 & Lights & FL4: FL 1X4 2F40T12 STD2 & FL52: FL 1X4 2F32T8 ELC2 & 3 & 186 & 451 & 636 & 2.4 \\
\hline
\end{tabular}




\section{Alternative Financing Energy Consumption by Fuel Type}

The modeled energy consumption for a typical year was 314,110 kwh before retrofits and 302,889 kwh after proposed retrofits are implemented. The energy use intensity goes from 49.3 MBtu/Ksf to 47.5 MBtu/Ksf after retrofits.

Medium $1960^{\prime}$ s admin 2155

Fuel

Electricity $(\mathrm{kWh})$

existing

post-retrofit

difference

$\%$ change

Total (MBtu)

existing

post-retrofit

difference

$\%$ change
Energy

314,110

302,889

$-11,221$

\section{1,072}

1,034

-38
-4
Energy

Intensity

(user units/1000ft2)

$14,445.2$

$13,929.1$

$-516.0$

\section{3}

47.5

-1.8
-4
Energy

Intensity (MBtu/1000ft2)

49.3

47.5

$-1.8$

49.3

47.5

$-1.8$

$-4$
Dollars

$(2009)$ *

55,650

53,519

$-2,131$

55,650

53,519

$-2,131$

* Dollar values for electricity include both energy and demand components. 


\section{Alternative Financing Energy Consumption by End Use}

Motors and miscellaneous equipment is the largest load in the building with 145,710 kWh/year, followed by space cooling with 92,995 kWh/year.

Annual Energy Use by Building Set, Fuel Type, and End Use medium 1960's admin 2155

Fuel
Electricity (kWh)
existing
post-retrofit
difference
$\%$ change
Total (MBtu)
existing
post-retrofit
difference
\% change
Total (MBtu/1000ft2)
existing
post-retrofit
difference
$\%$ change

$\begin{array}{rr}\text { Heating } & \text { Cooling } \\ & \\ 0 & 92,995 \\ 0 & 90,499 \\ 0 & -2,496 \\ 0 & -3 \\ & \\ 0 & 317 \\ 0 & 309 \\ 0 & -9 \\ 0 & -3 \\ & \\ 0 & 15 \\ 0 & 14 \\ 0 & 0 \\ 0 & -3\end{array}$

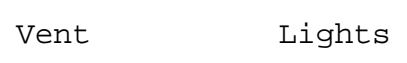

52,915
52,799
-116

$-116$

181

180

○

\section{Lights}

22,491
13,881
$-8,610$

$-38$

77
47
-29

$-38$
8
8
0
0
Motors and Misc Equip

145,710

145,710

$\odot$

497

497

$\odot$
$\odot$

23
23
9

$\odot$
Hot Water

0
0
0
0


0
0
0
0

$\odot$

0 


\section{Alternative Financing Emission Reduction}

The emission reductions from implemented the proposed retrofits are as follows:

medium 1960 's admin 2155

Sulfur Oxides (lb)

existing

post-retrofit

difference

2,840

2,738

$\%$ change

-101
-4

Nitrogen 0xides (lb)

existing

post-retrofit

difference

1,357

1,308

$\%$ change

$-48$

Carbon Monoxide (lb)

existing

post-retrofit

difference

2,334

$\%$ change

Carbon Dioxide (tons)

existing

post-retrofit

287

difference

277

$\%$ change

$-10$

Particulate Matter (lb)

existing

post-retrofit

difference

$\%$ change

Hydrocarbons (lb)

existing

post-retrofit

difference

931

$\%$ change

-35
-4 


\section{Building 502 Law Office}

The following information identifies the cost-effective energy- and cost-reducing retrofit projects for building 502 identified from the FEDS modeling and analysis. Key energy and economic results are presented for each cost-effective retrofit measure.

\section{Facility Description 502}

Building 502 is a small law office building built in 1971 that is served by two separate electric DX units with a courtroom in the center of the office space. Building 502 is 9,217 sf.

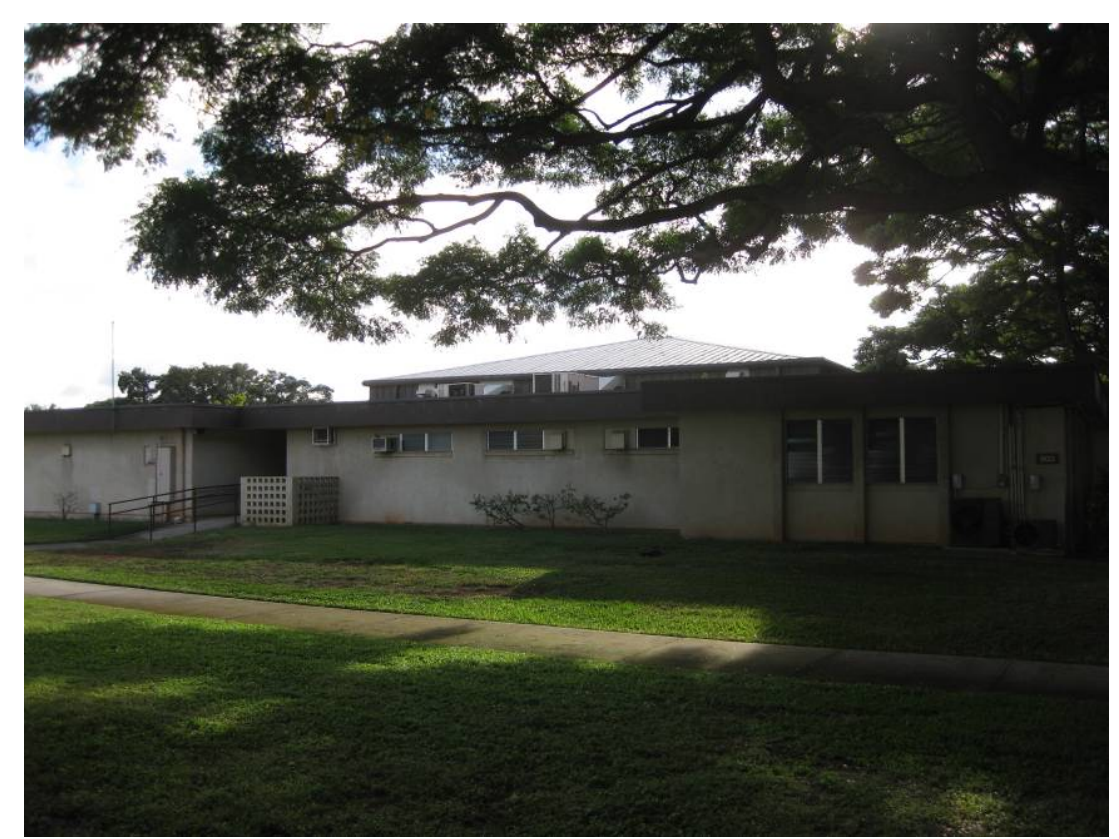




\section{Alternative Financing Results}

A FEDS analysis using alternative financing suggests increasing the insulation in the suspended ceiling as well as replacing the EXIT lighting.

Alternative financing FEDS results for building 502:

\begin{tabular}{|c|c|c|c|c|c|c|c|c|}
\hline Bldg. Set Description & $\begin{array}{l}\text { End } \\
\text { Use }\end{array}$ & Existing Technology & Retrofit Technology & $\begin{array}{l}\text { Energy } \\
\text { Savings } \\
\text { (MMBtu/yr) }\end{array}$ & $\begin{array}{l}\text { 1st Year } \\
\text { Savings } \\
(\$ / y r)\end{array}$ & $\begin{array}{l}\text { Installed } \\
\text { Cost (\$) }\end{array}$ & $\begin{array}{l}\text { Net } \\
\text { Present } \\
\text { Value (\$) } \\
\end{array}$ & SIR \\
\hline small 1960s admin 502 & Lights & EX1: EXIT - INC (2x20) & $\begin{array}{l}\text { EX12: EXIT - } \\
\text { ELECTROLUMINESCENT PANEL } \\
\text { RETRO KIT }\end{array}$ & 13 & 880 & 621 & 4,530 & 8.3 \\
\hline small 1960s admin 502 & Roof & Roof Insulation R-Value 0.00 & $\begin{array}{l}\text { Suspended Ceiling: Increase } \\
\text { Insulation by R-11 }\end{array}$ & 45 & 2,764 & 12,142 & 3,725 & 1.3 \\
\hline
\end{tabular}




\section{Alternative Financing Energy Consumption by Fuel Type}

The modeled energy consumption for a typical year was 143,177 kwh before retrofits and 126,257 kwh after proposed retrofits are implemented. The energy use intensity goes from 53.0 MBtu/Ksf to 46.8 MBtu/Ksf after retrofits.

Small 1960s admin 502

Fuel

Electricity $(\mathrm{kWh})$

existing

post-retrofit

difference

$\%$ change

Total (MBtu)

existing

post-retrofit

difference

$\%$ change
Energy

143,177

126,257

$-16,920$

$-12$

489
431
-58
-12

$$
\text { Energy }
$$$$
\text { Intensity }
$$$$
\text { (user units/1000ft2) }
$$$$
\text { Intensity }
$$$$
\text { (MBtu/1000ft2) }
$$

Dollars

$(2009)$ *

$$
\begin{array}{r}
15,534.1 \\
13,698.3 \\
-1,835.7 \\
-12
\end{array}
$$
53.0
46.8
$-6.3$
$-12$

53.0
46.8
-6.3
-12

25,366

22,309

$-3,057$

53.0

46.8

$-6.3$

$-12$
25,366

22,309

$-3,057$

* Dollar values for electricity include both energy and demand components. 


\section{Alternative Financing Energy Consumption by End Use}

Space cooling is the largest load in the building with $58,890 \mathrm{kWh} /$ year, followed by motors and miscellaneous equipment with 45,525 $\mathrm{kWh} /$ year.

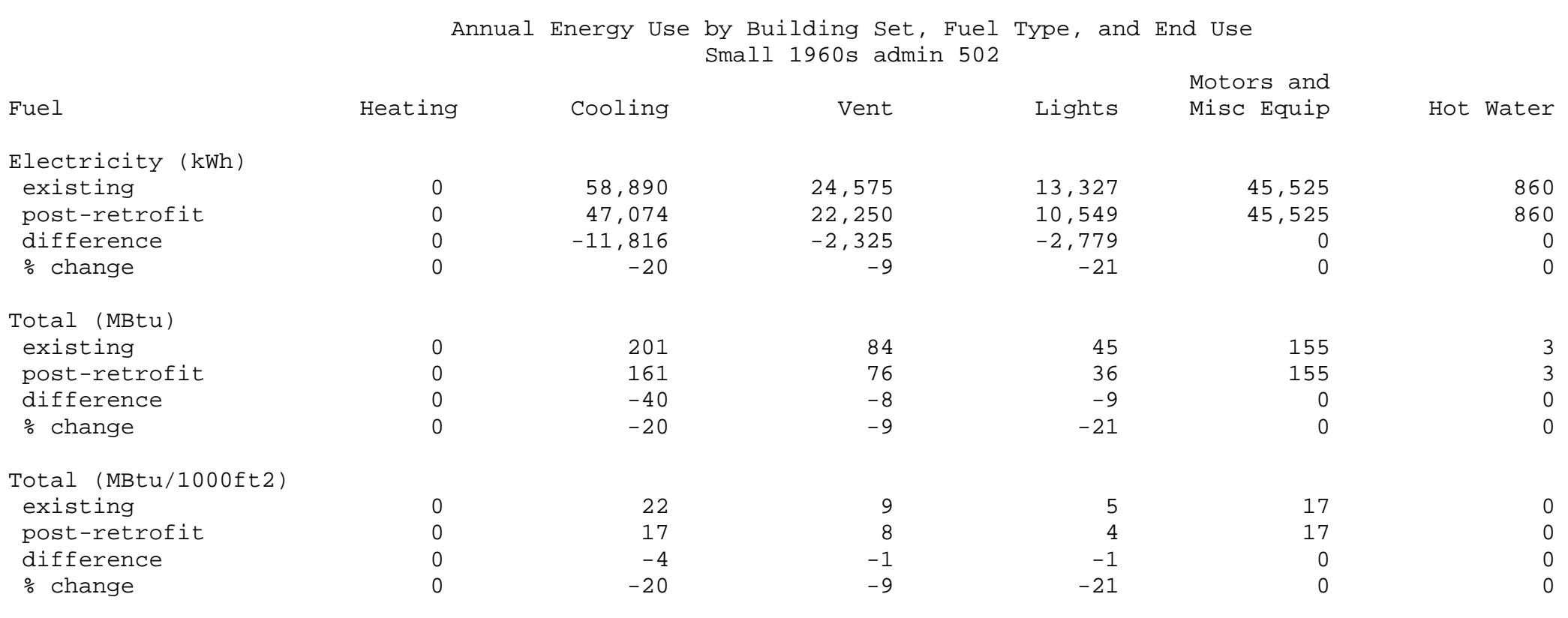




\section{Alternative Financing Emission Reduction}

The emission reductions from implemented the proposed retrofits are as follows:

Small 1960s admin 502

Sulfur Oxides (lb)

existing

post-retrofit

difference

1,294

1,141

$\%$ change

$-153$

$-12$

Nitrogen Oxides (lb)

existing

619

post-retrofit

545

difference

$-73$

$\%$ change

Carbon Monoxide (lb)

existing

post-retrofit

1,064

difference

$\%$ change

$-12$

Carbon Dioxide (tons)

existing

post-retrofit

difference

131

\% change

$-12$

Particulate Matter (lb)

existing

post-retrofit

difference

$\%$ change

Hydrocarbons (lb)

existing

post-retrofit 388

440

difference -52

$\%$ change

$-12$ 


\section{Building 2133 Administration Building}

The following information identifies the cost-effective energy- and cost-reducing retrofit projects for building 2133 identified from the FEDS modeling and analysis. Key energy and economic results are presented for each cost-effective retrofit measure.

\section{Facility Description 2133}

Building 2133 is a weapon systems management facility built in 2005. 2133 is cooled by an air cooled chiller and has some insulation in its building envelope. Building 2133 is 25,764 sf.

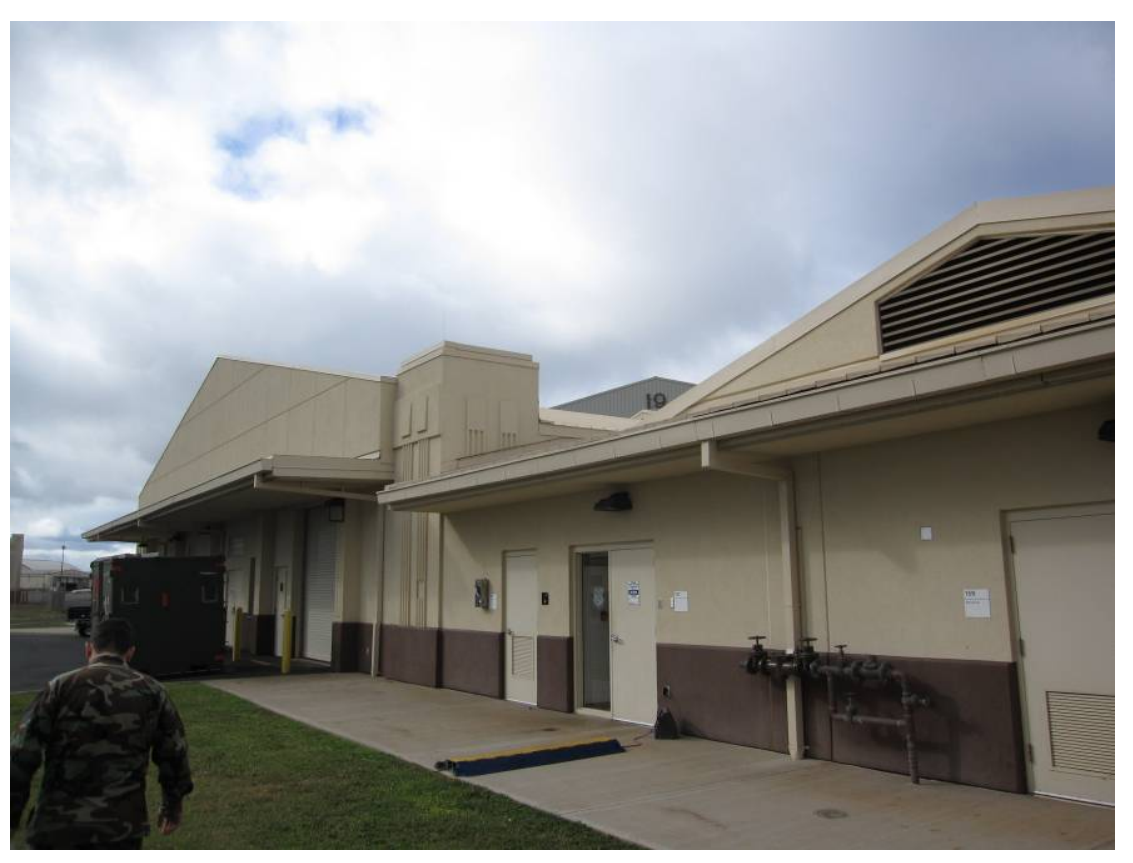




\section{Alternative Financing Results}

A FEDS analysis using alternative financing suggests replacing the electric hot water boiler with a heat pump water heater. FEDS also suggests replacing some of the lighting technologies.

Alternative financing FEDS results for building 2133:

\begin{tabular}{|c|c|c|c|c|c|c|c|c|}
\hline Bldg. Set Description & $\begin{array}{l}\text { End } \\
\text { Use } \\
\end{array}$ & Existing Technology & Retrofit Technology & $\begin{array}{l}\text { Energy } \\
\text { Savings } \\
\text { (MMBtu/yr) }\end{array}$ & $\begin{array}{l}\text { 1st Year } \\
\text { Savings } \\
(\$ / y r)\end{array}$ & $\begin{array}{l}\text { Installed } \\
\text { Cost (\$) }\end{array}$ & $\begin{array}{l}\text { Net } \\
\text { Present } \\
\text { Value (\$) } \\
\end{array}$ & SIR \\
\hline $\begin{array}{l}\text { medium 2000's admin } \\
2133\end{array}$ & $\begin{array}{l}\text { Hot } \\
\text { Water }\end{array}$ & Electric Central Boiler & $\begin{array}{l}\text { Central Heat Pump Hot Water } \\
\text { System, Wrap Tank }\end{array}$ & 249 & 12,188 & 8,565 & 61,475 & 8.2 \\
\hline $\begin{array}{l}\text { medium 2000's admin } \\
2133\end{array}$ & Lights & EX1: EXIT - INC $(2 \times 20)$ & $\begin{array}{l}\text { EX12: EXIT - } \\
\text { ELECTROLUMINESCENT PANEL } \\
\text { RETRO KIT }\end{array}$ & 14 & 937 & 621 & 4,854 & 8.8 \\
\hline $\begin{array}{l}\text { medium 2000's admin } \\
2133\end{array}$ & Lights & FL236: FL 2X4 3F32T8 ELC3 & $\begin{array}{l}\text { FL279: FL 2X4 2F32ST8 ELC2 } \\
\text { REF }\end{array}$ & 271 & 14,972 & 62,594 & 23,564 & 1.4 \\
\hline
\end{tabular}




\section{Alternative Financing Energy Consumption by Fuel Type}

The modeled energy consumption for a typical year was 586,408 kwh before retrofits and 429,858 kwh after proposed retrofits are implemented. The energy use intensity goes from 77.7 MBtu/Ksf to 56.9 MBtu/Ksf after retrofits.

\begin{tabular}{|c|c|c|c|c|}
\hline \multirow[b]{2}{*}{ Fuel } & \multicolumn{3}{|c|}{ Medium 2000's admin 2133} & \multirow[b]{2}{*}{$\begin{array}{l}\text { Dollars } \\
(2009) \text { * }\end{array}$} \\
\hline & Energy & $\begin{array}{c}\text { Energy } \\
\text { Intensity } \\
\text { (user units/1000ft2) }\end{array}$ & $\begin{array}{c}\text { Energy } \\
\text { Intensity } \\
\text { (MBtu/1000ft2) }\end{array}$ & \\
\hline \multicolumn{5}{|c|}{ Electricity (kWh) } \\
\hline existing & 586,408 & $22,760.8$ & 77.7 & 103,892 \\
\hline post-retrofit & 429,858 & $16,684.4$ & 56.9 & 75,953 \\
\hline difference & $-156,550$ & $-6,076.3$ & -20.7 & $-27,938$ \\
\hline$\%$ change & -27 & -27 & -27 & -27 \\
\hline \multicolumn{5}{|l|}{ Total (MBtu) } \\
\hline existing & 2,001 & 77.7 & 77.7 & 103,892 \\
\hline post-retrofit & 1,467 & 56.9 & 56.9 & 75,953 \\
\hline difference & -534 & -20.7 & -20.7 & $-27,938$ \\
\hline$\%$ change & -27 & -27 & -27 & -27 \\
\hline
\end{tabular}

* Dollar values for electricity include both energy and demand components. 


\section{Alternative Financing Energy Consumption by End Use}

Space cooling is the largest load in the building with 170,993 kWh/year, followed by lighting with $149,077 \mathrm{kWh} /$ year.

$\begin{array}{lcr} & & \\ & & \\ & \text { Heating } & \text { Cooling } \\ \text { Fuel } & & \\ \text { Electricity (kWh) } & 0 & 170,993 \\ \text { existing } & 0 & 149,639 \\ \text { post-retrofit } & 0 & -21,354 \\ \text { difference } & 0 & -12 \\ \text { \% change } & & \\ \text { Total (MBtu) } & 0 & 584 \\ \text { existing } & 0 & -73 \\ \text { post-retrofit } & 0 & -12 \\ \text { difference } & 0 & \\ \text { \% change } & & 23 \\ \text { Total (MBtu/1000ft2) } & & -3 \\ \text { existing } & 0 & -12 \\ \text { post-retrofit } & 0 & \\ \text { difference } & 0 & \\ \text { \% change } & 0 & \end{array}$

by Building Set, Fuel Type, and End Use

Medium 2000's admin 2133

Motors and

Misc Equip

Hot Water

Lights

132,355

132,355

89,861

$-59,216$

50,710

$-3,062$

$-40$

80,211

$-72,917$

$-91$

$\begin{array}{rr}84 & 184 \\ 11 & 173 \\ 73 & -10 \\ 12 & -6\end{array}$

509

307

$-202$

$-40$

452

452

274

25

$-249$

$\begin{array}{ll}0 & -91\end{array}$




\section{Alternative Financing Emission Reduction}

The emission reductions from implemented the proposed retrofits are as follows:

Medium 2000's admin 2133

Sulfur Oxides (lb)

existing

post-retrofit

difference

5,301

3,886

$\%$ change

$-1,415$
-27

Nitrogen Oxides (lb)

existing

post-retrofit

difference

1,857

$-676$

$\%$ change

$$
-27
$$

Carbon Monoxide (lb)

existing

post-retrofit

4,357

difference

3,194

$-1,163$
-27

$\%$ change

$-27$

Carbon Dioxide (tons)

existing

537

post-retrofit

393

difference

$-143$

$\%$ change

$-27$

Particulate Matter (lb)

existing

post-retrofit

difference

$\%$ change

105

77
-28

$-27$

Hydrocarbons (lb)

existing

post-retrofit $\quad 1,322$

1,803

difference

$-481$

$\%$ change

$-27$ 


\section{Building 2125 Administration Building}

The following information identifies the cost-effective energy- and cost-reducing retrofit projects for building 2125 identified from the FEDS modeling and analysis. Key energy and economic results are presented for each cost-effective retrofit measure.

\section{Facility Description 2125}

Building 2125 is an administration building built in 1994. This petroleum operations building is cooled by an electric package unit and has little to no insulation in its building envelope. Building 2125 is 3,867 sf.

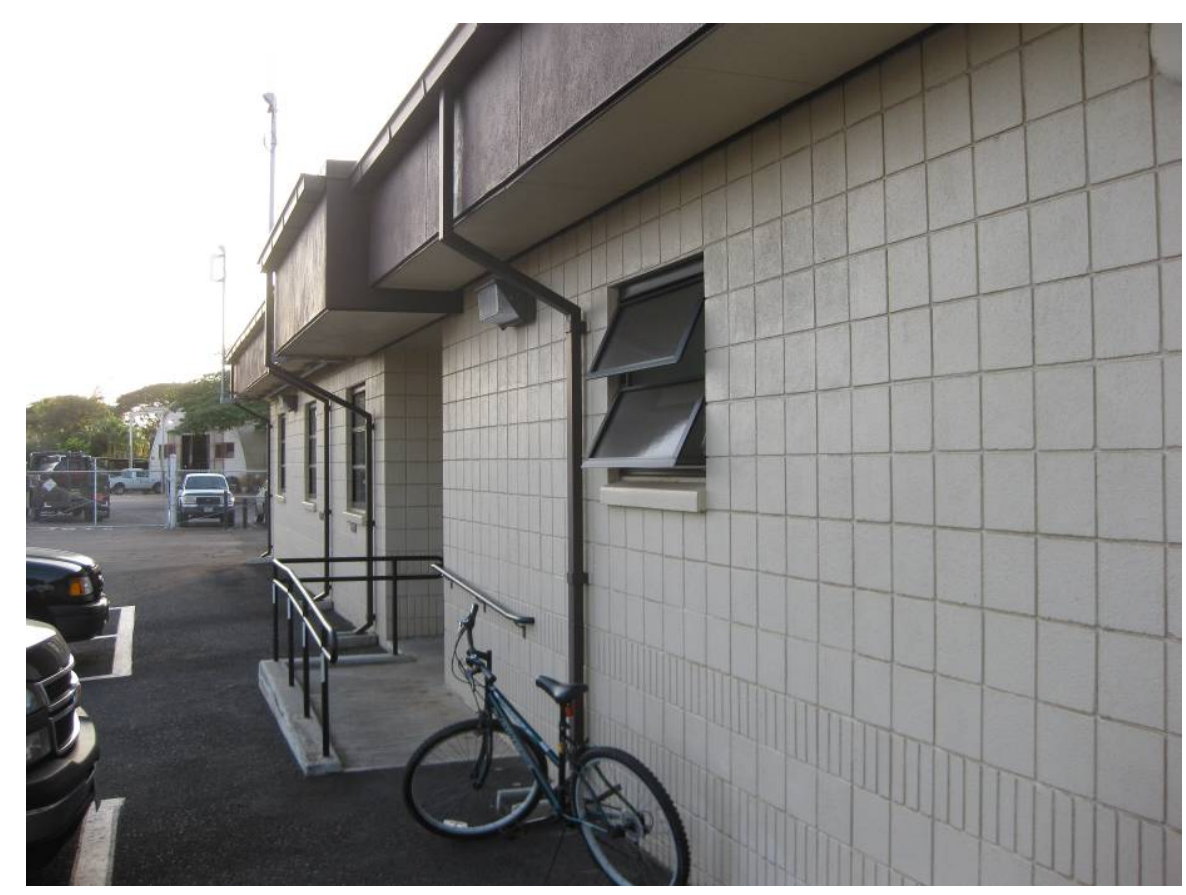




\section{Alternative Financing Results}

A FEDS analysis using alternative financing suggests replacing the 32W T8 lighting with 25W Super T8 lighting as well as replacing the exit lighting and increasing the insulation in the roof to 4 inches of fiberglass.

Alternative financing FEDS results for building 2125:

\begin{tabular}{|c|c|c|c|c|c|c|c|c|}
\hline Bldg. Set Description & $\begin{array}{l}\text { End } \\
\text { Use } \\
\end{array}$ & Existing Technology & Retrofit Technology & $\begin{array}{l}\text { Energy } \\
\text { Savings } \\
\text { (MMBtu/yr) } \\
\end{array}$ & $\begin{array}{c}\text { 1st Year } \\
\text { Savings } \\
(\$ / y r)\end{array}$ & $\begin{array}{l}\text { Installed } \\
\text { Cost (\$) }\end{array}$ & $\begin{array}{l}\text { Net } \\
\text { Present } \\
\text { Value (\$) } \\
\end{array}$ & SIR \\
\hline Small 1990's admin 2125 & Lights & EX6: EXIT - LED & $\begin{array}{l}\text { EX12: EXIT - } \\
\text { ELECTROLUMINESCENT PANEL } \\
\text { RETRO KIT }\end{array}$ & - & 61 & 311 & 56 & 1.2 \\
\hline Small 1990's admin 2125 & Lights & FL38: FL 2X4 3F32T8 EEF1,2 & $\begin{array}{l}\text { FL304: FL 2X4 3F25ST8 ELC3 } \\
\text { REF }\end{array}$ & 26 & 1,960 & 5,508 & 6,011 & 2.1 \\
\hline
\end{tabular}




\section{Alternative Financing Energy Consumption by Fuel Type}

The modeled energy consumption for a typical year was 56,331 kwh before retrofits and 48,664 kwh after proposed retrofits are implemented. The energy use intensity goes from $49.7 \mathrm{MBtu} / \mathrm{Ksf}$ to $43.0 \mathrm{MBtu} / \mathrm{Ksf}$ after retrofits.

Small 1990 's admin 2125

\begin{tabular}{|c|c|c|c|c|}
\hline Fuel & Energy & $\begin{array}{c}\text { Energy } \\
\text { Intensity } \\
\text { (user units/1000ft2) }\end{array}$ & $\begin{array}{c}\text { Energy } \\
\text { Intensity } \\
\text { (MBtu/1000ft2) }\end{array}$ & $\begin{array}{l}\text { Dollars } \\
(2009) \text { * }\end{array}$ \\
\hline \multicolumn{5}{|c|}{ Electricity (kWh) } \\
\hline existing & 56,331 & $14,567.1$ & 49.7 & 9,957 \\
\hline post-retrofit & 48,664 & $12,584.4$ & 43.0 & 8,592 \\
\hline difference & $-7,667$ & $-1,982.7$ & -6.8 & $-1,365$ \\
\hline$\%$ change & -14 & -14 & -14 & -14 \\
\hline \multicolumn{5}{|l|}{ Total (MBtu) } \\
\hline existing & 192 & 49.7 & 49.7 & 9,957 \\
\hline post-retrofit & 166 & 43.0 & 43.0 & 8,592 \\
\hline difference & -26 & -6.8 & -6.8 & $-1,365$ \\
\hline$\%$ change & -14 & -14 & -14 & -14 \\
\hline
\end{tabular}

* Dollar values for electricity include both energy and demand components. 


\section{Alternative Financing Energy Consumption by End Use}

Space cooling is the largest load in the building with 18,138 kWh/year, followed by lighting with 16,214 kWh/year.

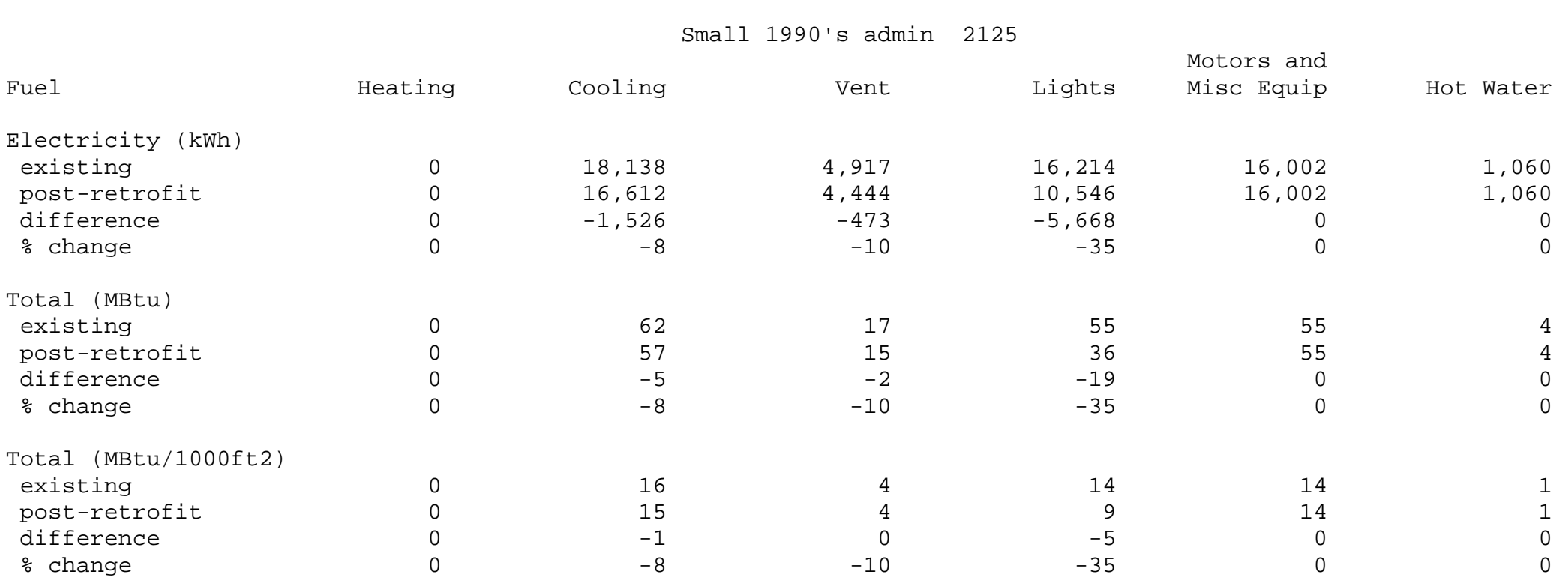




\section{Alternative Financing Emission Reduction}

The emission reductions from implemented the proposed retrofits are as follows:

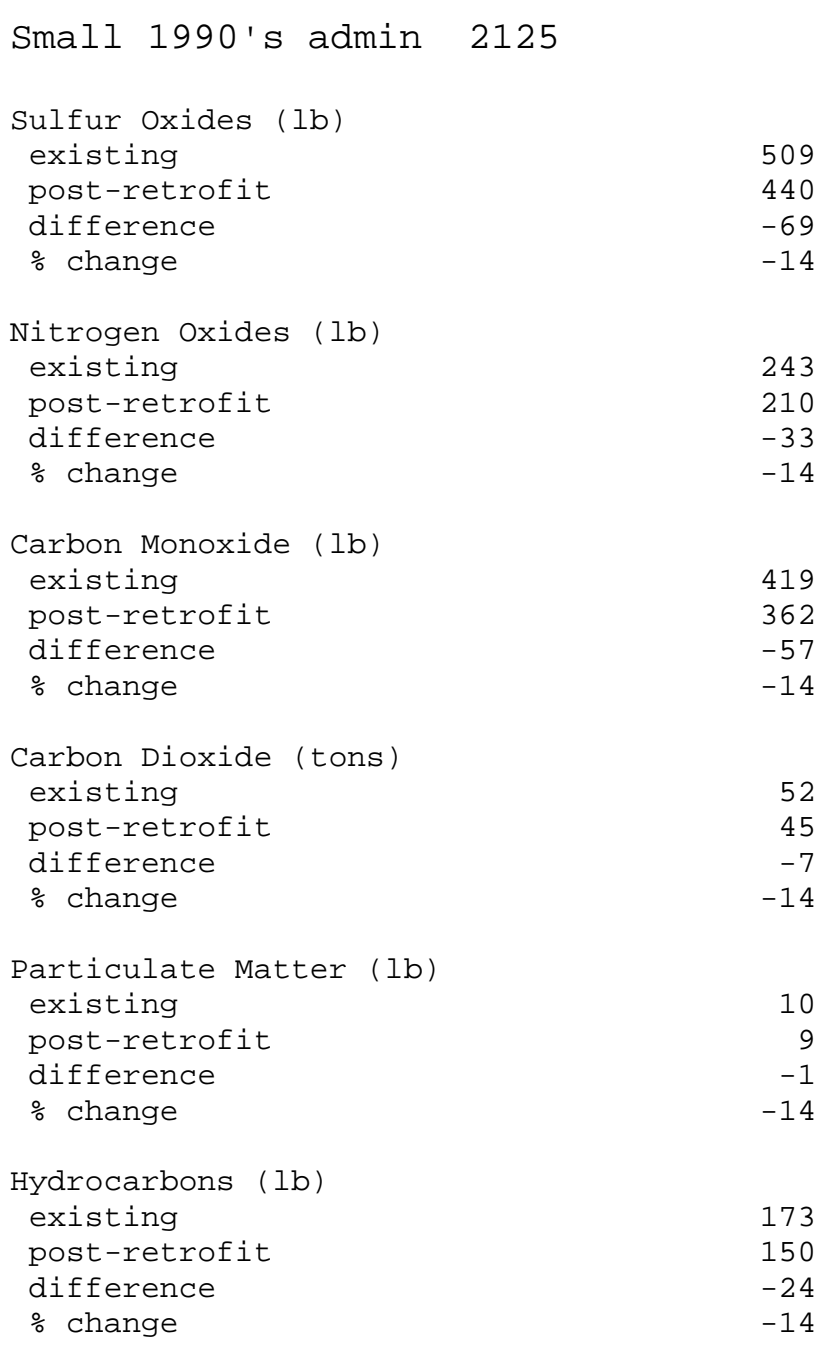




\section{Building 559 Clinic}

The following information identifies the cost-effective energy- and cost-reducing retrofit projects for building 559 identified from the FEDS modeling and analysis. Key energy and economic results are presented for each cost-effective retrofit measure.

\section{Facility Description 559}

Building 559 is the air force clinic built in 1942. This building is cooled by water cooled chillers and has an electric central hot water system. Building 559 is 78,823 sf.

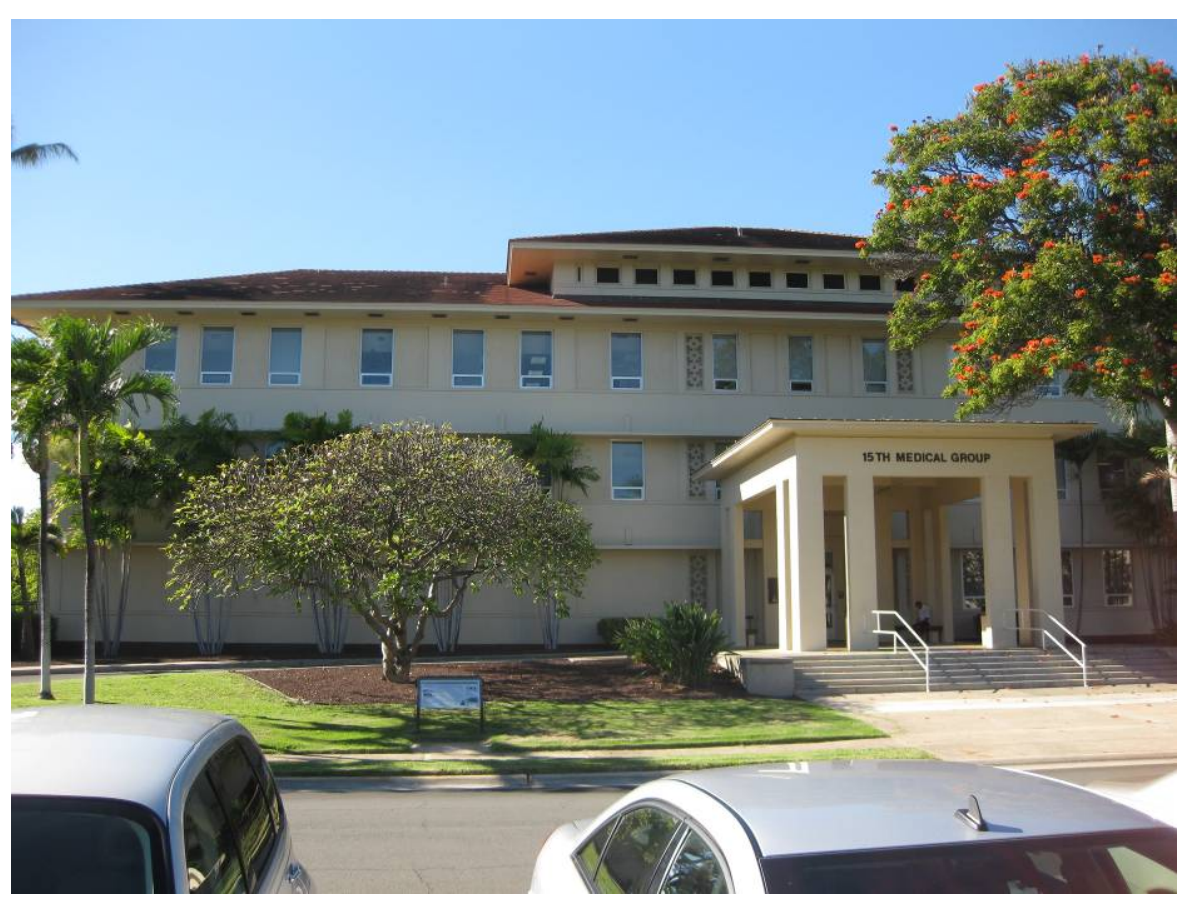




\section{Alternative Financing Results}

A FEDS analysis using alternative financing suggests replacing the electric boiler with a heat pump water heater as well as various improvements to the lighting in the building.

Alternative financing FEDS results for building 559:

\begin{tabular}{|c|c|c|c|c|c|c|c|c|}
\hline Bldg. Set Description & $\begin{array}{l}\text { End } \\
\text { Use }\end{array}$ & Existing Technology & Retrofit Technology & $\begin{array}{l}\text { Energy } \\
\text { Savings } \\
\text { (MMBtu/yr) } \\
\end{array}$ & $\begin{array}{l}\text { 1st Year } \\
\text { Savings } \\
(\$ / y r)\end{array}$ & $\begin{array}{l}\text { Installed } \\
\text { Cost (\$) }\end{array}$ & $\begin{array}{l}\text { Net } \\
\text { Present } \\
\text { Value (\$) }\end{array}$ & SIR \\
\hline Medical facilities 559 & $\begin{array}{l}\text { Hot } \\
\text { Water }\end{array}$ & Electric Central Boiler & $\begin{array}{l}\text { Central Heat Pump Hot Water } \\
\text { System, Wrap Tank, Aerators, } \\
\text { LFSHs }\end{array}$ & 939 & 47,853 & 74,876 & 199,891 & 3.7 \\
\hline Medical facilities 559 & Lights & EX1: EXIT - INC $(2 \times 20)$ & $\begin{array}{l}\text { EX12: EXIT - } \\
\text { ELECTROLUMINESCENT PANEL } \\
\text { RETRO KIT }\end{array}$ & 54 & 3,674 & 2,795 & 18,739 & 7.7 \\
\hline Medical facilities 559 & Lights & FL1: FL 2X4 4F40T12 STD2 & FL244: FL 2X4 4F32T8 ELC4 & 445 & 31,658 & 57,052 & 127,748 & 3.2 \\
\hline Medical facilities 559 & Lights & FL39: FL 2X4 2F32T8 EEF2 & $\begin{array}{l}\text { FL303: FL 2X4 2F25ST8 ELC2 } \\
\text { REF }\end{array}$ & 127 & 10,803 & 43,662 & 19,937 & 1.5 \\
\hline
\end{tabular}




\section{Alternative Financing Energy Consumption by Fuel Type}

The modeled energy consumption for a typical year was 1,458,222 kwh before retrofits and 999,686 kwh after proposed retrofits are implemented. The modeled other fuels (propane) consumption for a typical year was $34 \mathrm{MBtu}$ before retrofits and $34 \mathrm{MBtu}$ after proposed retrofits are implemented. The energy use intensity goes from $63.6 \mathrm{MBtu} / \mathrm{Ksf}$ to $43.7 \mathrm{MBtu} / \mathrm{Ksf}$ after retrofits.

\begin{tabular}{|c|c|c|c|c|}
\hline \multirow[b]{2}{*}{ Fuel } & \multicolumn{3}{|c|}{ Medical facilities 559} & \multirow[b]{2}{*}{$\begin{array}{l}\text { Dollars } \\
(2009)^{*}\end{array}$} \\
\hline & Energy & $\begin{array}{c}\text { Energy } \\
\text { Intensity } \\
\text { (user units/1000ft2) }\end{array}$ & $\begin{array}{l}\text { Energy } \\
\text { Intensity } \\
\text { (MBtu/1000ft2) }\end{array}$ & \\
\hline \multicolumn{5}{|c|}{ Electricity (kWh) } \\
\hline existing & $1,458,222$ & $18,500 . \odot$ & 63.1 & 258,347 \\
\hline post-retrofit & 999,686 & $12,682.7$ & 43.3 & 176,639 \\
\hline difference & $-458,535$ & $-5,817.3$ & -19.9 & $-81,708$ \\
\hline$\%$ change & -31 & -31 & -31 & -32 \\
\hline \multicolumn{5}{|c|}{ Other Fuels (MBtu) } \\
\hline existing & 34 & 0.4 & 0.4 & 1,100 \\
\hline post-retrofit & 34 & 0.4 & 0.4 & 1,100 \\
\hline difference & 0 & 0.0 & 0.0 & 0 \\
\hline$\%$ change & 0 & 0 & 0 & 0 \\
\hline \multicolumn{5}{|l|}{ Total (MBtu) } \\
\hline existing & 5,011 & 63.6 & 63.6 & 259,447 \\
\hline post-retrofit & 3,446 & 43.7 & 43.7 & 177,739 \\
\hline difference & $-1,565$ & -19.9 & -19.9 & $-81,708$ \\
\hline$\%$ change & -31 & -31 & -31 & -31 \\
\hline
\end{tabular}

* Dollar values for electricity include both energy and demand components. 


\section{Alternative Financing Energy Consumption by End Use}

Motors and miscellaneous equipment is the largest load in the building with 396,160 kWh/year, followed by lighting with 363,057 $\mathrm{kWh} /$ year.

Annual Energy Use by Building Set, Fuel Type, and End Use

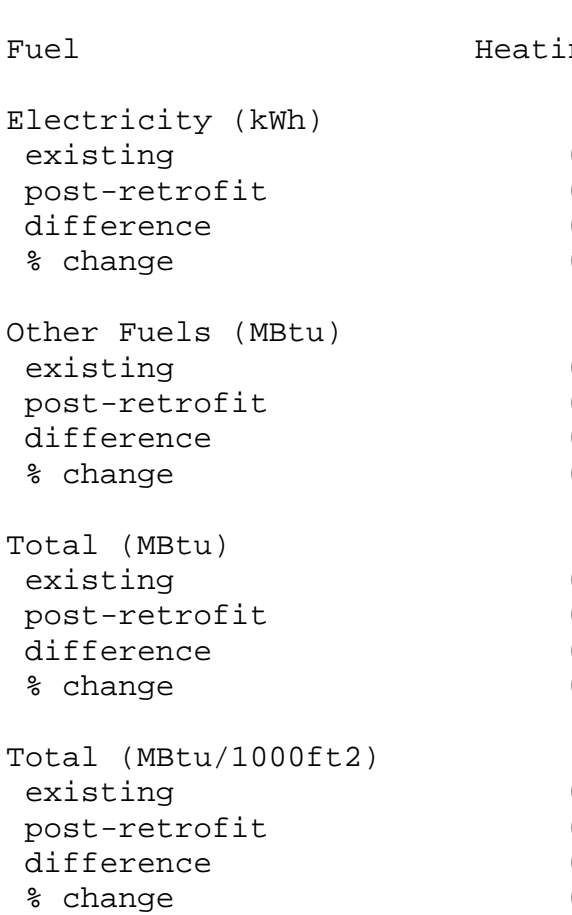
Medical facilities 559

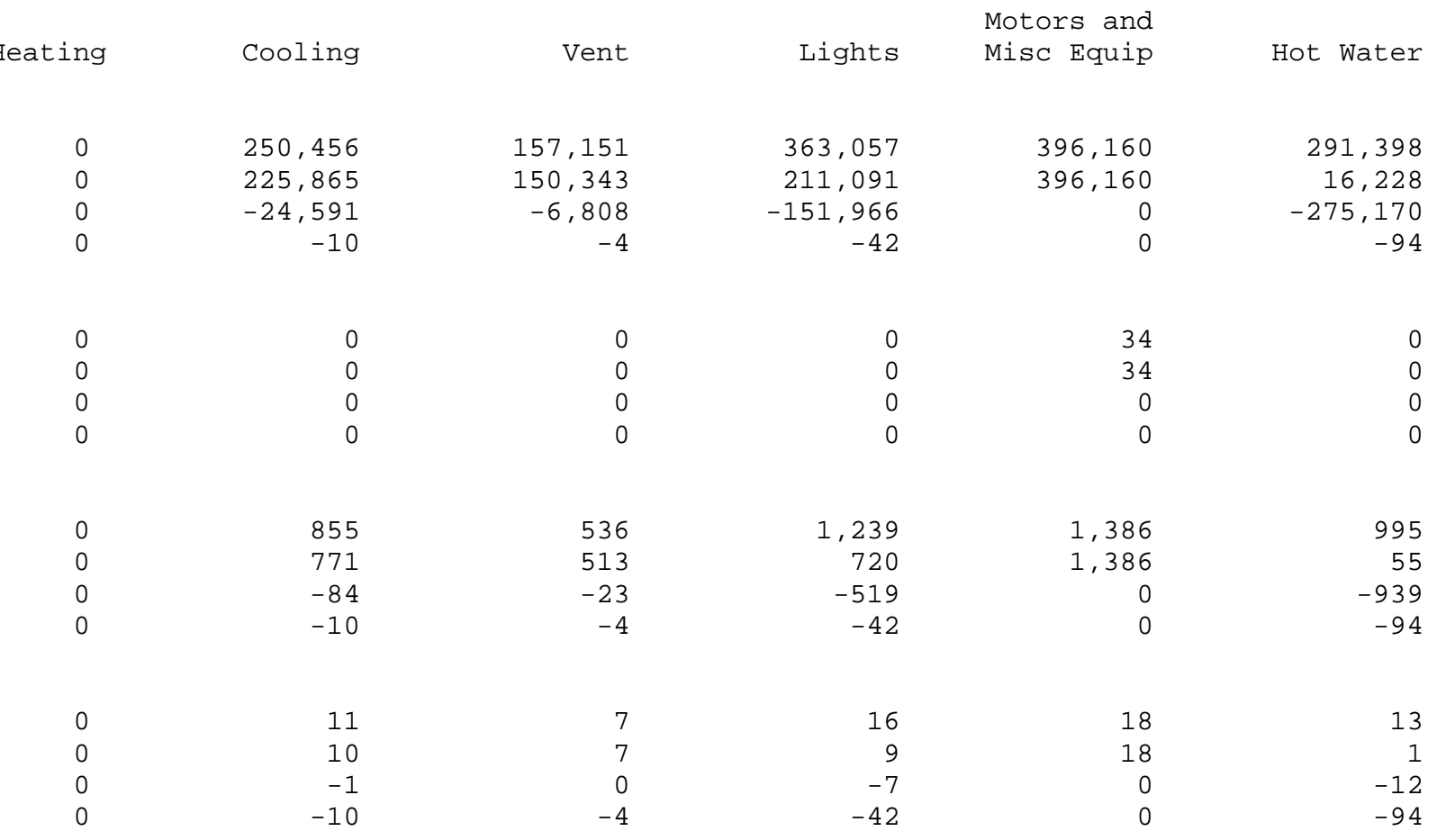




\section{Alternative Financing Emission Reduction}

The emission reductions from implemented the proposed retrofits are as follows:

Medical facilities

559

Sulfur Oxides (lb)

existing

post-retrofit

difference

13,191

9,046

$-4,145$

$\%$ change

$-31$

Nitrogen Oxides (lb)

existing

6,311

post-retrofit

4,330

difference

$-1,981$

$\%$ change

$-31$

Carbon Monoxide (lb)

existing

post-retrofit

difference

10,861

$\%$ change

7,454

$-3,407$

Carbon Dioxide (tons)

existing

post-retrofit

difference

1,337

918

$\%$ change

$-420$

$-31$

Particulate Matter (lb)

existing

261

post-retrofit

179

difference

$-82$

$\%$ change

$-31$

Hydrocarbons ( $1 b$ )

existing

post-retrofit

4,493

post-retrofi

3,083

$-1,410$

$\%$ change

$-31$ 


\section{Building 1060 Laboratory}

The following information identifies the cost-effective energy- and cost-reducing retrofit projects for building 1060 identified from the FEDS modeling and analysis. Key energy and economic results are presented for each cost-effective retrofit measure.

\section{Facility Description 1060}

Building 1060 is a lab built in 1943. This lab is cooled by an air cooled chiller and has an electric water heater. 1060 is 14,920 sf.

\section{Alternative Financing Results}

A FEDS analysis using alternative financing suggests replacing various lighting technologies in the building. FEDS suggests replacing T12, T8 and EXIT lights.

Alternative financing FEDS results for building 1060:

\begin{tabular}{|c|c|c|c|c|c|c|c|c|}
\hline Bldg. Set Description & $\begin{array}{l}\text { End } \\
\text { Use }\end{array}$ & Existing Technology & Retrofit Technology & $\begin{array}{l}\text { Energy } \\
\text { Savings } \\
\text { (MMBtu/yr) }\end{array}$ & $\begin{array}{l}\text { 1st Year } \\
\text { Savings } \\
(\$ / y r)\end{array}$ & $\begin{array}{l}\text { Installed } \\
\text { Cost (\$) }\end{array}$ & $\begin{array}{l}\text { Net } \\
\text { Present } \\
\text { Value (\$) }\end{array}$ & SIR \\
\hline Labs 1060 & Lights & EX1: EXIT - INC $(2 \times 20)$ & $\begin{array}{l}\text { EX12: EXIT - } \\
\text { ELECTROLUMINESCENT PANEL } \\
\text { RETRO KIT }\end{array}$ & 14 & 905 & 621 & 4,669 & 8.5 \\
\hline Labs 1060 & Lights & FL39: FL 2X4 2F32T8 EEF2 & $\begin{array}{l}\text { FL303: FL 2X4 2F25ST8 ELC2 } \\
\text { REF }\end{array}$ & 9 & 743 & 3,516 & 844 & 1.2 \\
\hline Labs 1060 & Lights & FL3: FL 2X4 2F40T12 STD2 & FL51: FL 2X4 2F32T8 ELC2 & 12 & 935 & 3,249 & 2,233 & 1.7 \\
\hline
\end{tabular}




\section{Alternative Financing Energy Consumption by Fuel Type}

The modeled energy consumption for a typical year was 292,009 kwh before retrofits and 281,462 kwh after proposed retrofits are implemented. The energy use intensity goes from 66.8 MBtu/Ksf to 64.4 MBtu/Ksf after retrofits.

\begin{tabular}{|c|c|c|c|c|}
\hline \multirow[b]{2}{*}{ Fuel } & \multirow[b]{2}{*}{ Energy } & 1060 & \multirow[b]{2}{*}{$\begin{array}{c}\text { Energy } \\
\text { Intensity } \\
\text { (MBtu/1000ft2) }\end{array}$} & \multirow[b]{2}{*}{$\begin{array}{l}\text { Dollars } \\
(2009) \text { * }\end{array}$} \\
\hline & & $\begin{array}{c}\text { Energy } \\
\text { Intensity } \\
\text { (user units/1000ft2) }\end{array}$ & & \\
\hline \multicolumn{5}{|c|}{ Electricity (kWh) } \\
\hline existing & 292,009 & $19,571.7$ & 66.8 & 51,734 \\
\hline post-retrofit & 281,462 & $18,864.8$ & 64.4 & 49,733 \\
\hline difference & $-10,547$ & -706.9 & -2.4 & $-2,001$ \\
\hline$\%$ change & -4 & -4 & -4 & -4 \\
\hline \multicolumn{5}{|l|}{ Total (MBtu) } \\
\hline existing & 997 & 66.8 & 66.8 & 51,734 \\
\hline post-retrofit & 961 & 64.4 & 64.4 & 49,733 \\
\hline difference & -36 & -2.4 & -2.4 & $-2,001$ \\
\hline$\%$ change & -4 & -4 & -4 & -4 \\
\hline
\end{tabular}

* Dollar values for electricity include both energy and demand components. 


\section{Alternative Financing Energy Consumption by End Use}

Space cooling is the largest load in the building with $116,645 \mathrm{kWh} /$ year, followed by motors and miscellaneous equipment with 77,382 kWh/year.

Annual Energy Use by Building Set, Fuel Type, and End Use

Fuel
Electricity (kWh)
existing
post-retrofit
difference
\% change
Total (MBtu)
existing
post-retrofit
difference
\% change
Total (MBtu/10००ft2)
existing
post-retrofit
difference
$\%$ change

$\begin{array}{rr}\text { Heating } & \text { Cooling } \\ & \\ \odot & 116,645 \\ 0 & 114,048 \\ 0 & -2,596 \\ \odot & -2 \\ & \\ \odot & 398 \\ \odot & 389 \\ \odot & -9 \\ 0 & -2 \\ & \\ \odot & 27 \\ \odot & 26 \\ 0 & -1 \\ 0 & -2\end{array}$

Labs 1060

Motors and

Misc Equip

Hot Water

$\begin{array}{rrr}52,192 & 44,584 & 77,382 \\ 51,676 & 37,150 & 77,382 \\ -516 & -7,434 & 0 \\ -1 & -17 & \\ & & \\ 178 & 152 & 264 \\ 176 & 127 & 264 \\ -2 & -25 & 0 \\ -1 & -17 & 0 \\ & & \\ 12 & 10 & 18 \\ 12 & 8 & 18 \\ 0 & -2 & 0 \\ -1 & -17 & 0\end{array}$

1,207

1,207

$\odot$
$\odot$

264

0

18
18
0
0 


\section{Alternative Financing Emission Reduction}

The emission reductions from implemented the proposed retrofits are as follows:

Labs 1060

Sulfur Oxides (lb)

existing

post-retrofit

difference

2,640

2,544

-95
-4

$\%$ change

1,261

1,216

$-46$

post-retrofit

difference

$-4$

Carbon Monoxide (lb)

existing

post-retrofit

2,170

difference

2, 091

-78
-4

$\%$ change

(tons)

existing

post-retrofit

258
-10

difference

$\%$ change

$-4$

Particulate Matter (lb)

existing

post-retrofit

difference

$\%$ change

Hydrocarbons (lb)

existing

post-retrofit 866

898

difference $\quad-32$

$\%$ change

$-4$ 


\section{Building 1805 Dormitory}

The following information identifies the cost-effective energy- and cost-reducing retrofit projects for building 1805 identified from the FEDS modeling and analysis. Key energy and economic results are presented for each cost-effective retrofit measure.

\section{Facility Description 1805}

Building 1805 is a dormitory built in 1970. The dormitory is cooled by an air cooled chiller and has little to no insulation in its building envelope. This building has a desuperheater system, providing some of the hot water to the building. 1805 is 55,187 sf.

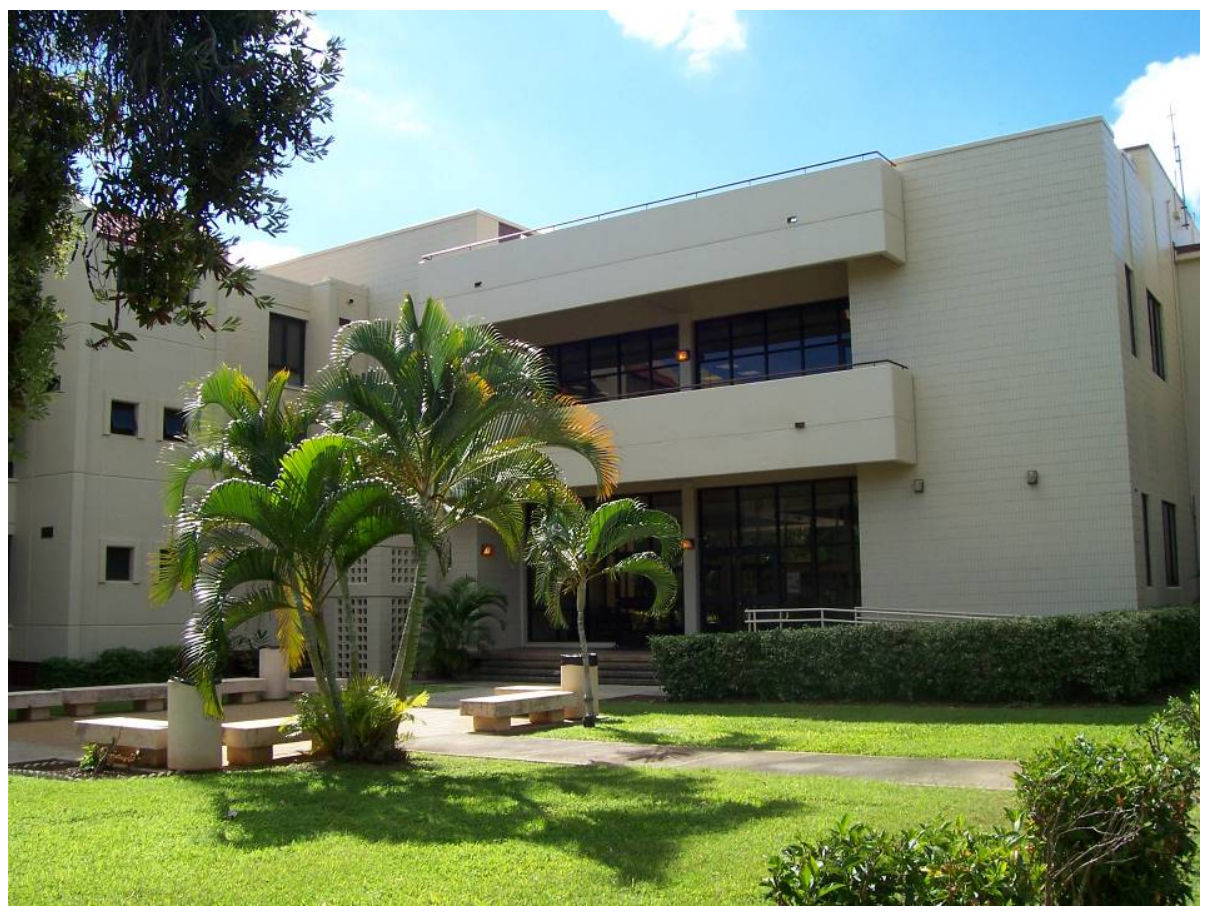




\section{Alternative Financing Results}

A FEDS analysis using alternative financing suggests replacing the electric hot water heater with a heat pump water heater and replacing the EXIT lighting.

Appropriated funding FEDS analysis results for building 1805:

\begin{tabular}{|c|c|c|c|c|c|c|c|c|}
\hline Bldg. Set Description & $\begin{array}{l}\text { End } \\
\text { Use }\end{array}$ & Existing Technology & Retrofit Technology & $\begin{array}{l}\text { Energy } \\
\text { Savings } \\
\text { (MMBtu/yr) }\end{array}$ & $\begin{array}{l}\text { 1st Year } \\
\text { Savings } \\
(\$ / y r)\end{array}$ & $\begin{array}{l}\text { Installed } \\
\text { Cost (\$) }\end{array}$ & $\begin{array}{l}\text { Net } \\
\text { Present } \\
\text { Value (\$) }\end{array}$ & SIR \\
\hline Dorms 1970's 1805 & Lights & EX1: EXIT - INC (2x20) & $\begin{array}{l}\text { EX12: EXIT - } \\
\text { ELECTROLUMINESCENT PANEL } \\
\text { RETRO KIT }\end{array}$ & 62 & 4,053 & 2,795 & 20,914 & 8.5 \\
\hline
\end{tabular}




\section{Alternative Financing Energy Consumption by Fuel Type}

The modeled energy consumption for a typical year was 515,140 kwh before retrofits and 391,677 kwh after proposed retrofits are implemented. The energy use intensity goes from 31.9 MBtu/Ksf to 24.2 MBtu/Ksf after retrofits.

Fuel

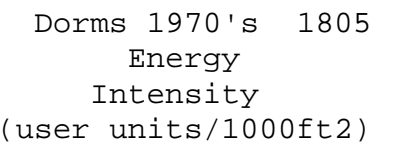

$$
\begin{aligned}
& \text { Energy } \\
& \text { Intensity }
\end{aligned}
$$

Energy

Dollars

$(2009)$ *

Electricity (kWh)

existing

post-retrofit

difference

$\%$ change

$$
\begin{array}{r}
515,140 \\
496,775 \\
-18,365 \\
-4 \\
\\
1,758 \\
1,695 \\
-63 \\
-4
\end{array}
$$$$
\begin{aligned}
& 9,334.4 \\
& 9,001.7
\end{aligned}
$$$$
\begin{array}{r}
-332.8 \\
-4
\end{array}
$$$$
31.9
$$$$
30.7
$$$$
-1.1
$$

$$
\begin{array}{r}
31.9 \\
30.7 \\
-1.1 \\
-4 \\
\\
31.9 \\
30.7 \\
-1.1 \\
-4
\end{array}
$$$$
91,013
$$$$
\begin{array}{r}
-3,237 \\
-4
\end{array}
$$

Total (MBtu)

post-retrofit

differenc
91,013

87,776

$-3,237$

* Dollar values for electricity include both energy and demand components. 


\section{Alternative Financing Energy Consumption by End Use}

Space cooling is the largest load in the building with 188,139 kWh/year, followed by hot water with 121,790 kWh/year.

Fuel
Electricity (kWh)
existing
post-retrofit
difference
$\%$ change
Total (MBtu)
existing
post-retrofit
difference
$\%$ change
Total (MBtu/1000ft2)
existing
post-retrofit
difference
\% change

$\begin{array}{rr}\text { Heating } & \text { Cooling } \\ & \\ 0 & 188,139 \\ 0 & 182,899 \\ 0 & -5,240 \\ 0 & -3 \\ & \\ 0 & 642 \\ 0 & 624 \\ 0 & -18 \\ 0 & -3 \\ & \\ 0 & 12 \\ 0 & 11 \\ 0 & 0 \\ 0 & -3\end{array}$

Dorms 1970's 1805

$\begin{array}{rrrr}\text { Vent } & \text { Lights } & \begin{array}{r}\text { Motors and } \\ \text { Misc Equip }\end{array} & \text { Hot water } \\ & & & \\ 9,012 & 37,709 & 71,49 \odot & 121,79 \odot \\ 5,392 & 25,205 & 71,49 \odot & 121,79 \odot \\ -620 & -12,504 & 0 & \odot \\ -1 & -33 & 0 & \odot \\ & & & \\ 328 & 129 & 244 & 416 \\ 326 & 86 & 244 & 416 \\ -2 & -43 & 0 & \odot \\ -1 & -33 & 0 & \odot\end{array}$

$$
\begin{array}{r}
2 \\
2 \\
-1 \\
-33
\end{array}
$$

$$
\begin{aligned}
& 4 \\
& 4 \\
& 0 \\
& 0
\end{aligned}
$$

8 


\section{Alternative Financing Emission Reduction}

The emission reductions from implemented the proposed retrofits are as follows:

Sulfur oxides (lb)

Dorms 1970 's 1805

existing

post-retrofit

$\%$ change

$$
\begin{array}{r}
4,657 \\
4,491 \\
-166 \\
-4 \\
\\
2,225 \\
2,146 \\
-79 \\
-4 \\
\\
3,828 \\
3,691 \\
-136 \\
-4 \\
\\
471 \\
455 \\
-17 \\
-4 \\
\\
92 \\
89 \\
-3 \\
-4 \\
\\
1,584 \\
1,528 \\
-56 \\
-4
\end{array}
$$$$
\text { Nitrogen 0xides (lb) }
$$$$
\text { existing }
$$$$
\text { post-retrofit }
$$

difference$$
\% \text { change }
$$

Carbon Monoxide ( $1 b)$

existing

post-retrofit

$\%$ change

Carbon Dioxide (tons)

existing

post-retrofit

difference

$\%$ change

Particulate Matter (lb)

existing

post-retrofit

difference

$\%$ change

Hydrocarbons (lb)

existing

post-retrofit

difference

$\%$ change 


\section{Building 1856 Dormitory}

The following information identifies the cost-effective energy- and cost-reducing retrofit projects for building 1856 identified from the FEDS modeling and analysis. Key energy and economic results are presented for each cost-effective retrofit measure.

\section{Facility Description 1856}

Building 1856 is a dormitory built in 1995. The dormitory is cooled by an electric air cooled chiller and has substantial roofing and wall insulation in its building envelope. The central hot water system runs on diesel fuel and works in conjunction with a desuperheater. Building 1856 is 43,187 sf.

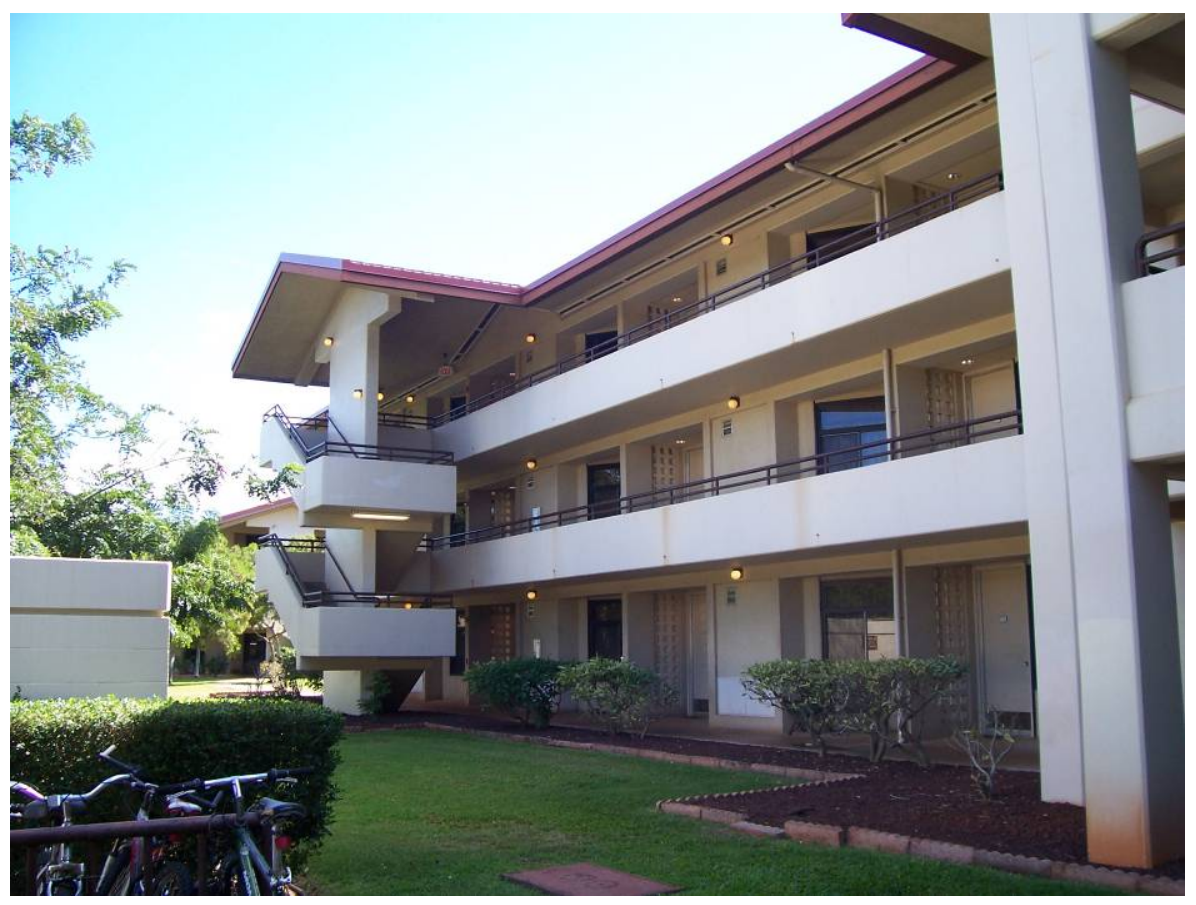




\section{Alternative Financing Results}

A FEDS analysis using alternative financing suggests replacing the air cooled chiller with a very high efficiency water cooled chiller, replacing the diesel hot water boiler with a heat pump hot water system and replacing the EXIT lighting.

Alternative financing FEDS analysis results for building 1856:

\begin{tabular}{|c|c|c|c|c|c|c|c|c|}
\hline Bldg. Set Description & $\begin{array}{l}\text { End } \\
\text { Use }\end{array}$ & Existing Technology & Retrofit Technology & $\begin{array}{l}\text { Energy } \\
\text { Savings } \\
\text { (MMBtu/yr) } \\
\end{array}$ & $\begin{array}{l}\text { 1st Year } \\
\text { Savings } \\
(\$ / y r)\end{array}$ & $\begin{array}{l}\text { Installed } \\
\text { Cost (\$) }\end{array}$ & $\begin{array}{l}\text { Net } \\
\text { Present } \\
\text { Value (\$) }\end{array}$ & SIR \\
\hline $\begin{array}{l}\text { Dorms } 1990 \text { 's } 1856 \text { - heat } \\
\text { recovery }\end{array}$ & Cooling & $\begin{array}{l}\text { Electric Air-Cooled Chiller } \\
\{\mathrm{C} 1\}\end{array}$ & $\begin{array}{l}\text { Water-Cooled Reciprocating } \\
\text { Electric Chiller (very high } \\
\text { efficiency) and Cooling Tower }\end{array}$ & 286 & 15,031 & 75,277 & 10,924 & 1.1 \\
\hline $\begin{array}{l}\text { Dorms } 1990 \text { 's } 1856 \text { - heat } \\
\text { recovery }\end{array}$ & $\begin{array}{l}\text { Hot } \\
\text { Water }\end{array}$ & Distillate Oil Central Boiler & $\begin{array}{l}\text { Central Heat Pump Hot Water } \\
\text { System }\end{array}$ & 151 & 5,505 & 22,540 & 11,662 & 1.5 \\
\hline $\begin{array}{l}\text { Dorms } 1990 \text { 's } 1856 \text { - heat } \\
\text { recovery }\end{array}$ & Lights & EX1: EXIT - INC $(2 \times 20)$ & $\begin{array}{l}\text { EX12: EXIT - } \\
\text { ELECTROLUMINESCENT PANEL } \\
\text { RETRO KIT }\end{array}$ & 61 & 4,021 & 2,795 & 20,726 & 8.4 \\
\hline
\end{tabular}




\section{Alternative Financing Energy Consumption by Fuel Type}

The modeled energy consumption for a typical year was 418,237 kwh before retrofits and 320,744 kwh after proposed retrofits are implemented. The modeled distillate oil consumption for a typical year was 1,252 gallons before retrofits and 0 gallons after proposed retrofits are implemented. The energy use intensity goes from 37.1 MBtu/Ksf to 25.3 MBtu/Ksf after retrofits.

Dorms 1990's 1856

Fuel

Electricity (kWh)

existing

post-retrofit

difference

$\%$ change

Distillate oil (gal)

existing

post-retrofit

difference

$\%$ change

Total (MBtu)

existing

post-retrofit

difference

$\%$ change
Energy

418,237
320,744

$-97,494$

$-23$

1,252

$-1,252$

$-100$

1,601
1,095
-506
-32
Energy

Intensity

(user units/1000ft2)
$9,684.3$

$7,426.9$

$-2,257.5$

$-23$

29.0

0.0

$-100$

$-100$

37.1

25.3

$-11.7$

$-32$

\section{Energy}

Intensity

(MBtu/1000ft2)

Dollars

(2009) *

33.1

25.3

-7.7
-23

$-23$

4.0

0.0

$-100$

37.1

25.3

$-11.7$

$-32$
73,892

56,633

$-17,259$

$-23$

6,385

$-6,385$

$-100$

80,278

56,633

$-23,645$

$-29$

* Dollar values for electricity include both energy and demand components. 


\section{Alternative Financing Energy Consumption by End Use}

Space cooling is the largest load in the building with $233,630 \mathrm{kWh} /$ year, followed by ventilation with $66,184 \mathrm{kWh} /$ year.

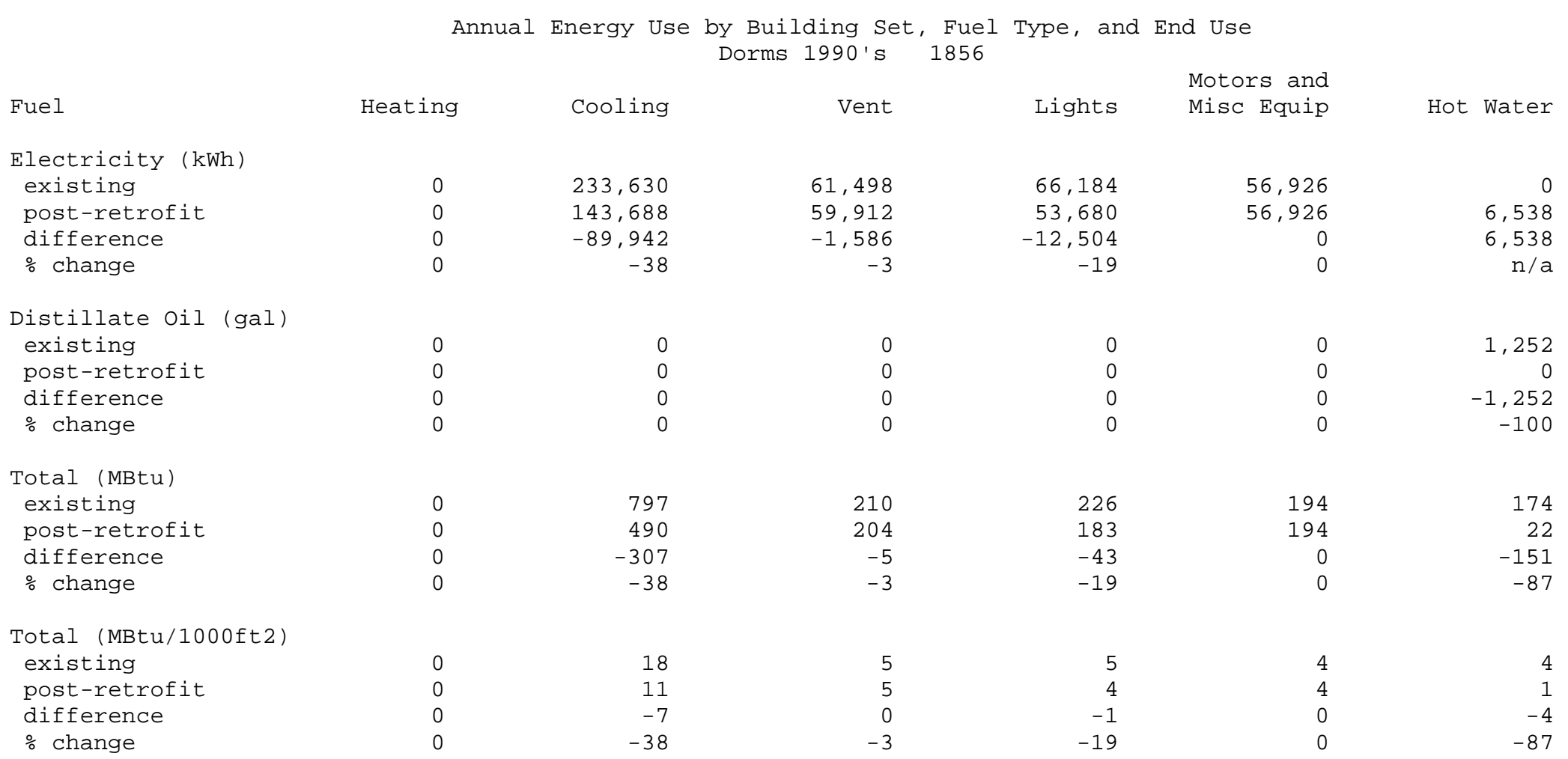




\section{Alternative Financing Emission Reduction}

The emission reductions from implemented the proposed retrofits are as follows:

Dorms 1990 's

1856

Sulfur Oxides (lb)

existing

post-retrofit

difference

3,868

2,900

$-968$

$\%$ change

$-25$

Nitrogen 0xides (lb)

existing

1,866

post-retrofit

1,386

$-480$

differenc

$-26$

Carbon Monoxide (lb)

existing

post-retrofit

, 246

difference

2,383

$-863$

$\%$ change

$-27$

Carbon Dioxide (tons)

existing

post-retrofit

399

difference

293

$\%$ change

$-106$

Particulate Matter (lb)

existing

post-retrofit

difference

$\%$ change

Hydrocarbons (lb)

existing

post-retrofit

1,331

difference

986

$\%$ change

-345
-26 


\section{Building 1166 Lodging Facility}

The following information identifies the cost-effective energy- and cost-reducing retrofit projects for building 1166 identified from the FEDS modeling and analysis. Key energy and economic results are presented for each cost-effective retrofit measure.

\section{Facility Description 1166}

Building 1166 is a hotel style building used as a temporary lodging facility and was built in 1968 . The building is cooled by an air cooled chiller and has little to no insulation in the building envelope. Building 1166 is 25,113 sf.

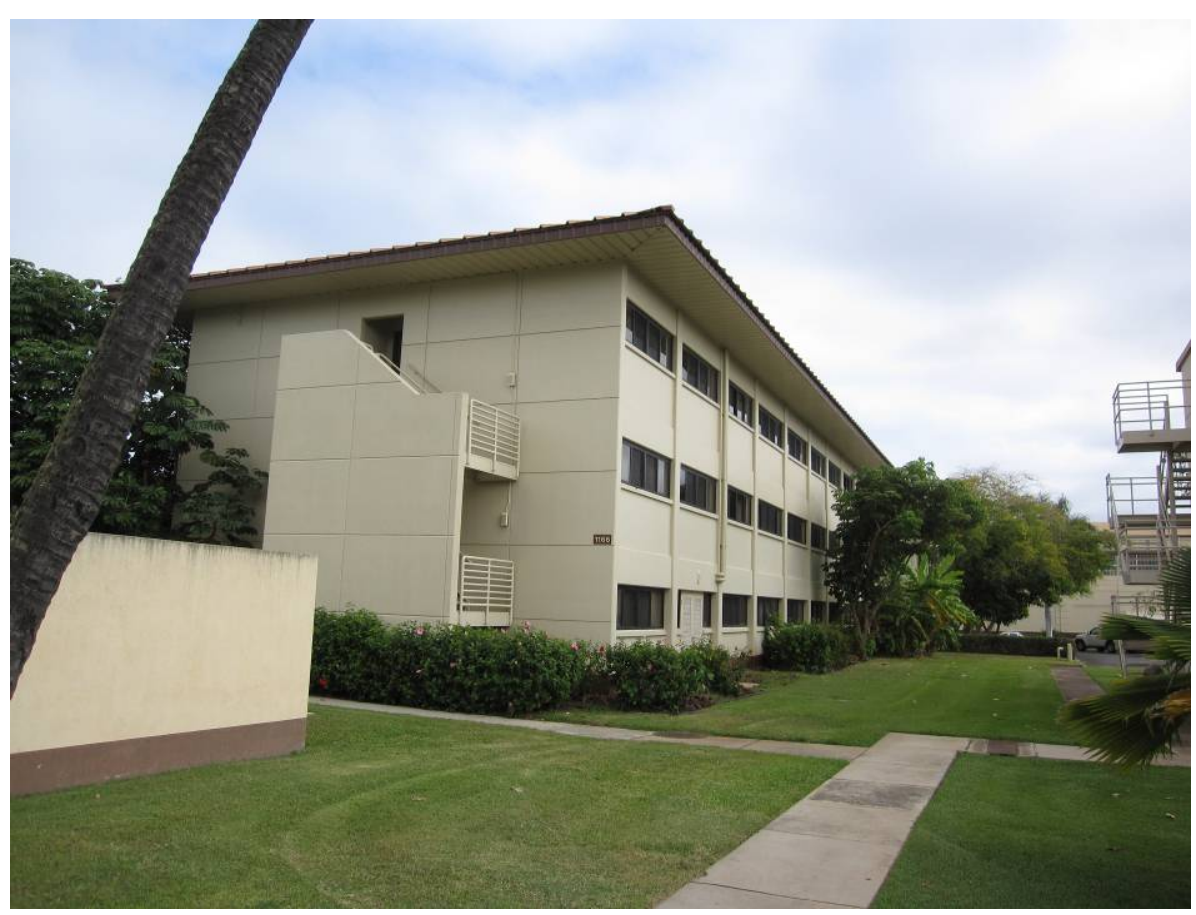




\section{Alternative Financing Results}

A FEDS analysis using alternative financing suggests replacing the hot water systems with a heat pump water heater. FEDS also suggests replacing the EXIT lighting and adding insulation to the interior surface of the metal roof.

Alternative financing FEDS analysis results for building 1166:

\begin{tabular}{|c|c|c|c|c|c|c|c|c|}
\hline Bldg. Set Description & $\begin{array}{l}\text { End } \\
\text { Use }\end{array}$ & Existing Technology & Retrofit Technology & $\begin{array}{l}\text { Energy } \\
\text { Savings } \\
\text { (MMBtu/yr) }\end{array}$ & $\begin{array}{l}\text { 1st Year } \\
\text { Savings } \\
(\$ / y r)\end{array}$ & $\begin{array}{l}\text { Installed } \\
\text { Cost (\$) }\end{array}$ & $\begin{array}{l}\text { Net } \\
\text { Present } \\
\text { Value (\$) }\end{array}$ & SIR \\
\hline Lodging facilities 1166 & Lights & EX1: EXIT - INC $(2 \times 20)$ & $\begin{array}{l}\text { EX12: EXIT - } \\
\text { ELECTROLUMINESCENT PANEL } \\
\text { RETRO KIT }\end{array}$ & 58 & 3,898 & 2,795 & 20,020 & 8.2 \\
\hline
\end{tabular}




\section{Alternative Financing Energy Consumption by Fuel Type}

The modeled energy consumption for a typical year was 425,234 kwh before retrofits and 374,596 kwh after proposed retrofits are implemented. The modeled other fuels (propane) consumption for a typical year was $48 \mathrm{MBtu}$ before retrofits and $0 \mathrm{MBtu}$ after proposed retrofits are implemented. The energy use intensity goes from 59.7 MBtu/Ksf to 50.9 MBtu/Ksf after retrofits.

Lodging facilities 1166

Fuel

Electricity (kWh)

existing

post-retrofit

difference

$\%$ change

Other Fuels (MBtu)

existing

post-retrofit

difference

$\%$ change

Total (MBtu)

existing

post-retrofit

difference

$\%$ change
Energy

425,234

407,904

$-17,330$

$-4$

48

48

$\odot$

1,499
1,440
-59
-4
Energy Intensity

(user units/1000ft2)

\section{$16,932.8$}

$16,242.8$

$-690.1$

$-4$

1.9
1.9
0.0
0

59.7

57.3

-2.4
-4

Energy

Intensity

(MBtu/1000ft2)

Dollars

(2009) *

\section{8}

55.4

-2.4
-4

75,129

72,073

$-3,055$
-4

1,531

1,531

1.9

0.0

59.7

57.3

-2.4
-4

* Dollar values for electricity include both energy and demand components. 


\section{Alternative Financing Energy Consumption by End Use}

Space cooling is the largest load in the building with 166,650 kWh/year, followed by ventilation with 117,053 kWh/year.

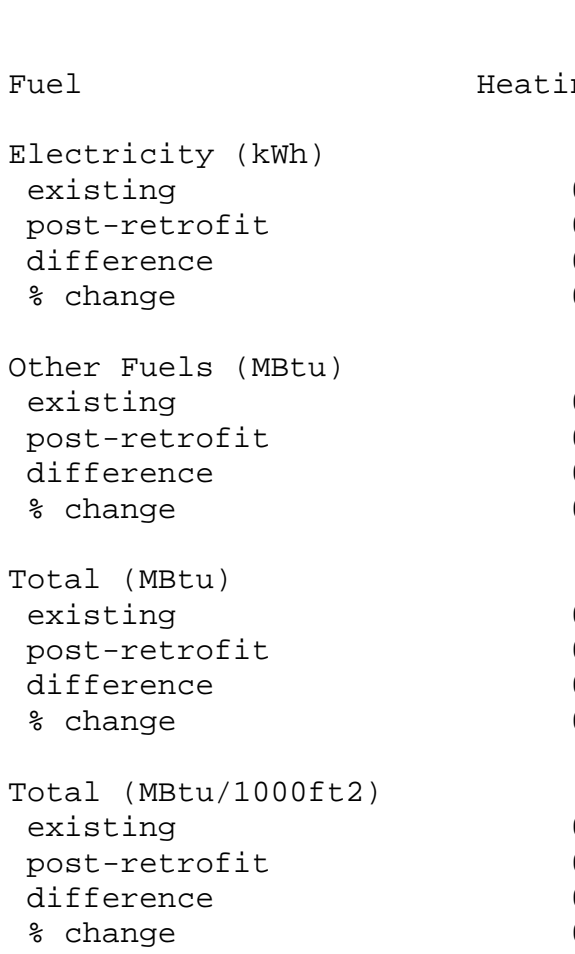

\begin{tabular}{|c|c|c|}
\hline & faciliti & \\
\hline Cooling & Vent & Lights \\
\hline 166,650 & 117,053 & 61,726 \\
\hline 161,824 & 117,053 & 49,222 \\
\hline$-4,826$ & 0 & $-12,504$ \\
\hline-3 & $\odot$ & -20 \\
\hline$\odot$ & $\odot$ & $\odot$ \\
\hline$\odot$ & $\odot$ & $\odot$ \\
\hline$\odot$ & $\odot$ & $\odot$ \\
\hline$\odot$ & $\odot$ & $\odot$ \\
\hline 569 & 400 & 211 \\
\hline 552 & 400 & 168 \\
\hline-16 & 0 & -43 \\
\hline-3 & $\odot$ & -20 \\
\hline 23 & 16 & 8 \\
\hline 22 & 16 & 7 \\
\hline-1 & $\odot$ & -2 \\
\hline-3 & 0 & -20 \\
\hline
\end{tabular}

Motors and Misc Equip

Hot Water

$\begin{array}{rrrrrr}\odot & 166,650 & 117,053 & 61,726 & 38,238 & 41,566 \\ \odot & 161,824 & 117,053 & 49,222 & 38,238 & 41,566 \\ \odot & -4,826 & 0 & -12,504 & 0 & 0 \\ \odot & -3 & 0 & -20 & 0 & 0\end{array}$

$\begin{array}{lr}\odot & \odot \\ \odot & \odot \\ \odot & \odot \\ \odot & \odot \\ & \\ \odot & 569 \\ \odot & 552 \\ \odot & -16 \\ \odot & -3 \\ & \\ \odot & \\ \odot & 23 \\ \odot & 22 \\ \odot & -1 \\ & -3\end{array}$

$$
131
$$$$
\odot
$$$$
\odot
$$

48 


\section{Alternative Financing Emission Reduction}

The emission reductions from implemented the proposed retrofits are as follows:

Lodging facilities

1166

Sulfur oxides (lb)

existing

post-retrofit

3,856

3,699

difference

$-157$

$\%$ change

$-4$

Nitrogen 0xides (lb)

existing

post-retrofit

1,854

difference

1,779

$\%$ change

-75
-4

Carbon Monoxide (lb)

existing

post-retrofit

3,196

3,067

difference

$-129$

$\%$ change

$-4$

Carbon Dioxide (tons)

existing

393

post-retrofit

377

difference

$-16$

$\%$ change

$-4$

Particulate Matter (lb)

existing

post-retrofit

difference

77

$\%$ change

Hydrocarbons (lb)

existing

post-retrofit

1,320

difference

1,266

$\%$ change

$-53$ 


\section{Building 2040 Aircraft Maintenance Shop}

The following information identifies the cost-effective energy- and cost-reducing retrofit projects for building 2040 identified from the FEDS modeling and analysis. Key energy and economic results are presented for each cost-effective retrofit measure.

\section{Facility Description 2040}

Building 2040 is an aircraft maintenance shop built in 1937. 2040 is cooled by an air cooled chiller and has little to no insulation in its building envelope. Building 2040 is 77,439 sf.

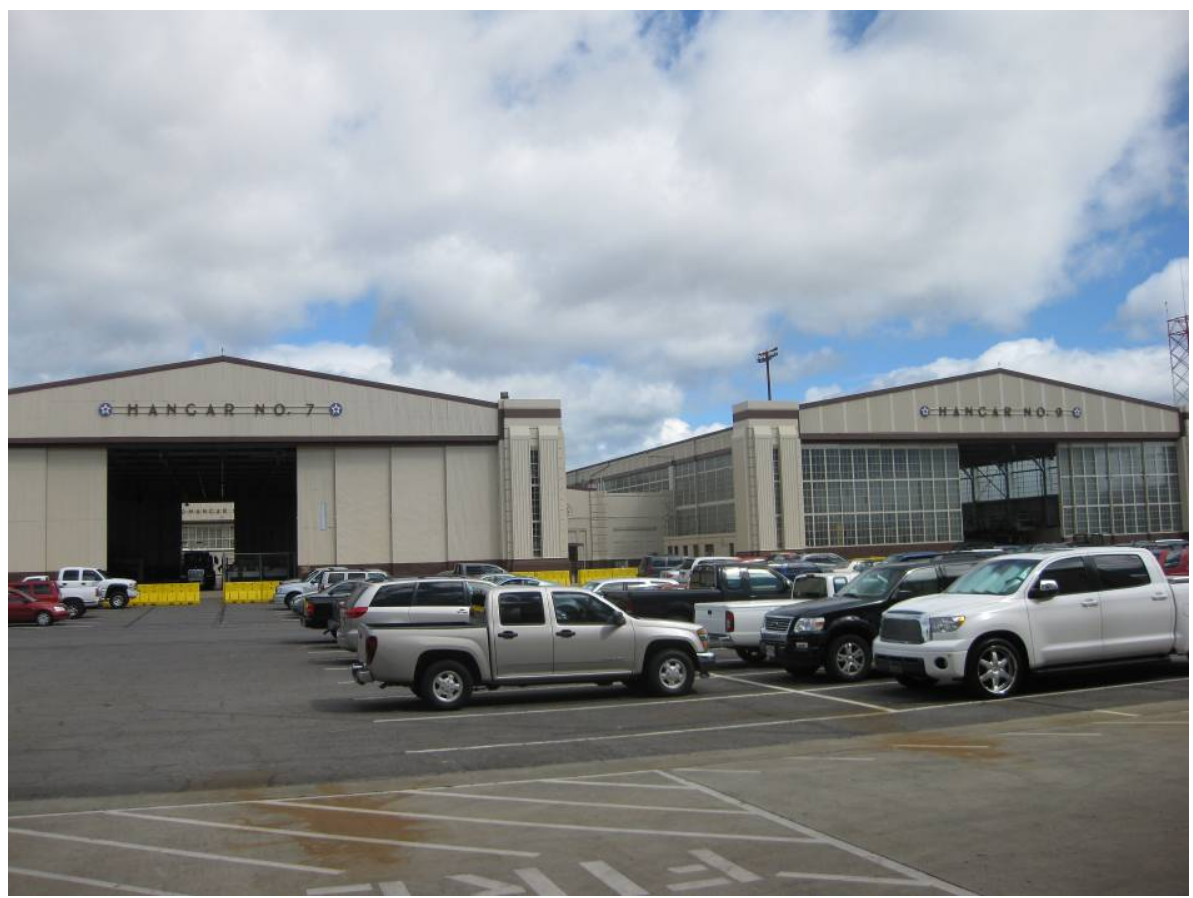




\section{Alternative Financing Results}

A FEDS analysis using alternative financing suggests replacing some of the lighting technologies, and various upgrades to the electric water heater system for the conditioned spaces. FEDS had no life cycle cost effective retrofits for the unconditioned space.

Appropriated funding FEDS analysis results for building 2040 conditioned space:

\begin{tabular}{|c|c|c|c|c|c|c|c|c|}
\hline Bldg. Set Description & $\begin{array}{l}\text { End } \\
\text { Use }\end{array}$ & Existing Technology & Retrofit Technology & $\begin{array}{l}\text { Energy } \\
\text { Savings } \\
\text { (MMBtu/yr) } \\
\end{array}$ & $\begin{array}{l}\text { 1st Year } \\
\text { Savings } \\
(\$ / y r)\end{array}$ & $\begin{array}{l}\text { Installed } \\
\text { Cost (\$) }\end{array}$ & $\begin{array}{l}\text { Net } \\
\text { Present } \\
\text { Value (\$) }\end{array}$ & SIR \\
\hline 1940's shops 2040 & $\begin{array}{l}\text { Hot } \\
\text { Water }\end{array}$ & Electric Water Heater & $\begin{array}{l}\text { Faucet Aerators, Lower Tank } \\
\text { Temperature }\end{array}$ & 1 & 89 & 46 & 465 & 11.1 \\
\hline 1940's shops 2040 & Lights & EX6: EXIT - LED & $\begin{array}{l}\text { EX12: EXIT - } \\
\text { ELECTROLUMINESCENT PANEL } \\
\text { RETRO KIT }\end{array}$ & - & 58 & 311 & 47 & 1.2 \\
\hline 1940's shops 2040 & Lights & FL2: FL 2X4 3F40T12 STD1,2 & FL236: FL 2X4 3F32T8 ELC3 & 8 & 599 & 1,764 & 1,742 & 2.0 \\
\hline 1940's shops & Lights & FL1: FL 2X4 4F40T12 STD2 & FL244: FL 2X4 4F32T8 ELC4 & 56 & 4,034 & 9,524 & 13,994 & 2.5 \\
\hline 1940 's shops 2040 & Lights & FL3: FL 2X4 2F40T12 STD2 & FL51: FL 2X4 2F32T8 ELC2 & 9 & 695 & 2,821 & 1,253 & 1.4 \\
\hline
\end{tabular}




\section{Alternative Financing Energy Consumption by Fuel Type}

The modeled energy consumption for a typical year was 352,637 kwh. No proposed retrofits were suggested for the unconditioned space. The energy use intensity is $18.3 \mathrm{MBtu} / \mathrm{Ksf}$.

\begin{tabular}{|c|c|c|c|c|}
\hline \multirow[b]{2}{*}{ Fuel } & \multirow[b]{2}{*}{ Energy } & \multicolumn{2}{|c|}{$2 \odot 4 \odot$ unconditioned space } & \multirow[b]{2}{*}{$\begin{array}{l}\text { Dollars } \\
(2009) \text { * }\end{array}$} \\
\hline & & $\begin{array}{c}\text { Energy } \\
\text { Intensity } \\
\text { (user units/1000ft2) }\end{array}$ & $\begin{array}{c}\text { Energy } \\
\text { Intensity } \\
\text { (MBtu/1000ft2) }\end{array}$ & \\
\hline \multicolumn{5}{|c|}{ Electricity (kWh) } \\
\hline existing & 352,637 & $5,362.8$ & 18.3 & 62,302 \\
\hline post-retrofit & 352,637 & $5,362.8$ & 18.3 & 62,264 \\
\hline difference & 0 & 0.0 & $\odot .0$ & -38 \\
\hline$\%$ change & $\odot$ & $\odot$ & 0 & 0 \\
\hline \multicolumn{5}{|l|}{ Total (MBtu) } \\
\hline existing & 1,204 & 18.3 & 18.3 & 62,302 \\
\hline post-retrofit & 1,204 & 18.3 & 18.3 & 62,264 \\
\hline difference & 0 & 0.0 & 0.0 & -38 \\
\hline$\%$ change & $\odot$ & 0 & 0 & $\odot$ \\
\hline
\end{tabular}

* Dollar values for electricity include both energy and demand components. 
The modeled energy consumption for the conditioned space a typical year was 156,928 kwh before retrofits and 134,998 kwh after proposed retrofits are implemented. The energy use intensity goes from $46.2 \mathrm{MBtu} / \mathrm{Ksf}$ to $39.7 \mathrm{MBtu} / \mathrm{Ksf}$ after retrofits.

\begin{tabular}{|c|c|c|c|c|}
\hline \multirow[b]{2}{*}{ Fuel } & 1940 's shops & \multicolumn{2}{|c|}{2040 conditioned space } & \multirow[b]{2}{*}{$\begin{array}{l}\text { Dollars } \\
(2009)^{*}\end{array}$} \\
\hline & Energy & $\begin{array}{c}\text { Energy } \\
\text { Intensity } \\
\text { (user units/1000ft2) }\end{array}$ & $\begin{array}{c}\text { Energy } \\
\text { Intensity } \\
\text { (MBtu/1000ft2) }\end{array}$ & \\
\hline \multicolumn{5}{|c|}{ Electricity (kWh) } \\
\hline existing & 156,928 & $13,523.7$ & 46.2 & 27,725 \\
\hline post-retrofit & 134,998 & $11,633.7$ & 39.7 & 23,836 \\
\hline difference & $-21,931$ & $-1,889.9$ & -6.5 & $-3,889$ \\
\hline$\%$ change & -14 & -14 & -14 & -14 \\
\hline \multicolumn{5}{|l|}{ Total (MBtu) } \\
\hline existing & 536 & 46.2 & 46.2 & 27,725 \\
\hline post-retrofit & 461 & 39.7 & 39.7 & 23,836 \\
\hline difference & -75 & -6.5 & -6.5 & $-3,889$ \\
\hline$\%$ change & -14 & -14 & -14 & -14 \\
\hline
\end{tabular}

* Dollar values for electricity include both energy and demand components. 


\section{Alternative Financing Energy Consumption by End Use}

Motors and miscellaneous equipment is the largest load in the unconditioned space of the building with 308,331 kWh/year, followed by lighting with $44,307 \mathrm{kWh} /$ year.

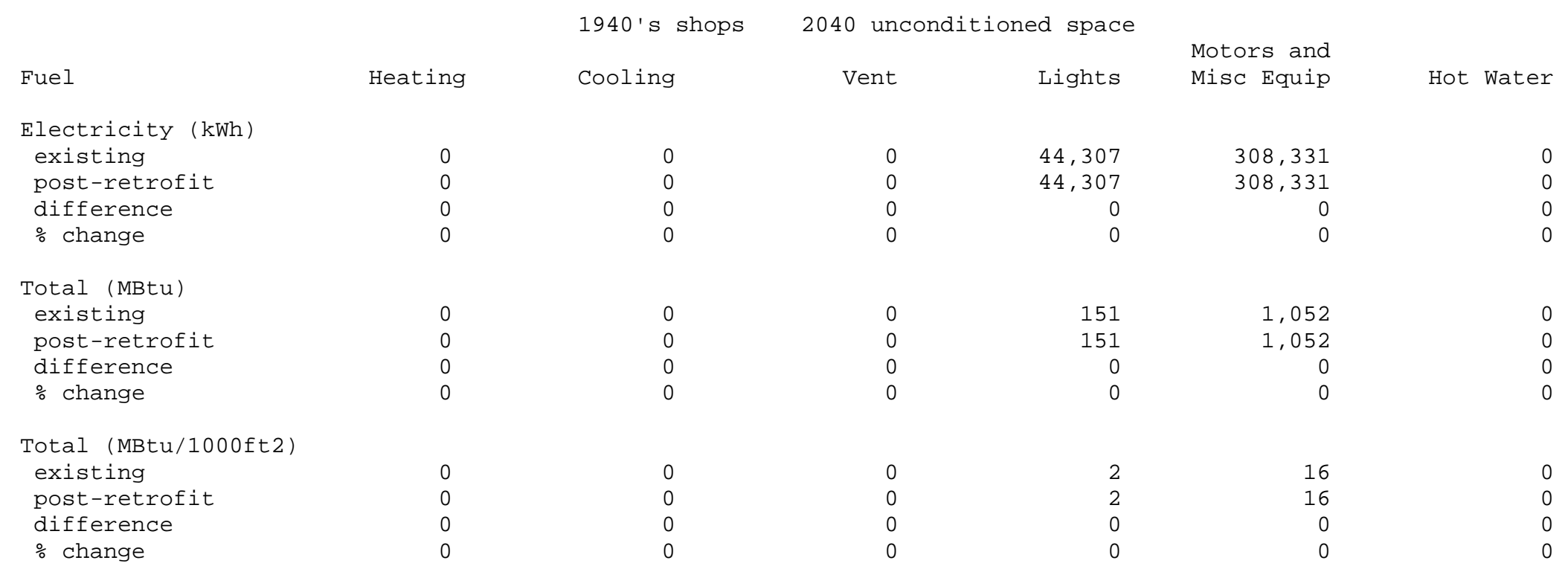


Motors and miscellaneous equipment is the largest load in the conditioned space of the building with 56,167 kWh/year, followed by space cooling with 52,533 kWh/year.

1940's shops 2040
Fuel
Electricity (kWh)
existing
post-retrofit
difference
\% change
Total (MBtu)
existing
post-retrofit
difference
\% change
Total (MBtu/1000ft2)
existing
post-retrofit
difference
\% change

Heatin

$\begin{array}{rr}\text { Vent } & \text { Lights } \\ 2,983 & \\ 2,757 & 43,930 \\ -226 & 26,536 \\ -8 & -17,394 \\ & -40 \\ 10 & \\ 9 & 150 \\ -1 & 91 \\ -8 & -59 \\ & -40 \\ 1 & \\ 1 & 13 \\ \odot & 8 \\ -8 & -5 \\ & -40\end{array}$

Motors and

Misc Equip

Hot Water

52,533
48,660
$-3,874$
-7

179
166
-13
-7

$$
\begin{array}{r}
56,167 \\
56,167 \\
\odot \\
\odot
\end{array}
$$

(1)

$\begin{array}{ll}\odot & 15 \\ \odot & 14 \\ \odot & -1 \\ \odot & -7\end{array}$

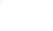




\section{Alternative Financing Emission Reduction}

The emission reductions from implemented the proposed retrofits are as follows:

1940 's shops 2040 unconditioned space

Sulfur Oxides (lb)

existing

post-retrofit

3,188

difference

3,188

$\%$ change

3,188
0

Nitrogen oxides (lb)

existing

post-retrofit

difference

1,523
1,523

$\%$ change

$\odot$

Carbon Monoxide (lb)

existing

post-retrofit

difference

$\%$ change

2,620

2,620

$\odot$

Carbon Dioxide (tons)

existing

post-retrofit

323

difference

323

$\%$ change

$\odot$

Particulate Matter (lb)

existing

post-retrofit

difference

63

$\%$ change

Hydrocarbons (lb)

existing

post-retrofit

1,084

difference

1,084

$\%$ change

$\odot$
$\odot$ 
Sulfur Oxides (lb)

existing

post-retrofit

1,419

difference

1,220
-198

$\%$ change

$-14$

Nitrogen oxides (lb)

existing

post-retrofit $\quad 583$

difference $\quad-95$

$\%$ change

$-14$

Carbon Monoxide (lb)

existing

post-retrofit

1,166

1,003

$-163$

$\%$ change

$-14$

Carbon Dioxide (tons)

existing

144

post-retrofit

difference $\quad-20$

$\%$ change

Particulate Matter (lb)

existing

post-retrofit

difference -4

$\%$ change

Hydrocarbons ( $1 b$ )

existing

483

post-retrofit $\quad 415$

difference $\quad-67$

$\%$ change

$-14$ 


\section{Building 1715 Recycling Center}

The following information identifies the cost-effective energy- and cost-reducing retrofit projects for building 1715 identified from the FEDS modeling and analysis. Key energy and economic results are presented for each cost-effective retrofit measure.

\section{Facility Description 1715}

1715 is a recycling center built in 1944. The majority of the space is unconditioned, with a small office that is served by an electric package unit. The building was modeled as two linked buildings, one conditioned, one unconditioned. Building 1715 is 30,400 sf.

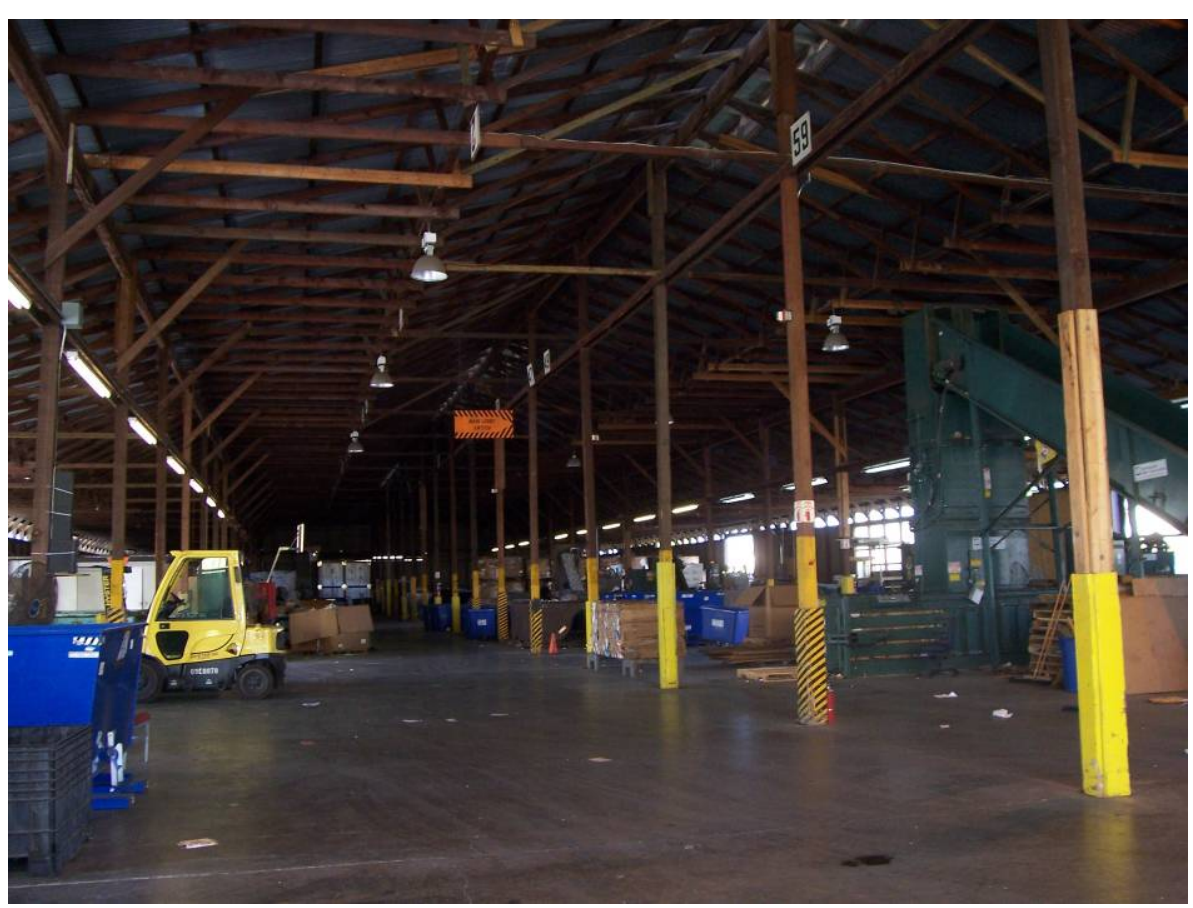




\section{Alternative Financing Results}

A FEDS analysis using alternative financing suggests replacing lights in the unconditioned space as well as replacing lights in the conditioned space.

Alternative financing FEDS results for building 1715 unconditioned space:

\begin{tabular}{|c|c|c|c|c|c|c|c|c|}
\hline Bldg. Set Description & $\begin{array}{l}\text { End } \\
\text { Use }\end{array}$ & Existing Technology & Retrofit Technology & $\begin{array}{l}\text { Energy } \\
\text { Savings } \\
\text { (MMBtu/yr) }\end{array}$ & $\begin{array}{l}\text { 1st Year } \\
\text { Savings } \\
(\$ / y r)\end{array}$ & $\begin{array}{l}\text { Installed } \\
\text { Cost (\$) }\end{array}$ & $\begin{array}{l}\text { Net } \\
\text { Present } \\
\text { Value (\$) }\end{array}$ & SIR \\
\hline 1940's shops & Lights & EX6: EXIT - LED & $\begin{array}{l}\text { EX12: EXIT - } \\
\text { ELECTROLUMINESCENT PANEL } \\
\text { RETRO KIT }\end{array}$ & - & 112 & 621 & 62 & 1.1 \\
\hline 1940's shops 1715 & Lights & FL62: FL 1X8 2F96T12 STD2 & FL74: FL 1X8 2F96T12 ELC2 & 14 & 1,129 & 5,809 & 796 & 1.1 \\
\hline
\end{tabular}

Alternative financing FEDS results for building 1715 conditioned space:

\begin{tabular}{|c|c|c|c|c|c|c|c|c|}
\hline Bldg. Set Description & $\begin{array}{l}\text { End } \\
\text { Use }\end{array}$ & Existing Technology & Retrofit Technology & $\begin{array}{l}\text { Energy } \\
\text { Savings } \\
\text { (MMBtu/yr) } \\
\end{array}$ & $\begin{array}{l}\text { 1st Year } \\
\text { Savings } \\
(\$ / y r)\end{array}$ & $\begin{array}{l}\text { Installed } \\
\text { Cost (\$) }\end{array}$ & $\begin{array}{l}\text { Net } \\
\text { Present } \\
\text { Value (\$) } \\
\end{array}$ & SIR \\
\hline 1940's shops 1715 & Lights & EX6: EXIT - LED & $\begin{array}{l}\text { EX12: EXIT - } \\
\text { ELECTROLUMINESCENT PANEL } \\
\text { RETRO KIT }\end{array}$ & - & 59 & 311 & 50 & 1.2 \\
\hline 1940's shops 1715 & Lights & FL37: FL 2X4 4F32T8 EEF2 & $\begin{array}{l}\text { FL280: FL 2X4 3F32ST8 ELC3 } \\
\text { REF }\end{array}$ & 115 & 8,684 & 25,173 & 25,616 & 2.0 \\
\hline
\end{tabular}




\section{Alternative Financing Energy Consumption by Fuel Type}

The modeled energy consumption for a typical year was 147,909 kwh before retrofits and 143,661 kwh after proposed retrofits are implemented. The energy use intensity goes from 18.0 MBtu/Ksf to 17.5 MBtu/Ksf after retrofits.

\begin{tabular}{|c|c|c|c|c|}
\hline \multirow[b]{2}{*}{ Fuel } & 1940 's shops & \multicolumn{2}{|c|}{1715 unconditioned space } & \multirow[b]{2}{*}{$\begin{array}{l}\text { Dollars } \\
(2009)^{*}\end{array}$} \\
\hline & Energy & $\begin{array}{c}\text { Energy } \\
\text { Intensity } \\
\text { (user units/1000ft2) }\end{array}$ & $\begin{array}{c}\text { Energy } \\
\text { Intensity } \\
\text { (MBtu/1000ft2) }\end{array}$ & \\
\hline \multicolumn{5}{|c|}{ Electricity (kWh) } \\
\hline existing & 147,909 & $5,288.5$ & 18.0 & 26,132 \\
\hline post-retrofit & 143,661 & $5,136.6$ & 17.5 & 25,366 \\
\hline difference & $-4,248$ & -151.9 & -0.5 & -766 \\
\hline$\%$ change & -3 & -3 & -3 & -3 \\
\hline \multicolumn{5}{|l|}{ Total (MBtu) } \\
\hline existing & 505 & 18.0 & 18.0 & 26,132 \\
\hline post-retrofit & 490 & 17.5 & 17.5 & 25,366 \\
\hline difference & -14 & -0.5 & -0.5 & -766 \\
\hline$\%$ change & -3 & -3 & -3 & -3 \\
\hline
\end{tabular}

* Dollar values for electricity include both energy and demand components. 
The modeled energy consumption for a typical year was 331,402 kwh before retrofits and 299,027 kwh after proposed retrofits are implemented. The energy use intensity goes from 466.5.6 MBtu/Ksf to 419.6 MBtu/Ksf after retrofits.

\begin{tabular}{|c|c|c|c|c|}
\hline Fuel & Energy & $\begin{array}{c}\text { Energy } \\
\text { Intensity } \\
\text { (user units/1000ft2) }\end{array}$ & $\begin{array}{c}\text { Energy } \\
\text { Intensity } \\
\text { (MBtu/1000ft2) }\end{array}$ & $\begin{array}{l}\text { Dollars } \\
(20 \odot 9)^{*}\end{array}$ \\
\hline \multicolumn{5}{|c|}{ Electricity (kWh) } \\
\hline existing & $332,4 \odot 2$ & $136,678.6$ & 466.5 & 58,727 \\
\hline post-retrofit & 299,027 & $122,955.2$ & 419.6 & 52,798 \\
\hline difference & $-33,375$ & $-13,723.4$ & -46.8 & $-5,929$ \\
\hline$\%$ change & -10 & -10 & -10 & -10 \\
\hline \multicolumn{5}{|l|}{ Total (MBtu) } \\
\hline existing & 1,134 & 466.5 & 466.5 & 58,727 \\
\hline post-retrofit & 1,021 & 419.6 & 419.6 & 52,798 \\
\hline difference & -114 & -46.8 & -46.8 & $-5,929$ \\
\hline$\%$ change & -10 & -10 & -10 & -10 \\
\hline
\end{tabular}

* Dollar values for electricity include both energy and demand components. 


\section{Alternative Financing Energy Consumption by End Use}

Motors and miscellaneous equipment is the largest load in the unconditioned space of the building with 131,142 kWh/year, followed by lighting with 16,767 kWh/year.

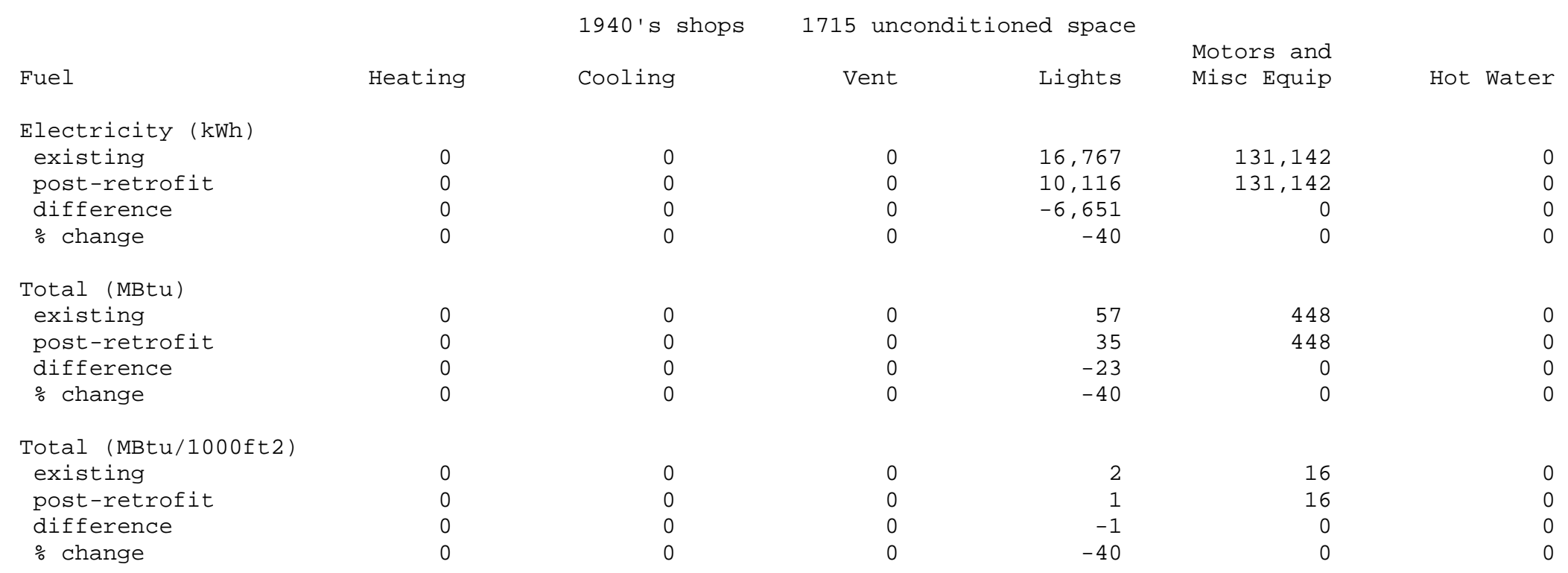


Lighting is the largest load in the conditioned space of the building with 234,179 $\mathrm{kWh} / \mathrm{year}$, followed by space cooling with 82,158 $\mathrm{kWh}$ /year.

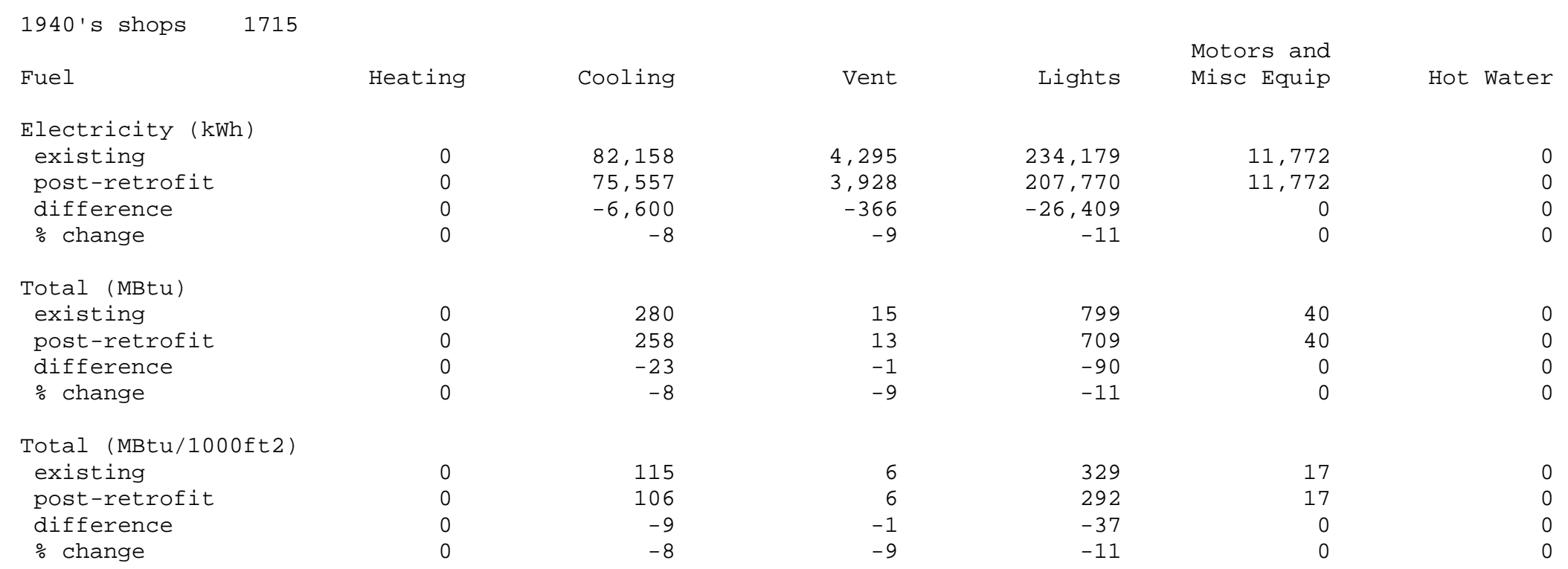




\section{Alternative Financing Emission Reduction}

The emission reductions from implemented the proposed retrofits are as follows:

1940 's shops 1715 unconditioned space

Sulfur oxides (lb)

existing

post-retrofit

1,337

difference

1,299

299
-38

$\%$ change

$-3$

Nitrogen Oxides (lb)

existing

639

post-retrofit

621

difference

$-18$

$\%$ change

$-3$

Carbon Monoxide (lb)

existing

post-retrofit

1,099

difference

1,067

$\%$ change

-32
-3

Carbon Dioxide (tons)

existing

135

post-retrofit

131

difference

$-4$

$\%$ change

$-3$

Particulate Matter (lb)

existing

post-retrofit

difference

$\%$ change

Hydrocarbons (lb)

existing

post-retrofit

difference

455

$\%$ change

442

$-13$

change 
Sulfur Oxides (lb)

existing

post-retrofit

3,005

difference

2,703

$\%$ change

$-10$

Nitrogen 0xides (lb)

existing

post-retrofit

, 436

1,292

$-144$

difference

$-10$

Carbon Monoxide (lb)

existing

post-retrofit

difference

2,470

2,222

$-248$

$\%$ change

Carbon Dioxide (tons)

existing

post-retrofit

304

difference

274

$\%$ change

-31
-10

Particulate Matter (lb)

existing

post-retrofit

59

difference

53
-6

$\%$ change

Hydrocarbons (1b)

existing

1, 022

post-retrofit

difference

$\%$ change

-103
-10 


\section{Building 2177 Maintenance Shop}

The following information identifies the cost-effective energy- and cost-reducing retrofit projects for building 2177 identified from the FEDS modeling and analysis. Key energy and economic results are presented for each cost-effective retrofit measure.

\section{Facility Description 2177}

2177 is a base engineer maintenance shop built in 1944. This building is partially cooled. Building 2177 is 3,200 sf. 


\section{Alternative Financing Results}

A FEDS analysis using alternative financing suggests replacing T12 lighting with T8 lighting in the unconditioned spaces. Upgrades to the hot water system include insulating the tank and pipes as well as installing aerators and lowering the tank temperature for the unconditioned spaces. For the conditioned spaces FEDS suggests replacing the lighting, and upgrading the hot water system.

Alternative financing FEDS results for building 2177 unconditioned spaces:

\begin{tabular}{|c|c|c|c|c|c|c|c|c|}
\hline Bldg. Set Description & $\begin{array}{l}\text { End } \\
\text { Use }\end{array}$ & Existing Technology & Retrofit Technology & $\begin{array}{l}\text { Energy } \\
\text { Savings } \\
\text { (MMBtu/yr) }\end{array}$ & $\begin{array}{c}\text { 1st Year } \\
\text { Savings } \\
(\$ / y r)\end{array}$ & $\begin{array}{l}\text { Installed } \\
\text { Cost (\$) }\end{array}$ & $\begin{array}{l}\text { Net } \\
\text { Present } \\
\text { Value (\$) } \\
\end{array}$ & SIR \\
\hline 1940's shops & $\begin{array}{l}\text { Hot } \\
\text { Water }\end{array}$ & Electric Water Heater & $\begin{array}{l}\text { Faucet Aerators, Lower Tank } \\
\text { Temperature }\end{array}$ & - & 1 & 2 & 5 & 3.9 \\
\hline 1940's shops 2177 & Lights & FL4: FL 1X4 2F40T12 STD2 & FL52: FL 1X4 2F32T8 ELC2 & 7 & 585 & 2,437 & 1,000 & 1.4 \\
\hline
\end{tabular}

Alternative financing FEDS results for building 2177 conditioned spaces:

\begin{tabular}{|c|c|c|c|c|c|c|c|c|}
\hline Bldg. Set Description & $\begin{array}{l}\text { End } \\
\text { Use }\end{array}$ & Existing Technology & Retrofit Technology & $\begin{array}{l}\text { Energy } \\
\text { Savings } \\
\text { (MMBtu/yr) }\end{array}$ & $\begin{array}{l}\text { 1st Year } \\
\text { Savings } \\
(\$ / y r)\end{array}$ & $\begin{array}{l}\text { Installed } \\
\text { Cost (\$) }\end{array}$ & $\begin{array}{l}\text { Net } \\
\text { Present } \\
\text { Value (\$) }\end{array}$ & SIR \\
\hline 1940's shops 2177 & $\begin{array}{l}\text { Hot } \\
\text { Water }\end{array}$ & Electric Water Heater & $\begin{array}{l}\text { Faucet Aerators, Lower Tank } \\
\text { Temperature }\end{array}$ & - & 1 & 2 & 9 & 5.0 \\
\hline 1940's shops & Lights & EX6: EXIT - LED & $\begin{array}{l}\text { EX12: EXIT - } \\
\text { ELECTROLUMINESCENT PANEL } \\
\text { RETRO KIT }\end{array}$ & - & 7 & 43 & 7 & 1.2 \\
\hline
\end{tabular}




\section{Alternative Financing Energy Consumption by Fuel Type}

The modeled energy consumption for a typical year was 13,067 kwh before retrofits and 11,100 kwh after proposed retrofits are implemented. The energy use intensity goes from 27.9 MBtu/Ksf to 23.7 MBtu/Ksf after retrofits.

\begin{tabular}{|c|c|c|c|c|}
\hline \multirow[b]{2}{*}{ Fuel } & 1940 's shops & \multicolumn{2}{|c|}{2177 unconditioned space } & \multirow[b]{2}{*}{$\begin{array}{l}\text { Dollars } \\
(2009) \text { * }\end{array}$} \\
\hline & Energy & $\begin{array}{c}\text { Energy } \\
\text { Intensity } \\
\text { (user units/1000ft2) }\end{array}$ & $\begin{array}{c}\text { Energy } \\
\text { Intensity } \\
\text { (MBtu/1000ft2) }\end{array}$ & \\
\hline \multicolumn{5}{|c|}{ Electricity (kWh) } \\
\hline existing & 13,067 & $8,166.8$ & 27.9 & 2,310 \\
\hline post-retrofit & 11,100 & $6,937.4$ & 23.7 & 1,960 \\
\hline difference & $-1,967$ & $-1,229.4$ & -4.2 & -350 \\
\hline$\%$ change & -15 & -15 & -15 & -15 \\
\hline \multicolumn{5}{|l|}{ Total (MBtu) } \\
\hline existing & 45 & 27.9 & 27.9 & 2,310 \\
\hline post-retrofit & 38 & 23.7 & 23.7 & 1,960 \\
\hline difference & -7 & -4.2 & -4.2 & -350 \\
\hline$\%$ change & -15 & -15 & -15 & -15 \\
\hline
\end{tabular}

* Dollar values for electricity include both energy and demand components. 
The modeled energy consumption for a typical year was 42,463 kwh before retrofits and 27,009 kwh after proposed retrofits are implemented. The energy use intensity goes from 90.6 MBtu/Ksf to 57.6 MBtu/Ksf after retrofits.

1940 's shops 2177 conditioned space

Fuel

Electricity $(\mathrm{kWh})$

existing

post-retrofit

difference

$\%$ change

Total (MBtu)

existing

post-retrofit

difference

$\%$ change

\section{Energy}

Intensity

(user units/1000ft2)

Energy

Intensity

(MBtu/1000ft2)

$$
\begin{array}{r}
18,159.8 \\
18,146.8 \\
-12.9 \\
0
\end{array}
$$

29,056
29,035

-21
0

62.0

61.9

0.0
62.0

61.9

0.0

62.0

61.9

$\odot .0$
0
Dollars

$(2009)$ *

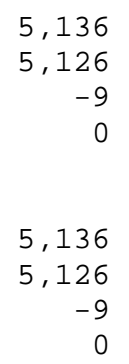

5,136

126

0

-9
0

* Dollar values for electricity include both energy and demand components. 


\section{Alternative Financing Energy Consumption by End Use}

Motors and miscellaneous equipment is the largest load in the unconditioned space of the building with 7,502 kWh/year, followed by lighting with 5,536 kWh/year.

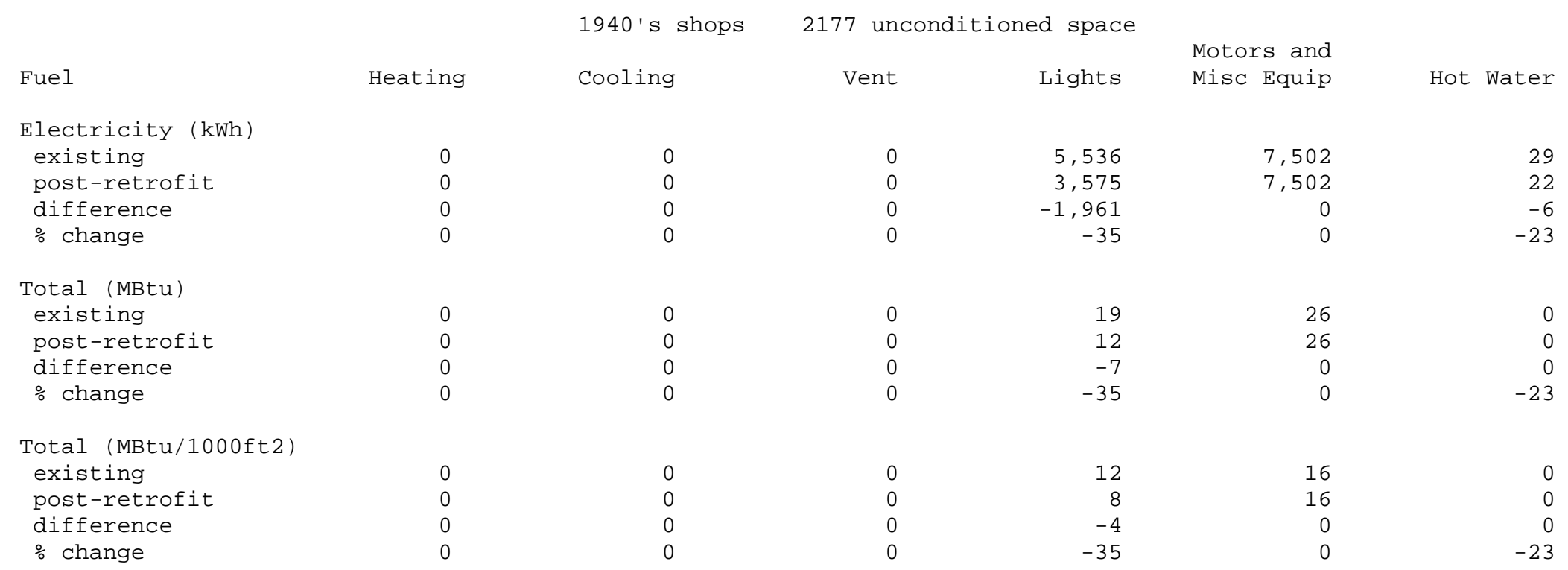


Space cooling is the largest load in the conditioned space of the building with 10,796 kWh/year, followed by lighting with 10,793 kWh/year.

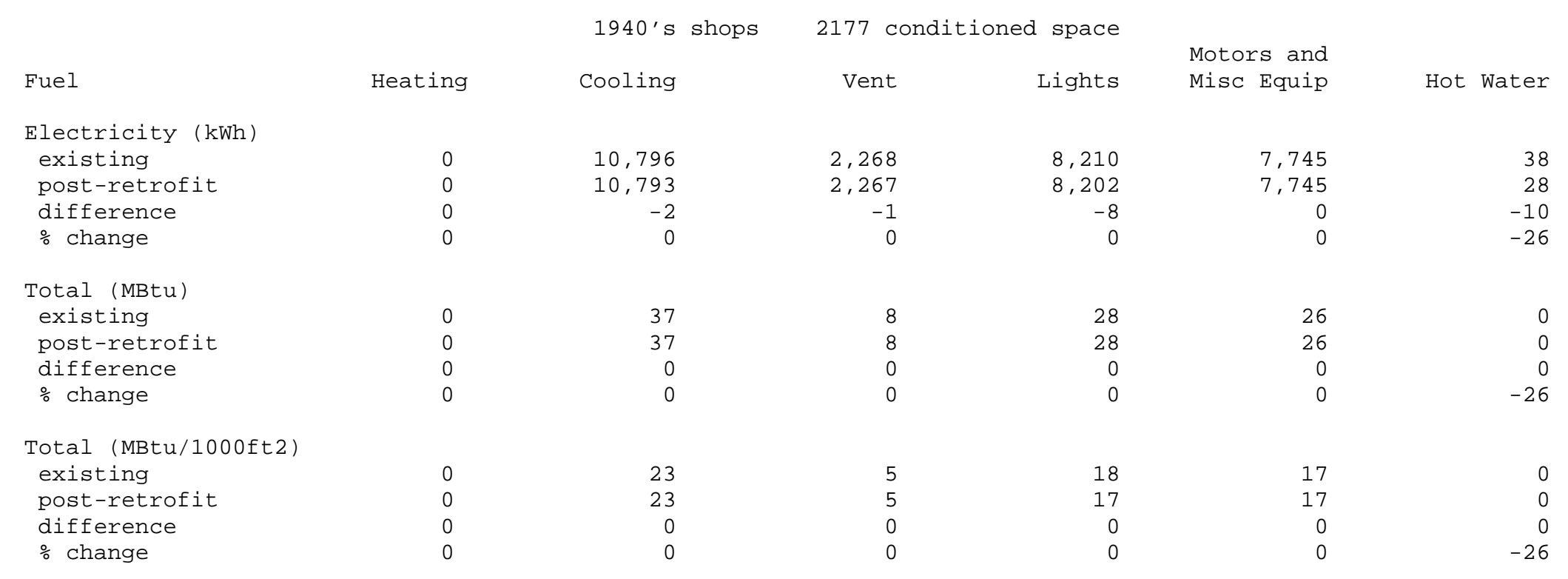




\section{Alternative Financing Emission Reduction}

The emission reductions from implemented the proposed retrofits are as follows:

1940 's shops 2177 unconditioned space

Sulfur Oxides (lb)

existing

post-retrofit

difference -18

\% change -15

Nitrogen Oxides (lb)

existing

post-retrofit

$\%$ change -15

Carbon Monoxide (lb)

existing

post-retrofit

difference

$\%$ change

Carbon Dioxide (tons)

existing

post-retrofit

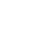

difference

12

$-2$

Particulate Matter (lb)

existing

post-retrofit

difference

$\%$ change

Hydrocarbons ( $1 b$ )

existing

post-retrofit

difference

$\%$ change

$-15$

2

2
0

$-15$ 
1940 's shops

post-retrofit
difference

$\%$ change

Nitrogen oxides (lb)

existing

post-retrofit

difference

$\%$ change

Carbon Monoxide (lb)

existing

post-retrofit

difference

$\%$ change

Carbon Dioxide (tons)

existing

post-retrofit

difference

$\%$ change

Particulate Matter (lb)

existing

post-retrofit

difference

$\%$ change

Hydrocarbons ( $1 b$ )

existing

post-retrofit

difference

$\%$ change
263

262

$\odot$

2177 conditioned space

126

125

$\odot$

216

216

$\odot$

27

27

0
0

5

5

0

89

89

$\odot$ 


\section{Building 4016 Maintenance Shop}

The following information identifies the cost-effective energy- and cost-reducing retrofit projects for building 4016 identified from the FEDS modeling and analysis. Key energy and economic results are presented for each cost-effective retrofit measure.

\section{Facility Description 4016}

Building 4016 is a base engineer maintenance shop built in 1973. 4016 is cooled by multiple package units and has little to no insulation in its building enveloped. Building 4016 is 7,701 sf.

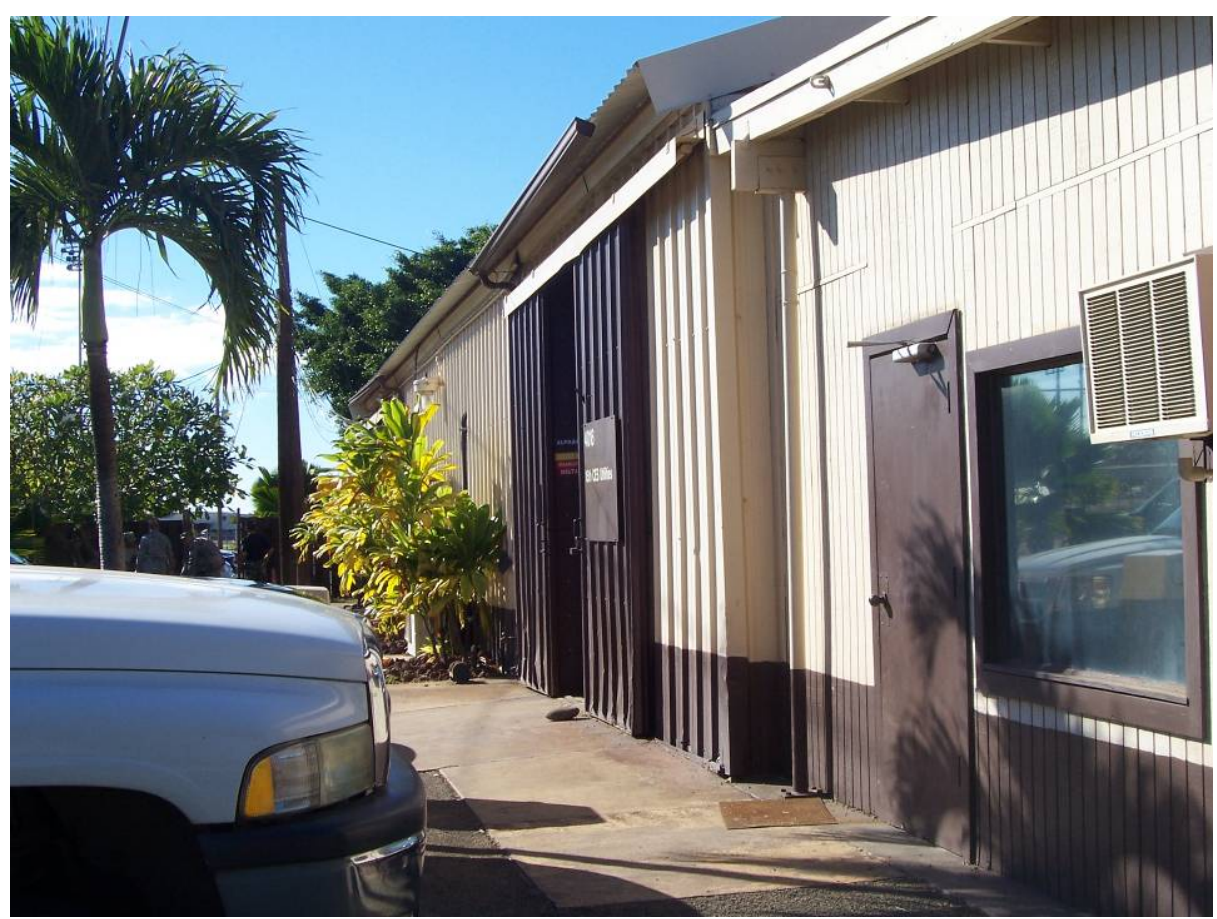

\section{Alternative Financing Results}


A FEDS analysis using alternative financing suggests replacing the EXIT lighting. In the unconditioned space FEDS suggests replacing the EXIT lighting as well.

Alternative financing FEDS results for building 4016 unconditioned spaces:

\begin{tabular}{|c|c|c|c|c|c|c|c|c|}
\hline Bldg. Set Description & $\begin{array}{l}\text { End } \\
\text { Use }\end{array}$ & Existing Technology & Retrofit Technology & $\begin{array}{l}\text { Energy } \\
\text { Savings } \\
\text { (MMBtu/yr) }\end{array}$ & $\begin{array}{c}\text { 1st Year } \\
\text { Savings } \\
\text { (\$/yr) }\end{array}$ & $\begin{array}{l}\text { Installed } \\
\text { Cost (\$) }\end{array}$ & $\begin{array}{l}\text { Net } \\
\text { Present } \\
\text { Value (\$) } \\
\end{array}$ & SIR \\
\hline 1970's shops 4016 & Lights & EX1: EXIT - INC $(2 \times 20)$ & $\begin{array}{l}\text { EX12: EXIT - } \\
\text { ELECTROLUMINESCENT PANEL } \\
\text { RETRO KIT }\end{array}$ & 14 & 863 & 621 & 4,433 & 8.1 \\
\hline
\end{tabular}

Alternative financing FEDS results for building 4016 unconditioned spaces:

\begin{tabular}{|c|c|c|c|c|c|c|c|c|}
\hline Bldg. Set Description & $\begin{array}{l}\text { End } \\
\text { Use }\end{array}$ & Existing Technology & Retrofit Technology & $\begin{array}{l}\text { Energy } \\
\text { Savings } \\
\text { (MMBtu/yr) }\end{array}$ & $\begin{array}{l}\text { 1st Year } \\
\text { Savings } \\
(\$ / y r)\end{array}$ & $\begin{array}{l}\text { Installed } \\
\text { Cost (\$) }\end{array}$ & $\begin{array}{l}\text { Net } \\
\text { Present } \\
\text { Value (\$) }\end{array}$ & SIR \\
\hline 1970 's shops 4016 & Lights & EX1: EXIT - INC $(2 \times 20)$ & $\begin{array}{l}\text { EX12: EXIT - } \\
\text { ELECTROLUMINESCENT PANEL } \\
\text { RETRO KIT }\end{array}$ & 5 & 348 & 311 & 1,742 & 6.6 \\
\hline
\end{tabular}




\section{Alternative Financing Energy Consumption by Fuel Type}

The modeled energy consumption for a typical year was 67,485 kwh before retrofits and 63,739 kwh after proposed retrofits are implemented. The energy use intensity goes from 39.9 MBtu/Ksf to 37.7 MBtu/Ksf after retrofits.

\begin{tabular}{|c|c|c|c|c|}
\hline Fuel & Energy & $\begin{array}{c}\text { Energy } \\
\text { Intensity } \\
\text { (user units/1000ft2) }\end{array}$ & $\begin{array}{c}\text { Energy } \\
\text { Intensity } \\
\text { (MBtu/1000ft2) }\end{array}$ & $\begin{array}{l}\text { Dollars } \\
(2009) \text { * }\end{array}$ \\
\hline \multicolumn{5}{|c|}{ Electricity (kWh) } \\
\hline existing & 67,485 & $11,685.8$ & 39.9 & 11,928 \\
\hline post-retrofit & 63,739 & $11,037.1$ & 37.7 & 11,254 \\
\hline difference & $-3,746$ & -648.7 & -2.2 & -674 \\
\hline$\%$ change & -6 & -6 & -6 & -6 \\
\hline \multicolumn{5}{|l|}{ Total (MBtu) } \\
\hline existing & 230 & 39.9 & 39.9 & 11,928 \\
\hline post-retrofit & 218 & 37.7 & 37.7 & 11,254 \\
\hline difference & -13 & -2.2 & -2.2 & -674 \\
\hline$\%$ change & -6 & -6 & -6 & -6 \\
\hline
\end{tabular}

* Dollar values for electricity include both energy and demand components. 
The modeled energy consumption for a typical year was 12,772 kwh before retrofits and 11,383 kwh after proposed retrofits are implemented. The energy use intensity goes from 22.6 MBtu/Ksf to 20.2 MBtu/Ksf after retrofits.

\begin{tabular}{|c|c|c|c|c|}
\hline \multirow[b]{2}{*}{ Fuel } & 1970 's shops & \multicolumn{2}{|c|}{4016 unconditioned space } & \multirow[b]{2}{*}{$\begin{array}{l}\text { Dollars } \\
(2009)^{*}\end{array}$} \\
\hline & Energy & $\begin{array}{c}\text { Energy } \\
\text { Intensity } \\
\text { (user units/1000ft2) }\end{array}$ & $\begin{array}{c}\text { Energy } \\
\text { Intensity } \\
\text { (MBtu/1000ft2) }\end{array}$ & \\
\hline \multicolumn{5}{|c|}{ Electricity (kWh) } \\
\hline existing & 12,772 & $6,634.9$ & 22.6 & 2,257 \\
\hline post-retrofit & 11,383 & $5,913.1$ & 20.2 & 2,010 \\
\hline difference & $-1,389$ & -721.7 & -2.5 & -248 \\
\hline$\%$ change & -11 & -11 & -11 & -11 \\
\hline \multicolumn{5}{|l|}{ Total (MBtu) } \\
\hline existing & 44 & 22.6 & 22.6 & 2,257 \\
\hline post-retrofit & 39 & 20.2 & 20.2 & 2,010 \\
\hline difference & -5 & -2.5 & -2.5 & -248 \\
\hline$\%$ change & -11 & -11 & -11 & -11 \\
\hline
\end{tabular}

* Dollar values for electricity include both energy and demand components. 


\section{Alternative Financing Energy Consumption by End Use}

Motors and miscellaneous equipment is the largest load in the conditioned space of the building with 27,381 kWh/year, followed by space cooling with 23,540 kWh/year.

\begin{tabular}{|c|c|c|c|c|c|c|}
\hline Fuel & Heating & 1970 's & $\begin{array}{l}4016 \\
\text { Vent }\end{array}$ & d space & $\begin{array}{l}\text { Motors and } \\
\text { Misc Equip }\end{array}$ & Hot water \\
\hline \multicolumn{7}{|c|}{ Electricity (kWh) } \\
\hline existing & $\odot$ & 23,540 & 5,159 & 10,761 & 27,381 & 643 \\
\hline post-retrofit & $\odot$ & 22,675 & 5,057 & 7,983 & 27,381 & 643 \\
\hline difference & $\odot$ & -865 & -103 & $-2,779$ & 0 & $\odot$ \\
\hline$\%$ change & $\odot$ & -4 & -2 & -26 & $\odot$ & $\odot$ \\
\hline \multicolumn{7}{|l|}{ Total (MBtu) } \\
\hline existing & $\odot$ & 80 & 18 & 37 & 93 & 2 \\
\hline post-retrofit & $\odot$ & 77 & 17 & 27 & 93 & 2 \\
\hline difference & $\odot$ & -3 & $\odot$ & -9 & $\odot$ & $\odot$ \\
\hline$\%$ change & $\odot$ & -4 & -2 & -26 & $\odot$ & $\odot$ \\
\hline \multicolumn{7}{|c|}{ Total (MBtu/1000ft2) } \\
\hline existing & $\odot$ & 14 & 3 & 6 & 16 & $\odot$ \\
\hline post-retrofit & $\odot$ & 13 & 3 & 5 & 16 & $\odot$ \\
\hline difference & $\odot$ & -1 & $\odot$ & -2 & $\odot$ & $\odot$ \\
\hline$\%$ change & $\odot$ & -4 & -2 & -26 & $\odot$ & $\odot$ \\
\hline
\end{tabular}


Motors and miscellaneous equipment is the largest load in the unconditioned space of the building with 8,620 kWh/year, followed by lighting with 3,738 kWh/year.

\begin{tabular}{|c|c|c|c|c|c|c|}
\hline \multirow{2}{*}{ Fuel } & \multirow[b]{2}{*}{ Heating } & \multirow{2}{*}{$\begin{array}{l}1970 \text { 's shops } \\
\text { Cooling }\end{array}$} & \multicolumn{2}{|c|}{4016 unconditioned space } & \multirow[b]{2}{*}{$\begin{array}{l}\text { Motors and } \\
\text { Misc Equip }\end{array}$} & \multirow[b]{2}{*}{ Hot Water } \\
\hline & & & Vent & Lights & & \\
\hline \multicolumn{7}{|c|}{ Electricity (kWh) } \\
\hline existing & 0 & $\Theta$ & 0 & 3,738 & 8,620 & 415 \\
\hline post-retrofit & 0 & $\odot$ & $\odot$ & 2,349 & 8,620 & 415 \\
\hline difference & $\odot$ & 0 & $\odot$ & $-1,389$ & 0 & 0 \\
\hline$\%$ change & 0 & $\odot$ & $\odot$ & -37 & $\odot$ & 0 \\
\hline \multicolumn{7}{|l|}{ Total (MBtu) } \\
\hline existing & 0 & 0 & $\Theta$ & 13 & 29 & 1 \\
\hline post-retrofit & 0 & $\Theta$ & $\Theta$ & 8 & 29 & 1 \\
\hline difference & 0 & $\Theta$ & $\Theta$ & -5 & $\Theta$ & 0 \\
\hline$\%$ change & 0 & 0 & 0 & -37 & 0 & 0 \\
\hline \multicolumn{7}{|c|}{ Total (MBtu/1000ft2) } \\
\hline existing & 0 & $\Theta$ & 0 & 7 & 15 & 1 \\
\hline post-retrofit & 0 & $\odot$ & $\odot$ & 4 & 15 & 1 \\
\hline difference & 0 & 0 & 0 & -2 & 0 & 0 \\
\hline$\%$ change & 0 & $\theta$ & $\Theta$ & -37 & 0 & 0 \\
\hline
\end{tabular}




\section{Alternative Financing Emission Reduction}

The emission reductions from implemented the proposed retrofits are as follows:

1970's shops 4016 conditioned space

Sulfur oxides (lb)

existing

post-retrofit

difference

$\%$ change

576

$-34$

$-6$

Nitrogen Oxides (lb)

existing

292

post-retrofit

275

difference

$-16$

$\%$ change

$-6$

Carbon Monoxide (lb)

existing

501

post-retrofit

difference

474

$\%$ change

-28
-6

Carbon Dioxide (tons)

existing

post-retrofit

difference

62

difference

$-3$

change

$-6$

Particulate Matter (lb)

existing

post-retrofit

difference

$\%$ change

Hydrocarbons (lb)

existing

post-retrofit 196

208

difference

$-12$

$\%$ change

$-6$ 
1970 's shops

Sulfur oxides (lb)

existing

post-retrofit

difference

$\%$ change

Nitrogen oxides (lb)

existing

post-retrofit

difference

$\%$ change

Carbon Monoxide (lb)

existing

post-retrofit

difference

$\%$ change

Carbon Dioxide (tons)

existing

post-retrofit

difference

$\%$ change

Particulate Matter (lb)

existing

post-retrofit

difference

$\%$ change

Hydrocarbons ( $1 b$ )

existing

post-retrofi

difference

$\%$ change
115

103

$-13$

$-11$

55

49

$-6$

$-11$

95

85

$-10$

$-11$

12

10

$-1$

$-11$

2

2

$-11$

39

35

-4
-11
4016 unconditioned space 


\section{Building 2131 Administrative Building}

The following information identifies the cost-effective energy- and cost-reducing retrofit projects for building 2131 identified from the FEDS modeling and analysis. Key energy and economic results are presented for each cost-effective retrofit measure.

\section{Facility Description 2131}

Building 2131 is a building with some administration space as well as some lab-space and unconditioned high-bay space. Building 2131 was built in 2008 and is 26,296 sf.

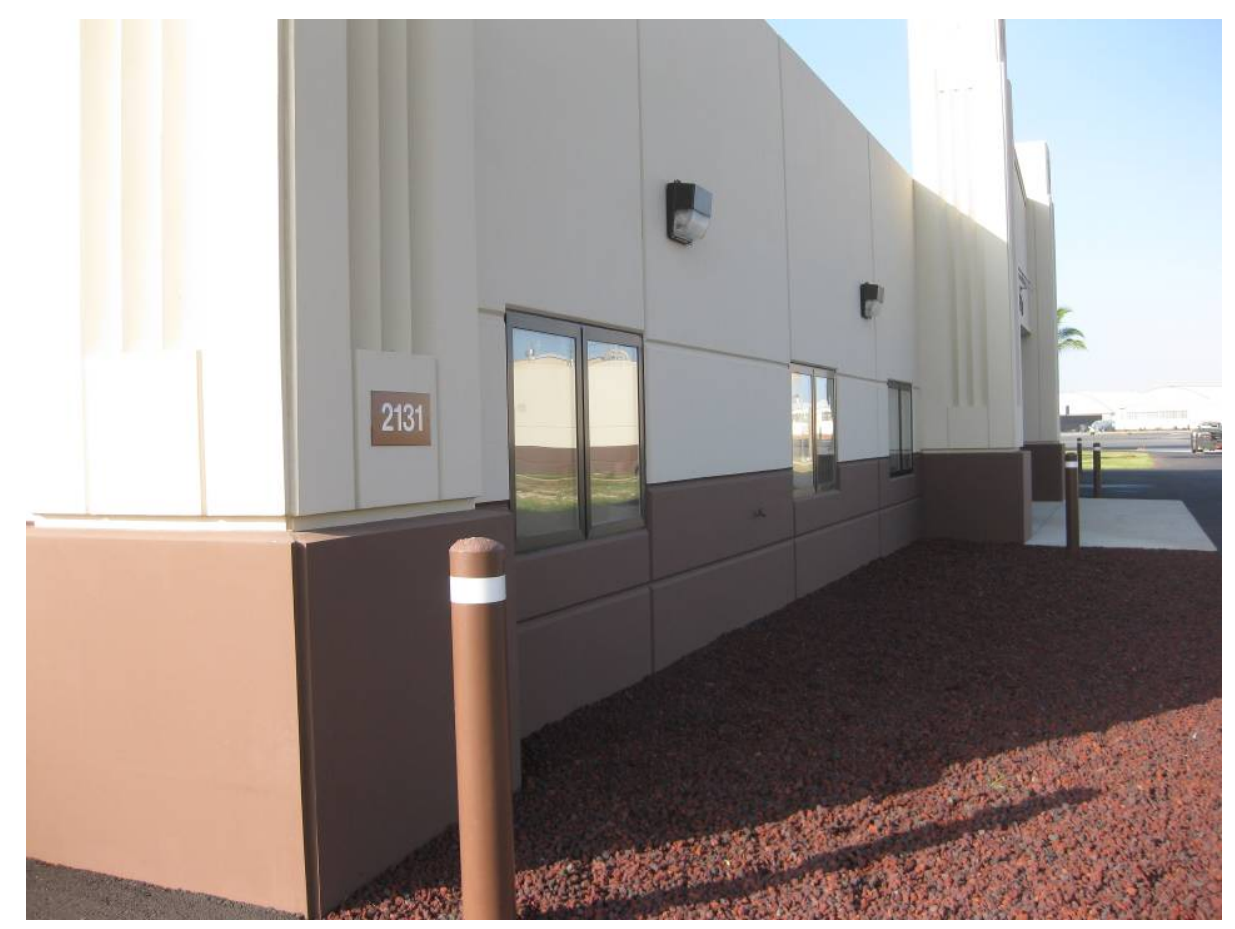




\section{Alternative Financing Results}

A FEDS analysis using alternative financing suggests replacing the EXIT lighting in the laboratory and administration spaces. FEDS also suggests replacing EXIT lighting and metal halide lighting in the high bay space.

Alternative financing FEDS results for building 2131 administration and laboratory spaces:

\begin{tabular}{|c|c|c|c|c|c|c|c|c|}
\hline Bldg. Set Description & $\begin{array}{l}\text { End } \\
\text { Use }\end{array}$ & Existing Technology & Retrofit Technology & $\begin{array}{l}\text { Energy } \\
\text { Savings } \\
\text { (MMBtu/yr) }\end{array}$ & $\begin{array}{c}\text { 1st } \\
\text { Year } \\
\text { Savings } \\
\text { (\$/yr) }\end{array}$ & $\begin{array}{l}\text { Installed } \\
\text { Cost (\$) }\end{array}$ & $\begin{array}{l}\text { Net } \\
\text { Present } \\
\text { Value } \\
\text { (\$) } \\
\end{array}$ & SIR \\
\hline 1990's shops & Lights & EX6: EXIT - LED & $\begin{array}{l}\text { EX12: EXIT - ELECTROLUMINESCENT PANEL RETRO } \\
\text { KIT }\end{array}$ & 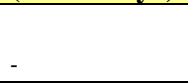 & 73 & 373 & 69 & 1.2 \\
\hline 1990's shops & Lights & EX6: EXIT - LED & $\begin{array}{l}\text { EX12: EXIT - ELECTROLUMINESCENT PANEL RETRO } \\
\text { KIT }\end{array}$ & - & 48 & 248 & 46 & 1.2 \\
\hline
\end{tabular}

Alternative financing FEDS results for building 2131 high bay spaces:

\begin{tabular}{|c|c|c|c|c|c|c|c|c|}
\hline Bldg. Set Description & $\begin{array}{l}\text { End } \\
\text { Use }\end{array}$ & Existing Technology & Retrofit Technology & $\begin{array}{l}\text { Energy } \\
\text { Savings } \\
\text { (MMBtu/yr) }\end{array}$ & $\begin{array}{c}\text { 1st } \\
\text { Year } \\
\text { Savings } \\
\text { (\$/yr) }\end{array}$ & $\begin{array}{l}\text { Installed } \\
\text { Cost (\$) }\end{array}$ & $\begin{array}{l}\text { Net } \\
\text { Present } \\
\text { Value } \\
\text { (\$) }\end{array}$ & SIR \\
\hline $\begin{array}{l}\text { 1990's shop highbay } \\
\text { space } 2131\end{array}$ & Lights & EX6: EXIT - LED & $\begin{array}{l}\text { EX12: EXIT - ELECTROLUMINESCENT PANEL RETRO } \\
\text { KIT }\end{array}$ & - & 112 & 621 & 10 & 1.1 \\
\hline
\end{tabular}




\section{Alternative Financing Energy Consumption by Fuel Type}

The modeled energy consumption for a typical year was 221,784 kwh before retrofits and 221,610 kwh after proposed retrofits are implemented. The energy use intensity goes from 57.6 MBtu/Ksf to 57.5 MBtu/Ksf after retrofits.

1990 's shops administration and laboratory space 2131

Fuel

Electricity $(\mathrm{kWh})$

existing

difference

$\%$ change

Total (MBtu)

existing

post-retrofit

difference

$\%$ change post-retrofit
Energy

221,784

221,610

$-174$

757
756
-1
$\odot$

Energy

Intensity

$16,868.3$

$16,855 . \odot$

$-13.2$

57.6
57.5
$\odot . \odot$

0 (user units/1000ft2)

Energy

Dollars

(MBtu/1000ft2) (2009)*

* Dollar values for electricity include both energy and demand components.
57.6

57.5

0.0

57.6

57.5

0.0

$\odot$
39,293

39,157

$-135$

39,293

39,157

$-135$ 
The modeled energy consumption for a typical year was 152,074 kwh before retrofits and 151,958 kwh after proposed retrofits are implemented. The energy use intensity goes from $39.5 \mathrm{MBtu} / \mathrm{Ksf}$ to $39.5 \mathrm{MBtu} / \mathrm{Ksf}$ after retrofits.

1990 's shop highbay space 2131

Fuel

Electricity $(\mathrm{kWh})$

existing

post-retrofit

difference

$\%$ change

Total (MBtu)

existing

post-retrofit

difference

$\%$ change
Energy

152,074

151,958

$-116$

519
519
0
0
Energy

Intensity

(user units/1000ft2)

Energy

Intensity

(MBtu/1000ft2)

$11,570.7$

$11,561.9$

$-8.8$

39.5

39.5

$\odot .0$
39.5

39.5

0.0

39.5

39.5

0.0
Dollars

(2009) *

26,948

26,933

$-15$

26,948

26,933

-15
0

* Dollar values for electricity include both energy and demand components. 


\section{Alternative Financing Energy Consumption by End Use}

Motors and miscellaneous equipment is the largest load in the building with 84,806 kWh/year, followed by space cooling with 62,357 $\mathrm{kWh} /$ year.

Annual Energy Use by Building Set, Fuel Type, and End Use 1990 's administration and laboratory space 2131

\begin{tabular}{|c|}
\hline Fuel \\
\hline $\begin{array}{l}\text { Electricity (kWh) } \\
\text { existing } \\
\text { post-retrofit } \\
\text { difference } \\
\% \text { change }\end{array}$ \\
\hline $\begin{array}{l}\text { Total (MBtu) } \\
\text { existing } \\
\text { post-retrofit } \\
\text { difference } \\
\% \text { change }\end{array}$ \\
\hline $\begin{array}{l}\text { Total (MBtu/1000ft2) } \\
\text { existing } \\
\text { post-retrofit } \\
\text { difference } \\
\% \text { change }\end{array}$ \\
\hline
\end{tabular}

Cooling Vent Lights Misc Equip

Hot Water *

$\%$ change

62,357
62,310
-47
$\odot$

213
213
$\odot$
$\odot$

16
16
$\odot$
$\odot$

14,183
14,171
-11
0
57,107
5692
-116
16
84,806
84,806

$\begin{array}{rr}\odot & 62,357 \\ \odot & 62,310 \\ \odot & -47 \\ \odot & \odot\end{array}$

48
48
$\odot$
$\odot$
195
195
$\odot$

$\begin{array}{rr}15 & 22 \\ 15 & 22 \\ \odot & 0 \\ \odot & 0\end{array}$

$\begin{array}{rr}289 & 11 \\ 289 & 11 \\ \odot & 0 \\ 0 & 0\end{array}$

Motors and miscellaneous equipment is the largest load in the building with 92,181 kWh/year, followed by space cooling with 57,524 kWh/year.

Annual Energy Use by Building Set, Fuel Type, and End Use 1990 's shop highbay space 2131

\begin{tabular}{|c|c|c|c|c|c|c|}
\hline Fuel & Heating & Cooling & Vent & Lights & $\begin{array}{l}\text { Motors and } \\
\text { Misc Equip }\end{array}$ & Hot Water \\
\hline \multicolumn{7}{|c|}{ Electricity (kWh) } \\
\hline existing & $\odot$ & $\odot$ & $\odot$ & 57,524 & 92,181 & 2,368 \\
\hline post-retrofit & $\odot$ & $\odot$ & $\odot$ & 57,409 & 92,181 & 2,368 \\
\hline
\end{tabular}


difference

$\%$ change

Total (MBtu)

existing

post-retrofit

difference

$\%$ change

Total (MBtu/100๑ft2)

existing

post-retrofit

difference

$\%$ change $\odot$

$\odot$

0

$\odot$

$\odot$

$\odot$

$\odot$

$\odot$

$\odot$

$\odot$

$\odot$

0
0
0
0
$-116$

$\odot$

196

196

$\odot$

15

15 $\odot$

$\odot$

8

315

315

$\odot$

8
$\odot$

$\odot$

24

1

24

0

$\odot$ 


\section{Alternative Financing Emission Reduction}

The emission reductions from implemented the proposed retrofits are as follows:

1990 's administration and lab space 2131

Sulfur oxides (lb)

existing

post-retrofit

2,005

difference

2,003

$\%$ change

-2
0

Nitrogen Oxides (lb)

existing

post-retrofit

difference

957

$\%$ change

$-1$

Carbon Monoxide (lb)

existing

post-retrofit

difference

$\%$ change

$-1$

-1
0

Carbon Dioxide (tons)

existing

post-retrofit

203

difference

203

$\%$ change

Particulate Matter (lb)

existing

post-retrofit

difference

40

$\%$ change

Hydrocarbons (lb)

existing

post-retrofit

difference 
Sulfur oxides (lb)

existing

post-retrofit

difference

$\%$ change

Nitrogen oxides (lb)

existing

post-retrofit

difference

\% change

Carbon Monoxide (lb)

existing

post-retrofit

difference

$\%$ change

Carbon Dioxide (tons)

existing

post-retrofit

difference

$\%$ change

1,375

1,374

-1
0

657

656

$\odot$

1,130

1,129

-1
0

139

139

$\odot$
$\odot$

Particulate Matter (lb)

existing

post-retrofit

difference

$\%$ change

27

$\odot$

Hydrocarbons (lb)

existing

post-retrofit

difference

468

467

$\odot$
$\odot$ 


\section{Building 1728 Warehouse}

The following information identifies the cost-effective energy- and cost-reducing retrofit projects for building 1728 identified from the FEDS modeling and analysis. Key energy and economic results are presented for each cost-effective retrofit measure.

\section{Facility Description 1728}

1728 is a warehouse building built in 1993. This building partially unconditioned with the office space being served by an electric air cooled chiller. Building 1728 is 140,383 sf.

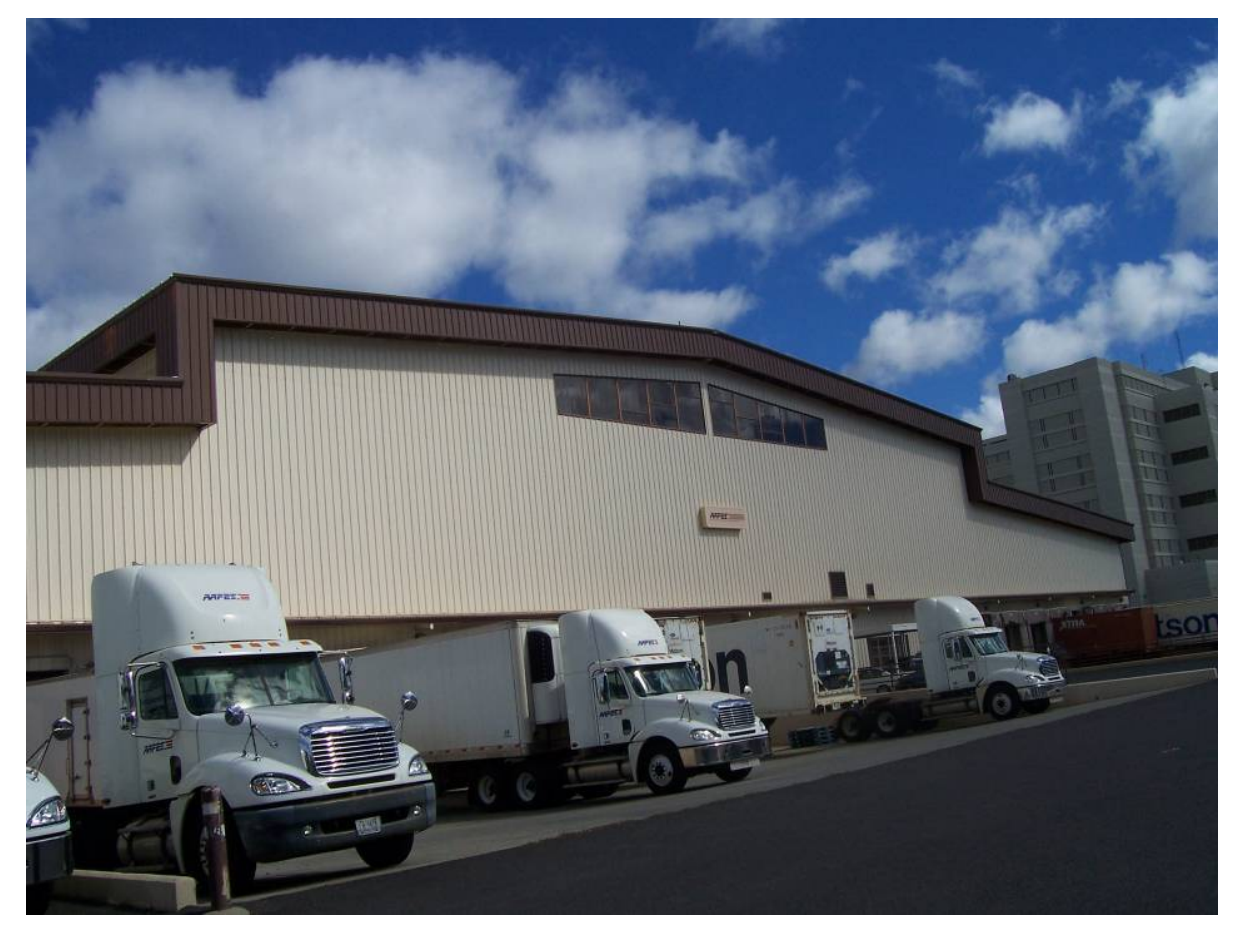




\section{Alternative Financing Results}

A FEDS analysis using alternative financing suggests replacing lights in the conditioned and unconditioned spaces. For the conditioned space, it is recommended to increase roof insulation as well as installing aerators.

Alternative financing FEDS results for building 1728 unconditioned space:

\begin{tabular}{|c|c|c|c|c|c|c|c|c|}
\hline Bldg. Set Description & $\begin{array}{l}\text { End } \\
\text { Use }\end{array}$ & Existing Technology & Retrofit Technology & $\begin{array}{l}\text { Energy } \\
\text { Savings } \\
\text { (MMBtu/yr) } \\
\end{array}$ & $\begin{array}{c}\text { 1st Year } \\
\text { Savings } \\
(\$ / y r)\end{array}$ & $\begin{array}{l}\text { Installed } \\
\text { Cost (\$) }\end{array}$ & $\begin{array}{l}\text { Net } \\
\text { Present } \\
\text { Value (\$) } \\
\end{array}$ & SIR \\
\hline $\begin{array}{l}1050 \text { 's Wharehouse/storage } \\
1728\end{array}$ & Lights & EX6: EXIT - LED & $\begin{array}{l}\text { EX12: EXIT - } \\
\text { ELECTROLUMINESCENT PANEL } \\
\text { RETRO KIT }\end{array}$ & - & 112 & 621 & 62 & 1.1 \\
\hline $\begin{array}{l}1050 \text { 's Wharehouse/storage } \\
1728\end{array}$ & Lights & MH6: MH 400 PEND & HS18: HPS 310 PEND & 175 & 8,758 & 38,531 & 11,889 & 1.3 \\
\hline
\end{tabular}

Alternative financing FEDS results for building 1728 conditioned space:

\begin{tabular}{|c|c|c|c|c|c|c|c|c|}
\hline Bldg. Set Description & $\begin{array}{l}\text { End } \\
\text { Use }\end{array}$ & Existing Technology & Retrofit Technology & $\begin{array}{l}\text { Energy } \\
\text { Savings } \\
\text { (MMBtu/yr) } \\
\end{array}$ & $\begin{array}{l}\text { 1st Year } \\
\text { Savings } \\
(\$ / y r)\end{array}$ & $\begin{array}{l}\text { Installed } \\
\text { Cost (\$) }\end{array}$ & $\begin{array}{l}\text { Net } \\
\text { Present } \\
\text { Value (\$) } \\
\end{array}$ & SIR \\
\hline $\begin{array}{l}1050 \text { 's Wharehouse/storage } \\
1728\end{array}$ & $\begin{array}{l}\text { Hot } \\
\text { Water }\end{array}$ & Electric Water Heater & Faucet Aerators & - & 21 & 14 & 109 & 8.7 \\
\hline $\begin{array}{l}1050 \text { 's Wharehouse/storage } \\
1728\end{array}$ & Lights & EX6: EXIT - LED & $\begin{array}{l}\text { EX12: EXIT - } \\
\text { ELECTROLUMINESCENT PANEL } \\
\text { RETRO KIT }\end{array}$ & - & 56 & 311 & 30 & 1.1 \\
\hline $\begin{array}{l}1050 \text { 's Wharehouse/storage } \\
1728\end{array}$ & Lights & FL37: FL 2X4 4F32T8 EEF2 & $\begin{array}{l}\text { FL280: FL 2X4 3F32ST8 ELC3 } \\
\text { REF }\end{array}$ & 55 & 3,428 & 3,312 & 16,684 & 6.0 \\
\hline $\begin{array}{l}1050 \text { 's Wharehouse/storage } \\
1728\end{array}$ & Roof & Roof Insulation R-Value 0.00 & $\begin{array}{l}\text { Add Insulation to Interior } \\
\text { Surface of Metal Roof: } 4 \\
\text { inches Fiberglass }\end{array}$ & 118 & 7,242 & 8,890 & 32,679 & 4.7 \\
\hline
\end{tabular}




\section{Alternative Financing Energy Consumption by Fuel Type}

The modeled energy consumption for a typical year was 493,902 kwh before retrofits and 442,488 kwh after proposed retrofits are implemented. The energy use intensity goes from 12.2 MBtu/Ksf to $11.0 \mathrm{MBtu} / \mathrm{Ksf}$ after retrofits.

\begin{tabular}{|c|c|c|c|c|}
\hline Fuel & Energy & $\begin{array}{c}\text { Energy } \\
\text { Intensity } \\
\text { (user units/1000ft2) }\end{array}$ & $\begin{array}{c}\text { Energy } \\
\text { Intensity } \\
\text { (MBtu/1000ft2) }\end{array}$ & $\begin{array}{l}\text { Dollars } \\
(2009)^{*}\end{array}$ \\
\hline \multicolumn{5}{|c|}{ Electricity (kWh) } \\
\hline existing & 493,902 & $3,584.7$ & 12.2 & 87,503 \\
\hline post-retrofit & 442,488 & $3,211.5$ & 11.0 & 78,185 \\
\hline difference & $-51,414$ & -373.2 & -1.3 & $-9,318$ \\
\hline$\%$ change & -10 & -10 & -10 & -11 \\
\hline \multicolumn{5}{|l|}{ Total (MBtu) } \\
\hline existing & 1,686 & 12.2 & 12.2 & 87,503 \\
\hline post-retrofit & 1,510 & 11.0 & 11.0 & 78,185 \\
\hline difference & -175 & -1.3 & -1.3 & $-9,318$ \\
\hline$\%$ change & -10 & -10 & -10 & -11 \\
\hline
\end{tabular}

* Dollar values for electricity include both energy and demand components. 
The modeled energy consumption for a typical year was 146,926 kwh before retrofits and 97,702 kwh after proposed retrofits are implemented. The energy use intensity goes from 192.8 MBtu/Ksf to 128.2 MBtu/Ksf after retrofits.

\begin{tabular}{|c|c|c|c|c|}
\hline Fuel & Energy & $\begin{array}{c}\text { Energy } \\
\text { Intensity } \\
\text { (user units/1000ft2) }\end{array}$ & $\begin{array}{l}\text { Energy } \\
\text { Intensity } \\
(\text { MBtu/1000ft2) }\end{array}$ & $\begin{array}{l}\text { Dollars } \\
\text { (2009)* }\end{array}$ \\
\hline \multicolumn{5}{|c|}{ Electricity (kWh) } \\
\hline existing & 146,926 & $56,488.3$ & 192.8 & 26,030 \\
\hline post-retrofit & 97,702 & $37,563.3$ & 128.2 & 17,263 \\
\hline difference & $-49,224$ & $-18,925.0$ & -64.6 & $-8,767$ \\
\hline$\%$ change & -34 & -34 & -34 & -34 \\
\hline \multicolumn{5}{|l|}{ Total (MBtu) } \\
\hline existing & 501 & 192.8 & 192.8 & 26,030 \\
\hline post-retrofit & 333 & 128.2 & 128.2 & 17,263 \\
\hline difference & -168 & -64.6 & -64.6 & $-8,767$ \\
\hline$\%$ change & -34 & -34 & -34 & -34 \\
\hline
\end{tabular}

* Dollar values for electricity include both energy and demand components. 


\section{Alternative Financing Energy Consumption by End Use}

Lighting is the largest load in the unconditioned space of the building with $253,372 \mathrm{kWh} /$ year, followed by motors and miscellaneous equipment with 240,530 kWh/year.

Annual Energy Use by Building Set, Fuel Type, and End Use 1050's Warehouse/storage 1728 unconditioned space

Fuel
Electricity (kWh)
existing
post-retrofit
difference
\% change
Total (MBtu)
existing
post-retrofit
difference
\% change
Total (MBtu/100०ft2)
existing
post-retrofit
difference
\% change

$\begin{array}{cr}\text { Heating } & \text { Cooling } \\ & \\ \odot & \odot \\ \odot & \odot \\ \odot & \odot \\ \odot & \odot \\ & \\ \odot & \\ \odot & \odot \\ \odot & \odot \\ \odot & \odot \\ & \odot \\ \odot & \\ \odot & \\ \odot & \odot \\ \odot & \odot \\ & \odot \\ & \\ & \\ & \end{array}$

Hot Water

$\begin{array}{rr}253,372 & 240,530 \\ 201,958 & 240,530 \\ -51,414 & \\ -20 & \\ & \\ 865 & 821 \\ 689 & 821 \\ -175 & \\ -20 & 0\end{array}$

$\odot$

$\odot$

$\odot$

9 
Space cooling is the largest load in the conditioned space of the building with 60,659 $\mathrm{kWh} /$ year, followed by motors and miscellaneous equipment with 31,893 kWh/year.

Annual Energy Use by Building Set, Fuel Type, and End Use 1050 's Warehouse/storage 1728 conditioned space

Fuel
Electricity (kWh)
existing
post-retrofit
difference
$\%$ change
Total (MBtu)
existing
post-retrofit
difference
$\%$ change
Total (MBtu/1000ft2)
existing
post-retrofit
difference
$\%$ change

$\begin{array}{rr}\text { Heating } & \text { Cooling } \\ & \\ \odot & 6 \odot, 659 \\ \odot & 27,215 \\ \odot & -33,444 \\ \odot & -55 \\ & \\ \odot & 207 \\ \odot & 93 \\ \odot & -114 \\ \odot & -55 \\ & \\ \odot & 80 \\ \odot & 36 \\ \odot & -44 \\ \odot & -55\end{array}$

Vent
27,479
23,026
4,453
-16

94
79
-15
-16

36
30
-6
-16

\section{Lights}

Misc Equip
26,560
15,339
$-11,222$

$-42$
91
52$$
-38
$$$$
-42
$$

35
20
-15
-42

Hot Water

334
229
-105
-31

1
1
0
-31


0
0
0
-31




\section{Alternative Financing Emission Reduction}

The emission reductions from implemented the proposed retrofits are as follows:

1050 's Wharehouse/storage 1728 unconditioned space

Sulfur Oxides (lb)

existing

post-retrofit

4,000

difference

$-465$

$\%$ change

$-10$

Nitrogen Oxides (lb)

existing

post-retrofit

2,134

difference

1,911

$\%$ change

$-222$

Carbon Monoxide (1b)

existing

post-retrofit

, 670

difference

3,288

$-382$

$\%$ change

$-10$

Carbon Dioxide (tons)

existing

452

post-retrofit

405

difference

$-47$

$\%$ change

$-10$

Particulate Matter (lb)

existing

post-retrofit

difference

$\%$ change

88

79
-9

$-10$

Hydrocarbons ( $1 b$ )

existing

post-retrofit $\quad 1,361$

, 519

difference

$-158$

$\%$ change

$-10$ 
Sulfur 0xides (lb)

existing

post-retrofit

difference

\% change

Nitrogen oxides (lb)

existing

post-retrofit

difference

$\%$ change

Carbon Monoxide (lb)

existing

post-retrofit

difference

$\%$ change

1,328

883

$-445$

$-34$

635

422

$-213$

$-34$

1, 092

726

$-366$

$-34$

134

89

$-45$

$-34$

difference

$\%$ change

Particulate Matter (1b)

existing

post-retrofit

difference

\% change

26

17

$-9$

$-34$

Hydrocarbons (lb)

existing

post-retrofit

difference

$\%$ change
452

300

$-151$

$-34$ 


\section{Building 1072 Supply Warehouse}

The following information identifies the cost-effective energy- and cost-reducing retrofit projects for building 1072 identified from the FEDS modeling and analysis. Key energy and economic results are presented for each cost-effective retrofit measure.

\section{Facility Description 1072}

1072 is a warehouse building built in 1941. The warehouse is largely unconditioned but has a few small offices that are conditioned by DX units. Building 1072 is 83,379 sf.

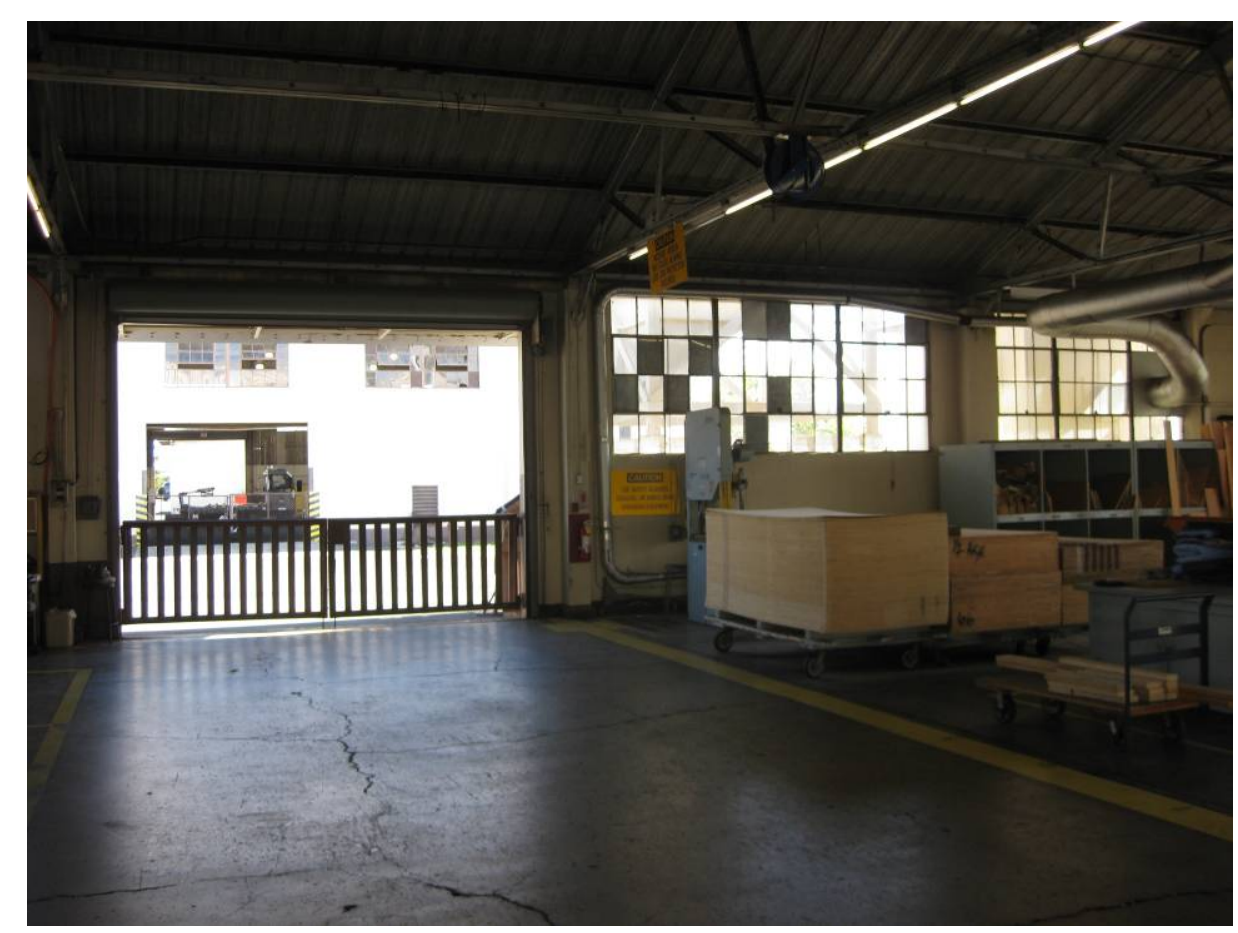




\section{Alternative Financing Results}

A FEDS analysis using alternative financing suggests replacing lights in the conditioned and unconditioned spaces of the building. FEDS also suggests adding insulation to the interior of the roof and replacing the single pane windows with double pane, super low-e windows in the conditioned space.

Alternative financing FEDS results for building 1072 unconditioned space:

\begin{tabular}{|c|c|c|c|c|c|c|c|c|}
\hline Bldg. Set Description & $\begin{array}{l}\text { End } \\
\text { Use }\end{array}$ & Existing Technology & Retrofit Technology & $\begin{array}{l}\text { Energy } \\
\text { Savings } \\
\text { (MMBtu/yr) } \\
\end{array}$ & $\begin{array}{l}\text { 1st Year } \\
\text { Savings } \\
(\$ / y r)\end{array}$ & $\begin{array}{l}\text { Installed } \\
\text { Cost (\$) }\end{array}$ & $\begin{array}{l}\text { Net } \\
\text { Present } \\
\text { Value (\$) } \\
\end{array}$ & SIR \\
\hline $\begin{array}{l}1050 \text { 's Wharehouse/storage } \\
1072\end{array}$ & Lights & EX6: EXIT - LED & $\begin{array}{l}\text { EX12: EXIT - } \\
\text { ELECTROLUMINESCENT PANEL } \\
\text { RETRO KIT }\end{array}$ & - & 112 & 621 & 62 & 1.1 \\
\hline $\begin{array}{l}1050 \text { 's Wharehouse/storage } \\
1072\end{array}$ & Lights & FL236: FL 2X4 3F32T8 ELC3 & $\begin{array}{l}\text { FL279: FL 2X4 2F32ST8 ELC2 } \\
\text { REF }\end{array}$ & 128 & 6,785 & 13,362 & 25,840 & 2.9 \\
\hline $\begin{array}{l}1050 \text { 's Wharehouse/storage } \\
1072\end{array}$ & Lights & MH6: MH 400 PEND & HS18: HPS 310 PEND & 69 & 3,445 & 15,160 & 4,677 & 1.3 \\
\hline
\end{tabular}

Alternative financing FEDS results for building 1072 conditioned space:

\begin{tabular}{|c|c|c|c|c|c|c|c|c|}
\hline Bldg. Set Description & $\begin{array}{l}\text { End } \\
\text { Use }\end{array}$ & Existing Technology & Retrofit Technology & $\begin{array}{l}\text { Energy } \\
\text { Savings } \\
\text { (MMBtu/yr) }\end{array}$ & $\begin{array}{l}\text { 1st Year } \\
\text { Savings } \\
(\$ / y r)\end{array}$ & $\begin{array}{l}\text { Installed } \\
\text { Cost (\$) }\end{array}$ & $\begin{array}{l}\text { Net } \\
\text { Present } \\
\text { Value (\$) }\end{array}$ & SIR \\
\hline $\begin{array}{l}1050 \text { 's Wharehouse/storage } \\
1072\end{array}$ & Lights & EX6: EXIT - LED & $\begin{array}{l}\text { EX12: EXIT - } \\
\text { ELECTROLUMINESCENT PANEL } \\
\text { RETRO KIT }\end{array}$ & - & 61 & 311 & 61 & 1.2 \\
\hline $\begin{array}{l}1050 \text { 's Wharehouse/storage } \\
1072\end{array}$ & Lights & FL236: FL 2X4 3F32T8 ELC3 & $\begin{array}{l}\text { FL279: FL 2X4 2F32ST8 ELC2 } \\
\text { REF }\end{array}$ & 41 & 2,214 & 3,158 & 9,617 & 4.0 \\
\hline $\begin{array}{l}\text { 1050's Wharehouse/storage } \\
1072\end{array}$ & Roof & Roof Insulation R-Value 0.00 & $\begin{array}{l}\text { Add Insulation to Interior } \\
\text { Surface of Metal Roof: } 4 \\
\text { inches Fiberglass }\end{array}$ & 299 & 15,650 & 22,799 & 67,034 & 3.9 \\
\hline
\end{tabular}




\section{Alternative Financing Energy Consumption by Fuel Type}

The modeled energy consumption for a typical year was 404,132 kwh before retrofits and 346,253 kwh after proposed retrofits are implemented. The energy use intensity goes from 17.5 MBtu/Ksf to 15.0 MBtu/Ksf after retrofits.

\begin{tabular}{|c|c|c|c|c|}
\hline Fuel & Energy & $\begin{array}{c}\text { Energy } \\
\text { Intensity } \\
\text { (user units/1000ft2) }\end{array}$ & $\begin{array}{c}\text { Energy } \\
\text { Intensity } \\
\text { (MBtu/1000ft2) }\end{array}$ & $\begin{array}{l}\text { Dollars } \\
(2009) \text { * }\end{array}$ \\
\hline \multicolumn{5}{|c|}{ Electricity (kWh) } \\
\hline existing & 404,132 & $5,134.6$ & 17.5 & 71,400 \\
\hline post-retrofit & 346,253 & $4,399.2$ & 15.0 & 61,137 \\
\hline difference & $-57,879$ & -735.4 & -2.5 & $-10,263$ \\
\hline$\%$ change & -14 & -14 & -14 & -14 \\
\hline \multicolumn{5}{|l|}{ Total (MBtu) } \\
\hline existing & 1,379 & 17.5 & 17.5 & 71,400 \\
\hline post-retrofit & 1,182 & 15.0 & 15.0 & 61,137 \\
\hline difference & -198 & -2.5 & -2.5 & $-10,263$ \\
\hline$\%$ change & -14 & -14 & -14 & -14 \\
\hline
\end{tabular}

* Dollar values for electricity include both energy and demand components. 
The modeled energy consumption for a typical year was 163,410 kwh before retrofits and 65,956 kwh after proposed retrofits are implemented. The energy use intensity goes from 83.6 MBtu/Ksf to 33.7 MBtu/Ksf after retrofits.

\section{0 's Wharehouse/storage 1072 conditioned space}

Fuel

Energy

$$
\begin{array}{r}
163,410 \\
65,956 \\
-97,454
\end{array}
$$

difference

$\%$ change

Total (MBtu)

existing

post-retrofit

difference

$\%$ change

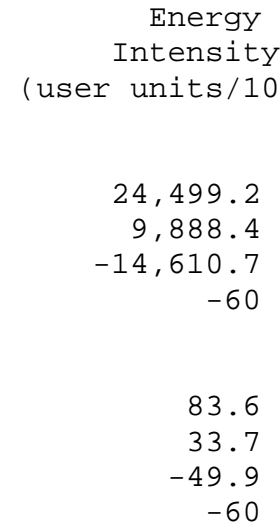

$-49.9$

$-60$

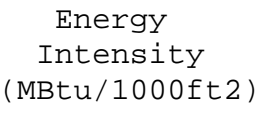

83.6

33.7

$-49.9$

$-60$

83.6

33.

$-49.9$

$-60$
Dollars

(2००9) *

28,870

11,646

$-17,225$

28,870

11,646

$-17,225$

* Dollar values for electricity include both energy and demand components. 


\section{Alternative Financing Energy Consumption by End Use}

Lighting is the largest load in the unconditioned space of the building with $266,729 \mathrm{kWh} /$ year, followed by motors and miscellaneous equipment with $137,403 \mathrm{kWh} /$ year.

Fuel

Electricity (kWh)

existing

post-retrofit

difference

$\%$ change

Total (MBtu)

existing

post-retrofit

difference

$\%$ change

Total (MBtu/1000ft2)

existing

post-retrofit

difference

$\%$ change
1050 's Warehouse/storage

Heating

Cooling

$\odot$
$\odot$
$\odot$
$\odot$

$\odot$

$\odot$

$\odot$

$\odot$

$\odot$

$\odot$

0

$\odot$

$\odot$
1072 unconditioned space

Vent

Lights

Motors and

Misc Equip

Hot Water

266,729
208,850
$-57,879$
-22

910
713
-198
-22

12
9
-3
-22

137,403

137,403

$\odot$

469

469

○

$\odot$

6
6
$\odot$
$\odot$

$\odot$

$\odot$
$\odot$ 
Space cooling is the largest load in the conditioned space of the building with 79,200 kWh/year, followed by ventilation with 53,432 kWh/year.

Fuel

Electricity $(\mathrm{kWh})$

existing

post-retrofit

difference

$\%$ change

Total (MBtu)

existing

post-retrofit

difference

$\%$ change

Total (MBtu/100๑ft2)

existing

post-retrofit

difference

$\%$ change 1050's Wharehouse/storage

Heating

cooling

79,200

25,968

$-53,232$

$-67$

270
89

$-182$

$-67$

41
13
-27
-67
1072 conditioned space

Motors and

Lights Misc Equip

Hot Water

19,134

11,081
$-8,052$

17,263

$-36,169$

$-68$

$-42$

11,644

$\odot$
$\odot$

40

40

$\odot$

$-123$

38
-27

-27
-42

27
9
-19
-68

10

6
-4

$-42$
0

$\odot$

0

$\odot$

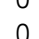

$\odot$

$\odot$

$\odot$
0 


\section{Alternative Financing Emission Reduction}

The emission reductions from implemented the proposed retrofits are as follows:

1050 's Wharehouse/storage

1072 unconditioned space

Sulfur Oxides (lb)

existing

post-retrofit

3,653

difference

3,130

$\%$ change

523

$-14$

Nitrogen Oxides (lb)

existing

post-retrofit

1,746

difference

1,496

$\%$ change

$-250$

Carbon Monoxide (lb)

existing

post-retrofit

difference

3,003

$\%$ change

2,573

-430
-14

Carbon Dioxide (tons)

existing

post-retrofit

370

difference

317

$\%$ change

$-53$

$-14$

Particulate Matter (1b)

existing

post-retrofit

difference

$\%$ change

$-14$

Hydrocarbons ( $1 b$ )

existing

post-retrofit

1,243

post-retro

1,065

$\%$ change

$-178$

$-14$ 
Sulfur 0xides (lb)

existing

post-retrofit

difference

$\%$ change

Nitrogen 0xides (lb)

existing

post-retrofit

difference

$\%$ change

Carbon Monoxide (lb)

existing

post-retrofit

difference

$\%$ change

1,477

596

$-881$

$-60$

706

285

$-421$

$-60$

1,214

490

$-724$

$-60$

Carbon Dioxide (tons)

existing

post-retrofit

difference

$\%$ change

150

60

$-89$

$-60$

Particulate Matter (lb)

existing

post-retrofit

difference

$\%$ change

$-17$

$-60$

Hydrocarbons ( $1 b$ )

existing

post-retrofit

503

difference

203

$-300$

$\%$ change 


\section{Building 1070 Warehouse Building}

The following information identifies the cost-effective energy- and cost-reducing retrofit projects for building 1070 identified from the FEDS modeling and analysis. Key energy and economic results are presented for each cost-effective retrofit measure.

\section{Facility Description 1070}

1070 is a warehouse building with some conditioned hazmat storage built in 1941 . Building 1070 is mostly unconditioned storage with a small office that is conditioned by a small DX unit. Building 1070 is 62,779 sf.

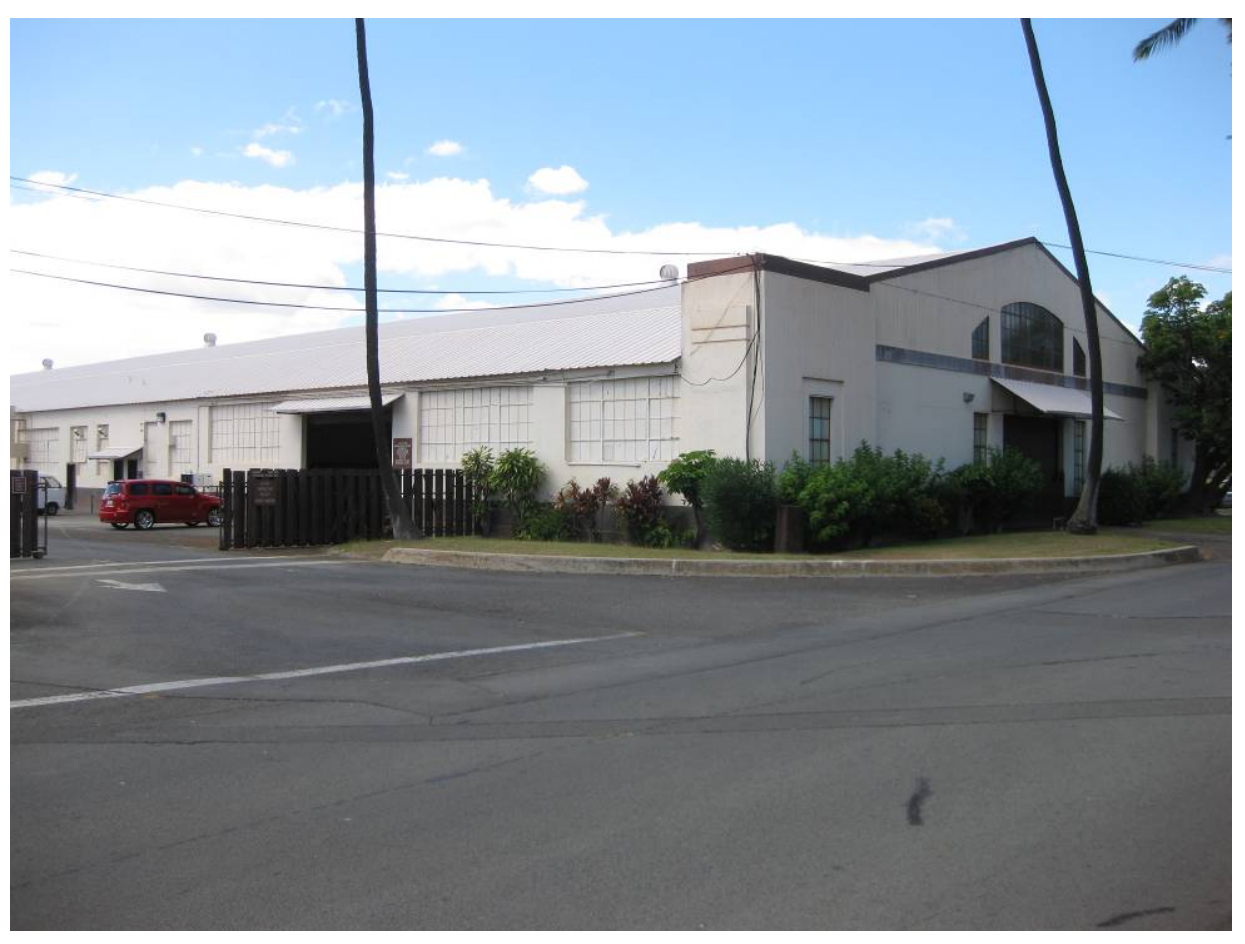




\section{Alternative Financing Results}

A FEDS analysis using alternative financing suggests replacing lighting in the unconditioned space as well as replacing the EXIT lighting fixtures in the conditioned and unconditioned spaces. FEDS also suggests increasing the insulation in the roof of the conditioned office space.

Alternative financing FEDS results for building 1070 unconditioned space:

\begin{tabular}{|c|c|c|c|c|c|c|c|c|}
\hline Bldg. Set Description & $\begin{array}{l}\text { End } \\
\text { Use }\end{array}$ & Existing Technology & Retrofit Technology & $\begin{array}{l}\text { Energy } \\
\text { Savings } \\
\text { (MMBtu/yr) }\end{array}$ & $\begin{array}{c}\text { 1st Year } \\
\text { Savings } \\
\text { (\$/yr) }\end{array}$ & $\begin{array}{l}\text { Installed } \\
\text { Cost (\$) }\end{array}$ & $\begin{array}{l}\text { Net } \\
\text { Present } \\
\text { Value (\$) } \\
\end{array}$ & SIR \\
\hline small storage & Lights & IN27: INC 100 WALL & CF9: CFL 26 INTEGRAL UNIT ELC & 9 & 663 & 2,530 & 1,347 & 1.5 \\
\hline small storage & Lights & EX1: EXIT - INC (2x20) & $\begin{array}{l}\text { EX12: EXIT - } \\
\text { ELECTROLUMINESCENT PANEL } \\
\text { RETRO KIT }\end{array}$ & 9 & 698 & 621 & 3,483 & 6.6 \\
\hline
\end{tabular}

Alternative financing FEDS results for building 1070 conditioned space:

\begin{tabular}{|c|c|c|c|c|c|c|c|c|}
\hline Bldg. Set Description & $\begin{array}{l}\text { End } \\
\text { Use }\end{array}$ & Existing Technology & Retrofit Technology & $\begin{array}{l}\text { Energy } \\
\text { Savings } \\
\text { (MMBtu/yr) }\end{array}$ & $\begin{array}{l}\text { 1st Year } \\
\text { Savings } \\
(\$ / y r)\end{array}$ & $\begin{array}{l}\text { Installed } \\
\text { Cost (\$) }\end{array}$ & $\begin{array}{l}\text { Net } \\
\text { Present } \\
\text { Value (\$) }\end{array}$ & SIR \\
\hline $\begin{array}{ll}\text { small storage } 1070 & \\
& \\
\text { small storage } 1070\end{array}$ & Lights & $\begin{array}{l}\text { EX1: EXIT - INC (2x20) } \\
\text { Roof Insulation R-Value } 0.00\end{array}$ & $\begin{array}{l}\text { EX12: EXIT - } \\
\text { ELECTROLUMINESCENT PANEL } \\
\text { RETRO KIT } \\
\text { Add Insulation to Interior } \\
\text { Surface of Metal Roof: } 4 \text { inches } \\
\text { Fiberglass }\end{array}$ & 20 & 426 & 311 & 2,186 & 8.0 \\
\hline
\end{tabular}




\section{Alternative Financing Energy Consumption by Fuel Type}

The modeled energy consumption for a typical year was 17,134 kwh before retrofits and 11,645 kwh after proposed retrofits are implemented. The modeled other fuels (propane) consumption for a typical year was $382 \mathrm{MBtu}$ before retrofits and $382 \mathrm{MBtu}$ after proposed retrofits are implemented. The energy use intensity goes from 7.2 MBtu/Ksf to 6.9 MBtu/Ksf after retrofits.

Small storage $\quad 1070$ unconditioned space

Fuel

Electricity (kWh)

existing

post-retrofit

difference

$\%$ change

Other Fuels (MBtu)

existing

post-retrofit

difference

$\%$ change

Total (MBtu)

existing

post-retrofit

difference

$\%$ change
Energy

17,134

11,645

$-5,489$
-32

382

382

$\odot$

440
422
-19

-19
-4

* Dollar values for electricity include both energy and demand components. 
The modeled energy consumption for a typical year was 25,042 kwh before retrofits and 17,627 kwh after proposed retrofits are implemented. The modeled other fuels (propane) consumption for a typical year was 8 MBtu before retrofits and 8 MBtu after proposed retrofits are implemented. The energy use intensity goes from 74.3 MBtu/Ksf to 54.1 MBtu/Ksf after retrofits.

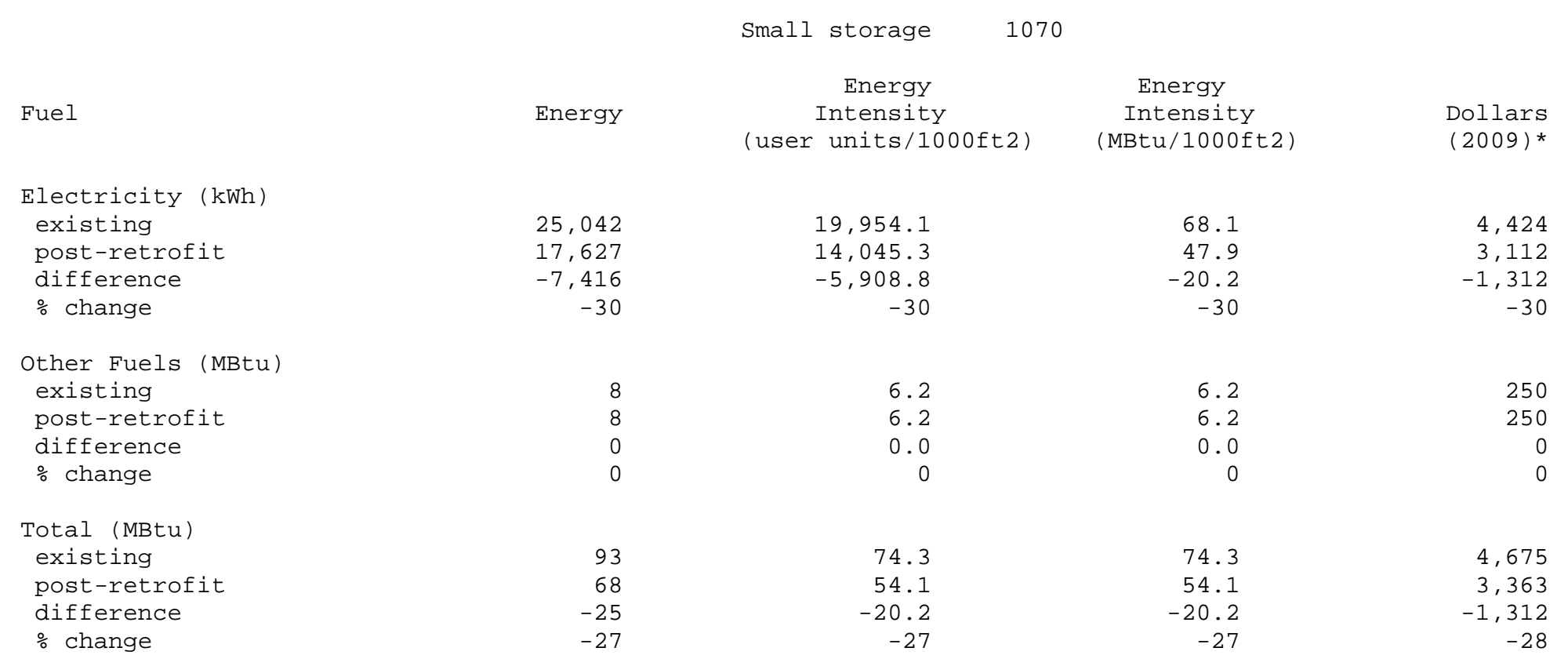

* Dollar values for electricity include both energy and demand components. 


\section{Alternative Financing Energy Consumption by End Use}

Lighting is the largest load in the building with $16,137 \mathrm{kWh} /$ year, followed by motors and miscellaneous equipment with 997 $\mathrm{kWh} /$ year.

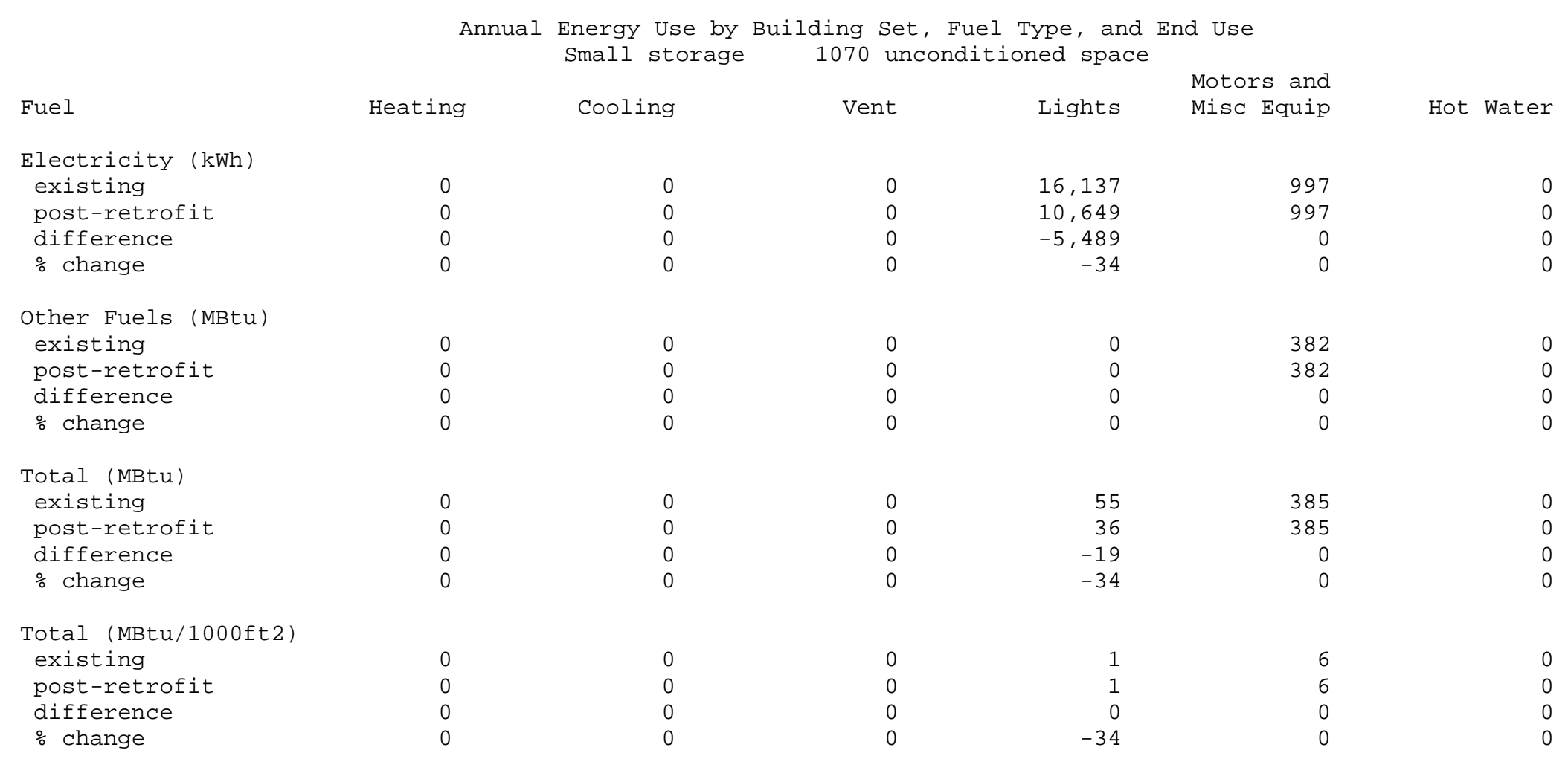


Space cooling is the largest load in the building with 12,471 $\mathrm{kWh} /$ year, followed by motors and miscellaneous equipment with 6,323 kWh/year.

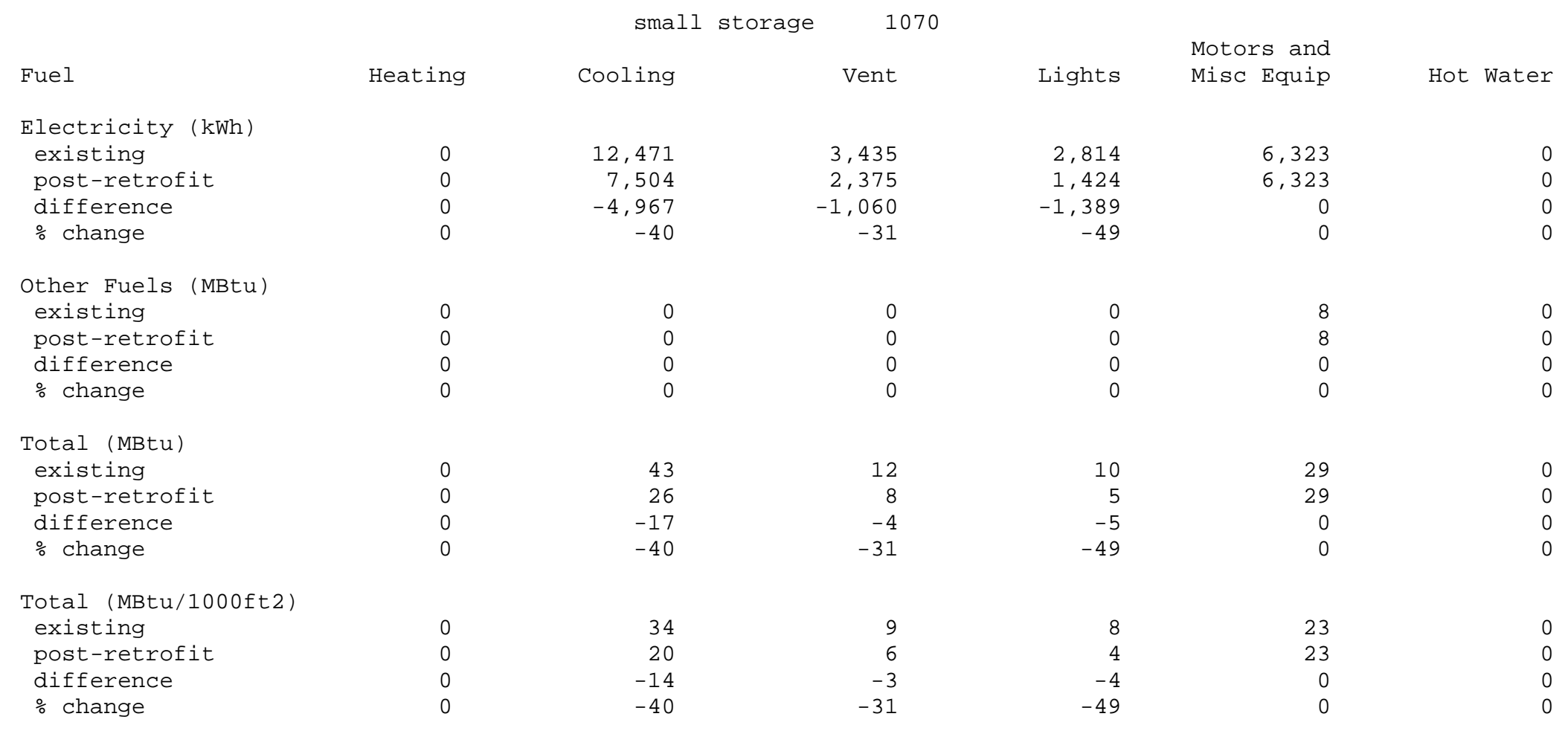




\section{Alternative Financing Emission Reduction}

The emission reductions from implemented the proposed retrofits are as follows:

Small storage

$107 \odot$ unconditioned space

Sulfur oxides (lb)

existing

post-retrofit

250

difference

201

$\%$ change

$-20$

Nitrogen Oxides (lb)

existing

post-retrofit

208

difference

184

$\%$ change

$-24$

Carbon Monoxide (lb)

existing

post-retrofit

difference

418

$\%$ change

377

-41
-10

Carbon Dioxide (tons)

existing

post-retrofit

difference

48

43

$\%$ change

Particulate Matter (lb)

existing

post-retrofit

difference

$\%$ change

Hydrocarbons (lb)

existing

post-retrofit

148

post-retro

131

$\%$ change

$-17$

$-11$ 
Sulfur oxides (lb)

existing

post-retrofit

161

difference

$\%$ change

-67
-29

Nitrogen 0xides (lb)

existing

post-retrofit

111

79

difference

$-32$

$\%$ change

$-29$

Carbon Monoxide (lb)

existing

post-retrofit

192

difference

137

-55
-29

$\%$ change

Carbon Dioxide (tons)

existing

post-retrofit

difference

24

17

$\%$ change

$-29$

Particulate Matter (lb)

existing

post-retrofit

difference

\% change

5

-1
-29

Hydrocarbons (1b)

existing

post-retrofit

difference

79

$\%$ change 


\section{Building 2002 Vehicle Maintenance Shop}

The following information identifies the cost-effective energy- and cost-reducing retrofit projects for building 2002 identified from the FEDS modeling and analysis. Key energy and economic results are presented for each cost-effective retrofit measure.

\section{Facility Description 2002}

2002 is a vehicle maintenance building with admin and workshop space built in 1940. Building 2002 generally has fluorescent lighting, an electric hot water system and its administration spaces are cooled by an electric package, or DX, unit. Building 2002 is 23,981 sf. 


\section{Alternative Financing Results}

A FEDS analysis using alternative financing suggests replacing the EXIT lighting as well as replacing the T12 Fluorescent lighting in the unconditioned space. In the conditioned space FEDS suggests replacing EXIT lighting, T12 Flourescent lighting and adding insulation to the interior surface of the metal roof.

Alternative financing FEDS results for building 2002 unconditioned space:

\begin{tabular}{|c|c|c|c|c|c|c|c|c|}
\hline Bldg. Set Description & $\begin{array}{l}\text { End } \\
\text { Use }\end{array}$ & Existing Technology & Retrofit Technology & $\begin{array}{l}\text { Energy } \\
\text { Savings } \\
\text { (MMBtu/yr) } \\
\end{array}$ & $\begin{array}{l}\text { 1st Year } \\
\text { Savings } \\
(\$ / y r)\end{array}$ & $\begin{array}{l}\text { Installed } \\
\text { Cost (\$) }\end{array}$ & $\begin{array}{l}\text { Net } \\
\text { Present } \\
\text { Value (\$) } \\
\end{array}$ & SIR \\
\hline Vehicle maintenance 2002 & Lights & EX1: EXIT - INC $(2 \times 20)$ & $\begin{array}{l}\text { EX12: EXIT - } \\
\text { ELECTROLUMINESCENT PANEL } \\
\text { RETRO KIT }\end{array}$ & 1 & 105 & 93 & 522 & 6.6 \\
\hline Vehicle maintenance 2002 & Lights & EX1: EXIT - INC $(2 \times 20)$ & $\begin{array}{l}\text { EX12: EXIT - } \\
\text { ELECTROLUMINESCENT PANEL } \\
\text { RETRO KIT }\end{array}$ & 8 & 593 & 528 & 2,961 & 6.6 \\
\hline Vehicle maintenance 2002 & Lights & FL1: FL 2X4 4F40T12 STD2 & FL244: FL 2X4 4F32T8 ELC4 & 16 & 1,244 & 3,265 & 4,014 & 2.2 \\
\hline Vehicle maintenance 2002 & Lights & FL4: FL 1X4 2F40T12 STD2 & FL52: FL 1X4 2F32T8 ELC2 & 3 & 259 & 993 & 534 & 1.5 \\
\hline Vehicle maintenance 2002 & Lights & FL62: FL 1X8 2F96T12 STD2 & FL74: FL 1X8 2F96T12 ELC2 & 6 & 487 & $2,195 `$ & 655 & 1.3 \\
\hline
\end{tabular}


Alternative financing FEDS results for building 2002 conditioned space:

\begin{tabular}{|c|c|c|c|c|c|c|c|c|}
\hline Bldg. Set Description & $\begin{array}{l}\text { End } \\
\text { Use }\end{array}$ & Existing Technology & Retrofit Technology & $\begin{array}{l}\text { Energy } \\
\text { Savings } \\
\text { (MMBtu/yr) }\end{array}$ & $\begin{array}{c}\text { 1st Year } \\
\text { Savings } \\
(\$ / y r)\end{array}$ & $\begin{array}{l}\text { Installed } \\
\text { Cost (\$) }\end{array}$ & $\begin{array}{l}\text { Net } \\
\text { Present } \\
\text { Value (\$) } \\
\end{array}$ & SIR \\
\hline Vehicle maintenance 2002 & Lights & EX1: EXIT - INC $(2 \times 20)$ & $\begin{array}{l}\text { EX12: EXIT - } \\
\text { ELECTROLUMINESCENT PANEL } \\
\text { RETRO KIT }\end{array}$ & 1 & 65 & 47 & 335 & 8.2 \\
\hline Vehicle maintenance 2002 & Lights & EX1: EXIT - INC $(2 \times 20)$ & $\begin{array}{l}\text { EX12: EXIT - } \\
\text { ELECTROLUMINESCENT PANEL } \\
\text { RETRO KIT }\end{array}$ & 5 & 367 & 264 & 1,888 & 8.2 \\
\hline Vehicle maintenance 2002 & Lights & FL1: FL 2X4 4F40T12 STD2 & FL244: FL 2X4 4F32T8 ELC4 & 5 & 386 & 816 & 1,435 & 2.1 \\
\hline Vehicle maintenance 2002 & Lights & FL4: FL 1X4 2F40T12 STD2 & FL52: FL 1X4 2F32T8 ELC2 & 1 & 65 & 248 & 134 & 1.5 \\
\hline Vehicle maintenance 2002 & $\begin{array}{l}\text { Lights } \\
\text { Roof }\end{array}$ & $\begin{array}{l}\text { FL62: FL 1X8 2F96T12 STD2 } \\
\text { Roof Insulation R-Value } 0.00\end{array}$ & $\begin{array}{l}\text { FL74: FL 1X8 2F96T12 ELC2 } \\
\text { Add Insulation to Interior } \\
\text { Surface of Metal Roof: } 4 \text { inches } \\
\text { Fiberglass }\end{array}$ & 139 & 7,145 & 16,393 & 413 & 1.4 \\
\hline
\end{tabular}




\section{Alternative Financing Energy Consumption by Fuel Type}

The modeled energy consumption for a typical year was 125,846 kwh before retrofits and 115,487 kwh after proposed retrofits are implemented. The energy use intensity goes from 22.4 MBtu/Ksf to 20.5 MBtu/Ksf after retrofits.

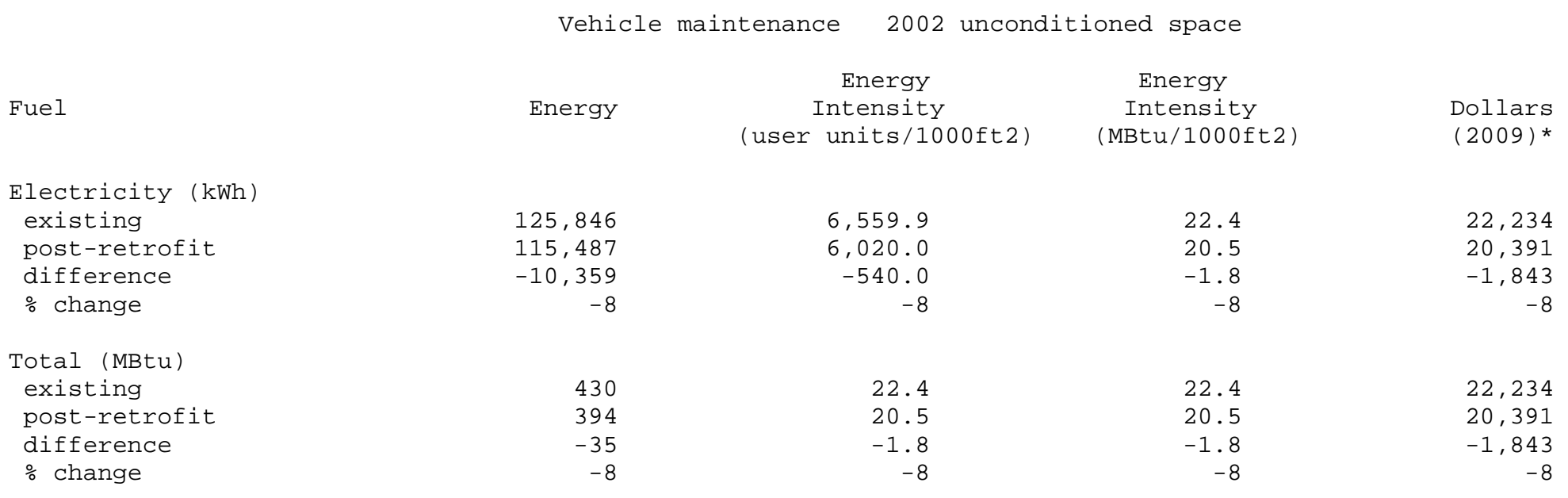

* Dollar values for electricity include both energy and demand components. 
The modeled energy consumption for a typical year was 97,300 kwh before retrofits and 52,706 kwh after proposed retrofits are implemented. The energy use intensity goes from 69.2 MBtu/Ksf to 37.5 MBtu/Ksf after retrofits.

\section{Vehicle maintenance 2002 conditioned space}

Fuel

Electricity $(\mathrm{kWh})$

existing

post-retrofit

difference

$\%$ change

Total (MBtu)

existing

post-retrofit

difference

$\%$ change
Energy

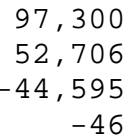

332
180
-152
-46

\section{Energy}

Intensity

(user units/1000ft2)

Intensity

(MBtu/1000ft2)
$20,287.8$
$10,989.5$
$-9,298.3$
$-46$
69.2
37.5
$-31.7$
$-46$

69.2

37.5

$-31.7$

46

69.2

37.5

$-31.7$

$-46$
Dollars

$(2 \odot \odot 9)$ *

17,191

9,306

$-7,885$

$-46$

17,191

9,306

$-7,885$

$-46$

* Dollar values for electricity include both energy and demand components. 


\section{Alternative Financing Energy Consumption by End Use}

Motors and miscellaneous equipment is the largest load in the building with 94,120 kWh/year, followed by lighting with 31 ,616 $\mathrm{kWh} /$ year.

Vehicle maintenance 2002 unconditioned space

\begin{tabular}{|c|c|c|c|c|c|c|}
\hline Fuel & Heating & Cooling & Vent & Lights & $\begin{array}{l}\text { Motors and } \\
\text { Misc Equip }\end{array}$ & Hot Water \\
\hline \multicolumn{7}{|c|}{ Electricity (kWh) } \\
\hline existing & $\odot$ & $\odot$ & $\odot$ & 31,616 & 94,120 & 110 \\
\hline post-retrofit & $\odot$ & $\odot$ & $\odot$ & 21,257 & 94,120 & 110 \\
\hline difference & $\odot$ & $\odot$ & $\odot$ & $-10,359$ & 0 & c \\
\hline$\%$ change & $\odot$ & $\odot$ & $\odot$ & -33 & $\odot$ & c \\
\hline \multicolumn{7}{|l|}{ Total (MBtu) } \\
\hline existing & $\odot$ & $\odot$ & $\odot$ & 108 & 321 & c \\
\hline post-retrofit & $\odot$ & $\odot$ & $\odot$ & 73 & 321 & c \\
\hline difference & $\odot$ & $\odot$ & $\odot$ & -35 & 0 & $\mathrm{c}$ \\
\hline$\%$ change & $\odot$ & $\odot$ & $\odot$ & -33 & $\odot$ & c \\
\hline \multicolumn{7}{|c|}{ Total (MBtu/1000ft2) } \\
\hline existing & $\odot$ & $\odot$ & $\odot$ & 6 & 17 & c \\
\hline post-retrofit & $\odot$ & $\odot$ & $\odot$ & 4 & 17 & c \\
\hline difference & $\odot$ & $\odot$ & $\odot$ & -2 & 0 & c \\
\hline$\%$ change & $\odot$ & $\odot$ & $\odot$ & -33 & $\odot$ & c \\
\hline
\end{tabular}

* Energy consumption values for both distributed and central SHW are reported for Hot Water annual energy use. 
Space cooling is the largest load in the building with 58,459 $\mathrm{kWh} /$ year, followed by motors and miscellaneous equipment with 23,530 kWh/year.

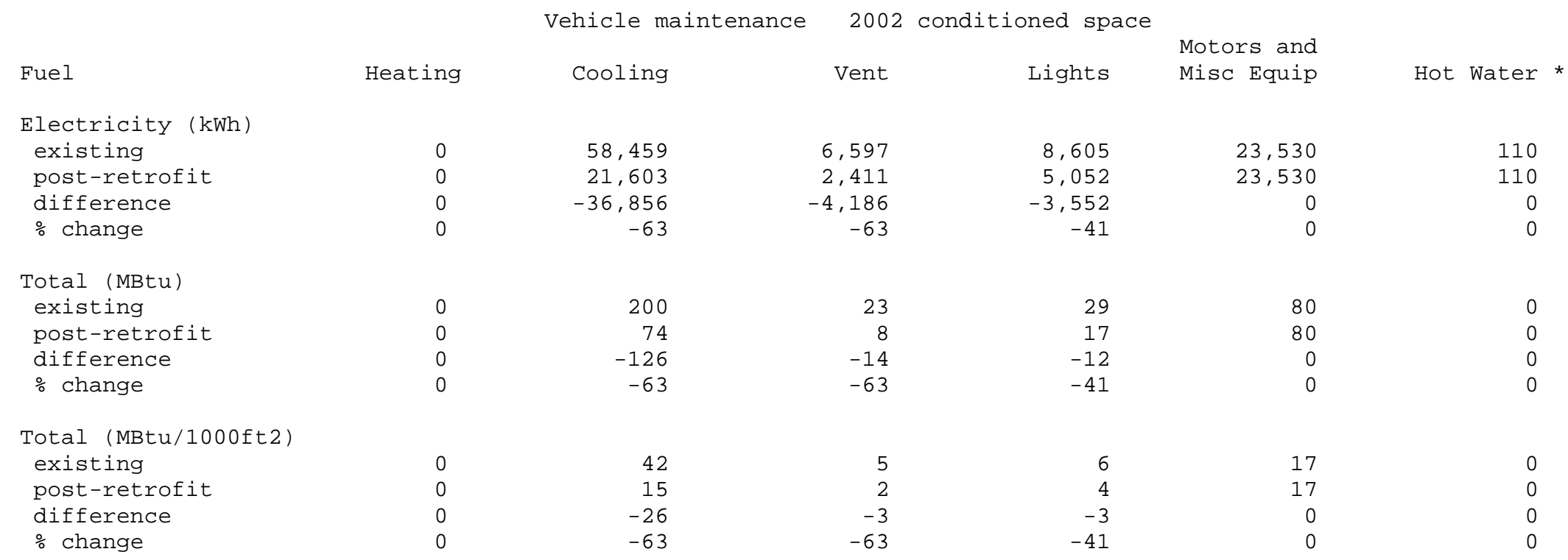

* Energy consumption values for both distributed and central SHW are reported for Hot Water annual energy use. 


\section{Alternative Financing Emission Reduction}

The emission reductions from implemented the proposed retrofits are as follows:

Vehicle maintenance 2002 unconditioned space

Sulfur Oxides (lb)

existing

post-retrofit

difference

1,044

$-94$

$\%$ change

$-8$

Nitrogen oxides (lb)

existing

544

post-retrofit

499

difference

$-45$

$\%$ change

$-8$

Carbon Monoxide (lb)

existing

935

post-retrofit

858

difference

$-77$

$\%$ change

$-8$

Carbon Dioxide (tons)

existing

115

post-retrofit

106

difference

$-9$

$\%$ change

$-8$

Particulate Matter (lb)

existing

post-retrofit

difference

$\%$ change

Hydrocarbons (lb)

existing

post-retrofit

difference

355

$\%$ change

-32
-8 
Sulfur Oxides (lb)

existing

post-retrofit

1,138

1,044

difference

$-94$

$\%$ change

544

Nitrogen 0xides (lb)

existing

post-retrofit

difference

$-45$

$\%$ change

$-8$

Carbon Monoxide (lb)

existing

post-retrofit

935

difference

858
-77

\% change

Carbon Dioxide (tons)

existing

post-retrofit

115

106

difference

-9
-8

$\%$ change

23

Particulate Matter (1b)

existing

post-retrofit

21

difference

$-2$

$\%$ change

ns (1b)

existing

355

post-retrofit

$-32$

$\%$ change

$-8$ 


\section{Building 1713 Warehouse}

The following information identifies the cost-effective energy- and cost-reducing retrofit projects for building 1713 identified from the FEDS modeling and analysis. Key energy and economic results are presented for each cost-effective retrofit measure.

\section{Facility Description 1713}

1713 is a warehouse building built in 1944. 1713 is the main recycling center on base and has a small conditioned office space served by an electric package, or DX, unit. Building 1713 is 30,400 sf.

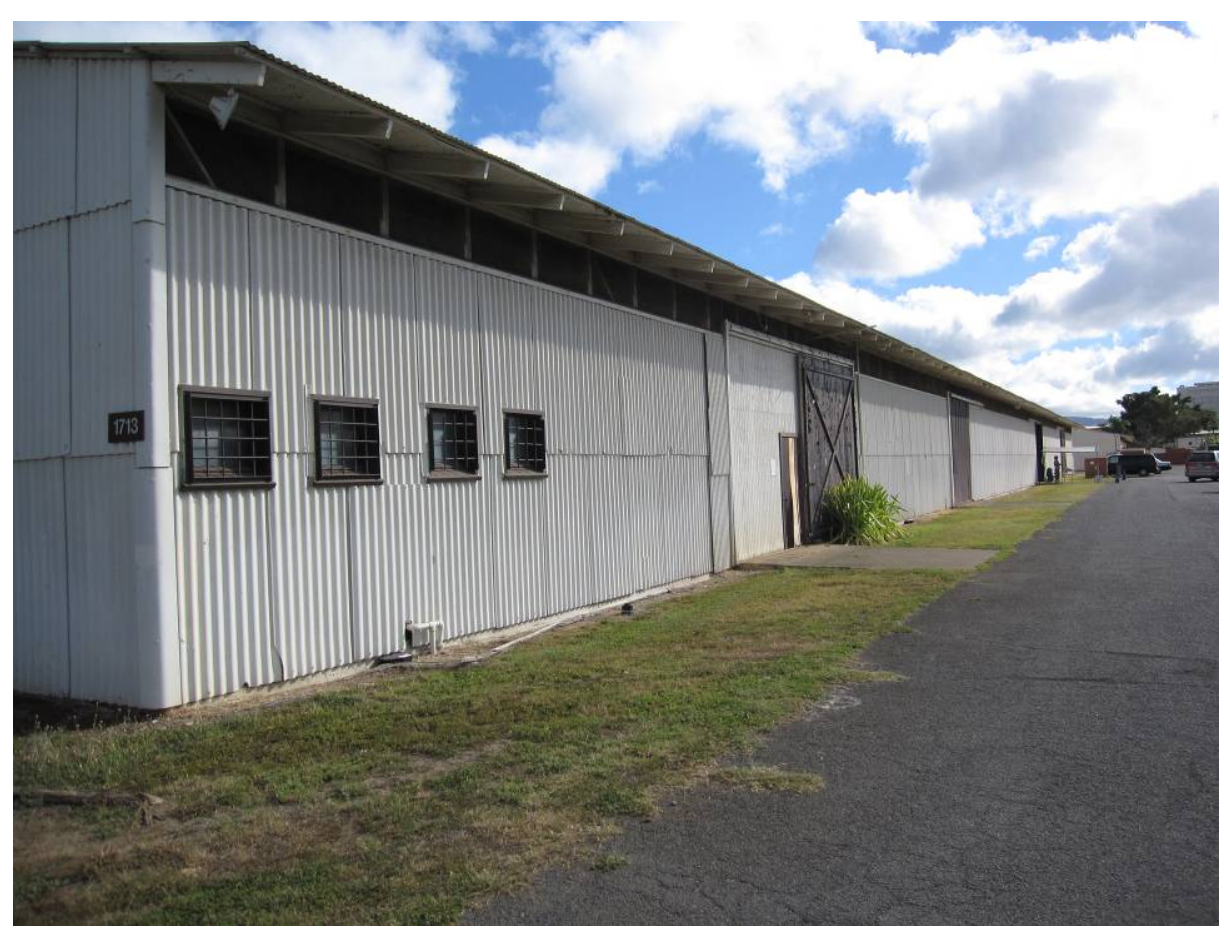




\section{Alternative Financing Results}

A FEDS analysis using alternative financing suggests replacing the lighting in the building in the conditioned and unconditioned spaces as well as increasing the interior insulation of the roof for the conditioned space only.

Alternative financing FEDS results for building 1713 unconditioned space:

\begin{tabular}{|c|c|c|c|c|c|c|c|c|}
\hline Bldg. Set Description & $\begin{array}{l}\text { End } \\
\text { Use }\end{array}$ & Existing Technology & Retrofit Technology & $\begin{array}{l}\text { Energy } \\
\text { Savings } \\
\text { (MMBtu/yr) }\end{array}$ & $\begin{array}{l}\text { 1st Year } \\
\text { Savings } \\
(\$ / y r)\end{array}$ & $\begin{array}{l}\text { Installed } \\
\text { Cost (\$) }\end{array}$ & $\begin{array}{l}\text { Net } \\
\text { Present } \\
\text { Value (\$) }\end{array}$ & SIR \\
\hline 1940's storage 1713 & Lights & EX6: EXIT - LED & $\begin{array}{l}\text { EX12: EXIT - } \\
\text { ELECTROLUMINESCENT PANEL } \\
\text { RETRO KIT }\end{array}$ & - & 56 & 311 & 31 & 1.1 \\
\hline
\end{tabular}

Alternative financing FEDS results for building 1713 conditioned space:

\begin{tabular}{|c|c|c|c|c|c|c|c|c|c|}
\hline Bldg. Set Descri & iption & $\begin{array}{l}\text { End } \\
\text { Use }\end{array}$ & Existing Technology & Retrofit Technology & $\begin{array}{l}\text { Energy } \\
\text { Savings } \\
\text { (MMBtu/yr) }\end{array}$ & $\begin{array}{l}\text { 1st Year } \\
\text { Savings } \\
(\$ / y r)\end{array}$ & $\begin{array}{l}\text { Installed } \\
\text { Cost (\$) }\end{array}$ & $\begin{array}{l}\text { Net } \\
\text { Present } \\
\text { Value (\$) }\end{array}$ & SIR \\
\hline 1940 's storage & 1713 & Lights & IN8: INC 75 CEIL & CF5: CFL 18 INTEGRAL UNIT ELC & 1 & 89 & 48 & 477 & 10.9 \\
\hline 1940 's storage & 1713 & Lights & IN11: INC 100 CEIL & CF9: CFL 26 INTEGRAL UNIT ELC & 1 & 110 & 48 & 594 & 13.3 \\
\hline 1940 's storage & 1713 & Lights & EX6: EXIT - LED & $\begin{array}{l}\text { EX12: EXIT - } \\
\text { ELECTROLUMINESCENT PANEL } \\
\text { RETRO KIT }\end{array}$ & - & 58 & 311 & 41 & 1.1 \\
\hline 1940 's storage & 1713 & Roof & Roof Insulation R-Value 0.00 & $\begin{array}{l}\text { Add Insulation to Interior } \\
\text { Surface of Metal Roof: } 4 \\
\text { inches Fiberglass }\end{array}$ & 14 & 961 & 2,078 & 3,438 & 2.7 \\
\hline
\end{tabular}




\section{Alternative Financing Energy Consumption by Fuel Type}

The modeled energy consumption for a typical year was 22,232 kwh before retrofits and 22,175 kwh after proposed retrofits are implemented. The energy use intensity goes from 2.5 MBtu/Ksf to 2.5 MBtu/Ksf after retrofits.

\begin{tabular}{|c|c|c|c|c|}
\hline Fuel & Energy & $\begin{array}{c}\text { Energy } \\
\text { Intensity } \\
\text { (user units/1000ft2) }\end{array}$ & $\begin{array}{c}\text { Energy } \\
\text { Intensity } \\
\text { (MBtu/1000ft2) }\end{array}$ & $\begin{array}{l}\text { Dollars } \\
(2009) \text { * }\end{array}$ \\
\hline \multicolumn{5}{|c|}{ Electricity (kWh) } \\
\hline existing & 22,232 & 746.3 & 2.5 & 3,930 \\
\hline post-retrofit & 22,175 & 744.3 & 2.5 & 3,915 \\
\hline difference & -58 & -1.9 & 0.0 & -14 \\
\hline$\%$ change & $\odot$ & $\odot$ & $\odot$ & $\odot$ \\
\hline \multicolumn{5}{|l|}{ Total (MBtu) } \\
\hline existing & 76 & 2.5 & 2.5 & 3,930 \\
\hline post-retrofit & 76 & 2.5 & 2.5 & 3,915 \\
\hline difference & $\odot$ & $\odot .0$ & $\odot .0$ & -14 \\
\hline$\%$ change & $\odot$ & 0 & $\odot$ & $\odot$ \\
\hline
\end{tabular}

* Dollar values for electricity include both energy and demand components. 
The modeled energy consumption for a typical year was 10,421 kwh before retrofits and 5,573 kwh after proposed retrofits are implemented. The energy use intensity goes from 58.5 MBtu/Ksf to 31.3 MBtu/Ksf after retrofits.

\begin{tabular}{|c|c|c|c|c|}
\hline \multirow[b]{2}{*}{ Fuel } & \multirow[b]{2}{*}{ Energy } & condi & ned space & \multirow[b]{2}{*}{$\begin{array}{l}\text { Dollars } \\
(2 \odot \odot 9)^{*}\end{array}$} \\
\hline & & $\begin{array}{c}\text { Energy } \\
\text { Intensity } \\
\text { (user units/1000ft2) }\end{array}$ & $\begin{array}{c}\text { Energy } \\
\text { Intensity } \\
\text { (MBtu/1000ft2) }\end{array}$ & \\
\hline \multicolumn{5}{|c|}{ Electricity (kWh) } \\
\hline existing & 10,421 & $17,140.2$ & 58.5 & 1,841 \\
\hline post-retrofit & 5,573 & $9,165.9$ & 31.3 & 984 \\
\hline difference & $-4,848$ & $-7,974.3$ & -27.2 & -857 \\
\hline \% change & -47 & -47 & -47 & -47 \\
\hline \multicolumn{5}{|l|}{ Total (MBtu) } \\
\hline existing & 36 & 58.5 & 58.5 & 1,841 \\
\hline post-retrofit & 19 & 31.3 & 31.3 & 984 \\
\hline difference & -17 & -27.2 & -27.2 & -857 \\
\hline$\%$ change & -47 & -47 & -47 & -47 \\
\hline
\end{tabular}

* Dollar values for electricity include both energy and demand components. 


\section{Alternative Financing Energy Consumption by End Use}

Lighting is the largest load in the unconditioned space of the building with $21,755 \mathrm{kWh} /$ year, followed by motors and miscellaneous equipment with $477 \mathrm{kWh} /$ year.

Fuel

Electricity (kWh)

existing

post-retrofit

difference

$\%$ change

Total (MBtu)

existing

post-retrofit

difference

$\%$ change

Total (MBtu/1000ft2)

existing

post-retrofit

difference

\% change
1940 's storage

Heating

Cooling

$\odot$

0

$\odot$

$\odot$

$\odot$

$\odot$

$\odot$

$\odot$

$\odot$

$\odot$
$\odot$
$\odot$
$\odot$

$\odot$

$\odot$
0

$\odot$
1713 unconditioned space

Vent

Lights

Motors and

Misc Equip

Hot Water
21,755

21,697

-58
0

477

477

$\odot$

$\odot$

$\odot$

74

74

$\odot$

0

$\odot$

2
2
0

$\odot$

$\odot$

2

$\odot$

$\odot$

2
$\odot$

$\odot$

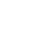

.


Space cooling is the largest load in the building with 5,318 kWh/year, followed by lighting with 4,901 kWh/year.

\begin{tabular}{|c|c|c|c|c|c|c|}
\hline Fuel & Heating & $\begin{array}{l}1940 \text { 's storage } \\
\text { Cooling }\end{array}$ & $\begin{array}{l}1713 \\
\text { Vent }\end{array}$ & $\begin{array}{c}\text { conditioned space } \\
\text { Lights }\end{array}$ & $\begin{array}{l}\text { Motors and } \\
\text { Misc Equip }\end{array}$ & Hot water \\
\hline \multicolumn{7}{|c|}{ Electricity (kWh) } \\
\hline existing & 0 & 5,318 & 193 & 4,901 & 10 & 0 \\
\hline post-retrofit & 0 & 1,345 & 46 & 4,172 & 10 & 0 \\
\hline difference & $\odot$ & $-3,973$ & -147 & -729 & 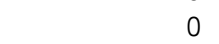 & 0 \\
\hline$\%$ change & 0 & -75 & -76 & -15 & 0 & 0 \\
\hline \multicolumn{7}{|l|}{ Total (MBtu) } \\
\hline existing & 0 & 18 & 1 & 17 & 0 & 0 \\
\hline post-retrofit & 0 & 5 & 0 & 14 & 0 & 0 \\
\hline difference & 0 & -14 & -1 & -2 & 0 & 0 \\
\hline$\%$ change & $\odot$ & -75 & -76 & -15 & $\odot$ & 0 \\
\hline \multicolumn{7}{|c|}{ Total (MBtu/1000ft2) } \\
\hline existing & 0 & 30 & 1 & 28 & 0 & 0 \\
\hline post-retrofit & $\odot$ & 8 & 0 & 23 & 0 & 0 \\
\hline difference & 0 & -22 & -1 & -4 & 0 & 0 \\
\hline$\%$ change & 0 & -75 & -76 & -15 & 0 & 0 \\
\hline
\end{tabular}




\section{Alternative Financing Emission Reduction}

The emission reductions from implemented the proposed retrofits are as follows:

1940's storage 1713 unconditioned space

\begin{tabular}{|c|c|}
\hline Sulfur Oxides (lb) & \\
\hline existing & 201 \\
\hline post-retrofit & 200 \\
\hline difference & -1 \\
\hline$\%$ change & $\odot$ \\
\hline Nitrogen Oxides (lb) & \\
\hline existing & 96 \\
\hline post-retrofit & 96 \\
\hline difference & $\odot$ \\
\hline$\%$ change & $\odot$ \\
\hline Carbon Monoxide (lb) & \\
\hline existing & 165 \\
\hline post-retrofit & 165 \\
\hline difference & $\odot$ \\
\hline$\%$ change & $\odot$ \\
\hline Carbon Dioxide (tons) & \\
\hline existing & 20 \\
\hline post-retrofit & 20 \\
\hline difference & $\odot$ \\
\hline$\%$ change & $\odot$ \\
\hline Particulate Matter (lb) & \\
\hline existing & 4 \\
\hline post-retrofit & 4 \\
\hline difference & $\odot$ \\
\hline$\%$ change & $\odot$ \\
\hline Hydrocarbons (lb) & \\
\hline existing & 68 \\
\hline post-retrofit & 68 \\
\hline difference & 0 \\
\hline$\%$ change & $\odot$ \\
\hline
\end{tabular}


Sulfur oxides (lb)

existing

post-retrofit

94

difference

50

$\%$ change

$-47$

Nitrogen 0xides (lb)

existing

post-retrofit

\% change

$-21$

Carbon Monoxide (lb)

existing

post-retrofit

difference

$\%$ change

77

41
-36

$-47$

Carbon Dioxide (tons)

existing

post-retrofit

difference

10
5

$-4$

$\%$ change

Particulate Matter (lb)

existing

post-retrofit

difference

$\%$ change

2

1
-1

$-47$

Hydrocarbons (1b)

existing

post-retrofit

difference

17

$\%$ change

$-47$ 


\section{Building 2130 Corrosion Control Hangar}

The following information identifies the cost-effective energy- and cost-reducing retrofit projects for building 2130 identified from the FEDS modeling and analysis. Key energy and economic results are presented for each cost-effective retrofit measure.

\section{Facility Description 2130}

2130 is a corrosion control facility for aircraft built in 2008. Building 2130 cleans aircraft of corrosion causing agents and has a large ventilation system to aid its mission. Building 2130 is 56,734 sf.

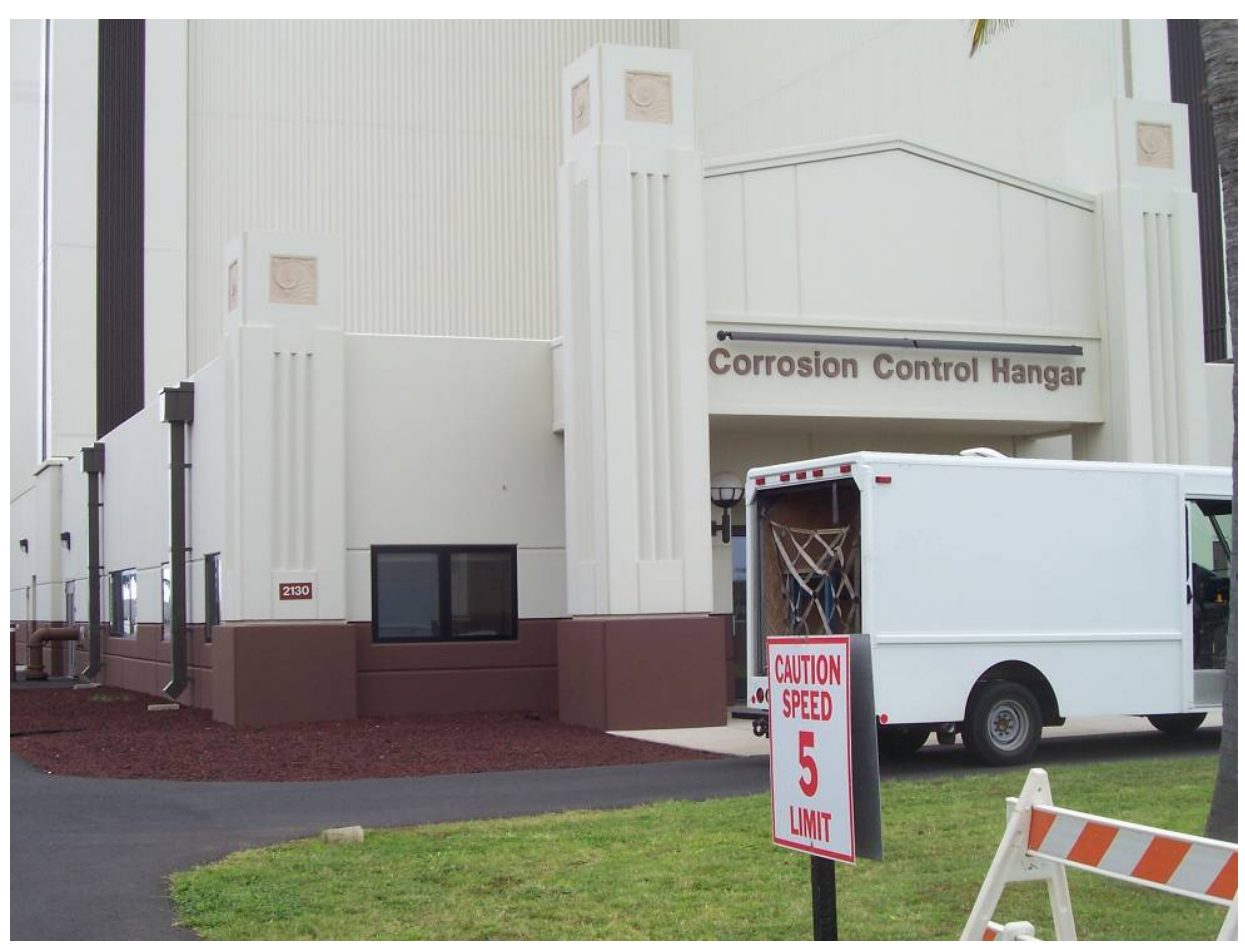




\section{Alternative Financing Results}

A FEDS analysis using alternative financing suggests replacing the lighting in the high bay space of the building. In the administration space FEDS also suggests replacing the air cooled chiller with a high efficiency water cooled chiller.

Alternative financing FEDS results for building 2130 high-bay space:

\begin{tabular}{|c|c|c|c|c|c|c|c|c|}
\hline Bldg. Set Description & $\begin{array}{l}\text { End } \\
\text { Use }\end{array}$ & Existing Technology & Retrofit Technology & $\begin{array}{l}\text { Energy } \\
\text { Savings } \\
\text { (MMBtu/yr) }\end{array}$ & $\begin{array}{c}\text { 1st } \\
\text { Year } \\
\text { Savings } \\
\text { (\$/yr) }\end{array}$ & $\begin{array}{l}\text { Installed } \\
\text { Cost (\$) }\end{array}$ & $\begin{array}{l}\text { Net } \\
\text { Present } \\
\text { Value } \\
\text { (\$) }\end{array}$ & SIR \\
\hline $\begin{array}{l}2008 \text { hanger unconditioned space } \\
2130\end{array}$ & Lights & EX6: EXIT - LED & $\begin{array}{l}\text { EX12: EXIT - } \\
\text { ELECTROLUMINESCENT PANEL } \\
\text { RETRO KIT }\end{array}$ & - & 56 & 311 & 31 & 1.1 \\
\hline $\begin{array}{l}2008 \text { hanger unconditioned space } \\
2130\end{array}$ & Lights & MH8: MH 1500 PEND & HS20: HPS 1000 PEND & 61 & 5,353 & 30,695 & 1,011 & 1.0 \\
\hline
\end{tabular}

Alternative financing FEDS results for building 2130 administration space:

\begin{tabular}{|c|c|c|c|c|c|c|c|c|}
\hline Bldg. Set Description & $\begin{array}{l}\text { End } \\
\text { Use }\end{array}$ & Existing Technology & Retrofit Technology & $\begin{array}{l}\text { Energy } \\
\text { Savings } \\
\text { (MMBtu/yr) }\end{array}$ & $\begin{array}{l}\text { 1st Year } \\
\text { Savings } \\
(\$ / y r)\end{array}$ & $\begin{array}{l}\text { Installed } \\
\text { Cost }(\$)\end{array}$ & $\begin{array}{l}\text { Net } \\
\text { Present } \\
\text { Value (\$) } \\
\end{array}$ & SIR \\
\hline $\begin{array}{l}2008 \text { hanger conditioned space } \\
2130\end{array}$ & Cooling & $\begin{array}{l}\text { Electric Air-Cooled Chiller } \\
\{\mathrm{C} 1\}\end{array}$ & $\begin{array}{l}\text { Water-Cooled Reciprocating } \\
\text { Electric Chiller (high efficiency) } \\
\text { and Cooling Tower }\end{array}$ & 106 & 7,130 & 39,495 & 2,124 & 1.1 \\
\hline
\end{tabular}




\section{Alternative Financing Energy Consumption by Fuel Type}

The modeled energy consumption for the unconditioned space of the building for a typical year was 194,224 kwh before retrofits and $176,277 \mathrm{kwh}$ after proposed retrofits are implemented. The energy use intensity goes from 13.7 MBtu/Ksf to $12.5 \mathrm{MBtu} / \mathrm{Ksf}$ after retrofits.

2008 hanger unconditioned space 2130

Fuel

Energy

Energy
Intensity
(user units/100०ft2)

\section{Energy}

Intensity

(MBtu/1000ft2)

\section{Dollars}

$(2009)$ *

Electricity (kWh)

existing

post-retrofit

194,224

176,277

$4,027.4$

$3,655.3$

$-372.1$

$\%$ change

$-17,946$
-9

$-9$

13.7

12.5

$-1.3$

34,417

31,244

$-3,173$
-9

Total (MBtu)

existing

post-retrofit

difference

13.7

12.5

663

602

$\%$ change

13.7

12.5

$-1.3$

1.3
-9

$-9$

34,417

31,244

$-3,173$

* Dollar values for electricity include both energy and demand components. 
The modeled energy consumption for the conditioned space of the building for a typical year was $402,873 \mathrm{kwh}$ before retrofits and $314,844 \mathrm{kwh}$ after proposed retrofits are implemented. The energy use intensity goes from 161.6 MBtu/Ksf to $126.3 \mathrm{MBtu} / \mathrm{Ksf}$ after retrofits.

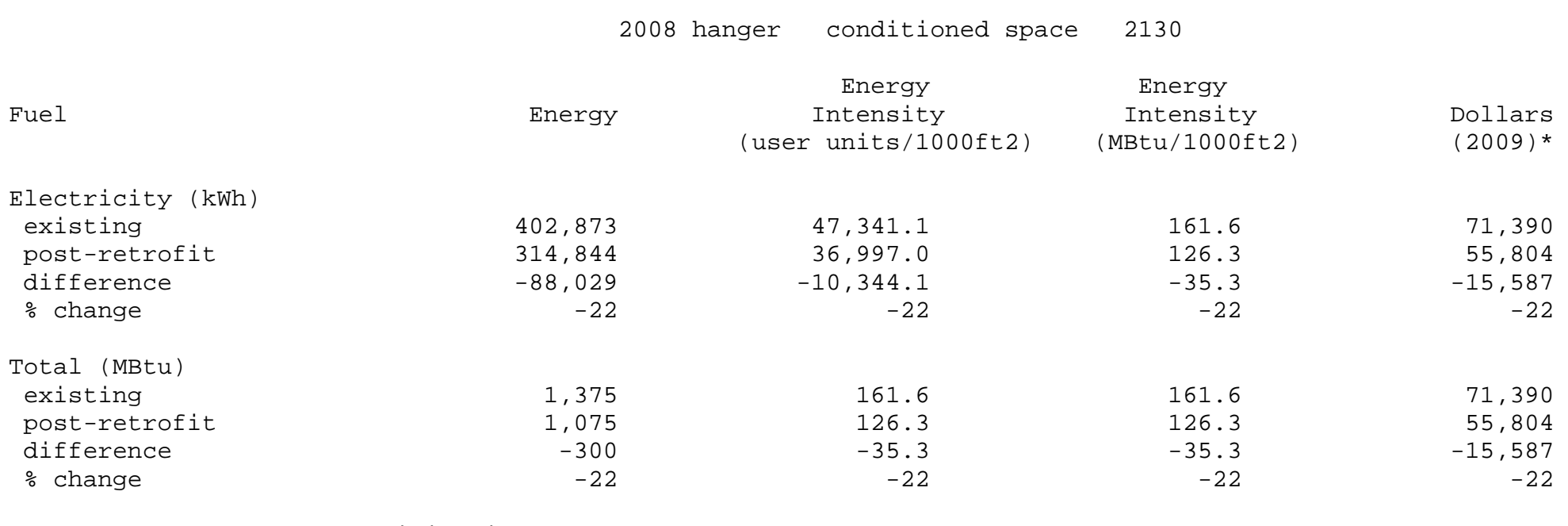

* Dollar values for electricity include both energy and demand components. 


\section{Alternative Financing Energy Consumption by End Use}

Motors and miscellaneous equipment is the largest load in the unconditioned space of the building with 117,194 kWh/year, followed by space cooling with $73,546 \mathrm{kWh} /$ year.

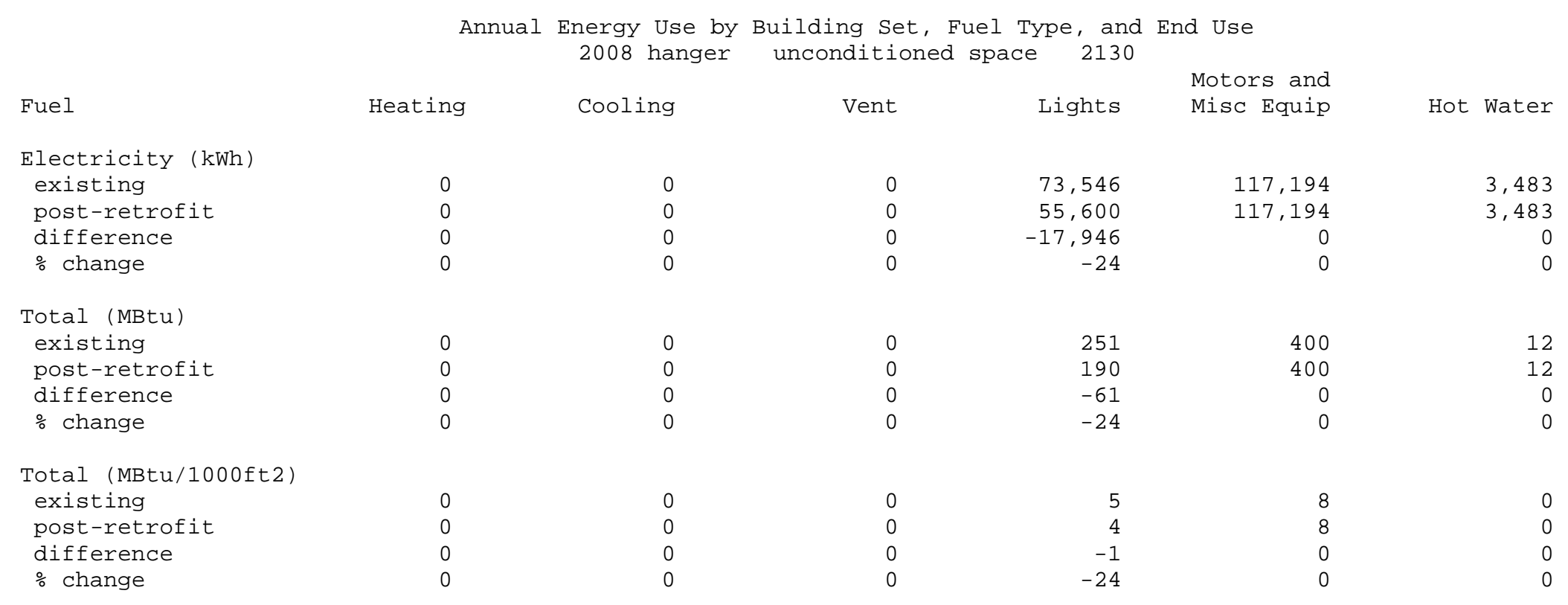


Lighting is the largest load in the conditioned space of the building with 172,239 $\mathrm{kWh} /$ year, followed by space cooling with 117,190 $\mathrm{kWh} /$ year.

Annual Energy Use by Building Set, Fuel Type, and End Use

2008 hanger conditioned space 2130

Fuel

Electricity $(\mathrm{kWh})$

existing

post-retrofit

difference

$\%$ change

Total (MBtu)

existing

post-retrofit

difference

$\%$ change

Total (MBtu/1000ft2)

existing

post-retrofit

difference

$\%$ change

$\begin{array}{rr}\text { Heating } & \text { Cooling } \\ & \\ \odot & 117,19 \odot \\ \odot & 70,813 \\ \odot & -46,377 \\ \odot & -4 \odot \\ & \\ \odot & 40 \odot \\ \odot & 242 \\ \odot & -158 \\ \odot & -4 \odot \\ & \\ \odot & 47 \\ \odot & 28 \\ \odot & -19 \\ \odot & -4 \odot\end{array}$

Vent
48,056
48,056
0
0

164
164
0
0

19
19
0
0

Lights

172,239
130,587

$-41,651$

$-24$

588

446

$-142$

$-24$

69
52
-17

-17
-24
Motors and

Misc Equip

62,803

62,803

$\odot$

214

214

$\odot$

25

25
Hot Water

2,584

2,584

0

9

9
$\odot$

$\odot$

1

1

$\odot$ 


\section{Alternative Financing Emission Reduction}

The emission reductions from implemented the proposed retrofits are as follows:

Sulfur Oxides (lb)

existing

post-retrofit

1,756

1,594

difference

$-162$

$\%$ change

$-9$

Nitrogen Oxides (lb)

existing

839

post-retrofit

761

difference

$-78$

$\%$ change

Carbon Monoxide (lb)

existing

post-retrofit

1,443

difference

1,310

$\%$ change

Carbon Dioxide (tons)

existing

post-retrofit

178

difference

161

$\%$ change

Particulate Matter (lb)

existing

post-retrofit

difference

$\%$ change

Hydrocarbons (1b)

existing

post-retrofit

difference

542

$\%$ change

$-9$ 
Sulfur oxides (lb)

existing

post-retrofit

difference

$\%$ change

Nitrogen Oxides (lb)

existing

post-retrofit

difference

\% change

Carbon Monoxide (lb)

existing

post-retrofit

difference

$\%$ change

Carbon Dioxide (tons)

existing

post-retrofit

difference

$\%$ change

3,642

2,846

$-796$

$-22$

1,740

1,360

$-380$

$-22$

2,993

2,339

-654
-22

369

288

$-81$

$-22$

Particulate Matter (lb)

existing

post-retrofit

difference

$\%$ change

Hydrocarbons (lb)

existing

post-retrofit

difference

$\%$ change

\section{2 \\ 56
-16 \\ $-22$}

1,239

968

$-271$ 


\section{Building 1860 Dining Hall}

The following information identifies the cost-effective energy- and cost-reducing retrofit projects for building 1860 identified from the FEDS modeling and analysis. Key energy and economic results are presented for each cost-effective retrofit measure.

\section{Facility Description 1860}

1860 is a dining hall built in 1969. It is lighted mostly by T8's and is cooled by an electric air cooled chiller Building 1860 is 12,941 sf.

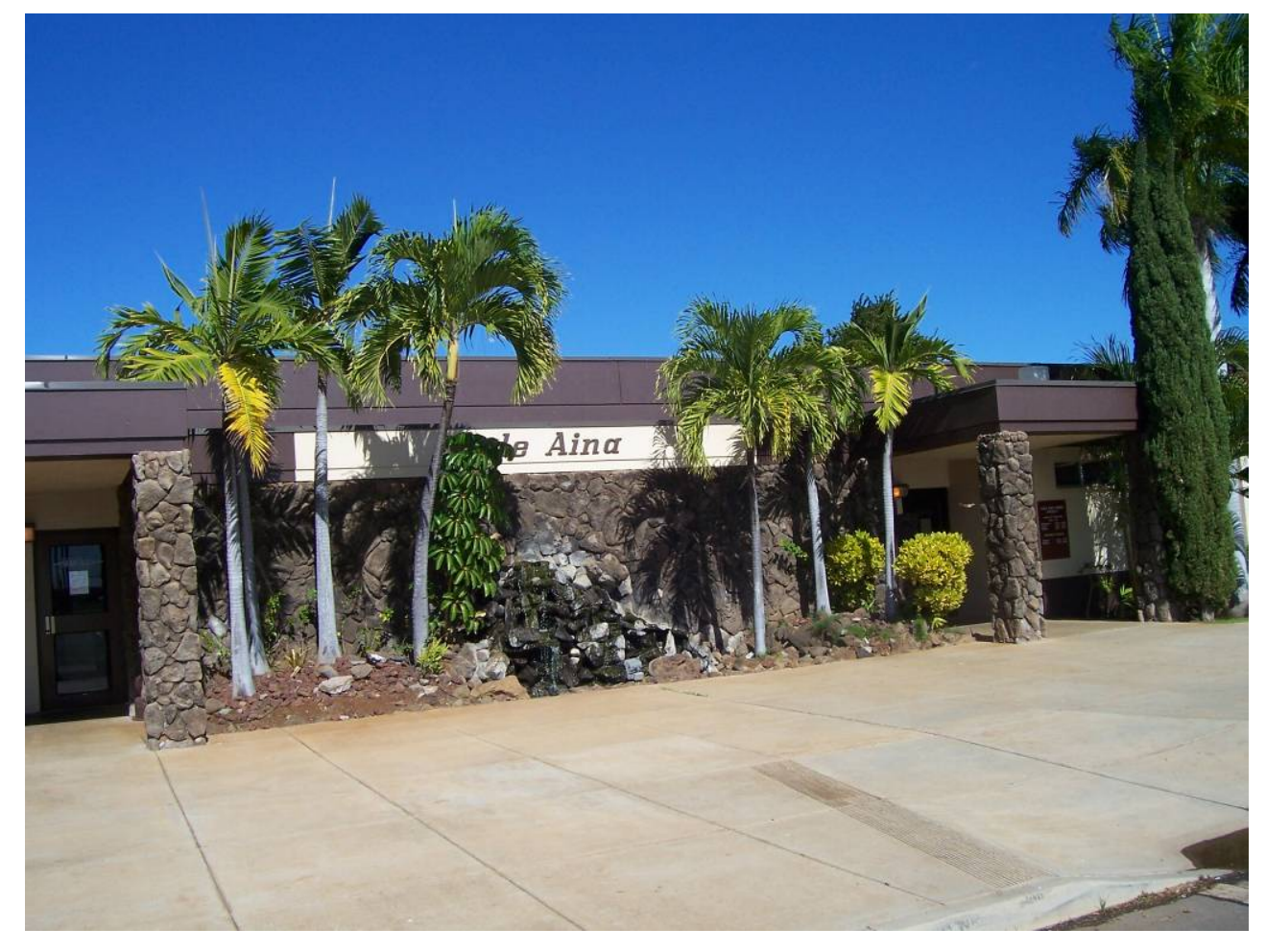




\section{Alternative Financing Results}

A FEDS analysis using alternative financing suggests replacing incandescent lights with CFL lights, replacing the EXIT lighting, replacing the air cooled chiller with a standard efficiency water cooled reciprocating chiller and wrapping the hot water tank with insulation.

Alternative financing FEDS results for building 1860:

\begin{tabular}{|c|c|c|c|c|c|c|c|c|}
\hline Bldg. Set Description & $\begin{array}{l}\text { End } \\
\text { Use }\end{array}$ & Existing Technology & Retrofit Technology & $\begin{array}{l}\text { Energy } \\
\text { Savings } \\
\text { (MMBtu/yr) }\end{array}$ & $\begin{array}{l}\text { 1st Year } \\
\text { Savings } \\
(\$ / y r)\end{array}$ & $\begin{array}{l}\text { Installed } \\
\text { Cost (\$) }\end{array}$ & $\begin{array}{l}\text { Net } \\
\text { Present } \\
\text { Value (\$) }\end{array}$ & SIR \\
\hline Dining Hall 1860 & Cooling & $\begin{array}{l}\text { Electric Air-Cooled Chiller } \\
\{\mathrm{C} 1\}\end{array}$ & $\begin{array}{l}\text { Water-Cooled Reciprocating } \\
\text { Electric Chiller (standard } \\
\text { efficiency) and Cooling Tower }\end{array}$ & 148 & 9,129 & 48,417 & 4,355 & 1.1 \\
\hline Dining Hall 1860 & $\begin{array}{l}\text { Hot } \\
\text { Water }\end{array}$ & Other Fuels Central Boiler & Wrap Tank with Insulation & 219 & 7,025 & 268 & 50,050 & 187.8 \\
\hline Dining Hall 1860 & Lights & IN18: INC 25 WALL & CF14: CFL 5 + BLST UNIT & 13 & 799 & 4,340 & 309 & 1.1 \\
\hline Dining Hall 1860 & Lights & EX1: EXIT - INC (2x20) & $\begin{array}{l}\text { EX12: EXIT - } \\
\text { ELECTROLUMINESCENT PANEL } \\
\text { RETRO KIT }\end{array}$ & 14 & 891 & 621 & 4,590 & 8.4 \\
\hline
\end{tabular}

Alternative financing FEDS results for building 1860:

\begin{tabular}{|c|c|c|c|c|c|c|c|c|}
\hline Bldg. Set Description & $\begin{array}{l}\text { End } \\
\text { Use }\end{array}$ & Existing Technology & Retrofit Technology & $\begin{array}{l}\text { Energy } \\
\text { Savings } \\
\text { (MMBtu/yr) }\end{array}$ & $\begin{array}{l}\text { 1st Year } \\
\text { Savings } \\
(\$ / y r)\end{array}$ & $\begin{array}{l}\text { Installed } \\
\text { Cost (\$) }\end{array}$ & $\begin{array}{l}\text { Net } \\
\text { Present } \\
\text { Value (\$) } \\
\end{array}$ & SIR \\
\hline Dining Hall 1860 & $\begin{array}{l}\text { Hot } \\
\text { Water }\end{array}$ & Other Fuels Central Boiler & Wrap Tank with Insulation & 219 & 7,025 & 268 & 50,050 & 187.8 \\
\hline Dining Hall 1860 & Lights & EX1: EXIT - INC $(2 \times 20)$ & $\begin{array}{l}\text { EX12: EXIT - } \\
\text { ELECTROLUMINESCENT PANEL } \\
\text { RETRO KIT }\end{array}$ & 14 & 928 & 621 & 4,802 & 8.7 \\
\hline
\end{tabular}




\section{Alternative Financing Energy Consumption by Fuel Type}

The modeled energy consumption for a typical year was 485,332 kwh before retrofits and 429,230 kwh after proposed retrofits are implemented. The modeled other fuels (propane) consumption for a typical year was $971 \mathrm{MBtu}$ before retrofits and $534 \mathrm{MBtu}$ after proposed retrofits are implemented. The energy use intensity goes from $203.1 \mathrm{MBtu} / \mathrm{Ksf}$ to $154.5 \mathrm{MBtu} / \mathrm{Ksf}$ after retrofits.

\begin{tabular}{|c|c|c|c|c|}
\hline & & Dining Hall 1860 & & \\
\hline Fuel & Energy & $\begin{array}{c}\text { Energy } \\
\text { Intensity } \\
\text { (user units/1000ft2) }\end{array}$ & $\begin{array}{c}\text { Energy } \\
\text { Intensity } \\
\text { (MBtu/1000ft2) }\end{array}$ & $\begin{array}{l}\text { Dollars } \\
(2009)^{*}\end{array}$ \\
\hline Electricity (kW & & & & \\
\hline existing & 485,332 & $37,503.4$ & 128.0 & 85,984 \\
\hline post-retrofit & 429,230 & $33,168.2$ & 113.2 & 75,842 \\
\hline difference & $-56,102$ & $-4,335.2$ & -14.8 & $-10,142$ \\
\hline$\%$ change & -11.6 & -11.6 & -11.6 & -11.8 \\
\hline Other Fuels (MB & & & & \\
\hline existing & 971 & 75.0 & 75.0 & 31,223 \\
\hline post-retrofit & 534 & 41.3 & 41.3 & 17,172 \\
\hline difference & -437 & -33.8 & -33.8 & $-14,051$ \\
\hline$\%$ change & -45 & -45 & -45 & -45 \\
\hline Total (MBtu) & & & & \\
\hline existing & 2,628 & 203.1 & 203.1 & 117,208 \\
\hline post-retrofit & 1,999 & 154.5 & 154.5 & 93,014 \\
\hline difference & -629 & -48.6 & -48.6 & $-24,194$ \\
\hline$\%$ change & -24 & -24 & -24 & -21 \\
\hline
\end{tabular}

* Dollar values for electricity include both energy and demand components. 


\section{Alternative Financing Energy Consumption by End Use}

Space cooling is the largest load in the building with 221,654 kWh/year, followed by motors and miscellaneous equipment with $160,038 \mathrm{kWh} /$ year.

Annual Energy Use by Building Set, Fuel Type, and End Use

Fuel
Electricity (kWh)
existing
post-retrofit
difference
$\%$ change
Other Fuels (MBtu)
existing
post-retrofit
difference
$\%$ change
Total (MBtu)
existing
post-retrofit
difference
$\%$ change
Total (MBtu/100○ft2)
existing
post-retrofit
difference
$\%$ change
Dining Hall 1860

\begin{tabular}{|c|c|c|c|c|c|}
\hline ing & Cooling & Vent & Lights & $\begin{array}{l}\text { Motors and } \\
\text { Misc Equip }\end{array}$ & Hot Water \\
\hline$\odot$ & 221,654 & $7 \odot, 6 \odot 7$ & 33,032 & 160,038 & $\odot$ \\
\hline$\odot$ & 174,142 & 70,209 & 24,842 & 160,038 & 0 \\
\hline$\odot$ & $-47,512$ & -398 & $-8,190$ & 0 & $\odot$ \\
\hline$\odot$ & -21 & -1 & -25 & $\odot$ & $\odot$ \\
\hline 0 & $\odot$ & $\odot$ & $\odot$ & 193 & 778 \\
\hline$\odot$ & $\odot$ & $\odot$ & $\odot$ & 193 & 340 \\
\hline$\odot$ & $\odot$ & $\odot$ & $\odot$ & 0 & -438 \\
\hline$\odot$ & $\odot$ & $\odot$ & $\odot$ & $\odot$ & -56 \\
\hline$\odot$ & 756 & 241 & 51 & 740 & 408 \\
\hline$\odot$ & 594 & 240 & $4 \odot$ & 740 & 175 \\
\hline$\odot$ & -162 & -1 & -11 & 0 & -248 \\
\hline$\odot$ & -21 & -1 & -22 & $\odot$ & -59 \\
\hline$\odot$ & 116 & 37 & 17 & 114 & 124 \\
\hline$\odot$ & 40 & 37 & 13 & 114 & 53 \\
\hline$\odot$ & -21 & 0 & -4 & 0 & -71 \\
\hline$\odot$ & -18 & $\odot$ & -24 & $\odot$ & -57 \\
\hline
\end{tabular}




\section{Alternative Financing Emission Reduction}

The emission reductions from implemented the proposed retrofits are as follows:

Dining Hall 1860

Sulfur Oxides (lb)

existing

post-retrofit

difference

48,430

43,439

$\%$ change

$-4,991$

$-10$

Nitrogen oxides (lb)

existing

post-retrofit

difference

4,630

4,014

$-616$

$\%$ change

$-13$

Carbon Monoxide (lb)

existing

2,437

post-retrofit

2,041

difference

$-749$

$\%$ change

$-16$

Carbon Dioxide (tons)

existing

post-retrofit

4,344

3,595

difference

$-749$

$\%$ change

$-17$

Particulate Matter (lb)

existing

post-retrofit

268

difference

165

165
-749

$\%$ change

$-38$

Hydrocarbons (lb)

existing

post-retrofit

1,736

1,453

difference

$-283$

\% change

$-16$ 


\section{Building 1804 Dining Facility}

The following information identifies the cost-effective energy- and cost-reducing retrofit projects for building 1804 identified from the FEDS modeling and analysis. Key energy and economic results are presented for each cost-effective retrofit measure.

\section{Facility Description 1804}

1804 is an open mess facility built in 2003. Building 1804 has incandescent and 32W T8 lilghts, an electric air cooled chiller and little to no insulation in its building envelope. Building 1804 is 27,579 sf.

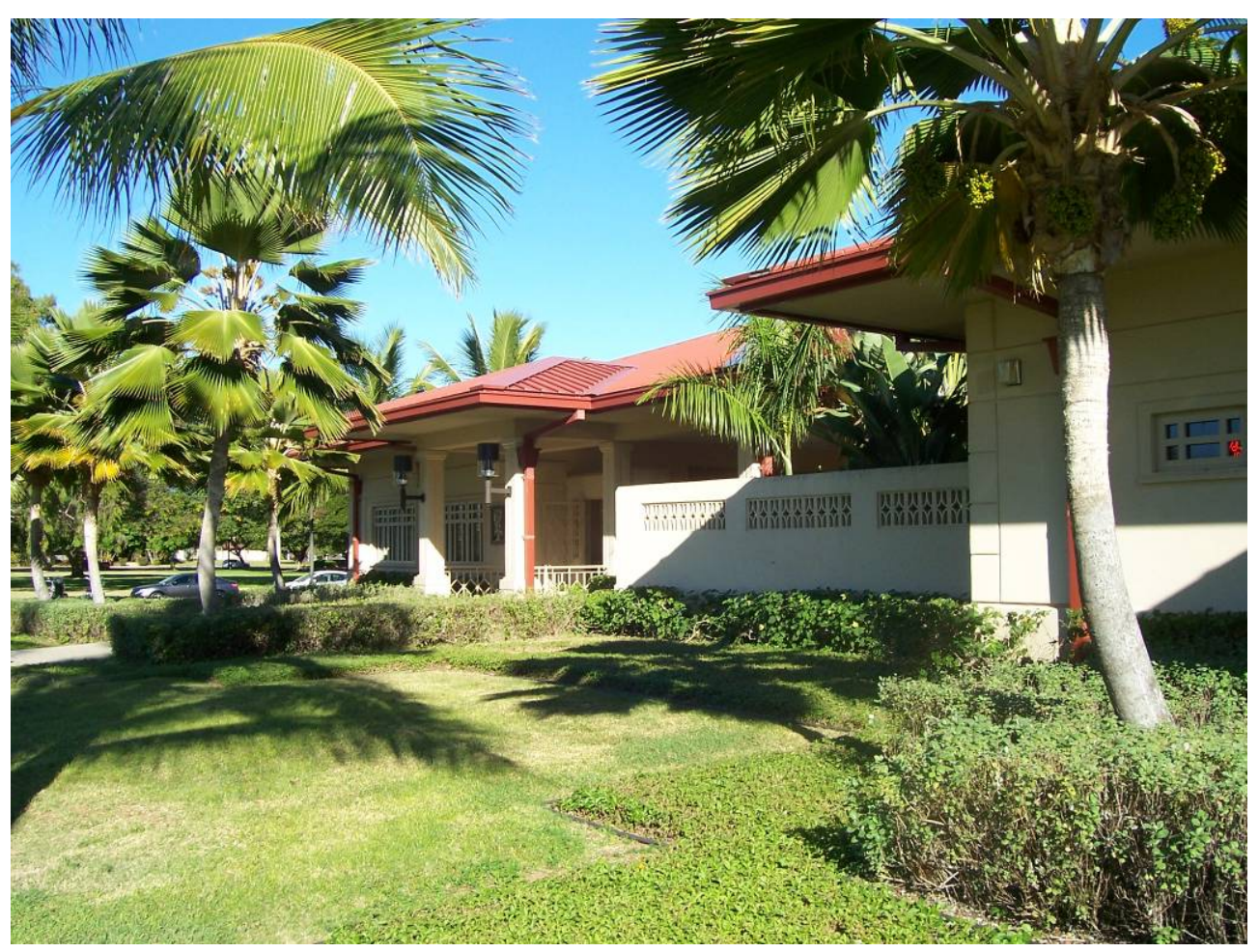




\section{Alternative Financing Results}

A FEDS analysis using alternative financing suggests wrapping the hot water tank with insulation, replacing incandescent lamps with CFL lamps and replacing the EXIT lighting.

Alternative financing FEDS results for building 1804:

\begin{tabular}{|c|c|c|c|c|c|c|c|c|c|}
\hline \multicolumn{2}{|c|}{ Bldg. Set Description } & $\begin{array}{l}\text { End } \\
\text { Use }\end{array}$ & Existing Technology & Retrofit Technology & $\begin{array}{l}\text { Energy } \\
\text { Savings } \\
\text { (MMBtu/yr) }\end{array}$ & $\begin{array}{l}\text { 1st Year } \\
\text { Savings } \\
(\$ / y r)\end{array}$ & $\begin{array}{l}\text { Installed } \\
\text { Cost (\$) }\end{array}$ & $\begin{array}{l}\text { Net } \\
\text { Present } \\
\text { Value (\$) }\end{array}$ & SIR \\
\hline Dining & 1804 & $\begin{array}{l}\text { Hot } \\
\text { Water }\end{array}$ & Other Fuels Water Heater & Wrap Tank with Insulation & 37 & 1,202 & 1,764 & 5,751 & 3.0 \\
\hline Dining & 1804 & Lights & IN25: INC 75 WALL & CF5: CFL 18 INTEGRAL UNIT ELC & 334 & 20,370 & 8,978 & 109,722 & 13.2 \\
\hline Dining & 1804 & Lights & EX1: EXIT - INC (2x20) & $\begin{array}{l}\text { EX12: EXIT - } \\
\text { ELECTROLUMINESCENT PANEL } \\
\text { RETRO KIT }\end{array}$ & 23 & 1,471 & 932 & 7,663 & 9.2 \\
\hline
\end{tabular}




\section{Alternative Financing Energy Consumption by Fuel Type}

The modeled energy consumption for a typical year was 809,008 kwh before retrofits and 704,303 kwh after proposed retrofits are implemented. The modeled other fuels (propane) consumption for a typical year was $855 \mathrm{MBtu}$ before retrofits and $818 \mathrm{MBtu}$ after retrofits. The energy use intensity goes from 131.1 MBtu/Ksf to 116.8 MBtu/Ksf after retrofits.

\begin{tabular}{|c|c|c|c|c|}
\hline \multirow[b]{2}{*}{ Fuel } & \multicolumn{2}{|r|}{ Dining } & \multirow[b]{2}{*}{$\begin{array}{c}\text { Energy } \\
\text { Intensity } \\
\text { (MBtu/1000ft2) }\end{array}$} & \multirow[b]{2}{*}{$\begin{array}{l}\text { Dollars } \\
(2009)^{*}\end{array}$} \\
\hline & Energy & $\begin{array}{c}\text { Energy } \\
\text { Intensity } \\
\text { (user units/1000ft2) }\end{array}$ & & \\
\hline \multicolumn{5}{|c|}{ Electricity (kWh) } \\
\hline existing & $809,0 \odot 8$ & $29,334.2$ & 100.1 & 143,329 \\
\hline post-retrofit & 704,303 & $25,537.7$ & 87.2 & 124,446 \\
\hline difference & $-104,705$ & $-3,796.5$ & -13.0 & $-18,882$ \\
\hline$\%$ change & -13 & -13 & -13 & -13 \\
\hline \multicolumn{5}{|c|}{ Other Fuels (MBtu) } \\
\hline existing & 855 & 31.0 & 31.0 & 27,501 \\
\hline post-retrofit & 818 & 29.7 & 29.7 & 26,299 \\
\hline difference & -37 & -1.4 & -1.4 & $-1,202$ \\
\hline$\%$ change & -4 & -4 & -4 & -4 \\
\hline \multicolumn{5}{|l|}{ Total (MBtu) } \\
\hline existing & 3,617 & 131.1 & 131.1 & 170,830 \\
\hline post-retrofit & 3,222 & 116.8 & 116.8 & 150,745 \\
\hline difference & -395 & -14.3 & -14.3 & $-20,085$ \\
\hline$\%$ change & -11 & -11 & -11 & -12 \\
\hline
\end{tabular}

* Dollar values for electricity include both energy and demand components. 


\section{Alternative Financing Energy Consumption by End Use}

Space cooling is the largest load in the building with $316,839 \mathrm{kWh} /$ year, followed by motors and miscellaneous equipment with 262,004 kWh/year.

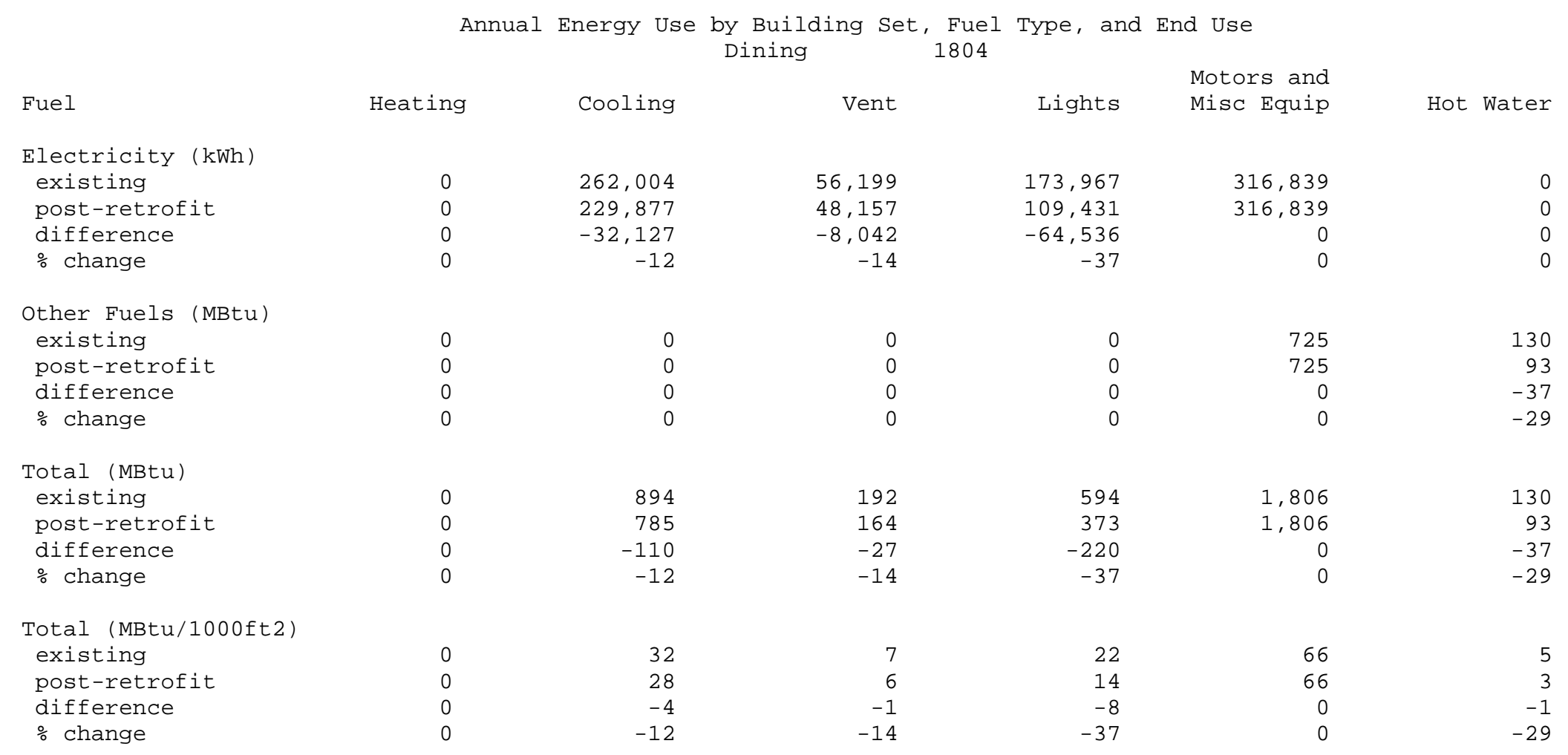




\section{Alternative Financing Emission Reduction}

The emission reductions from implemented the proposed retrofits are as follows:

Dining

1804

Sulfur Oxides (lb)

existing

post-retrofit

difference

7,527

6,571

$-956$

$\%$ change

$-13$

Nitrogen Oxides (lb)

existing

post-retrofit

3,794

difference

3,329

$\%$ change

$-465$

Carbon Monoxide (lb)

existing

post-retrofit

difference

6,661

$\%$ change

5,855

$-806$

$-12$

Carbon Dioxide (tons)

existing

post-retrofit

813

difference

714

$\%$ change

$-12$

Particulate Matter (lb)

existing

153

post-retrofit

134

difference

$-19$

$\%$ change

$-12$

Hydrocarbons (lb)

existing

post-retrofit

2,702

post-retrofi

2,370

$\%$ change

$-331$

$-12$ 


\section{Building 594 Lavatory Facility}

The following information identifies the cost-effective energy- and cost-reducing retrofit projects for building 594 identified from the FEDS modeling and analysis. Key energy and economic results are presented for each cost-effective retrofit measure.

\section{Facility Description 594}

594 is a lavatory by the baseball fields built in 1977. Building 594 is not cooled and has very little lighting. Building 594 is 293 sf.

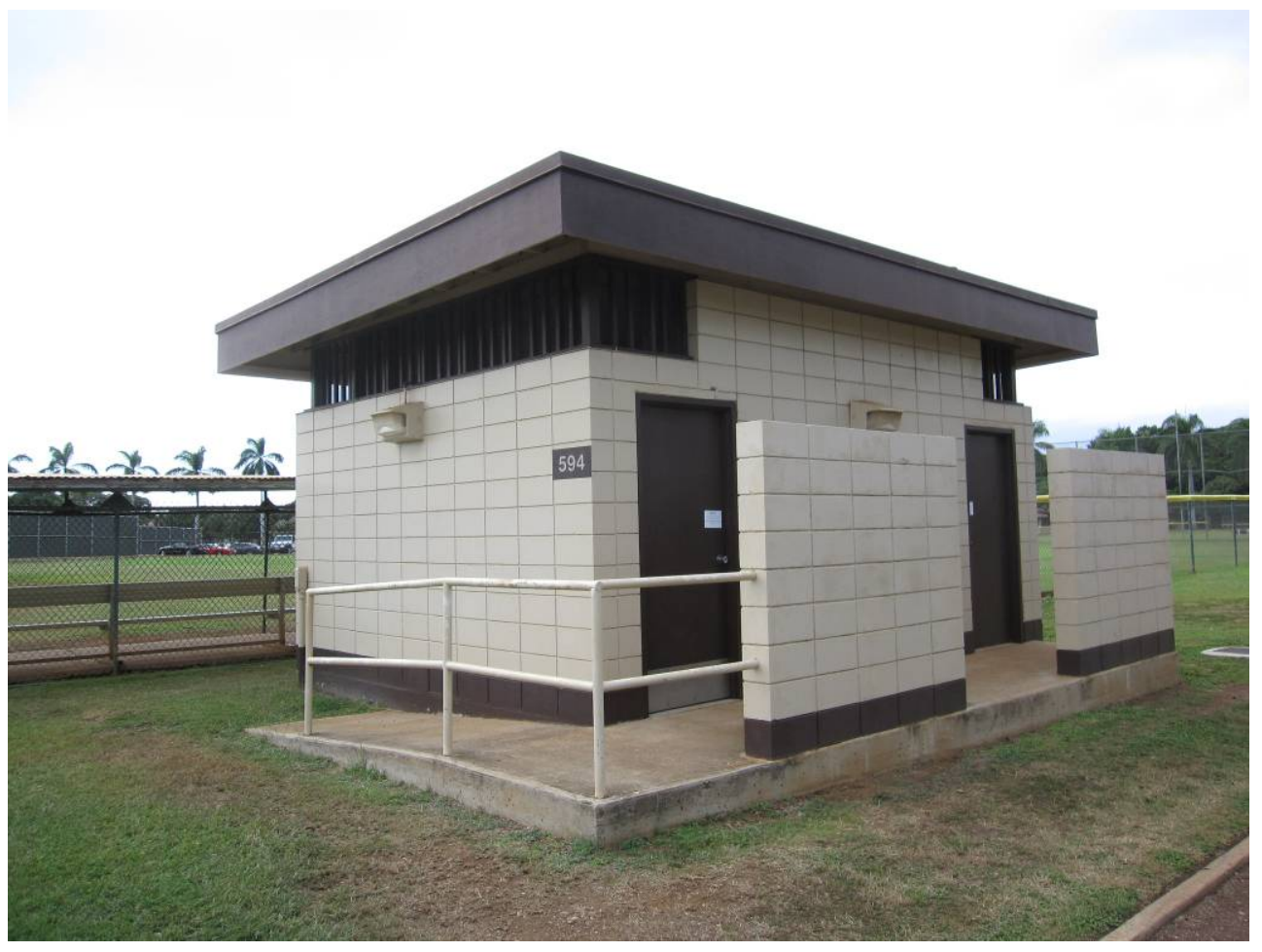




\section{Alternative Financing Results}

FEDS did not find any life cycle cost effective retrofits using alternative financing.

\section{Alternative Financing Energy Consumption by Fuel Type}

The modeled energy consumption for a typical year was 2,429 kwh before retrofits and 2,429 kwh after proposed retrofits are implemented. The energy use intensity goes from 34.9 MBtu/Ksf to 34.9 MBtu/Ksf after retrofits.

\begin{tabular}{|c|c|c|c|c|}
\hline \multirow[b]{2}{*}{ Fuel } & \multicolumn{2}{|c|}{ sanitary latrines/small storag } & \multirow{2}{*}{$\begin{array}{c}594 \\
\text { Energy } \\
\text { Intensity } \\
\text { (MBtu/1000ft2) }\end{array}$} & \multirow[b]{2}{*}{$\begin{array}{l}\text { Dollars } \\
(2009) \text { * }\end{array}$} \\
\hline & Energy & $\begin{array}{c}\text { Energy } \\
\text { Intensity } \\
\text { (user units/1000ft2) }\end{array}$ & & \\
\hline \multicolumn{5}{|c|}{ Electricity (kWh) } \\
\hline existing & 2,429 & $8,290.3$ & 28.3 & 430 \\
\hline post-retrofit & 2,429 & $8,290.3$ & 28.3 & 430 \\
\hline difference & 0 & 0.0 & $\odot$ & $\odot$ \\
\hline$\%$ change & $\odot$ & $\odot$ & $\odot$ & $\odot$ \\
\hline \multicolumn{5}{|c|}{ other Fuels (MBtu) } \\
\hline existing & 2 & 6.6 & 6.6 & 63 \\
\hline post-retrofit & 2 & 6.6 & 6.6 & 63 \\
\hline difference & $\odot$ & $\odot . \odot$ & $\odot . \odot$ & $\odot$ \\
\hline$\%$ change & $\odot$ & $\odot$ & $\odot$ & $\odot$ \\
\hline \multicolumn{5}{|l|}{ Total (MBtu) } \\
\hline existing & 10 & 34.9 & 34.9 & 493 \\
\hline post-retrofit & 10 & 34.9 & 34.9 & 493 \\
\hline difference & $\odot$ & $\odot . \odot$ & $\odot . \odot$ & $\odot$ \\
\hline$\%$ change & $\odot$ & 0 & 0 & $\odot$ \\
\hline
\end{tabular}

* Dollar values for electricity include both energy and demand components. 


\section{Alternative Financing Energy Consumption by End Use}

Lighting is the largest load in the building with 2,091 kWh/year, followed by motors and miscellaneous equipment with 338 $\mathrm{kWh} /$ year.

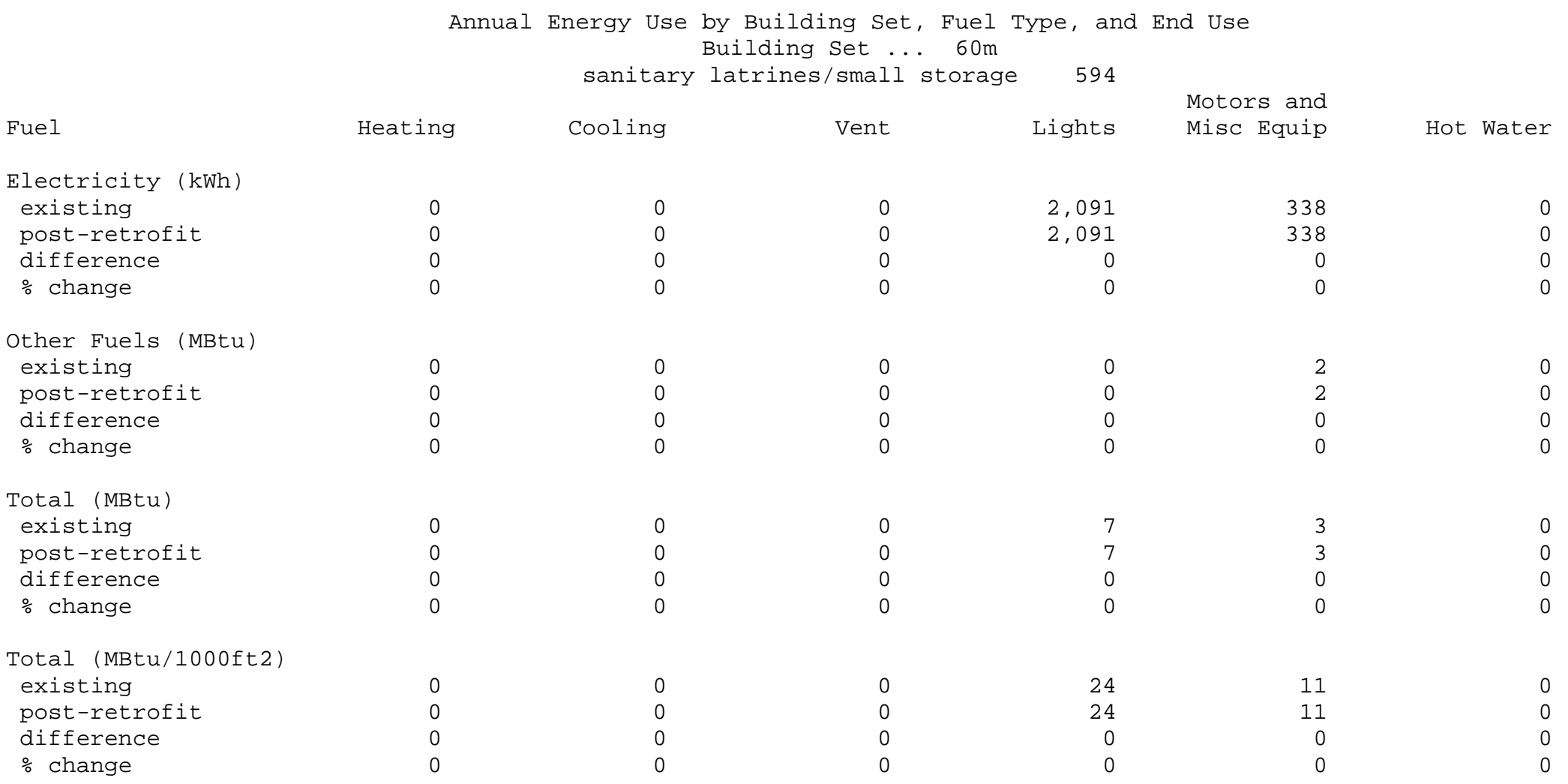




\section{Alternative Financing Emission Reduction}

The emission reductions from implemented the proposed retrofits are as follows:

sanitary latrines/small storage

Sulfur oxides (lb)

existing

post-retrofit

difference

$\%$ change

Nitrogen Oxides (lb)

existing

post-retrofit

difference

$\%$ change

Carbon Monoxide (lb)

existing

post-retrofit

difference

$\%$ change

Carbon Dioxide (tons)

existing

post-retrofit

difference

$\%$ change

Particulate Matter (lb)

existing

post-retrofit

difference

$\%$ change

Hydrocarbons ( $1 b$ )

existing

post-retrofit

difference

$\%$ change 


\section{Building 2093 Commissary}

The following information identifies the cost-effective energy- and cost-reducing retrofit projects for building 2093 identified from the FEDS modeling and analysis. Key energy and economic results are presented for each cost-effective retrofit measure.

\section{Facility Description 2093}

2093 is the commissary and was built in 1975. Building 2093 has large conditioned service spaces as well as large unconditioned storage spaces. Building 2093 is 115,408 sf.

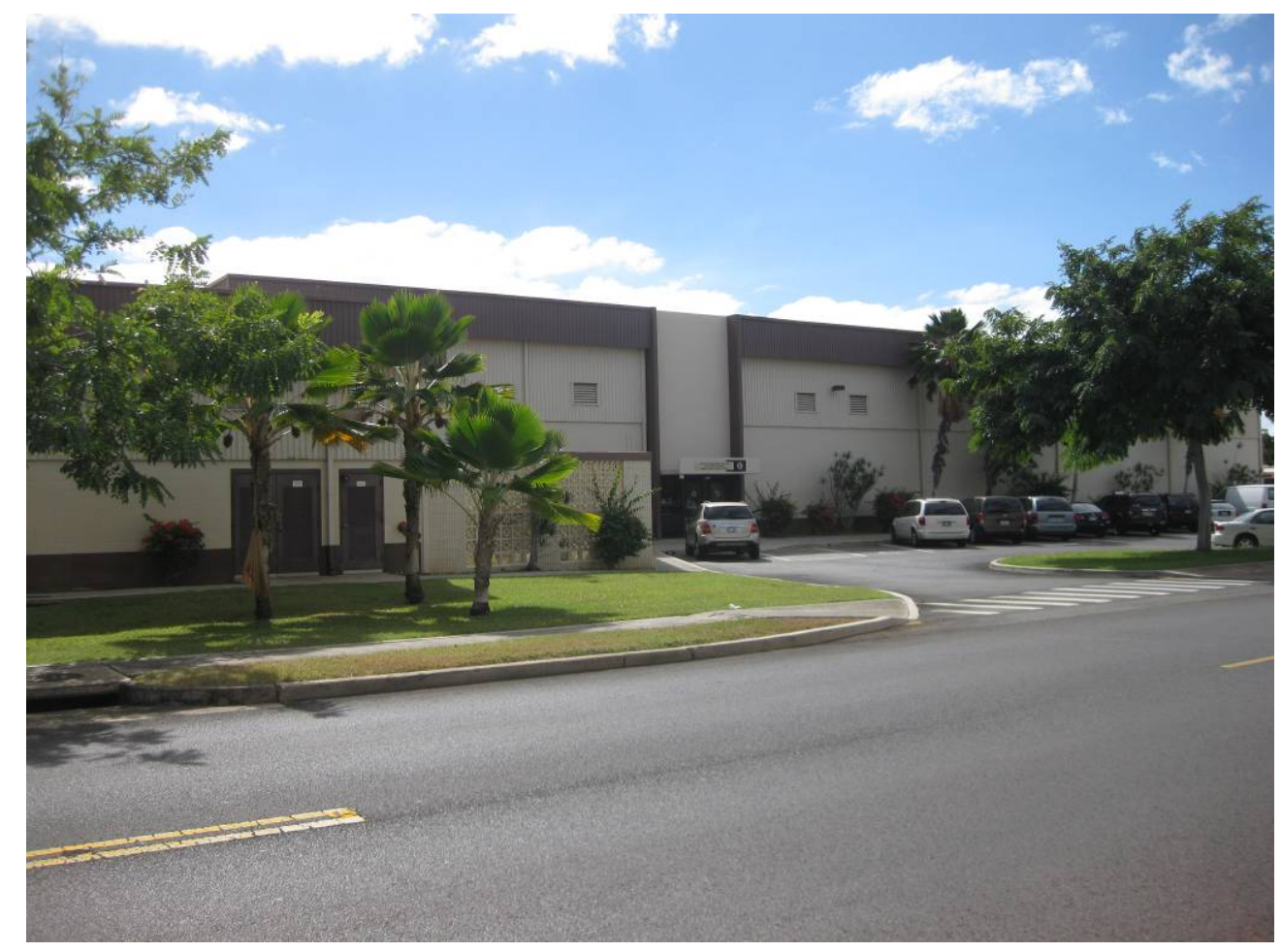




\section{Alternative Financing Results}

A FEDS analysis using alternative financing suggests replacing the electric water heater with a heat pump water heater and replacing some of the lighting.

Alternative financing FEDS results for building 2093

\begin{tabular}{|c|c|c|c|c|c|c|c|c|}
\hline Bldg. Set Description & $\begin{array}{l}\text { End } \\
\text { Use }\end{array}$ & Existing Technology & Retrofit Technology & $\begin{array}{l}\text { Energy } \\
\text { Savings } \\
\text { (MMBtu/yr) }\end{array}$ & $\begin{array}{l}\text { 1st Year } \\
\text { Savings } \\
(\$ / y r)\end{array}$ & $\begin{array}{l}\text { Installed } \\
\text { Cost (\$) }\end{array}$ & $\begin{array}{l}\text { Net } \\
\text { Present } \\
\text { Value (\$) } \\
\end{array}$ & SIR \\
\hline $\begin{array}{l}\text { Comissary, large sales } \\
2093\end{array}$ & $\begin{array}{l}\text { Hot } \\
\text { Water }\end{array}$ & Electric Water Heater & $\begin{array}{l}\text { Heat Pump Water Heater } \\
\text { (Com) }\end{array}$ & 168 & 8,496 & 47,981 & 643 & 1.0 \\
\hline $\begin{array}{l}\text { Comissary, large sales } \\
2093\end{array}$ & Lights & EX1: EXIT - INC (2x20) & $\begin{array}{l}\text { EX12: EXIT - } \\
\text { ELECTROLUMINESCENT PANEL } \\
\text { RETRO KIT }\end{array}$ & 23 & 1,560 & 1,118 & 8,017 & 8.2 \\
\hline $\begin{array}{l}\text { Comissary, large sales } \\
2093\end{array}$ & Lights & EX1: EXIT - INC $(2 \times 20)$ & $\begin{array}{l}\text { EX12: EXIT - } \\
\text { ELECTROLUMINESCENT PANEL } \\
\text { RETRO KIT }\end{array}$ & 3 & 174 & 124 & 891 & 8.2 \\
\hline $\begin{array}{l}\text { Comissary, large sales } \\
2093\end{array}$ & Lights & FL1: FL 2X4 4F40T12 STD2 & FL244: FL 2X4 4F32T8 ELC4 & 124 & 8,350 & 15,231 & 33,517 & 3.2 \\
\hline $\begin{array}{l}\text { Comissary, large sales } \\
2093\end{array}$ & Lights & FL1: FL 2X4 4F40T12 STD2 & $\begin{array}{l}\text { FL280: FL 2X4 3F32ST8 ELC3 } \\
\text { REF (FIX REPL) }\end{array}$ & 1,745 & 103,847 & 259,302 & 344,042 & 2.3 \\
\hline
\end{tabular}




\section{Alternative Financing Energy Consumption by Fuel Type}

The modeled energy consumption for a typical year was 6,735,356 kwh before retrofits and 6,130,693 kwh after proposed retrofits are implemented. The energy use intensity goes from 199.2 MBtu/Ksf to $181.3 \mathrm{MBtu} / \mathrm{Ksf}$ after retrofits.

\begin{tabular}{|c|c|c|c|c|}
\hline \multirow[b]{2}{*}{ Fuel } & \multicolumn{2}{|c|}{ Commissary, large sales } & \multicolumn{2}{|c|}{2093} \\
\hline & Energy & $\begin{array}{c}\text { Energy } \\
\text { Intensity } \\
\text { (user units/1000ft2) }\end{array}$ & $\begin{array}{c}\text { Energy } \\
\text { Intensity } \\
\text { (MBtu/1000ft2) }\end{array}$ & $\begin{array}{l}\text { Dollars } \\
(2009)^{*}\end{array}$ \\
\hline \multicolumn{5}{|c|}{ Electricity (kWh) } \\
\hline existing & $6,735,356$ & $58,361.3$ & 199.2 & $1,193,276$ \\
\hline post-retrofit & $6,130,693$ & $53,121.9$ & 181.3 & $1,083,257$ \\
\hline difference & $-604,664$ & $-5,239.4$ & -17.9 & $-110,018$ \\
\hline$\%$ change & -9 & -9 & -9 & -9 \\
\hline \multicolumn{5}{|l|}{ Total (MBtu) } \\
\hline existing & 22,988 & 199.2 & 199.2 & $1,193,276$ \\
\hline post-retrofit & 20,924 & 181.3 & 181.3 & $1, \odot 83,257$ \\
\hline difference & $-2,064$ & -17.9 & -17.9 & $-110,018$ \\
\hline$\%$ change & -9 & -9 & -9 & -9 \\
\hline
\end{tabular}

* Dollar values for electricity include both energy and demand components. 


\section{Alternative Financing Energy Consumption by End Use}

Motors and miscellaneous equipment is the largest load in the building with 4,432,117 kWh/year, followed by lighting with 1,096,681 $\mathrm{kWh} /$ year.

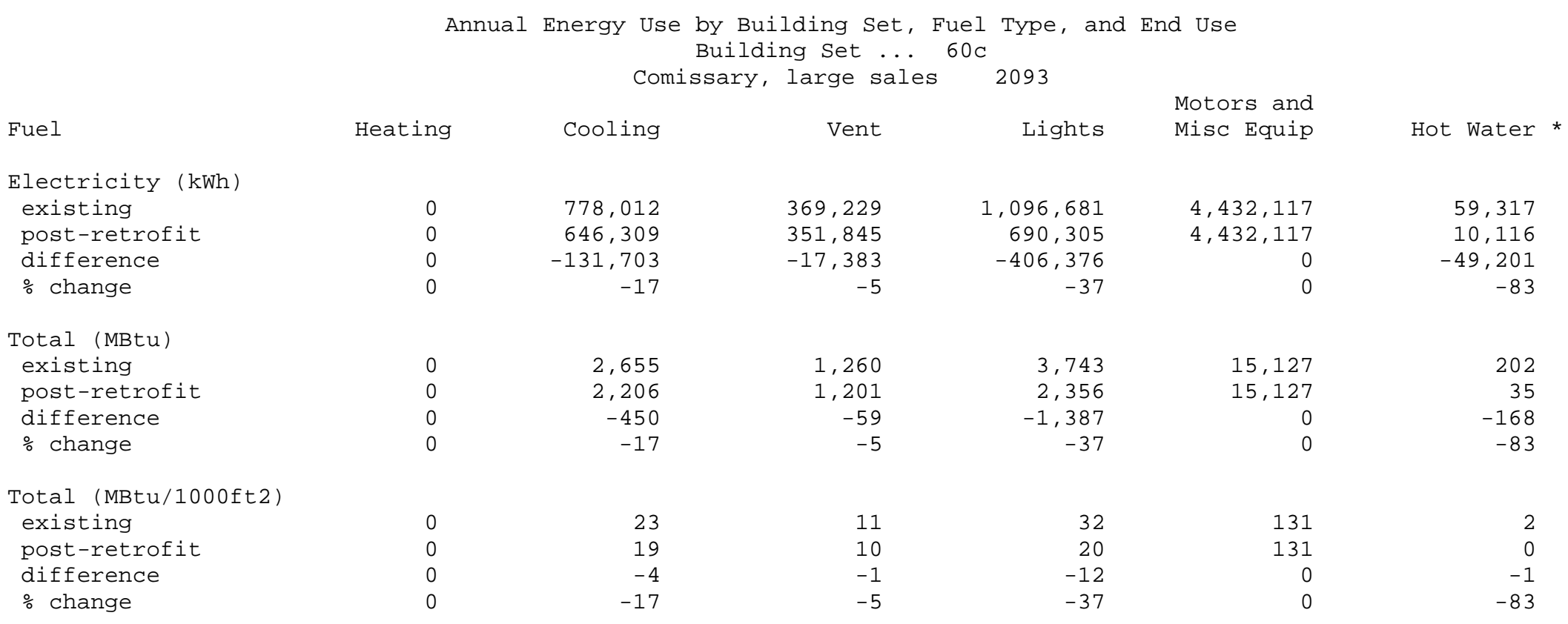

* Energy consumption values for both distributed and central SHW are reported for Hot Water annual energy use. 


\section{Alternative Financing Emission Reduction}

The emission reductions from implemented the proposed retrofits are as follows:

Sulfur Oxides (lb)

existing

post-retrofit

60,888

difference

55,422

$\%$ change

$-5,466$

Nitrogen Oxides (lb)

existing

post-retrofit

difference

26,484

$\%$ change

$-9$

Carbon Monoxide (lb)

existing

post-retrofit

50,044

difference

45,552

$-4,493$
-9

$\%$ change

\section{6,163}

5,610

post-retrofit

difference

$-9$

Particulate Matter (lb)

existing

post-retrofit

1,205

difference

1,096

$\%$ change

Hydrocarbons (lb)

existing

post-retrofit

18,853

difference

$-1,859$

$\%$ change

$-9$ 


\section{Building 2028 Passenger Terminal}

The following information identifies the cost-effective energy- and cost-reducing retrofit projects for building 2028 identified from the FEDS modeling and analysis. Key energy and economic results are presented for each cost-effective retrofit measure.

\section{Facility Description 2028}

2028 is the air passenger terminal built in 1973. Building 2028 has a water cooled reciprocating chiller, metal halide, fluorescent, incandescent and high pressure sodium lights and little to no insulation in the building envelope. Building 2028 is 46,128 sf.

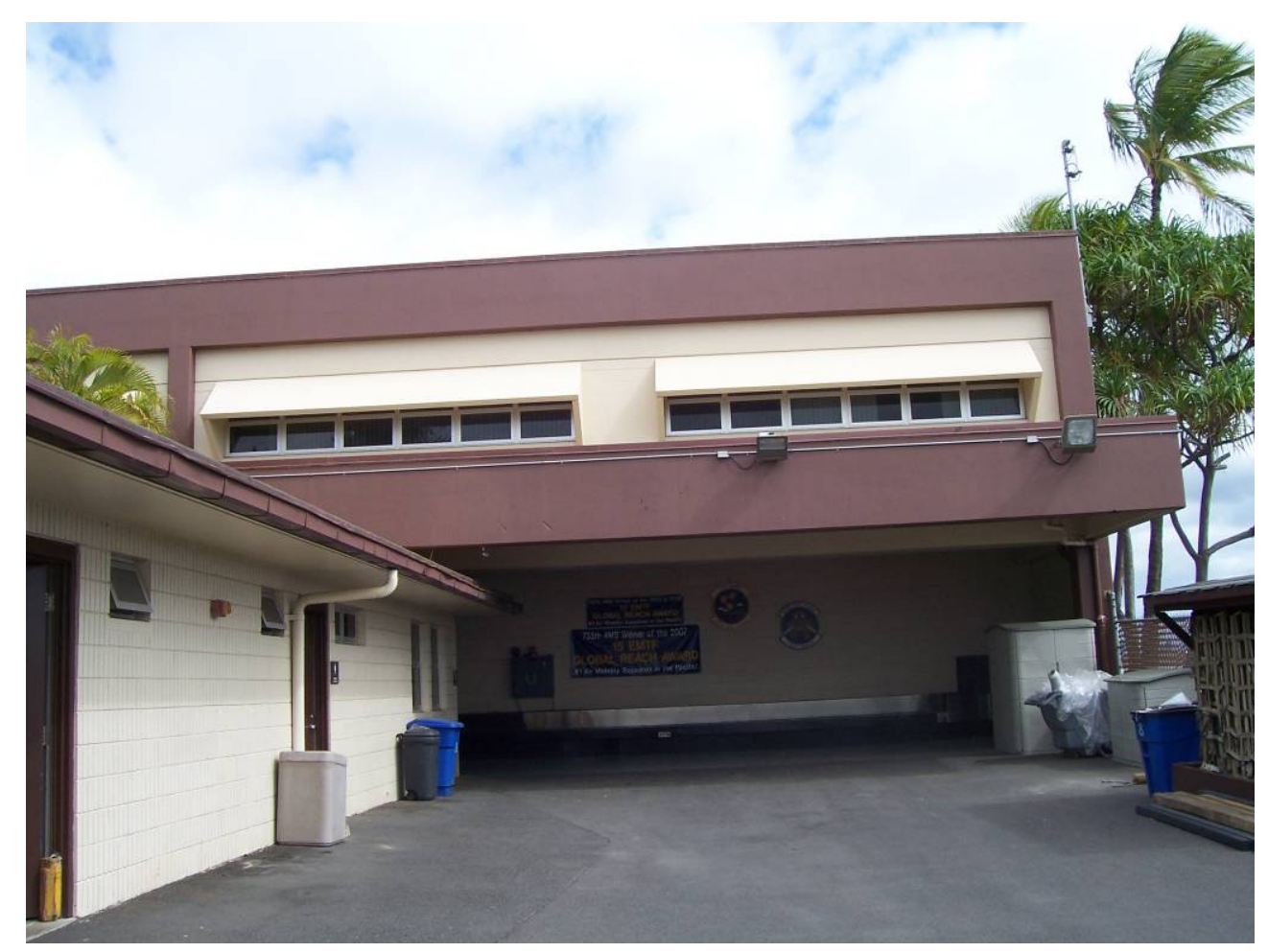




\section{Alternative Financing Results}

A FEDS analysis using alternative financing suggests replacing the lighting, replacing the electric water heater with a heat pump water heater and increasing the insulation in the suspended ceiling.

Alternative financing FEDS results for building 2028:

\begin{tabular}{|c|c|c|c|c|c|c|c|c|}
\hline Bldg. Set Description & $\begin{array}{l}\text { End } \\
\text { Use }\end{array}$ & Existing Technology & Retrofit Technology & $\begin{array}{l}\text { Energy } \\
\text { Savings } \\
\text { (MMBtu/yr) } \\
\end{array}$ & $\begin{array}{l}\text { 1st Year } \\
\text { Savings } \\
(\$ / y r)\end{array}$ & $\begin{array}{l}\text { Installed } \\
\text { Cost (\$) }\end{array}$ & $\begin{array}{l}\text { Net } \\
\text { Present } \\
\text { Value (\$) } \\
\end{array}$ & SIR \\
\hline Airport terminal 2028 & $\begin{array}{l}\text { Hot } \\
\text { Water }\end{array}$ & Electric Water Heater & $\begin{array}{l}\text { Heat Pump Water Heater } \\
\text { (Com) }\end{array}$ & 22 & 1,004 & 5,098 & 654 & 1.1 \\
\hline Airport terminal 2028 & Lights & EX1: EXIT - INC $(2 \times 20)$ & $\begin{array}{l}\text { EX12: EXIT - } \\
\text { ELECTROLUMINESCENT PANEL } \\
\text { RETRO KIT }\end{array}$ & 13 & 889 & 621 & 4,581 & 8.4 \\
\hline Airport terminal & Lights & FL1: FL 2X4 4F40T12 STD2 & $\begin{array}{l}\text { FL280: FL 2X4 3F32ST8 ELC3 } \\
\text { REF (FIX REPL) }\end{array}$ & 832 & 47,481 & 87,739 & 187,832 & 3.1 \\
\hline Airport terminal & Roof & Roof Insulation R-Value 0.00 & $\begin{array}{l}\text { Suspended Ceiling: Increase } \\
\text { Insulation by R-11 }\end{array}$ & 161 & 10,970 & 60,766 & 2,203 & 1.0 \\
\hline
\end{tabular}




\section{Alternative Financing Energy Consumption by Fuel Type}

The modeled energy consumption for a typical year was 804,699 kwh before retrofits and 512,174 kwh after proposed retrofits are implemented. The energy use intensity goes from 59.5 MBtu/Ksf to 37.9 MBtu/Ksf after retrofits.

\begin{tabular}{|c|c|c|c|c|}
\hline \multirow[b]{2}{*}{ Fuel } & \multicolumn{3}{|c|}{ Airport terminal 2028} & \multirow[b]{2}{*}{$\begin{array}{l}\text { Dollars } \\
(2009) \text { * }\end{array}$} \\
\hline & Energy & $\begin{array}{c}\text { Energy } \\
\text { Intensity } \\
\text { (user units/1000ft2) }\end{array}$ & $\begin{array}{c}\text { Energy } \\
\text { Intensity } \\
\text { (MBtu/1000ft2) }\end{array}$ & \\
\hline \multicolumn{5}{|c|}{ Electricity (kWh) } \\
\hline existing & 804,699 & $17,444.9$ & 59.5 & 142,565 \\
\hline post-retrofit & 512,174 & $11,103.3$ & 37.9 & 90,498 \\
\hline difference & $-292,525$ & $-6,341.6$ & -21.6 & $-52,067$ \\
\hline$\%$ change & -36 & -36 & -36 & -37 \\
\hline \multicolumn{5}{|l|}{ Total (MBtu) } \\
\hline existing & 2,746 & 59.5 & 59.5 & 142,565 \\
\hline post-retrofit & 1,748 & 37.9 & 37.9 & 90,498 \\
\hline difference & -998 & -21.6 & -21.6 & $-52,067$ \\
\hline$\%$ change & -36 & -36 & -36 & -37 \\
\hline
\end{tabular}

* Dollar values for electricity include both energy and demand components. 


\section{Alternative Financing Energy Consumption by End Use}

Lighting is the largest load in the building with 410,212 kWh/year, followed by space cooling with 235,351 kWh/year.

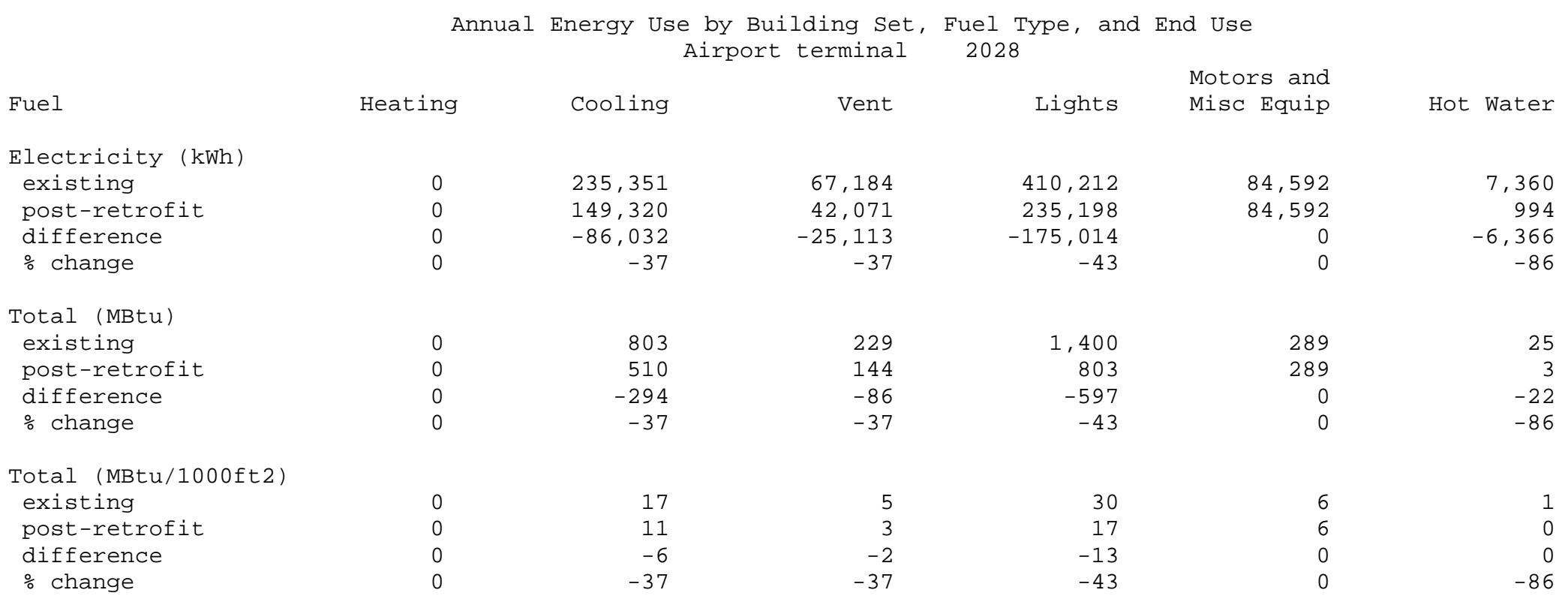




\section{Alternative Financing Emission Reduction}

The emission reductions from implemented the proposed retrofits are as follows:

Airport terminal

2028

Sulfur Oxides (lb)

existing

post-retrofit

difference

7,274

4,630

$\%$ change

$-2,644$
-36

Nitrogen 0xides (lb)

existing

post-retrofit

difference

3,476

2,213

$-1,264$

$\%$ change

$-36$

Carbon Monoxide (lb)

existing

post-retrofit

5,979

difference

3,806

$\%$ change

$-2,173$

Carbon Dioxide (tons)

existing

post-retrofit

736

difference

469

$\%$ change

$-268$

$-36$

Particulate Matter (lb)

existing

post-retrofit

difference

92

$\%$ change

$-36$

Hydrocarbons (1b)

existing

post-retrofit

2,475

post-retrof

1,575

$\%$ change

-900
-36 


\section{Building 1597 Child Care Center}

The following information identifies the cost-effective energy- and cost-reducing retrofit projects for building 1597 identified from the FEDS modeling and analysis. Key energy and economic results are presented for each cost-effective retrofit measure.

\section{Facility Description 1597}

1597 is a child care center built 1985. Building 1597 is conditioned by an electric air cooled chiller, has many fluorescent and some metal halide lights and has some insulation in the building envelope. Building 1597 is 12,760 sf.

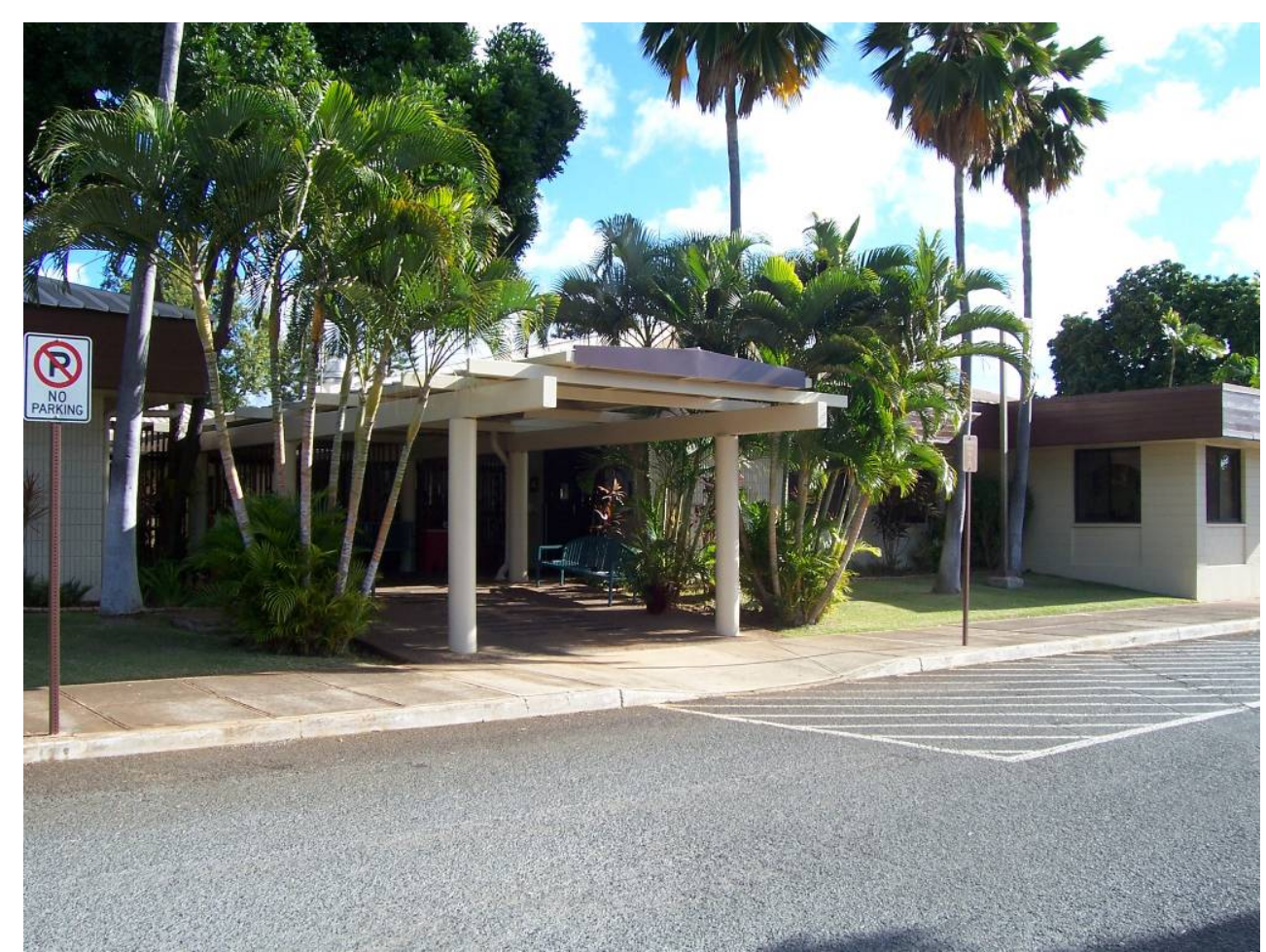




\section{Alternative Financing Results}

A FEDS analysis using alternative financing suggests replacing some of the lighting as well as replacing the electric water heater with a heat pump water heater.

Alternative financing FEDS results for building 1597:

\begin{tabular}{|c|c|c|c|c|c|c|c|c|}
\hline Bldg. Set Description & $\begin{array}{l}\text { End } \\
\text { Use }\end{array}$ & Existing Technology & Retrofit Technology & $\begin{array}{l}\text { Energy } \\
\text { Savings } \\
\text { (MMBtu/yr) }\end{array}$ & $\begin{array}{l}\text { 1st Year } \\
\text { Savings } \\
(\$ / y r)\end{array}$ & $\begin{array}{l}\text { Installed } \\
\text { Cost (\$) }\end{array}$ & $\begin{array}{l}\text { Net } \\
\text { Present } \\
\text { Value (\$) }\end{array}$ & SIR \\
\hline youth Center 1597 & $\begin{array}{l}\text { Hot } \\
\text { Water }\end{array}$ & Electric Water Heater & $\begin{array}{l}\text { Heat Pump Water Heater } \\
\text { (Com) }\end{array}$ & 42 & 2,455 & 6,854 & 7,220 & 2.1 \\
\hline youth Center 1597 & Lights & EX6: EXIT - LED & $\begin{array}{l}\text { EX12: EXIT - } \\
\text { ELECTROLUMINESCENT PANEL } \\
\text { RETRO KIT }\end{array}$ & 1 & 117 & 621 & 91 & 1.1 \\
\hline youth Center 1597 & Lights & MH40: MH 150 HE WALL & MH67: MH 150 HE WALL ELC & 1 & 310 & 1,376 & 502 & 1.4 \\
\hline
\end{tabular}




\section{Alternative Financing Energy Consumption by Fuel Type}

The modeled energy consumption for a typical year was 200,246 kwh before retrofits and 187,306 kwh after proposed retrofits are implemented. The energy use intensity goes from 53.6 MBtu/Ksf to $50.1 \mathrm{MBtu} / \mathrm{Ksf}$ after retrofits.

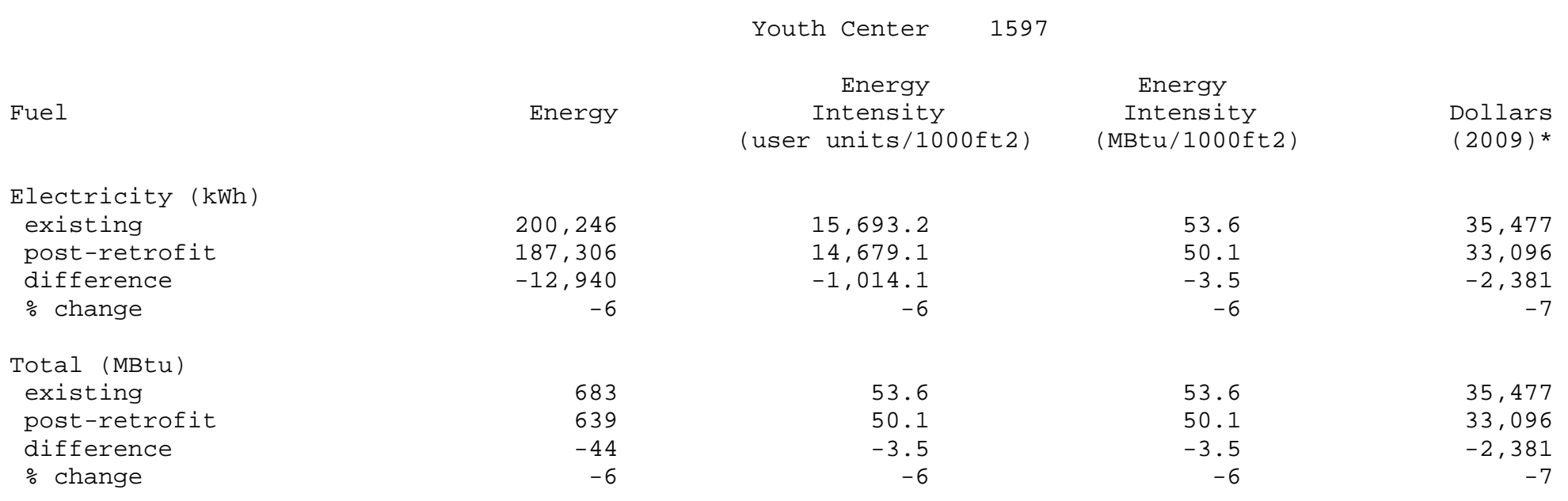

* Dollar values for electricity include both energy and demand components. 


\section{Alternative Financing Energy Consumption by End Use}

Space cooling is the largest load in the building with $84,926 \mathrm{kWh} /$ year, followed by motors and miscellaneous equipment with 36,565 $\mathrm{kWh} /$ year.

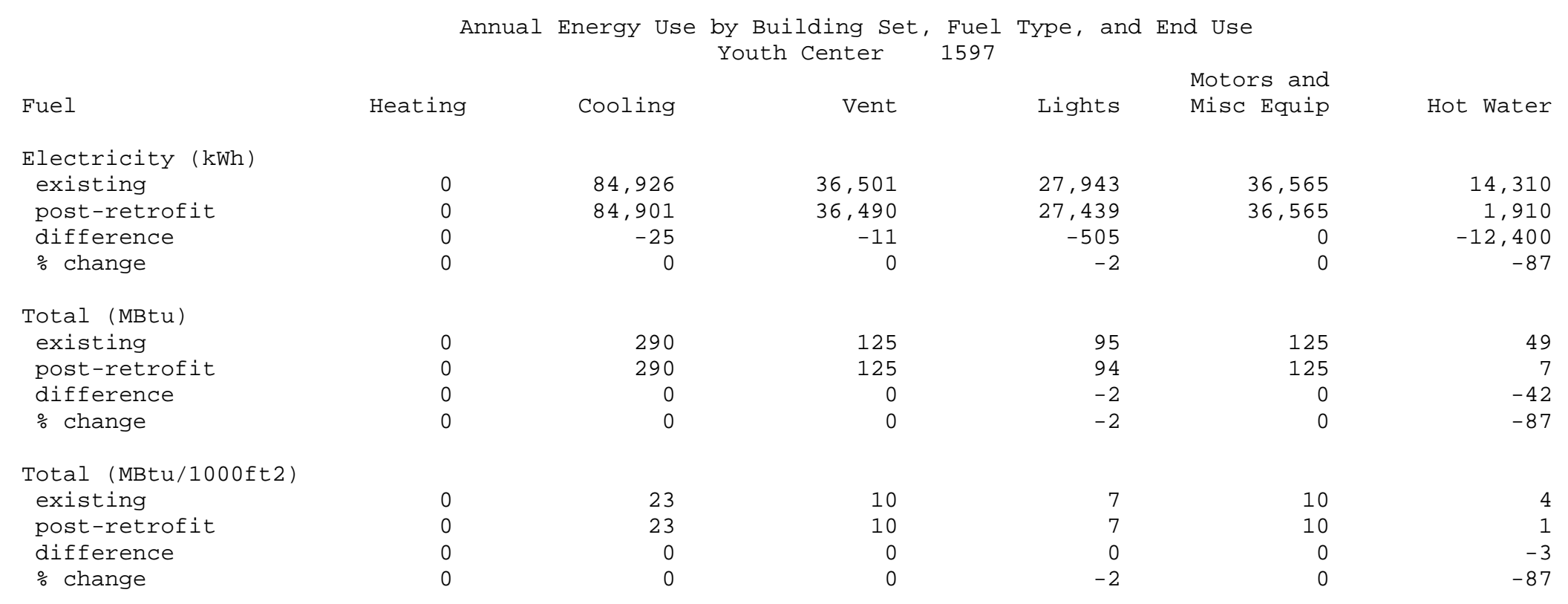




\section{Alternative Financing Emission Reduction}

The emission reductions from implemented the proposed retrofits are as follows:

Youth Center

1597

Sulfur Oxides (lb)

existing

post-retrofit

difference

1,810

1,693

$-117$

$\%$ change

$-6$

Nitrogen Oxides (lb)

existing

865

post-retrofit

809

difference

$-56$

$\%$ change

1,488

existing

post-retrofit

1,392

difference

-96
-6

$\%$ change

existing

171

post-retrofit

$-12$

difference

$-6$

Particulate Matter (lb)

existing

post-retrofit

difference

$\%$ change

Hydrocarbons (lb)

existing

post-retrofit

difference

616

$\%$ change

576

-40
-6 


\section{Building 1891 Bowling Facility}

The following information identifies the cost-effective energy- and cost-reducing retrofit projects for building 1891 identified from the FEDS modeling and analysis. Key energy and economic results are presented for each cost-effective retrofit measure.

\section{Facility Description}

1891 is a bowling facility built in 1971. The facility was once a gymnasium, but has since been converted to a bowling center.

Building 1891 is cooled by an electric package unit, has fluorescent, incandescent, and metal halide lights and some insulation in the building envelope. Building 1891 is 3,090 sf.

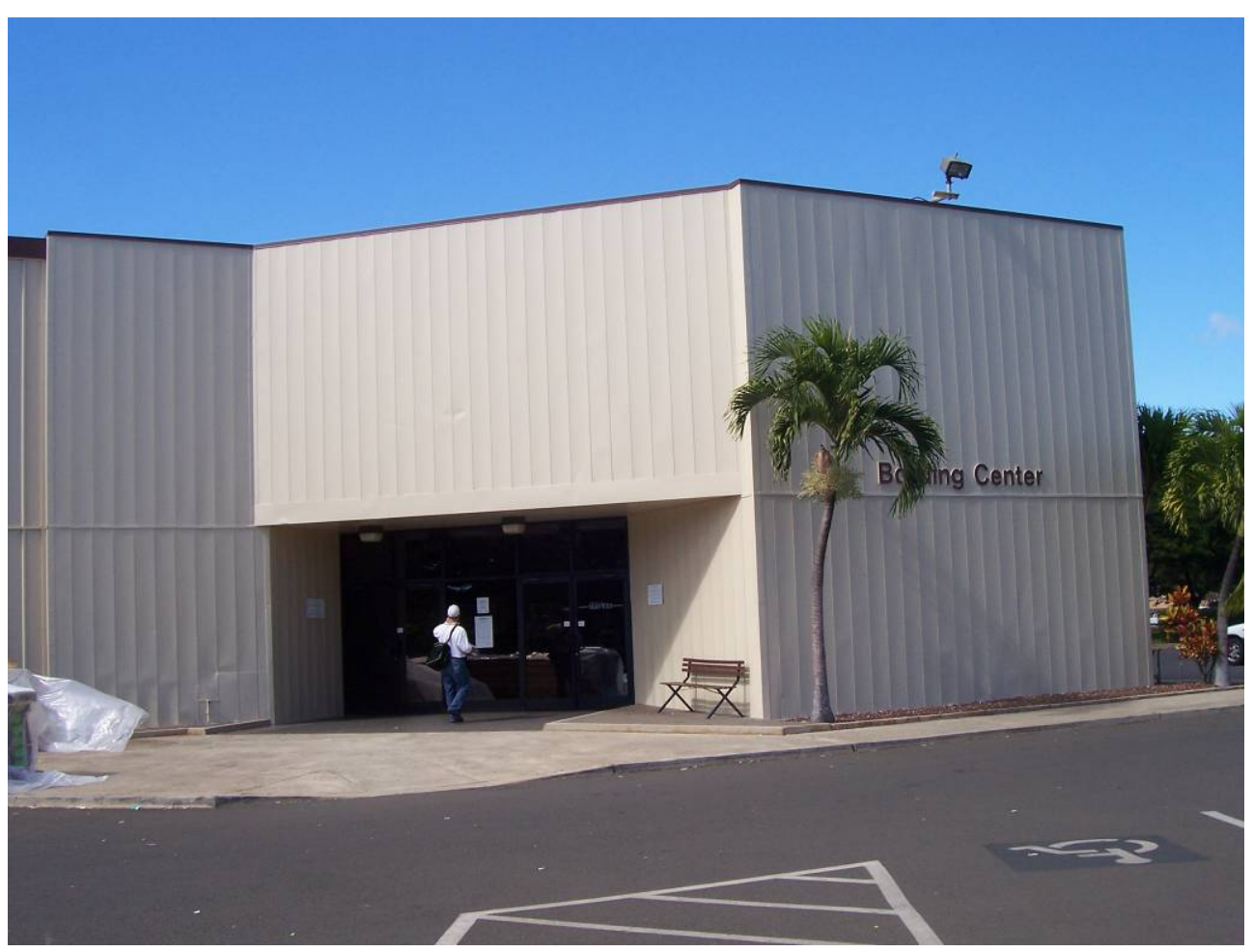




\section{Alternative Financing Results}

A FEDS analysis using alternative financing suggests replacing the electric package unit with a very high efficiency single zone package unit. FEDS also suggests delamping 4 tube T8 fixtures to 3 tube T8 fixtures as well as increasing insulation in the attic ceiling, and installing faucet aerators.

Alternative financing FEDS results for building 1891:

\begin{tabular}{|c|c|c|c|c|c|c|c|c|}
\hline Bldg. Set Description & $\begin{array}{l}\text { End } \\
\text { Use }\end{array}$ & Existing Technology & Retrofit Technology & $\begin{array}{l}\text { Energy } \\
\text { Savings } \\
\text { (MMBtu/yr) }\end{array}$ & $\begin{array}{l}\text { 1st Year } \\
\text { Savings } \\
(\$ / y r)\end{array}$ & $\begin{array}{l}\text { Installed } \\
\text { Cost (\$) }\end{array}$ & $\begin{array}{l}\text { Net } \\
\text { Present } \\
\text { Value (\$) } \\
\end{array}$ & SIR \\
\hline $\begin{array}{l}\text { misc recreation bldgs } \\
1891\end{array}$ & Cooling & Electric Package Unit $\{\mathrm{C} 1\}$ & $\begin{array}{l}\text { Single Zone Packaged AC Unit } \\
\text { (very high efficiency / small) }\end{array}$ & 119 & 10,538 & 31,186 & 31,012 & 2.0 \\
\hline $\begin{array}{l}\text { misc recreation bldgs } \\
1891\end{array}$ & $\begin{array}{l}\text { Hot } \\
\text { Water }\end{array}$ & Electric Water Heater & Faucet Aerators & 3 & 156 & 14 & 884 & 62.8 \\
\hline $\begin{array}{l}\text { misc recreation bldgs } \\
1891\end{array}$ & Lights & EX1: EXIT - INC $(2 \times 20)$ & $\begin{array}{l}\text { EX12: EXIT - } \\
\text { ELECTROLUMINESCENT PANEL } \\
\text { RETRO KIT }\end{array}$ & 7 & 456 & 311 & 2,358 & 8.6 \\
\hline $\begin{array}{l}\text { misc recreation bldgs } \\
1891\end{array}$ & Lights & FL37: FL 2X4 4F32T8 EEF2 & $\begin{array}{l}\text { FL280: FL 2X4 3F32ST8 ELC3 } \\
\text { REF }\end{array}$ & 21 & 1,206 & 4,416 & 2,550 & 1.6 \\
\hline $\begin{array}{l}\text { misc recreation bldgs } \\
1891\end{array}$ & Roof & $\begin{array}{l}\text { Roof Insulation R-Value } \\
11.00\end{array}$ & $\begin{array}{l}\text { Attic Ceiling: Increase } \\
\text { Insulation by R-13 (blow-in } \\
\text { cellulose) }\end{array}$ & 8 & 480 & 1,775 & 985 & 1.6 \\
\hline
\end{tabular}




\section{Alternative Financing Energy Consumption by Fuel Type}

The modeled energy consumption for a typical year was 158,242 kwh before retrofits and 104,256 kwh after proposed retrofits are implemented. The energy use intensity goes from 176.2 MBtu/Ksf to 116.6 MBtu/Ksf after retrofits.

\begin{tabular}{|c|c|c|c|c|}
\hline \multirow[b]{2}{*}{ Fuel } & \multicolumn{3}{|c|}{ Misc recreation bldgs 1891} & \multirow[b]{2}{*}{$\begin{array}{l}\text { Dollars } \\
(2009) \text { * }\end{array}$} \\
\hline & Energy & $\begin{array}{c}\text { Energy } \\
\text { Intensity } \\
\text { (user units/1000ft2) }\end{array}$ & $\begin{array}{c}\text { Energy } \\
\text { Intensity } \\
\text { (MBtu/1000ft2) }\end{array}$ & \\
\hline \multicolumn{5}{|c|}{ Electricity (kWh) } \\
\hline existing & 158,242 & $51,211.1$ & 174.8 & 27,976 \\
\hline post-retrofit & 101,296 & $32,782.0$ & 111.9 & 17,813 \\
\hline difference & $-56,946$ & $-18,429.1$ & -62.9 & $-10,162$ \\
\hline$\%$ change & -36 & -36 & -36 & -36 \\
\hline \multicolumn{5}{|c|}{ Other Fuels (MBtu) } \\
\hline existing & 4 & 1.4 & 1.4 & 143 \\
\hline post-retrofit & 4 & 1.4 & 1.4 & 143 \\
\hline difference & 0 & 0.0 & 0.0 & 0 \\
\hline$\%$ change & $\odot$ & 0 & 0 & $\odot$ \\
\hline \multicolumn{5}{|l|}{ Total (MBtu) } \\
\hline existing & 545 & 176.2 & 176.2 & 28,119 \\
\hline post-retrofit & 350 & 113.3 & 113.3 & 17,957 \\
\hline difference & -194 & -62.9 & -62.9 & $-10,162$ \\
\hline$\%$ change & -36 & -36 & -36 & -36 \\
\hline
\end{tabular}

* Dollar values for electricity include both energy and demand components. 


\section{Alternative Financing Energy Consumption by End Use}

Space cooling is the largest load in the building with $82,546 \mathrm{kWh} /$ year, followed by lighting with $38,734 \mathrm{kWh} /$ year.

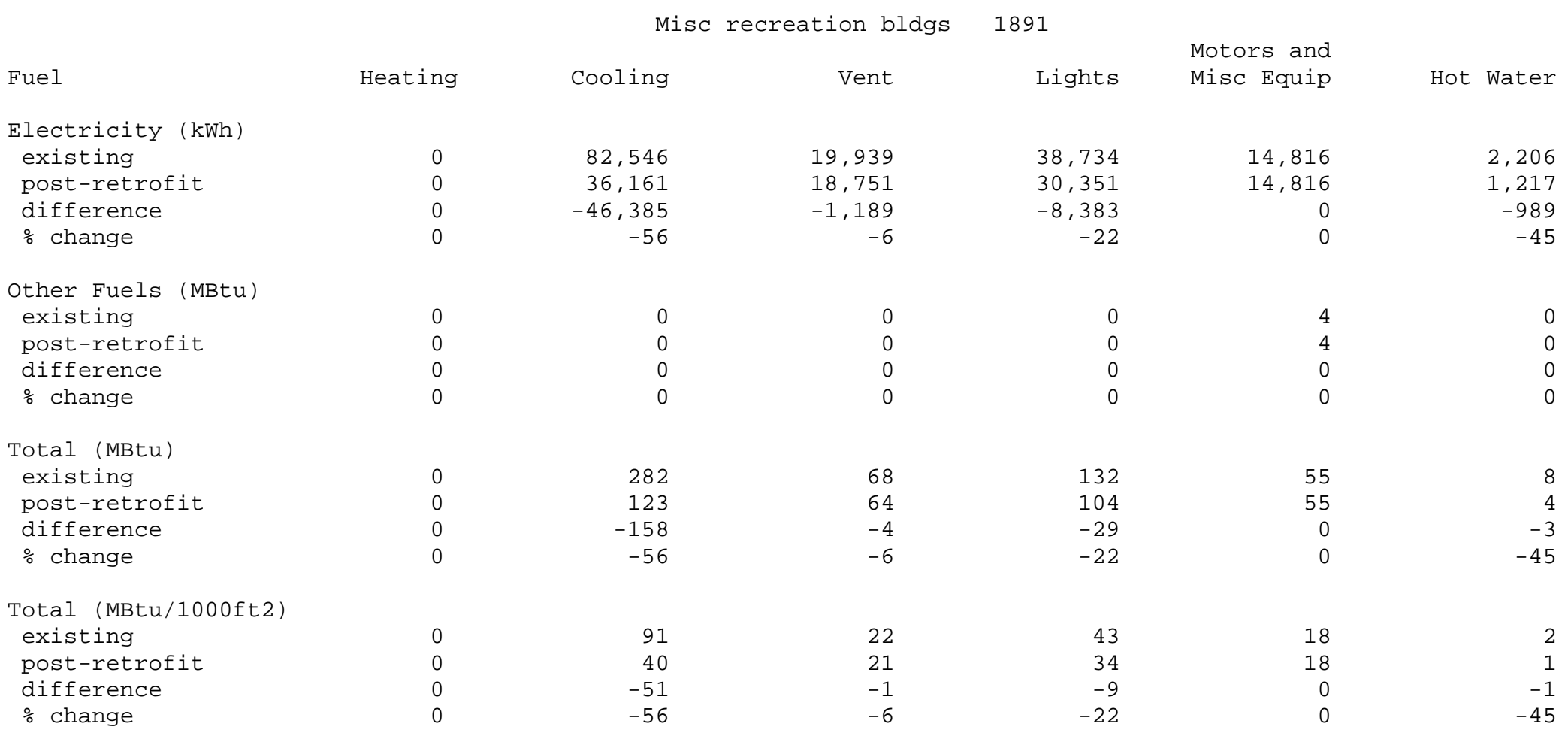




\section{Alternative Financing Emission Reduction}

The emission reductions from implemented the proposed retrofits are as follows:

Sulfur Oxides (lb)

existing

post-retrofit

1,432

difference

917

$\%$ change

-515
-36

Nitrogen 0xides (lb)

existing

difference

$-246$

$\%$ change

Carbon Monoxide (lb)

existing

post-retrofit

1,179

difference

756

$\%$ change

-423
-36

Carbon Dioxide (tons)

existing

post-retrofit

difference

$\%$ change

Particulate Matter (lb)

existing

post-retrofit

difference

$\%$ change

$-36$

Hydrocarbons (lb)

existing

post-retrofit

difference

$\%$ change

-175
-36 


\section{Building 1750 Religious Education Facility}

The following information identifies the cost-effective energy- and cost-reducing retrofit projects for building 1750 identified from the FEDS modeling and analysis. Key energy and economic results are presented for each cost-effective retrofit measure.

\section{Facility Description 1750}

1750 is a religious education facility built in 1977. Building 1750 is conditioned by an electric package unit. The building has incandescent, fluorescent, and metal halide lights and has little to no insulation in the building envelope. Building 1750 is 7,296 sf.

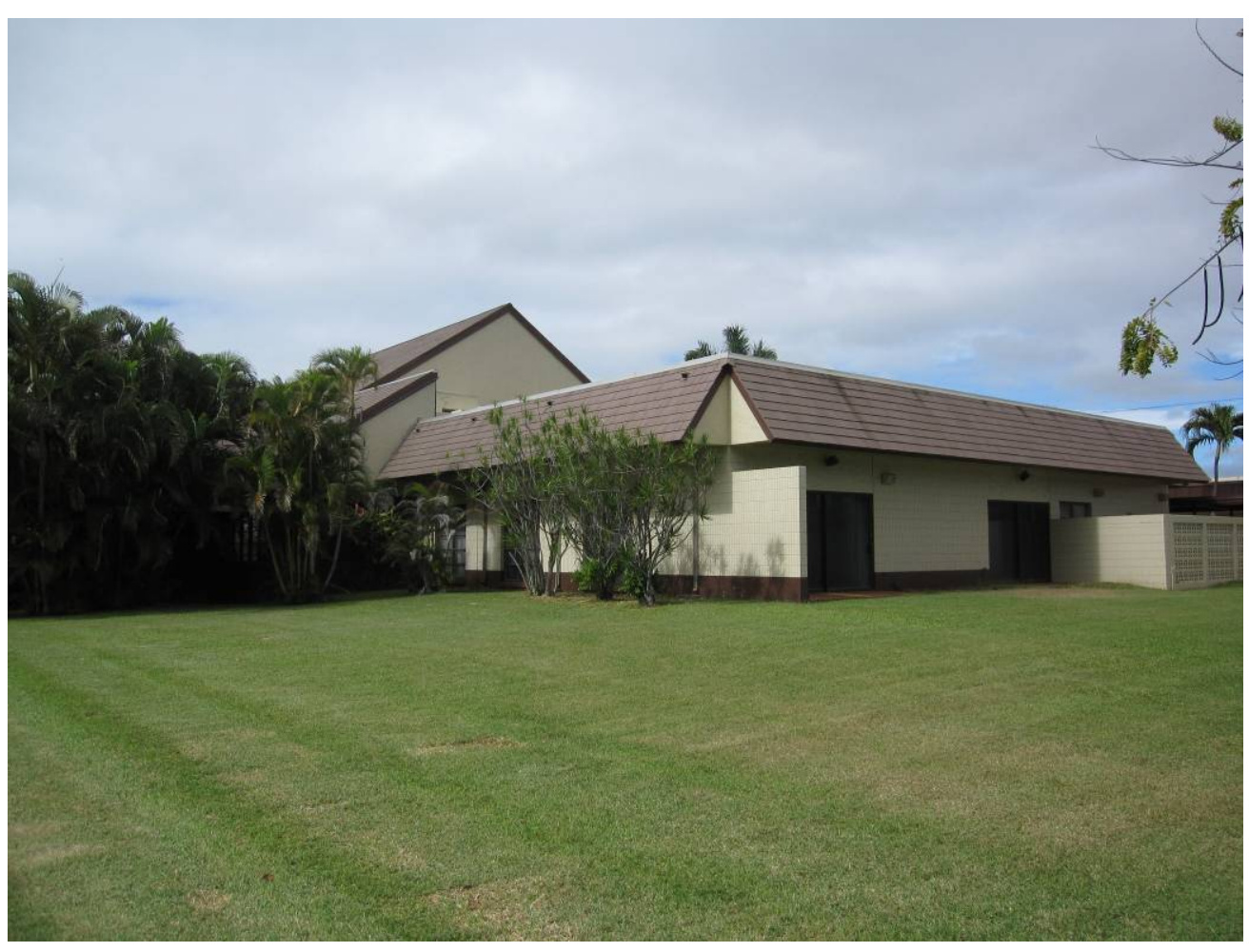




\section{Alternative Financing Results}

A FEDS analysis using alternative financing suggests replacing the lighting in the building, increasing the insulation in the roof and increasing the insulation on the hot water tank.

Alternative financing FEDS results for building 1750:

\begin{tabular}{|c|c|c|c|c|c|c|c|c|}
\hline Bldg. Set Description & $\begin{array}{l}\text { End } \\
\text { Use }\end{array}$ & Existing Technology & Retrofit Technology & $\begin{array}{l}\text { Energy } \\
\text { Savings } \\
\text { (MMBtu/yr) }\end{array}$ & $\begin{array}{l}\text { 1st Year } \\
\text { Savings } \\
(\$ / y r)\end{array}$ & $\begin{array}{l}\text { Installed } \\
\text { Cost (\$) }\end{array}$ & $\begin{array}{l}\text { Net } \\
\text { Present } \\
\text { Value (\$) }\end{array}$ & SIR \\
\hline Religious facilities 1750 & $\begin{array}{l}\text { Hot } \\
\text { Water }\end{array}$ & Distillate Oil Water Heater & Wrap Tank with Insulation & 1 & 30 & 53 & 96 & 2.1 \\
\hline Religious facilities 1750 & Lights & IN8: INC 75 CEIL & CF5: CFL 18 INTEGRAL UNIT ELC & 23 & 1,625 & 502 & 8,983 & 18.9 \\
\hline Religious facilities 1750 & Lights & IN11: INC 100 CEIL & CF9: CFL 26 INTEGRAL UNIT ELC & 3 & 228 & 58 & 1,271 & 22.9 \\
\hline Religious facilities 1750 & Lights & EX1: EXIT - INC $(2 \times 20)$ & $\begin{array}{l}\text { EX12: EXIT - } \\
\text { ELECTROLUMINESCENT PANEL } \\
\text { RETRO KIT }\end{array}$ & 13 & 866 & 621 & 4,449 & 8.2 \\
\hline Religious facilities 1750 & Lights & FL4: FL 1X4 2F40T12 STD2 & FL52: FL 1X4 2F32T8 ELC2 & 16 & 1,095 & 3,949 & 2,511 & 1.6 \\
\hline Religious facilities 1750 & Roof & Roof Insulation R-Value 0.00 & $\begin{array}{l}\text { Insulate Built-up Roof Surface } \\
\text { (R-10) and Re-Roof }\end{array}$ & 89 & 5,915 & 31,383 & 2,573 & 1.1 \\
\hline
\end{tabular}




\section{Alternative Financing Energy Consumption by Fuel Type}

The modeled energy consumption for a typical year was 113,294 kwh before retrofits and 91,403 kwh after proposed retrofits are implemented. The energy use intensity goes from 63.6 MBtu/Ksf to 43.9 MBtu/Ksf after retrofits.

\begin{tabular}{|c|c|c|c|c|}
\hline \multirow[b]{2}{*}{ Fuel } & \multirow[b]{2}{*}{ Energy } & \multicolumn{2}{|l|}{ ligious facilities } & \multirow[b]{2}{*}{$\begin{array}{l}\text { Dollars } \\
(2009)\end{array}$} \\
\hline & & $\begin{array}{c}\text { Energy } \\
\text { Intensity } \\
\text { (user units/1000ft2) }\end{array}$ & $\begin{array}{l}\text { Energy } \\
\text { Intensity } \\
\text { (MBtu/1000ft2) }\end{array}$ & \\
\hline \multicolumn{5}{|c|}{ Electricity (kWh) } \\
\hline existing & 133,294 & $18,269.4$ & 62.4 & 23,615 \\
\hline post-retrofit & 91,403 & $12,527.9$ & 42.8 & 16,150 \\
\hline difference & $-41,891$ & $-5,741.6$ & -19.6 & $-7,465$ \\
\hline$\%$ change & -31 & -31 & -31 & -32 \\
\hline \multicolumn{5}{|c|}{ Distillate Oil (gal) } \\
\hline existing & 64 & 8.7 & 1.2 & 324 \\
\hline post-retrofit & 58 & 7.9 & 1.1 & 294 \\
\hline difference & -6 & -0.8 & -0.1 & -30 \\
\hline$\%$ change & -9 & -9 & -9 & -9 \\
\hline \multicolumn{5}{|l|}{ Total (MBtu) } \\
\hline existing & 464 & 63.6 & 63.6 & 23,939 \\
\hline post-retrofit & 320 & 43.9 & 43.9 & 16,445 \\
\hline difference & -144 & -19.7 & -19.7 & $-7,494$ \\
\hline$\%$ change & -31 & -31 & -31 & -31 \\
\hline
\end{tabular}

* Dollar values for electricity include both energy and demand components. 


\section{Alternative Financing Energy Consumption by End Use}

Space cooling is the largest load in the building with $60,705 \mathrm{kWh} /$ year, followed by lighting with 31,784 kWh/year.

\begin{tabular}{|c|c|c|c|c|c|c|}
\hline Fuel & Heating & $\begin{array}{r}\text { Energy Us } \\
\text { R }\end{array}$ & $\begin{array}{l}\text { ding Se } \\
\text { facilit } \\
\text { Vent }\end{array}$ & $\begin{array}{l}\text { Type, an } \\
50\end{array}$ & $\begin{array}{l}\text { Id Use } \\
\text { Motors and } \\
\text { Misc Equip }\end{array}$ & Hot water \\
\hline \multicolumn{7}{|c|}{ Electricity (kWh) } \\
\hline existing & $\odot$ & 60,705 & 29,785 & 31,784 & 11,019 & $\odot$ \\
\hline post-retrofit & $\odot$ & 32,588 & 29,173 & 18,623 & 11,019 & $\odot$ \\
\hline difference & $\odot$ & $-28,117$ & -613 & $-13,161$ & 0 & $\odot$ \\
\hline$\%$ change & $\odot$ & -46 & -2 & -41 & $\odot$ & $\odot$ \\
\hline \multicolumn{7}{|c|}{ Distillate Oil (gal) } \\
\hline existing & $\odot$ & $\odot$ & $\odot$ & $\odot$ & $\odot$ & 64 \\
\hline post-retrofit & $\odot$ & $\odot$ & $\odot$ & $\odot$ & $\odot$ & 58 \\
\hline difference & $\odot$ & $\odot$ & $\odot$ & $\odot$ & $\odot$ & -6 \\
\hline$\%$ change & $\odot$ & $\odot$ & $\odot$ & $\odot$ & $\odot$ & -9 \\
\hline \multicolumn{7}{|l|}{ Total (MBtu) } \\
\hline existing & $\odot$ & 207 & 102 & 108 & 38 & 9 \\
\hline post-retrofit & $\odot$ & 111 & 100 & 64 & 38 & 8 \\
\hline difference & $\odot$ & -96 & -2 & -45 & 0 & -1 \\
\hline$\%$ change & $\odot$ & -46 & -2 & -41 & 0 & -9 \\
\hline \multicolumn{7}{|c|}{ Total (MBtu/100๑ft2) } \\
\hline existing & $\odot$ & 28 & 14 & 15 & 5 & 1 \\
\hline post-retrofit & $\odot$ & 15 & 14 & 9 & 5 & 1 \\
\hline difference & $\odot$ & -13 & $\odot$ & -6 & $\odot$ & $\odot$ \\
\hline$\%$ change & $\odot$ & -46 & -2 & -41 & $\odot$ & -9 \\
\hline
\end{tabular}




\section{Alternative Financing Emission Reduction}

The emission reductions from implemented the proposed retrofits are as follows:

Sulfur Oxides (lb)

existing

post-retrofit

1,209

difference

830

$\%$ change

-379
-31

Nitrogen 0xides (lb)

existing

post-retrofit

difference

398

$\%$ change

$-181$

-181
-31

Carbon Monoxide (lb)

existing

post-retrofit

difference

997

$\%$ change

686

$-312$

$-31$

Carbon Dioxide (tons)

existing

post-retrofit

123

difference

84

$\%$ change

-38
-31

Particulate Matter (lb)

existing

post-retrofit

difference

$\%$ change

Hydrocarbons (lb)

existing

post-retrofit 283

difference $\quad-129$

$\%$ change

$-31$ 


\section{Building 1120 Gymnasium}

The following information identifies the cost-effective energy- and cost-reducing retrofit projects for building 1120 identified from the FEDS modeling and analysis. Key energy and economic results are presented for each cost-effective retrofit measure.

\section{Facility Description 1120}

1120 is the main gymnasium built in 1949. Building 1120 is conditioned by an electric air cooled chiller. It has fluorescent, metal halide and high pressure sodium lights as well as little to no insulation in its building envelope. Building 1120 is 46,719 sf.

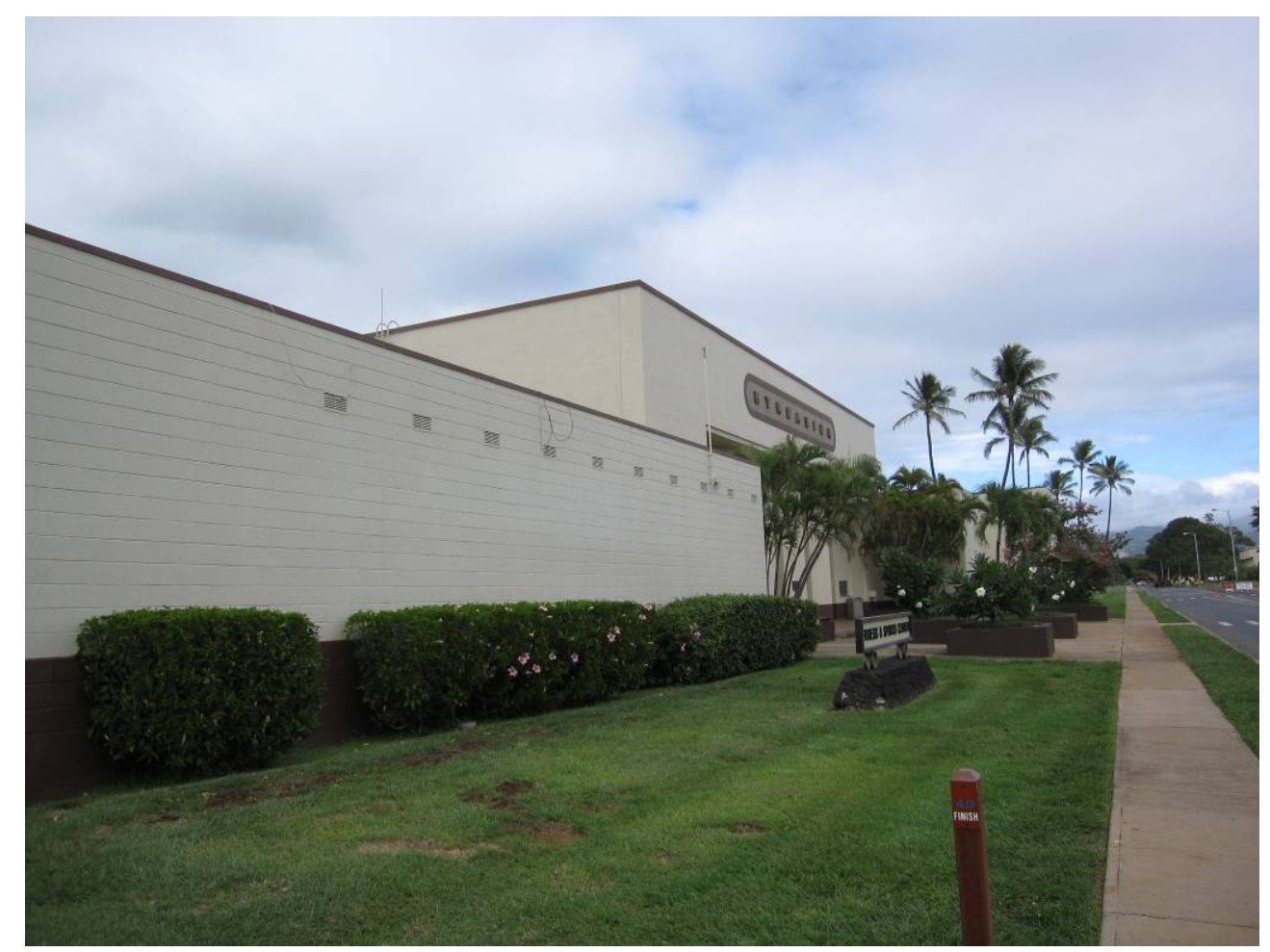




\section{Alternative Financing Results}

A FEDS analysis using alternative financing suggests replacing the air cooled chiller with a very high efficiency water cooled chiller, replacing T12 lighting with T8 and Super T8 lighting, replacing the EXIT lighting and insulating the hot water tank.

Alternative financing FEDS results for building 1750:

\begin{tabular}{|c|c|c|c|c|c|c|c|c|}
\hline Bldg. Set Description & $\begin{array}{l}\text { End } \\
\text { Use }\end{array}$ & Existing Technology & Retrofit Technology & $\begin{array}{l}\text { Energy } \\
\text { Savings } \\
\text { (MMBtu/yr) }\end{array}$ & $\begin{array}{l}\text { 1st Year } \\
\text { Savings } \\
(\$ / y r)\end{array}$ & $\begin{array}{l}\text { Installed } \\
\text { Cost (\$) }\end{array}$ & $\begin{array}{l}\text { Net } \\
\text { Present } \\
\text { Value (\$) } \\
\end{array}$ & SIR \\
\hline gymnasium 1120 & Cooling & $\begin{array}{l}\text { Electric Air-Cooled Chiller } \\
\{\mathrm{C} 1\}\end{array}$ & $\begin{array}{l}\text { Water-Cooled Reciprocating } \\
\text { Electric Chiller (very high } \\
\text { efficiency) and Cooling Tower }\end{array}$ & 545 & 28,385 & 116,055 & 46,568 & 1.4 \\
\hline gymnasium 1120 & $\begin{array}{l}\text { Hot } \\
\text { Water }\end{array}$ & Distillate Oil Water Heater & $\begin{array}{l}\text { Wrap Tank with Insulation, } \\
\text { Aerators }\end{array}$ & 21 & 783 & 296 & 4,510 & 16.2 \\
\hline gymnasium 1120 & Lights & EX1: EXIT - INC $(2 \times 20)$ & $\begin{array}{l}\text { EX12: EXIT - } \\
\text { ELECTROLUMINESCENT PANEL } \\
\text { RETRO KIT }\end{array}$ & 13 & 884 & 621 & 4,551 & 8.3 \\
\hline gymnasium 1120 & Lights & FL1: FL 2X4 4F40T12 STD2 & $\begin{array}{l}\text { FL280: FL 2X4 3F32ST8 ELC3 } \\
\text { REF (FIX REPL) }\end{array}$ & 120 & 7,159 & 11,663 & 29,900 & 3.6 \\
\hline gymnasium 1120 & Lights & FL3: FL 2X4 2F40T12 STD2 & FL51: FL 2X4 2F32T8 ELC2 & 28 & 2,059 & 5,820 & 6,258 & 2.1 \\
\hline
\end{tabular}




\section{Alternative Financing Energy Consumption by Fuel Type}

The modeled energy consumption for a typical year was 1,054,786 kwh before retrofits and 843,837 kwh after proposed retrofits are implemented. The energy use intensity goes from 78.1 MBtu/Ksf to 62.2 MBtu/Ksf after retrofits.

\begin{tabular}{|c|c|c|c|c|}
\hline \multirow[b]{2}{*}{ Fuel } & \multirow[b]{2}{*}{ Energy } & Gymnasium & \multirow[b]{2}{*}{$\begin{array}{c}\text { Energy } \\
\text { Intensity } \\
\text { (MBtu/1000ft2) }\end{array}$} & \multirow[b]{2}{*}{$\begin{array}{l}\text { Dollars } \\
(2009) \text { * }\end{array}$} \\
\hline & & $\begin{array}{c}\text { Energy } \\
\text { Intensity } \\
\text { (user units/1000ft2) }\end{array}$ & & \\
\hline \multicolumn{5}{|c|}{ Electricity (kWh) } \\
\hline existing & $1,054,786$ & $22,577.2$ & 77.1 & 186,872 \\
\hline post-retrofit & 843,837 & $18,062.0$ & 61.6 & 149,101 \\
\hline difference & $-210,949$ & $-4,515.3$ & $-15 \cdot 4$ & $-37,771$ \\
\hline$\%$ change & -20 & -20 & -20 & -20 \\
\hline \multicolumn{5}{|c|}{ Distillate Oil (gal) } \\
\hline existing & 356 & 7.6 & 1.1 & 1,814 \\
\hline post-retrofit & 202 & 4.3 & 0.6 & 1,031 \\
\hline difference & -154 & -3.3 & -0.5 & -783 \\
\hline$\%$ change & -43 & -43 & -43 & -43 \\
\hline \multicolumn{5}{|l|}{ Total (MBtu) } \\
\hline existing & 3,649 & 78.1 & 78.1 & 188,686 \\
\hline post-retrofit & 2,908 & 62.2 & 62.2 & 150,132 \\
\hline difference & -741 & -15.9 & -15.9 & $-38,554$ \\
\hline$\%$ change & -20 & -20 & -20 & -20 \\
\hline
\end{tabular}

* Dollar values for electricity include both energy and demand components. 


\section{Alternative Financing Energy Consumption by End Use}

Space cooling is the largest load in the building with $445,860 \mathrm{kWh} /$ year, followed by motors and miscellaneous equipment with $256,750 \mathrm{kWh} /$ year.

Annual Energy Use by Building Set, Fuel Type, and End Use

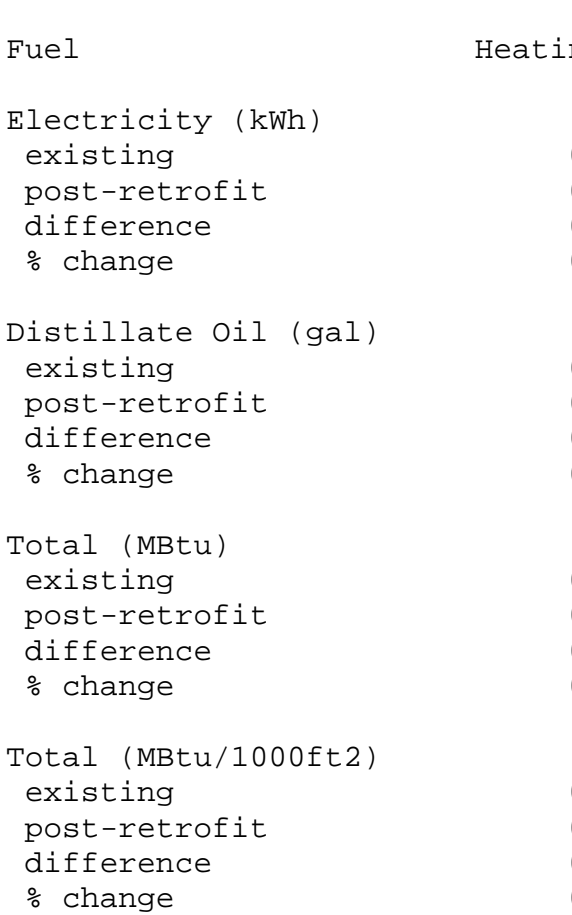

\begin{tabular}{|c|c|c|c|c|c|}
\hline$n g$ & Cooling & Vent & Lights & $\begin{array}{l}\text { Motors and } \\
\text { Misc Equip }\end{array}$ & Hot water \\
\hline 0 & 445,860 & 141,105 & 211,070 & 256,750 & $\odot$ \\
\hline 0 & 287,173 & 124,835 & 175,079 & 256,750 & 0 \\
\hline 0 & $-158,688$ & $-16,270$ & $-35,991$ & $\odot$ & 0 \\
\hline$\odot$ & -36 & -12 & -17 & $\odot$ & 0 \\
\hline$\odot$ & $\odot$ & $\odot$ & $\odot$ & $\odot$ & 356 \\
\hline$\odot$ & $\odot$ & $\odot$ & $\odot$ & $\odot$ & $2 \odot 2$ \\
\hline$\odot$ & $\odot$ & $\odot$ & $\odot$ & $\odot$ & -154 \\
\hline$\odot$ & $\odot$ & $\odot$ & $\odot$ & $\odot$ & -43 \\
\hline$\odot$ & 1,522 & 482 & 720 & 876 & 49 \\
\hline$\odot$ & 980 & 426 & 598 & 876 & 28 \\
\hline$\odot$ & -542 & -56 & -123 & $\odot$ & -21 \\
\hline$\odot$ & -36 & -12 & -17 & $\odot$ & -43 \\
\hline$\odot$ & 33 & 10 & 15 & 19 & 1 \\
\hline$\odot$ & 21 & 9 & 13 & 19 & 1 \\
\hline$\odot$ & -12 & -1 & -3 & $\odot$ & 0 \\
\hline$\odot$ & -36 & -12 & -17 & $\odot$ & -43 \\
\hline
\end{tabular}

Motors and

Misc Equip

Hot Water 


\section{Alternative Financing Emission Reduction}

The emission reductions from implemented the proposed retrofits are as follows:

Gymnasium

1120

Sulfur Oxides (lb)

existing

post-retrofit

difference

7,642

$\%$ change

$-1,918$

$-20$

Nitrogen Oxides (lb)

existing

post-retrofit

4,573

3,655

difference

$-919$

$\%$ change

7,877

Carbon Monoxide (lb)

existing

post-retrofit

6,292

difference

$-1,584$
-20

$\%$ change

Carbon Dioxide (tons)

existing

post-retrofit

970

difference

775

$\%$ change

Particulate Matter (lb)

existing

190

post-retrofit

151

difference

$-38$

$\%$ change

3,256

Hydrocarbons ( $1 b$ )

existing

post-retrofit

difference

, 602

$\%$ change

$-20$ 


\section{Building 2003 Vehicle Maintenance Facility}

The following information identifies the cost-effective energy- and cost-reducing retrofit projects for building 2003 identified from the FEDS modeling and analysis. Key energy and economic results are presented for each cost-effective retrofit measure.

\section{Facility Description 2003}

2003 is a vehicle maintenance administration facility built in 1994. Building 2003 is conditioned by an electric package unit, is lit by 32 watt fluorescent T8's and has little to no insulation in its building envelope. Building 2003 is 6,848 sf.

\section{Alternative Financing Results}

A FEDS analysis using alternative financing suggests replacing the fluorescent lighting, the EXIT lighting and increasing the insulation in the roof on the interior surface.

Alternative financing FEDS results for building 2003:

\begin{tabular}{|c|c|c|c|c|c|c|c|c|}
\hline Bldg. Set Description & $\begin{array}{l}\text { End } \\
\text { Use }\end{array}$ & Existing Technology & Retrofit Technology & $\begin{array}{l}\text { Energy } \\
\text { Savings } \\
\text { (MMBtu/yr) }\end{array}$ & $\begin{array}{l}\text { 1st Year } \\
\text { Savings } \\
(\$ / y r)\end{array}$ & $\begin{array}{l}\text { Installed } \\
\text { Cost (\$) }\end{array}$ & $\begin{array}{l}\text { Net } \\
\text { Present } \\
\text { Value (\$) }\end{array}$ & SIR \\
\hline Small 1990's admin & Lights & EX6: EXIT - LED & $\begin{array}{l}\text { EX12: EXIT - } \\
\text { ELECTROLUMINESCENT PANEL } \\
\text { RETRO KIT }\end{array}$ & 1 & 123 & 621 & 122 & 1.2 \\
\hline Small 1990's admin & Lights & FL41: FL 1X4 1F32T8 EEF1 & $\begin{array}{l}\text { FL302: FL 1X4 1F25ST8 ELC1 } \\
\text { REF }\end{array}$ & 4 & 411 & 2,310 & 141 & 1.1 \\
\hline Small 1990's admin & Lights & FL39: FL 2X4 2F32T8 EEF2 & $\begin{array}{l}\text { FL303: FL 2X4 2F25ST8 ELC2 } \\
\text { REF }\end{array}$ & 98 & 5,828 & 14,843 & 19,000 & 2.3 \\
\hline Small 1990's admin & Roof & Roof Insulation R-Value 0.00 & $\begin{array}{l}\text { Add Insulation to Interior } \\
\text { Surface of Metal Roof: } 4 \\
\text { inches Fiberglass }\end{array}$ & 299 & 16,487 & 23,407 & 71,229 & 4.0 \\
\hline
\end{tabular}




\section{Alternative Financing Energy Consumption by Fuel Type}

The modeled energy consumption for a typical year was 234,145 kwh before retrofits and 121,374 kwh after proposed retrofits are implemented. The energy use intensity goes from 116.7 MBtu/Ksf to $60.5 \mathrm{MBtu} / \mathrm{Ksf}$ after retrofits.

Small 1990 's admin 2003

Fuel

Electricity $(\mathrm{kWh})$

existing

post-retrofit

difference

$\%$ change

Total (MBtu)

existing

post-retrofit

difference

$\%$ change
Energy

234,145

121,374

$-112,771$

$-48$

799
414
-385

Energy
Intensity
(user units/10

$34,191.8$
$17,724.0$
$-16,467.8$
-48

116.7

60.5

$-56.2$

$-48$
Energy

Intensity

(MBtu/1000ft2)

Dollars

(2009) *

41,483

21,446

$-20,037$

$-56.2$

$-48$

116.7

60.5

$-56.2$

$-48$
41,483

21,446

$-20,037$

* Dollar values for electricity include both energy and demand components. 


\section{Alternative Financing Energy Consumption by End Use}

Space cooling is the largest load in the building with $91,143 \mathrm{kWh} /$ year, followed by ventilation with $56,887 \mathrm{kWh} / \mathrm{year}$.

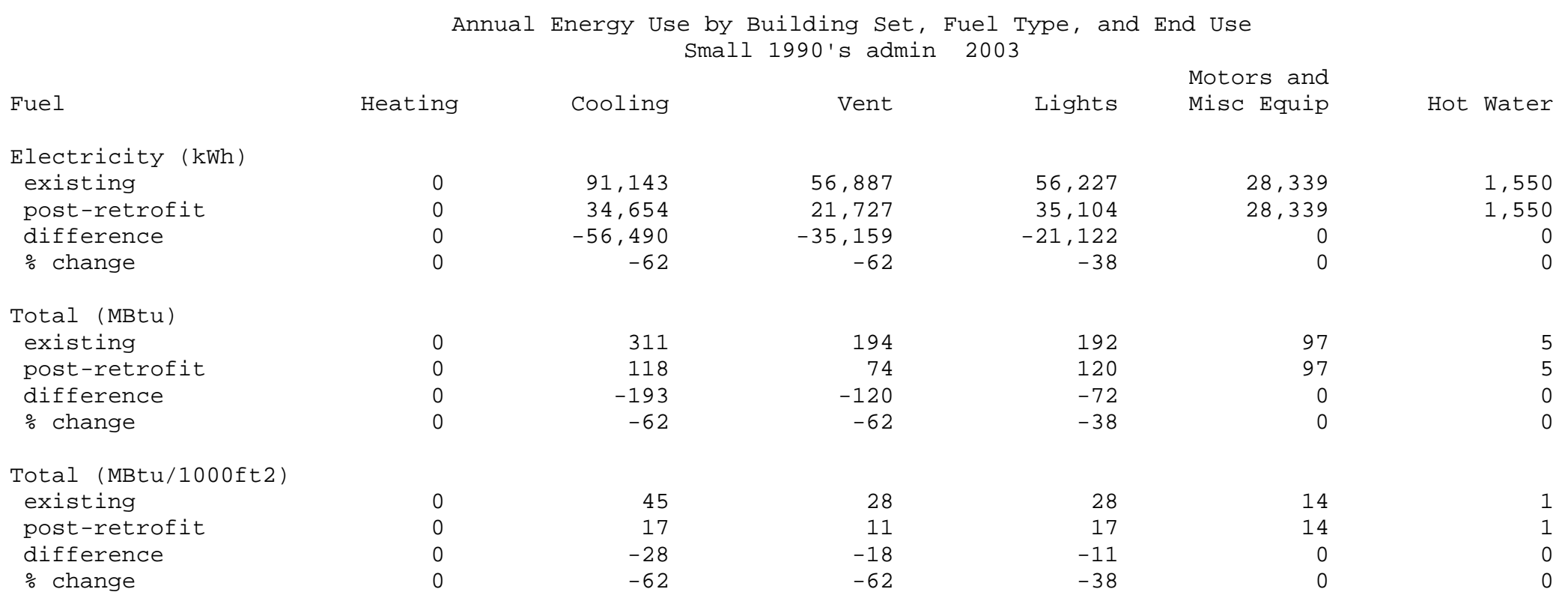




\section{Alternative Financing Emission Reduction}

The emission reductions from implemented the proposed retrofits are as follows:

Small 1990's admin 2003

Sulfur Oxides (lb)

existing

post-retrofit

difference

2,117

1,097

$-1,019$

$\%$ change

$-48$

Nitrogen 0xides (lb)

existing

post-retrofit

1, 011

524

difference

$-487$

$\%$ change

$-48$

Carbon Monoxide (lb)

existing

post-retrofit

difference

1,740

902

$\%$ change

$-48$

Carbon Dioxide (tons)

existing

post-retrofit

214

difference

111

$\%$ change

$-103$

Particulate Matter (lb)

existing

post-retrofit

difference

$\%$ change

$-48$

Hydrocarbons (lb)

existing

post-retrofit

post-retrofit

373

$\%$ change

$-347$

$-48$ 


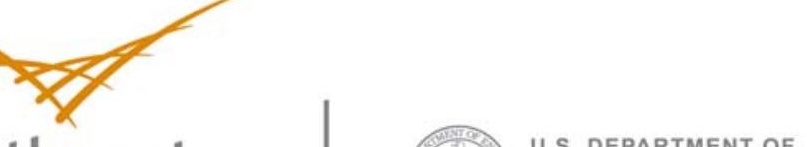




\section{Appendix E}

\section{Conversion to Water-Cooled Chillers for Building Space Cooling}

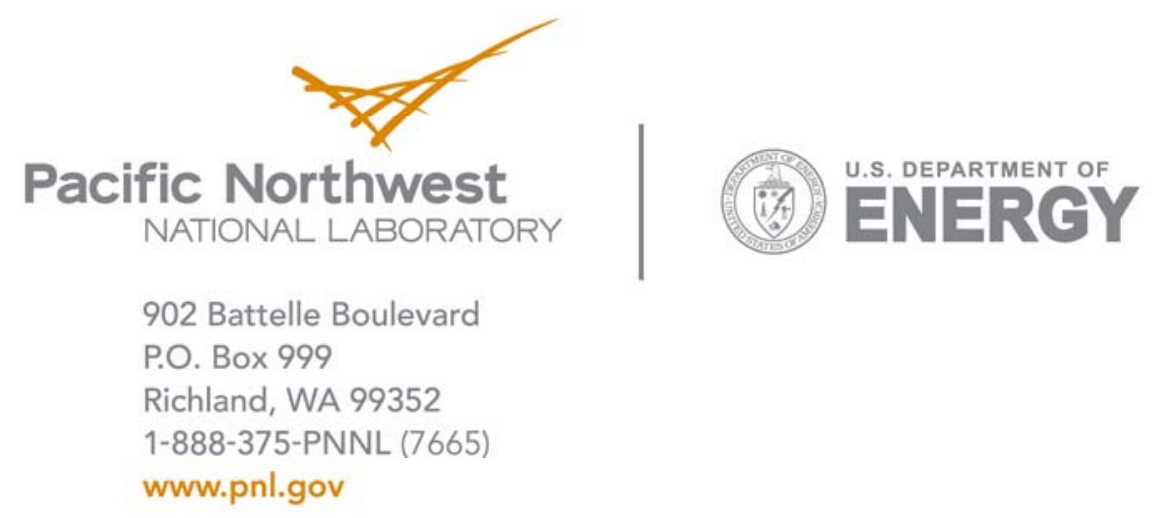




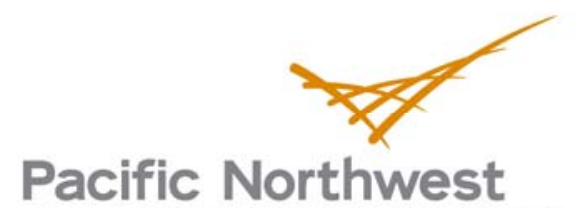

NATIONAL LABORATORY

902 Battelle Boulevard

P.O. Box 999

Richland, WA 99352

1-888-375-PNNL (7665)

www.pnl.gov
U.S. DEPARTMENT OF ENERGY 


\section{Appendix E Conversion to Water-Cooled Chillers for Building Space Cooling}

Water-cooled condensing of cooling equipment refrigerant results in a significant improvement in efficiency compared to air-cooled condensing. This advantage stems from two factors. Condenser water from an evaporative cooling tower is generally cooler than ambient air (except when the relative humidity is very high), and water is a more effective heat transfer fluid than air. The two factors work together to lower the refrigerant condensing temperature, hence improving both theoretical and actual refrigeration cycle efficiency. Combining cooling loads met by multiple smaller cooling units into fewer central units allows additional efficiency gains by using centrifugal compressors, a more efficient technology than alternative compressor types commonly used in smaller cooling equipment. These advantages do come at a price, however. Condensing refrigerant with water requires additional costs associated with a cooling tower, condenser water pumps and piping, and a shell to enclose the water as it passes by the condenser tubing. The condenser pump also represents an additional power consuming device that an air-cooled unit does not have. Finally, the distribution of centrally chilled water incurs pumping and piping costs and pumping energy not required by distributed direct expansion coolers (e.g., window air conditioner [AC] and packaged rooftop AC).

For the reasons noted above, water-cooled chillers offer significant performance advantages over air-cooled equipment that must be weighed against their additional capital costs. During the last few decades, space cooling has become much more common in Hawaiian military facilities because internal heating loads (e.g., personal computers and other office equipment) have increased, building designs have become less suitable for natural ventilation, and occupants expect a more comfortable working environment. The paragraphs that follow document the expected costs and energy savings associated with example conversions to water-cooled chillers at Hickam, Pearl, and Smith. Many other similar conversions are possible at these three facilities, but additional analysis was not possible with the assessment resources available. The installations are encouraged to consider additional opportunities for using water-cooled chillers where the economics are justified.

\section{Hickam AFB}

Buildings 2130, 2131, and 2133 are currently served by a small central cooling plant comprised of two air-cooled chillers. The proposed retrofit would replace the existing air-cooled chillers with two water-cooled chillers, a cooling tower, and condenser water pumps and piping. The existing chilled water pumps and piping would not change and the electrical service to the central plant should be adequate for the retrofit.

The peak and annual building cooling loads were estimated with the FEDS model, and the performance of the existing chillers was estimated from manufacturer's specifications for the two units. From this information, the annual $\mathrm{kWh}$ and peak $\mathrm{kW}$ electrical loads 
were calculated and then combined with Hickam's electricity rates to calculate the current annual electricity costs. The existing system performance and electricity cost figures are presented in Table E1.

Although the FEDS model estimates a peak of only 61 tons for the three buildings, two 40-ton water-cooled chillers were assumed for the retrofit to match the existing nameplate capacity of the two air-cooled chillers. In this size range, the water-cooled chillers were assumed to use a rotary screw compressor rated at $0.73 \mathrm{~kW} / \mathrm{ton}$. In addition, the condenser water pump and cooling tower fan would be expected to consume $0.12 \mathrm{~kW} /$ ton for a total cooling plant performance of $0.85 \mathrm{~kW} /$ ton. The annual electricity bill for the water-cooled system was calculated to be $\$ 35,360$ based on these assumptions, resulting in an annual savings of about $\$ 15,000$ and a peak electric load reduction of $22 \mathrm{~kW}$.

Table E 1. Hickam Buildings 2130, 2131, 2133 Existing System Performance and Electricity Cost

\begin{tabular}{|c|c|c|c|c|c|c|c|}
\hline & $\begin{array}{c}\text { Peak } \\
\text { Load, } \\
\text { Tons }\end{array}$ & $\begin{array}{c}\text { Annual } \\
\text { Load, } \\
\text { Ton- } \\
\text { hours }\end{array}$ & $\begin{array}{c}\text { Annual } \\
\text { Capacity } \\
\text { Factor }\end{array}$ & $\begin{array}{c}\text { Existing } \\
\text { Air } \\
\text { Cooled } \\
\text { kW/ton }\end{array}$ & $\begin{array}{c}\text { Existing } \\
\text { Annual } \\
\text { Electricity } \\
\text { kWh }\end{array}$ & $\begin{array}{c}\text { Existing } \\
\text { Peak } \\
\text { Electricity } \\
\text { kW }\end{array}$ & $\begin{array}{c}\text { Existing } \\
\text { Annual } \\
\text { Electricity } \\
\text { Cost }\end{array}$ \\
\hline 2130 & 18.1 & 73,335 & 0.46 & 1.204 & 88,296 & 21.8 & \\
\hline 2131 & 10.3 & 40,647 & 0.45 & 1.204 & 48,939 & 12.4 & \\
\hline 2133 & 32.7 & 100,092 & 0.35 & 1.204 & 120,511 & 39.3 & \\
\hline Totals & $\mathbf{6 1 . 0}$ & $\mathbf{2 1 4 , 0 7 4}$ & $\mathbf{0 . 4 0}$ & $\mathbf{1 . 2 0 4}$ & $\mathbf{2 5 7 , 7 4 5}$ & $\mathbf{7 3 . 5}$ & $\mathbf{\$ 5 0 , 0 8 7}$ \\
\hline
\end{tabular}

The two new 40-ton water-cooled chillers were estimated to cost \$88,200 and the cooling tower, condenser pump, and piping an additional \$26,100. These figures include all direct construction costs, but do not include any allowance for design or SIOH costs. Based on the direct cost, the payback period is 8 years. With an additional $16 \%$ for design and $\mathrm{SIOH}$, the payback period rises to 9 years.

\section{Pearl Harbor}

Building 631, the Navy Exchange (NEX) and Commissary, is currently served by a collection of packaged rooftop direct expansion (DX) AC units. The proposed retrofit would replace the existing DX units with a new chilled water coil (in the existing airhandler units [AHU]), two water-cooled chillers, a cooling tower, condenser water pumps and piping, and chilled water pumps and piping. The new chiller plant was assumed to be sited on the ground on the southeast side of the building, next to the Commissary.

The peak and annual building cooling loads were estimated with the FEDS model and the performance of the existing packaged DX units was estimated from the vintage of the existing equipment. From this information, the annual $\mathrm{kWh}$ and peak $\mathrm{kW}$ electrical loads were calculated and then combined with Pearl's electricity rates to calculate the current annual electricity costs. The existing system performance and electricity cost figures are presented in Table E2. 
Table E 2. Pearl Building 631 Existing System Performance and Electricity Cost

\begin{tabular}{|c|c|c|c|c|c|c|c|}
\hline & $\begin{array}{c}\text { Peak } \\
\text { Load, } \\
\text { Tons }\end{array}$ & $\begin{array}{c}\text { Annual } \\
\text { Load, } \\
\text { Ton- } \\
\text { hours }\end{array}$ & $\begin{array}{c}\text { Annual } \\
\text { Capacity } \\
\text { Factor }\end{array}$ & $\begin{array}{c}\text { Existing } \\
\text { Air } \\
\text { Cooled } \\
\text { kW/ton }\end{array}$ & $\begin{array}{c}\text { Existing } \\
\text { Annual } \\
\text { Electricity } \\
\text { kWh }\end{array}$ & $\begin{array}{c}\text { Existing } \\
\text { Peak } \\
\text { Electricity } \\
\text { kW }\end{array}$ & $\begin{array}{c}\text { Existing } \\
\text { Annual } \\
\text { Electricity } \\
\text { Cost }\end{array}$ \\
\hline $\begin{array}{c}\text { Navy } \\
\text { Exchange } \\
\text { (NEX) }\end{array}$ & 275.1 & 918,580 & 0.38 & 1.2859 & $1,181,180$ & 354 & \\
\hline $\begin{array}{c}\text { NEX Food } \\
\text { Court }\end{array}$ & 125.0 & 342,737 & 0.31 & 1.2859 & 440,717 & 161 & \\
\hline Commissary & 194.4 & 716,633 & 0.42 & 1.2859 & 921,501 & 250 & \\
\hline Totals & 594.5 & $1,977,950$ & 0.38 & 1.2859 & $2,543,446$ & 764 & $\$ 493,300$ \\
\hline
\end{tabular}

In this size range, the water-cooled chillers were assumed to use a centrifugal compressor rated at $0.51 \mathrm{~kW} /$ ton. In addition, the chilled water pumps, condenser water pumps, and cooling tower fan would be expected to consume $0.18 \mathrm{~kW} /$ ton for a total cooling plant performance of $0.69 \mathrm{~kW} / \mathrm{ton}$. The annual electricity bill for the water-cooled system was calculated to be $\$ 264,700$ based on these assumptions, resulting in an annual savings of $\$ 228,600$ and a peak electric load reduction of $354 \mathrm{~kW}$.

A new 600-ton water-cooled chiller plant (chillers, cooling tower, pumps, plant piping, electrical, controls, and structure) was estimated to cost $\$ 656,000$. Chilled water piping running to and from the ground to every rooftop air-handling unit was estimated to cost $\$ 225,000$. The cost of the new chilled water coils was estimated to be $\$ 180,000$. These figures include all direct construction costs, but do not include any allowance for design or SIOH costs. Based on the direct cost, the payback period is 4 years. With an additional $16 \%$ for design and $\mathrm{SIOH}$, the payback period rises to 4.5 years.

\section{Camp Smith}

Buildings 401, 402, 403, and 404 are currently served by window DX AC units. The proposed retrofit would replace the window units with room fan coil units, external chilled water supply and return piping and a central water-cooled chiller plant serving all four buildings. The same plant would also serve Building 20, which already has aircooled chillers, hence chilled water piping within the building, but will need chilled water supply and return piping from the new central plant to Building 20. The new chiller plant was assumed to be sited on the West side of Bailey Road, opposite Building 401.

The peak and annual building cooling loads were estimated with the FEDS model and the performance of the existing window DX AC units and air-cooled chillers were estimated from manufacturer's specifications for the two types of units. From this information, the annual kWh and peak kW electrical loads were calculated and then combined with Smith's electricity rates to calculate the current annual electricity costs. The existing system performance and electricity cost figures are presented in Table E3. 
Table E 3. Smith Buildings 401-404, and Building 20 Existing System Performance and Electricity Cost

\begin{tabular}{|c|c|c|c|c|c|c|c|}
\hline & $\begin{array}{c}\text { Peak } \\
\text { Load, } \\
\text { Tons }\end{array}$ & $\begin{array}{c}\text { Annual } \\
\text { Load, } \\
\text { Ton- } \\
\text { hours }\end{array}$ & $\begin{array}{c}\text { Annual } \\
\text { Capacity } \\
\text { Factor }\end{array}$ & $\begin{array}{c}\text { Existing } \\
\text { Air } \\
\text { Cooled } \\
\text { kW/ton }\end{array}$ & $\begin{array}{c}\text { Existing } \\
\text { Annual } \\
\text { Electricity } \\
\text { kWh }\end{array}$ & $\begin{array}{c}\text { Existing } \\
\text { Peak } \\
\text { Electricity } \\
\text { kW }\end{array}$ & $\begin{array}{c}\text { Existing } \\
\text { Annual } \\
\text { Electricity } \\
\text { Cost }\end{array}$ \\
\hline 401 & 65.7 & 147,804 & 0.26 & 1.16 & 171,515 & 76.2 & \\
\hline 402 & 65.7 & 147,804 & 0.26 & 1.16 & 171,515 & 76.2 & \\
\hline 403 & 65.7 & 147,804 & 0.26 & 1.16 & 171,515 & 76.2 & \\
\hline 404 & 65.7 & 147,804 & 0.26 & 1.16 & 171,515 & 76.2 & \\
\hline 20 & & & & & & & \\
\hline Totals & 142.8 & 419,327 & 0.34 & 1.44 & 603,203 & 205.3 & \\
\hline
\end{tabular}

In this size range, the water-cooled chillers were assumed to use a centrifugal compressor rated at $0.57 \mathrm{~kW} /$ ton. In addition, the chilled water pumps, condenser water pumps, and cooling tower fan would be expected to consume $0.18 \mathrm{~kW} /$ ton for a total cooling plant performance of $0.75 \mathrm{~kW} /$ ton. The annual electricity bill for the water-cooled system was calculated to be $\$ 164,200$ based on these assumptions, resulting in an annual savings of $\$ 111,300$ and a peak electric load reduction of $206 \mathrm{~kW}$.

A new 400-ton water-cooled chiller plant (chillers, cooling tower, pumps, plant piping, electrical, controls, and structure) was estimated to cost $\$ 520,000$. Chilled water piping that would be mounted on the exterior of Buildings 401-404 was estimated to cost $\$ 85,000$. Chilled water piping running to and from the new central plant to Buildings 401-404 and 20 was estimated to cost $\$ 189,000$. The cost of the new chilled water coils for Buildings 401-404 was estimated to be $\$ 75,000$. These figures include all direct construction costs, but do not include any allowance for design or SIOH costs. Based on the direct cost, the payback period is 8 years. With an additional 16\% for design and $\mathrm{SIOH}$, the payback period rises to 9 years.

Before implementing this project, Camp Smith should consider other possible means of serving these five buildings with water-cooled chillers. An expansion of the chilled water plant serving Building 700 may offer some economies over the new plant proposed here, but the chilled water distribution piping would be longer. Integration with a new chilled water plant serving the eventual replacement of the Old Hospital Complex would probably be ideal if the Complex is going to be replaced relatively soon. 


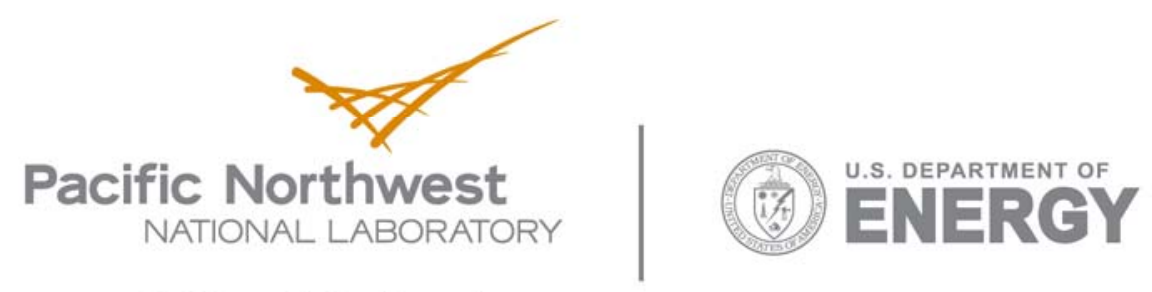

902 Battelle Boulevard

P.O. Box 999

Richland, WA 99352

1-888-375-PNNL (7665)

www.pnl.gov 